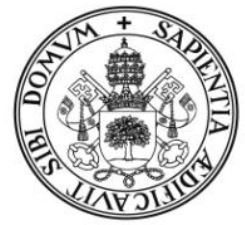

Universidad deValladolid

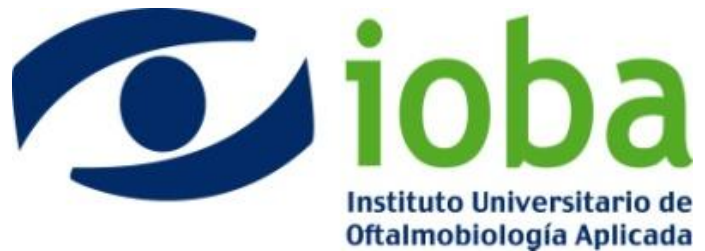

Faculty of Medicine, Eye Institute (IOBA), University of Valladolid (UVa)

DOCTORAL THESIS:

\title{
The potential of human adipose tissue derived mesenchymal stem cells in cell therapy for retinal degenerative diseases
}

Potencial terapéutico de las células madre mesenquimales humanas derivadas de tejido adiposo en enfermedades degenerativas retinianas

\author{
Presented by \\ Amar Kumar Singh \\ For obtaining $\mathrm{PhD}$ degree awarded by \\ University of Valladolid (UVa), \\ Valladolid, Spain
}

Guided by

Dr. Girish Kumar Srivastava 


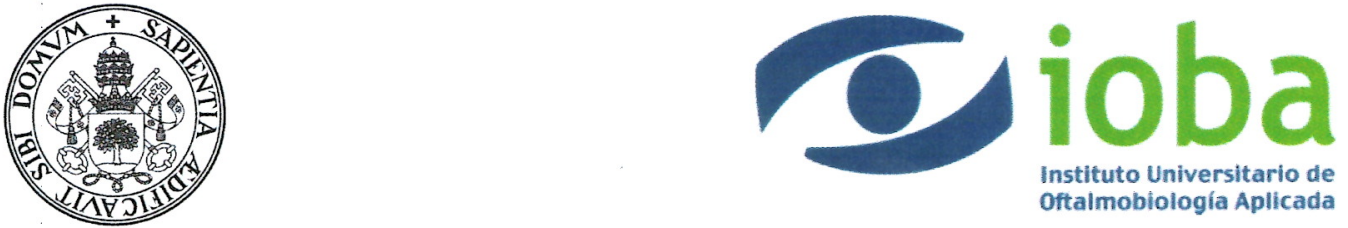

Universidad deValladolid

\section{CERTIFICATE BY THE DIRECTOR OF PhD THESIS}

Dr. GIRISH KUMAR SRIVASTAVA, principal investigator of University Institute of Applied Ophthalmobiology (IOBA), University of Valladolid (UVa), as the Director of $\mathrm{PhD}$ Thesis of International level with the title "The potential of human adipose tissue derived mesenchymal stem cells in cell therapy for retinall degenerative diseases" presented by Mr. AMAR KUMAR SINGH, student under the $\mathrm{PhD}$ programme in Vision Science of IOBA, UVa, authorizes for the presentation of the thesis after considering it suitable for its defense.

Valladolid, 18th December, 2013

Director of the Thesis,

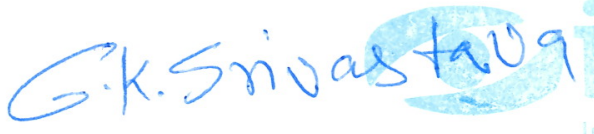

Signed: Dr. Girish Kumar Srivastava

THE CHAIRMAN OF THE DOCTORAL COMMITTEE OF THE UNIVERSITY OF VALLADOLIID 



\section{Universidad deValladolid}

Impreso 2T

\section{AUTORIZACIÓN DEL DIRECTOR DE TESIS}

(Art. 2.1. c de la Normativa para la presentación y defensa de la Tesis Doctoral en la UVa)

D. GIRISH KUMAR SRIVASTAVA, con D.N.I. $\mathrm{n}^{\circ}$ X5428926Y investigador principal del Instituto Universitario de Oftalmobiología Aplicada (IOBA) de la Universidad de Valladolid como Director de la Tesis Doctoral titulada "The potential of human adipose tissue derived mesenchymal stem cells in cell therapy for retinal degenerative diseases" presentada por D. AMAR KUMAR SINGH, alumno del programa de Doctorado en Ciencias de la Visión impartido por el IOBA, autoriza la presentación de la misma, considerando que es APTA para su defensa

Valladolid, 18 de diciembre de 2013

El Director de la Tesis,

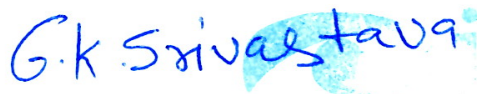

Fdo.: Dr. Girish Kumar Srivastava

SR. PRESIDENTE DE LA COMISIÓN DE DOCTORADO 



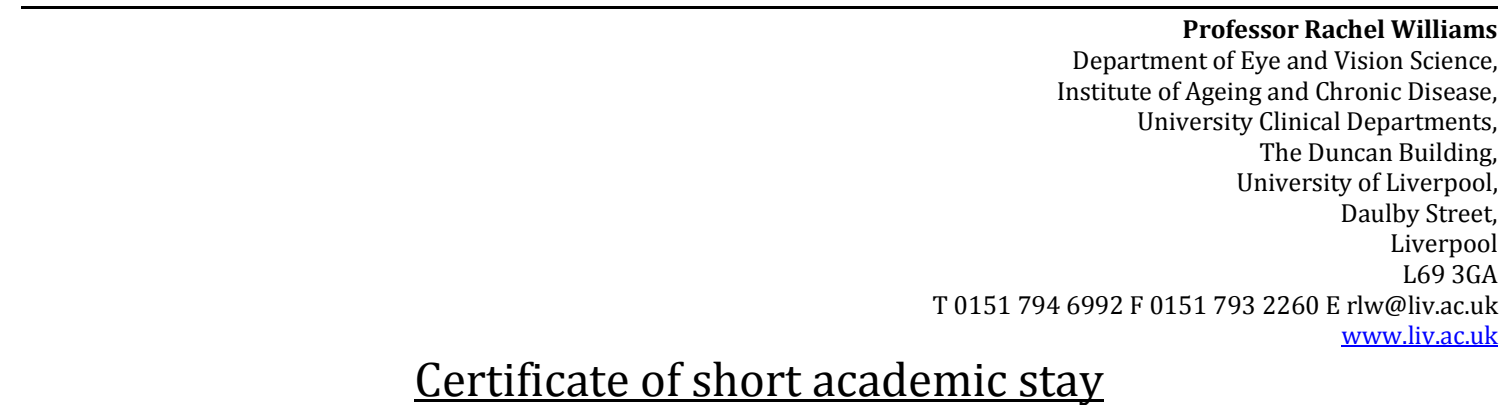

\section{Applicant}

Name: Amar Kumar Singh

Passport Number/NIE: G0074079/Y0728689V

Home Institution: Institute of Applied Ophthalmobiology (IOBA), University of Valladolid (UVa), Valladolid

\section{Host Institution}

Name: Department of Eye and Vision Science, Institute of Ageing and Chronic Diseases, University of Liverpool

Address: University Clinical Departments, The Duncan Building, University of Liverpool, Daulby Street, Liverpool L69 3GA UK

Responsible person in the host institution: Professor Rachel Williams

Post:Head of the Department

This is to certify that Amar Kumar Singh, a pre-doctoral student, from the IOBA, University of Valladolid, worked in our laboratories at the University of Liverpool from $3^{\text {rd }}$ October 2012 to 11 the January 2013 (101 days).

Within the framework of the collaboration with the IOBA, UVa, Mr. Amar Kumar Singh (responsible supervisor: Dr. Girish Kumar Srivastava) visited our laboratory of Eye and Vision Science for the above mentioned period. The project aims to develop a new treatment for age-related macular degeneration by harvesting the paracrine and differentiation potential of human adipose tissue derived mesenchymal stem cells (hADMSCs). He worked over the differentiation of hAD-MSCs to RPE phenotypes by stimulating the hAD-MSC with BMP-4 in 50\% foetal RPE condition medium with photoreceptor outer segments (POS). During his stay he produced some preliminary interesting results which will be the part of his thesis and could be further extended in the future. He also delivered a talk over his work here in different departmental meetings. The short stay helped him to work under international environment and to develop the skills to deliver his results in public. He was a hard working and enthusiastic person with focused objectives and was very dedicated to his work. I wish him all the best for his doctoral thesis and future career.

Yours sincerely,

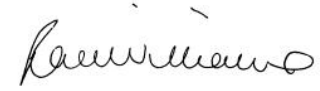

Professor Rachel Williams

Cc Professor Simon Harding, Head of Department Eye and Vision Science 



\section{Funding}

I would like to acknowledge to the following funded projects without which the works could not be performed during my doctoral thesis.

1. Evaluación de la seguridad y eficacia de células mesenquimales derivadas de tejido adiposo trasplantado en el espacio subretiniano para el tratamiento de la DMAE. 2012-2014, Spain.

2. Terapia Celular para la forma seca de la Degeneración Macular Asociada a la Edad (DMAE) (Cell therapy for dry forms of Age related Macular Degeneration), Institute of Health Carlos III, Spanish Ministry of Science and Innovation, P509/00938, 20102012, Spain.

3. Terapia celular con células madre para la degeneración macular asociada a la edad (DMAE) (Cell therapy with stem cells for AMD), Programa General de Apoyo a Proyectos de Investigación de Junta de Castilla y León (JCyL), 2010-2011, Spain.

4. Regenerative medicine and Cell Therapy Network Centre of Castilla and León, (Centro en Red de medicina regenerativa y Terapia celular de Castilla y León), Spanish Instituto de Health Carlos III y Castilla y León Council, the national R\&D+I Plan 200811. The ISCIII-Subdirección General de Evaluación y Fomento de la Investigación (PS09/00938) (MICNN), with co-financing by FEDER (Federación Española de Enfermedades Raras) and JCYL BIO/39/VA26/10, Junta de Castilla and León, Spain

5. Foundation for the prevention of blindness, Department of Eye and Vision Science, Institute of Ageing and Chronic Diseases, University of Liverpool, UK.

I would like to give my honest thanks to the following agencies for awarding me the scholarship to complete my International level doctoral thesis in the University of Valladolid, Spain.

1. Scholarship funded by MAEC-AECID (Ministerio de Asuntos Exteriores y de Cooperación - Agencia Española de Cooperación Internaciónal para el Desarrollo), Spanish Ministry of External Affairs and Cooperation-Spanish Agency for International Cooperation and Development for three years from Oct, 2009 to Sept, 2012.

2. Scholarship funded by FGUVA (Fundación General de la Universidad de Valladolid), UVa, Valladolid October 2012-December 2013.

3. Partial scholarship for short stay from $3^{\text {rd }}$ October 2011to $11^{\text {th }}$ January 2013 in Department of Eye and Vision Science, UoL, partly funded by the UVa under the partial fellowship support programme for attending a course, conferences and short stay (3-6 months) in the Foreign University outside Spain during doctoral thesis (Ayuda para la asistencia a cursos, congresos y jornadas relevantes para el desarrollo de tesis doctorales, de la Universidad de Valladolid, Convocatoria 2012). 



\section{Patent}

The part of the work of this thesis has given one patent which has been applied in the Spain, OEPM (Officina Española de Patentes y Marcas):

Spanish patent application number: P201331743. Composición farmacéutica para el tratamiento y/o prevención de enfermedades retinianas degenerativas (Pharmaceutical composition for the treatment and/or prevention of retinal degenerative diseases) Applicant: University of Valladolid. Inventors: Singh AK, Srivastava GK, Fernandez-Bueno I, Gayoso MJ, Pastor JC.

\section{Publications}

The some parts of work have been published as following articles in different journals.

Singh AK, Srivastava GK, García-Gutiérrez MT, Pastor JC. Adipose derived mesenchymal stem cells partially rescue mitomycin $C$ treated ARPE19 cells from death in co-culture condition. Histol Histopathol. (IF: 2.281) 2013 May 30. [Epub ahead of print]

Singh AK, Srivastava GK, Martín L, Alonso M, Pastor JC. Bioactive substrates for human retinal pigment epithelial cell growth from elastin-like recombinamers. J Biomed Mater Res A. 2013 Mar 29. doi: 10.1002/jbm.a.34726. [Epub ahead of print]

Srivastava GK, Reinoso R, Singh AK, Fernandez-Bueno I, Martino M, GarciaGutierrez MT, Pastor JC, Corell A. Flow cytometry assessment of the purity of human retinal pigment epithelial primary cell cultures. J Immunol Methods. (IF: 2.447) 2013 Mar 29; 389(1-2):61-8. doi: 10.1016/j.jim.2013.01.003. Epub 2013 Jan 11.

Srivastava GK, Reinoso R, Singh AK, Fernandez-Bueno I, Hileeto D, Martino M, Garcia-Gutierrez MT, Merino JM, Alonso NF, Corell A, Pastor JC. Trypan Blue staining method for quenching the autofluorescence of RPE cells for 
improving protein expression analysis. Exp Eye Res. 2011 (IF: 2.817) Dec; 93(6):956-62. doi: 10.1016/j.exer.2011.07.002. Epub 2011 Jul 18.

Srivastava GK, Martín L, Singh AK, Fernandez-Bueno I, Gayoso MJ, GarciaGutierrez MT, Girotti A, Alonso M, Rodríguez-Cabello JC, Pastor JC. Elastin-like recombinamers as substrates for retinal pigment epithelial cell growth. J Biomed Mater Res A. (IF: 3.044) 2011 Jun 1;97(3):243-50. doi: 10.1002/jbm.a.33050. Epub 2011 Mar 25.

\section{Scientific communications}

The some parts of works have been presented in the following various conferences and seminars as a poster or oral presentation.

1. G. K. Srivastava, Amar K. Singh, D. Rodríguez-Crespo, I. Fernández-Bueno, M.T. Garcia-Gutierrez, M.J. Gayoso, J.C. Pastor. Mesenchymal stem cells isolated from adipose tissues partially rescue human neuroretina and RPE cells degenerating in organotypic and co-culture models. World congress of Regenerative medicine. Leipzig, Germany, Oct 23-25, 2013. (poster)

2. Fernandez-Bueno I, Srivastava GK, Rodríguez-Crespo D, Singh AK, GarcíaGutierrez MT, Pastor JC. Improved method for obtaining retinal stem cells from ciliary body. European Society of Veterinary Ophthalmology (ESVO), Bucharest, Rumania, Meeting 2013. (Oral presentation)

3. I. Fernández-Bueno, G.K. Srivastava, D. Rodríguez-Crespo, A. K. Singh, C. Casado-Coterillo, M.T. Garcia-Gutierrez, J. Coronas, J.C. Pastor. Porcine retinal stem cells (prscs) growth feasibilitiy on chitosan membranes. ECVO meeting, Poster, 2013 Barcelona, Spain.

4. Lecture delivered on report of project of retina laboratory on advance cell therapy in the CDTI (Spain)-DBT (India) 2013 project call meeting. 2013, Madrid, Spain.

5. G.K. Srivastava, D. Rodríguez-Crespo, C. Casado-Coterillo, A. K. Singh, I. Fernández-Buenoํㅗㄹ. M.T. Garcia-Gutierrez, J. Coronas, J.C. Pastor. Evaluation of retinal stem cells adhesion, growth, viability and proteins expressions on chitosan surface. Poster, Julio, ISER2012 congress, Berlin, Germany. 
6. José Carlos Pastor, Amar K. Singh, G.K. Srivastava, David Rodríguez-Cres, Maria T. Garcia-Gutierrez., Adipose tissue derived Mesenchymal stem cells partially rescue the Mitomycin C treated ARPE19 from cell death in coculture. Invest Ophthalmol Vis Sci ARVO 2012;53: E-Abstract 5843. Poster, USA.

7. DMAE seca, nuevos tratamientos. VIII Congreso Internacional Sociedad Argentina de Retina y Vitreo. Lecture, 2012, Salta, Argentina.

8. Desprendimiento de Retina, lo peor de mi y como lo resolvi. XXXI Congreso de la Sociedad Mexicana de Oftalmología. Lecture, 2012, Guadaljara, Mexico.

9. G.K. Srivastava, Laura Martin, Amar K. Singh, José C. Rodriguez-Cabello, Jose C. Pastor., Evaluation of human retinal pigment epithelial cells growth on elastin-like recombinamers substrates., Acta Oftalmologica DOI: 10.1111/j.1755-3768.2011.204.x Poster, 5-8 Oct, 2011, EVER congress, Crete, Greece.

10. G.K. Srivastava, R Reinoso, Amar K. Singh, A Corell, Ivan Fernández- Bueno, D Hileeto, Jose C. Pastor.,Trypan blue staining method for quenching the autofluorescence of RPE cells for improving protein expression analysis., Acta Oftalmologica DOI: 10.1111/j.1755-3768.2011.205.x Poster, 5-8 Oct, 2011, EVER congress, Crete, Greece.

11. G.K. Srivastava, Laura Martin, Amar K. Singh, Ivan Fernández- Bueno, Maite T. Garcia-Gutierrez, José C. Rodriguez-Cabello, Jose C. Pastor., Retinal pigment epithelial (RPE) cell growth on Elastin-like recombinamers substrates., Poster, 11-16 July, 2010, World Polymer Congress, Glasgow, UK.

12. Amar Kumar Singh, Laura Martin, G.K. Srivastava, Jesús Gómez Escudero, Cristhian Urzua, Maite Garcia, Jose Carlos. Elastin-like polymers as substrates for retinal pigment epithelial (RPE) cell growth. Poster, IOBA Day, 6 Nov, 2009, IOBA, UVa, Valladolid, Spain 



\section{List of Abbreviations}

AAV Adeno-Associated Virus

AB Alamar Blue

ABCA4 ATP-Binding Cassette

subfamily A member 4

ADNP Activity Dependent

Neuroprotective Protein

ADP Adenosine Diphosphate

AFU Arbitrary Fluorescence

intensity Unit

AGEs polycyclic Advanced Glycation

End products

atRDH All-trans Retinol

Dehydrogenase

BAT Brown Adipose Tissue-good fat

BCA Bicinchoninic Acid assay

B cell origin from bursa of fabricious

in bird but now Bone marrow

BDNF Brain Derived Neurotrophic

Factor

AMD Age related Macular

Degeneration

BEST1 Bestrophin 1

bFGF basic Fibroblast Growth Factor

Ang-1 Angiopoietin-1

BM Bruch's Membrane

AP Apical Processes

BM- $\quad$ Bone Marrow derived MSCs

APC Antigen Presenting Cell

MSCS

aRCM ARPE19 RCM

BMP-4 Bone Morphogenetic Factors-4

ARMS2 Age Related Maculopathy

Susceptibility 2

ARPE1 an RPE cell line derived from a

BMPRI/BMP type I/II Receptors

II- $\boldsymbol{A} / \boldsymbol{B}$

919 yr old boy died in an

accident

ATCC American Type Culture

Collection

BRAF a proto-oncogene, serine/threonine kinase B-Raf (rapidly accelerated fibrosarcoma)

ATP Adenosine Triphosphate

ATP1B $\mathrm{Na}^{+} / \mathrm{K}^{+}$ATPase

C2/C3 Complement Component 2/3

1 convertase

ATRA All trans-retinoic acid 
Calcein Calcein Acetoxymethyl Ester AM

CC Choroidocapillaris

CCL2 Chemokine (C-C motif) Ligand 2

CD90 Cluster of differentiation 90

CFB Complement Factor B

CFH Complement Factor $\mathrm{H}$

CFU-F Colonu Forming Unit

Fibroblast

CK8 Cytokeratin 8

CNTF Ciliary Neurotrophic growth Factor

CNV Choroidal Neo-Vascularization

CRALBP Cellular Retinaldehyde Binding Protein

CRBP Cellular Retinol Binding Protein

CXCL-2 Chemokine (C-X-C motif) Ligand 2

11- 11-cis Retinol Dehydrogenase cRDH

DAPI 4', 6-diamindino-2phenylindole dihydrochloride

DCs Dendritic Cells

DHA Docosahexanoic Acid

DMEM Dulbecco's modified Eagle's medium

DMSO Dimethyl Sulfoxide

DNA Deoxyribonucleic acid
EC European Commission News

News

ECL Enhanced Chemiluminescence

ECM Extra Cellular Matrix

EDTA Tetrasodium

Ethylenediaminetetraacetate

EdU 5-Ethynyl-2'-DeoxuUridine

EGF Epidermal Growth Factor

ELISA Enzyme-Linked

Immunosorbant Assay

ELOVL- Elongation of Very Long chain

4 fatty acid-4

ELR- Elastin Like Recombinamer

RGD with RGD bioactive domain

EMA European Medicine Agency

EPA Eicosapentanoic Acid

EPO Erythropoietin

ERCC6 Excision-Repair Cross

Complementing,

Complementation Group 6

EthD-III Ethidium homodimer III

FACS Fluorescence Activated Cell Sorting

FAS-L CD95 apoptosis receptor ligand (Focal adhesions/contacts-Fas)

FBLN5 Fibulin 5 (an ECM protein)

FC Flow Cytometry

FCS/FB Fetal Calf/Bovine serum

$\mathbf{S}$

FDA Food and Drug Administration, USA

FGF Fibroblast Growth Factor 


\begin{tabular}{|c|c|c|c|}
\hline FGF-R & $\begin{array}{l}\text { Fibroblast Growth Factor } \\
\text { Receptor }\end{array}$ & hRCM & $\begin{array}{l}\text { human RPE Conditioned } \\
\text { Medium }\end{array}$ \\
\hline $\begin{array}{l}\text { FITC } \\
\text { fRCM }\end{array}$ & $\begin{array}{l}\text { Fluorescein isothiocyanate } \\
\text { foetal RPE Conditioned }\end{array}$ & hRPE & $\begin{array}{l}\text { Human Retinal Pigment } \\
\text { epithelium }\end{array}$ \\
\hline FSC & $\begin{array}{l}\text { Medium } \\
\text { Forward Scatter Channel }\end{array}$ & HRP-Ab & $\begin{array}{l}\text { Horse Radish Peroxidase } \\
\text { conjugated antibody }\end{array}$ \\
\hline $\begin{array}{l}\text { G/S } \\
\text { phase }\end{array}$ & $\begin{array}{l}\text { Growth/Synthesis Phase of cell } \\
\text { cycle }\end{array}$ & HTRA1 & $\begin{array}{l}\text { HtrA (high temperature } \\
\text { requirement factor A) serine }\end{array}$ \\
\hline GA & Geographic Atrophy & & peptidase 1 \\
\hline Gas6 & $\begin{array}{l}\text { Growth Arrest Specific protein } \\
6\end{array}$ & $\begin{array}{l}\text { HYADD } \\
3 / 4\end{array}$ & $\begin{array}{l}\text { Hyluronan based hydrogel } \\
\text { with different degree of }\end{array}$ \\
\hline GCL & Ganglion Cell Layer & & amidation \\
\hline G-CSF & $\begin{array}{l}\text { Granulocyte-Colony } \\
\text { Stimulating Factor }\end{array}$ & $\begin{array}{l}\text { HYAFF } \\
\text { IDO }\end{array}$ & $\begin{array}{l}\text { Ester of Hyaluronic Acid } \\
\text { Indoleamine-2,3-Dioxygenase }\end{array}$ \\
\hline GDNF & $\begin{array}{l}\text { Glial cell line-Derived } \\
\text { Neurotrophic Factor }\end{array}$ & IFATS & $\begin{array}{l}\text { International Federation for } \\
\text { Adipose Therapeutics and }\end{array}$ \\
\hline GFAP & Glial Fibrillary Acidic Protein & & Science \\
\hline GVHD & Graft Versus Host Disease & IFN- $\gamma$ & Interferon- $\gamma$ \\
\hline hAD- & Human Adipose tissue derived & IGF-1 & Insulin-like Growth Factor-1 \\
\hline MSCs & Mesenchymal Stem Cells & IGF-R & Insulin like Growth Factor \\
\hline HBSS & Hank's Balanced Salt Solution & & Receptor \\
\hline HB-EGF & $\begin{array}{l}\text { Heparin Binding-Epidermal } \\
\text { Growth Factor }\end{array}$ & $\begin{array}{l}\text { IgG1 } \\
\text { IL }\end{array}$ & $\begin{array}{l}\text { Immunoglobulin G1 } \\
\text { Interleukin }\end{array}$ \\
\hline $\begin{array}{l}\text { HEK } \\
\text { cells }\end{array}$ & $\begin{array}{l}\text { Human Embryonic Kidney } \\
\text { cells }\end{array}$ & $\begin{array}{l}\text { IL-R } \\
\text { ILM/OL }\end{array}$ & $\begin{array}{l}\text { Interleukin Receptor } \\
\text { Inner/Outer Limiting }\end{array}$ \\
\hline hESCS & human Embryonic Stem Cells & $\mathbf{M}$ & Membrane \\
\hline HFF1 & Human Foreskin Fibroblast1 & IPE & Iris Pigment Epithelium \\
\hline HGF & Hepatocyte Growth Factor & INL/ON & Inner/Outer Nuclear Layer \\
\hline HLA- & Human Luecocyte Antigen- & $\mathbf{L}$ & \\
\hline DR/G & Locus DR/G & IPL/OP & Inner/Outer Plexiform Layer \\
\hline hNR & Human Neuroretina & $\mathbf{L}$ & \\
\hline HO-1 & Heme Oxygenase-1 & iPSCs & $\begin{array}{l}\text { Induced Pluriopotent Stem } \\
\text { cells }\end{array}$ \\
\hline
\end{tabular}




\begin{tabular}{|c|c|c|c|}
\hline \multirow[t]{2}{*}{ IRBP } & \multirow{2}{*}{$\begin{array}{l}\text { Interstitial Retinal Binding } \\
\text { Protein }\end{array}$} & MERTK & $\begin{array}{l}\text { C-Mer Proto-oncogene } \\
\text { tyrosine-protein kinase }\end{array}$ \\
\hline & & \multirow[t]{2}{*}{ MFI } & Median/Mean Fluorescence \\
\hline IS/OS & Inner/Outer Segment & & Intensity \\
\hline \multirow[t]{2}{*}{ ISCT } & International Society for & \multirow[t]{2}{*}{ M-CSF } & Macrophage-Colony \\
\hline & Cellular Therapy & & Stimulating Factor \\
\hline \multirow{4}{*}{$\begin{array}{l}\text { ITGAM } \\
\text { KGF } \\
\text { LASAF }\end{array}$} & Integrin Alpha M & \multirow{4}{*}{$\begin{array}{l}\text { MHC } \\
\text { mHCN1 }\end{array}$} & Major Histocompatibility \\
\hline & Keratinocyte Growth Factor & & Mammalian \\
\hline & Leica Application Suite & & Hyperpolarization-activated \\
\hline & Advanced Fluorescence & & and Cyclic Nucleotide-gated \\
\hline \multirow[t]{2}{*}{ LEDGF } & Lens Epithelium-Derived & & channel 1 \\
\hline & Growth Factor & \multirow[t]{2}{*}{ MIP } & Macrophage Inflammatory \\
\hline \multirow[t]{2}{*}{ LDGF } & Leukocyte-derived growth & & Protein-1 \\
\hline & factor & \multirow[t]{2}{*}{ MITF } & Microphthalmia-associated \\
\hline LIF & Leukemia Inhibitory Factor & & Transcription Factor \\
\hline LIPC & hepatic Lipase C & MMC & Mitomycin C \\
\hline \multirow[t]{6}{*}{ LL37 } & A Cathelicidin, hCAP18 & MMP & Matrix Metallo-Proteinase \\
\hline & (human cationic antibacterial & \multirow[t]{2}{*}{ MPOD } & Macular Pigment Ocular \\
\hline & protein of 18kDa) of 37 amino & & Density \\
\hline & acid residues with & MP & Macular pigment \\
\hline & antimicrobial effect at its C- & mRNA & messenger Ribonucleic Acid \\
\hline & terminal & MSCs & Mesenchymal Stem/Stromal \\
\hline LPAs & Lipoaspirates & & cells \\
\hline LPLD & Lipoprotein Lipase Deficiency & $\mu \mathrm{g} / \mathrm{mL}$ & micro gram per mili litre \\
\hline \multirow[t]{2}{*}{ LRAT } & \multirow[t]{2}{*}{ Lecithin Retinol Acyltransferase } & $\mathbf{m M}$ & mili-Mole \\
\hline & & $\boldsymbol{\mu} \mathbf{M}$ & micro-Mole \\
\hline \multirow[t]{2}{*}{ LRP6 } & Low density lipoprotein & \multirow[t]{2}{*}{ NAD $^{+}$} & $\beta$-Nicotinamide Adenine \\
\hline & Receptor-related Protein 6 & & Dinucleotide \\
\hline \multirow[t]{3}{*}{ MCP-1 } & Monocyte & \multirow[t]{2}{*}{ NADP $^{+}$} & Nicotinamide Adenine \\
\hline & Chemotactic/Chemoattractant & & Dinucleotide Phosphate \\
\hline & Protein 1 & NCT & National Clinical Trial \\
\hline \multirow[t]{2}{*}{ MEM } & Minimum Essential Medium by & NFL & Nerve Fibre Layer \\
\hline & Harry Eagle & NGF & Nerve Growth Factor \\
\hline
\end{tabular}




\begin{tabular}{|c|c|c|c|}
\hline NIC & Nicotinamide & PET & Polyethylene Terephthalate \\
\hline NK cells & Natural Killer cells & PGE2 & Prostaglandin E2 \\
\hline NT-3 & Neurotrophin-3 & PI & Propidium Iodide \\
\hline $\mathbf{n M}$ & nano-Mole & PIGF & Placental Growth Factor \\
\hline NO & Nitric Oxide & PLA & Processed Lipoaspirate \\
\hline OS & Outer Segments & $\begin{array}{l}\text { PLGA } \\
\text { PN-1 }\end{array}$ & $\begin{array}{l}\text { Poly(lactic-co-glycolic) acid } \\
\text { Protease Nexin-1 }\end{array}$ \\
\hline OCT & Optical Coherence & PRs & Photoreceptors \\
\hline & $\begin{array}{l}\text { Tomography/Optimum cutting } \\
\text { temperature compound }\end{array}$ & $\begin{array}{l}\text { POS } \\
\text { PUFA } \\
\text { RANTE }\end{array}$ & $\begin{array}{l}\text { Photoreceptor Outer Segment } \\
\text { Polyunsaturated Fatty Acid } \\
\text { Regulated on Activation, }\end{array}$ \\
\hline Oct4 & $\begin{array}{l}\text { Octamer-binding transcription } \\
\text { factor } 4\end{array}$ & $\mathbf{S}$ & $\begin{array}{l}\text { Normal T Cell Expressed and } \\
\text { Secreted }\end{array}$ \\
\hline & & RARs & Retinoic Acid Receptors \\
\hline PACAP & Pitutary Adenylate Cyclase- & RAX2 & $\begin{array}{l}\text { Retina and Anterior neural fold } \\
\text { homeobox } 2\end{array}$ \\
\hline & Activating Polypeptide & $R B P / T T$ & Retinol Binding \\
\hline PAIs & Plasminogen Activator & $\boldsymbol{R}$ & Protein/Transthyretin complex \\
\hline & Inhibitors & $\operatorname{Rex1}$ & $\begin{array}{l}\text { Reduced expression 1-a marker } \\
\text { of pluripotency Syn:- Zfp42 }\end{array}$ \\
\hline $\begin{array}{l}\text { PARP } \\
\text { Pax6 }\end{array}$ & $\begin{array}{l}\text { Poly (ADP-ribose) Polymerase } \\
\text { Paired box protein } 6\end{array}$ & RGD & Arginine-Glycine-Aspartic acid \\
\hline $\begin{array}{l}\text { PBS } \\
\text { PC }\end{array}$ & $\begin{array}{l}\text { Phosphate Buffered Saline } \\
\text { Polycarbonate }\end{array}$ & $R G R$ & $\begin{array}{l}\text { RPE retinal G-protein coupled } \\
\text { receptor }\end{array}$ \\
\hline PCK & Pan Cytokeratin & ROS & Rod Outer Segment \\
\hline PDGF & $\begin{array}{l}\text { Platelet Derived Growth } \\
\text { Factor }\end{array}$ & ROS/I & $\begin{array}{l}\text { Reactive Oxygen } \\
\text { Species/Intermediates }\end{array}$ \\
\hline PDT & Photodynamic Therapy & $\mathbf{R P}$ & Retinitis Pigmentosa \\
\hline PE & Phycoerythrin & RPC & Retinal Progenitor Cell \\
\hline PEDF & Pigment Epithelium Derived & RPE & Retinal Pigment Epithelium \\
\hline & Factor (SERPINF1) & RPE65 & Retinal Pigment Epithelial \\
\hline PEG & Polyethylene Glycol & & protein of $65 \mathrm{kDa}$ \\
\hline
\end{tabular}




\begin{tabular}{|c|c|c|c|}
\hline RXRs & Retinoid X Receptors & TNF-R & Tumor Necrosis Factor \\
\hline RT & Room Temperature & & Receptor \\
\hline SCF & Stem Cell Factor & Toll-R & Toll Receptors \\
\hline SCLC & Small Cell-Lung Carcinoma & TPO & Thrombopoietin \\
\hline SD & Standard Deviation & TSG-6 & TNF-inducible/Stimulated \\
\hline SDF-1 & Stromal cell Derived Factor-1 & & Gene 6 protein \\
\hline SDS- & Sodium Dodecyl Sulfate Poly & UCP & Uncoupling Protein \\
\hline \multirow[t]{2}{*}{ PAGE } & Acryl amide Gel & UV & Ultra Violet \\
\hline & Electrophoresis & VEGF & Vascular Endothelial Growth \\
\hline \multirow[t]{2}{*}{ SEE } & Spanish Eyes Epidemiological & & Factor \\
\hline & group & VEGF-R & Vascular Endothelial Growth \\
\hline SEM & Standard Error of Mean & & Factor Receptor \\
\hline \multirow[t]{2}{*}{ SNPs } & Single Nucleotide & VIP & Vasoactive Intestinal Peptides \\
\hline & Polymorphisms & VLDLR & Very Low Density Lipoprotein \\
\hline \multirow[t]{2}{*}{ Sox2 } & SRY (sex determining region & & Receptor \\
\hline & Y)-box 2-a transcription factor & VPAC1/ & Fusion of name for VIP and \\
\hline SSC & Side Scatter Channel & 2 & PACAP receptor $1 / 2$ \\
\hline SVF & Stromal Vascular Fractions & WAT & White Adipose Tissue- bad fat \\
\hline \multirow[t]{2}{*}{ TA } & Tejido Adiposo (Adipose & WB & Western Blotting \\
\hline & Tissue) & Zfp42 & Zink finger protein 2 or Rex1 \\
\hline \multirow[t]{2}{*}{ TAP } & Treatment of AMD with & zo1 & Zonula Occludens \\
\hline & Photodynamic therapy & & \\
\hline TB & Trypan Blue & & \\
\hline TBS & Tris Buffer Saline & & \\
\hline \multirow[t]{2}{*}{ T-cell } & Thymus/Thymocyte derived & & \\
\hline & cell & & \\
\hline TER & Trans Epithelial Resistance & & \\
\hline TGF- $\beta$ & Transforming Growth Factor $\beta$ & & \\
\hline Thy1 & Thymocyte antigen 1 & & \\
\hline \multirow[t]{2}{*}{ TIMP3 } & Tissue Inhibitor of Metallo- & & \\
\hline & Proteases/Peptidase 3 & & \\
\hline
\end{tabular}

TLR3/4 Toll-Like Receptor 3/4 


\section{$\underline{\text { Index }}$}

Abstract of thesis...............................................................................

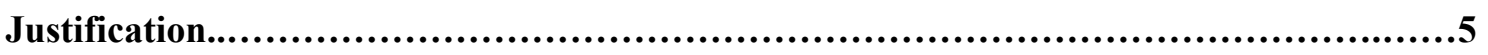

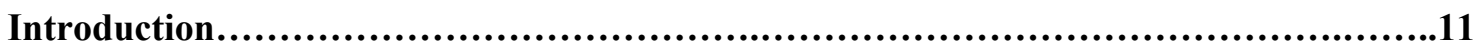

1. Age Related Macular Degeneration..........................................13

1.1. Definition...................................................................

1.2. Prevalence.................................................................

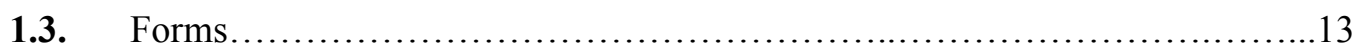

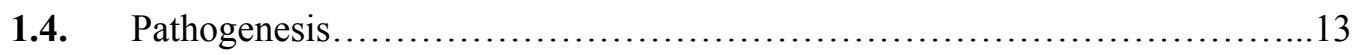

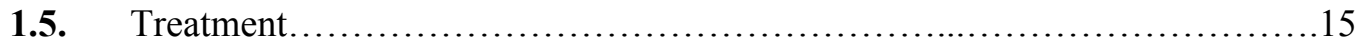

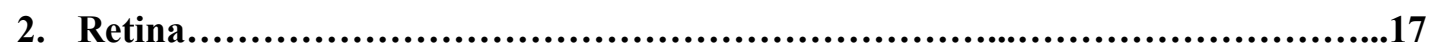

$2.1 \quad$ Human Neuroretina........................................................... 17

Central and peripheral retina.................................... 18

2.2 Human Retinal Pigment Epithelium (hRPE)...............................21

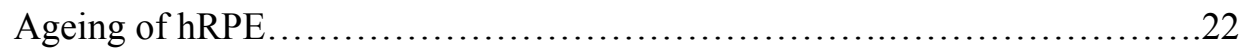

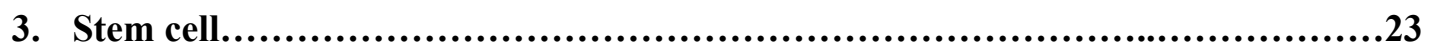

4. Advance Therapy..........................................................24

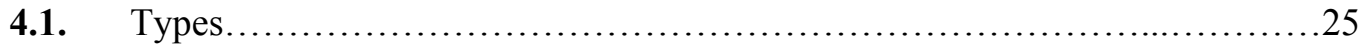

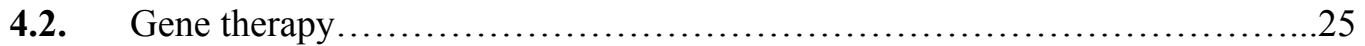

4.3. Somatic cell therapy/cell therapy.......................................26

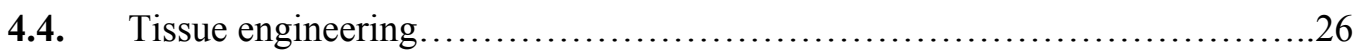

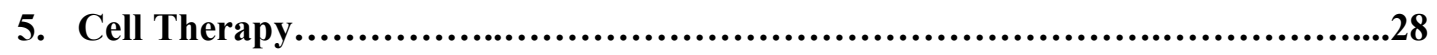

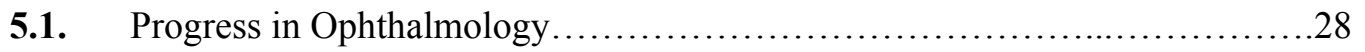

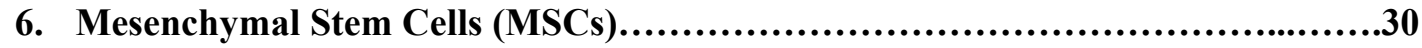

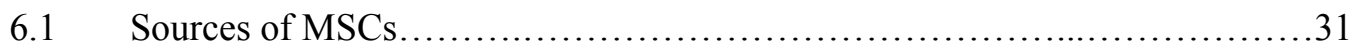

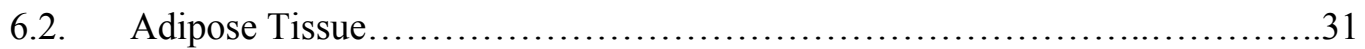

6.3. Therapeutic Aspects.....................................................

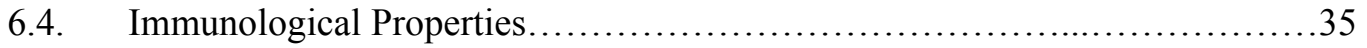

6.5. Paracrine effects......................................................... 37

6.6. Differentiation Potential................................................. 38

6.7. Currant status of MSCs in Ophthalmology ................................... 39

7. Factors of Differentiation used in this study....................................40

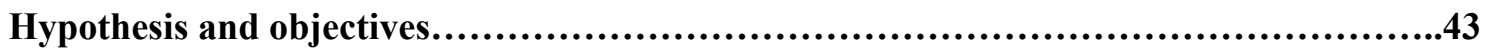

Hypothesis...................................................................45

Objectives.............................................................. 47

Materials and Methods................................................................

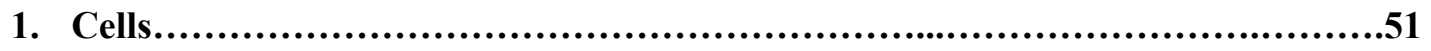

$1.1 \quad$ Cell line............................................................ 51

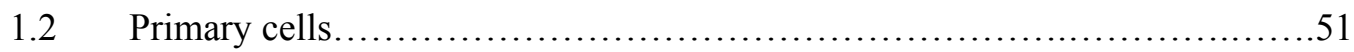

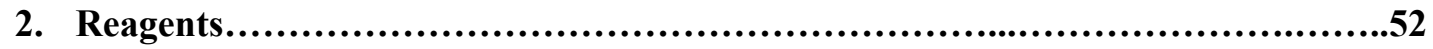

3. Cell culture..................................................................54

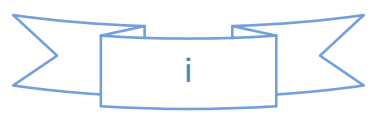


4. Cell/Tissue Isolation.

4.1 Isolation of RPE and neural retina and preparation of neuroretinal

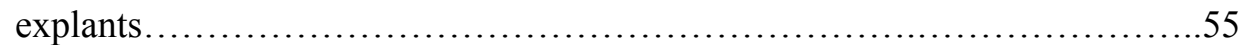

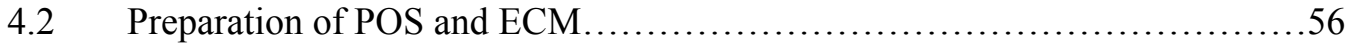

4.3 Preparation of pig retinal tissue sections and treatment with Trypan Blue

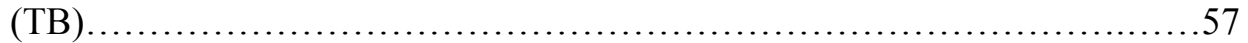

4.4 Treatment of RPE cells with MMC .......................................58

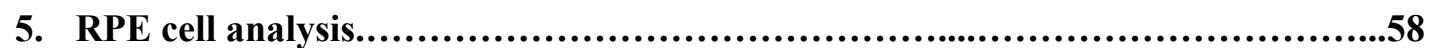

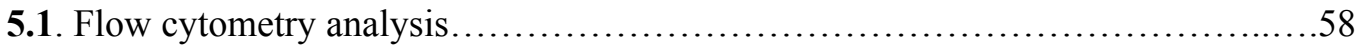

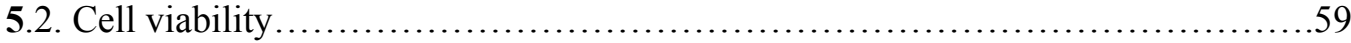

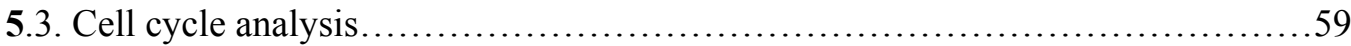

6. Human adipose derived mesenchaymal stem cells (hAD-MSCs)..................59

6.1 Isolation and culture of hAD-MSCs.......................................59

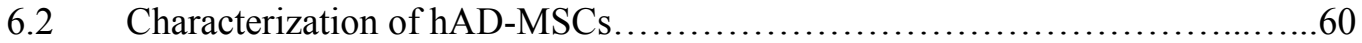

6.2.1. Immunophenotyping...........................................6

6.2.2. In vitro multilineage differentiation of hAD-MSCs...................61

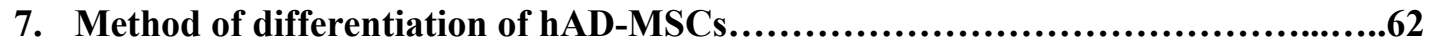

7.1. Culture of hAD-MSCs in hRCM with factors............................62

7.2. Culture of hAD-MSCs in fRCM and POS with BMP-4 .....................662

7.3. Direct co-culture of hAD-MSCs with inactivated RPE cells.................62

7.4. Indirect co-culture of hAD-MSCs with inactivated RPE cells along with factors of differentiation............................................................63

7.5. Indirect co-culture of hAD-MSCs with human neuroretinal (hNR) explants.............................................................63

8. Treatment with factors.........................................................65

9. Analysis of effects of different experimental conditions on RPE cells, hAD-MSCs

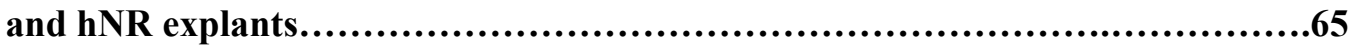

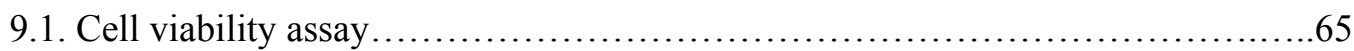

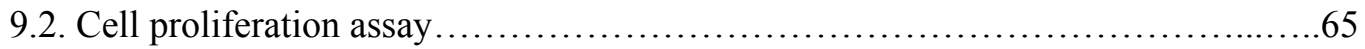

9.3. Protein preparation, PAGE and Western blotting...........................66

9.4. Immunofluorescence study................................................66

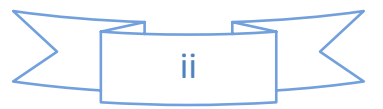


9.4.1.Immunocytochemistry.

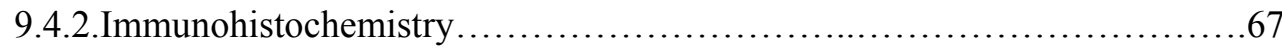

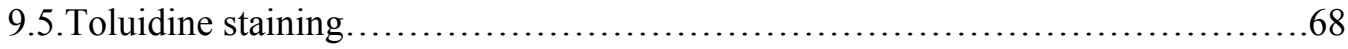

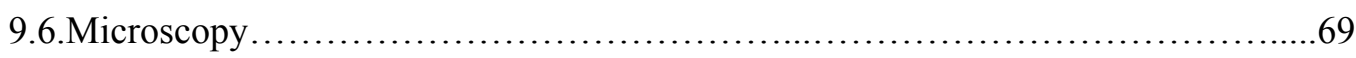

10. Arbitrary evaluation method for preservation status of human neuroretinal explants explants.....................................................................69

11. Statistical analysis....................................................................71

Results..............................................................................................73

1. Characterization of RPE cells and undifferentiated hAD-MSCs..................75

1.1. RPE cells.....................................................

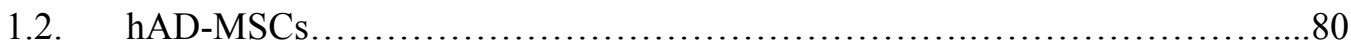

1.2.1. Isolation, Expansion and Maintenance............................80

1.2.2. Cell viability and cell cycle analysis................................ 82

1.2.3. Characterization of undifferentiated hAD-MSCs......................82

1.2.4. Comparison between higher and lower passages of undifferentiated hADMSCs.

2. Assessment of effects of hAD-MSCs over degenerating RPE cells in indirect coculture.

3. Assessment of differentiation of hAD-MSCs to RPE phenotypes .91

3.1. Induction of differentiation of hAD-MSCs with factors and their combinations in hRCM.

3.2. Induction of differentiation of hAD-MSCs to RPE phenotypes by culturing in fRCM supplemented with BMP-4 and POS... .95

3.3. Induction of differentiation of hAD-MSCs to RPE phenotypes by direct coculture with inactivated RPE cells.

3.4. Induction of differentiation of hAD-MSCs to RPE phenotypes by indirect coculture with inactivated RPE cells in the medium supplemented with factors and their combinations.

3.5. Induction of differentiation of hAD-MSCs to RPE phenotypes by indirect coculture with human neuroretinal in RCM supplemented with factors and their combinations 106

4. Assessment of status of hNR explants cultured with hAD-MSCs along with factors (VIP, NIC and ATRA) and their combination.

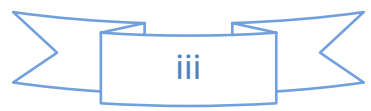


1. Limitations of works.

2. Characterization of RPE cells and undifferentiated hAD-MSCs.

3. The effects of hAD-MSCs over MMC treated RPE cells under indirect co-culture condition.

4. Cultivation of hAD-MSCs in RCM supplemented with factors and their combinations.

5. Induction of differentiation of hAD-MSCs to RPE phenotypes with BMP-4 and POS and fRCM.

6. Direct co-culture of hAD-MSCs with inactivated RPE cells

7. Indirect co-culture of hAD-MSCs with MMC treated RPE cells along with factors and their combinations

8. Indirect co-culture of hAD-MSCs with human neuroretinal explants in RCM supplemented with three differentiating factors and their combinations.

9. Differentiation of hAD-MSCs into RPE like cells. 139

10. Status of neuroretinal explants cultivated with hAD-MSCs along with factors and their combinations.

Conclusions and Future perspectives...............................................143

Conclusions 145

Future perspectives.

Spanish summary (Resumen español)

1. Justificación

2. Introducción.

3. Hipótesis y objetivos

4. Material y métodos

5. Resultados.

6. Discusión.

7. Conclusiones

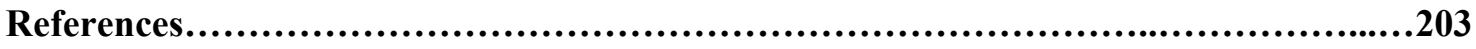




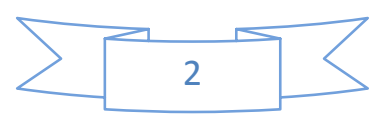


In the dry form of age related macular degeneration (AMD) disease, the subretinal environment consists of damaged and dysfunctional retinal pigment epithelium (RPE) and degenerating neuroretina. The subretinal injection of human adipose tissue derived mesenchymal stem cells (hAD-MSCs) to delay the process of disease progression in early onset of AMD could be one of the promising strategies under advance cell therapy options. The fate of injected hAD-MSCs and their paracrine immunomodulatory effects might be beneficial for AMD and other retinal degenerative diseases. It would be better if these cells could be directed to RPE phenotypes before moving to their subretinal injection. There is one published report, in which vasoactive intestinal peptide (VIP) has been used to differentiate hAD-MSCs towards RPE phenotypes but only partial success was achieved. It shows that there is a possibility to differentiate hAD-MSCs to RPE cells but it requires to explore other differentiation inducing factors and their combinations with the previously used factor (VIP), which could stimulate hADMSCs to RPE like cells especially in the artificial culture environment partially similar to the subretinal environment of AMD. Along the process of differentiation, it would be logical and novel to investigate that hAD-MSCs themselves in these environments may produce their positive or negative effects over the degenerating retina (neuroretina and RPE) under in vitro condition.

The purpose of this work is to explore the potential of hAD-MSCs as a future candidate for retinal tissue repair and replacement together with neuroprotection in cellular therapy for retinal degenerative diseases like dry AMD and others.

In this work, hAD-MSCs and RPE cells had been isolated from donated human lipoaspirates and post-mortem eyes respectively and they had been characterized and maintained in culture condition and finally they had been subjected to many different environments slightly similar to the in vivo subretinal environment present in the AMD patients where both RPE and neural retina are under degeneration process; further these in vitro environments were supplemented with different differentiation factors (VIP, NIC and ATRA) and their combinations to analyze their influences on cell behavior of hAD-MSCs and retinal tissue. For this purpose, the hAD-MSCs had been co-cultivated directly and indirectly with the inactivated and degenerating RPE and with degenerating human neuroretina (hNR) in the presence and absence of chosen factors (VIP, NIC and ATRA) and their 
combinations. In all these different conditions of differentiation, the fate of hADMSCs along with their effects on degenerating RPE cells and neural retina had been investigated by using different methods. The fate of hAD-MSCs subjected to different conditions was investigated by phase contrast observations and by analyzing the expression of RPE protein markers in them by immunofluorescence and western blot (WB) studies. The effects of these differentiation cells along with mentioned factor(s) were studied over the in vitro degenerating hNR explants through histochemical staining and immunohistostaining with toluidine blue and with different neuroretinal markers respectively. The effects of some of these differentiating conditions over the MMC inactivated (stressed and dying cells) RPE cells were evaluated by cell viability/cytotoxicity assay and cell proliferation assay (alamarBlue ${ }^{\circledR}$ assay).

It has been demonstrated in these experiments that hAD-MSCs enhance their expression of RPE markers such as RPE65, Ezrin, Bestrophin, CK8/18, PEDF and others while they reduces their expression of MSCs-specific marker CD90 significantly when they are co-cultured directly or indirectly with MMC treated RPE cells (hRPE and ARPE19) and these effects of differentiation was further enhanced when supplemented with combinations of differentiating factors in the culture medium. These experiments demonstrated that hAD-MSCs stimulate the proliferation and survival of MMC treated RPE cells in co-culture conditions. The hAD-MSCs have also demonstrated the neuroprotective effects over the in vitro degenerating neuroretinal explants and these neuroprotective effects were significantly enhanced in the presence of combinations of factors (VIP+NIC and VIP+NIC+ATRA)

This study concludes that the hAD-MSCs in combination with the factors of differentiation (VIP, NIC and ATRA) along with molecules secreted by the RPE cells not only can partially delay or rescue the degeneration of RPE and neural retina but also can induce the differentiation in hAD-MSCs to RPE phenotypes and hence could replace the diseased and degenerated RPE cells to some extent. 


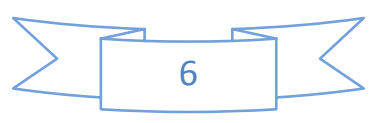


AMD is a constant major threat to blindness worldwide in elderly people for decades to come. The dry form of AMD constitutes around 60\% to $90 \%$ of AMD population as against wet from of AMD (advanced form is also ka CNV). Dry AMD represents a market size of \$25-30 billion in the U.S. and Europe and poses a drastic negative impact of around $\$ 30$ billion annually on gross domestic product (GDP) of U.S. (Matthew L. Brown Nov 30, 2010, Brown et al. 2005). As the population of old people in industrialized country is going to increase in coming decades, so it would alarm the social health and increase the economic and social burden of health problems. Thus, AMD is posing a major public health problem that can have a devastating effect upon the quality of life of the patients and hence is leading to adverse financial consequences for the economy of industrialized world. Moreover, other retinal degenerative diseases such as retinitis pigmentosa, Stargardt disease and others also exert significant negative impact on the quality of life globally.

Current management options for AMD show very modest advance especially in CNV but do not arrest the unrelenting course and prognosis of the disease itself very significantly. In the case of dry AMD, there is no any treatment option available to date. Retinal pigment epithelium (RPE) plays extremely important role in the normal visual function as well as in preserving structural integrity of the retina. RPE is considered as one of the main culprits in pathogenesis of AMD as a result of detrimental structural and biochemical changes occurred throughout the life time. All these detrimental changes in RPE progressively affect the normal health of the retina. Thus the neural retina and photoreceptors start to degenerate gradually with the increase in the degree of RPE malfunction.

It seems logical in this case that if the damaged RPE could be replaced with healthy and fresh RPE, especially in the early form of AMD, where the photoreceptors (PRs) are still functioning, may get benefits in the form of delay or prevention of PRs degeneration. There are several examples where researchers have used RPE and IPE (iris pigment epithelium) transplantation from autologous, homologous or heterologous sources in animal models of retinal degenerative disease. In case of homologous and heterologous sources there were strong immune rejections but autologous sources showed better results than that of other two previous sources. Although the autologous sources showed significant immune tolerance but they did not bring any significant improvement in visual functions because of carrying the same genetic and environmental imprints inside the cells.

There are three major components which get affected very badly in AMD namely: Bruch's membrane (BM), RPE and neural retina especially PRs. The advanced therapy aims to look forward to engineer both cellular/tissue part and the non-cellular part 
together. It is well known that stem cells have capacity to self-renew perpetually and differentiate to form any kind of mature terminal tissues or cells upon getting appropriate cues. So, stem cells are potential sources of getting cellular part i.e. RPE and PRs or even entire retina. Stem cells create a tremendous hope in the generation of retinal cells. There are significant advances towards getting fresh and functional RPE even PRs from ESCs and iPSCs. A number of clinical trials are under recruiting phase for ESCs derived RPE cell transplantation in the patients of retinal degenerative diseases (NCT01345006, NCT01344993, NCT01469832, NCT01625559, and NCT01691261). Riken Centre for Developmental Biology, Japan launched a pilot study (by Masayo Takahashi) to assess the safety and feasibility of the transplantation of autologous iPSCs-derived RPE cell sheets in patients with exudative AMD, which was scheduled to start on $1^{\text {st }}$ Aug 2013 . The use of ESCs in research is obviously hindered due to ethical problem and teratoma formation. Regarding iPSCs, the safety issue and the risks of tumor formation are major concern areas. While in case of using adult stem cell source like MSCs, these problems seem to be very minimal.

Although there are diverse sources of obtaining MSCs, out of which two are major sources namely: bone marrow and adipose tissue (lipoaspirate). As discussed further in the introduction section, adipose tissue takes advantage over bone marrow regarding the ease of harvest and the availability of large number of cells without any significant differences between BM-MSCs and AD-MSCs. The AD-MSCs have already been shown to differentiate into neuroectodermal origin (Zuk et al. 2001, Zuk et al. 2002) and have been recently shown to be able to differentiate into cells having RPE phenotype or retinal progenitor cells type (Vossmerbaeumer et al. 2009, Huang et al. 2012a, Moviglia et al. 2012a). In some reports, MSCs have been demonstrated to rescue the degeneration of PRs and to improve visual functions in animal models. The proof of concept was already tested by using intravitreal autologous bone marrow-derived mononuclear cell transplantation in retinal disease (Jonas et al. 2010, Jonas et al. 2008, Siqueira et al. 2011). In one of our results, hAD-MSCs have shown rescuing effect over death/degeneration of RPE cells under in vitro condition (Singh et al. 2013a). The use of MSCs for retinal degeneration diseases is not only limited to its differentiation towards RPE or PRs but it extends the benefits to the effects of neurotrophic, immunomodulatory or anti-inflammatory factors. There are several growth factors which play important roles in maintaining the health of neuroretina as well as RPE, which include PEDF, BDNF, VEGF, CNTF, HGF, NGF, LIF etc. RPE provides nutrients to the neural retina from the choroidal circulation, renew outer segments of photoreceptors and secretes different growth and neurotrophic factors, among them are the PEDF, BDNF and VEGF. These factors have very important roles in maintaining the neuroretinal homeostasis, and even some of them are already being used as drugs. It has 
been found that these factors are also constitutively secreted by MSCs even after partial differentiation. Recently in some clinical trials (NCT00062765, NCT00447954, NCT00447980, NCT00447993, NCT01530659), paracrine properties of engineered RPE cells (human NTC-201 derived from ARPE19 cell line) have been utilized in an intraocular encapsulated cell implant (ECT) for retinal degenerative diseases (RP and GA) by Neurotech (Sieving et al. 2006, Kauper et al. 2012, Thanos et al. 2004, Zhang et al. 2011). In these ECT implants, the use of MSCs might provide advantage over RPE cells. In several ways, MSCs therapy offers a great opportunity in looking for new therapy against AMD.

Finally this work is aimed on looking forward to use the differentiation potential of hAD-MSCs towards RPE phenotypes and simultaneously using their beneficiary paracrine effects against the degeneration of RPE and neuroretina under in vitro conditions. This work is trying to create microenvironment by using some biomolecules, which can guide the differentiation pathway of hAD-MSCs towards RPE phenotype and can also protect the degenerating retina (especially photoreceptors and RPE) under indirect co-culture systems. Finally this would give some cues to use these results for in vivo experimentation before going ahead to human clinical trials in AMD. 
Justification 


\section{Introduction}

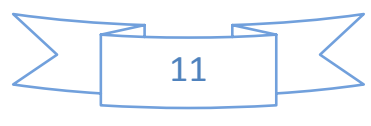




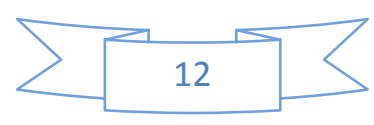




\section{Age Related Macular Degeneration (AMD)}

1.1. Definition: AMD is an ocular disease in which macula, central retinal area, required for the sharp and detailed vision, starts to deteriorate progressively with increasing age in people after 50 years. The symptoms in affected persons start from mild blurring or distortion to complete irreversible central vision loss but with the retention of blurred peripheral (side) vision. The condition, which is bilateral, comes under legal blindness (visual acuity of 20/200 or worse) and the affected individuals are not able to do activities like driving, reading, recognizing face etc.

1.2. Prevalence: AMD which was first described 85 years ago, (Holloway, Verhoeff 1928 ) is currently a leading cause of irreversible vision loss among the old age people over 50 years in industrialized countries (Ratnapriya, Chew 2013, Pascolini et al. 2004, Ambati et al. 2003, Ambati, Fowler 2012) and is increasing exponentially after each decade (Rein et al. 2009, Cheung, Eaton 2013). The prevalence of the disease increases with ageing (Klein et al. 2010, Klein et al. 2004, Klein et al. 2007, Friedman et al. 2004). The prevalence also depends on the race and ethnicity (genetic makeup of population) of the population (Klein et al. 2006). Around 3.4\% of Spanish elderly population of 65 years or older is suffering from AMD (Spanish Eyes Epidemiological (SEE) Study Group 2011). AMD is the primary cause of blindness in developed countries and the third leading cause of blindness worldwide after cataract and glaucoma, constituting 8-7\% of all legal blindness (central visual acuity of 20/200 or worse) worldwide (Resnikoff et al. 2004).

1.3. Forms: There are two forms of AMD: dry (also known as non-neovascular or atrophic) form which is most frequent (60-90\%) and wet (also known as neovascular or exudative) form (10-40\%) (Velez-Montoya et al. 2012). The wet form is also synonymously called Choriodal Neo-vascularization (CNV). The more severe and advanced form of dry AMD is also called geographic atrophy (GA) (Mata, Vogel 2010).

1.4. Pathogenesis: Despite huge recent advances in clinical research, the pathogenesis of AMD is yet poorly understood. It includes complicated interaction of genetic, environment, metabolic and functional factors (Nowak 2006). Risk factors for the disease like advance age, smoking, lack of some ingredients in the diet and obesity seems to be related to AMD by increasing oxidative stress (Klein et al. 2007, Klein et al. 2010). Over the course of human life, various environmental insults such as ageing, oxidative stress, smoking and excessive light exposure cause progressive damage to RPE cells while simultaneously affecting the functional integrity of BM beneath the RPE (Beatty et al. 2000, Cai et al. 2000, Boulton et al. 2004b, Liang, Godley 2003). This damage is directly 
attributed to the progressive atrophy of the retina leading to AMD and other debilitating retinal diseases (Mata, Vogel 2010, Munoz et al. 2000). The model of pathogenesis of AMD based on various clinical, pathological and experimental observations is shown in the Figure 1.

Several growth factors contribute to the development of AMD. Basically an imbalance between pro-angiogenic and anti-angiogenic (angiostatic) factors gives rise to the CNV (Gao et al. 2001, Tong, Yao 2006). There are mainly two important players in this regards namely vascular endothelial growth factor A (VEGF-A) and pigment epithelium derived growth factor (PEDF). VEGF-A is a mitogen as well as chemotactic factor which promotes survival (inhibits apoptosis) of endothelial and glial cells and it enhances vascular permeability and hence plays very important role in physiological and pathological angiogenesis (Ferrara et al. 2003, Alon et al. 1995). It is secreted by basolateral side of RPE as well as by accumulated microglia (Blaauwgeers et al. 1999, Adamis et al. 1993, Combadiere et al. 2007). Under normoxic condition, VEGF secretion towards basal side (choriocapillaris side) by RPE is 2 to 7 times higher than apical side (Blaauwgeers et al. 1999). This disparity is exaggerated by hypoxia, advanced glycation end products (AGEs) and reactive oxygen intermediates (ROIs) leading to pathological CNV (Lu et al. 1998, Kuroki et al. 1996, Beerepoot et al. 1996, Shima et al. 1995). PEDF is an anti-angiogenic factor secreted by apical side of RPE, which stabilizes neuroretina by inhibiting apoptosis and also enhance pathological angiogenesis in AMD (Ogata et al. 2001, Cayouette et al. 1999, Cai et al. 2006, Becerra et al. 2004, Dawson 1999). In vitro and in vivo results show that senescent RPE reduces PEDF secretion drastically with some exception (TombranTink et al. 1995, Holekamp et al. 2002, Matsunaga et al. 1999). In healthy individuals both proangiogenic factors and anti-angiogenic factors are well-balanced but this balance gets disturbed in AMD, leading to the mass production of VEGF and hence leads to CNV (Mousa, Mousa 2010, Velez-Montoya et al. 2012, Velez-Montoya et al. 2010, Tong, Yao 2006, Gao et al. 2001). Pathogenesis of dry form is even less understood, but probably same factors might be implicated, acting on different substrates. 


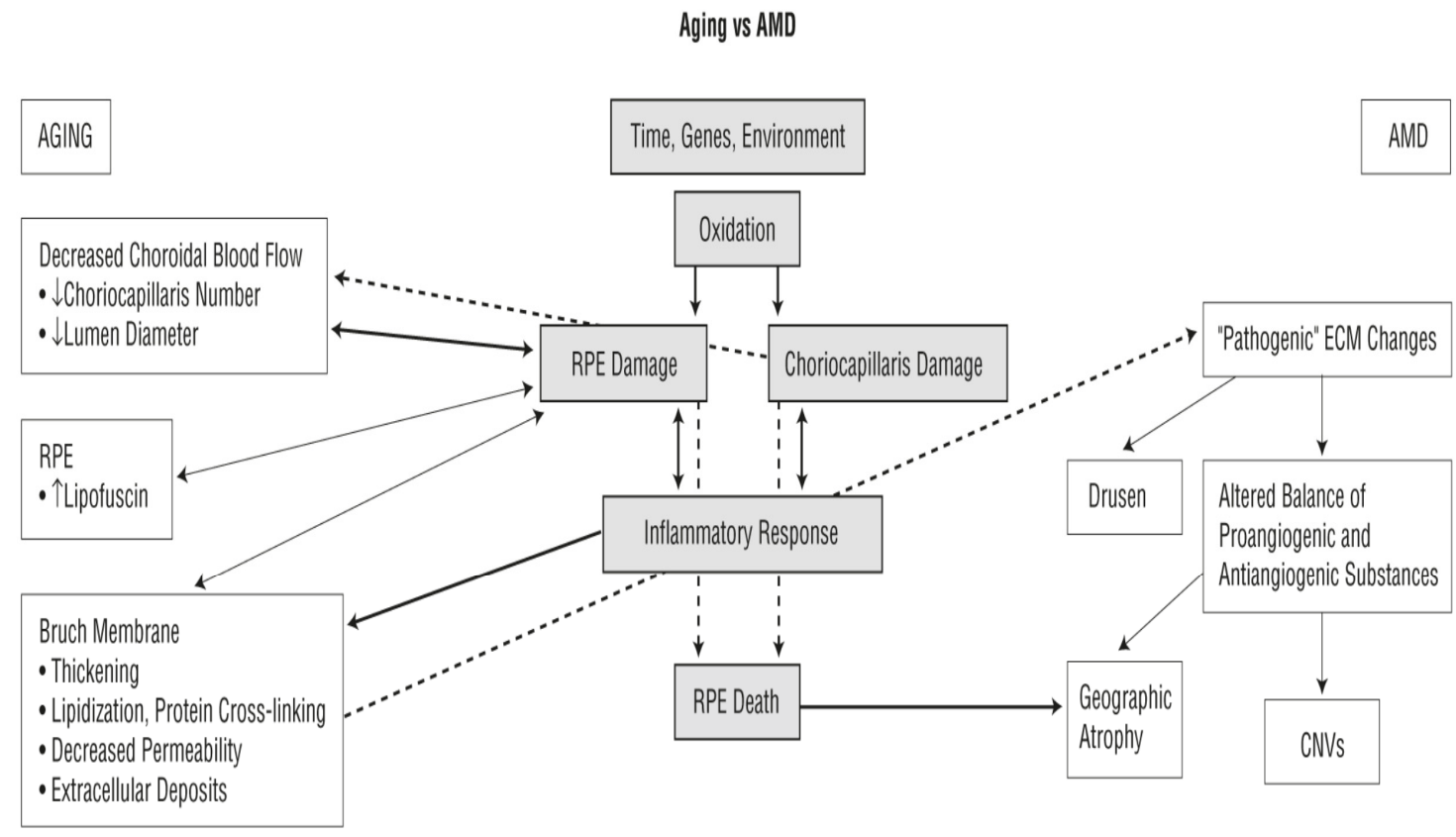

Fig.1. Aging vs. age related macular degeneration (AMD) (Zarbin 2004)

A number of genes are directly and indirectly involved in the pathogenesis of both forms of AMD. At least $25 \%$ of patients have been reported to have this disease due to their genetic predispositions (Recalde et al. 2008, Garcia Layana et al. 2011, Meyers et al. 1995). Several single nucleotide polymorphism (SNPs) and allelic variations are linked to AMD (Gold et al. 2006). Lots of candidate genes like ELOVL4, VEGF, VLDR, LRP6, ABCA4, ARMS2, C2, C3, CFB, CFH, ERCC6, FBLN5, mHCN1, HTRA1, LIPC, TIMP3, RAX2, TLR3 and TLR4 have been reported to be associated with AMD (Jorgenson et al. 2013, Stone et al. 2001, Meyers et al. 1995, Seddon et al. 2011, Haines et al. 2006, Biarnés et al. 2011, Gold et al. 2006). The role of these genes and associated polymorphism helped in understanding the pathogenesis of AMD and in the development of new therapies against AMD.

1.5. Treatment: Along the last 20 years, the wet form of AMD, the most aggressive clinical type has been getting various treatments including direct laser photocoagulation of neo-vessels and photodynamic therapy (PDT) (Randomized Clinical Trial 1982, Macular Photocoagulation Study Group 1991, Macular Photocoagulation Study Group 1986, Wormald et al. 2007, Wormald et al. 2005, Wormald et al. 2003, Wormald et al. 2001, Bressler, Treatment of Age-Related Macular Degeneration with Photodynamic Therapy (TAP) Study Group 2001, Bressler 2000, Garcia Layana 2000). Surgical approaches such as submacular surgery and retinal translocation came later but with more prospects (Aisenbrey et al. 2002, Gelisken et al. 2007, Suesskind et al. 2008, Toth, Freedman 2001). The arrival of anti-VEGF agents (e.g. pegaptanib, ranibizumab, bevacizumab,) has limited 
the use of previous treatment options drastically (Cheung, Wong 2013, Lim et al. 2012, Cook et al. 2008, Ambati et al. 2003, Wong et al. 2007).

There is a new US Food and Drug administration (FDA) and European Medicines Agency (EMA) approved (2011 and 2013) anti-VEGF agent called aflibercept (VEGF tap-eye), which binds to all isoforms of VEGF-A, VEGF-B and placental growth factors (PLGF-1 and 2) with high affinity (Mousa, Mousa 2010, Thomas et al. 2013, Stewart et al. 2012, Bakall et al. 2013, Browning et al. 2012, Verner-Cole et al. 2012, FDA 2012, Kaiser 2013, Ohr, Kaiser 2012, Sivaprasad, Hykin 2013, Stewart 2012, Traynor 2012, Ciombor et al. 2013). It is a fusion protein of key binding domain of VEGF receptor1 (VEGFR1) and VEGFR2 combined with human immunoglobulin G1 (IgG1) Fc fragment (an entirely human soluble recombinant VEGFR) (Ciombor et al. 2013, Ohr, Kaiser 2012, Stewart et al. 2012). Its binding affinity for VEGF-A is higher than that of native VEGFR (Brown et al. 2011). It promises to decrease the frequency of injections for patients with CNV and also to provide at a cheaper cost than that of ranibizumab (Thomas et al. 2013, Ciombor et al. 2013, Stewart et al. 2012).

In spite of some significant advances towards the treatment of wet form, there is no any effective treatment option till date in case of dry form of AMD (Cheung, Eaton 2013, VelezMontoya et al. 2012, Lim et al. 2012, Zarbin, Szirth 2007). There are some preventive measures against early form of AMD like cessation of smoking and use of antioxidant vitamin supplements (Vitamin C, E, $\beta$-carotene) and minerals (zinc, copper), and $\omega$-3 long chain polyunsaturated fatty acids (PUFA) such as docosahexanoic acid (DHA), and its precursor eicosapentaenoic acid (EPA), which have been shown in some cases to slow down the progress of AMD but the beneficial effect was not global (SanGiovanni et al. 2007, SanGiovanni et al. 2008, Age-Related Eye Disease Study Research Group et al. 2007, Johnson et al. 2008, Richer et al. 2004, Aslam et al. 2013, Garcia Layana 2002, Age-Related Eye Disease Study Research Group 2001a, Age-Related Eye Disease Study Research Group 2001b, Augood et al. 2008, Swenor et al. 2010, SanGiovanni et al. 2009a, SanGiovanni et al. 2009b, Seddon et al. 1994, Seddon et al. 2001, Seddon et al. 2003, Seddon et al. 2011, Tan et al. 2008). In some in vitro studies, Lipofuscin accumulation in RPE has been shown to reduce significantly by the use of lutein, zeaxanthin, lycopene or tocopherol (Sundelin, Nilsson 2001) and reversed by centrophenoxine treatment (Dylewski et al. 1983). Macular pigment ocular density (MPOD), which is believed to retard visual loss and AMD progression has been reported to increase upon intake of nutritional supplement of lutein, zeaxanthin and DHA (Garcia-Layana et al. 2013, Aslam et al. 2013, Johnson et al. 2008). Because of having very important role of these nutritional supplements in AMD pathogenesis, their inclusions as antioxidant pills in AMD therapy have been 
recommended by some authors (Garcia Layana 2002, Aslam et al. 2013). In spite of lot of variations in outcome of the nutritional supplements measures, it cannot be ruled out that good food keeps overall good health.

Currently, there are considerable basic and clinical researches going on against AMD worldwide which is reflected by the number of articles coming in PubMed constantly leading to better understanding of pathophysiology of AMD, which in turn guide the AMD research towards new treatment options such as advance cell therapies and tissue engineering, one step ahead of traditional drug therapies.

2. Retina: It is multi-layered (10 layers) photo sensory tissue at the back of the eye as shown in the diagram (Fig.2) containing several different types of interconnected neurons by synapses. It can be roughly divided into two parts: neuronal parts ( 9 layers) and non neuronal parts (RPE). The cellular and layer structure of whole retina is presented in Figure 3.

2.1. Human neuroretina: It consists of two types of photo sensory cells (PRs): rods and cones. PRs have two parts dangled in subretinal space: Outer segment (also known as photoreceptor outer segment-POS) juxtaposed with microvilli of RPE and the inner segment (IS). Rods are responsible for the intensity of the vision and cones are responsible for the detailed, sharp and colored vision. Besides PRs, some ganglion cells present in ganglion cell layer (GCL) are photosensitive. These ganglion cells are called by different names e.g. melanopsin-containing ganglion cells or photosensitive retinal ganglion cells (Provencio et al. 2000, Science Web News by Carl Zimmer 2012). Rods are more abundant in the peripheral retina and responsible for peripheral vision. Cones are more abundant in the central retina and responsible for sharp and clear vision. Retina contains three layers of cell bodies: the outer nuclear layer (ONL) containing cell bodies of rods and cones, the inner nuclear layer (INL) containing cell bodies of bipolar, horizontal and amacrine cells and the ganglion cell layer (GCL) containing cell bodies of ganglion cell and displaced amacrine cells (Fig.3). Intervening these cell bodies layers, there are two neuropils/neuropiles or synaptic layers containing interneuronal synapses. First neuropil is called outer plexiform layer (OPL), where bipolar and horizontal cells make synapses with rods and cones and second one is called inner plexiform layer (IPL), where bipolar cells connect with ganglion cells and some amacrine cells also connect with bipolar and ganglion cells (Fig.3). For vision process, light travels through the thickness to the retina and strikes to the PRs, where light is absorbed by the visual pigments of the PRs and gets converted to the electrical signals and these electrical signals travel from PRs to bipolar, 
from bipolar to ganglion cells finally transmitted to the visual cortex of the brain through optic nerve fibres.

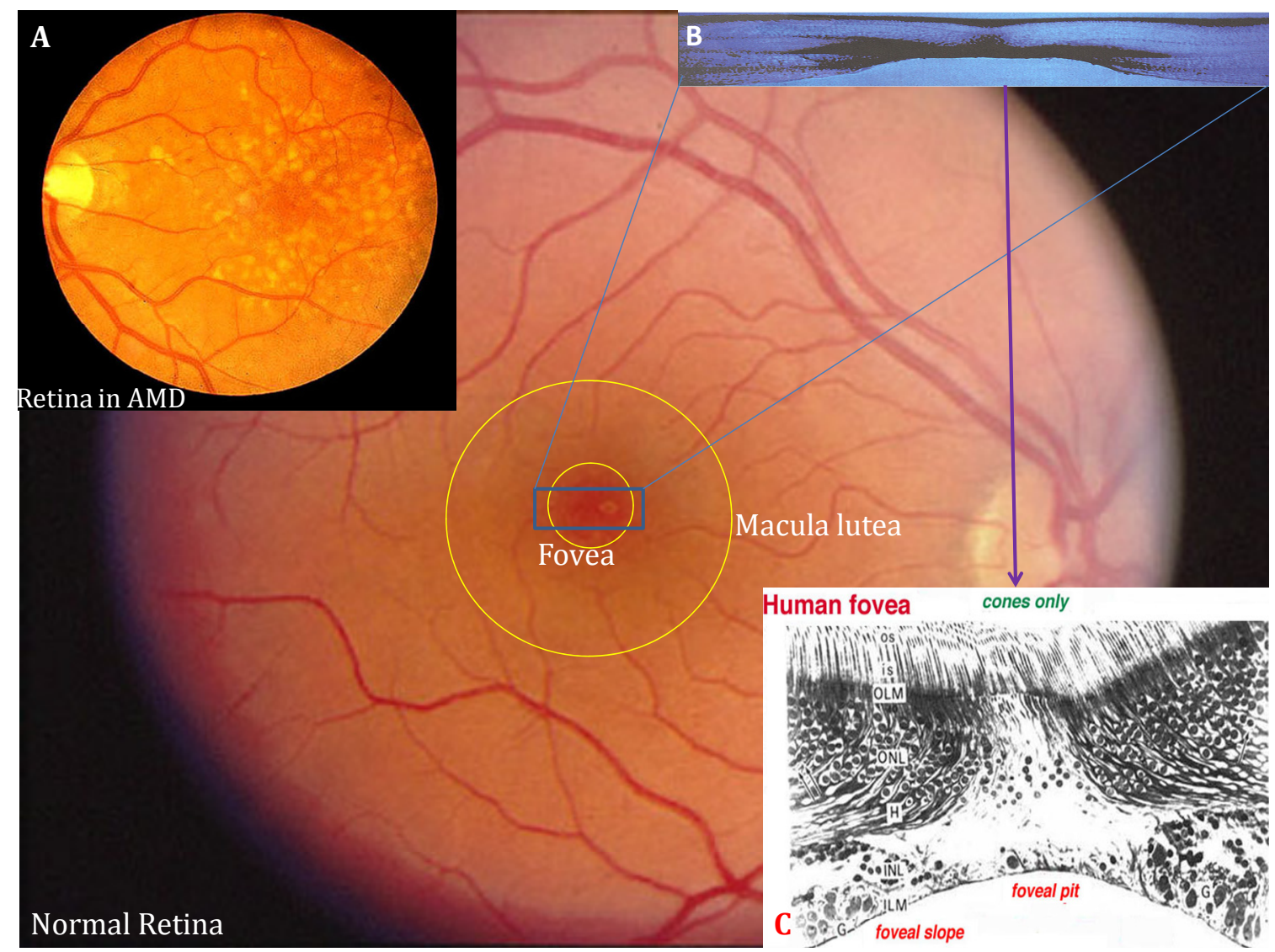

Fig.2. Ophthalmoscopic appearance of normal retina, Macula lutea [yellowish area around the fovea (inner circle) inside the outer circle area] Inside panel A. Retina with drusen (yellow white) in AMD B. Vertical section of fovea C. Vertical section of human fovea with cellular structure from Yamada 1969 Ref: Picture adapted and modified from (www.webvision.med.utah.edu)

\section{Central and peripheral retina}

Structure and function of entire retina is not homogenous so it is roughly divided into central part and peripheral part. Central retina is more pigmented having high cone density than the peripheral retina where rods are abundant. In the central retina, axons of the cones are oblique and accompany with Müller cells processes and form yellowish fibrous like area called Henle fibre layer, which is absent in the peripheral retina. The INL, IPL, GCL and nerve fibre layer (NFL) are thicker in central retina compared with peripheral one, due to high density of cone connecting second order neuron (cone bipolar cells), horizontal, amacrine and ganglion cells concerned with cone pathways, which shows that this layer is more central and crucial for the clear vision due to having high activity of the retinal neurons. Müller cells are type of radial glial cells which nourish the retinal neurons and make outer limiting membrane (OLM) by forming adherens junctions with the inner segment (IS) of PRs and similarly form inner limiting membrane (ILM) by

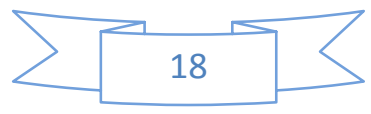


connecting end feet through associated membrane constituents. The OLM forms very important barrier to limit subretinal space, where outer segment of PRs are in very close contact with apical microvilli of RPE (non-neuronal layer of retina).

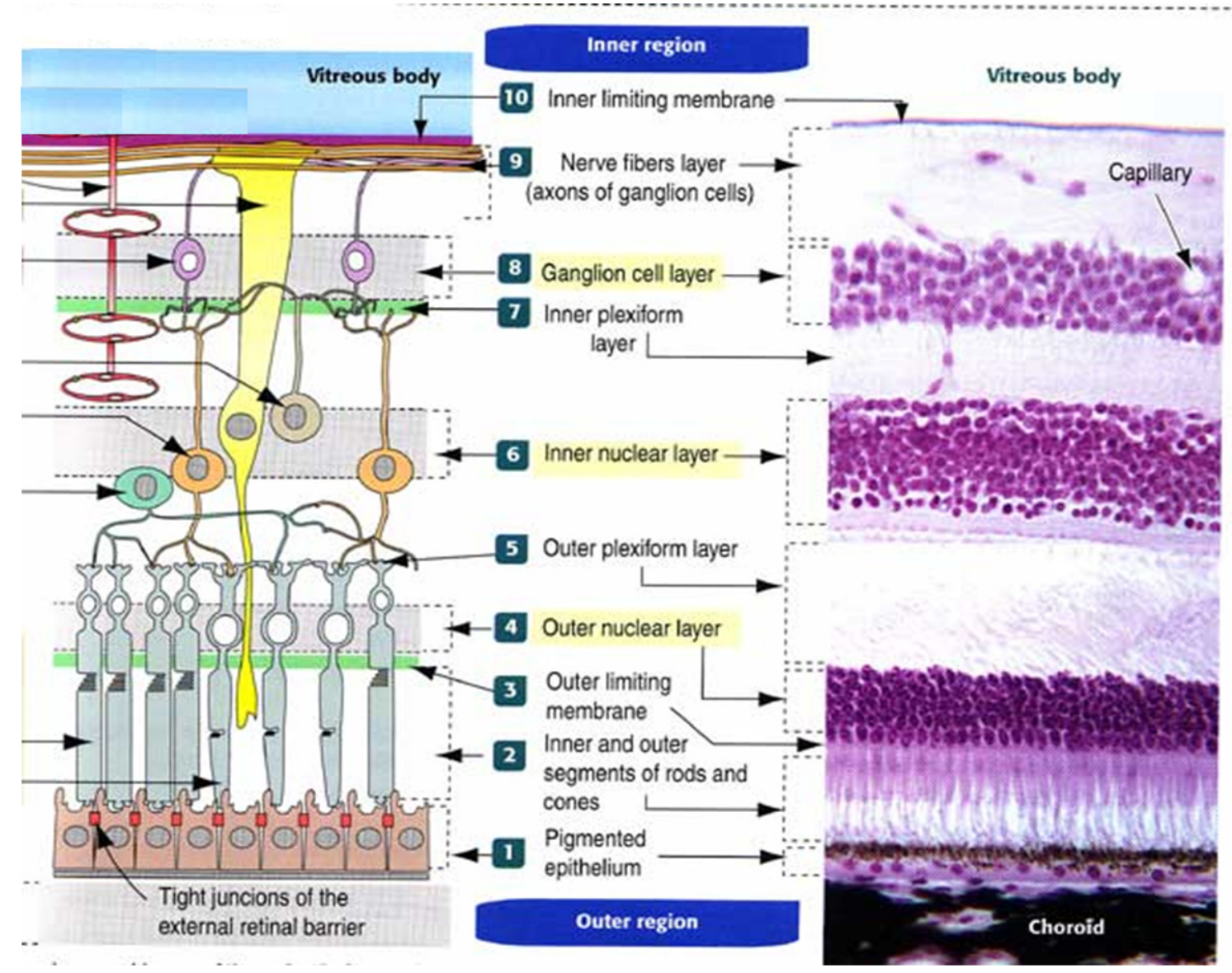

Fig.3. Diagram of Retina with histological stained section of entire retina Ref: Adapted and modified from web page (http://www.bio.miami.edu/tom/courses/protected/bil265/retina.jpg)

The macula (macula lutea) is dark yellow pigmented oval area (around $12 \mathrm{~mm}$ in diameter) at the centre of the human retina (Fig.2). It contains three carotenoid pigments named lutein, zeaxanthin and meso-zeaxanthin collectively called as macular pigment, which gives its yellow color (Loskutova et al. 2013, Weale 2007, Whitehead et al. 2006, Landrum, Bone 2001). The macular pigment is mainly present in the cone axons in Henle fibre layer and protects the macular region by absorbing harming part (blue light) of the light (Loskutova et al. 2013, Weale 2007, Whitehead et al. 2006, Wooten, Hammond 2002, Snodderly 1995). The centre of macula, also called fovea (about $2 \mathrm{~mm}$ in diameter) has highest concentration of cone PRs but devoid of rod PRs and is essentially required for the sharp and colored vision (Hornan et al. 2007). The retinal degeneration in AMD is more focused at macula and gradually spreads peripherally and hence finally leads to irreversible painless central vision loss (Hornan et al. 2007, Quillen 1999, Elner 1999).

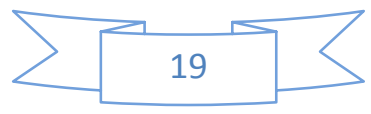




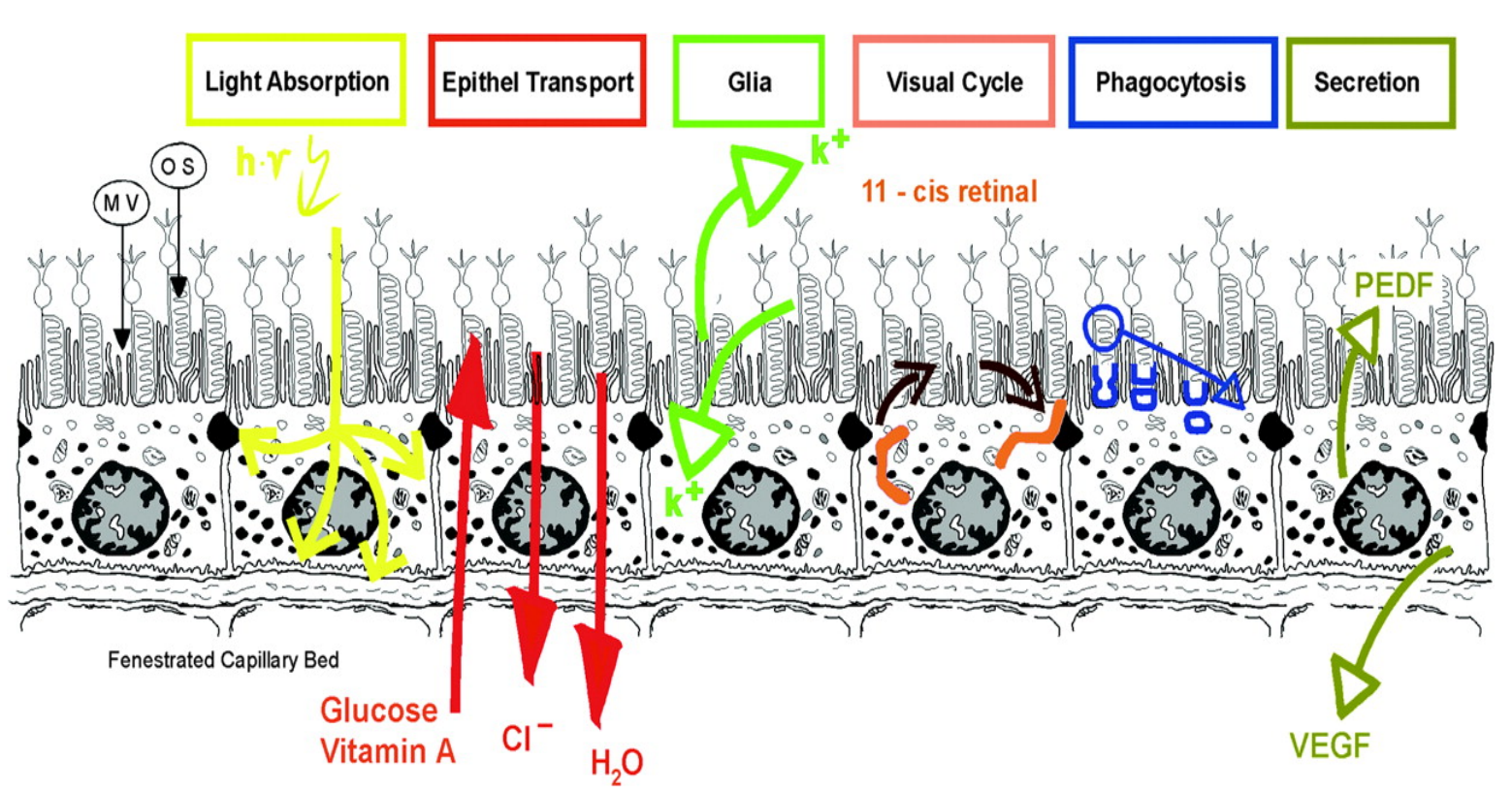

Fig.4. Summary of retinal pigment epithelium (RPE) functions. Ref: Adapted from (Strauss 2005)

Table1. Trophic factors and their biological effect with their cell source

\begin{tabular}{|c|c|c|}
\hline Trophic factor & $\begin{array}{l}\text { Source } \\
\text { RPE/MSCs }\end{array}$ & Biological Effect \\
\hline VEGF & both & $\begin{array}{l}\text { Photoreceptor development, Endothelial cell proliferation } \\
\text { and survival, permeability factor stabilizing fenestration } \\
\text { of endothelium }\end{array}$ \\
\hline PEDF & RPE & Antiangiogenic, Neuroprotection \\
\hline BDNF & both & Neurotrophic/Neuroprotection \\
\hline CNTF & both & Neuroprotection/Neuroprotection \\
\hline $\begin{array}{l}\text { bFGF (FGF2/FGF- } \\
\beta), \text { FGF1, FGF5 }\end{array}$ & both & $\begin{array}{l}\text { Proangiogenic, PRs rescue, Anti-apoptotic for RPE, } \\
\text { Neuroprotection }\end{array}$ \\
\hline HB-EGF & RPE & VEGF secretion, RPE proliferation \\
\hline HGF & both & RPE survival, Neuroprotection \\
\hline NGF & both & Neurotrophic, Inflammation \\
\hline LIF & both & PRs rescue and RPE survival \\
\hline TIMP-1,2,3 & $\begin{array}{l}1 \text { both but } \\
2 \text { (MSCs) } \\
3 \text { (RPE) }\end{array}$ & Stabilize Endothelium and ECM \\
\hline Gas6 & RPE & Stimulator of Phagocytosis by RPE (Karl 2008) \\
\hline LEDGF & RPE & Endocrine/intracrine survival factor of RPE \\
\hline LL37 & MSCs & Antibacterial effect \\
\hline $\begin{array}{l}\text { GDNF, gelectin-1, } \\
\text { NT-3 }\end{array}$ & MSCs & Neuroprotection \\
\hline G-CSF, M-CSF & MSCs & Antiapoptotic, Chemoattractant \\
\hline $\begin{array}{l}\text { IL-6, -7, -8, -10, - } \\
11,-20\end{array}$ & $\begin{array}{l}\text { MSCs and } \\
\text { RPE }\end{array}$ & Immunomodulatory and Neurotrophic chemokines \\
\hline $\begin{array}{l}\text { IDO, PGE2, HLA- } \\
\text { G, TSG-6, }\end{array}$ & MSCs & Immune modulation \\
\hline $\begin{array}{l}\text { EPO, Ang-1 and - } \\
\text { 2, PDGF, PIGF, } \\
\text { MCP-1 }\end{array}$ & MSCs & Angiogenesis \\
\hline SDF-1 & MSCs & Chemokine (migration) \\
\hline IL-8, CFH, MCP-1 & Both & Immune modulation \\
\hline
\end{tabular}


2.2. Human Retinal Pigment Epithelium (hRPE): RPE is the outermost part of retina sandwiched between PRs and choroid as shown in the diagram (Fig.3\&4). This cellular monolayer is comprised of tightly packed highly specialized cuboidal hexagonal pigmented epithelial cells. Inside the eye, RPE has polarized morphology with densely populated pigmented apical microvilli, which embed the OS of neuroretinal PRs (cones and rods) (Fig.3\&4) for efficient physicochemical communication between them. Basolateral infoldings towards choriocapillaris which help RPE to attach to the Bruch's membrane (BM) (in vivo natural Extracellular Matrix (ECM) for RPE) and to facilitate molecular transport between RPE and blood circulation by increasing the contact surface area between RPE and BM. One RPE cell is connected to around 20 PRs (PRs) (Sparrow et al. 2010). RPE cells are connected to each other by tight junction (Zonula occludens; ZOs) making this sheet impermeable to any macromolecule (outer blood retinal barrier function) and hence help in maintaining immune privilege within the eye (Rizzolo et al. 2011, Rizzolo 2007). In the course of retinal development both RPE and PRs are strictly dependent on each other but in case of selective loss of RPE, PRs in neuronal retina is the most affected tissue (Marmorstein 2001, Marmorstein et al. 1998, Raymond, Jackson 1995, Rizzolo 1997). Furthermore, RPE plays various crucial roles that are indispensable for structure, function and survival of neural retina as well as choriocapillaris and endothelium as shown in the pictorial summary of RPE functions in Fig.4 (Boulton, Dayhaw-Barker 2001, Strauss 2009, Strauss 2005). Pigment of RPE such as melanin and others absorb extra scattered light to improve spatial resolution in vision as well as to protect retina against photo-oxidation. It maintains the PRs excitability by digesting shed POS and by taking up the all-trans retinol and converting it to the 11-cis retinal through the visual cycle enzymes (LRAT, RPE65, 11-cis RDH, CRALBP and CRBP) and returning the 11-cis retinal back to the PRs (Strauss et al. 1998, Nandrot et al. 2012, Finnemann 2003, Gal et al. 2000, Karl et al. 2008, Kevany, Palczewski 2010). It transports nutrients e.g. retinal, glucose, $\omega-3$ fatty acid from blood to PRs (Ban, Rizzolo 2000, Bazan et al. 1994), ions, water and metabolite end products e.g. lactic acid (Hamann 2002) from subretinal space to blood. In this way it maintains chemical homeostasis in the subretinal space to keep the functional state of PRs. It secrets several immunosuppressive factors like IL-8, MCP-1, CFH, TGF- $\beta$ and others to establish immune privilege inside the eye (Kehrl et al. 1986a, Kehrl et al. 1986b, Ishida et al. 2003, Streilein et al. 2002, Streilein 2003). RPE secretes some other cytokines, growth factors and immune modulators (PEDF, VEGF, PDGF, LDGF, BDNF, NGF, HGF, NT-3, FGF-1/2/5, ATP, IGF-1, TIMP3, CNTF, GAS-6, FAS-L) towards apical (PEDF) and basolateral (VEGF) side to stabilize PRs as well as choroids and endothelial cells (Holtkamp et al. 2001, Holtkamp et al. 1998, Bharti et al. 2006, Matsui et al. 2001, Campochiaro et al. 1996, Dawson 1999, Ogata et al. 2001). The summary of these 
factors with their biological activity is given in the table 1 . From the above summary of RPE functions (also depicted in Fig.4), it is clear that this non-neuronal layer of retina plays extremely important roles to maintain the structural and functional integrity of neuronal retina as well as choriocapillaris.

\subsubsection{Ageing of hRPE}

The hRPE is a sheet of non-dividing terminally differentiated cells. With increasing age it accumulates random damage from variety of direct environmental insults (e.g. age, oxidative stress, smoking, light exposure etc.) and undergoes numerous structural and biochemical changes inside RPE, which are generally pathologic to the visual function (Beatty et al. 2000, Cai et al. 2000, Boulton, Dayhaw-Barker 2001, Boulton et al. 2004b). Structural changes in RPE include loss of shape (polarity), hyperplasia, atrophy, and decrease in cell density usually by up to $0.3 \%$ per year (Panda-Jonas et al. 1996, Boulton et al. 1990, Boulton et al. 2004b). Lipofuscin (an RPE senescent marker; autofluorescent lipid protein aggregate) accumulation within lysosome in the basal side of RPE, which fills the entire cytoplast by the $5^{\text {th }}$ decade of life is due to inability of complete digestion of POS and normal metabolic tear and wear resulting from oxidative stress (Eldred, Katz 1988, Feeney-Burns et al. 1984, Feeney 1978, Sparrow, Boulton 2005, Weiter et al. 1986b, Kennedy et al. 1995, Boulton et al. 2004a, Boulton et al. 2004b). It is also commonly called as an age pigment/senescent marker; it contains 10 or more fluorophores (golden yellow fluorescence), which facilitate clinical monitoring (Eldred, Katz 1988, HaralampusGrynaviski et al. 2003, Bindewald et al. 2005, Delori et al. 1995, Delori et al. 2001, Holz et al. 2001, von Ruckmann et al. 1995). The increase in pigment complexes is associated (e.g. melanolipofuscin, melanolysosomes) with decrease in melanosome number in RPE (an event in RPE senescence) with increasing age (Boulton et al. 1990, Weiter et al. 1986a, Feeney-Burns et al. 1984). Polycyclic advance glycation endproducts (AGEs) accumulate inside RPE and interfere in the functions of lysosome and enhance RPE cell death by apoptosis, and are reported to be associated with lipofuscin accumulation (Schutt et al. 2003, Wassell et al. 1999, Holz et al. 1999, Mahaffy et al. 2003) . RPE ageing is also been associated with pathological rounded yellowish extracellular deposits (called drusen) beneath the RPE and within BM, which are considered as a hallmark of AMD (Fig.2A) (Buschini et al. 2011, Mullins et al. 2000, Gass 2003, Gass 1972, Abdelsalam et al. 1999, Russell et al. 2000). Besides these other age related changes include, decrease in level of vitamin $\mathrm{E}$, decrease in phagocytosis, increase in catalase activity due to $\mathrm{H}_{2} \mathrm{O}_{2}$-induced oxidative stress, telomere loss, decrease in melanin content, altered gene expression due to accumulation of mutation, mitochondrial DNA deletion and rearrangement, altered cell- 
ECM interaction etc (Ambati et al. 2003, Wallace et al. 1999, Robert 1998, Yu et al. 1997, Wassell et al. 1999, Holz et al. 1999). All these age related changes (mostly pathologic) in RPE, make RPE non-functional and promotes RPE cell death and simultaneously also affect BM very adversely, which together lead to progressive pathogenesis of AMD.

3. Stem cell: In 1908, the term "stem cell" was proposed for scientific use by a Russian histologist Alexander Maksimov at the Congress of hematologic society in Berlin. Stem cells are present in all multicellular organisms and have capacity to self-renew perpetually and can differentiate to form any mature terminal tissue or cells upon getting appropriate cues. They do self renewal constantly and maintain their undifferentiated state by repeatedly undergoing stochastic cell division without any senescence or telomere shortening. One stem cell produces one mother cell (stem cell) and another some more differentiated stem cells by asymmetric cell division under developmental pathway inside the body. This goes further in the same manner till terminal differentiated cells as depicted in the figure 5. Cell potency of stem cells means capacity to differentiate into different types of terminal cells. According the potency of stem cells they are termed differently as follows: A) Totipotent/Omnipotent cells are present in the embryo until the 8-cell stage or zygote itself and can give rise to embryo or extra embryonic cells hence can form complete new individual if provided with appropriate maternal support. B) Pluripotent cells are present in the internal cell mass of the blastocyst or in the higher stage of morula. They can differentiate into all multiple-cell types representative of all 3 embryonic germ layers. C) Multipotent stem cells are present in all tissues of an organism, which are committed to ectodermic, mesodermic, or endodermic differentiations for maintaining the organ tissue homeostasis. They can give rise to multiple cell types of closely related family of cells. D) Oligopotent stem cells can differentiate into only few cells such as myeloid and lymphoid stem cells. E) Unipotent stem cells can give rise to only one cell type such as all progenitor or precursor cells. F) Nullipotent cells have no capacity of differentiation to any cell type but they may self-renew or propagate.

The property of stem cells can be demonstrated by clonogenic assay, in which single cells are assessed for their differentiation and self renewal capacity (Friedenstein et al. 1974, Friedenstein et al. 1976). On the basis of origin, the stem cells are divided into three categories: a) embryonic stem cells (ESCs) derived from inner cell mass of blastocyst an early stage embryo. The hESCs can give rise to any of the 200 or so somatic cells found in the human body; b)adult stem cell derived from non-embryonic origin from any part of the body; c)induced pluripotent stem cells (iPSCs) derived by transforming any somatic 
cells by genetic reprogramming through genetic engineering method. The iPSCs are equivalent to ESCs regarding pluripotency.

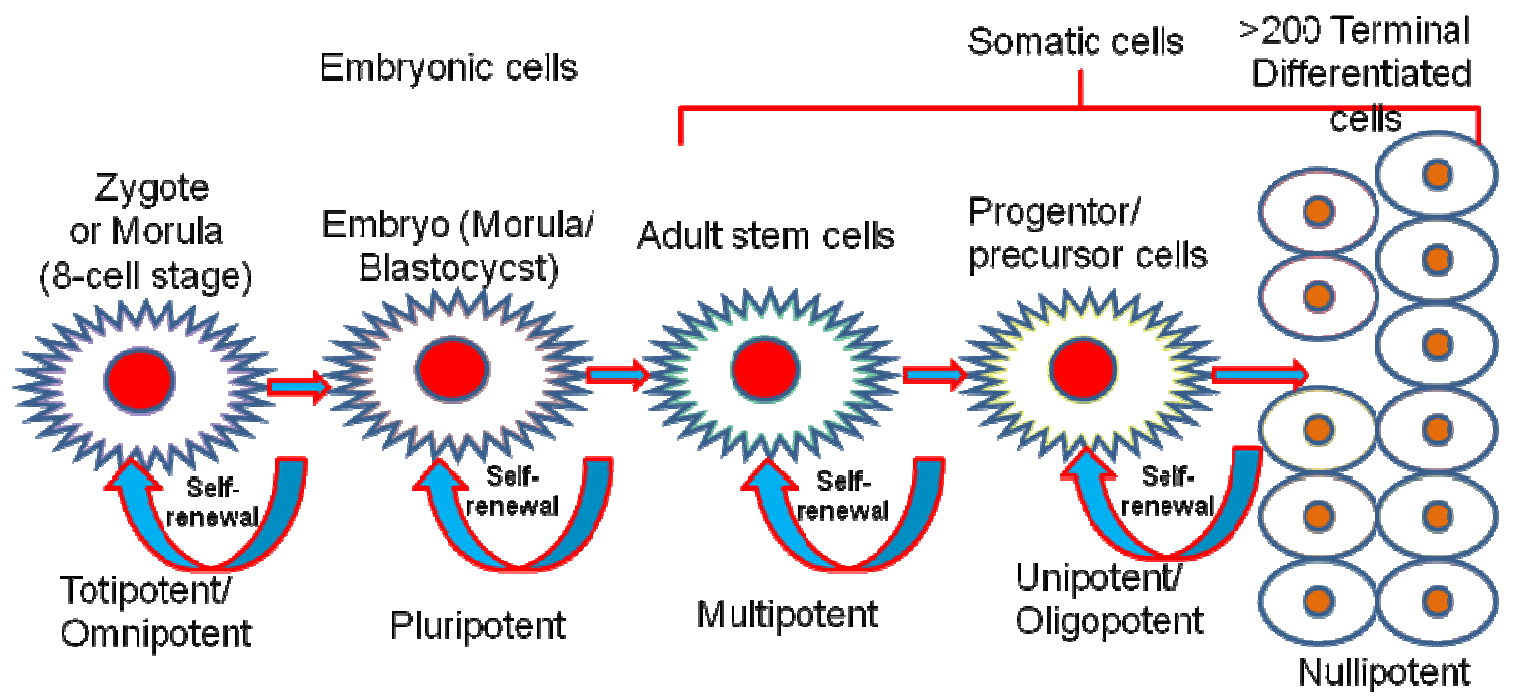

Fig 5: Schematic representation of the functional potency and developmental pathway of stem cells. The turning arrow indicates the clonogenic self-renewal capacity of stem cells (self sketched).

\section{Advance therapy}

Unlike traditional drug therapy, advance therapy includes gene therapy, somatic cell therapy, tissue engineering or regenerative medicine. It may include as well personalized treatments and nanomedicine. The legal definitions and regulations have been formulated in European pharmaceutical legislation approved in 2001, 2004, 2007 and 2009 by the European Parliament [EC/83/2001, EC/726/2004, EC/1394/2007, EC/219/2009 (European Commission News 2013b, European Commission News 2013c) ].

The term 'regenerative medicine' was first coined by William Haseltine (founder of Human Genome Sciences). Now it is very frequent used term in the field of the basic science keeping the same meaning and sense. Although there are lots of definitions of regenerative medicine by different researchers but there is one extensive definition mentioned by Daar et al., which envisage almost all the aspects properly as follows: Regenerative medicine is an interdisciplinary field of research and clinical applications focused on the repair, replacement or regeneration of cells, tissues or organs to restore impaired function resulting from any cause, including congenital defects, disease, trauma and ageing. It uses a combination of several converging technological approaches, both existing and newly emerging, that moves it beyond traditional transplantation and replacement therapies. The approaches often stimulate and support the body's own self-healing capacity. These approaches may include, but are not limited to, the use of soluble molecules, gene therapy, 
stem and progenitor cell therapy, tissue engineering and the reprogramming of cell and tissue types (Daar, Greenwood 2007, Mason, Dunnill 2008a). In this definition, regenerative medicine includes: cell, tissue and organ transplantation; stem cell biology; tissue engineering; material science or nanomedicine; genetics and molecular biology; developmental biology. They have huge potential to offer groundbreaking new treatment opportunities for many incurable diseases and injuries.

\subsection{Types}

Advanced therapies are different from conventional therapies, which are based on chemicals or proteins. According to the European Medicine Agency (EMA) of European Union, advance therapy has been categorized in three main groups (European Commission News 2013a).

Gene therapy: This involves manipulation of genes that lead to therapeutic effect. Gene therapy is used to treat a variety of diseases, including genetic disorders, cancer or longterm diseases by inserting 'recombinant' genes into cells. A recombinant gene is a piece of DNA which is created in the laboratory by ligating the DNA pieces from different sources. Gene therapy medicinal products contain genes, administration of which into the human body, serves the therapeutic, prophylactic or diagnostic effect via expression of inserted recombinant nucleic acid. Usually new genes are added to the patient's cells using modified virus vectors (commonly used vector-harmless adeno-associated virus -AAV) or non-viral vectors like lipoplexes, polyplexes and dendrimers to replace missing or defective copies, for restoring or imparting new functions of genetic origin to overcome a disease.

In the Western world, glybera is the first of its kind of gene therapy treatment, which was approved by EMA on Nov 2nd, 2012 for a disease named Lipoprotein Lipase Deficiency (LPLD) (also known as type 1 hyperlipidemia, primary hyperlipoproteinemia or familial (hyper) chylomicronemia) caused by various mutations in one gene (Yla-Herttuala 2012, Gruber 2012). Although the result of Glybera is disappointing as it did not cure the disease but the milestone achieved in the era of gene therapy is really encouraging for other disease and also gave several lessons for the next step in gene therapy treatment (Bryant LM 2013). First global approval on gene therapy was done recently on May 29, 2013, by U.S. Food and Drug Administration (FDA) for Trametinib and Dabrafenib for unresectable or metastatic BRAF-Mutated (a proto-oncogene known as serine/threonine kinase B-Raf (Rapidly Accelerated Fibrosarcoma)) Melanoma (U.S.Food and Drug Administration 2013, 
U.S.Food and Drug Administration May 29, 2013, U.S.Food and Drug Administration May29, 2013).

Gene therapy in ophthalmology seems very promising because there is a significant advantage to use gene therapy in the eyes. First eye offers reduced chances of vector rejection due to immune privileged organ while in other cases, vectors are rejected rapidly; second it is relatively easy to monitor eye through electroretinography, visual acuity, optical coherence tomography (OCT), fundus photography and ophthalmoscopy than the other organs. According to eyeGene National Ophthalmic Disease Genotyping Network, to date, more than 110 ocular disease genes are identified, which are associated with 34 ocular disorders. There are 17 registered clinical trials, out of which 11 are under recruiting phase associated to retinal degeneration including AMD directly or indirectly and rests are either completed or unknown status or not active (ClinicalTrials.gov page by using the search term 'gene therapy in age related macular degeneration' accessed on $18^{\text {th }}$ Dec, 2013). The two completed clinical trials used the gene for VEGF receptor, sFlt-1 and PEDF by Genzyme (NCT01024998) and GenVec (NCT00109499) respectively but the results are not published yet.

Somatic cell therapy/Cell therapy: Somatic cell therapy includes use of humans somatic cells, that may be autologous/syngenic (from the patient), homologous/allogenic (from another person) and heterologous/xenogeneic (from animal), biological characteristics of which have been substantially altered by different manipulations to obtain a desired therapeutic, diagnostic or preventive metabolic effects. This manipulation includes the expansion or activation of autologous/syngeneic cell populations (e.g. Adoptive immunotherapy), ex vivo or in vivo use of allogeneic and xenogeneic cells associated with medical devices used e.g. microcapsules, biodegradable or nonbiodegradable intrinsic matrices and scaffolds.

Tissue engineering: This involves cells or tissues that have been modified so that they can be used to repair, regenerate or replace portion or whole tissue such as bone, cartilage, blood vessels, skin, bladder, muscle etc.

The term regenerative medicine sometimes used vaguely with tissue engineering, although those involved in regenerative medicine pay more emphasis on the use of stem cells to produce tissues (Kaiser LR, 1992). Tissue engineering is the use of combination of engineering and material science with genetics, molecular cell biology, cell biology and developmental biology in organ transplant and regeneration (Langer, Vacanti 1999). Engineered tissue must be easily transplantable and capable of being integrated 
structurally, mechanically and functionally to the transplantable site in the host. For making particular tissue, two things are important: source of cells and support (extracellular structure). Cell source must have the capacity to offer the fresh and healthy alternative to the diseased and damaged tissue.

The biomaterial for the tissue engineering must be biocompatible e.g. the degree of biodegradability must be matched with the in vivo tissue structure and ECM development and must be friendly enough to the cells to be carried over it. So the cells must remain to the desired phenotype on these biomaterials. Various biomaterials may be divided according to the use and structure of the tissue to be engineered like porous 3D scaffold, sheet like structure, cell encapsulating hydrogels or injectable hydrogels or microsphere as cell carriers. AD-MSCs engineering is a one of the example where researchers are trying to make soft tissue (adipose tissue) for reconstructive and plastic surgery resulting from postoperative, congenital or post traumatic loss of the subcutaneous fat layer (Patrick et al. 1999, Patrick et al. 2002, Bauer-Kreisel et al. 2010). A variety of biomaterials like poly(lactic-co-glycolic) acid (PLGA), collagen/gelatin, ester of hyaluronic acid (HYAFF), silk, polyethylene terephthalate (PET), polyethylene glycol (PEG) based hydrogels, fibrin, hyaluronan based hydrogels with different degree of amidation (HYADD3, HYADD4), matrigels, genetically engineered biomaterials (hyaluronic acid and fibrin tissue glue, elastin like polymer with bioactive domain of RGD, laminin etc.) and others have been used for research for making tissue constructs (Bauer-Kreisel et al. 2010, Singh et al. 2013b, Srivastava et al. 2011a, Allan 1999). The specific requirements for biocompatibility vary according to the specific application and site of implantation. For stable tissue integration surface adhesion property of biomaterial is very much required while in case of fluid containing application (blood vessels) the adhesion property significantly impairs its use. Biocompatible means it must be biologically compatible for the specific use in different site with desirable physiological and biological functions. Depending on the site and function of implant or device, bio-inert or bioactive materials are required. Engineered replacement tissue constructs have been under development for use in a variety of tissues e.g. skin, cartilage, nerve, liver, kidney, muscle, cardiac muscle and valves, blood vessels and soft tissues (Bauer-Kreisel et al. 2010, Yannas et al. 1982, Bell et al. 1981, Cao et al. 1997, Cao et al. 2005, Baiguera et al. 2012, Baiguera et al. 2010, Davis, Vacanti 1996, Humes 1996, Okano, Matsuda 1998, Shinoka et al. 1996, L'Heureux et al. 1998, Zhao et al. 2012, Grayson et al. 2011, Syedain et al. 2011, Allan 1999, Franquesa et al. 2013, Lu et al. 2001).

For RPE transplantation in retinal degenerative diseases, the RPE substrate (ECM) must resemble like BM (natural in vivo substrate for RPE). The substrate must be flexible and 
porous enough to allow the nutrients and oxygen to pass across it. It must have appropriate thickness to make the cells accessible to the nutrient and oxygen source e.g. cells are unable to survive over the ECM of thickness more than $500 \mu \mathrm{m}$ from the diffusible nutrient source (blood, aqueous, synovial or cerebrospinal fluid) (Sittinger et al. 1996). Tissue engineering hopes to solve the problems of biocompatibility of ECMs or scaffolds, tissue supply problems, rejection problems, problems of iatrogenic disease transmission etc. soon within few decades.

\section{Cell therapy}

The cell therapy is considered as the fourth and most recent emerging therapeutic pillar of global health care (Mason, Dunnill 2008b). According to Spanish Ministry of Health and Consumer, cell-based therapies cover a wide range of cell therapies, including somatic cell and tissue-engineered products that have been manufactured from a patient's own cells (autologous), donor cells (allogeneic) or animal cells (xenogeneic). Cells have usually undergone substantial manipulation and could be used to perform different essential function.

Cell therapy can be divided into two branches 1) Somatic cell therapy or cell replacement therapy; 2) Stem cell therapy or regenerative medicine. While the former one just uses any terminally differentiated healthy somatic cells to replace the disease and damaged cells in the body, the later uses any type of stem cells. Stem cells are different from somatic cells as they have capacity to self renew constantly and have the capacity to form any somatic cells upon induction. There are usually two main types of strategies under cell therapies: (1) those focused at restoring structure and function of specific cells or tissues by using cells or genetically modified cells; (2) those directed to restore functional homeostasis by taking the advantage of trophic, immunomodulatory, other biochemical effects of transplanted cells. However there is functional overlap between these two types of applications. Fresh healthy somatic cells and stem cells of all types come under first type and the use of immune cells or mesenchymal stem cells comes under the second type.

\subsection{Cell therapy progress in ophthalmology}

Presently there is a significant attention towards using stem cell therapy against AMD, which includes the possibility to use retinal progenitor cells, ESCs, iPSCs, adult stem cells. Theoretically these cells can be used to replace RPE and PRs in AMD patients and a number of in vitro and in vivo animal studies have shown significant successes towards it. 
One of the major reasons of neuroretina degeneration in AMD is senescent, nonfunctional and damaged RPE. Cell therapy is focused at replacing this deteriorated RPE with fresh and healthy one. Delay in photoreceptor degeneration and improvement in visual function has been reported in various autologous/syngenic, homologous/allogenic, and heterologus/xenogenic-transplantation of RPE in animal models (Gouras et al. 1989, Lopez et al. 1989, Li, Turner 1988, Gouras et al. 1984, Girman et al. 2005, Girman et al. 2003, Gias et al. 2007, Yamamoto et al. 1993, Sauve et al. 1998, Sheedlo et al. 1989, Lane et al. 1989), which finally led to several clinical trials using fetal and adult homologous RPE transplant (Algvere et al. 1999, Weisz et al. 1999, Del Priore et al. 2001, Peyman et al. 1991, Valtink et al. 1999, Algvere et al. 1994, Algvere et al. 1997, Gouras, Algvere 1996). Rejection was reported in almost all cases. To avoid immune rejection problems, autologous adult RPE and IPE cells transplant have been tried by several groups and fetched some temporary beneficial effects (Binder et al. 2002, Binder et al. 2004, Binder et al. 2007, Krebs et al. 2008, Ma et al. 2009, Thumann et al. 2000, Abe et al. 2000a, Abe et al. 2000b, Abe et al. 2007, Stanga et al. 2001, Stanga et al. 2002, van Meurs, Van Den Biesen 2003, Caramoy et al. 2010). In case of autologous source, RPE carries the same level of genetic and environmental abuse due to ageing, which limits the use of autologous source in RPE transplantation. Some groups also transplanted the whole fetal retina with RPE in animal model and even in human successfully and got some encouraging results (Aramant et al. 1999, Aramant, Seiler 2004, Aramant, Seiler 2002a, Aramant, Seiler 2002b, Radtke et al. 1999, Radtke et al. 2008, Radtke et al. 2002, Seiler, Aramant 2012). Getting fetal retina for transplantation is very complicated issue. Due to supply issue of fresh retinal tissue, the cell therapy towards AMD is shifting rapidly to new promising source like stem cells transplant. Stem cell therapy has been used in several animal models for retinal degenerative diseases (Lund et al. 2001b, Lund et al. 2001a, Lund et al. 2007, Arnhold et al. 2006, Arnhold et al. 2007).

Human embryonic stem cells (hESCs) have the potential to differentiate into all kinds of cell and are considered as an unlimited source of cells for cell replacement therapies. The hESCs have been used to differentiate into RPE under guided conditions by several researchers, where they got significant success in getting functional RPE, Retinal Progenitor Cell (RPC) and PRs (Vugler et al. 2008, Haruta 2004, Klimanskaya et al. 2004, Idelson et al. 2009b, Corneo, Temple 2009, Carr et al. 2009b, Lamba et al. 2006, Meyer et al. 2009, Clarke et al. 2012). The RPE cells derived from ESCs have been used by several labs in experimental animal models and got interesting positive results in terms of PRs rescue and improve in visual functions (Lund et al. 2006, Lu et al. 2009, Vugler et al. 2007). This led to the currently seven registered clinical trials in which one is closed and rests are 
under recruiting phase (accessed clinicaltrials.gov on $18^{\text {th }}$ Dec, 2013 by search term 'Embryonic stem cells + Age Related Macular Degeneration'), where they are using ESCs derived RPE cells for RPE transplantation, among them three are directly in AMD (NCT01344993, NCT01674829, and NCT01691261 (Schwartz et al. 2012)). A critical issue related to the use of hESCs, however, is the risk of teratoma formation after transplantation (Arnhold et al. 2004, Chaudhry et al. 2009). To avoid complicated ethical quandaries and other complications like widely reported tumorigenicity (Blum, Benvenisty 2008, Cao et al. 2007, Blum, Benvenisty 2007, Nussbaum et al. 2007) and availability issues associated with hESCs, some researchers used iPSCs for obtaining RPE and other retinal cells like RPCs and PRs and they obtained interesting results (Bharti et al. 2011, Barbara 2009, Boucherie et al. 2011, Buchholz et al. 2013, Jin et al. 2009, John et al. 2013, Kuroda et al. 2012, Mekala et al. 2013, Kokkinaki et al. 2011, Lamba et al. 2010, Parameswaran et al. 2010, Meyer et al. 2011). The RPE and other retinal cells derived from iPSC also rescued PRs degeneration and improved visual function in animal model (Wang et al. 2010a, Carr et al. 2009a, Tucker et al. 2011, Lamba et al. 2010, Meyer et al. 2011). Although there is a significant advancement in the use of hESCs and iPSCs for retinal degenerative disease, still they carry the issue of ethical problems and teratoma formation and issue of safety. Thus adult stem cells like MSCs, which lack these concerns, could be a promising source for obtaining new and fresh RPE.

\section{Mesemchymal Stem Cells (MSCs)}

Mesenchymal stem cell also known as mesenchymal stromal cell or marrow stromal cell (all abbreviated to MSC) has been defined by the International Society for Cellular Therapy as multipotent adult stem cells which are (Horwitz et al. 2005): (i) plastic-adherent under standard culture conditions; (ii) positive for expression of stem cell markers CD105 (endoglin), CD73 (5'-nucleotidase/ecto-5'-nucleotidase-5'-NT), and CD90 (Thy1) and negative for expression of hematopoietic cell surface markers CD34, CD45 (protein tyrosine phosphate receptor type c-PTPRC), CD14, CD11b (Integrin alpha M-ITGAM), CD19, CD79a, and HLA-DR (MHC class II surface receptor molecule); (iii) under specific stimulus, these cells can differentiate into osteocytes, adipocytes, and chondrocytes under in vitro conditions (Dominici et al. 2006, Horwitz et al. 2005, Gimble et al. 2007). Other markers used for marking MSCs are Stro-1, Sca-1(stem cell antigen-1), PDGFR $\alpha$ (Plateletderived growth factor receptor $\alpha$ ), nestin, CD29, CD144, CD146, CD-166, CD-115, CD44, CD49a-f, CD51, CD106, HLA-ABC (Phinney, Prockop 2007, Kalinina et al. 2011, Morikawa et al. 2009, Mendez-Ferrer et al. 2010). MSCs have also been found to express stem cell markers including Oct4, Rex1, and Sox2 (Izadpanah et al. 2006). MSCs reside in the 
connective tissue of most organs and have ability to migrate to the site of injury and tumor, especially to hypoxic, apoptotic and inflamed areas (homing capacity) and can alter microenvironment via secretion of soluble factors. MSCs are not as plastic as ESCs or iPSCs but they have been differentiated into a variety of terminal differentiated cells. Due to lack of tumorigenicity and ethical problems, MSCs' plasticity, immunomodulatory properties, homing capacity, paracrine effects have created much excitement giving hope for their therapeutic use in wide range of diseases.

\subsection{Sources of MSCs}

Almost all organs contain niche of mesenchymal stem cells to maintain the cellular homeostasis inside that organs (da Silva Meirelles Lindolfo et al. 2006). First time, MSCs were isolated from guinea pig bone marrow by Friedenstein and colleagues in 1970 (Friedenstein et al. 1970). Till date, successful isolation of MSCs have been reported from several sources like bone marrow, adipose tissue (lipoaspirate), dental pulp of deciduous teeth, periosteum, placenta, amniotic fluid, umbilical cord blood, hair follicle, skeletal muscle, synovium, brain, amniotic fluid, thymus, liver, spleen, pancreas, aorta, vena cava, kidney, kidney glomeruli, lungs, cornea, retina, ciliary body etc (In 't Anker et al. 2004, Bieback et al. 2004, Bieback, Brinkmann 2010, Kern et al. 2006, Wagner et al. 2005, Sethe et al. 2006, Prockop 1997, Beyer Nardi, da Silva Meirelles 2006, Bianco, Gehron Robey 2000, Zarnett, Salter 1989, Wickham et al. 2003, Tuli et al. 2003, Noth et al. 2002, Noort et al. 2002, Nakahara et al. 1990, O'Driscoll et al. 2001, Miura et al. 2003, Sottile et al. 2002, De Bari et al. 2001, De Ugarte et al. 2003, Dragoo et al. 2003, Fukumoto et al. 2003, Gronthos et al. 2001, Jankowski et al. 2002). Although bone marrow source remains primary source of MSCs for most preclinical and clinical studies, fat sources are gaining more momentum day by day due to its ease and abundance of isolation.

\subsection{Adipose tissue}

Adipose tissue is the most abundant in human body comprising $10-29 \%$ of the body mass in normal adult human (Kahn 2008). It has two major forms: brown adipose tissue (BATgood fat), which is thermogenic, generating heat through uncoupling protein (UCP) by short circuiting mitochondrial pH gradient, mainly present in new born babies and white adipose tissue (WAT-bad fat) store energy, maintain body contours providing mechanical protection and insulation and represents a source of multiple autocrine, paracrine, and endocrine factors such as proteins (adipokines), fatty acid, steroid hormone and prostaglandins (Gimble et al. 2013, Guerre-Millo 2002). All metabolic hormones like adiponectin, leptin, resistin and others together secreted by adipocytes are called 
adipokines. WAT is distributed throughout the body mainly in intra-abdominal depots around the omentum, intestine and perirenal area as well as in subcutaneous depots in buttock, thigh and abdomen (Gesta et al. 2007). On cellular level WAT consists of mature adipocytes (containing large lipid droplets which is up to $90 \%$ of total cytoplasm components), endothelial cells, pericytes, smooth muscle cells, fibroblasts, committed adipose progenitor cells (preadipocytes) and mesenchymal stem cells in the stromal vascular fractions of the tissue, out of which only mesenchymal stem cells and preadipocytes are capable of further proliferation and differentiation under proper conditions (Bauer-Kreisel et al. 2010). The AD-MSCs can be extracted from the human lipoaspirates with 99.99\% purity obtained from donor's cosmetic surgery byproducts. Although AD-MSCs have propensity to differentiate into mesodermal lineage but they can give rise to several types of cells (Boquest et al. 2005).

Several different terms with merits have been used to describe plastic adherent cell populations isolated from collagenase digest of adipose tissue such as adipose derived mesenchymal stem/stromal cells (AD-MSCs); adipose derived stem/stromal cells (ASCs); adipose tissue derived stem cells (ADSCs); adipose-derived adult stem/stromal (ADAS) cells; adipose-derived adult stromal cells; adipose-derived stromal cells (ADSC); adipose stem/stromal cells (ASC); adipose mesenchymal stem cells (AdMSC); preadipocytes; processed lipoaspirate (PLA) cells; adipose-derived stromal/stem cells (ASCs); lipoblast; pericyte, fat stem cell, fat stromal cells. International Federation for Adipose Therapeutics and Science (IFATS) recommends for using the term 'adipose derived stromal cells (ASCs)' (Gimble et al. 2007). However we have been using the term Adipose tissue Derived Mesenchymal Stem Cells (AD-MSCs) since the beginning and maintaining the same term throughout the thesis. The origin of AD-MSCs may be bone marrow as the AD-MSCs are considered to be colony forming unit fibroblast (CFU-F) derived from the bone marrow and distributed through circulation (Castro-Malaspina et al. 1980, Crossno et al. 2006, Hausman, Hausman 2006). The stem cell recovery and their differential potential depend upon the site of extraction of lipoaspirate and the age of donors (Schipper et al. 2008). The arms have been found to give higher yield of MSCs than that of thigh, abdomen and breast (Schipper et al. 2008).

Bone marrow stem cells (BM-MSCs) is the most traditional and principal MSCs used in research and clinics. In spite of most frequent use of BM-MSCs, AD-MSCs are gaining more momentum in clinical and basic research gradually due to lots of advantage of the later over the former. Both BM-MSCs and AD-MSCs show considerable similarity in respect of morphology, marker gene expression, gene expression profile and cytokine profile (De Ugarte Daniel a. et al. 2003, Kilroy et al. 2007, Lee et al. 2004a, Lee et al. 2004b). MSCs can 
be expanded in culture for 15-40 population doublings, period during which subpopulations of cells can undergo localized changes in DNA copy number (demonstrated by comparative genomic hybridization) and changes in epigenetic DNA patterns (methylation patterns) (Meza-Zepeda et al. 2008, Dahl et al. 2008). Interestingly, these analysis shows that BM-MSCs seem more prone to genetic alterations than AD-MSCs. BM-MSCs demonstrated better differentiation towards bone and cartilage whereas slightly better differentiability towards adipocytes is attributed to AD-MSCs (Liu et al. 2007). Bone marrow stem cells are typically obtained from the mononuclear layer of bone marrow after separation by density gradient centrifugation and give rise to two stem cell populations: adherent (MSCs) and non-adherent (hematopoietic cells) stem cells (Colter et al. 2000, Ogawa 1993). Due to considerably low frequency of MSCs present in bone marrow, extensive ex-vivo expansion of BM-MSCs is required to obtained clinicaly significant number (Fraser et al. 2006). Additionally, bone marrow harvest is relatively inconvenient for the donor than that of liposuction or abdominoplastic procedure (Auquier et al. 1995). Moreover the removal of extra fat seems more desirable and attractive option for the donor than the painful bone marrow harvest and has easier accessibility and minimum morbidity (Bauer-Kreisel et al. 2010). Adipose tissue can be obtained in large quantity and yields large number of stem cells compared to bone marrow aspirates (Bauer-Kreisel et al. 2010). Clonogenic assay for fibroblast like colonies (CFU-F) give a frequency of 1-3\% AD-MSCs of the nucleated cells, which is 100-500 times as high as in bone marrow (Fraser et al. 2006). These evidences show that AD-MSCs have significant advantages over BM-MSCs regarding ease of harvest and availability of large number of cells.

\subsection{Therapeutic aspects of MSCs}

Mesenchymal stem cells (MSCs) have received significant attention in recent years due to their huge interest for cell replacement and for their paracrine and immunomodulatory effects in addition to their differentiation potential in advance therapies. These stem cells have been currently used in 414 clinical trials under different phases (414 registered clinical trials using search term 'mesenchymal' 377 registered clinical trials using search term 'mesenchymal stem cells' and 'mesenchymal stromal cells' in ClinicalTrial.gov accessed on 18 ${ }^{\text {th }}$ Dec, 2013) and some of them are approved for final application. All these clinical trials are in the area of Myocardial Infarction (ca 23\%), Graft Versus Host Disease (ca 16\%), Diabetes (ca 10\%), Liver Cirrhosis (ca 10\%), Spinal Cord Injury (ca 9\%), Osteoarthritis (ca 8\%) and rests around 23\% clinical trials in other diseases e.g. Crohn's disease, Multiple Sclerosis, Aplastic Anemia, Systemic Lupus, Rheumatoid Arthritis, 
Parkinson's disease, Brain Injury etc (Wei et al. 2013). The clinical trials in phase wise are as follows: Phase I (safety studies) - ca 31\%; Phase II (proof of concept for efficacy in human patients) - ca 50\%; Phase III (comparing a newer treatment to the standard or the best known treatment) - ca 15\% Phase IV (Post marketing studies like treatment risks, benefits and optimal use) - ca 4\% (Wei et al. 2013). Out of these 414 trials, 14 trials are related to ocular diseases directly or indirectly and 5 are for retinal diseases (two for Retinitis Pigmentosa). One clinical trial by Retinal Associates of South Florida (NCT01920867) is using autologous BM-MSCs for the condition of Retinal Disease; Macular Degeneration; Hereditary Retinal Dystrophy; Optic Nerve Disease; Glaucoma. The most frequent source of MSCs used in these clinical trials is bone marrow followed by adipose tissue and umbilical cord.

Particularly, AD-MSCs have attracted huge attention in the clinical use due to easier isolation in clinically significant number. They have been expected to fill the gap between the demand and supply of fresh donor cells for cell replacement therapy. In part, these cells rely on their paracrine factors secretion and immunomodulatory function. The current features in favor of use of AD-MSCs as a biological therapeutic for clinical applications include: relative abundance of the source, ease of isolation in clinically significant numbers, culture and preservations, ease of maintenance, high proliferative capacity, presumptive plasticity, immunomodulatory properties, use in allogeneic transplant, paracrine mediated effects, home-in capacity to the site of injury, well characterized and studied, ease of transfection and lack of ethical concerns.

MSCs are present ubiquitously in almost all organs or tissues which renew the mesenchymal lineages both under normal and pathological conditions (Young et al., 1995). In addition to replace the dead and damaged cells, the MSCs may provide as a source of trophic and biochemical positive effects due to capable of releasing lots of immunomodulatory and neuroprotective growth factor molecules (Doorn et al., 2011; Levkovitch-Verbin et al., 2010). There are several growth factors like CNTF, BDNF, IGF1, NGF and bFGF secreted by MSCs which might protect neuroretina and RPE from further degeneration (Labouyrie et al., 1999; Lin et al., 2009).

The reprogramming efficiency of hAD-MSCs have been found 100 times higher than that of fibroblasts for making iPSCs and further this does not require any feeder cells to grow as hAD-MSCs themselves secrete a high level of self-renewal supporting factors like FGF2, LIF, fibronectin and vitronectin (Sugii et al. 2011, Sugii et al. 2010). It certainly extends its therapeutic spectrum further in stem cell therapy. 
Due to high proliferative capacity and long time survival compared with other somatic cells, genetic modification of MSCs is an attractive target for combined stem cell and gene therapy. The transfected MSCs have been demonstrated to express exogenous proteins (e.g. Factor VIII and IL-3) for longer period and maintained this ability even after transplantation in mice (Allay et al. 1997).

\subsection{Immunological properties of MSCs}

There is increasing in vivo and in vitro experimental evidences to accept MSCs as universal donor cells due to absence of class II major histocompatibility complex (MHC class II/HLADR) and other co-stimulatory molecules like CD40-, CD80- (B7-1), and CD86-(B7-2) (Tse et al. 2003). MSCs are not only immune privilege but they can also modulate and suppress the host immune response. So, immunological properties of MCSs can be divided into three categories: 1) Non-immunogenic or hypo-immunogenic 2) Immunomodulation 3) Immunosuppression.

MSCs are considered MHC class I positive and MHC class II negative (Pittenger et al. 1999). Although the presence of MHC class I on the cell surface of MSCs protects it from certain natural killer (NK) cell mediated deletion, it may activate alloreactive $\mathrm{T}$ cells but due to absence of Fas-ligands or co-stimulatory molecules like CD40-, CD80-(B7-1), and CD86(B7-2) on MSCs, a secondary signal do not engage, leaving the T cells anergic. MHC class II is a potent alloantigen and their absence on MSCs makes it non-recognizable from effector CD4+ T cells. Thus immunological characteristics of cell surface of MSCs make them hypoimmunogenic or non-immunogenic.

The immunomodulatory characteristics of MSCs was first recognized in 2000, when Liechty and colleagues found that unique immunologic characteristics of MSCs facilitate their persistence in a xenogeneic environment (Liechty et al. 2000). MSCs have been considered to modulate immune response in both ways by direct cell-to-cell contact and release of soluble immunosuppressive factors (paracrine effects). Pittenger and Aggarwal found that MSCs secrete constitutively prostaglandin E2 (PGE2), which alters the secretion profile of dendritic cells (DCs), naïve and cytotoxic T lymphocytes, and NK cells by inhibiting the TNF- $\alpha$ and interferon- $\gamma$ (IFN- $\gamma$ ) and by stimulating interleukin-10 (IL-10) secretion to modulate the immune cell response (Aggarwal, Pittenger 2005, English et al. 2009). MSCs inhibit the differentiation, maturation and migration of several antigen presenting cells (APCs), suppress B-cell, T-cell and NK cell proliferation, activation, differentiation, induce suppressor $\mathrm{T}$ cell formation and alter expression of several receptors necessary for antigen capture and processing (Aggarwal, Pittenger 2005, Atoui 
et al. 2008, Di Nicola et al. 2002, English et al. 2009, Augello et al. 2005, Asari et al. 2009, Sotiropoulou et al. 2006, Spaggiari et al. 2008, Zhang et al. 2004, Chen et al. 2007, Zhang et al. 2009).

MSCs have been found to express mRNA of IL-1, $-6,-7,-8,-10,-11,-12,-15,-27$, leukemia inhibitory factor (LIF), macrophage colony stimulating factor (M-CSF), stem cell factor, some of which could play role in creating immunosuppressive environment (Rasmusson 2006, Aggarwal, Pittenger 2005). MSCs express constitutively IL-10, which play very important role in $\mathrm{T}$ cell regulation which promote suppressor phenotype and also antagonize the IL-2 activity during inflammatory response (Aggarwal, Pittenger 2005). There is evidence that MSCs interact directly with T cells to suppress alloreactivity and direct CD4+ T cells to suppressive phenotype (Di Nicola et al. 2002, Tse et al. 2003). MSCs have found to evade NK-cell targeting mechanism by inhibiting the formation of CD8+ $\mathrm{T}$ cell (Glennie et al. 2005). MSCs appear to reduce IL-2-induced NK-cell proliferation, and INF- $\gamma$ production (Kassis et al. 2011, Aggarwal, Pittenger 2005, Rasmusson et al. 2003, Spaggiari et al. 2006, Spaggiari et al. 2008). It has been shown that MSCs prevent maturation, differentiation and function of DCs locking them into immature state by downregulating the expression of CD1a, CD40, CD80, CD86 and HLA-DR (Beyth et al. 2005, Zhang et al. 2004). HGF, NO, HO-1, HLA-G5, indoleamine 2,3-dioxygenase (IDO), IL-6, IL10, kynurenine, and TGF- $\beta$ secreted by MSCs together may contribute local immunosuppressive environment by suppressing T-cell activity (Di Nicola et al. 2002, Rasmusson 2006, Barry et al. 2005, Toubai et al. 2009, Kwidzinski et al. 2005, Kogler et al. 2005, Sato et al. 2007, Meisel et al. 2004, Selmani et al. 2008, Casaroli-Marano et al. 2013, Aggarwal, Pittenger 2005, Le Blanc, Ringden 2005, Uccelli et al. 2008b, Paul, Anisimov 2013, Salgado et al. 2010, Cawthorn et al. 2012). MSCs can also modulate immune response by inhibiting B-cell proliferation, as well as their chemotactic behavior and antibody production (Nauta, Fibbe 2007, Glennie et al. 2005). In brief, MSCs causes suppression function of immune cells in vivo by means of following ways: through the activation and differentiation of T cells, the differentiation of DCs, proliferation of B cells, and by means of cytolytic activity of NK cells. These functions are achieved either by secreting soluble factors like IL-10, PGE2, NO, TGF- $\beta_{1}$, gelectin-1 and -3 or through direct or indirect intercellular contact with immune cells (Uccelli et al. 2008a, Uccelli et al. 2008b, Uccelli et al. 2006a, Uccelli et al. 2007, Uccelli, Prockop 2010, Uccelli et al. 2006b, Sioud 2011). All these evidences explains that why MSCs have been successful to evade, modulate and suppress host immune response in case of solid autologous, homologous and heterologous organ transplant and producing clinical successes in graft-versus-host disease GVHD (Popp et al. 2008, Popp et al. 2009, Eggenhofer et al. 2011, Casiraghi et al. 
2008, Berman et al. 2010, Figliuzzi et al. 2009, Longoni et al. 2010, Ding et al. 2009, Le Blanc et al. 2008, Le Blanc K et al. 2003, Le Blanc et al. 2004).

\subsection{Paracrine effects of MSCs}

MSCs produce large amount of secreted factors (secretome) or paracrine factors like cytokines, chemokines, neurotrophic growth factors, and ECM proteins, which play several important and diverse roles through cross talk between different cell types and is increasingly appreciated for their therapeutic potential. (Salgado et al. 2010, Doorn et al. 2012, Meirelles Lda et al. 2009). These soluble trophic factors play very important roles in reducing tissue damage, in tissue regeneration and repair, inhibiting fibrosis and apoptosis, promoting angiogenesis, stimulating endogenous stem cell recruiting and proliferation and in remodeling immune response (Maumus et al. 2013). The secretome includes angiogenic factors (VEGF, HGF, Ang-1, , bFGF, IGF1, PDGF, PIGF, IL6, EPO, MCP1), antiangiogenic factors (PAIs-1, angiostatin, and thrombospondin), antiapoptotic factors (IGF-1, IL-6, GM-CSF, TGF- $\beta$, bFGF, HGF), hematopoietic factors (M-CSF, ILs, Ang-1, and -2, SCF, TPO, LIF, SDF-1, TGF- $\beta$ ), immunomodulators (IDO, PGE2, HGF, LIF, HLA-G, TGF- $\beta$, TSG-6, NO, HO-1, HLA-G5, IL-10, IL-6), migratory factors/chemoattractants (SDF-1, HGF, VEGF, CCL-2 to -6,-20, LIF, IGF, G-CSF, M-CSF, CXCL-2,-3,-5,-8,-10,-11), anti-fibrotic factors (HGF, bFGF, Ang-1, KGF, MMP-2/9, TIMP-1/2), neuroprotective factors (BDNF, NGF, GDNF, gelectin-1) (Meirelles Lda et al. 2009, Salgado et al. 2010, Doorn et al. 2012, Maumus et al. 2013, Lopatina et al. 2011, Rubina et al. 2009, Cai et al. 2009, Uccelli et al. 2011b, Uccelli et al. 2011a, Puissant et al. 2005, Di Nicola et al. 2002, Aggarwal, Pittenger 2005, Uccelli et al. 2008b, Kogler et al. 2005, Sato et al. 2007, Meisel et al. 2004, Selmani et al. 2008, Le Blanc, Ringden 2005, Paul, Anisimov 2013).

MSCs derived from adipose tissue (AD-MSCs) differ significantly over the secretion of several paracrine factors from the BM-MSCs, which may provide us clue to choose the source for particular therapeutic purpose. AD-MSCs secrete higher level of pro-angiogenic factors like VEGF, HGF, bFGF, Ang-1 and -2 and PDGF making them attractive for cardiovascular disease (Nakanishi et al. 2011, Kachgal, Putnam 2011, Hsiao et al. 2012). AD-MSCs also secrete significantly high amount of several neurotrophic factors like BDNF, NGF and GDNF making them attractive for neurodegenerative disease therapies (Lopatina et al. 2011). There are also in vitro and in vivo evidences which show that AD-MSCs protect neuronal degeneration and slow disease progression in neurodegenerative disease model of Huntington disease (Wei et al. 2009, Lee et al. 2009). 


\subsection{Differentiation potential of MSCs}

In addition to paracrine therapeutic potential of MSCs, they offer significant possibilities in regenerative medicine by giving rise to several terminal differentiated somatic cells. MSCs have been used primarily in the regeneration of bone and cartilage in several clinical trials (Bieback et al. 2012). Use of AD-MSCs in engineering soft tissue transplants for reconstructive and plastic surgery is one of the interesting uses of MSC in tissue engineering (Bauer-Kreisel et al. 2010).

Under normal characterization, MSCs are used to differentiate into mesenchymal lineages including chondrocytes, osteocytes and adipocytes both under in vivo and in vitro conditions shown by multiple laboratories (Dan et al. 2006, Muraglia et al. 2000, Pittenger et al. 1999, Bruder et al. 1998a, Bruder et al. 1998b, Barry et al. 2001, Digirolamo et al. 1999, Johnstone et al. 1998). Apart from this, MSCs including hAD-MSCs have been differentiated into several cell types such as hepatocytes, cardiomyocytes, smooth muscle, skeletal myocytes, hematopeotic supporting cells, endothelial cells, pancreatic cells, neuronal cells, retinal progenitor cells, RPE like cells, corneal epithelial like cells (Banas et al. 2007, Hong et al. 2005, Drosos, Kolios 2013, Chiou et al. 2005, Ong et al. 2006, SanchezRamos et al. 2000, Sato et al. 2005, Seo et al. 2005, Vossmerbaeumer et al. 2009, NietoMiguel et al. 2013, Gupta et al. 2013, Tombran-Tink, Johnson 1989, Anghileri et al. 2008, Zuk et al. 2001, Zuk et al. 2002, Krampera et al. 2007, Timper et al. 2006, Safford, Rice 2005, Talens-Visconti et al. 2007, Talens-Visconti et al. 2006, Mizuno et al. 2002, Lee, Kemp 2006, Moviglia et al. 2012, Zhang, Dong 2008, Huang et al. 2012, Cao et al. 2005, Banas et al. 2009, Konno et al. 2010, Tan et al. 2010, Park et al. 2011). MSCs have also been demonstrated to differentiate into RPE cells, skin epithelial cells, sebaceous duct cells and tubular duct cells in kidney in animal models (Arnhold et al. 2006, Arnhold et al. 2007, Nakagawa et al. 2005, Fu et al. 2006, Morigi et al. 2004, Herrera et al. 2004). These evidences show that MSCs including AD-MSCs have huge potential to give rise different kind of tissues and cells. However, the in vitro multipotency of BM-MSCs gets lost gradually during their cultivation (Muraglia et al. 2000, Tallone et al. 2011). AD-MSCs have been reported to express the cell surface markers of at least five progenitors cells such as sub-endothelial progenitor, supra-adventitional progenitor, transient progenitor, preadipocyte, progenitor and endothelial progenitor cells, on the their cell surface (Tallone et al. 2011). 


\subsection{Current status of MSCs in Ophthalmology}

As mentioned earlier, 14 clinical trials are related to the use of MSCs in ocular diseases directly or indirectly. One clinical trial (NCT01562002) executed by IOBA, use allogeneic BM-MSCs along with amniotic membrane for Limbal Insufficiency Syndrome. Eight clinical trials are associated with retinal diseases directly or indirectly. The use of MSCs in ophthalmology is gaining importance day by day. In ocular surface disease, lots of works have been done on MSCs towards corneal diseases for engineering corneal tissue (McIntosh Ambrose et al. 2010, De Miguel et al. 2010, Arnalich-Montiel et al. 2008). MSCs have shown significant healing capacity in corneal epithelial burns mainly due to antiinflammatory and anti-angiogenic effects (Ma et al. 2006, Oh et al. 2009, Oh et al. 2008). Recently, AD-MSCs have been shown to differentiate into corneal epithelial like cells, which may give hope to fulfill the supply of stem cells in case of limbal stem cell deficiency (Nieto-Miguel et al. 2013). The advances in MSCs research in ophthalmology point preferably towards the back of the eye i.e. retinal diseases based on the preferential use of MSCs in clinical trial in eye diseases. Several reports show that MSCs including AD-MSCs can differentiate partially towards the phenotype of retinal origin cells such as RPE, retinal progenitor cells and PRs under in vitro and in vivo condition (Arnhold et al. 2006, Vossmerbaeumer et al. 2009, Huang et al. 2012a, Moviglia et al. 2012b, Kicic et al. 2003, Tomita et al. 2002, Tomita et al. 2006, Zhang, Dong 2008). MSCs have been found to differentiate into cells expressing PR protein (rhodopsin) when injected into rat subretinal space (Gong et al. 2008b, Castanheira et al. 2008). Epidermal growth factor (EGF) has been found to induce rat MSCs into PRs like cells expressing rhodopsin (Zhang, Dong 2008). MSCs not only differentiate to retinal neuron like cells, they also have been found to rescue and delay retinal degeneration when injected into the vitreous cavity by releasing neuroprotective factors like CNTF, BDNF and bFGF (Li et al. 2009). Subretinal injection of MSCs has been found to rescue and delay the retinal or PR degeneration (Inoue et al. 2007). The systemic intravenous (tail vein) injection of MSCs in RCS rat model of retinitis pigmentosa (RP) has been found to integrate into retina partially and rescued PR loss and preserved visual function (Wang et al. 2010b). This showed the homing capacity of MSCs. So, they can migrate from one part to another part of body especially to repair the damaged tissue through blood circulation. In another example, intravenous injection of genetically engineered mouse BM-MSCs to secrete PEDF reduced the size of neo-vascular complexes induced by retinal laser burns, which point towards the potential of MSCs in CNV treatment (Hou et al. 2011, Hou et al. 2010). MSCs have been shown to express a number of neuroprotective factors like BDNF, CNTF, bFGF, NGF, GDNF, IGF1, gelectin-1 and anti-inflammatory factors (Maumus et al. 2013, Mundra et al. 2013). The neurotrophic 
secretion profile including other anti-inflammatory and immunomodulatory factors of MSCs advocates their potential use in all types of diseases related to neuronal or neuroretinal degeneration including neuroinflammation like panuveitis, glaucoma, retinal degenerative diseases (e.g. RP, AMD etc.) and other neurodegenerative diseases (Joe, Gregory-Evans 2010, Paul, Anisimov 2013). Intravitreal injection of BM-MSCs in glaucoma model of Wistar rat showed significant neuroprotection by increasing ganglion cell survival (Yu et al. 2006a). The therapeutic potential of MSCs in general and in ophthalmology is more attributed to their neuroprotection, immunomodulation, homing capacity, anti-inflammatory and wound healing properties rather than to plasticity.

\section{Factors of differentiation used in this work}

There are four differentiation factors used in this study. Their roles in various differentiation processes and other physiological process are described here briefly.

Vasoactive intestinal peptide (VIP), a neuropeptide belonging to the VIP-glucagon growth hormone release secretin superfamily, is found in the retina (amacrine and displaced amacrine cells), choroids and other places e.g. cerebral cortex, pituitary body, adrenal glands, nerve endings of respiratory system, gastrointestinal tract and reproductive system (Koh 2000, Dejda et al. 2005). It has been shown to promote growth and differentiation of many cells in tissue and organ culture. The effect of VIP over the proliferation and differentiation and neuro-protection is in a concentration dependent manner. The in vivo neuroprotective effect of VIP has been found to be mediated by BDNF (Rangon et al. 2006). VIP has also been found a potent secretagogue that promotes release of astroglia-derived factors (chemokines) such as IL-1, IL-6, NT-3, protease nexin-1 (PN1), RANTES, macrophage inflammatory protein-1 (MIP-1), activity dependent neuroprotective factor (ADNF) and activity dependent neuroprotective protein (ADNP)(Dejda et al. 2005). The VIP receptors (VPAC-1, VPAC-2 and PAC-1) have been reported in RPE, neuron and glial cells. The expression of VIP receptor PAC-1 has been shown to increase in the pathological states such as ischemic injury (Shioda et al. 2006). VIP has been shown to protect the retina effectively at nano-molar concentration in Wistar rat under ischemic retinal degeneration. VIP has been used also for differentiation of stem cells to RPE like cells (Buchholz et al. 2013, Koh 2000, Vossmerbaeumer et al. 2009, Vossmerbaeumer et al. 2008). The concentration of VIP used for neuroprotection (10-16_ $\left.10^{-9} \mathrm{M}\right)$ is much less than that used for differentiation towards RPE phenotypes $\left(10^{-6}-10^{-}\right.$ $\left.{ }^{3} \mathrm{M}\right)$. 
Nicotinamide or Vitamin B3 (NIC), is a precursor of the coenzyme $\beta$-nicotinamide adenine dinucleotide $\left(\mathrm{NAD}^{+}\right)$, which plays role in the production of ATP in the mitochondria. NIC has been reported to induce differentiation in iPSCs and ESCs towards RPE phenotype (Buchholz et al. 2013, Buchholz et al. 2009, Idelson et al. 2009a). NIC acts as an inhibitor of the enzyme poly (ADP-ribose) polymerase (PARP), which plays important role in DNA repair in cell death as well as in retinal cell death (Paquet-Durand et al. 2007, Uehara et al. 2006, Surjana et al. 2010a). NIC also inhibits NAD-dependent histone deacetylase (Peled et al. 2012, Imai et al. 2000), which plays an important role in differential gene expression and silencing, genome stability, signal transduction, cell growth and cell death. NIC have several effects on cell culture, including PARP inhibition, which can protect cells from oxidative stress (Idelson et al. 2009a, Zahabi et al. 2012a). There are number of articles which support the neuroprotective roles of NIC. PARP activation plays role in certain apoptotic pathway (Virag 2005, Moncada, Bolanos 2006) and its upregulation has been suggested to involve in certain type of retinal cell death (Ji et al. 2008). Activation of PARP is dependent upon $\mathrm{NAD}^{+}$and during process of poly-ADP ribosylation, PARP dependent mechanism gets activated and leads to apoptosis in cells(Yu et al. 2006b, Paquet-Durand et al. 2007). Nicotinamide may be involved in inhibiting cell death or reducing retinal damage in culture by inhibiting PARP, modulating the $\mathrm{NAD}^{+}$level in the cells (SadanagaAkiyoshi et al. 2003, Gale 1996, Ayoub et al. 1999, Sakakibara et al. 2002, Uehara et al. 2006), reducing ATP depletion (Ayoub et al. 1999), inhibiting lipid peroxidation (Klaidman et al. 1996, Klaidman et al. 2001, Lindner et al. 1998) or reducing inflammation (Ungerstedt et al. 2003a, Ungerstedt et al. 2003b). These multiple effects of NIC may be due to being essential substrate for NADH and NADPH.

All-trans-retinoic acid (ATRA) is a lipophilic molecule and can traverse easily through the cellular membrane. It is synthesized by retinol in brain tissue. ATRA is presumed to involve in the differentiation of optic vesicle into RPE (Zahabi et al. 2012b). It is the natural metabolite of retinoic acid. It plays important role in growth and differentiation. It is widely used in the differentiation of embryonic stem cells to different types of cells such as neurons, cardiac and smooth muscles, germ cells differentiation (Kennedy et al. 2009, Silva et al. 2009, Lin et al. 2010). It has been used in differentiation of MSCs to epithelial phenotypes and neuronal phenotypes (Wan et al. 2012, Li et al. 2012a, Brzoska et al. 2005, Bi et al. 2013, Huang et al. 2012b, Li et al. 2012b). It is also involved in neuroprotection in vivo as well as in vitro (Ahlemeyer et al. 2000, Cheung et al. 2009, Li et al. 2008, Mey 2006). It has been shown that ATRA reduces oxidative stress in embryonic neurons by increasing the activity of superoxide dismutase, catalase and glutathione reductase (Ahlemeyer et al. 2001). It has been reported to modulate proliferation, differentiation and 
apoptosis in abnormal and normal cells in vitro (Defer et al. 1997). It has potentiated the neuroprotective effects of NGF (Ahlemeyer et al. 2000). It influences the early developmental events such as patterning, neurogenesis, neuronal specification, axonal growth by binding to nuclear retinoic acid receptors (RARs) and retinoid X receptors (RXRs) that regulate gene transcription (Maden 2001, Mangelsdorf, Evans 1995). It has been reported to have potent anti-apoptotic effect in spontaneous eosinophil death (Ueki et al. 2008). It plays an important role in stem cell differentiation by altering epigenetic changes in DNA as well as histone protein (Gudas 2013). It has been reported to show neuroprotective effects in different in vivo and in vitro experiments (Sato et al. 2008). It has also been reported to induce pigmentation in melanoma cells line (Lotan, Lotan 1980b).

Bone morphogenetic factors-4 (BMP-4) is a secreted signaling protein of transforming growth factor $\beta$ (TGF $\beta$ ) superfamily that elicits its effects by binding to a heterodimer receptor complex composed of a BMP type I receptor (BMPRIA or BMPRIB) and a BMP type II receptor (BMPRII) (Nohe et al. 2004). BMP-4 signaling reported to play important role in multiple biological events including neural induction (Harland 2000), epithelialmesenchymal interactions in organogenesis (Kulessa et al. 2000), and in the creation of stem cell "niches" in developing and adult organs (Lim et al. 2000, Watt, Hogan 2000). The functions of BMP-4 are regulated in the extracellular space by several secreted antagonistic regulators such as noggin, chordin, follistatin, neurogenesin-1, gremlin, which are thought to bind BMP-4 and inhibit its interactions with the receptors (Balemans, Van Hul 2002, Ueki et al. 2003). It is involved in the differentiation and survival of retinal neurons (Liu et al. 2003, Huillard et al. 2005, Murali et al. 2005, Trousse et al. 2001, Adler, Belecky-Adams 2002). It is also involved in VEGF secretion from RPE (Vogt et al. 2006). It is shown to involve in the neuroprotective effect in retina (Fischer et al. 2004). It has been shown to involve in specification of RPE and neuroretina in eye development (Müller et al. 2007, Du et al. 2010, Furuta, Hogan 1998). 


\section{Hypothesis and Objectives}

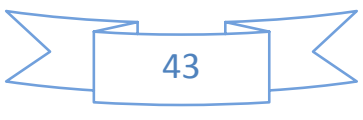




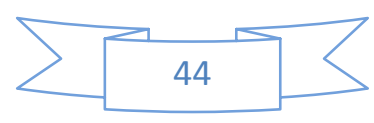




\section{Hypothesis}

In the dry form of AMD disease, the subretinal environment consists of damaged and dysfunctional RPE and degenerating neuroretina. The subretinal injection of hAD-MSCs to delay the process of disease progression in early onset of AMD could be one of the promising strategies under advance cell therapy options. The fate of injected hAD-MSCs and their paracrine immunomodulatory effects might be beneficial for AMD and other retinal degenerative diseases. It would be better if these cells could be directed to RPE phenotypes before moving to their subretinal injection. There is one published report, in which VIP has been used to differentiate hAD-MSCs towards RPE phenotypes but only partial success was achieved. It shows that there is a possibility to differentiate hAD-MSCs to RPE cells but it requires to explore other differentiation inducing factors and their combinations with the previously used factor (VIP), which could stimulate hADMSCs to RPE like cells especially in the artificial culture environment slightly similar to the subretinal environment of AMD. Along the process of differentiation, it would be logical and novel to investigate that hAD-MSCs themselves in these environments may produce their positive or negative effects over the degenerating retina (neuroretina and RPE) under in vitro condition.

The hypothesis of this work is that it is possible to create an artificial microenvironment using hAD-MSCs and different factors of differentiation (VIP, NIC, ATRA, and BMP-4) and their combinations along with RPE cells or medium conditioned by them, which would induce the differentiation of hAD-MSCs towards RPE phenotypes and simultaneously can protect the degenerating neural retina and dying RPE cells, where the pathogenesis lies in case of retinal degenerative diseases including dry form of AMD in aged population. 


\section{Objectives}

The general objective of this work was to assess the effects of different factors or bio-molecules and their combinations along with RPE cells or growth medium conditioned by them (RPE condition medium-RCM) over the differentiation of hAD-MSCs towards RPE phenotype and over the degenerating neuroretina and dying RPE cells under in vitro condition.

The specific objectives were as follows:

1. Isolation, expansion and characterization of hAD-MSCs and RPE cells from donated human lipoaspirates and post-mortem eye globes respectively

a) Extraction, expansion and maintenance of MSCs from donated human lipoaspirates

b) Characterization of above hAD-MSCs at different passages

i) Immunophenotyping of hAD-MSCs by flow cytometry

ii) Evaluation of in vitro differentiation capacity of hAD-MSCs into three lineages e.g. adipocytes, chondrocytes, osteocytes.

c) Extraction, expansion and maintenance of RPE cells from human and pig eye globes

d) Characterization of hRPE cells by flow cytometry, immunofluorescence and western blot study

\section{Examination of effect of hAD-MSCs over the proliferation and death of} RPE cells

a) Optimization of concentration and period of treatment of mitomycin C (MMC) treatment for inducing degeneration process in RPE cell line (ARPE19).

b) Indirect co-cultivation of MMC treated RPE cells with hAD-MSCs and analysis of the effect of hAD-MSCs on viability and proliferation of the treated RPE cells 
3. Induction of differentiation of hAD-MSCs towards RPE phenotypes and their characterization under different conditions

A) Induction of differentiation in hAD-MSCs towards RPE phenotypes by different methods.

a) Cultivation of hAD-MSCs in 50\% hRCM along with VIP, NIC, ATRA in different combinations.

b) Cultivation of hAD-MSCs in 50\% fetal RPE condition medium (fRCM) along with bone morphogenetic protein-4 (BMP-4) and human neuroretinal extracts.

c) Direct co-cultivation of hAD-MSCs with MMC treated ARPE19 cells to analyze the possible induction of RPE phenotypes in hAD-MSCs.

d) Indirect co-cultivation of hAD-MSCs along with combination of different factor molecules like vasoactive intestinal peptide (VIP), nicotinamide (NIC) and all trans-retinoic acid (ATRA) in $50 \%$ hRPE condition medium.

e) Indirect co-cultivation of human neuroretinal explants with hADMSCs in 50\% RCM along with different combinations of VIP, NIC and ATRA or alone.

B) Assessment of RPE phenotypes in the hAD-MSCs cultivated under above mentioned culture conditions by immunofluorescence and western blot study.

\section{Analysis and evaluation of above neuroretinal explants cultured with} hAD-MSCs along with mentioned factors (VIP, NIC and ATRA) and their combinations in $50 \%$ RCM (3Ae.)

a) Assessment of structural changes (damage and degeneration) in the different layers of above co-cultured human neuroretinal explants by histochemistry.

b) Assessment of structural and pathological changes in the different layers of above co-cultivated human neuroretinal explants by immunohistochemistry. 


\section{Materials and Methods}




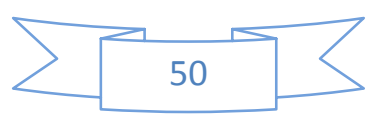




\section{Cells}

\section{Cell line}

ARPE19 cells: This cell line was purchased from the American Type Culture Collection (Manassas, VA, USA). It is a spontaneously arising RPE cell line derived from the normal eyes of a 19-year-old male who died from head trauma in a motor vehicle accident in 1986 by Amy Aotaki-Keen (Dunn et al. 1996). Cells are diploid and can be carried for over 30 passages. Progeny were found to undergo an additional 48 population doublings in longevity trials performed during characterization at ATCC.

Human foetal fore skin fibroblast (HFF1) cells: This cell line was purchased from the American Type Culture Collection (Manassas, VA, USA). This cell line is also called BJ cell line. This cell line had been established form skin taken from normal foreskin of a newborn male. It has a long lifespan in comparison with other normal human fibroblast cell lines with population doubling time of 2-3 days. These cells have a reported normal diploid karyotype at population doubling 61 but an abnormal karyotype at population doubling 82. Cells have the capacity to proliferate to a maximum of 72 population doublings before the onset of senescence. This cell line was used in various experiments as negative controls.

\section{Primary culture}

Retinal pigment epithelial (RPE) cells: These cells were isolated from donated postmortem human eye in the lab regularly depending on the availability of donors. The isolation procedure is discussed later in the next section. The hRPE cells grow very slowly in the culture and normally take 8-12 days to become confluent if seeded at $5000 \mathrm{cell} / \mathrm{cm}^{2}$. The rate of growth and other RPE characteristics also depend upon the age and health status of the donor at the time of death. The hRPE cells are very vulnerable to any change in the culture condition and start to lose the pigmentation and other RPE characteristics very rapidly just after passage one. Unlike the RPE cell line (ARPE19), the primary RPE cells i.e. hRPE cells cannot be maintained very long in the culture without losing their phenotypes. These cells are not recommendable to use in the experiment after passage 8.

Adipose tissue derived mesenchymal stem cells (AD-MSCs): MSCs were isolated from the donated lipoaspirates obtained in the cosmetic liposuction surgery. The isolation procedure is discussed later in this section. These cells have plastic adherent fibroblastic morphology and grow as fast as RPE cell line (ARPE19). The hAD-MSCs are maintained at 
sub-confluence level $(<85 \%)$. These are adult stem cells and are also very vulnerable to any change in their culture condition leading to dedifferentiated phenotypes. Cryopreservation also affects them very considerably especially in their growth rate. The donor is indicated by the abbreviation TA (Tejido adiposo) e.g. MSCs P3/TA05 means MSCs of passage third (P3) and donor fifth (TA05).

\section{Reagents}

Mitomycin C (MMC): MMC also called Ametycine, Mutamycin, and Mitocin- $\mathrm{C}$, was purchased from Sigma-Aldrich, USA. It has empirical molecular formula $\mathrm{C}_{15} \mathrm{H}_{18} \mathrm{~N}_{4} \mathrm{O}_{5}$ with mol. wt. of 334.33. The name mitomycin comes from the action of mitosis inhibitor. MMC is produced by a strain of actinomyces, Streptomyces caespitosus, which contains three anticancer moieties, quinone, urethane, and aziridine groups. It is widely used mitomycin antibiotics due to least toxicity and with the most potent antitumor activity. It has strong bactericidal action against Gram-positive and Gram-negative organisms. It inhibits DNA synthesis. It reacts covalently with DNA, in vivo and in vitro, forming cross-links between the complementary strands of DNA. This interaction prevents the separation of the complementary DNA strands, thus inhibiting DNA replication and hence proliferation of cells. It was dissolved in PBS $(0.5 \mathrm{mg} / \mathrm{mL}$ ) (Gibco, Invitrogen, Paisley, UK). It is most likely to retain activity in aqueous solutions at pH 6-9. Since it is readily decomposed by light, it is kept in a dark vial. A study has shown that the amount of MMC in a culture medium (at $38{ }^{\circ} \mathrm{C}$ ) containing antibiotics with fetal calf serum was reduced by $29 \%$ after 30 minutes and by 53\% after 60 minutes (Proctor, Gaulden 1986). Its solution was made according to manufacturer's instructions and used within one week at $4{ }^{\circ} \mathrm{C}$ or preserved at $-80{ }^{\circ} \mathrm{C}$ for later use.

Vasoactive Intestinal Peptide (VIP): VIP was earlier purchased from Sigma-Aldrich later was purchased from Calbiochem, Germany due to cheaper availability. VIP, also known as vasoactive intestinal polypeptide, is a peptide hormone of 28 amino acids (HHis-Ser-Asp-Ala-Val-Phe-Thr-Asp-Asn-Tyr-Thr-Arg-Leu-Arg-Lys-Gln-Met-Ala-Val-Lys-LysTyr-Leu-Asn-Ser-Ile-Leu-Asn- $\mathrm{NH}_{2}$ ) with empirical formula $\mathrm{C}_{147} \mathrm{H}_{238} \mathrm{~N}_{44} \mathrm{O}_{42} \mathrm{~S}$ (Mol. Wt. 3325.9). It is a neuropeptide that belongs to a member of glucagon/secretin superfamily (Umetsu et al. 2011). The neurons which respond to VIP are called VIP-ergic neurons. It acts as a mitogenic factor for embryonic neurons in the sympathetic nervous system. It stimulates cAMP production in rat peritoneal macrophages. It is recommended as a less toxic chemotherapeutic agent for the treatment of human small-cell lung carcinoma (SCLC) because it inhibits the growth of both in vitro and in vivo SCLC. Nitric oxide stimulates the release of this peptide. Its solution was made according to manufacturer's 
instructions and kept at $-20 \stackrel{\circ}{\mathrm{o}} \mathrm{C}$ and used within one month. It was used at $5 \mu \mathrm{M}$ concentration in all the experiments in this study.

Nicotinamide (NIC): NIC was purchased from Sigma-Aldrich, USA. NIC, also known as niacinamide or nicotinic acid amide, is water soluble amide form of nicotinic acid (Vit B3/Niacin). Its chemical name is pyride-3-carboxamide with empirical formula $\mathrm{C}_{6} \mathrm{H}_{6} \mathrm{~N}_{2} \mathrm{O}$. It is the primary precursor of nicotinamide adenine dinucleotide $\left(\mathrm{NAD}^{+}\right)$and phosphorylated derivative $\mathrm{NADP}^{+}$, an essential coenzyme in ATP production and the sole substrate of the nuclear enzyme poly-ADP-ribose polymerase-1 (PARP-1). It plays important role in DNA damage repair (Surjana et al. 2010b). Its solution was made according to manufacturer's instructions and kept at $4{ }^{\circ} \mathrm{C}$ and used within one month. It was used at $10 \mathrm{mM}$ concentration in all the experiments in this study.

All-trans-retinoic acid (ATRA): ATRA was purchased from Sigma-Aldrich, USA. It is also called as Retinoic acid or Vitamin A acid. Its empirical formula is $\mathrm{C}_{20} \mathrm{H}_{28} \mathrm{O}_{2}$ (Mol. Wt. 300.4). It is extremely sensitive to UV light, air, and oxidizing agents and even more vulnerable in solution form. Any unused portion must be protected by an inert gas atmosphere. It is practically insoluble in water but soluble in DMSO at about $40 \mathrm{mg} / \mathrm{mL}$. It is involved in the control of cellular differentiation and cellular proliferation in normal and transformed cells (may control oncogenes) (Orfanos et al. 1987, Lotan, Lotan 1980a). The pharmaceutical grade has been used in the treatment of different skin disorders. It can be an immunomodulator and a preventive and therapeutic anticancer agent. It also acts as a teratogen effecting different patterns of malformations when mammalian embryos at different developmental stages are exposed to it (Creech Kraft et al. 1989, Creech Kraft et al. 1991, Nau 2001). It inhibits the cell-substrate adhesion and motility in melanocytes (Situ et al. 1993). It has also been used to induce epithelial cell phenotypes and pigment in some cell lines (Brzoska et al. 2005, Lotan, Lotan 1980a). Its solution was made according to manufacturer's instructions and kept at $-20{ }^{\circ} \mathrm{C}$ and used within one month. It was used at $5 \mu \mathrm{M}$ concentration in all the experiments in this study.

Bone Morphogenetic Protein-4 (BMP-4): The BMP-4 is the part of the transforming growth factor-beta superfamily. The superfamily includes large families of growth and differentiation factors. This particular family member plays an important role in the onset of endochondral bone formation in humans and involved in bone and cartilage development, specifically tooth and limb development and fracture repair. Human Recombinant produced BMP-4 in HEK cells is a glycosylated disulfide linked homodimer, having a total molecular weight of 34kDa. BMP-4 was purchased from an Isreal based recombinant protein manufacturer biotechnology company ProSpec (Protein-Specialists) 
during short academic stay in the department of EVS, UoL. Its solution was prepared freshly according to the manufacturer's instructions and kept at $-20{ }^{\circ} \mathrm{C}$ and was used within two weeks. In the experiment it was used at concentration of 200nM. This concentration was chosen on the basis of previous results obtained by Dr. Sharon in the department of Eye and Vision Science (EVS), University of Liverpool (UoL).

\section{Cell culture}

All the different types of cells (ARPE19, HFF1, hAD-MSC, hRPE, pRPE) were cultured under standard culture conditions of $5 \% \mathrm{CO}_{2}$ in the humidified air at $37^{\circ} \mathrm{C}$ with medium renewal at every 2-3 days interval in respective culture mediums as given in the table 1 . The complete medium for hAD-MSCs does not contain antimycotic or amphotericine B because amphotericine B has been found to induce differentiation of MSCs to myotubes (Phinney et al. 1999). After reaching 90\% confluence, cells were trypsinized by incubating them with $0.05 \%$ trypsin and $0.02 \%$ tetrasodium ethylenediaminetetraacetate (Trypsin-EDTA, Gibco, Invitrogen, Paisley, UK), or with TrypLE express (Gibco, Invitrogen, Paisley, UK) for 5-10 minutes and re-suspended in culture mediums. The cells were used for further subculture at 1:3 to $1: 5$ ratio or cryopreserved in chilled culture medium with $25 \%$ FBS (Gibco, Invitrogen, Paisley, UK) and 5\% DMSO (Sigma, USA). Cell numbers and viability for seeding the cells for each experiment were determined by standard Trypan Blue exclusion assay. It is essential to maintain the culture of hAD-MSCs at sub-confluence $(<90 \%)$ level otherwise they may change their stemness (potency).

Table2. Cells and their growth medium

\begin{tabular}{|c|c|}
\hline Cells & Medium with the supplements (Complete medium) \\
\hline ARPE19 & $\begin{array}{l}\text { DMEM/F12 with } 10 \% \text { FBS, } 1 \% \text { antibiotic (penicilin and } \\
\text { streptomycin) and } 1 \% \text { antimycotic (amphotericin) } \\
\text { solution (Gibco, Invitrogen, Paisley, UK) }\end{array}$ \\
\hline $\begin{array}{l}\text { hRPE, pRPE, Fibroblast } \\
\text { (HFF1/BJ cell line), }\end{array}$ & $\begin{array}{l}\text { DMEM with } 10 \% \text { FBS, } 1 \% \text { antibiotic and } 1 \% \text { antimycotic } \\
\text { (amphotericin) solution (Gibco, Invitrogen, Paisley, UK) }\end{array}$ \\
\hline hAD-MSCs & $\begin{array}{l}\text { DMEM low glucose with glutamine with } 10 \% \text { FBS, } 1 \% \\
\text { antibiotic (penicilin and streptomycin) } \\
\text { (Gibco, } \\
\text { Invitrogen, Paisley, UK) }\end{array}$ \\
\hline $\begin{array}{l}\text { RPE (special medium)* } \\
\text { * Used once for optimization }\end{array}$ & $\begin{array}{l}\text { RPE medium-500 mL of MEM, } 5 \mathrm{~mL} \text { of } \mathrm{N} 1 \text { Supplement, } \\
5 \mathrm{~mL} \text { of Glutamine-Penicillin-Streptomycin, } 5 \mathrm{~mL} \text { of Non } \\
\text { essential amino acids, } 125 \mathrm{mg} \text { of Taurine, } 10 \mathrm{ug} \text { of } \\
\text { Hydrocortisone and } 0.0065 \mathrm{ug} \text { of Triiodothyronin, heat } \\
\text { inactivated FBS }(1 \%, 5 \%, 10 \% \text {, or } 15 \%) \text { (Sigma-Aldrich) }\end{array}$ \\
\hline
\end{tabular}




\section{Cell/Tissue isolation}

4.1. hRPE/pRPE and neuroretinal explants: Intact post-mortem human eye globes or anterior segment extracted eye globes were obtained after getting specific informed consents from the family of the deceased person and kept in PBS (Gibco, Invitrogen, Paisley, UK) with $10 \%$ serum and $5 \%$ antibiotics for transporting to lab. The eye globes were bathed in Betadine $₫$ solution for 5 minutes then rinsed quickly with PBS before flash dip in 70\% alcohol. In case of pig eye globes, excessive muscle and connective tissue were trimmed to clean after Betadine ${ }^{\circledR}$ bath outside the laminar hood and then again it was kept in Betadine ${ }^{\circledR}$ for one min. In case of human eye globes all the processes were always carried out inside the hood due to already exposed anterior portion of eye globes. Then the eye globes were rinsed for 5 minutes serially in each three wide-mouthed $50 \mathrm{~mL}$ tubes/bottles with 20\%,10\%, and 5\% antibiotic-antimycotic (diluted to 10X; cat. no. 15240-096; Invitrogen, USA). The globes were transferred to $90 \mathrm{~mm}$ Petri dish coated with silicon (ca $1 \mathrm{~cm}$ thick) and they were fixed by piercing tangentially with three $27 \mathrm{G}$ needles to the silicon coat to avoid any slipping during further processing. Using a blade an incision was made through the sclera below the limbus. This incision was made to start a circular cut for removal of the anterior eye portion using curved scissors. The vitreous was removed from the cut globe carefully with the help of forceps and by inverting the eye downward. The neural retina was detached gently from RPE-choroids with the help of soft brush and forceps and put in the supplemented neural basal medium. Peripheral retina (around $6 \mathrm{~mm}$ ) was removed and central retina was cut through radially into around 6X8 $\mathrm{mm}$ size explants (numbers in the diagram) for co-culture experiments as shown in Fig6. Although maximum of 32 neuroretinal explants could be made from one neuroretina but we only made maximum of $20 \mathrm{hNR}$ explants from one neuroretina. After the neural retina was removed, the posterior pole cup with RPE-choroid was washed thrice with PBS and incubated with $0.05 \%$ trypsin- $0.02 \%$ EDTA for $30-45$ minutes at $37^{\circ} \mathrm{C}-5 \% \mathrm{CO}_{2}$ in the cell incubator. After 45 minutes of incubation, the trypsin solution present in eye cup was pipetted up and down vigorously several times to separate RPE from the choroids. RPE cells were spun down at $1200 \mathrm{rpm}$ for 5 minutes after the solution became very dark, which shows the presence of pigmented RPE cells in the solution. Pellets of hRPE cells were re-suspended in complete growth medium and then put into $25 \mathrm{~cm}^{2}$ flasks (Nunc, Fisher Scientific, USA) in the incubator for one week. After proper cell attachment to the surface of the flask, the cell monolayer was washed properly to remove the tissue debris and the medium was changed at 2-3 days interval. The pigmented cells grown in this flask are termed as primary cell culture, which was trypsinized at 85 to $95 \%$ uniform cell confluence and further sub-cultured from passage zero (P-0). Total of 12 eyes were 
obtained from 6 different deceased persons without any known concomitant ocular disease. All the procedures related to human samples were performed cautiously after the approval of Ethical committee of Clinical Hospital of University of Valladolid. I would like to thank to all the residents of Clinical Hospital, who really made great efforts to obtain the post-mortem eye globes.

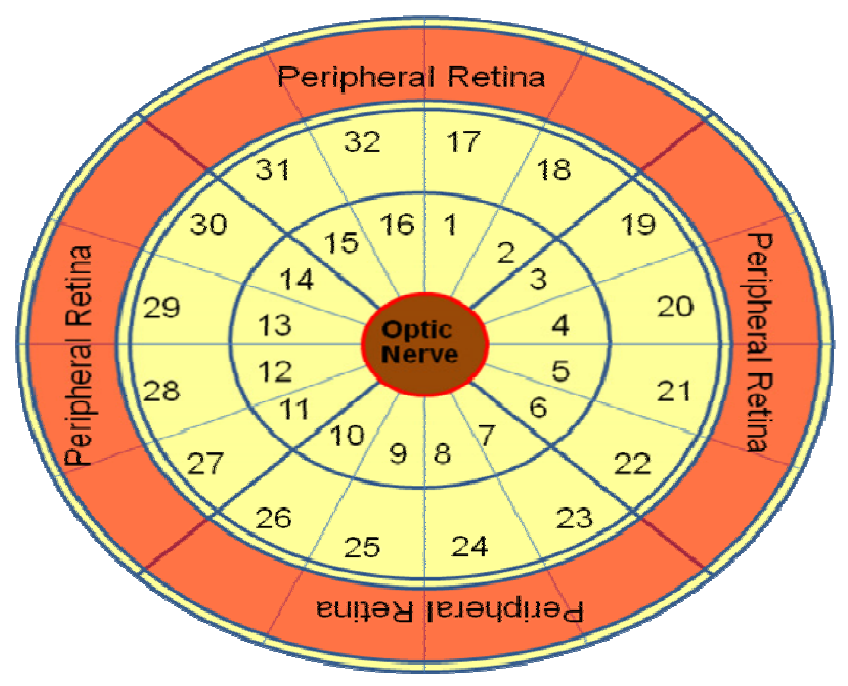

Fig.6. Pattern of section of human neural retina for making co-culture explants. Numbering just shows the counting of maximum possible neuroretinal explants taken from one neuroretina (self sketched).

\subsection{Photoreceptor outer segment (POS) and RPE condition medium (RCM)}

Preparation of POS: The procedure for preparing a suspension of POS from pig eyes was similar to that used by some others (Hall 1978, Huang et al. 2012a). The pig eye globes were obtained from local slaughter house of Laguna de Duero, Valladolid. POS preparation was used to start within 6-8 hrs after enucleation. The neural retina was removed from the eyecup and transferred to HBSS or PBS. The neuroretinal tissue was fragmented in $0.25 \%$ Trypsin- $0.02 \%$ EDTA with a magnetic stirrer for 30 minutes at $4{ }^{\circ} \mathrm{C}$. Large tissue fragments were eliminated by precipitation for $15-20$ minutes $\left(4^{\circ} \mathrm{C}\right)$ and the supernatant was centrifuged at $800 \mathrm{xg}$ for 10 minutes. The pellet of the sample was rinsed thrice with HBSS/PBS and re-suspended in complete DMEM/F12 medium at $1 \times 10^{8}$ $\mathrm{POS} / \mathrm{mL}$ and stored at $-80^{\circ} \mathrm{C}$ for later use.

Preparation of RCM: RPE cells were first seeded in T-75 flask and cultured until complete confluence. After reaching confluence, the spent medium from hRPE (P1 to P3), ARPE19 and fetal RPE (fRPE) were collected after $48 \mathrm{hrs}$. These spent mediums were centrifuged at $1000 \mathrm{rpm}$ for 10 minutes to remove any cell/tissue debris and then

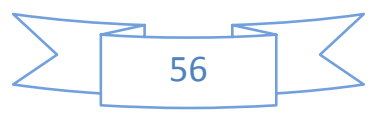


supernatant were stored at $-80{ }^{\circ} \mathrm{C}$ for later use. The mediums collected from hRPE, ARPE, and fRPE are termed as hRCM (hRPE condition medium), aRCM (ARPE19 condition medium), and fRCM (fRPE condition medium) respectively. The fRCM was obtained from Dr. Paul with the kind permission of Dr. Luminita Paraon in the department of Eye and Vision Science, University of Liverpool (Thank to Dr. Luminita and Dr. Paul).

\subsection{Preparation of pig retinal tissue sections (RTSs) and treatment with}

Trypan blue (TB): Pig eye globes were collected from local slaughter house and transported in $4 \%$ p-formaldehyde for $48 \mathrm{hrs}$. The anterior segment was removed and the posterior segment of each eye globe was cut into $3 \times 5 \mathrm{~mm}$ small pieces and rehydrated by washing with PBS. Each piece was incubated with 0.5\% TritonX-100 for 10 minutes at RT to permeabilize the ocular tissue and later washed and subsequently incubated with 0,20 , 200 and $250 \mu \mathrm{g} / \mathrm{mL}$ of TB for 15 minutes at RT (pre-treatment). All pieces were washed twice with PBS after incubation, dehydrated and embedded in paraffin wax. Retinal tissue sections (RTSs) of $3 \mu \mathrm{m}$ thick were cut from each piece, mounted on slide with $50 \%$ glycerol under cover-slip and observed using a microscope (Leica Microsystems, Mannheim, Germany) with three filters viz. A (Blue, excitation filter (EF) of 360/40入), I3

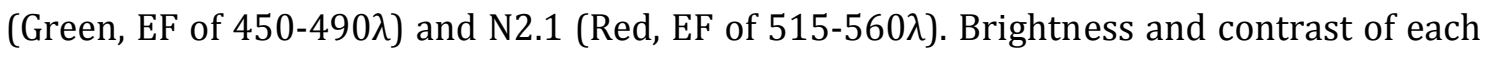
photo were adjusted using Adobe Photoshop 7.0 (Adobe Systems, San Jose, CA).

In another set of experiments, retinal pieces were not treated with TB and were embedded in paraffin wax. RTSs of $3 \mu \mathrm{m}$ thick were cut from these untreated paraffin embedded pieces. Subsequently these RTSs were incubated with different concentrations of TB (posttreatment), mounted and observed using a microscope. This method was developed for establishing the protocols of FC and immunofluorescence studies for detecting the expression of different protein markers by quenching the cellular autofluorescence (Srivastava et al. 2011b).

4.4. Treatment of RPE cells with Mitomycin C: ARPE19 cells were seeded at the density of 60,000 cells $/ \mathrm{cm}^{2}$ in 8 -chambers slide of permanox (Nunc® Lab-Tek®, USA) at standard culture conditions and incubated for $24 \mathrm{hrs}$ to adhere on tissue culture treated polystyrene surface properly and to take morphology. ARPE19 cells were then incubated with $\mathrm{MMC}$ at 25, 50, 100 and $200 \mu \mathrm{g} / \mathrm{mL}$ concentration dissolved in the growth medium for 2 hours for optimizing the appropriate concentration for our experiments. After optimization $50 \mu \mathrm{g} / \mathrm{mL}$ concentration was chosen to inactivate the cells as well as to induce the cell death in the long run. After MMC incubation the ARPE19 cells were washed with PBS three times to remove any residual MMC over the ARPE19 monolayer. 


\section{RPE cell analysis}

5.1. Flow cytometry (FC) analysis of RPE cells: Cells obtained by trypsinization of cell culture were analyzed by FC to determine the cell viability, cell cycle (DNA content) and expression of different proteins. All FC analysis were performed in triplicate, but only the representative experiment was shown. For each assay, at least 5000 cells were analyzed. FC analysis was performed with a cytometer (Cytomics FC 500 ) using $488 \mathrm{~nm}$ excitation with an argon-ion laser for FITC, PE, and PI. The data were analyzed with a software program (Cytomics RXP, Beckman-Coulter). Controls included cross-reactivity of the fluorescence signals of each channel and isotypematched non-specific monoclonal antibodies (MoAbs) used as negative controls. The mean fluorescence intensities (MFIs) were quantified and expressed as arbitrary units. The MFI value is directly related to the mean density of a given protein per cell. The relative MFI index was calculated as the ratio of MFI (+)/MFI (-), where MFI $(+)$ was the MFI value of the cells stained with the corresponding MoAbs and MFI (-) was the MFI value of the cells stained with the isotype-matched unspecific negative controls. Controls included cross-reactivity of the fluorescence signals of each channel, as well as isotype-matched unspecific MoAbs used as negative staining controls.

RPE cells were washed with $2 \mathrm{~mL}$ of Cell Wash Solution and centrifuged at $500 \mathrm{x} \mathrm{g}$ for 5 minutes; the pellet was resuspended and incubated with $50 \mu \mathrm{l}$ of non-specific IgG (40 $\mu \mathrm{g} / \mathrm{mL}$ ) (Sigma-Aldrich) for 15 minutes at room temperature RT to block Fc receptors for unspecific binding. Cells were then resuspended in $250 \mu \mathrm{l}$ of a fixation/permeabilization solution (Cytofix/Cytoperm ${ }^{\mathrm{TM}}$ ) for 20 minutes in the dark at $4{ }^{\circ} \mathrm{C}$ to fix the cells and permeabilize the cell membrane. After incubation, cells were washed twice with $1 \mathrm{~mL}$ of washing solution. Then cells were incubated with $1 \mu \mathrm{l}$ of anti-RPE65 (IgG1) mouse MoAb or with $5 \mu$ of anti-human epithelial/fibroblasts primary MoAb conjugated with R-PE (IgG2a) for $30 \mathrm{~min}$ at $4{ }^{\circ} \mathrm{C}$ in the dark. Cells were then washed with washing solution. The cells undergoing RPE65 staining were incubated in the dark for 30 min at $4{ }^{\circ} \mathrm{C}$ with $20 \mu \mathrm{l}$ of goat anti-mouse IgG secondary antibody conjugated with FITC previously diluted at 1:50 in washing solution (BD Perm/Wash). At this step, cells undergoing staining with anti-human epithelial/fibroblasts primary MoAb did not need incubation with a secondary antibody. Cells were washed with $1 \mathrm{~mL}$ of $1 \%$ BSA in PBS after incubation with secondary antibody and then resuspended and incubated in $500 \mu \mathrm{l}(10,20$ and $40 \mu \mathrm{g} / \mathrm{mL}$ solution) 
ice-cold TB solution for 10 minutes at $4{ }^{\circ} \mathrm{C}$ (post-treatment). This step was made to get rid of autofluorescence of RPE (to quench the autofluorescence given by the pigments of RPE) (Srivastava et al. 2011b). Finally the cells were washed, resuspended in $500 \mu \mathrm{l}$ PBS containing 1\% p-formaldehyde and cell fluorescence was measured in FC.

Quantitative analysis of RPE cells (hRPE, pRPE and ARPE19) expressing RPE65 (Lee et al. 2010, Steindl-Kuscher et al. 2009), a specific protein marker of RPE cells, was performed to determine the deviation of cells from its natural characteristics in primary RPE cell cultures. The hRPE cells were exposed to UV-B light for 48 hours and used as positive controls for apoptosis. The autofluorescence observed in the early passages of the hRPE cell primary cultures was quenched using trypan blue as previously described (Srivastava et al. 2011b).

5.2. Cell Viability: For cell viability analysis, the cells were stained with an annexin VFITC and propidium iodide (PI) commercial kit (Beckman-Coulter, Marseille, France) as previously described (Reinoso et al. 2011, Martinez-Osorio et al. 2009). Cell suspension were washed with $2 \mathrm{~mL}$ cold PBS and centrifuged at $500 \mathrm{x}$ g for 5 minutes. The supernatant was removed and cell suspension was made in cold $100 \mu \mathrm{l}$ annexin binding buffer $\left(4^{\circ} \mathrm{C}\right)$. Cells were incubated with $1 \mu \mathrm{l}$ of annexin V-FITC $(25 \mu \mathrm{g} / \mathrm{mL})$ and $5 \mu \mathrm{l}$ of PI (250 $\mu \mathrm{g} / \mathrm{mL})$ at $4 \stackrel{\circ}{ } \mathrm{C}$ for 10 minutes in the dark. Then cold $400 \mu \mathrm{l}$ of binding buffer was added and agitated gently and FC analysis was done.

Annexin $\mathrm{V}$ is an anticoagulant that binds with negatively charged phosphatidylserine. Phosphatidylserine is an internal plasma membrane phospholipid that is exposed on the outer layer of plasma membrane in the early apoptotic phase before the cells become permeable to PI. Therefore the cells in early apoptotic phase only show staining for annexin V, while cells in late apoptotic phase show double staining with annexin V and PI. The necrotic cells show staining with PI only. The viable cells are negative for both annexin V and PI i.e. neither stain with annexin V nor with PI.

5.3. Cell cycle analysis: For cell-cycle analysis, the DNA content of the cells was determined by staining with PI after cell permeabilization (Coulter DNA Prep Reagents Kit, Beckman-Coulter) as described (Reinoso et al. 2011, Martinez-Osorio et al. 2009). Cell suspension was centrifuged at $500 \mathrm{x} g$ for 5 minutes. The cells were permeabilized with 50 $\mu$ of DNA-Prep LPR (Coulter® DNA Prep Reagents Kit; Beckman Coulter) at RT after decanting the supernatant. $1 \mathrm{~mL}$ of DNA-Prep Stain containing PI and RNA-ase III-A was added and agitated gently and incubated for 30 minutes at RT in the dark. The preparations were kept in dark before FC analysis. The mean proliferation index (MPI) 
was calculated as the ratio of $(\mathrm{S}+\mathrm{G} 2 / \mathrm{M})$ to $(\mathrm{S}+\mathrm{G} 2 / \mathrm{M}+\mathrm{G} 0 / \mathrm{G} 1)$, where $\mathrm{S}$ is the percentage of cells ( $>2 n$ ) in S-phase, G2/M is the percentage of cells (4n) in G2 and M phases, and $\mathrm{G} 0 / \mathrm{G} 1$ is the percentage of cells $(2 \mathrm{n})$ in $\mathrm{G} 0$ and $\mathrm{G} 1$ phases.

\section{6. hAD-MSCs}

6.1. Isolation and culture of hAD-MSCs: The procedure for extraction, expansion and characterization of MSCs from lipoaspirates (LPAs) was identical to that one used by Nieto and coleagues (Nieto-Miguel et al. 2013). LPAs were obtained from subcutaneous human adipose tissue of six different donors, as byproducts of liposuction procedures in cosmetic surgery after obtaining the written informed consents from the donors. LPAs were centrifuged to separate soluble fractions and washed twice with PBS to remove residual serum and anesthetic liquid. Then LPAs were digested with $0.075 \%$ collagenase type I (Gibco-Invitrogen, USA) $(0.75 \mathrm{mg} / \mathrm{mL}$ ) by stirring at $37^{\circ} \mathrm{C}$ for $45-60$ minutes in orbital shaker. The high density pellets of stromal vascular fractions (SVF) were obtained after neutralizing the enzyme activity with FBS and by centrifuging at 1200rpm for 5 minutes. After one wash with PBS, SVF was incubated with $160 \mathrm{mM} \mathrm{NH} 4 \mathrm{Cl}$ (Sigma-Aldrich, USA) for $10 \mathrm{~min}$ to lyse RBC (Erythrocytes). The SVF was collected by centrifuging and re-suspended in growth medium and filtered serially through $100 \mu$ and $40 \mu$ nylon filters (BD Bioscience, USA) to remove any tissue debris. After cell count including viability determination by trypan blue exclusion, cells were seeded at $2 \times 10^{7}$ cells/T-75 flask and left for incubation in the cell incubator for 2-3 days. Then the adherent cells were washed with PBS extensively to remove non-adherent cells. These adherent cells are called primary culture and further subculturing starts from passage zero (P-0) till passage three (P-3). The hAD-MSCs of P3 to P-5 including higher passages were used further for their characterization by panel of cell surface markers by flow cytometer (immunophenotyping) and by potency assay into three lineages. Under standard culture conditions the hAD-MSCs were splitted at $90 \%$ of confluence and were further sub-cultured at a density of 3000-6000 cells $/ \mathrm{cm}^{2}$. Depending on culture mediun and passage number, cell doubling time of hAD-MSCs have been found 2 to 4 days by us as found by others (Mitchel et al. 2006, Izadpanah et al. 2006) . The passages of hADMSCs used in different experiments were from P-3 to P-6. To avoid spontanous differentiation, hAD-MSCs need to maintained subconfluent i.e less than $95 \%$ confluency. 


\subsection{Characterization of hAD-MSCs}

6.2.1. Immunophenotyping of hAD-MSCs: Because of lack of specific marker for MSCs, cells are required to be positive in more than 95\% for CD105, CD73, and CD90 and negative for the expression of CD34, CD45, CD14 and CD19 according to the International Society for Cellular Therapy (ISCT) in flow cytometry analysis (Dominici et al. 2006, Horwitz et al. 2005). The hAD-MSCs (P3 to P5) were harvested and washed. 200,000 cells were taken in each tube ( 6 tubes) for cytometry analysis and washed twice with cold cell wash buffer or PBS. Additional two tubes were taken for the cell viability and cell cycle analysis. The cells were blocked with $40 \mu \mathrm{g} / \mathrm{mL}$ IgG (Sigma-Aldrich, USA) for 15 minutes at room temperature (RT) then cells were incubated with $10 \mu$ l of anti-human monoclonal antibodies and with matching isotype controls at $4{ }^{\circ} \mathrm{C}$ for 30 minutes in darkness as follows:

$$
\begin{aligned}
& \text { Isotype controls } \\
& \text { Tube 1: IgG2a- FITC / IgG1- PE / IgG2a- APC (5 } \mu \text { l each) } \\
& \text { Tube 2: IgG1- FITC / IgG1- PE / IgG1- PC7 (5 } \mu \text { l each) } \\
& \text { Tube 3: IgG2a- FITC / IgG1- PE/ IgG1- PC7 (5 } \mu \text { l each) } \\
& \text { Test antibodies (Antibody panel for MSCs) } \\
& \text { Tube 1:CD19-FITC/CD105- PE/ HLADR-APC (10 } \mu \text { each) } \\
& \text { Tube 2: CD45- FITC/ CD90- PE/ CD34- PC7 (10 } \mu \text { l each) } \\
& \text { Tube 3: CD14- FITC/ CD73- PE/ CD45- PC7 (10 } \mu \text { l each) }
\end{aligned}
$$

Afterwards the cells were washed with cold PBS and resuspended in $500 \mu \mathrm{l}$ PBS and analyzed in Flow Cytometer (Cytomics FC 500 Cytometer, Beckman Coulter, Inc., Fullerton, CA).

MFI can be defined as medium (not mean) fluorescence intensity per events. In FC analysis, MFI reflects the average amount of protein expression (density) per cells (events) in arbitrary unit (fluorescence signal). The relative MFI is the ratio of MFI of test antibody to MFI of isotype control. Further percentage of cells expressing the antibody, MFI and relative MFI were plotted as bar graph. 
6.2.2. In vitro multi-lineage differentiation of hAD-MSCs: The hAD-MSCs (P3 to P5 and higher) were seeded at 10,000 cells $/ \mathrm{cm}^{2}$ in four 2-chamber permanox slides (LabTek, USA). Two slides were incubated with adipogenic and osteogenic differentiation medium, provided in StemPro Adipogenesis Differentiation Kit and Stem Pro Osteogenesis Differentiation Kit (Gibco Invitrogen A10070-01) for 15 days and for 29 days respectively according to the Kit's instructions. Two slides were incubated with normal nondifferentiating medium as controls. After completion of incubation period, both controls and test slides were stained with Oil Red 0 (Sigma 00625) to detect triglycerides and lipids in oil droplets of adipocytes and von Kossa staining to precipitate (black) phosphate salt present as calcium phosphate in osteocytes respectively. For chondrocytes differentiation, hAD-MSCs were pelleted in two $15 \mathrm{~mL}$ tubes $\left(1 \times 10^{6}\right.$ cells in each) and one incubated in StemXVivo ${ }^{\mathrm{TM}}$ Chondrogenic Base Media with StemXVivo ${ }^{\mathrm{TM}}$ Chondrogenic Supplement (RD Systems, Minneapolis, USA) and another in normal low glucose complete medium for 27 days. Afterward pellets were fixed with 4\% paraformaldehyde (PFA) at RT for $3 \mathrm{hrs}$, and washed twice with distilled water then left in 30\% saccharose (sucrose) overnight $(\mathrm{ON})$ at $4 \stackrel{\circ}{\circ}$. The next day it was again incubated with fresh $30 \%$ saccharose at RT for 30 minutes and embedded in optimal cutting temperature (OCT) compound (Aname, Madrid, Spain) on dry ice or in $-20{ }^{\circ} \mathrm{C}$. Finally they were cryo-sectioned, and stained with Alcian blue dye. In all the above three case they were counterstained with Hematoxylin Gill III (Sigma-Aldrich, USA). Alcian blue dye is used to stain acidic polysaccharides such as glycosaminoglycans present in chondrocytes or in cartilages.

\section{Methods of differentiation of hAD-MSCs}

7.1. Culture of hAD-MSCs in hRCM supplemented with factors: The hAD-MSCs were seeded at cell density of 30,000 cells $/ \mathrm{cm}^{2}$ in 6 -well plates or in 8-chamber slides (Permanox $®$, LabTek $\AA$, USA). After 24 hours, adhered cells were washed and cultured further in 50\% hRCM in DMEMF/12 along with factors in seven different combination excluding control. These seven combinations were as follows VIP, NIC, ATRA, VIP+NIC, VIP+ATRA, NIC+ATRA, VIP+NIC+ATRA. VIP, NIC and ATRA were used at concentration of $5 \mu \mathrm{M}, 10 \mathrm{mM}$, and $5 \mu \mathrm{M}$ respectively. RPE special medium was used for differentiation in some samples but this medium was having some problem. We did not find satisfactory growth and differentiation in it. So later we removed this medium from our experiments.

7.2. Culture of hAD-MSCs in fRCM and POS with BMP-4: The hAD-MSCs were seeded at cell density of 30,000 cells $/ \mathrm{cm}^{2}$ in 6-well plates as well as in 8-chamber slide (Permanox®, LabTek®, USA) as shown below. After 24 hours, adhered cells were washed 
and cultured further in 50\% fRCM in DMEM/F12 with POS (1x105 POS $/ \mathrm{mL})$ along with BMP-4 (200ng/mL) for two weeks. There were four plus four combinations as shown below: 1. Basal growth medium (complete DMEM/F12 without antifungal agent); 2. Basal

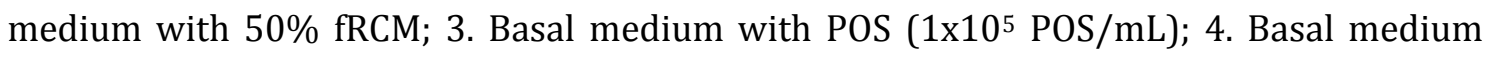
with $50 \%$ fRCM and POS $\left(1 \times 10^{5} \mathrm{POS} / \mathrm{mL}\right)$ and the next four setup was all these four setup supplemented with BMP-4. The medium was changed at exactly 2 days interval. This experiment was performed in the Department of Eye and Vision Sciences, University of Liverpool.

8-Chamber slides (experimental design)

\begin{tabular}{|l|l|l|l|l|}
\hline \multirow{2}{*}{$\begin{array}{l}\text { Without BMP- } \\
4\end{array}$} & DMEM/F12 & $50 \%$ fRCM & POS $\left(1 \times 10^{5} / \mathrm{mL}\right)$ & $50 \%$ fRCM+POS \\
\cline { 2 - 5 } & DMEM/F12 & $50 \%$ fRCM & POS $\left(1 \times 10^{5} / \mathrm{mL}\right)$ & $50 \%$ fRCM+POS \\
\hline
\end{tabular}

\begin{tabular}{|l|l|l|l|l|}
\hline $\begin{array}{l}\text { With BMP-4 } \\
(200 \mathrm{ng} / \mathrm{mL})\end{array}$ & DMEM/F12 & $50 \%$ fRCM & POS $\left(1 \times 10^{5} / \mathrm{mL}\right)$ & $50 \%$ fRCM+POS \\
\cline { 2 - 5 } & DMEM/F12 & $50 \%$ fRCM & POS $\left(1 \times 10^{5} / \mathrm{mL}\right)$ & $50 \%$ fRCM+POS \\
\hline
\end{tabular}

7.3. Direct co-culture of hAD-MSCs with inactivated RPE cells: In direct co-culture, the RPE cells (ARPE19 or hRPE P2) were seeded in 8-chamber permanox slide at cell density of 60,000 cells $/ \mathrm{cm}^{2}$ and left for 24 hours to attach well and to take morphology. Next day the attached RPE cells were washed and incubated with freshly prepared MMC at 50ug/mL for 2 hours in the cell incubator. After 2 hours of incubation the cells were washed properly with PBS or growth medium thrice and hAD-MSCs were seeded at cell density of 30,000 cells $/ \mathrm{cm}^{2}$ over MMC treated RPE cells. MMC treated RPE cells and hAD-MSCs have been co-cultured directly in 2:1 ratio as shown in the diagram (Fig. 7A\&B). The size of RPE cells is much smaller than that of hAD-MSCs. We have initially found that cell density of 60,000 cells $/ \mathrm{cm}^{2}$ is required at the time of seeding RPE cells to obtain the confluent monolayer of RPE immediately after 24 hours. The medium used for the direct co-culture was complete DMEM/F12 as shown in table1 and changed at 2-3 days interval at $0.350 \mathrm{~mL} /$ chamber for 30 days. For demonstrating the two cells distinctly, the RPE cells were marked with EdU (Click-iT EdU imaging Kit, Invitrogen, USA) according to manufacturer's instructions before the MMC treatment to RPE cells.

7.4. Indirect co-culture of hAD-MSCs with inactivated RPE cells: ARPE19 cells and AD-MSCs have been co-cultivated in the same wells of 6-well plates in 2:1 ratio for 3 weeks, sharing the same growth medium (complete DMEM/F12 medium) but physically separated by trans-membrane of BD Falcon ${ }^{\mathrm{TM}}$ cell culture inserts as shown in the diagram (Fig. 7B\&C). The ARPE 19 cells were cultured in the bottom of the 24-well plates (BD Falcon) and the AD-MSCs were cultured on the trans-well inserts (BD Falcon) having tissue culture treated track-etched 
polyethylene terephthalate (PET) membrane of $0.4 \mu \mathrm{m}$ pore size (pore density of $2.0 \pm 0.2 \times 10^{6} / \mathrm{cm}^{2}$ ). In another set of experiments, AD-MSCs were co-cultivated with ARPE19 cells treated with different concentrations of MMC $(50,100,200 \mu \mathrm{g} / \mathrm{mL})$.

\subsection{Indirect co-culture of hAD-MSCs with human neuroretinal explants (hNR):}

The hAD-MSCs were seeded on the bottom of the 6-well plate at least 24 hours before putting human neural retina explants on trans-insert. The neural retina explants (size 6x8mm) were placed with PRs side downwards over the membrane of trans-insert (Corning Inc. New York, USA) as demonstrated in the diagram (Fig. 7D). The material of trans-membrane is tissue culture treated polycarbonate (PC) having diameter of $24 \mathrm{~mm}$, thickness of $10 \mu \mathrm{m}$, and pore size of $0.4 \mu \mathrm{m}$ with density of $1 \times 10^{8}$ pores $/ \mathrm{cm}^{2}$. The trans-well was lifted by putting the laboratory made sterile rings of thick filter paper to adjust more growth medium $(5 \mathrm{~mL})$. The growth medium with 50\% hRPE condition medium (hRCM) was strictly replaced at 2 days interval to avoid excessive change in the $\mathrm{pH}$ and nutrient loss. This tissue-cell co-culture was incubated for one and two weeks' period with different factors and their combinations.

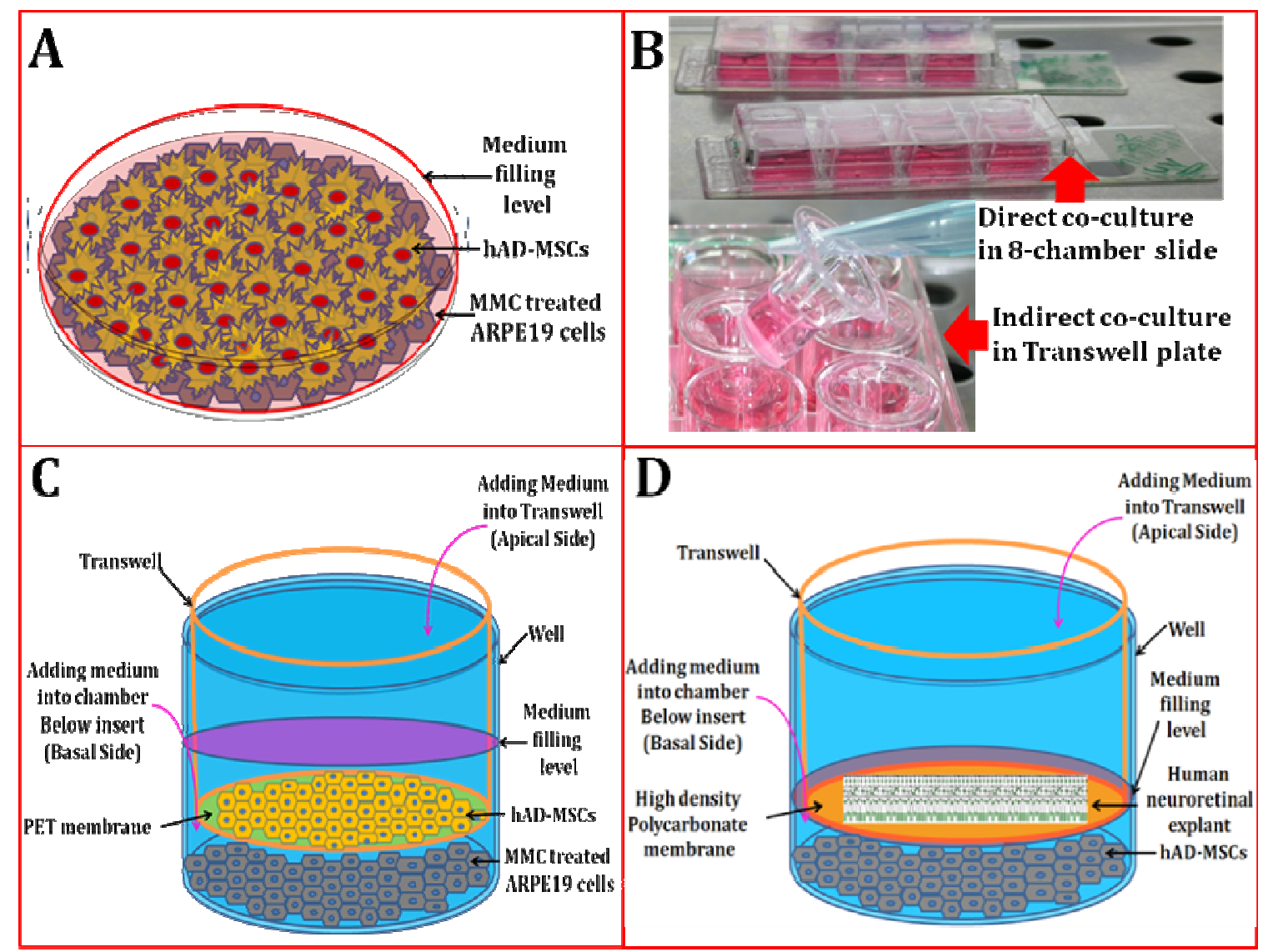

Fig.7. Co-culture diagrams. Direct co-culture of hAD-MSCs with MMC treated RPE cells (A) Direct co-culture in 8 chamber slides and indirect co-culture in trans-well plates (B) Indirect co-culture of hADMSCs with MMC treated RPE cells (C) Indirect co-culture with hAD-MSCs with human neuroretinal (hNR) explants (D) (Self Sketched) 
8. Treatment with factors: VIP, NIC, and ATRA were used in the growth medium along with $50 \%$ hRCM in different experiments at $5 \mu \mathrm{M}, 10 \mathrm{mM}$, and $5 \mu \mathrm{M}$ respectively. There were seven different combinations of these factors and one control without any factor as follows: VIP, NIC, ATRA, VIP+NIC, VIP+ATRA, NIC+ATRA, VIP+NIC+ATRA. All these combinations were used in different separate experiments. Bone morphogenetic protein 4 (BMP-4) (ProSpec, Ness Ziona, Israel) were used in conjugation with POS and human fetal RCM (hfRCM). BMP-4 was used at the concentration of $200 \mathrm{ng} / \mathrm{mL}$ based on the previous experimental results done by Dr. Carl Sheridan Group in the department of Eye and Vision Science, University of Liverpool.

\section{Analysis of effects of different experimental conditions on RPE cells, hAD- MSCs and neuroretinal explants}

9.1. Cell viability assay: The cell viability/cytotoxicity Assay Kit for live \& dead cells (Biotium Inc., USA) was used to evaluate the cell viability after three weeks of MMC treatment to ARPE19 cells in accordance with manufacturer protocol. The kit provides a two-color fluorescent staining of live (green) and dead (red) cells using two probes; Calcein acetoxymethyl ester (calcein AM) stains live cells green while EthD-III (Ethidium homodimer III) stains dead and damaged cells red. After staining, both cells, APRE19 and hAD-MSCs, were visualized using a fluorescence microscope (Leica Microsystems, Mannheim, Germany). Cell viability was measured by Trypan blue exclusion method as well.

9.2. Cell proliferation assay: AlmarBlue $(A B)$ assay is used for measuring the cell activity as result of cell proliferation and viability in terms of fluorescence intensity. Cells were washed with PBS and complete DMEM/F12 medium with 10\% AB (AbD Serotec, Oxford, UK) was added to each sample and incubated under standard condition of incubation for 5 hours. The fluorescence intensity of $\mathrm{AB}$ was measured (Spectra Max M5 Multi-Mode Microplate Reader, Molecular Devices LLC, US) with excitation wavelength at 560nm and emission wavelength at $590 \mathrm{~nm}$. The degree of $A B$ reduction would correspond to the number of live cells and cell proliferation activity. The negative control for the measurement of fluorescence intensity was taken as the medium with $\mathrm{AB}$ reagent without any cells. The viability and proliferation activity of ARPE19 cells in different culture conditions is directly correlated with the degree of reduction of $A B$, which is reflected by the fluorescence intensity measured for each sample. The mean value of arbitrary fluorescence intensity unit (AFU) \pm standard error of mean (SEM) has been plotted against all the different samples of the experiments. 


\subsection{Protein preparation, polyacrylamide gel electrophoresis (PAGE) and}

Western blotting (WB): The adherent cells were trypsinized with trypsin (TrypLE ${ }^{\mathrm{TM}}$ Express, Invitrogen, USA) to collect the cells efficiently. The cells were collected by centrifuging at $1200 \mathrm{rpm}$ and the supernatant was decanted and the residual supernatant was taken out carefully by small pipette. The cells were left at minus $80{ }^{\circ} \mathrm{C}$ for minimum of two hours or directly lyzed with RIPA lysis buffer (Santa Cruz Biotech Inc., USA) and incubated for 30 minutes on ice. The lyzed cell samples were centrifuged at 14,000 rpm for 30 minutes at $4{ }^{\circ} \mathrm{C}$ to remove any cell debris. The supernatant were diluted in $2 \mathrm{x}$ Laemmli sample (Sigma-Aldrich, USA) buffer and heated at $100{ }^{\circ} \mathrm{C}$ for $5 \mathrm{~min}$. The protein concentration was measured by BCA (Bicinchoninic acid assay) method (Thermo Scientific Pierce BCA Protein Assay Kit). The sample were loaded in the wells of SDS-PAGE $(10 \%$ gel) in equal concentration $(20-40 \mu \mathrm{g} /$ well) to separate the cellular proteins along with dual colour protein markers (protein ladder of Biorad, USA). The separated proteins in gel were transferred to Nylon membrane by wet transfer method. The membrane was stained with Ponceau stain (Sigma-Aldrich, USA) to ensure whether the protein is transferred to the membrane or not. Membranes were washed with DDW and blocked by incubating it in blocking buffer containing 5\% skimmed milk (casein), 2.5\% BSA, and 0.05\% Tween-20 (Biorad, USA) in Tris buffer saline (TBS) for 1 hour at RT on rocker at gentle rocking speed and then it is washed once lightly with TBS. The membranes were incubated with primary antibodies diluted in $(0.05 \%$ Tween-20 in TBS with $1 \%$ BSA) overnight at optimized dilution shown in Table 3. Next day membranes were washed 5 times with $0.1 \%$ Tween20 in TBS (TBS-T) and then incubated with secondary antibody conjugated with HRP diluted in buffer (0.05\% Tween-20 in TBS with 1\% BSA) as mentioned in Table 3 for $1 \mathrm{hr}$ at RT. The membranes were again washed 6 times. The specific protein, against which primary antibody was used, was detected by incubation of the membrane in enhanced chemiluminiscent solution (ECL; Santa Cruz Biotech Inc., USA) for one minute and signals were captured by CCD camera in chemidoc (Biorad Inc., USA) at exposure from 2 to 8 minutes. The intensity of each band was calculated and analyzed by densitometry method by Quantity One 4.4.1 software (Biorad, USA), where, at first, all bands were selected by the rectangular select tools and background global subtraction mode was selected to get the densitometry analysis reports for each lane present in the image obtained from the exposure of each blot membrane. The relative protein expression was calculated by normalizing the average density of signals data obtained from each sample from different experiments with that of the bands intensities of GAPDH expression in them and subjected to statistical analysis. 


\subsection{Immunofluorescence study}

9.4.1. Immunocytochemistry: After induction of differentiation of the hAD-MSCs by above mentioned methods, RPE markers were analyzed by immunofluorescence study. For immunostaining, cells were fixed either in 4\% paraformaldehyde (PFA) for 15 minutes or in chilled methanol for 20 minutes at RT. The fixed adherent cells were washed with PBS for three times for 5 minutes in each wash. The cells were permeabilized with $0.5 \%$ Triton X-100 in PBS (PBT) for 10 minutes in case of PFA fixed cells. In case of methanol fixed samples there is no need of permeabilization step. After three wash with PBT, the cells were blocked in blocking buffer (10\% normal goat serum (NGS), 1\% BSA in PBT) for one hour at RT. Then blocked cells were incubated with primary antibody diluted in blocking buffer (according to Table 3) for overnight (ON). The next day the cells were washed with PBT three times for 5 minutes each. The cells were incubated with corresponding secondary antibody diluted in 1\% BSA in PBT (according to Table 3) for 1 hour at RT. The cells were washed again with PBT three times for minutes each and incubated with Rhodamine Phalloidin (1:40) for 30 minutes at RT. The cells were washed again three times with PBT and incubated again with 496-diamindino-2-phenylindole dihydrochloride (DAPI) at 1:500 dilution for 2 minutes. The cells were washed one time with PBT and mounted in fluorescence mounting medium (Dako, Spain). The immunostained slides were watched under inverted fluorescence microscope (Leica Microsystem Mannheim, Germany). The cells were immunostained against RPE makers like CRALBP (cellular retinaldehyde binding protein found in apical microvilli of RPE and in Müller glial cells), BEST1 (Bestrophin, Basal marker of RPE), RPE65 (a 65-kDa cytoplasmic protein involved in retinoid metabolism), CK8+18+19 (Specific cytokeratin of RPE), pan-cytokeratin (epithelial marker), Ezrin (Apical marker of RPE), PAX6 (considered as immature RPE cell marker), and Z01 (Zonula occludens present in tight junction by which RPE cells are attached compactly). Cells were also stained with one MSCs maker CD90 (Thy1) to show residual mesenchymal characteristics. Secondary antibodies were FITC or Alexa Fluor conjugated. Cells were also counterstained with rhodamine phalloidin and DAPI to visualize normal morphology of cells (by staining F-actin) and to visualize nuclei respectively. Non-specific staining was tested by omission of the primary antibodies. Images of immunologically stained cells (differentiated hAD-MSCs) were obtained by Leica digital inverted florescence microscope and analyzed with Leica Application Suite Advanced Fluorescence (LASAF) software.

9.4.2. Immunohistochemistry: The neuroretinal explants were embedded in OCT compound on dry ice or at $-20{ }^{\circ} \mathrm{C}$ after serial treatment with $5 \%, 15 \%$, and $30 \%$ saccharose for 30 minutes, $2 \mathrm{hrs}$, and overnight respectively. Then the embedded neuroretinal explants were cryosectioned $4 \mu \mathrm{m}$ thick sections and placed over the special treated slides for further 
processing for immunohistostaining. These sections were stained against the CRALBL, GFAP, Calbindin, Synaptophysin, and rhodopsin with primary antibodies and then with secondary antibodies conjugated with Alexa Fluor 488 or 568 (Invitrogen, USA). All the antibodies are used in the dilutions as mentioned in Table 3.

Table 3. Antibody list with reference and dilution (mm-mouse monoclonal, rm-rabbit monoclonal, rp-rabbit polyclonal, WB-Western blot, ICC- Immunocytochemistry, IHCimmunohistochemistry, ab-abcam, sc-santa cruz, NB- novus biological, MA/PA- Monoclonal or polyclonal antibody in Thermofisher)

\begin{tabular}{|c|c|c|}
\hline Antibody & Trade mark & Dilution \\
\hline RPE65- (mm) & $\begin{array}{l}\text { NB100-355, ab13826, MA-1- } \\
16578\end{array}$ & ICC-1:50, WB-1:1000 \\
\hline CRALBP (mm) & ab15051 & ICC-1:50, IHC-1:500, \\
\hline CD-90 (rm) & ab92574 & WB-1:1000 \\
\hline Bestrophin (mm) & ab2182, & ICC-1:100, WB-1:1000 \\
\hline B-actin $(\mathrm{mm})$ & ABIN387703 & WB-1:1000 \\
\hline Cytokeratin8+18+19 (mm) & ab41825/ab90102 & ICC-1:100, WB-1:1000 \\
\hline Pancytokeratin $(\mathrm{mm})$ & ab7753 & ICC-1:100, WB-1:1000 \\
\hline Calbindin (rm) & Swant CB38a & IHC-1:500 \\
\hline Pan-cadherin (mm) & C-1821(sigma) & ICC-1:100, WB-1:1000 \\
\hline Ezrin (C-4) (mm) & sc-271541 & ICC-1:100, WB-1:1000 \\
\hline GAPDH (10B8) (mm) & sc-51905 & WB-1:1000 \\
\hline GFAP (rp) & Dako IR524/IS524 & IHC-1:500, WB-1:1000 \\
\hline MITF (rp) & NB100-92181/ab3201 & ICC-1:50, WB-1:1000 \\
\hline MERTK (rm) & ab52968 & ICC-1:50, WB-1:1000 \\
\hline Pax-6 (r/m) & Covance PRB-278P/ab78545 & ICC-1:100, WB-1:1000 \\
\hline PEDF/SERPINF1 (rp) & bs-0731R & ICC-1:50, WB-1:1000 \\
\hline RBP (G4E4) (mm) & ab24090 & ICC-1:50, WB-1:1000 \\
\hline Synaptophysin SY38 (mm) & MASB5258 & IHC-1:200, WB-1:1000 \\
\hline Rhodopsin & AB9279 & IHC-1:500 \\
\hline Tyrosinase (rp) & bs-0819R & ICC-1:100, WB-1:1000 \\
\hline $\mathrm{ZO}-1(\mathrm{~mm})$ & Invitrogen;339100 & ICC-1:50, WB-1:1000 \\
\hline CD14-FITC & IM0645 Beckman Coulter & $\mathrm{FC}$ \\
\hline CD19-PE & 345789 BD Biosciences & $\mathrm{FC}$ \\
\hline CD34-PC7 (mm) & A21691 Beckman Coulter & FC \\
\hline CD45-FITC & A07782 Beckman Coulter & $\mathrm{FC}$ \\
\hline CD45 PC7 & IM3548 Beckman Coulter & FC \\
\hline HLADR-FITC & $\begin{array}{l}1638 \text { Immunotech, Beckman } \\
\text { Coulter }\end{array}$ & FC \\
\hline CD73-PE (mm) & 550257 BD Biosciences & $\mathrm{FC}$ \\
\hline CD90-PE (mm) & IM1840U Beckman Coulter & FC \\
\hline CD105-APC (mm) & 090608/0105 BioLegend & FC \\
\hline $2^{\circ} \mathrm{Ab}$ GAM-FITC & JIR 115-095-164 & ICC-1:100 \\
\hline $2 \div$ Ab GAR-FITC & ab6717 & ICC-1:100 \\
\hline 2 Ab GAM-Alexa Fluor 488 & A11029 & ICC- $1: 100$ \\
\hline $2^{\circ} \mathrm{Ab}$ GAR- Alexa Fluor 488 & A11034 & ICC-1:100 \\
\hline 2ㅇ Ab GAM-Alexa Fluor 568 & A11031 & ICC- $1: 100$ \\
\hline 2 Ab GAR-Alexa Fluor 568 & A11036 & ICC-1:100 \\
\hline $2^{\circ} \mathrm{Ab}$ GAR-HRP & JIR 115-035-003 & WB-1:3000 \\
\hline 20 Ab GAM-HRP & JIR 111-035-003 & WB-1:3000 \\
\hline
\end{tabular}


9.5. Toluidine staining: The neuroretinal explants co-cultured with hAD-MSCs in above experiments were fixed in PALAY (1\% glutaraldehyde, 1\% paraformaldehyde, $0.002 \%$ Calcium chloride, in 0.4M phosphate buffer with $7.4 \mathrm{pH}$ ) (Palay, Chan-Palay 1974) and then in $1 \%$ osmium tetra oxide in phosphate buffer. Then fixed nueroretinal explants were embedded in Araldite epoxy resin 506 (Sigma-Aldrich, USA) in special block at $60{ }^{\circ} \mathrm{C}$ overnight. Ultrathin sections were made in a microtome LKB, model Ultratome III, using glass knives at $45^{\circ}$ angle. The selection of the cutting area, was determined from the study of semi-thin sections ( 1 micron thick). These sections were stained with the following solution: $1 \mathrm{~g}$ Toluidine Blue in $100 \mathrm{~mL}$ solution of 3\% Sodium tetraborate (Borax). These sections were mounted in mounting medium and observed and photographed under the light microscope (Axiophot, Zeiss). A total of 128 samples of hNR explants were processed from 6 different experiments to around 300 slides with 4-5 sections on each for toluidine staining.

9.6. Light microscopy: Several microscopes were used in this works for different purposes. Phase contrast light microscope (compound) with digital sight camera and monitor (Nikon) (up to 40x) was used to watch and take picture of normal live cell culture. The normal small light microscope (up to 10x) was used to count the cells in normal trypsinization, seeding and maintenance of the cells. The Leica inverted fluorescence digital microscope (up to 63X) with multiple filters and phase contrast option was used to take the picture of immunostained cells. Leica upright fluorescence digital microscope (up to $100 \mathrm{x}$ ) was used to take pictures of immunostained histological sections of retinal explants as well as to take picture of chemically stained fixed cells. Upright light digital microscope (Axiophot, Zeiss) (up to 100x) was used to take the picture of toluidine stained ultrathin section of neuroretinal explants. The picture in this microscope was captured with the digital camera (Axiocam HRc) and processed with the help of Axiovision Software (with version 4.8.1 (11-2009).

\section{Arbitrary evaluation method for the status of neuroretinal explants}

The neuroretinal explants were evaluated by observing the photomicrographs obtained by toluidine staining and immunostaining against CRALBP and GFAP, Calbindin and Synaptophysin, and Rhodopsin. We have decided to give arbitrary number from 0 to 10 depending upon the degree of preservation of global health status of neuroretinal explants including all the layer and cellular status. The points 0-0.5 were given for the retinal explants which were completely destroyed and disorganized (no tissue structure) and 10 points were given for the well-structured and well-preserved retinal explants in very good health. The intermediate points (numbers) are given for the global status of neuroretinal 
explants for one condition by observing the photomicrographs from histochemical and immunohistochemical staining. For each condition there were 1-2 representative photomicrographs in each staining. There were 6 experiments performed and evaluated to give points for each sample of each experiment. The evaluation was made by three different experts having knowledge of neuroretina and their degeneration patterns. The points were assigned for preservation status of neuroretinal explants in each condition by observing the following aspects.

10:- Perfect architecture of retina

9-8: Similar to 0 day retinal explants (status of retina at the time of the beginning of the experiment)

7-8: PR degeneration, early degeneration of outer segment (OS), shortening/disruption/truncation of rods, swelling of inner segment (IS) and cell body, late degeneration of IS, complete loss of PRs, ganglion cell vacuolization

5-6: Nuclei layers (ONL/INL)- Early and late pyknosis, karyorrhexis and karyolysis, degree of compaction and number of nuclei in the layers, retraction of PRs axon, pyknosis and loss of horizontal cells (HCs), descending of bipolar across INL, reduction of axon terminal, damage of postsynaptic bipolar and HCs

3-4: Synaptic layers (OPL/IPL)- Thinning/Swelling of OPL/IPL, retinal edema, disappearance of OPL, discontinuity of OPL/IPL, degree of density of plexus, sprouting of HCs processes in IPL, mislocation of expression of specific protein e.g. Calbindin (CB), Synaptophysin (SYP), Rhodopsin (RHO) etc. Sprouting of bipolar cell dendrites, reactive gliosis, glial cell death, hypertrophic proliferation and migration, loss of horizontal cell dendrites

1-2: External and internal limiting membrane- Rosette formation, disappearance of ELM/ILM, optic nerve fibre layer swelling and swelling or degeneration of astrocytes

0-0.5: Complete disorganization/destruction of retinal layers to look like disorganized cell mass

Finally all the points from three experts for all the 6 experiments were subjected to statistical analysis to obtain a final summarized view of the results. 
Table 4. Example of Evaluation table

\begin{tabular}{|l|l|l|l|l|l|}
\hline Experimenta No. & $\begin{array}{l}\text { Toluidine } \\
\text { Blue }\end{array}$ & $\begin{array}{l}\text { CRALBP\& } \\
\text { GFAP }\end{array}$ & $\begin{array}{l}\text { Calbindin \& } \\
\text { Synaptophysin }\end{array}$ & Rhodopsin & Mean \\
\hline 0 Day & 9 & 8 & 7 & 9 & 8.25 \\
\hline RCM (R) & 3 & 3 & 2 & 3 & 2.75 \\
\hline R+MSCs (B) & 5 & 4 & 4 & 5 & 4.5 \\
\hline B+VIP & 3 & 4 & 2 & 5 & 3.5 \\
\hline B+NIC & 4 & 4 & 3 & 6 & 4.25 \\
\hline B+ATRA & 6 & 4 & 3 & 5 & 4.5 \\
\hline B+VIP+NIC & $\mathbf{6}$ & 6 & 4 & 7 & 6.25 \\
\hline B+VIP+ATRA & 6 & 6 & 6 & 5 & 5.76 \\
\hline B+NIC+ATRA & 5 & 5 & 5 & 6 & 5.25 \\
\hline B+VIP+NIC+ATRA & 7 & 5 & 6 & 8 & 6.5 \\
\hline
\end{tabular}

11. Statistical analysis: All the experiments have been repeated three to six times in duplicates or triplicates to check the reproducibility of the trends observed. The readings obtained from three or more experiments were subjected to the statistical analysis through MS Excel programme and online GraphPad Software. Standard Deviations (SD), Standard errors of means (SEM), means and p-values were calculated and the observed statistical significance was adjusted to $\mathrm{P}<0.05, \mathrm{P}<0.01$ and $\mathrm{P}<0.001$. The t-test (paired/two tailed) was used to compare the different geometrical means of each treatment group obtained by the three experiments. 


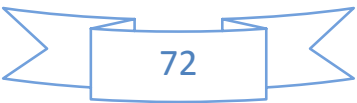




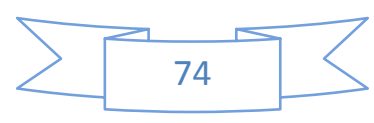




\section{Characterization of RPE cells and undifferentiated hAD-MSCs}

Before starting the main work, the principle cells used in this study such as hRPE cells, ARPE19 cells and hAD-MSCs have been worked out for getting familiar with them. The isolation of these cells from their source organs or tissues, their expansion and maintenance have been established properly. They have also been studied for their morphological and cellular characteristics. Various methods and protocols have been optimized for each of them. Additionally, they have been tested for purity (FC method), viability and cell cycle phase analysis and other inherent cellular characteristics during splitting and sub-culturing.

\subsection{RPE cells}

\section{Cell isolation, expansion and maintenance}

RPE cells were isolated from donated post-mortem human eyes. The hRPE cells along with RPE cell line (ARPE19) had been expanded and maintained in the culture as described in materials and methods. The phase contrast optical microscopic observation showed that hRPE cells at lower passage (P0-P1) acquired hexagonal epithelial morphology with lots of pigment in the cytoplasm after being confluent (Fig.8 A \& B). But during further subcultures, hRPE showed decreased pigmentation in cytoplasm and loss of epithelial characteristics e.g. epithelial to fibroblast like morphology (data not shown). The growth rate of hRPE cells were significantly less in comparison to other cells e.g. ARPE19 and hAD-MSCs and the doubling time was found 4-5 days (Fig.8 D). In further study, these RPE cells showed significant adherence and proliferation and maintained their cobblestone morphology with RPE65 expression on the surface of biocompatible elastin like recombinamers with integrated RGD bioactive domain (ELR-RGD) in comparison to ELR without any specific sequence (ELR-IK) (Singh et al. 2013b, Srivastava et al. 2011a). 


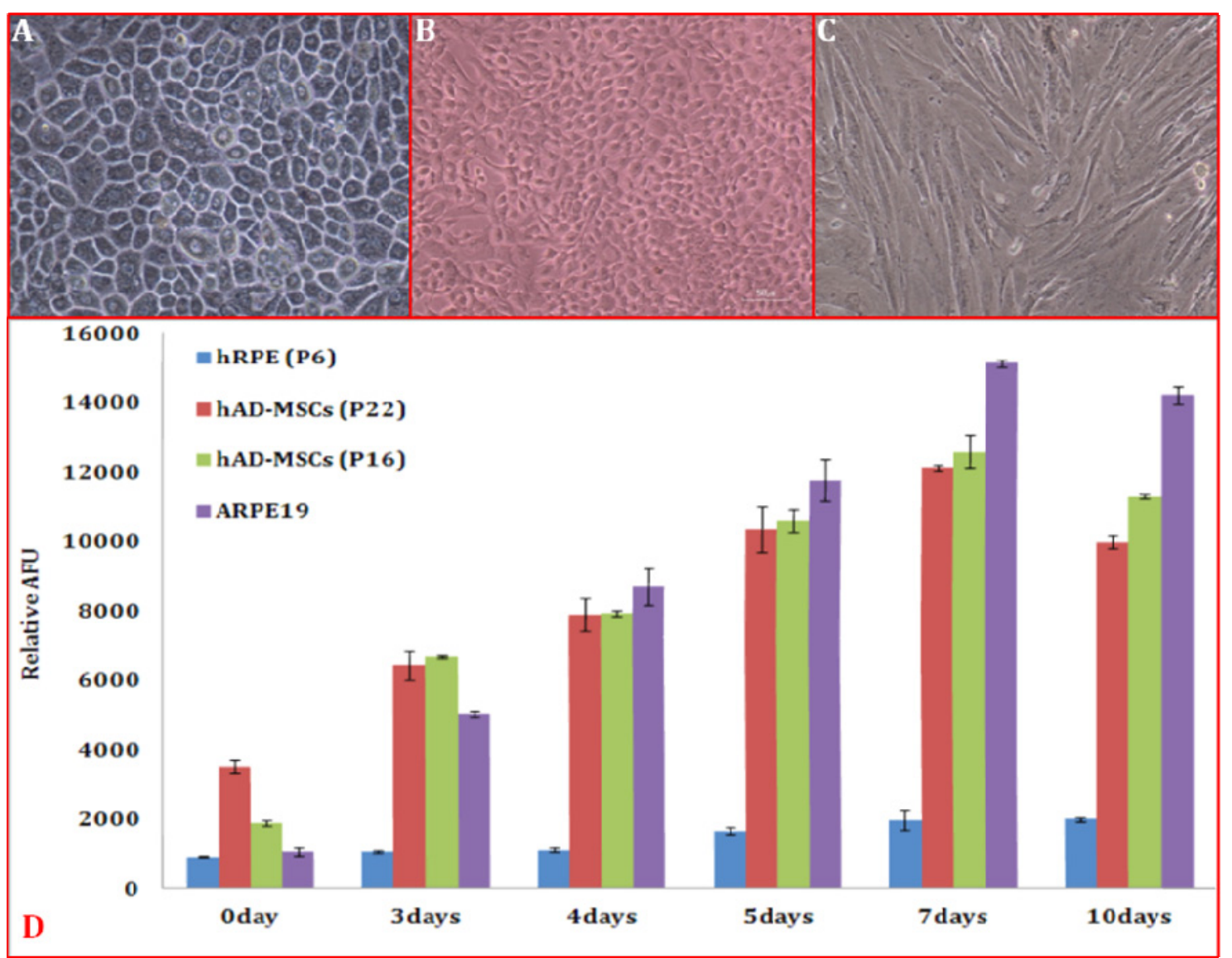

Fig.8. Cell morphology and growth of hRPE, ARPE19 and hAD-MSCs in their corresponding growth medium. Light photomicrographs of hRPE cells at 40x (A), ARPE19 cells at 10x (B) and hAD-MSCs at 10x (C) and their cell growth analysis, plotted as a bar graph (D) by measuring arbitrary fluorescence signal unit (AFU) in Almar blue assay at different time points. All cells were seeded at equal density of 15,000 cells $/ \mathrm{cm}^{2}$. Error bar is indicated as $\pm S D(n=3)$.

\section{Cell viability and cell cycle analysis}

Flow cytometry (FC) analysis was performed for determining the hRPE cell viability and cell cycle in different passages of RPE cells by staining against annexin V and propidium iodide (PI). Homogenous cell population (88.3\%) of each passage was selected for cell analysis (Fig. 9A). The results showed that 99.5\% hRPE cells (P2-P10) were found viable. The percent of cells in early and late apoptotic phase, and final dead phase were $0.1 \%$, $0.0 \%$ and $0.3 \%$ respectively (Fig. 9B). The Cell cycle analysis showed that the percent of cells in the $\mathrm{G}_{0} / \mathrm{G}_{1}$ phase $(2 \mathrm{n})$, S-phase and $\mathrm{G}_{2} / \mathrm{M}$ phase $(4 \mathrm{n}$ ) were $86.3 \%, 3.5 \%$ and $7.4 \%$ respectively (Fig. 9C). The hRPE cells were exposed to UV-B for 48 hours for taking positive control for apoptosis. The results showed that percent of early and late apoptotic cells and live and dead cells were 19.4\%, 54.7\%, 9.2\% and 16.6\% respectively (Fig. 9D) (Srivastava et al. 2013). 

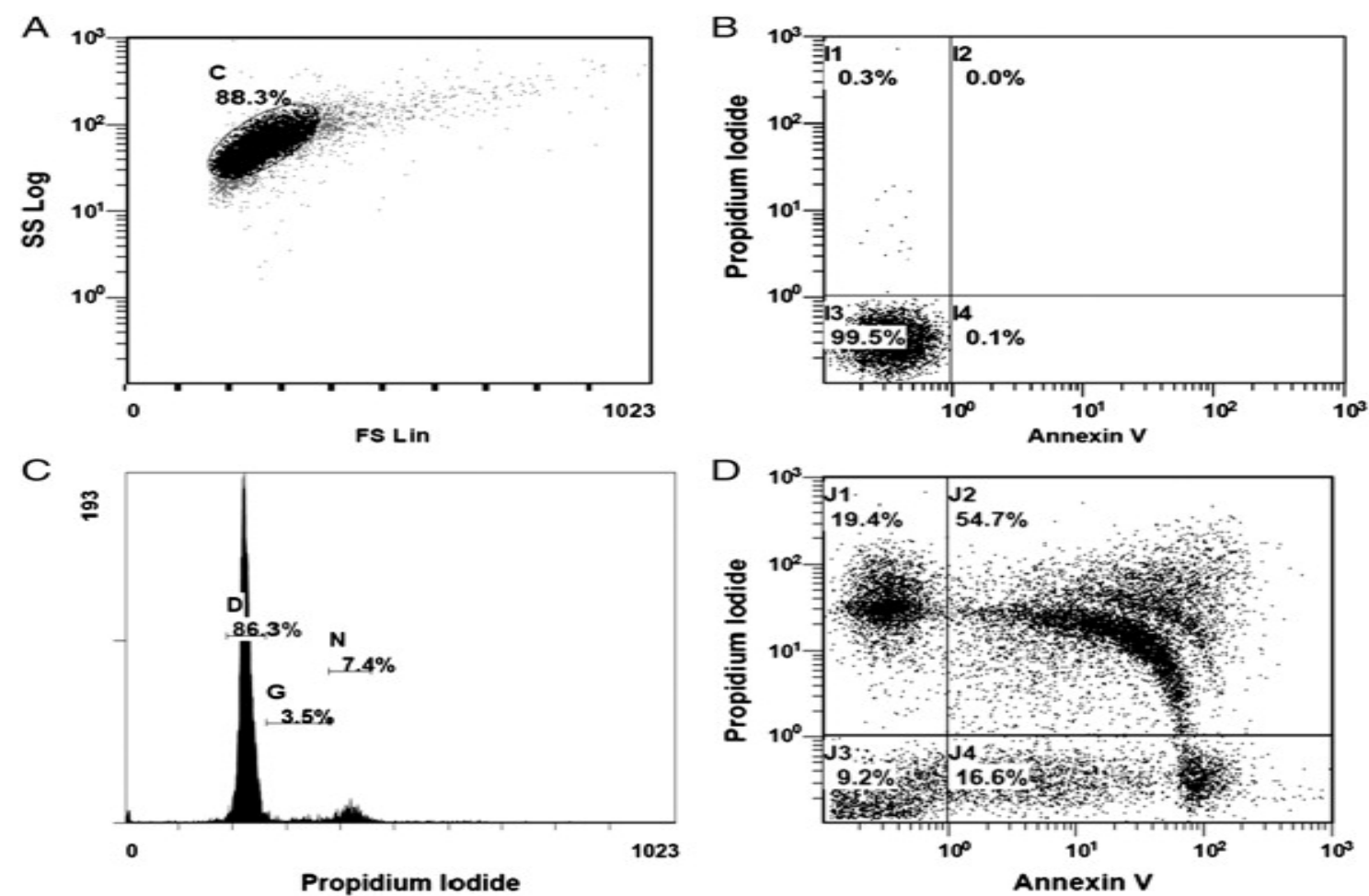

Fig.9. Cell viability and cell-cycle analysis of hRPE cells (passage 5) using FC. Selection of homogeneous hRPE cells for FC analysis (A), detection of their viability and death by annexin $\mathrm{V}$ and PI staining (B) and their cell cycle phase analysis by PI staining (C). Cell viability analysis of hRPE stimulated with UV-B light for 48 hours as a positive control for apoptosis (D). All FC histograms are representative to different passages of hRPE (P5-P10). SS log is side scatters at logarithmic scale and FS Lin is forward scatter at linear scale. The SS Log vs. FS Lin plot shows the complexity (shape and size) of cells in the cell population.

\section{Assessment of RPE and fibroblastic characteristics}

The FC analysis of hRPE cells of different passages (P5, P8 and P10) and ARPE19 cells (control) were done to assess the percentage of cells expressing RPE65 protein and the degree of RPE65 protein expression per cell. In FC analysis, mean fluorescence index (MFI) reflects the average amount of protein expression (density) per cell (event) in arbitrary unit (fluorescence signal). The relative MFI is the ratio of MFI of test antibody to MFI of isotype control. The FC analysis showed that 91.7\% of ARPE19 cells were positive for cytoplasmic RPE65 protein with relative MFI of 6 and only $0.07 \%$ of them were positive for the fibroblast-epithelial protein with relative MFI of 1 (no expression) (Fig. 10A and B). In case of hRPE cells, 93.6\% of cells of passage 5 were positive for RPE65 protein with relative MFI of 6.3 and negative for the fibroblast-epithelial protein (Fig. 10C and D). Further the percentage and intensity of RPE65 protein decreased in passage 8 and 10 with 90.1\% (relative MFI=3.3) and 85.3\% (relative MFI=3.0) respectively from the higher value of percentage (93.6\%) and relative MFI (6.3) of passage 5. The percentage of hRPE cells expressing RPE65 protein and the intensity of RPE65 protein expression decreased with 
increase in passage number from 93.6\% with MFI of 6.3 (P5) to 90.1\% with MFI of 3.3 (P8) and 85.3\% with MFI of 3.0 (P10) (Fig. 10C, E and G) without any significant changes in their fibroblast-epithelial protein expressions (Fig. 10D, F and H). This decrease in RPE65 expression of hRPE cells with increase in their passage number were further confirmed by WB analysis that showed the decrease in band intensity of RPE65 protein expression from lower passage (P2) to higher one (P9) (Fig. 11). ARPE19 cells including other cells e.g. human skin fibroblast, human corneal epithelial cells were used as positive controls in this study (data not shown) (Srivastava et al. 2013).

The hRPE cells contain pigments that can emit strong autofluorescence. This autofluorescence interfered to detect signals of RPE65 antibody conjugated fluorescence in FC analysis and other immunofluorescence studies. A novel method was developed to quench cellular autofluorescence in RPE cells by using different concentration $(10 \mu \mathrm{g} / \mathrm{mL}$, $20 \mu \mathrm{g} / \mathrm{mL}, 200 \mu \mathrm{g} / \mathrm{mL}, 250 \mu \mathrm{g} / \mathrm{mL}, 2000 \mu \mathrm{g} / \mathrm{mL}$ ) of trypan blue (TB). The pRPE cells and pig retinal tissues were used in this study for optimizing the concentration and protocol steps (Srivastava et al. 2011b). FC analysis showed that pRPE cells (P1-P6) were emitting autofluorescence. Due to autofluorescence, there was significant overlap between the positive (test signal) and negative signal (noise) of RPE65 staining of pRPE cells in FC diagram. The overlapping curve of RPE65 positive and negative signals were separated when pRPE cells were treated with TB. The results demonstrated that $74.94 \%$ (relative MFI = 10.6), 80.01\% (relative MFI = 19.2) and 53.58\% (relative MFI = 5.7) of pRPE cells were RPE65 antigen positive (clear and separated signal) when treated with $10 \mu \mathrm{g} / \mathrm{mL}$, $20 \mu \mathrm{g} / \mathrm{mL}$ and $40 \mu \mathrm{g} / \mathrm{mL}$ ice-cold TB solution respectively. The quenching of autofluorescence by TB at $20 \mu \mathrm{g} / \mathrm{mL}$ and higher concentrations was further confirmed in immunohistochemical staining of pig retina against RPE65 protein (Srivastava et al. 2011b). This method worked well in quenching hRPE cells as well and it was used in further immunofluorescence studies (data not shown).

The hRPE cells were successfully isolated, expanded, maintained and characterized. These cells including ARPE19 cells showed significant growth, viability, adhesion and morphological characteristics on the surface of biocompatible ELR-RGD polymer. The hRPE cells have been found to lose their RPE characteristics such as pigmentation, RPE65 protein and cobblestone morphology rapidly during sub-culturing in cell culture. An FC method was established for testing the purity of hRPE cells (contamination with fibroblast). A method was established for quenching cellular pigment associated autofluorescence in FC analysis and other immunofluorescence studies using trypan blue stain. There were four articles published from this section (Singh et al. 2013b, Srivastava et al. 2013, Srivastava et al. 2011b, Srivastava et al. 2011a). 
A

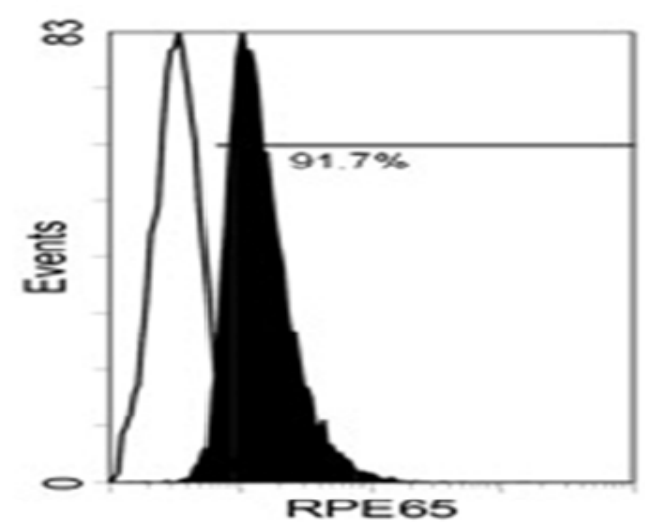

C

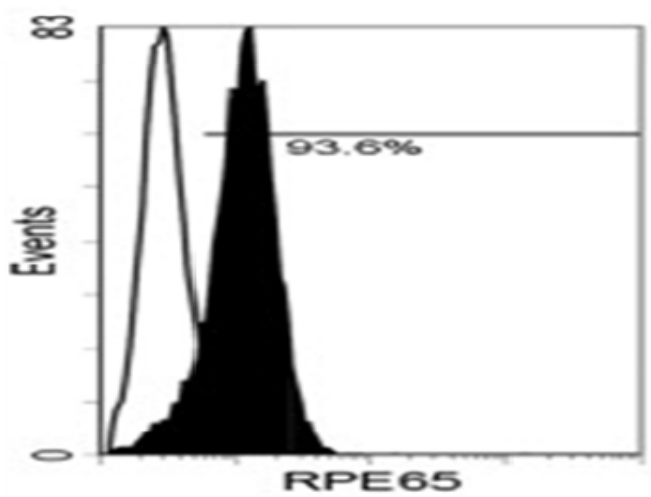

$\mathbf{E}$

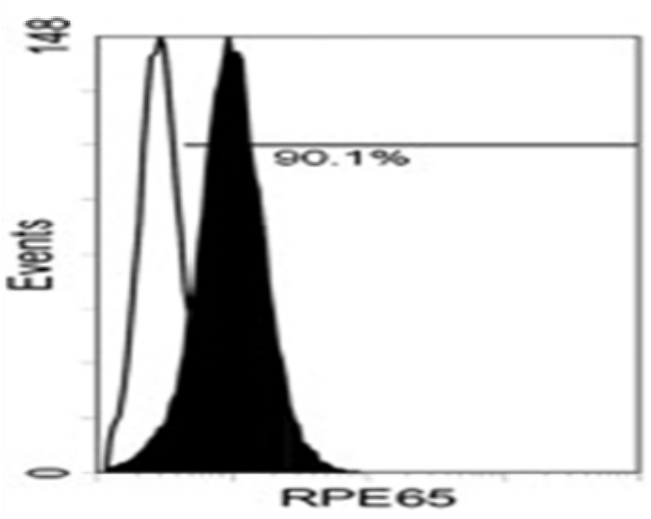

G

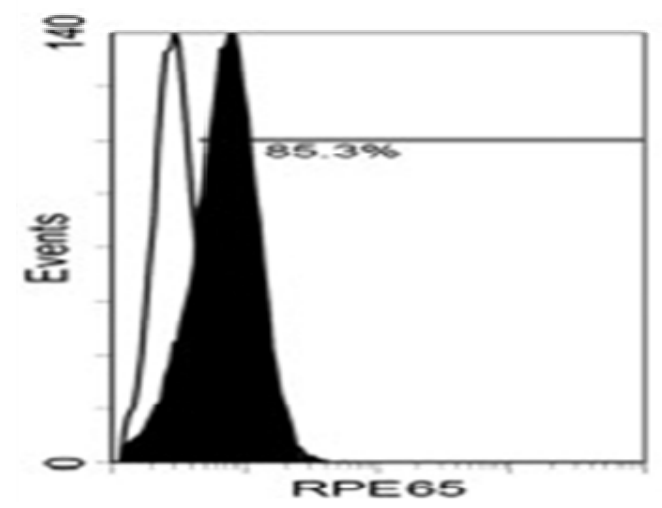

B

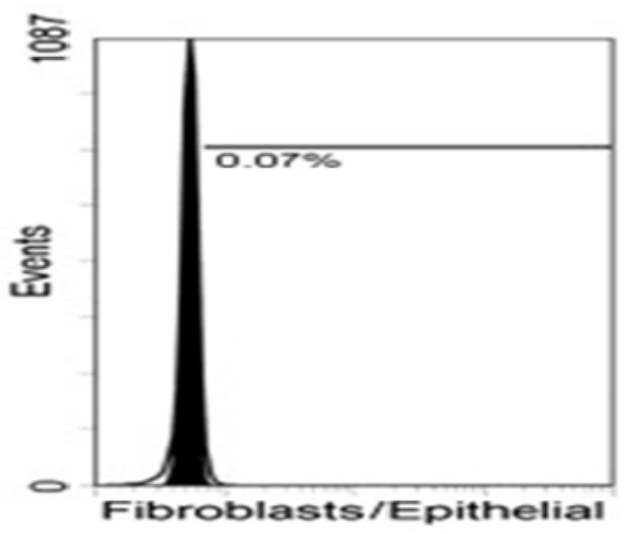

D

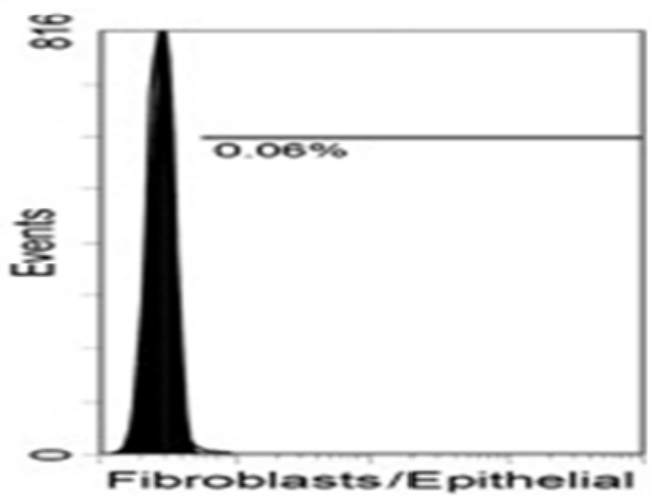

$\mathbf{F}$

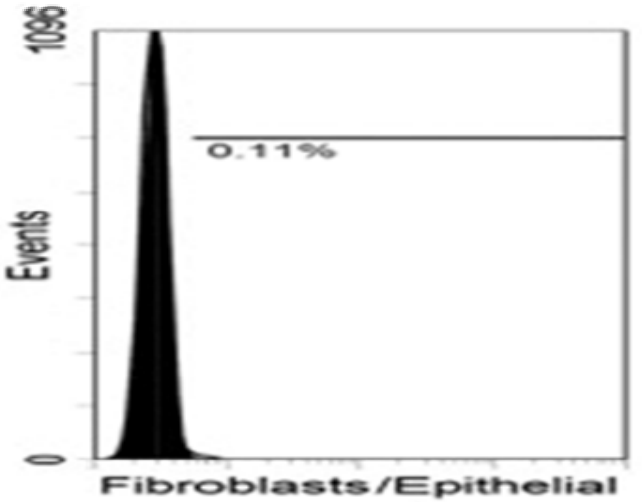

$\mathbf{H}$

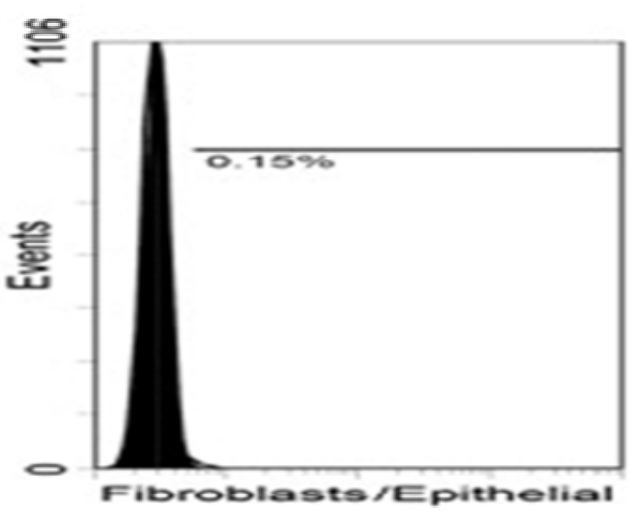

Fig.10. FC analysis of expression of RPE65 and epithelial/fibroblastic proteins in RPE cells by staining with anti-RPE65 and anti-human MoAb. Percentage of cells expressing RPE65 protein in ARPE19 cells (A) and in hRPE cells of passage 5 (C), passage 8 (E), passage $10(\mathbf{G})$ and the percentage of cells expressing epithelial/fibroblast protein in ARPE19 cells (B) and in hRPE cells of passage 5 (D), passage $8(\mathbf{F})$ and passage $10(\mathbf{H})$.

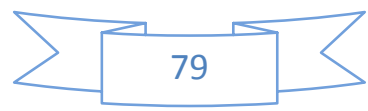




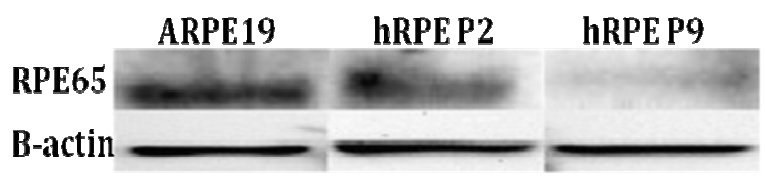

Fig.11. RPE65 protein expression analysis in hRPE cells of primary cell cultures of passages 2 and 9 by WB method. ARPE19 cells are used as a control for detecting RPE65 (65kDa) protein and $\beta$ actin $(42 \mathrm{kDa})$ is used to determine equal loading of proteins.

\section{2. hAD-MSCs}

\section{Cell isolation, expansion and maintenance}

MSCs were isolated from donated lipoaspirates (adipose tissues) obtained in cosmetic liposuction procedure by using the described procedure (Nieto-Miguel et al. 2013) from six different donors. The isolation, expansion, growth and maintenance of lower as well as higher passages of hAD-MSCs were established at the beginning of the work. The hADMSCs showed the normal plastic adherent fibroblastic morphology in culture condition (Fig. 8C). The growth characteristics of hAD-MSCs were also performed including higher passages (Fig. 8D). The results showed that the growth rate of hAD-MSCs is similar to that of ARPE19 cells (data not shown). Depending on culture condition and seeding density/sub-culture splitting ratio, cell doubling time of hAD-MSCs was found to be between 2 to 4 days (data not shown) as reported by others as well (Mitchell et al. 2006, Izadpanah et al. 2006). Although the hAD-MSCs were grown till passage 30 to compare the potency and other growth characteristics (data not shown), in all the differentiation experiments, only lower passages (P3-P6) of hAD-MSCs had been used. 

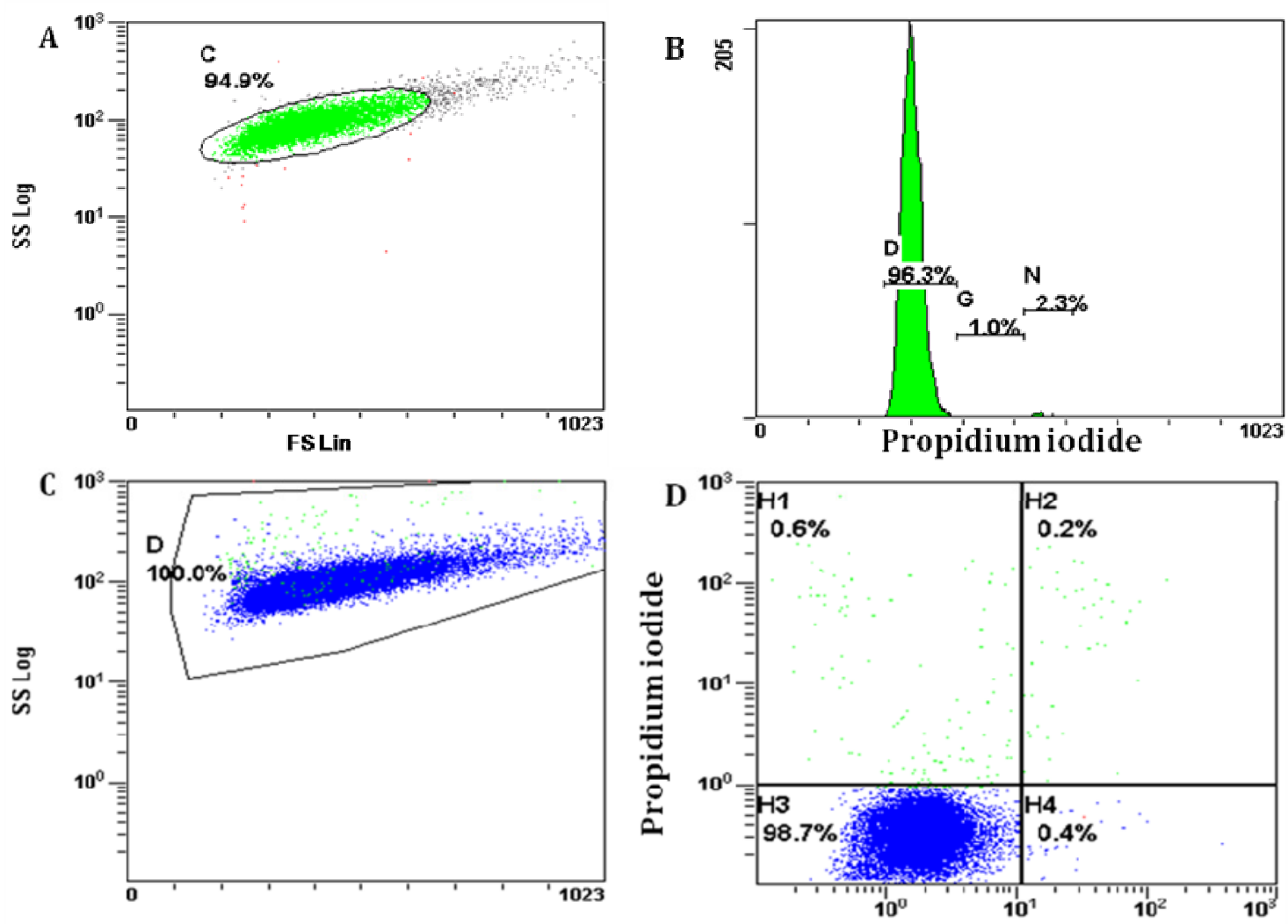

FS Lin

Annexin V

Fig.12. FC analysis of hAD-MSCs determination of cell cycle phase (cell proliferation), cell death (apoptosis) and viability in standard cell culture condition. 94.9\% of hAD-MSCs population were homogenous (GREEN) observed in the flow cytometer gate C (A). 96.3\%, 1\% and 2.3\% of hAD-MSCs were in $\mathrm{G}_{0} / \mathrm{G}_{1}$ phase (2n) (gate D), S-phase ( $>2 n$ ) (gate $\mathrm{G}$ ), and $\mathrm{G}_{2} / \mathrm{M}$ phase (4n) (gate N) respectively (B). All the cells (100\%) were selected for cell viability analysis through apoptotic markers (C). $98.7 \%$ smaller and less complex cells (BLUE) were negative for annexin $\mathrm{V}$ as well as for propidium iodide as observed in the flow cytometer gate $\mathrm{H} 3$, indicating very high viability of hAD-MSCs in standard culture condition (D). [Forward scatter (FS) is the laser light that is scattered in the forward direction (along the same axis the laser is traveling) and is detected in the Forward Scatter Channel (FSC). The intensity of this signal has been attributed to cell size and refractive index (membrane permeability). Side scatter (SS) is the laser light that is scattered at 90 degrees to the axis of the laser path and is detected in the Side Scatter Channel (SSC). The intensity of this signal is proportional to the amount of cytosolic structure in the cell (e.g. granules, cell inclusions, etc.). So, FS vs. SS (cell size vs. internal structure), a correlated measurements between them allow for distinguishing cell types in a heterogeneous cell population. Gating is used to isolate a subset of cells on a plot that allows to look at parameters specific to only that subset of cells and can use Boolean logic to include or exclude multiple gates.] 


\section{Cell viability and cell cycle analysis}

Morphological parameters of hAD-MSCs were analyzed by shape and size complexity, 94.9\% cell population was found homogenous when observed in flow cytometer gate C, which is used to select for cell cycle analysis of total cell population (Fig. 12A). The cell cycle analysis of hAD-MSCs showed that vast majority (96.3\%) of cells were in $G_{0} / G_{1}$ phase $(2 \mathrm{n})$ and smaller portions of cells i.e. $1 \%$ and $2.3 \%$ were in S-phase $(>2 n)$ and $G_{2} / M$ phase (4n) respectively (Fig. 12B). Entire cell population (100\%) was selected for the cell viability analysis through apoptotic markers (annexin $\mathrm{V}$ and propidium iodide). A large portion of cells $(98.7 \%)$ were negative for annexin $\mathrm{V}$ as well as for propidium iodide, which implicate that hAD-MSCs maintained their high viability in standard in vitro culture condition (Fig. 12C\&D).

\section{Characterization of undifferentiated hAD-MSCs}

The passages of hAD-MSCs between P3-P5 were used to characterize according to the minimal criteria established by ISCT (Horwitz et al. 2005). Further the higher passages of hAD-MSCs were also characterized as well to compare the characteristics of uninduced hAD-MSCs and to decide if the higher passage hAD-MSCs could be used for the differentiation experiments.

\section{Immunophenotypic Profile}

For immunophenotypic profiling, $97.1 \%$ of homogenous hAD-MSCs population was selected for FC analysis (Fig. 13A). Results showed that the large portions of hAD-MSCs (>99\% population) were shown negative for cell surface markers e.g. CD14, CD19, CD34, CD45 and HLA-DR (Fig. 13B, C, D, E \& F) and the vast majority of the hAD-MSCs (>97\% population) were shown positive for MSCs-specific glycoprotein surface markers e.g. CD73, CD90, and CD105 with relative MFI of around 38, 32, and 6 respectively (Fig. 13G, H \& I).

\section{Differentiation potential of hAD-MSCs}

The hAD-MSCs were subjected to routine differentiation procedure into three mesodermal lineages e.g. adipocytes, osteocytes, and chondrocytes as established under the minimal criteria by ISCT. When these cells were cultured in adipogenic differentiation medium for 15 days, they differentiated into adipocytes as shown by numerous Oil Red 0 stained lipid droplets inside the cells in comparison to the control (1st column of Fig. 14). Similarly they were differentiated into chondrocytes with light blue stained sulfated proteoglycans 
(glycosaminoglycans present in chondrocytes and cartilage) present in the ECM of dense nodule of micromass differentiation culture (Alcian blue) after culturing them in chondrocytes differentiation medium for 27 days, while in control condition they could not form the firm micromass because of absence of any differentiation to chondrocytes ( $2^{\text {nd }}$ column of Fig. 14). They have also been differentiated well into osteocytes with blackish brownish deposits of calcium phosphate salts in the cytoplasm stained with von Kossa as compared to their controls after culturing them in osteocytes differentiation medium for 29 days ( $3^{\text {rd }}$ column of Fig. 14). The hAD-MSCs in controls of these routine differentiation procedures were incubated with normal non-differentiating medium for the same time period as for the differentiation in each case and did not show any spontaneous differentiation into these three mesodermal lineages. 


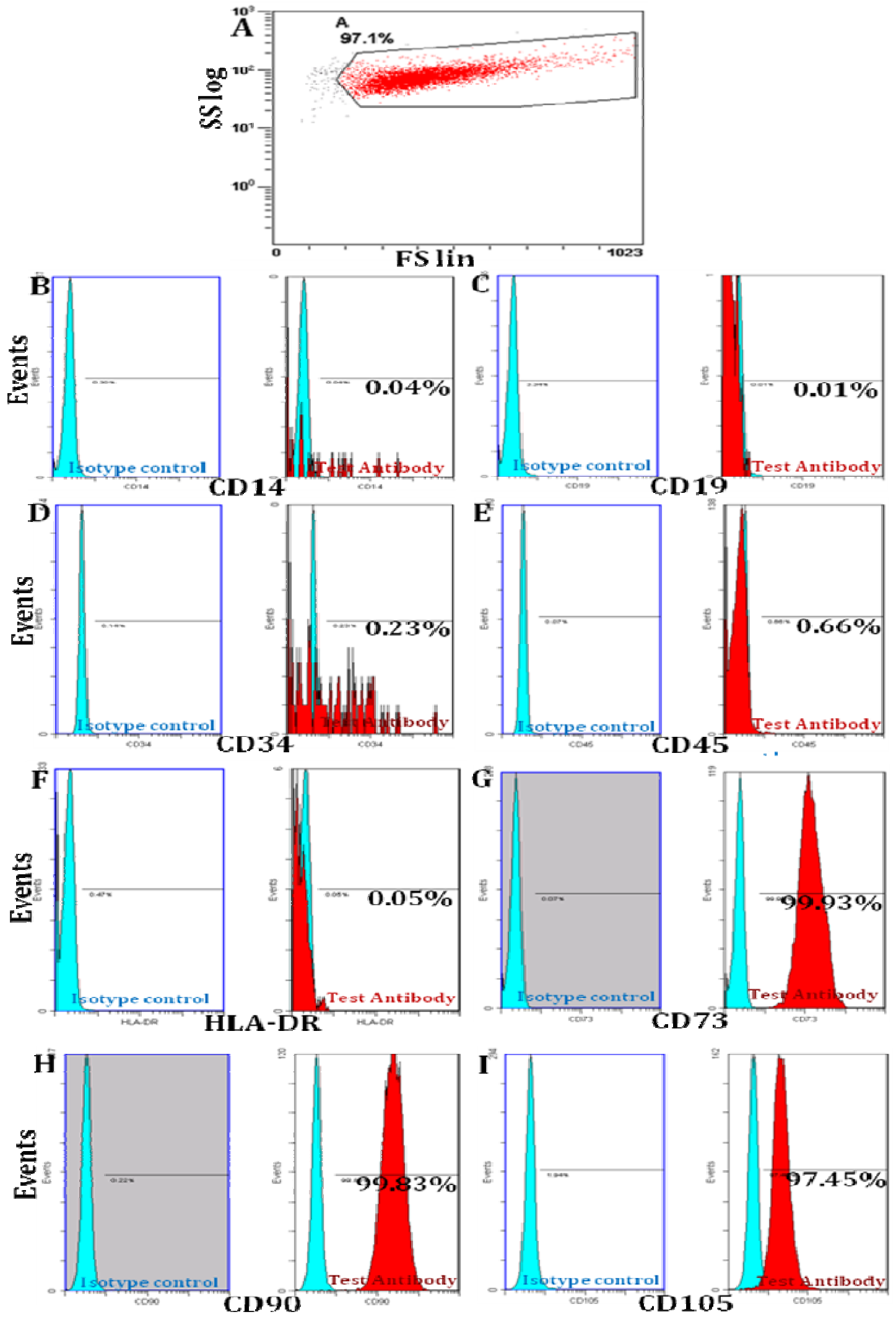

Fig.13. Immunophenotypic profile of hAD-MSCs (P3-P5) by FC analysis. The expression (red colour) of selected panel of cell surface markers (CD14, CD19, CD34, CD45, HLA-DR, CD73, CD90, and CD105) compared with corresponding isotype control (sky blue colour) by percentage in the selected homogenous population of hAD-MSCs (A). Percentage of cells expressing CD14 (B), CD19 (C), CD34 (D), CD45 (E), HLA-DR (F), CD73 (G), CD90 (H) and CD105 (I).

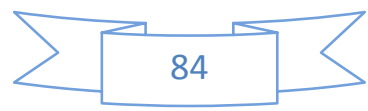




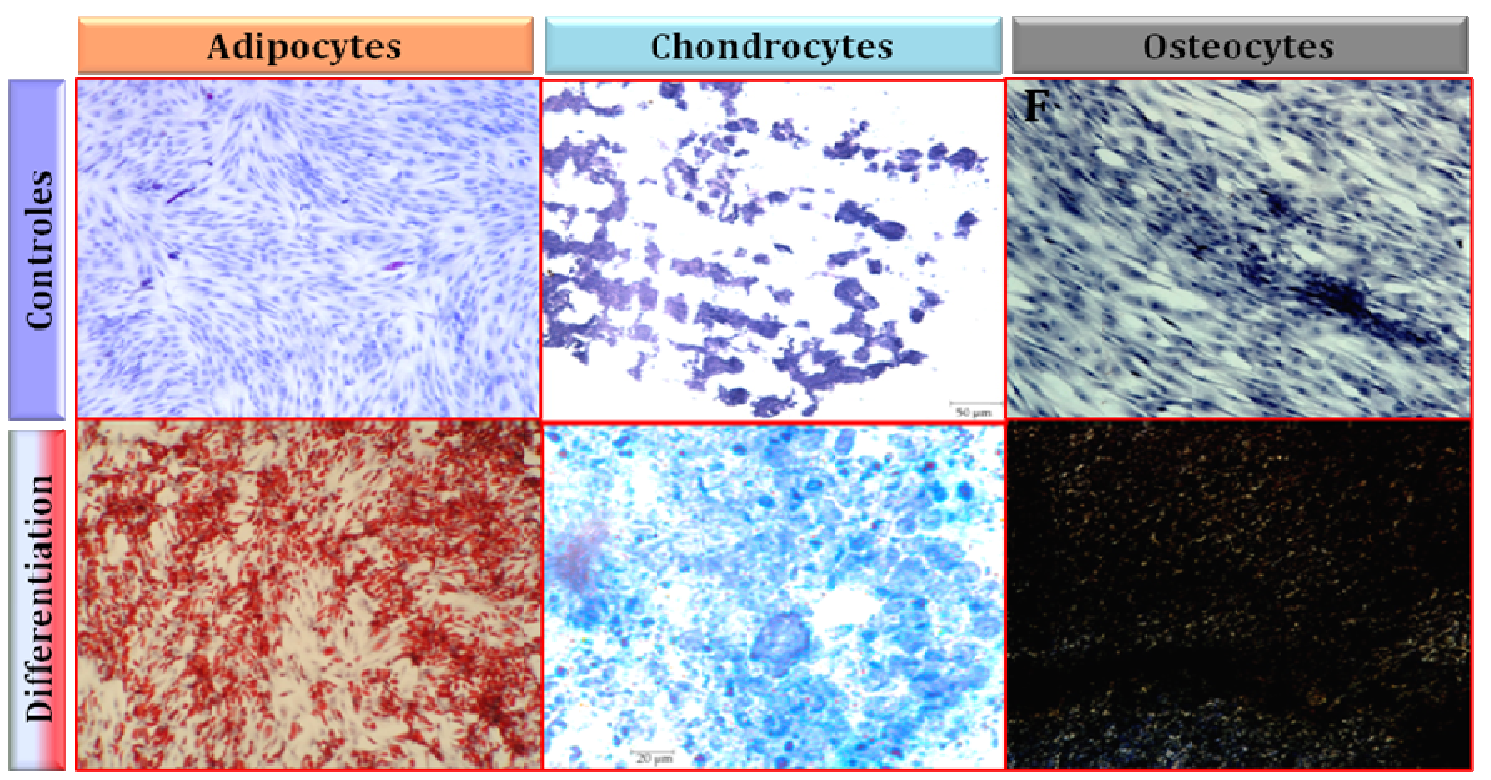

Fig.14. Differentiation potential of hAD-MSCs cultured in three different differentiation medium for three mesodermal lineages (adipocytes, chondrocytes and osteocytes). The differentiation of hAD-MSCs into Adipocytes stained with Oil Red 0, Chondrocytes stained with Alcian blue, and Osteocytes stained with von Kossa. All the cells were counterstained with Hematoxylin Gill III. The induction period for adipocytes, chondrocytes and osteocytes were 15, 27 and 29 days respectively.

\section{Comparison between higher and lower passages of undifferentiated hAD-} MSCs

The hAD-MSCs of higher passages (P16 and P22) were characterized similarly as mentioned previously for lower passages to test whether the immunophenotypes and differentiation potential of hAD-MSCs were maintained along the subculturing. FC analysis showed the repetition of results for cell surface markers e.g. CD14, CD19, CD34, CD45 and HLA-DR in hAD-MSCs of higher passages (data not shown). However, there was increase in the population of cells, expressing MSCs-specific glycoprotein surface markers e.g. CD73, CD90, and CD105 in comparison to that of lower passages (Fig. 15A). These cells showed higher relative MFI of around 58, 83 and 7 for CD73, CD90 and CD105 expression respectively in comparison to those $(43,44$ and 7) of lower passages hAD-MSCs (Fig. 15B). The hAD-MSCs are usually heterogeneous fibroblastoid population of adult stem cells. During subsequent culturing and expansion they became more homogenous as shown by the presence of higher percentage of expression of MSCs-specific markers in hAD-MSCs' population at higher passages (Fig. 15A).

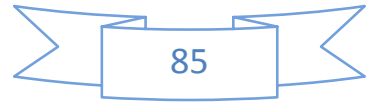




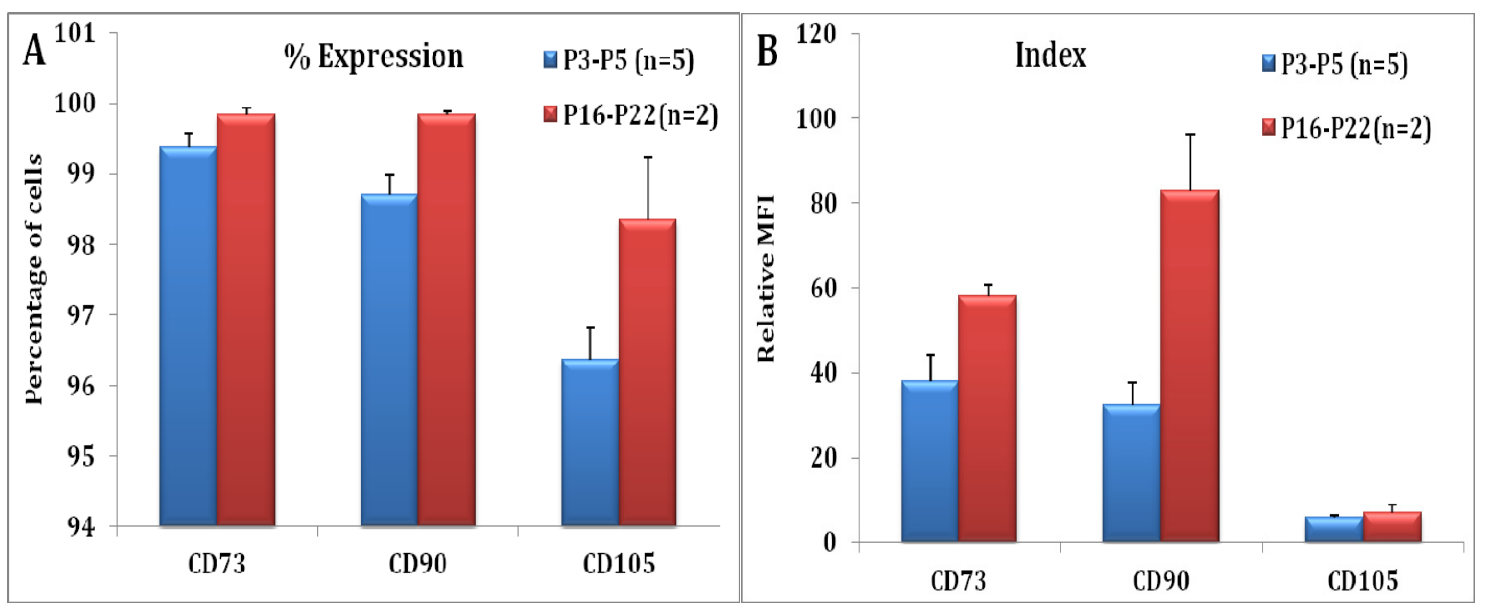

Fig.15. Comparison of expression of MSCs-specific markers between lower and higher passages. Percentage of hAD-MSCs' population expressing three MSCs-specific markers (CD73, CD90 and CD105) in lower passages (P3-P4) and higher passages (P16-P22) (A) and the comparison of their relative MFI (B) Note:- some of the data of lower passages were taken from the work of Sara Galindo (with her kind permission) working under Dr. M. Teresa Nieto in Ocular Surface group of IOBA.

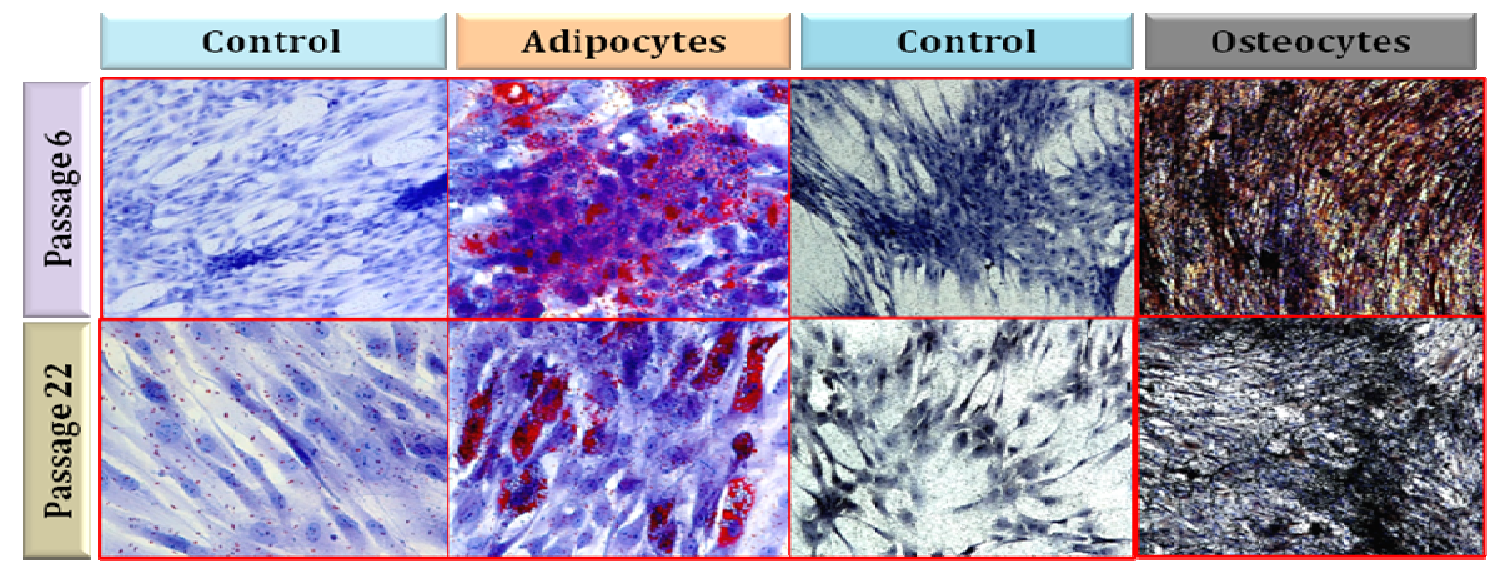

Fig.16. Comparative differentiation potential of lower passage (P6) and higher passage of hAD-MSCs (P22) cultured in differentiation medium for adipocytes and osteocytes. The differentiation of hAD-MSCs into Adipocytes stained with Oil Red $\mathbf{O}$ and Osteocytes stained with von Kossa. All the cells were counterstained with Hematoxylin Gill III. The induction period for adipocytes and osteocytes were 15 and 29 days respectively.

The differentiation capacity of hAD-MSCs (P22) into three mesodermal lineages (adipocytes, osteocytes and chondrocytes) was found lesser than that of lower passage hAD-MSCs (P6), is evident from the experimental results (Fig. 16). The frequency of lipid vacuole with lipid droplets in adipocytes in higher passages was lesser than that of lower passages (adipocytes column of Fig. 16). Similarly the deposits of calcium phosphate salts in osteocytes of higher passages were lesser than that of lower passage one (Osteocytes column of Fig. 16). In the chondrocytes differentiation capacity of higher passages was

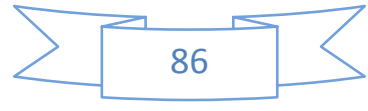


found very low and they were not able to form firm cell mass in micromass culture at passage 22 (data not shown).

The cell growth analysis results showed that the hAD-MSCs of higher passages (P16 and P22) had slower growth rate in comparison to the hAD-MSCs of lower passages (P2-P8) (Fig. 8D). Lower passages hAD-MSCs (P2-P8) had almost similar growth rate of ARPE19 cells (data not shown). They needed 4-6 days to be confluent depending upon the cell seeding density and culture conditions. In Fig. 8, the bars for ARPE19 cells (in the bar graph) were representative for the growth of lower passages of hAD-MSCs (P2-P8) as well due to similar growth curve (data not shown).

MSCs were successfully isolated from lipoaspirates and expanded, maintained and characterized according to the criteria laid by international society for cellular therapy (ISCT) including higher passages (P16-P22). The hAD-MSCs of higher passages were found more homogeneous and showed higher expression of MSCs-specific markers but showed slower growth and lower differentiation capacity in comparison to that of lower passages.

\section{Assessment of effects of hAD-MSCs on MMC treated RPE cells (ARPE19) under indirect co-culture conditions}

The hAD-MSCs were co-cultured on trans-well with the MMC treated RPE (ARPE19) cells in the wells of 6-well plates (indirect co-culture system) to test the effect of hAD-MSCs on MMC treated RPE cells.

\section{MMC treatment triggers cell death and reduces proliferation of RPE cells}

For optimizing the concentration of MMC and their treatment period to RPE cells, the RPE cells were exposed to different concentrations of MMC for different time period. Later the treated RPE cells were assessed for proliferation and viability at different time points after treatment. It was observed that MMC treatment triggered programmed cell death (apoptosis) in RPE cells at higher dose (>50 $\mathrm{g} / \mathrm{mL}$ ) as seen in microscopic observations of cellular morphology of MMC treated RPE cells. Microscopic observation showed the formation of apoptotic vesicle/bodies (dim green fluorescent bodies indicated by blue arrows), shrinkage of cells and bleb formation in MMC treated RPE cells at two weeks (Fig. 17A). The cells were found almost dead (red fluorescent cells) after 3 weeks (Fig. 17B). Florescence microscopy showed that RPE cells in control without any treatment of MMC were $100 \%$ viable (Fig. 18A), while almost all MMC (50 $\mu \mathrm{g} / \mathrm{mL})$ treated RPE cells were dead after 3 weeks of treatment (Fig. 18B). The results of $A B$ analysis (to measure 
proliferation activity of cells) showed that MMC (50 $\mu \mathrm{g} / \mathrm{mL}, 100 \mu \mathrm{g} / \mathrm{mL}$ and $200 \mu \mathrm{g} / \mathrm{mL})$ treatment reduced the proliferation activity of RPE cells in 3 days significantly $(\mathrm{p}<0.05$, $n=3)$. Fluorescence intensity decreased significantly $(p<0.05, n=3)$ with the increase in concentration of MMCs (50 $\mu \mathrm{g} / \mathrm{mL}, 100 \mu \mathrm{g} / \mathrm{mL}$ and $200 \mu \mathrm{g} / \mathrm{mL}$ ) (Fig. 18E). After 3 days, a significant high fluorescence intensity $(\mathrm{p}<0.05, \mathrm{n}=3)$ was found in the untreated RPE cells compared to the MMC treated RPE cells (Fig. 18E, F \& G).

\section{hAD-MSCs partially rescue the MMC-treated RPE cells (ARPE19) from cell death}

Cell viability/toxicity assay demonstrated that $50 \mu \mathrm{g} / \mathrm{mL}$ MMC treated RPE cells after three weeks retained significant viability, when co-cultured with hAD-MSCs (Fig. 18C) and there were no adverse effects after three weeks on the hAD-MSCs grown on trans-well due to death of MMC treated RPE cells in the well (Fig. 18D).

$\mathrm{AB}$ analysis showed that MMC treated and untreated RPE cells co-cultured with hAD-MSCs had significantly higher fluorescence intensity $(\mathrm{p}<0.05, \mathrm{n}=3)$ than those of MMC treated and untreated RPE cells alone (Fig. 18E). In further experiment, a comparative study using $\mathrm{AB}$ assay kit, was done to determine the effects of hAD-MSCs and human fore skin fibroblast cell line (HFF1/BJ cell) separately over the MMC $(50 \mu \mathrm{g} / \mathrm{mL})$ treated RPE cells. The AB assay results showed that the MMC treated RPE cells with hAD-MSCs had significantly higher fluorescence intensity $(\mathrm{p}<0.05, \mathrm{n}=3$ ) than those with HFF1 (Fig 18F). hAD-MSCs produced the same growth stimulating effects over MMC treated RPE cells through its spent medium used in culture (Fig.18F) and stimulated significantly higher proliferation activity in MMC treated RPE cells in comparison to HFF1 at 3 and 6 days periods (Fig. 18G).

It was observed that the proliferation of MMC treated RPE cells decreases, as the concentration of MMC increases from $50 \mu \mathrm{g} / \mathrm{mL}$ to $200 \mu \mathrm{g} / \mathrm{mL}$. Co-culturing of these MMC treated RPE cells with hAD-MSCs showed that the proliferative capacity of these cells were significantly higher than those MMC treated RPE cells alone but lesser than those of untreated RPE cells with or without co-culture with hAD-MSCs. This indicates that the hAD-MSCs cells were secreting protective factors in co-culture medium that created a microenvironment which might be conducive to rescue partially RPE cells from cell death. 


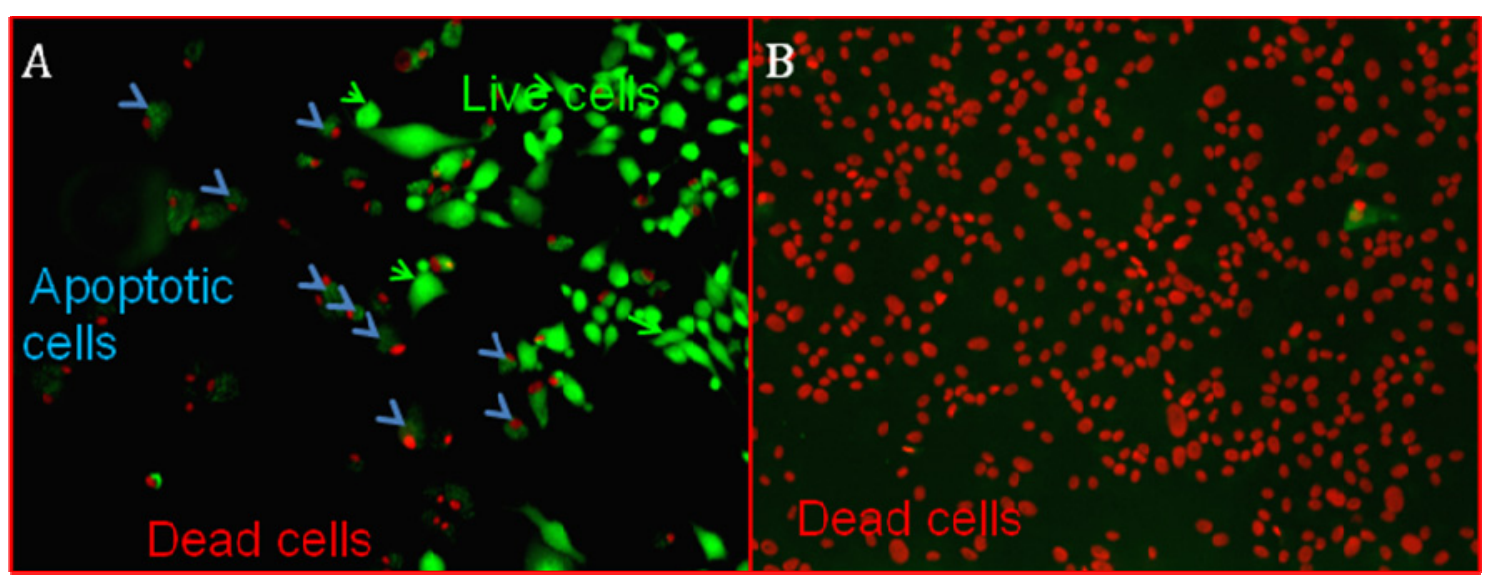

Fig.17. Cell viability/Cytotoxicity assay of MMC treated ARPE19 cells. The process triggers in ARPE 19 cells treated with MMC (50 $\mu \mathrm{g} / \mathrm{mL})$ after 2 weeks (A) and after 3 weeks (B) Green fluorescence indicates live cells, red fluorescence indicates dead cells and granulated dim green fluorescence indicate Apoptotic cell death (shown by blue arrow head) and early apoptotic vesicles/bodies formation, shrinkage of cells and bleb formation (shown by green arrow head).

MMC was found to trigger programmed cell death (apoptosis) in RPE cells. The hAD-MSCs partially rescued the MMC treated RPE cells from entering into cell death in indirect coculture system and stimulated the proliferation of RPE cells. The similar effect was observed when supernatant of hAD-MSCs was used in culture of the MMC treated RPE cells. 

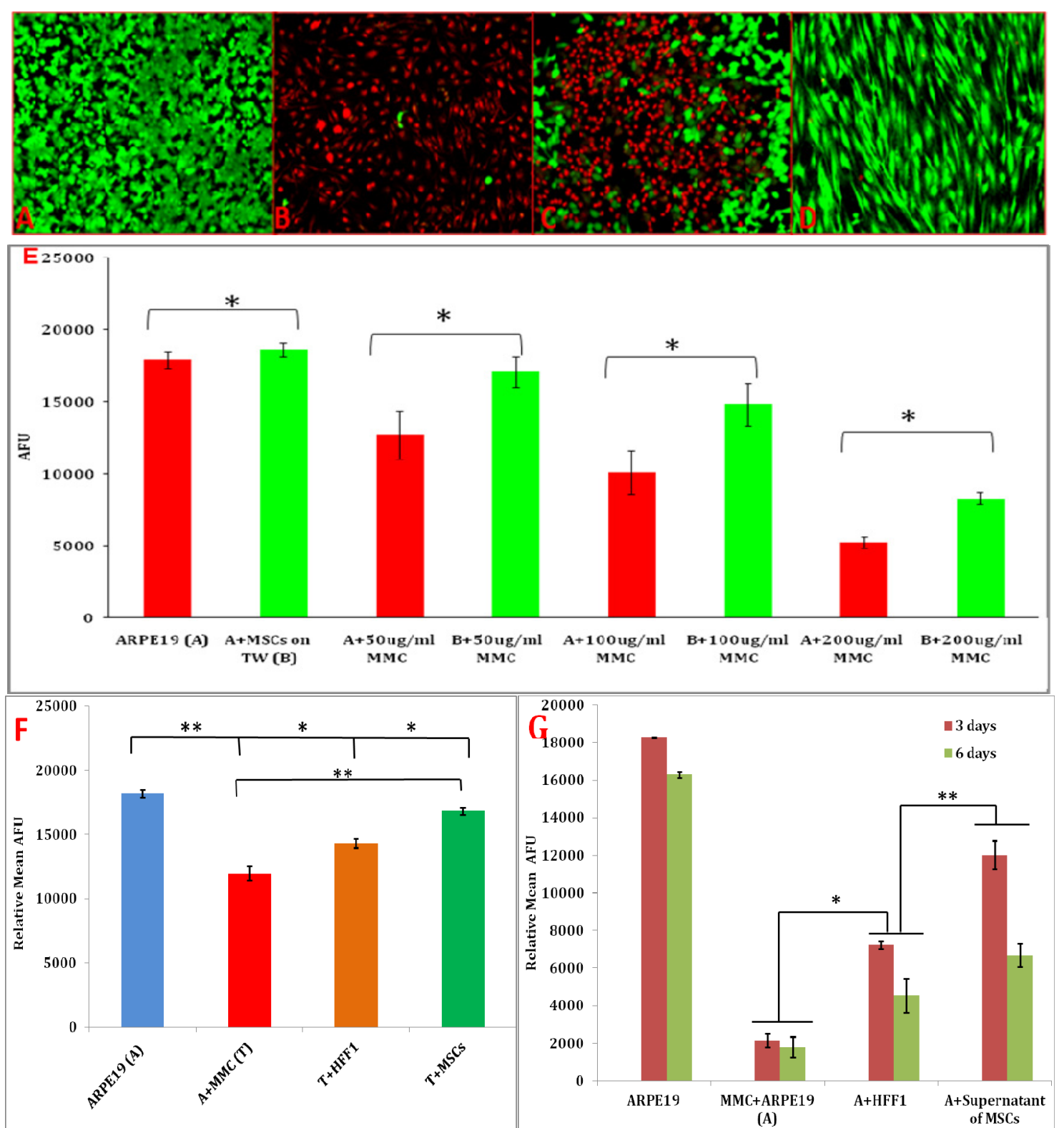

Fig.18. Cell viability/Cytotoxicity assay and cell proliferation assay of MMC treated and untreated RPE (ARPE19) cells co-cultivated with hAD-MSCs in indirect co-culture condition. Green fluorescence indicates live cells and red fluorescence indicates dead cells. Cell viability of untreated RPE cells (A), MMC treated $(50 \mu \mathrm{g} / \mathrm{mL})$ RPE cells (B), MMC treated RPE cells and cocultured with hAD-MSCs (C) and hAD-MSCs on Falcon trans-well (D). AB assay for cell proliferation and activity of MMC treated (50 $\mathrm{gg} / \mathrm{mL}, 100 \mu \mathrm{g} / \mathrm{mL}$ and $200 \mu \mathrm{g} / \mathrm{mL})$ and untreated RPE cells cocultured with and without hAD-MSCs (E), the comparison of effects of hAD-MSCs with that of fibroblast (HFF1/BJ Cell line) on MMC treated RPE cells (F) and the comparison of effects of supernatant of hAD-MSCs and fibroblast cells on MMC treated RPE cells at 3 and 6 days (G). All values shown are corrected with background fluorescence of the reagent in media alone. Error bar is indicated as \pm SEM. Single asterisk $\left({ }^{*}\right)$ represents the significant P-value between MMC treated or untreated RPE cells vs. MMC treated or untreated RPE cells co-cultivated with AD-MSCs. Double asterisk $\left(^{* *}\right)$ represents the significant P-value between untreated vs. MMC treated RPE cells. Statistical significance is adjusted at $\mathrm{P}<0.05\left(*{ }^{* *}\right)$. The panel $\mathrm{A}, \mathrm{B}, \mathrm{C}, \mathrm{D}, \mathrm{E}$ and $\mathrm{F}$ are adapted from the published article (Singh et al. 2013a)

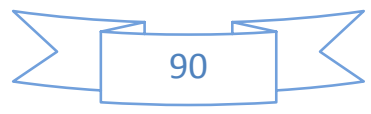




\section{Assessment of differentiation of hAD-MSCs towards RPE phenotype}

Five different methods were used to stimulate differentiation of hAD-MSCs to RPE phenotype:

1. Cultivation of hAD-MSCs in the growth medium with $50 \%$ hRCM in the presence of three different factors (VIP, NIC and ATRA) and their combinations

2. Cultivation of hAD-MSCs in the growth medium with $50 \%$ fRCM and POS $\left(1 \times 10^{5} / \mathrm{mL}\right)$ in the presence of BMP-4 (Department of Eye and Vision Science, University of Liverpool)

3. Direct co-cultivation of hAD-MSCs with MMC inactivated RPE cells

4. Indirect co-cultivation of hAD-MSCs with MMC inactivated RPE cells in the presence of three different factors (VIP, NIC and ATRA) and their combinations

5. Indirect co-cultivation of hAD-MSCs with human neuroretinal (hNR) explants in the growth medium with 50\% RCM in the presence of three different factors (VIP, NIC and ATRA) and their combinations

\subsection{Cultivation of hAD-MSCs in hRCM supplemented with factors and their combinations}

The hAD-MSCs were stimulated for differentiation towards RPE phenotypes by culturing them in DMEM/F12 medium with 50\% hRCM supplemented with factors (VIP, NIC, and ATRA) and their combinations (VIP+NIC and NIC+ATRA) for 21 days. WB analysis for the expression of four RPE markers (RPE65, Bestrophin, CK8/18 and Pancytokeratin) has been carried out for the hAD-MSCs under stimulated and unstimulated conditions along with RPE cells as a positive control (Fig. 19). The summary of significant increase in the expressions of the RPE specific proteins is given in the Table 5. There was a significant increase in expression of RPE65 in hAD-MSCs when stimulated with the combinations of $\mathrm{VIP}+\mathrm{NIC}$ and VIP+ATRA in comparison to other factors alone including RCM only. The epithelial markers CK8/18 and PCK were increased in the presence of ATRA which already acted as an epithelial differentiation factor in hAD-MSCs (Brzoska et al. 2005).

The immunofluorescence study of hAD-MSCs stimulated by factors and their combination showed that the combinations (VIP+NIC and NIC+ATRA) induced the significant increase in the expression of RPE marker proteins (RPE65 and Bestrophin) in comparison to other conditions including basal condition of stimulation by hRCM only (Fig. 20). 

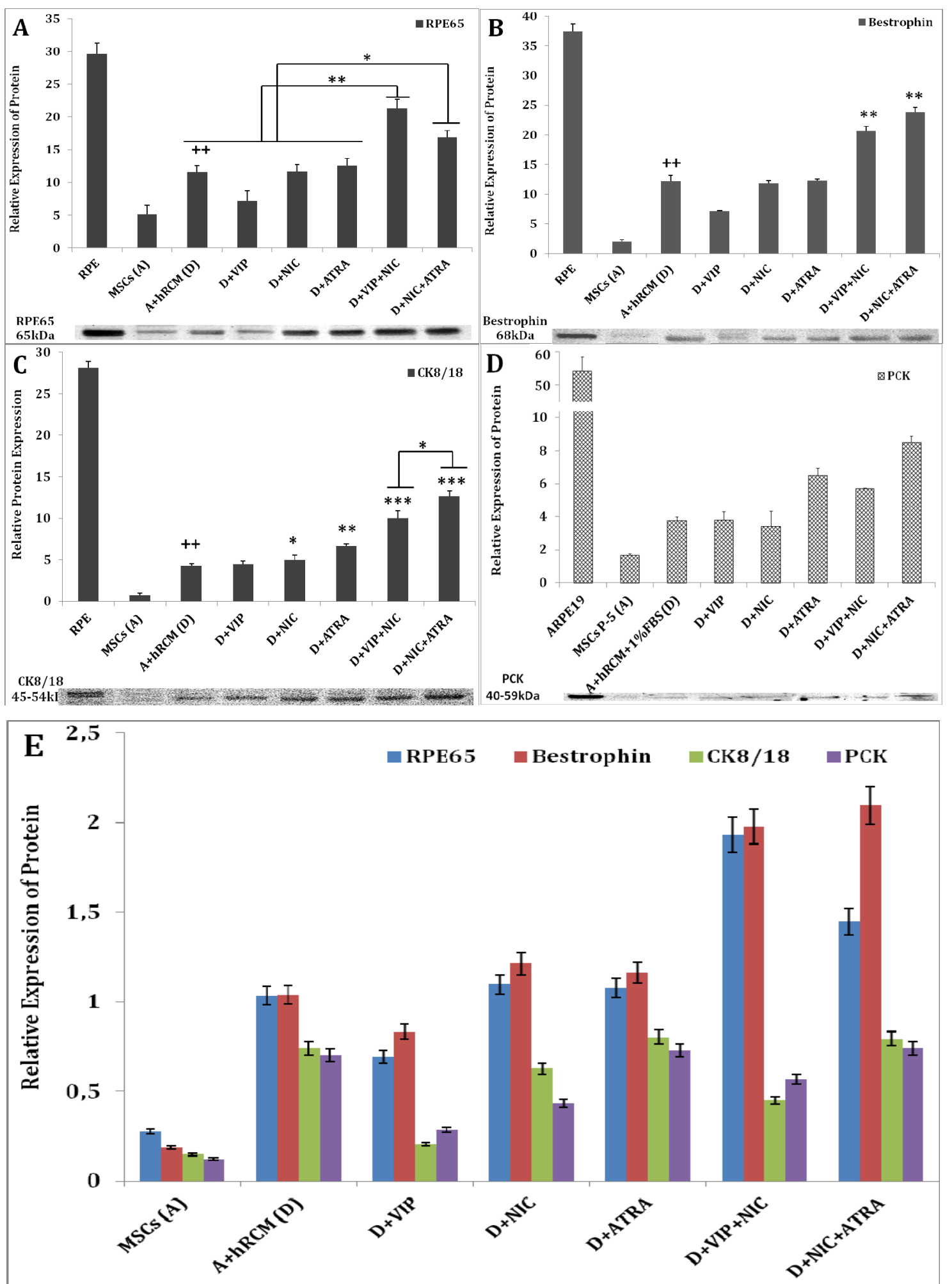

Fig.19. Assessment of differentiation of hAD-MSCs to RPE phenotypes when cultured for 21 days in $50 \% \mathrm{hRCM}$ and with different factors (VIP, NIC and ATRA) and their combinations (VIP+NIC and NIC+ATRA) by WB analysis. Relative expression of RPE protein markers such as RPE65 (A), Bestrophin (B), CK8/18 (C), and PCK (D) and their comparative expression plotted together (E), in unstimulated and stimulated hAD-MSCs. The statistical significance is set at $\mathrm{P} \leq 0.05\left({ }^{*}\right) /(+), P \leq 0.01$ $\left({ }^{* *}\right) /(++)$ and $\mathrm{P} \leq 0.001\left(^{* * *}\right)$. Plus sign indicates the significant difference between the uninduced and induced hAD-MSCs with hRCM only and asterisk show the significant difference between with and without factors treatment. Error bar is indicated as $\pm \mathrm{SD}(\mathrm{n}=3)$.

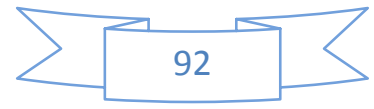


Table 5. Summary of WB analysis protein expression (from Fig. 25) in case of culture of MSCs in different environments Note: Here the increase in number of plus sign indicates the difference at statistical significance ( $\mathrm{P} \leq 0.05, \mathrm{n}=3$ ) except in the case of PCK (only indicative difference).

\begin{tabular}{|l|l|l|l|l|}
\hline WB analysis & RPE65 & Bestrophin & CK8/18 & PCK \\
\hline MSCs (A) & + & $+/-$ & $+/$ & $+/$ \\
\hline A+RCM (D) & +++ & +++ & ++ & ++ \\
\hline D+VIP & ++ & ++ & ++ & ++ \\
\hline D+NIC & +++ & +++ & +++ & ++ \\
\hline D+ATRA & +++ & +++ & ++++ & +++ \\
\hline D +VIP+NIC & +++++ & +++++ & +++++ & +++ \\
\hline D+NIC+ATRA & ++++ & +++++ & +++++ & +++ \\
\hline
\end{tabular}




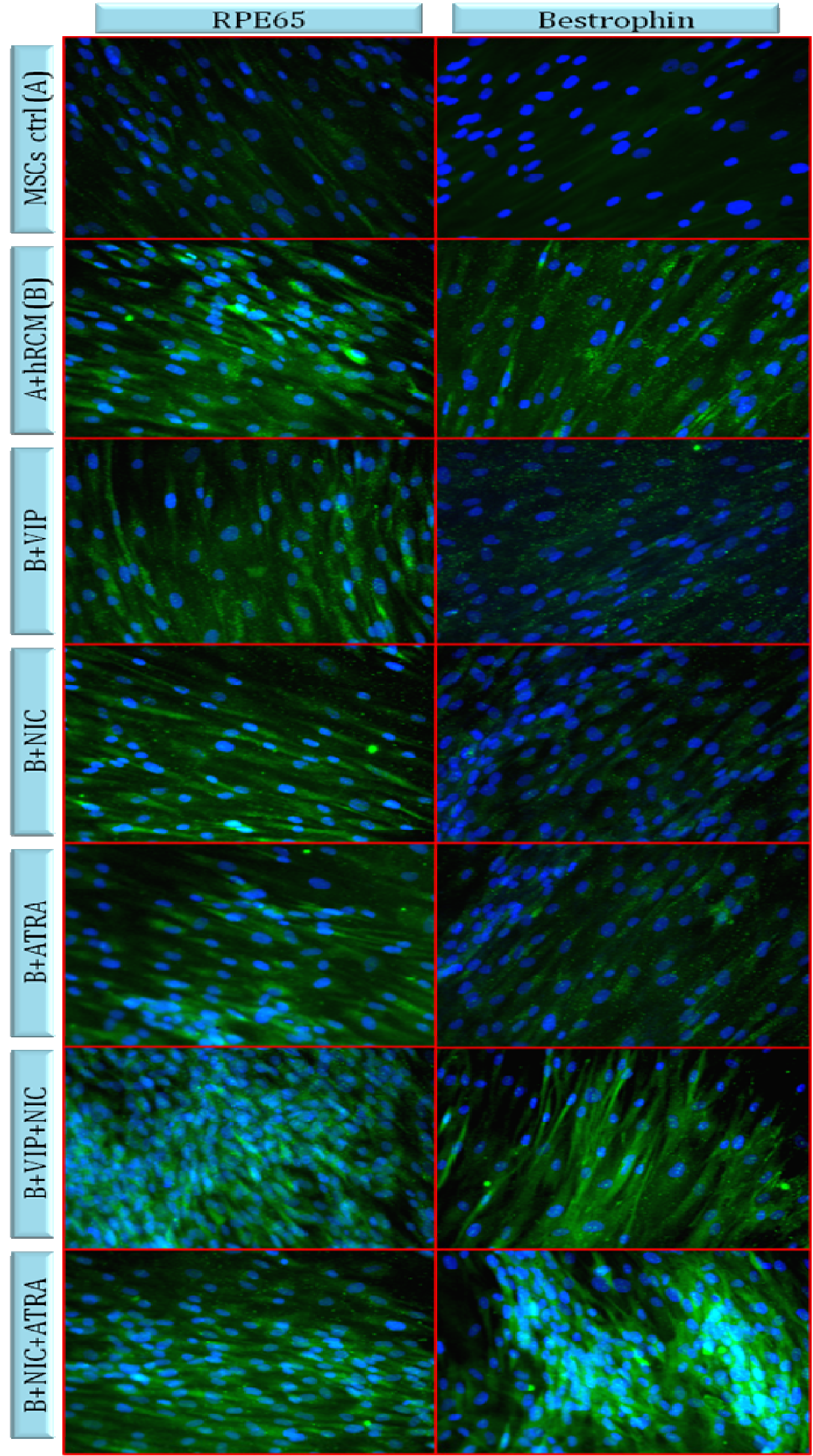

Fig.20. Immunocytochemical fluorescence analysis of differentiation status of hAD-MSCs cultured in 50\% hRCM supplemented with factors (VIC, NIC and ATRA) and their combinations (VIP+NIC and NIC+ATRA) for 21 days. Expression of RPE protein markers such as RPE65 and Bestrophin in stimulated and unstimulated hAD-MSCs. All images are taken at 40X magnification.

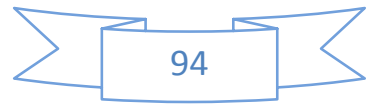


The hAD-MSCs increased the RPE markers expression when induced by NIC, ATRA, VIP+NIC, and NIC+ATRA along with 50\% hRCM based on WB and immunocytofluorescence studies, indicating that they differentiated towards RPE phenotype partially.

\subsection{Induction of differentiation of hAD-MSCs to RPE phenotype with BMP-4 and POS and fRCM (This part of study was performed in the department of Eye and Vision Science, Institute of Chronic and Ageing Disease, University of Liverpool)}

The hAD-MSCs were stimulated for differentiation to RPE phenotypes by culturing them with 50\% fRCM and POS with and without BMP-4 (200ng/mL) for 15 days as described in material and method section. Interestingly the combinations with POS showed some toxic effects on hAD-MSCs proliferations and finally these combinations with POS did not work. But other combinations with fRCM alone and fRCM with BMP-4 did give some preliminary interesting results. The hAD-MSCs were found to form pigmented attached dense cell mass sphere when stimulated with fRCM and BMP4 alone or together (Fig. 21B \&C) in comparison to control (Fig. 21A). Immunocytochemical studies showed a significant increase in RPE markers (RPE65 and CK8/18) expressions in hAD-MSCs grown in fRCM and the fRCM supplemented with BMP-4 (Fig. 22). The preliminary data of WB analysis did not show any signals of changes in RPE markers (Tyrosinase and CK8/18) expression (Fig. 23). There is a need to repeat these experiments to conclude about the differentiation of hAD-MSCs towards RPE phenotype in these conditions but the normal culture studies and immunocytochemical studies indicates the increase of RPE phenotype in stimulated hAD-MSCs.

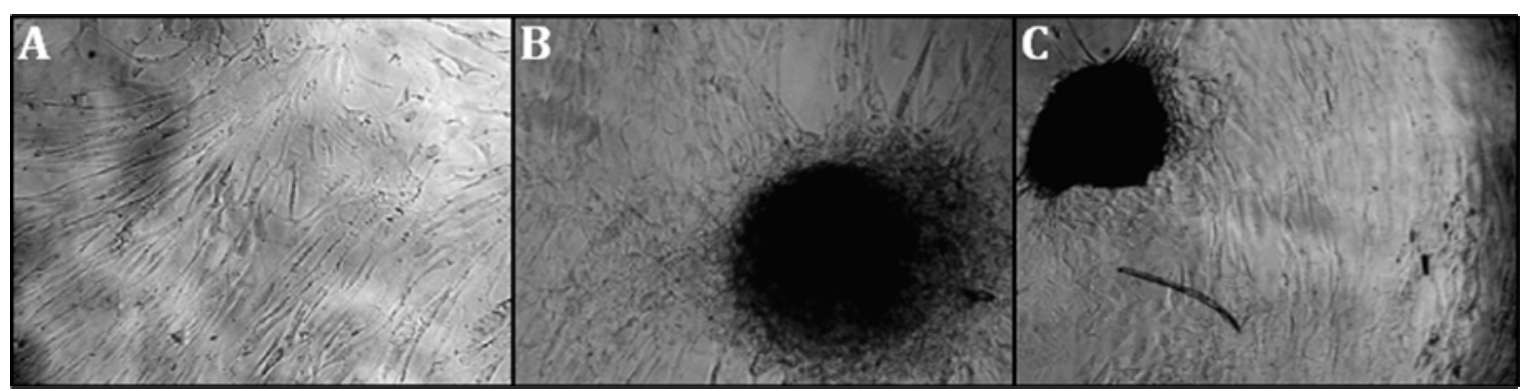

Fig.21. Pigmented attached sphere formation by hAD-MSCs. Light photomicrograph of hAD-MSCs in standard growth medium (A), dense cell mass (like attached sphere) formation by hAD-MSCs stimulated with 50\% fRCM (B) and dark pigmented dense cell mass (like attached sphere) formation by hAD-MSCs stimulated with BMP4 in 50\% fRCM (C). All figures are at 10x magnification and modified to black to increase the visibility of cells. 


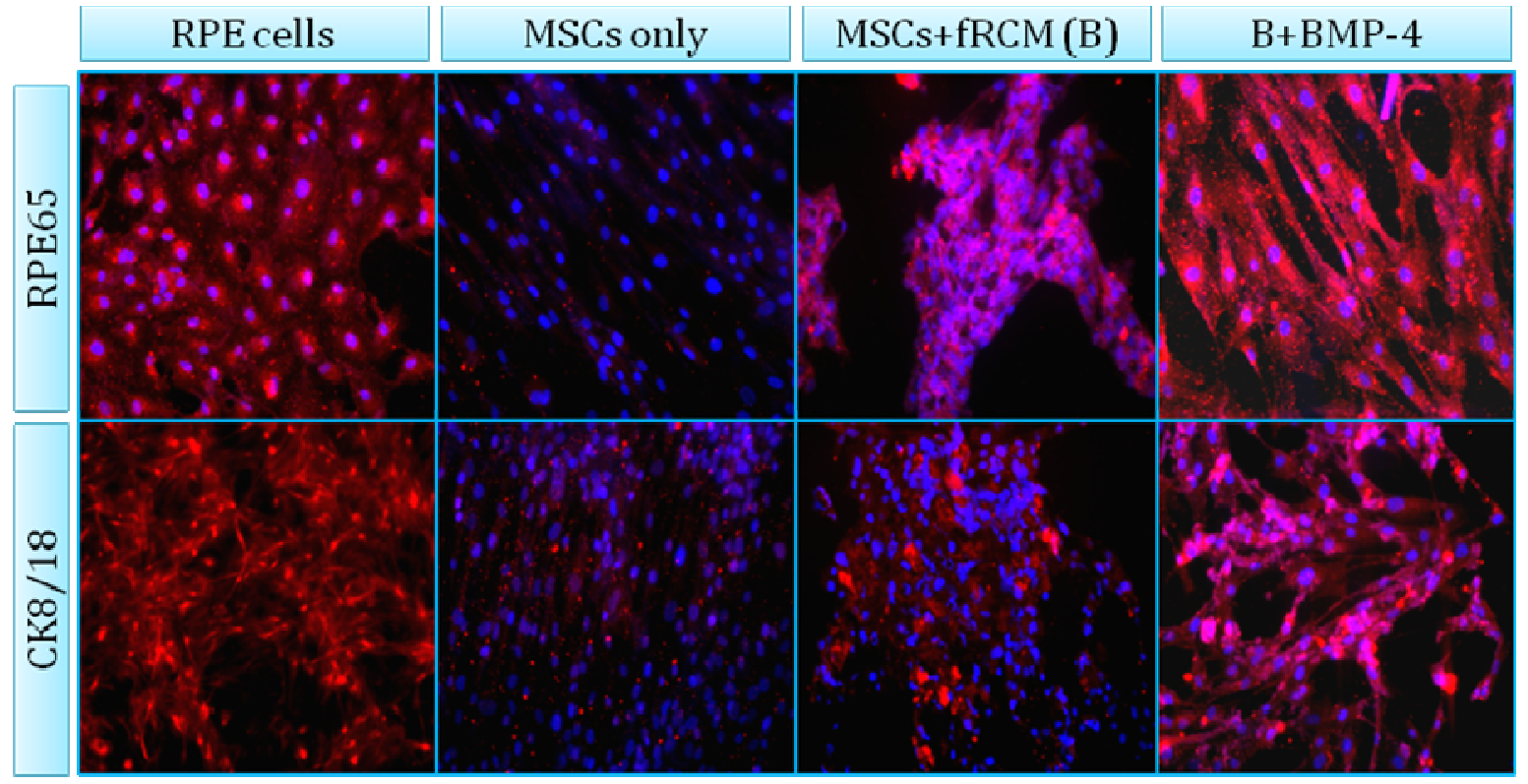

Fig.22. Immunocytochemical analysis of status of differentiation (RPE markers) of hAD-MSCs cultured in the standard growth medium with 50\% fRCM supplemented with BMP-4 (200ng/mL) for 15 days. First row has $40 \mathrm{x}$ magnifications and the second row has $20 \mathrm{x}$ magnifications. Red colour indicates the expressed protein marker and blue colour indicates DAPI stained cell nuclei.

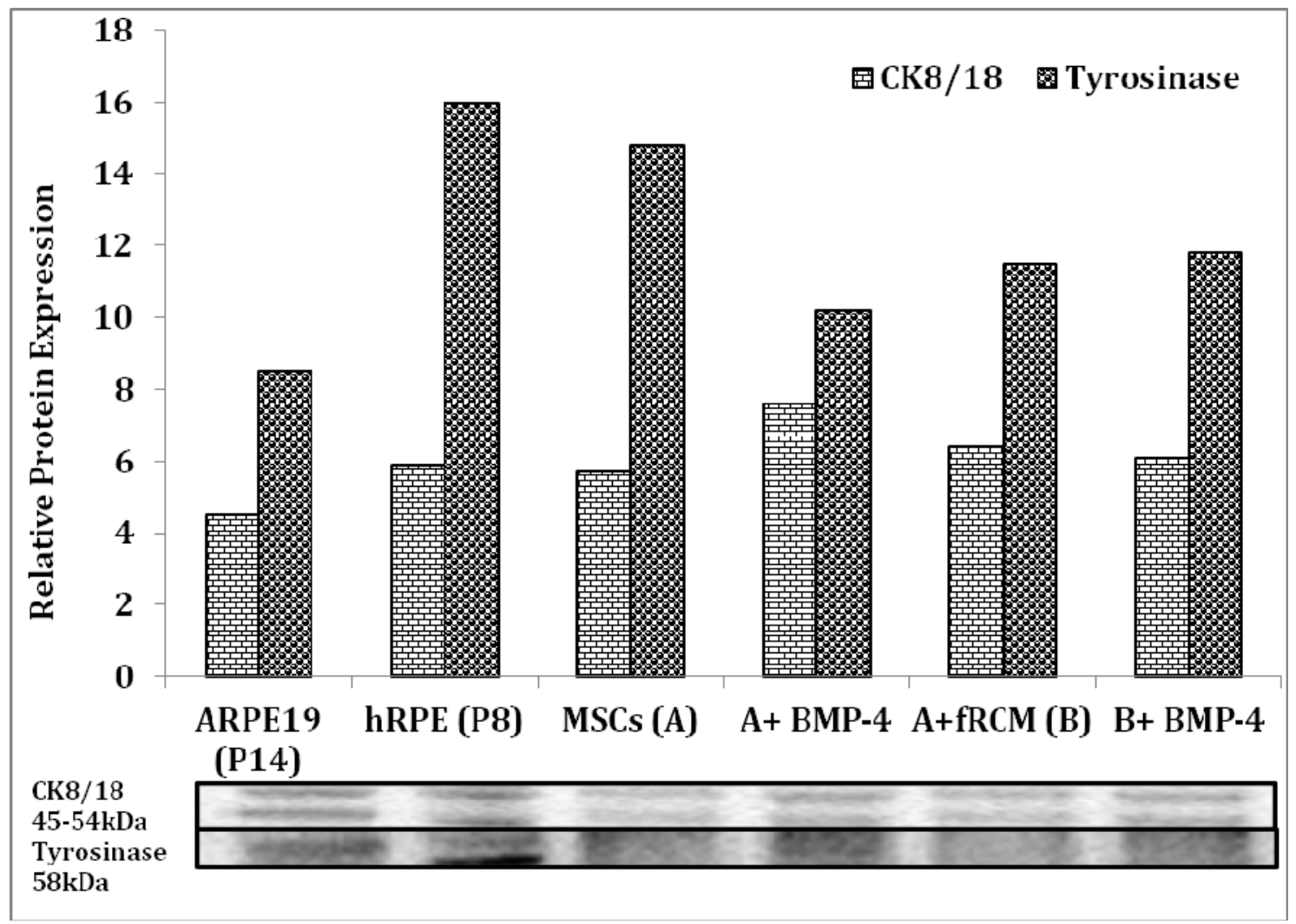

Fig.23. WB analysis of expression of RPE protein markers (CK8/18 and Tyrosinase) in hAD-MSCs under unstimulated and stimulated conditions with fRCM and BMP-4 $(200 \mathrm{ng} / \mathrm{mL})$.

BMP-4 and fRCM both together induced the pigmented sphere formation and RPE proteins expression in hAD-MSCs as observed in the preliminary results.

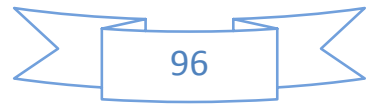




\subsection{Direct co-culture of hAD-MSCs with MMC inactivated RPE cells}

In this case, the hAD-MSCs were co-cultured directly over the inactivated RPE cells (ARPE19/hRPE) for one month period in 1:2 ratio. The hAD-MSCs alone (control) (Fig. 24 A) and hAD-MSCs with inactivated RPE cells (Fig. 24 B) were in monolayer form till two weeks. hAD-MSCs alone were found to form dense cell mass like attached spheres at super confluence in longer culture condition (Fig. 24 C \& E) but in the case of direct co-culture with inactivated RPE cells they formed more pigmented spheres (Fig. 24 D \& F). In case of direct co-culture, the size and pigment of dense cell spheres was found more than that of overgrown hAD-MSCs alone (control). The number of these dense cell spheres are also found more in case of direct co-culture than that of hAD-MSCs alone (data not shown). When these spheres were immunostained by MSCs-specific surface marker e.g. CD90 they showed reduced expression (based on fluorescence intensity) in case of direct co-culture with inactivated RPE cells. They have been also demonstrated positive for RPE marker (RPE65) (Fig. 25). This pigmented sphere formation seems to resemble the characteristics sphere or dense cell disc formation in the case of pluripotent stem cells or embryonic stem cells (Gong et al. 2008a). These events of formation of pigmented spheres in case of direct co-culture of hAD-MSCs with inactivated RPE cells may points towards the differentiation of hAD-MSCs to RPE like cells.

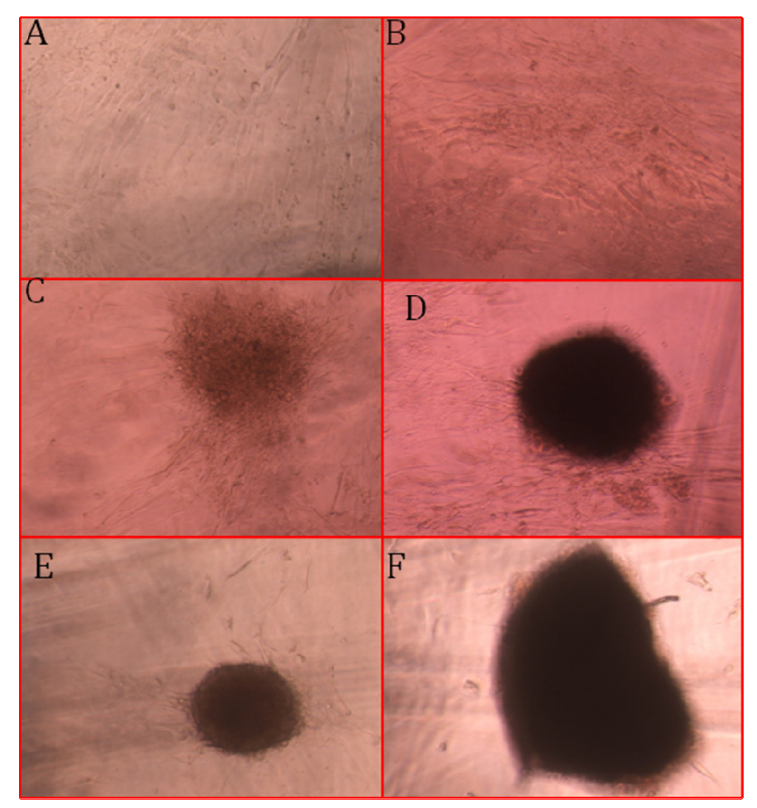

Fig.24. Characteristic sphere formation in culture of hAD-MSCs. Light photomicrograph of hADMSCs alone (A) and hAD-MSCs with RPE cells (B) in two weeks and attached pigmented cell spheres or dense cell mass formed by hAD-MSCs alone (C) and (E) at 30 days, Dark pigmented spheres formed by hAD-MSCs in direct co-culture with MMC treated RPE cells at 30 days (D) \& (F). 


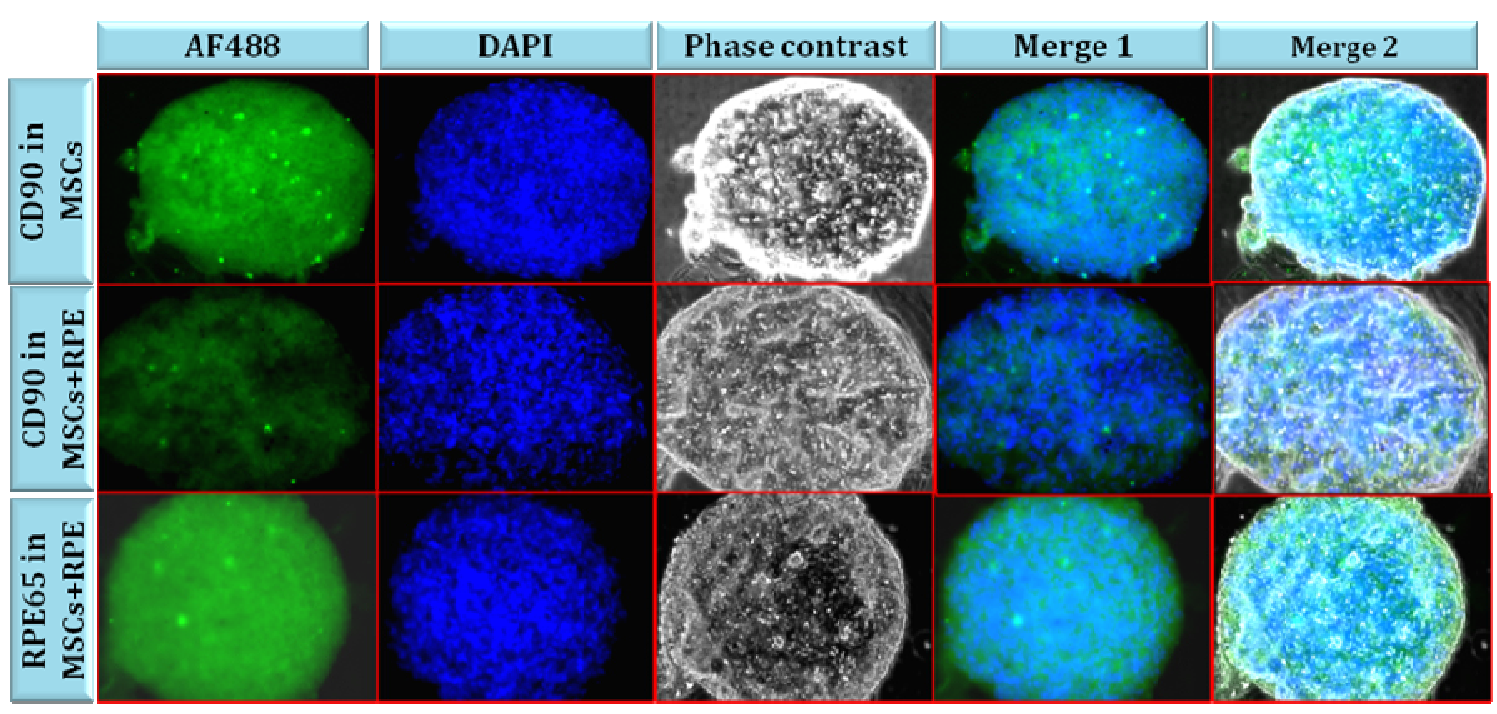

Fig.25. Immunofluorescence analysis of cell spheres formed by hAD-MSCs alone and hAD-MSCs co-cultivated with MMC treated RPE cells. Merge 1 column is the merge between green (AF488) and blue (DAPI) image and Merge2 (column) is the merge between phase contrast and Merge 1. First row- Sphere formed by hAD-MSCs alone and stained by CD90 (Thy1) and second and third rows are hAD-MSCs co-cultivated with MMC treated RPE cells marked with CD90 and RPE65 respectively.

In further immunofluorescence studies, hAD-MSCs showed marked increase in RPE markers (RPE65, Z01 and CK8/18) and reduction in MSCs specific marker Thy-1 (CD90) (Fig. 26) in the direct co-culture with inactivated RPE cells. Interestingly, hAD-MSCs showed expression of almost all RPE markers in normal growth condition at basal level and some in significantly higher level (data not shown). The differentiation tendency of hAD-MSCs towards RPE phenotypes was basically variable depending on the donors but they showed similar patterns (data not shown). 


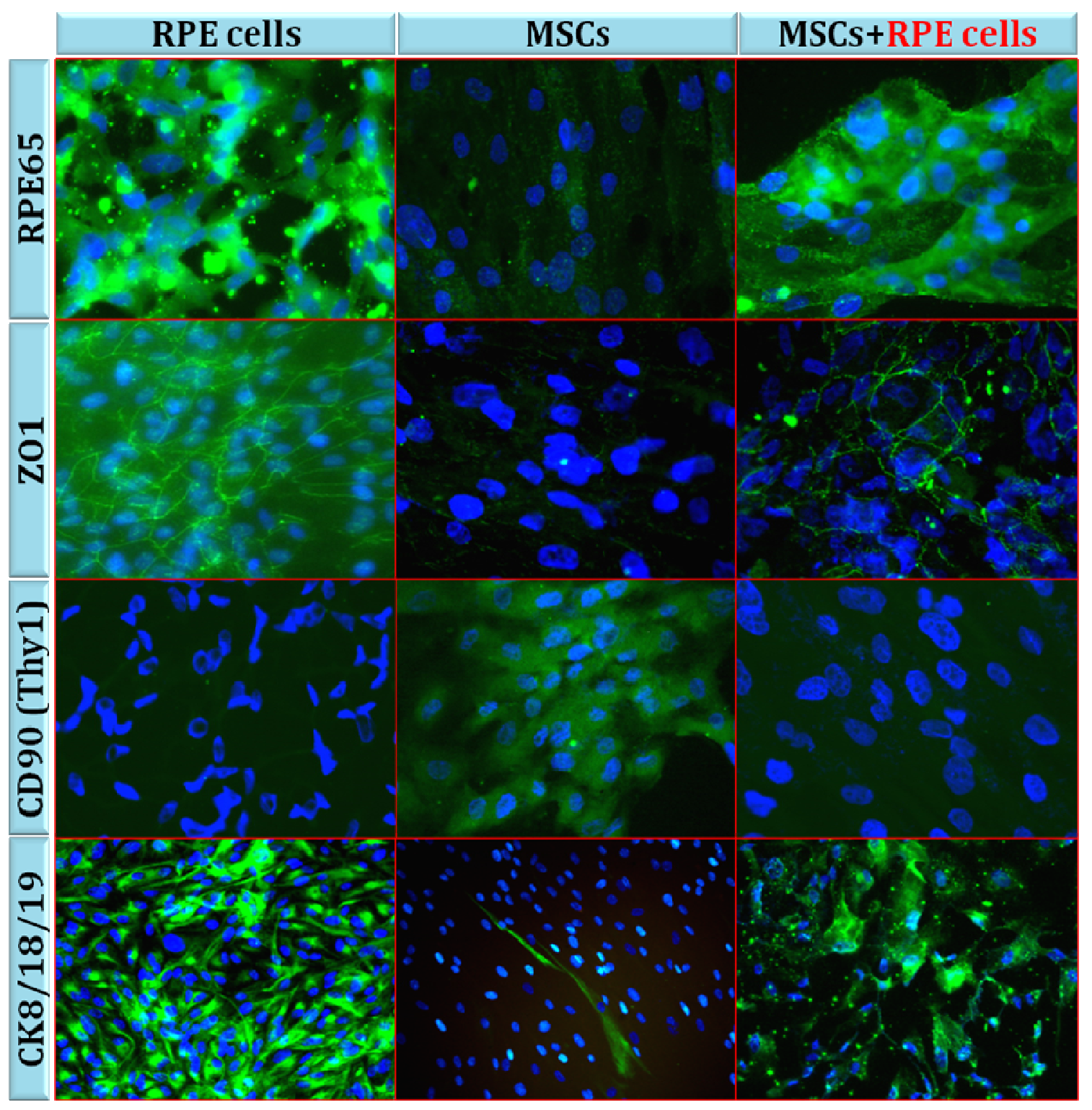

Fig.26. Immunocytochemical fluorescence analysis of stem cell status (CD90) and differentiation status towards RPE phenotypes (RPE65, Z01 and Cytokeratin 8+18+19) in hAD-MSCs alone (control) and in hAD-MSCs in direct co-culture with inactivated RPE cells. Markers are shown in rows. First column shows expression of markers in ARPE19 cells, second column shows the markers expression in hAD-MSCs alone and third column shows the markers expression in hADMSCs when co-cultivated with MMC treated RPE cells (red font colour). These images are representative images of all the cell culture and direct co-culture for 30 days.

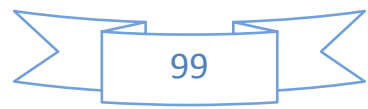




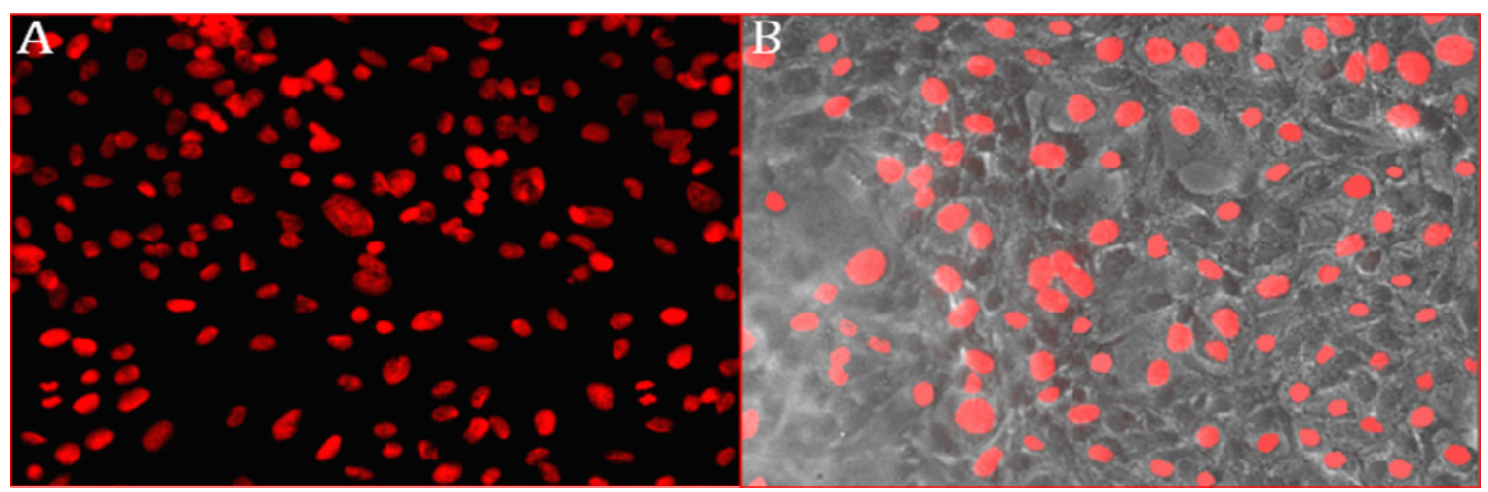

Fig.27. Marking of ARPE19 cells with EdU. EdU marked RPE cells (EdU marked nuclei and stained with Alexa Fluor 647 (red fluorescence) (A) and EdU marked nuclei merged with phase contrast image of the cells $(\mathbf{B})$.

In case of immunofluorescence results described above (Fig. 26), there was no clarity between the expression of RPE markers in stimulated hAD-MSCs and that in inactivated RPE cells. So, the RPE cells were first marked with 5-ethynyl-2'-deoxyuridine (EdU) then inactivated with mitomycin C (MMC) to track the MMC inactivated RPE cells in direct coculture. The inactivated RPE cells marked with EdU and stained with Alexa Fluor 647 azide is shown in Fig. 27A \& B as red stained nuclei.

It was previously shown that in case of higher dose of MMC ( $\geq 50 \mathrm{ug} / \mathrm{mL})$ treatment, RPE cells (ARPE19) start to die after 2-3 weeks (Fig. 17 A \& B) (Singh et al. 2013a) . The same results were again confirmed in the cell tracking experiment by the presence of red fluorescent EdU marked condensed and fragmented nuclei of MMC treated RPE cells (Pyknosis, Karyolysis, Karyorrhexis) in the direct co-culture conditions as shown by white arrows in Fig.28A. The immunofluorescence image in z-stack shows that the red fluorescent EdU marked nucleus of lower monolayer of inactivated RPE cells is below the nuclei of hAD-MSCs (globally stained blue with DAPI) (Fig. 28 G). RPE65 staining of stimulated hAD-MSCs in the direct co-culture with inactivated RPE cells are shown in Fig.28 from panel D to G. The DAPI was used here to counterstain globally to all the nuclei of both the cells i.e. hAD-MSCs and inactivated RPE cells (Fig. 28 B). The merged fluorescence photomicrographs of EdU marked and DAPI marked are shown to demonstrate the presence of both the cells together (Fig. $28 \mathrm{C} \& \mathrm{~J}$ ). Another RPE marker, Ezrin also known as cytovillin or villin-2 (an apical cytoplasmic peripheral membrane protein present in RPE microvilli), was also detected significantly in the hAD-MSCs directly co-cultivated with inactivated RPE (Fig. $28 \mathrm{~K} \& \mathrm{~L}$ ). The positive (RPE cells) and experimental control (hAD-MSCs) for RPE65 staining is not shown here to avoid the repetition as it is shown previously (First two panel of First Row of Fig. 26). Ezrin expression in RPE cell (positive control) and hAD-MSCs (experimental control) is shown in figures (Fig. $28 \mathrm{H} \& \mathrm{I}$ ). 


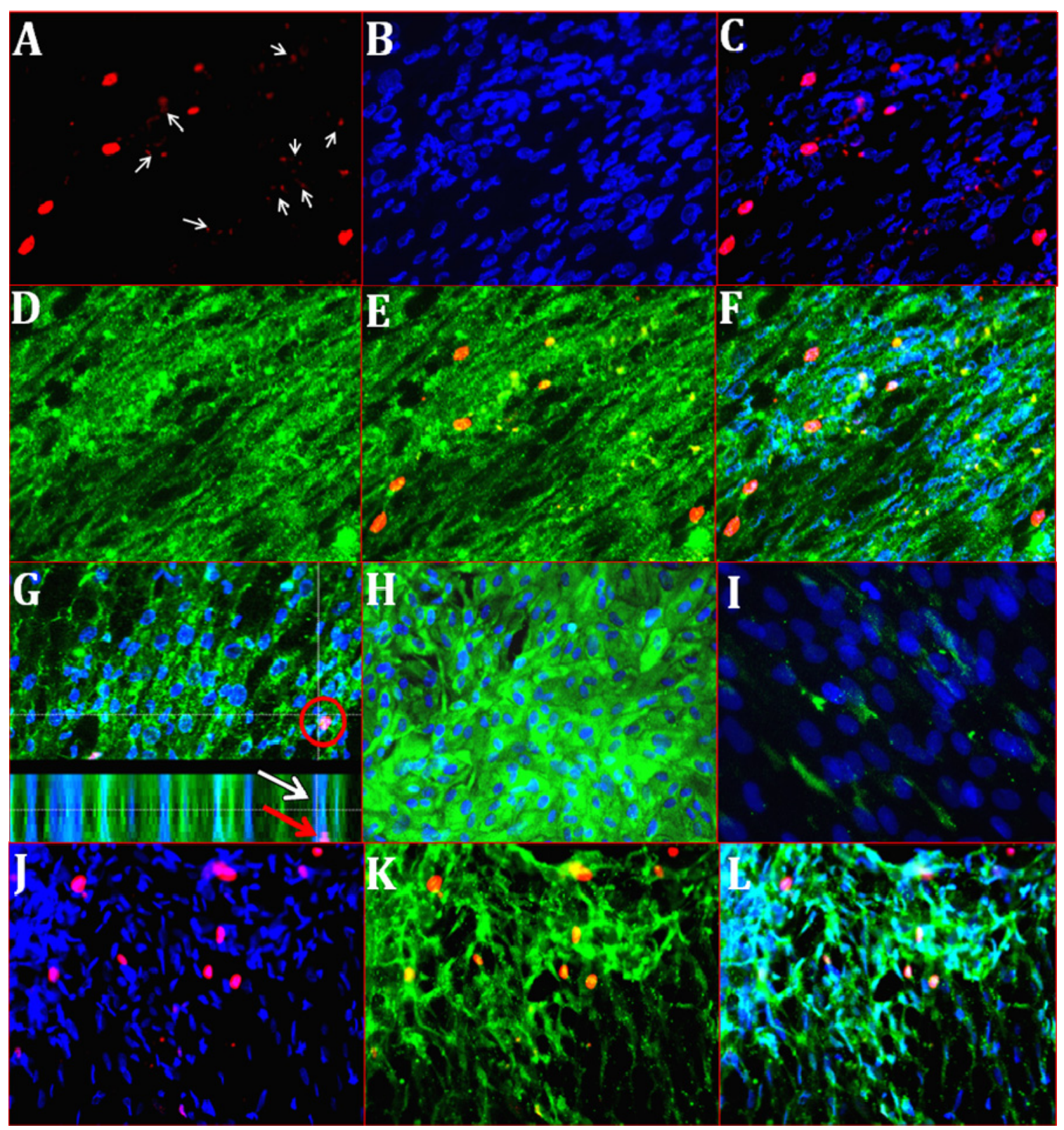

Fig.28. Immunocytochemical analysis of direct co-culture of hAD-MSCs with EdU marked MMC treated RPE cells (Cell tracking experiment). EdU marked MMC treated RPE cells' nuclei in direct coculture (A), the white arrows show the fragmented nuclei (karyorrhexis) of recently died RPE cells. DAPI stained nuclei of hAD-MSCs and MMC treated RPE cells in direct co-culture (B). Merge of (A) \& (B) is (C). RPE65 protein expression (green fluorescence) in the cells of direct co-culture (D). Merge of (A) \& (D) is (E). Merge of (B) \& (E) is (F). Immunofluorescent image of cells and their nuclei in z-axis to show EdU marked pink fluorescent nuclei of MMC treated RPE cells in lower cell monolayer (red arrow) and DAPI marked blue nuclei of hAD-MSCs in upper cell layer (white arrow) (G). Ezrin expression (green fluorescence) in RPE cells as positive control (H). Ezrin Expression (green fluorescence) in hAD-MSCs alone (I). EdU marked MMC treated RPE cells' nuclei and DAPI marked cells' nuclei together in direct co-culture for Ezrin panels (J). Ezrin expression (green fluorescence) in cell of direct co-culture merged with EdU marked nuclei of RPE cells (K). Merged fluorescent picture of Ezrin expression (green fluorescence) with EdU marked (orange) and DAPI marked (blue) nuclei in direct co-culture of hAD-MSCs with RPE cells (L).

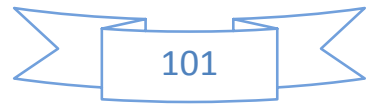


The immunocytological analysis of hAD-MSCs in direct co-culture with inactivated RPE cells showed the differentiation of hAD-MSCs towards RPE cells. This was further confirmed by WB analysis of direct co-culture experiments. The results confirmed that there was a significant increase in RPE retinoid cycle marker; RPE65 and an epithelial marker of RPE; tight junction protein, zonula occludens 1 (ZO1) in hAD-MSCs co-cultivated with inactivated hRPE cells at P $\leq 0.001$ (Fig.29). The increase in RPE markers (RPE65 and ZO1) in hAD-MSCs stimulated in direct co-culture with inactivated RPE cells was higher than that of indirect co-culture at $\mathrm{P} \leq 0.001$ (Fig.29). This indicates the crucial role of intercellular signaling induced by direct cell to cell contacts in the differentiation process of stem cells apart from added exogenous factors for differentiation.

The results based on immunocytochemical studies and western blot analysis showed that hAD-MSCs was differentiating towards RPE like cells when directly co-cultured with inactivated RPE cells.

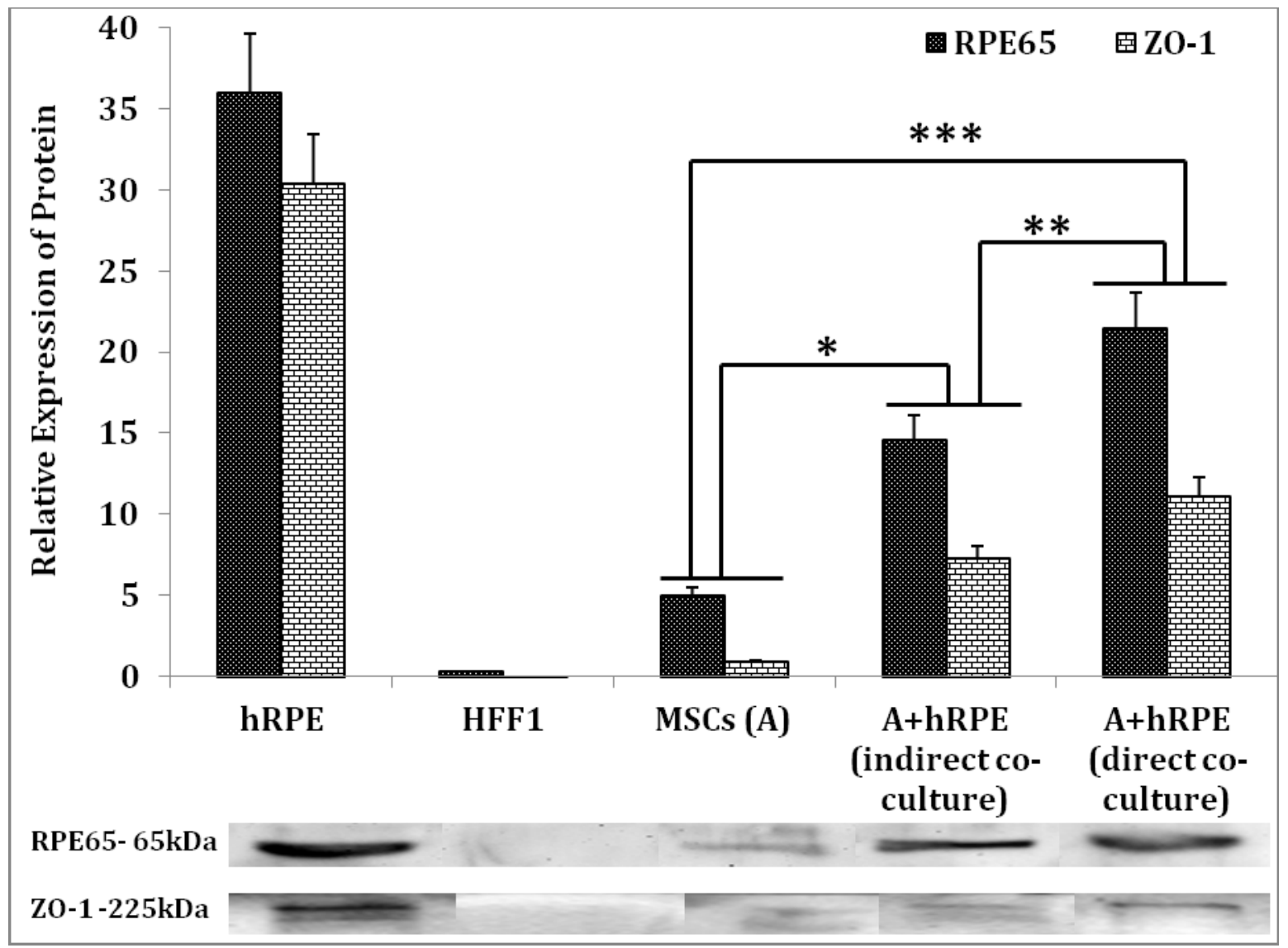

Fig.29. Assessment of differentiation of hAD-MSCs to RPE phenotypes under direct and indirect co-culture conditions with inactivated hRPE cells by WB analysis. Expression of RPE markers e.g. RPE65 (dark grid bar) and Z01 (light bricked bar). The statistical significance is set at P $\leq 0.05\left({ }^{*}\right)$, $\mathrm{P} \leq 0.01\left(^{* *}\right)$ and $\mathrm{P} \leq 0.001\left(^{* * *}\right)$. Error bar is indicated as \pm SD $(\mathrm{n}=3)$. Here, hRPE and human fore skin fibroblast (HFF1) are taken as positive and negative controls respectively. 


\subsection{Indirect co-culture of hAD-MSCs with MMC treated RPE cells along with factors}

The hAD-MSCs were stimulated for differentiation towards RPE phenotypes by coculturing them indirectly in the trans-well system of 6-well plates with MMC treated RPE (ARPE19) cells (on the bottom of the well) in the presence of factors (VIP, NIC, and ATRA) and their combinations (VIP+NIC, VIP+ATRA, NIC+ATRA and VIP+NIC+ATRA) for 12 days.

\section{Evaluation of proliferation of MMC inactivated RPE cells}

In each condition, the proliferation activity of RPE cells was measured by using Alamar blue at 3 and 5 days period. All the factors and their combinations were found to have significant anti-proliferative effects $(\mathrm{P} \leq 0.01)$ in comparison to that of control (MMC treatment but without factors) (Fig.30). The increase in anti-proliferative effects was more pronounced or significant $(\mathrm{P} \leq 0.01)$ at 5 days in comparison to that at 3 days period (shown by plus sign in the bar diagram) except in the case of VIP and VIP+ATRA (Fig.30). When the same combinations were used with hAD-MSCs co-cultivation, these antiproliferative effects were cancelled by the presence of hAD-MSCs (Fig.31). However there was no significant difference in the proliferation activity between stimulated hAD-MSCs with and without factors and their combinations except in the case of NIC and VIP+ATRA. Interestingly, it was noted that in case of hAD-MSCs at 5 days, RPE cell proliferation activity was higher than that at 3 days (Fig.31) while without hAD-MSCs, they are in opposite way (Fig.30).

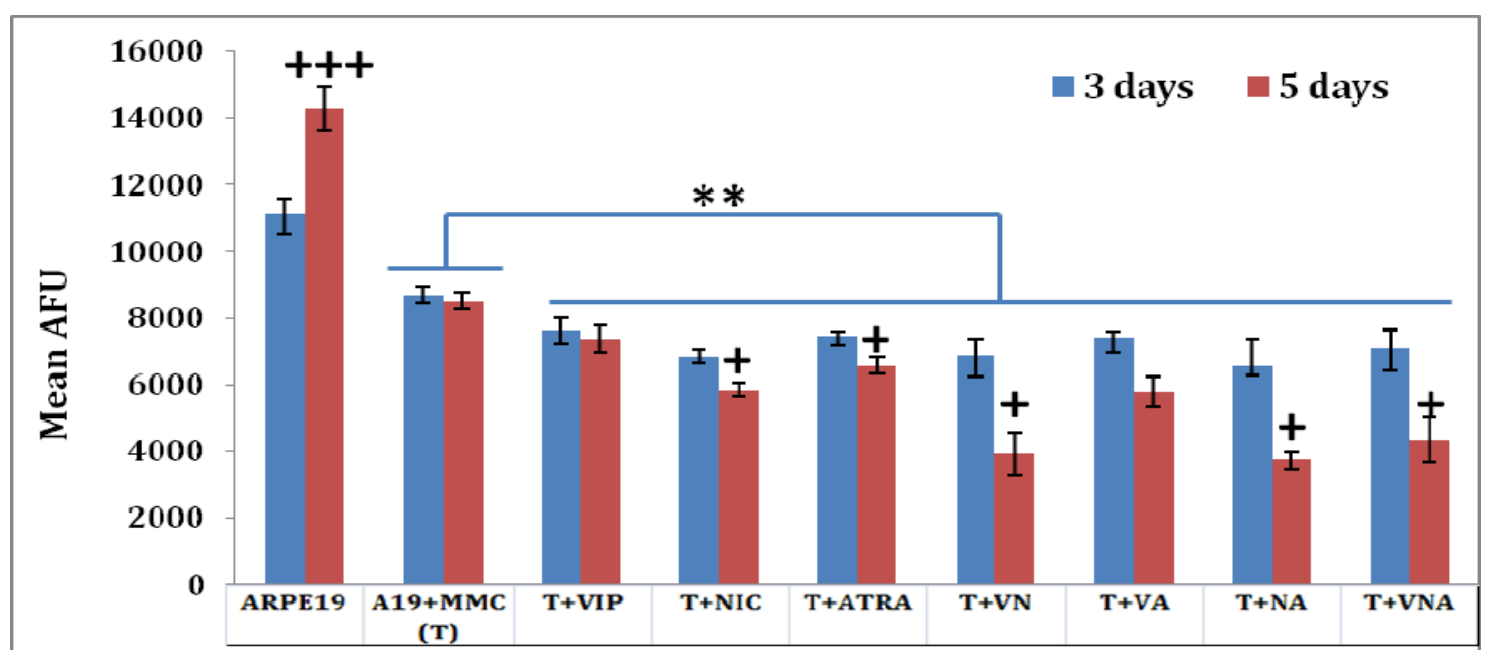

Fig.30. Cell proliferation activity of MMC treated RPE (ARPE19) cells cultured in the growth medium supplemented with VIP, NIC and ATRA and their combinations $(\mathrm{VIP}+\mathrm{NIC}=\mathbf{V N}$, VIP+ATRA $=\mathbf{V A}$, $\mathrm{NIC}+\mathrm{ATRA}=\mathbf{N A}$ and $\mathrm{VIP}+\mathrm{NIC}+\mathrm{ATRA}=\mathbf{V N A}$ ) by Alamar blue assay. $\mathrm{T}$ indicates the MMC treatment (50ug/mL) of RPE cells. The statistical significance is set at $\mathrm{P} \leq 0.01(* *) /(+)$ and $\mathrm{P} \leq 0.001(+++)$. Plus 
signs are used to indicate the significant difference between 3 and 5 days period samples. Error bar is indicated as $\pm \mathrm{SD}(\mathrm{n}=3)$.

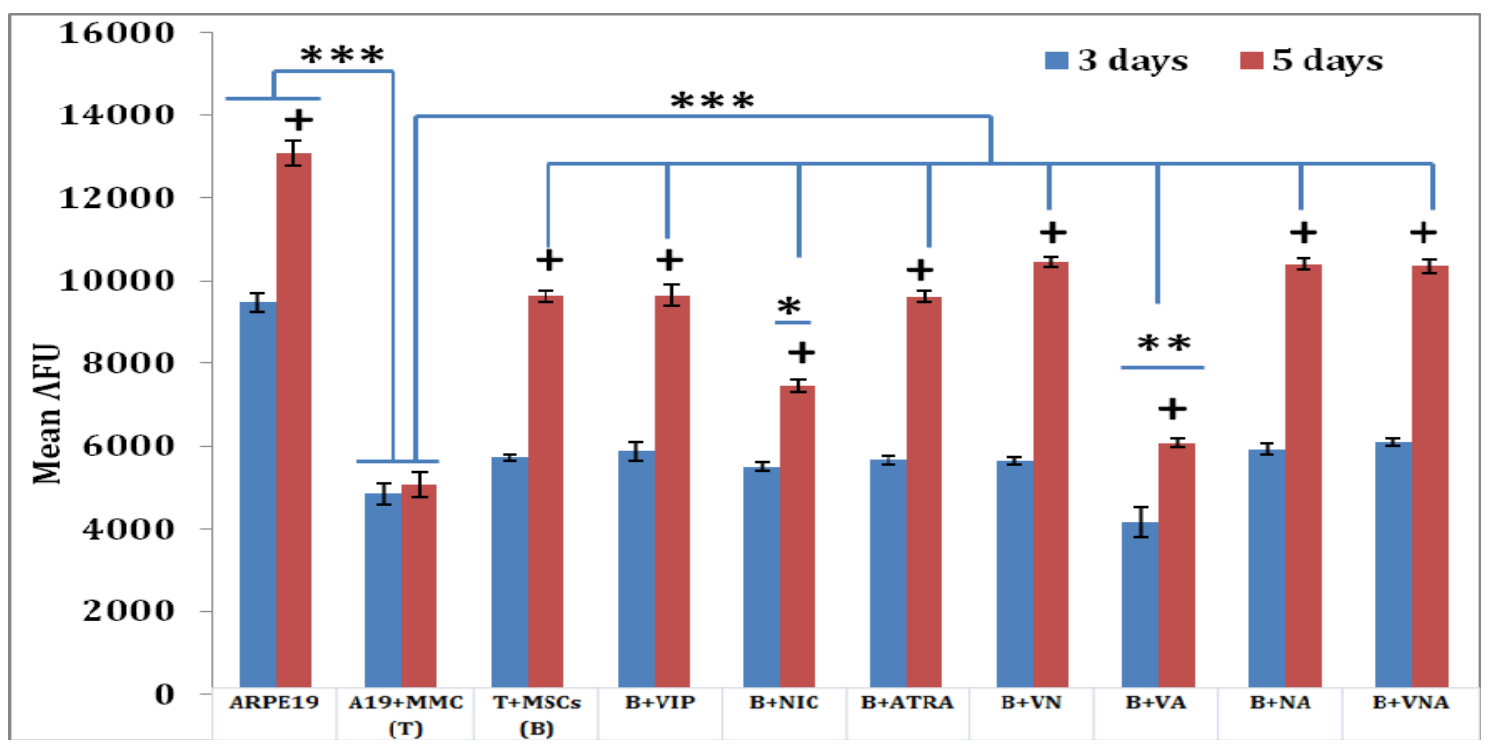

Fig.31. Cell proliferation activity of MMC treated RPE (ARPE19) cells under indirect co-culture with hAD-MSCs measured by Alamar blue assay in the presence of factors (VIP, NIC and ATRA) and their combinations $(\mathrm{VIP}+\mathrm{NIC}=\mathbf{V N}, \mathrm{VIP}+\mathrm{ATRA}=\mathbf{V A}, \mathrm{NIC}+\mathrm{ATRA}=\mathbf{N A}$ and VIP+NIC+ATRA=VNA$)$. Here, $\mathrm{T}$ indicates the MMC treatment $(50 \mathrm{ug} / \mathrm{mL})$ of ARPE19 cells. The statistical significance is set at $\mathrm{P} \leq 0.05\left(^{*}\right), \mathrm{P} \leq 0.01\left(^{* *}\right)$ and $\mathrm{P} \leq 0.001\left(^{* * *}\right) /(+)$. Error bar is indicated as $\pm \mathrm{SD}(\mathrm{n}=3)$.

\section{Evaluation of differentiation status of hAD-MSCs towards RPE phenotypes}

The differentiation status of hAD-MSCs was evaluated by measuring the amount of expression of RPE marker proteins in hAD-MSCs under each stimulated condition mentioned above by WB method. The WB analysis of MSCs-specific marker Thy-1 (CD90) and RPE markers (RPE65, Ezrin, CK8/18, Tyrosinase, MerTK, MiTF, Pax6) was done for the stimulated hAD-MSCs (Fig.32). Induction of epithelial characteristics of RPE in stimulated hAD-MSCs was evaluated by analyzing the expression of RPE specific epithelial proteins e.g. cytokeratin 8/18 (CK8/18), Z01 and pan-cytokeratin (PCK). The results of significant comparative changes (increase or decrease) of all the markers in the hAD-MSCs under stimulated and unstimulated conditions from the figure 25 are summarized in Table 6. The WB results (Fig.32) and summary Table 6 showed that the expression of MSCsspecific marker Thy-1 (CD90) decreased significantly in stimulated hAD-MSCs, this significant decrease was more prominent especially in the case of combinations of factors. The expression of RPE65, Ezrin, CK8/18, Tyrosinase, MerTK, MiTF, Pax6 proteins increased significantly in stimulated hAD-MSCs in comparison to unstimulated hAD-MSCs. 


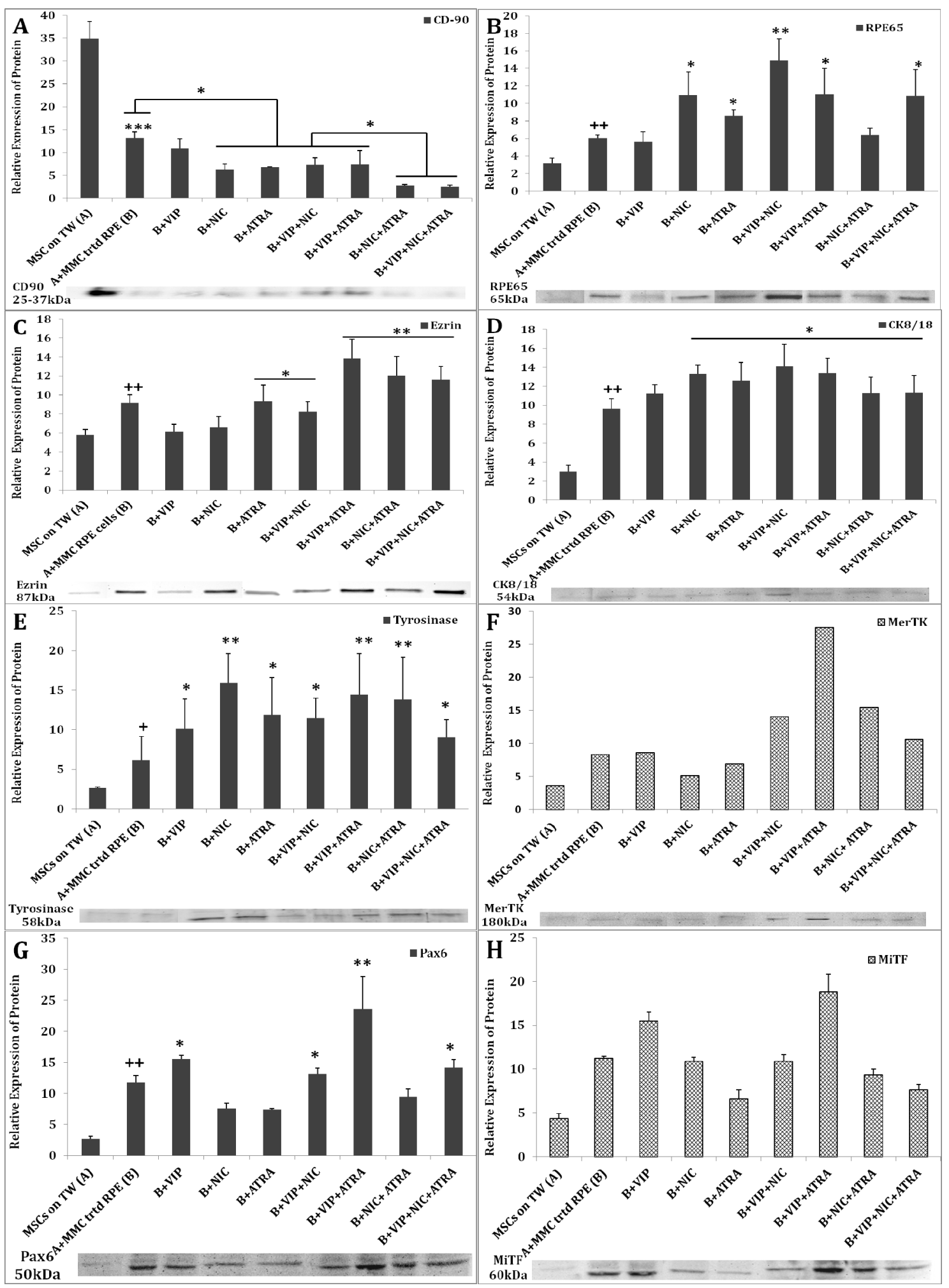

Fig.32. Assessment of differentiation of hAD-MSCs to RPE phenotypes under indirect co-culture with inactivated ARPE19 cells in the presence of factors (VIP, NIC \& ATRA) and their combinations by WB analysis. Relative expression of MSCs-specific protein marker CD 90 (A) and RPE protein markers such as RPE65 (B), Ezrin (C), CK8/18 (D), Tyrosinase (E), MerTK (F), Pax6 (G) and MiTF (H) in stimulated and unstimulated hAD-MSCs. The statistical significance is set at $\mathrm{P} \leq 0.05\left({ }^{*}\right) /(+), \mathrm{P} \leq 0.01(* *) /(++)$ and $\mathrm{P} \leq 0.001\left({ }^{* * *}\right)$. Here, asterisks indicate the significant difference between hAD-MSCs in co-culture without factors and hAD-MSCs with factors and the plus signs show the significant difference between $\mathrm{hAD}-\mathrm{MSCs}$ in co-culture only and hAD-MSCs alone (control). Error bar is indicated as $\pm \mathrm{SD}(\mathrm{n}=3)$.

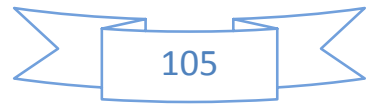


Table 6. Summary of WB analysis of expression of all the markers (from Fig.32) in stimulated hAD-MSCs under indirect co-cultivation with MMC treated RPE cells along with factors (VIP, NIC, and ATRA) and their combinations. The increase in number of plus sign is based on the significant increase at $\mathrm{P} \leq 0.05(\mathrm{n}=3)$ and + /- shows the infrequent expression. In case of MerTK and MiTF, the data are preliminary and indicative only.

\begin{tabular}{|l|l|l|l|l|l|l|l|l|}
\hline WB analysis & CD90 & RPE65 & Ezrin & CK8/18 & Tyrosinase & MerTK & MiTF & Pax6 \\
\hline MSCs (A) & +++++ & + & + & $+/-$ & $+/-$ & $+/-$ & $+/-$ & $+/-$ \\
\hline $\begin{array}{l}\text { A+MMC treated RPE } \\
\text { (B) }\end{array}$ & +++ & +++ & +++ & +++ & ++ & ++ & ++ & ++ \\
\hline B+VIP & +++ & +++ & ++ & +++ & +++ & ++ & +++ & +++ \\
\hline B+NIC & ++ & ++++ & ++ & ++++ & ++++ & + & ++ & + \\
\hline B+ATRA & ++ & ++++ & ++++ & ++++ & +++ & + & + & + \\
\hline B+VIP+NIC & ++ & +++++ & ++++ & ++++ & +++ & +++ & ++ & +++ \\
\hline B+VIP+ATRA & ++ & ++++ & +++++ & ++++ & ++++ & ++++ & +++ & ++++ \\
\hline B+NIC+ATRA & + & +++ & +++++ & ++++ & ++++ & +++ & ++ & ++ \\
\hline B+VIP+NIC+ATRA & + & ++++ & +++++ & ++++ & +++ & +++ & ++ & +++ \\
\hline
\end{tabular}

All factors were found to show anti-proliferative effects on the MMC treated RPE cells in the indirect co-culture method while these negative effects were annulled by the presence of hAD-MSCs. The hAD-MSCs in the indirect co-culture with inactivated RPE cells along with factors were found to differentiate towards RPE phenotypes to different extent, when stimulated by different combinations of factors based on WB results. The combinations of factors were found better in stimulating the differentiation in comparison to the individual factors.

\subsection{Indirect co-culture of hAD-MSCs with hNR explants in RCM along with differentiating factors and their combinations}

The hAD-MSCs were stimulated for differentiation towards RPE phenotypes by culturing them (on the bottom of the well) indirectly in the trans-well system of 6-well plates with human neuroretinal explants $(6 \times 8 \mathrm{~mm})$ on high density porous $(0.4 \mu)$ trans-membrane of the trans-well/trans-insert in the growth medium with 50\% hRCM supplemented with three factors (VIP, NIC, and ATRA) and their combinations (VIP+NIC, VIP+ATRA, NIC+ATRA and VIP+NIC+ATRA) for 15 days period. The WB analysis of expression of RPE markers such as Ezrin, RPE65, CK8/18, Bestrophin, PEDF, MiTF and Pax6 was done for the stimulated hAD-MSCs under each condition (Fig.33 \& 34). The results of comparative increase or decrease of all the markers (without statistical significance) are shown in Table 7 and 8 . The difference between Table 7 and 8 are the difference in factors and their combinations and different donors for hAD-MSCs and hNR. 
In another set of experiments with more combinations of factors, the indirect co-culture of hAD-MSCs with hNR was carried out for 6 and 12 days period. The WB analysis of expression of MSCs-specific marker CD90 and RPE markers (RPE65, Ezrin, Bestrophin, CK8/18, MerTK, Pax6, MiTF, and PEDF) was done in three different experiments with duplicates and their relative expressions based on band intensities of WB were plotted as a bar graph (Fig.35 \& 36). The summary of significant increase or decrease in the expression of all markers in stimulated hAD-MSCs under different combinations is given in the Table 9.

The hAD-MSCs enhanced the expression of RPE markers when they were co-cultivated with hNR in 50\% RCM significantly but this effect was potentiated by the presence of the factors and their combinations. The combinations of factors like VIP+NIC, NIC+ATRA, and $\mathrm{VIP}+\mathrm{NIC}+\mathrm{ATRA}$ were overall more effective in inducing RPE protein expression in hADMSCs than when they were used alone. The third set of experiments showed that the expression of RPE markers is getting increased significantly at 12 days period than that on 6 days except some cases (Fig. 35).

It is interesting to observe that hAD-MSCs have significant amount of cytoplasmic PEDF expression, which is a very important neuroprotective factor secreted by RPE cells.

The results from WB analysis showed that hAD-MSCs is induced partially towards RPE phenotypes under the presence of combinations of factors (VIP+NIC, VIP, NIC+ATRA and $V I P+N I C+A T R A)$ in the indirect co-culture with hNR explants with growth medium supplemented with 50\% RCM. 


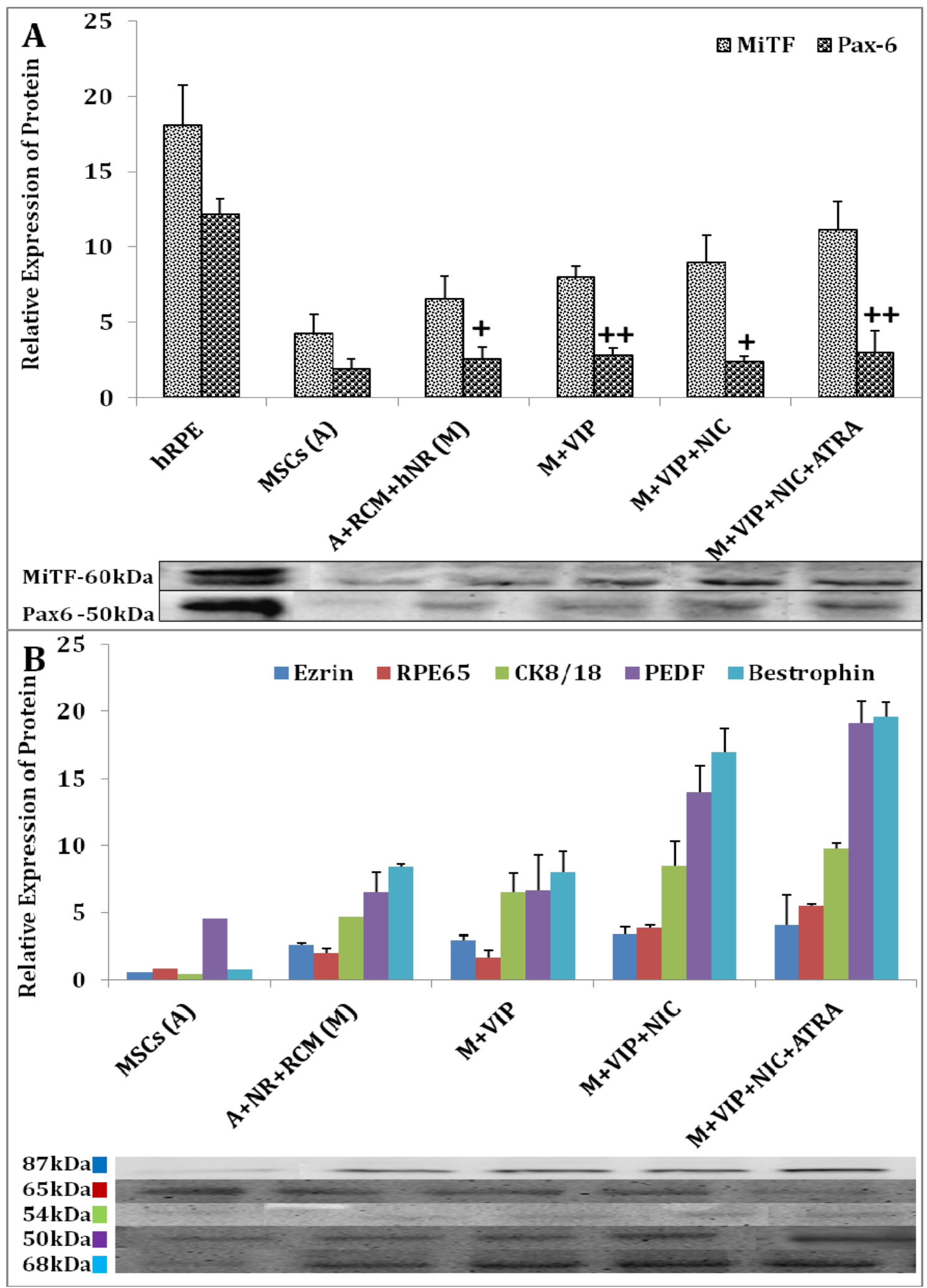

Fig.33. Assessment of status of differentiation of hAD-MSCs to RPE phenotypes under indirect coculture with human NR explants in the presence of factors (VIP, VIP+NIC and VIP+NIC+ATRA) in $50 \%$ hRCM after 15 days by WB analysis. Relative expression of RPE protein markers such as MiTF and Pax6 (A) and Ezrin, RPE65, CK8/18, PEDF and Bestrophin (B) in stimulated and unstimulated hAD-MSCs. Single or double plus sign $(+/++)$ shows the comparative increase in Pax6 (indicative only). Here, $\mathrm{hRPE}$ is used as a positive control. Error bar is indicated as $\pm \mathrm{SD}(\mathrm{n}=2)$. 
Table 7. Summary of WB analysis of RPE marker protein expression (from Fig. 33) of co-culture of hAD-MSCs with human neuroretina (hNR) along with combination of factors for 15 days Note: here the increase in number of plus sign is just indicative increase they do not have any statistical significance due to insufficient number of experiments.

\begin{tabular}{|l|l|l|l|l|l|l|l|}
\hline WB analysis & RPE65 & Bestrophin & Ezrin & CK8/18 & PEDF & MiTF & Pax6 \\
\hline MSCs (A) & + & + & + & + & ++ & + & + \\
\hline A+RCM+hNR (M) & ++ & ++ & ++ & ++ & ++ & ++ & ++ \\
\hline M+VIP & ++ & ++ & ++ & +++ & ++ & +++ & +++ \\
\hline M+VIP+NIC & +++ & ++++ & +++ & +++ & +++ & +++ & ++ \\
\hline M+VIP+NIC+ATRA & ++++ & ++++ & +++ & ++++ & ++++ & ++++ & +++ \\
\hline
\end{tabular}

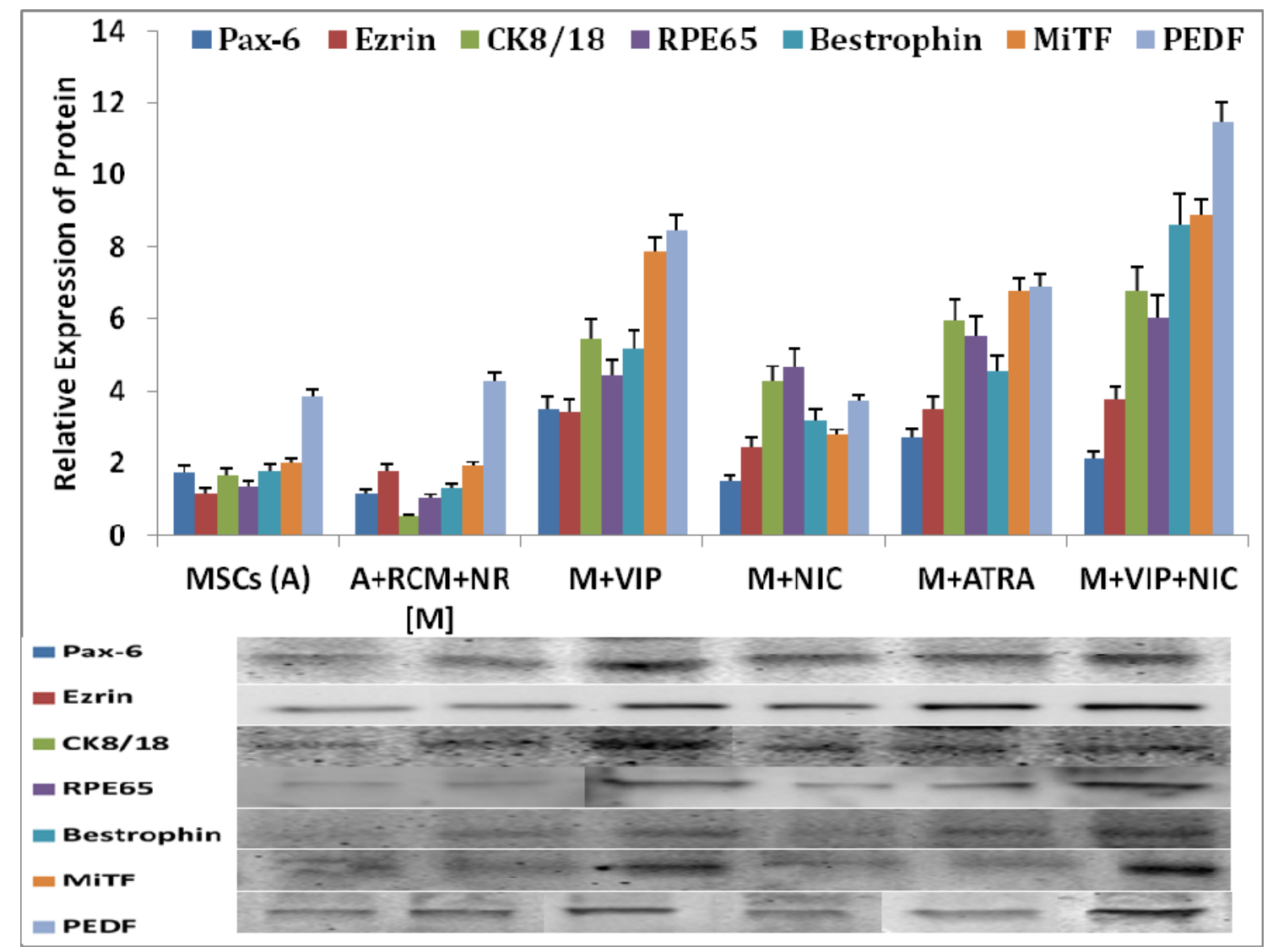

Fig.34. Assessment of differentiation of hAD-MSCs to RPE phenotypes under indirect co-culture with human NR explants in presence of factors (VIP, NIC and ATRA) and their combination (VIP+NIC) in 50\% RCM after 15 days by WB analysis. Relative expression of RPE protein markers such as Pax6 (50kDa), Ezrin (87kDa), CK8/18 (45-54kDa), RPE65 (65kDa), Bestrophin (68kDa), MiTF (60kDa) and PEDF (50kDa) in stimulated and unstimulated hAD-MSCs. Error bar is indicated as $\pm \mathrm{SD}(\mathrm{n}=2)$. 
Table 8. Summary of WB analysis (from Fig. 34) of co-culture of hAD-MSCs with human neuroretina (hNR) along with combination of factors for 15 days Note: Here the increase in number of plus sign is just indicative increase they do not have any statistical significance due to insufficient number of experiments.

\begin{tabular}{|l|l|l|l|l|l|l|l|}
\hline WB analysis & RPE65 & Bestrophin & Ezrin & CK8/18 & PEDF & MiTF & Pax6 \\
\hline MSCs (A) & + & + & + & + & +++ & $+/-$ & $+/-$ \\
\hline A+RCM+hNR (M) & + & + & ++ & + & +++ & + & + \\
\hline M+VIP & +++ & +++ & +++ & ++++ & ++++ & +++ & ++ \\
\hline M+NIC & +++ & ++ & +++ & +++ & +++ & ++ & + \\
\hline M+ATRA & ++ & +++ & +++ & ++++ & ++++ & +++ & ++ \\
\hline M+VIP+NIC & ++++ & ++++ & ++++ & ++++ & +++++ & +++ & ++ \\
\hline
\end{tabular}




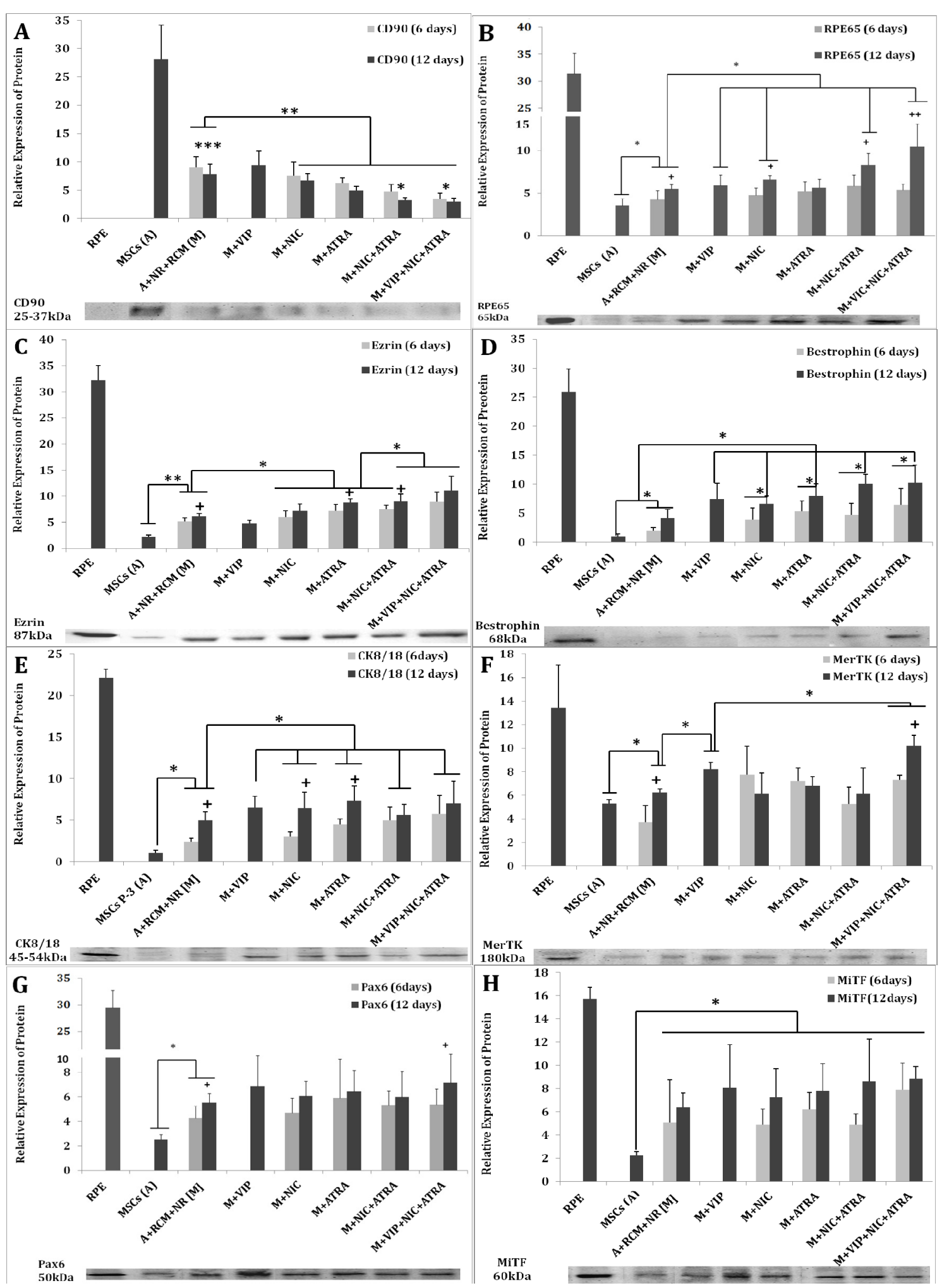

Fig.35. Assessment of differentiation of hAD-MSCs to RPE phenotypes under indirect co-culture with human NR explants in presence of factors (VIP, NIC and ATRA) and their combinations in 50\% RCM by WB analysis. Relative expression of MSCs-specific protein marker CD 90 (A) and RPE protein markers such as RPE65 (B), Ezrin (C), Bestrophin (D), CK8/18 (E), MerTK (F), Pax6 (G) and MiTF (H) in stimulated and unstimulated hAD-MSCs. The statistical significance is set at $\mathrm{P} \leq 0.05$ $\left({ }^{*}\right) /(+), P \leq 0.01\left(^{* *}\right) /(++)$ and $\mathrm{P} \leq 0.001\left(^{* * *}\right)$. The plus is used to show the significant difference between 6 and 12 days period samples. Error bar is indicated as $\pm S D(n=3)$. 


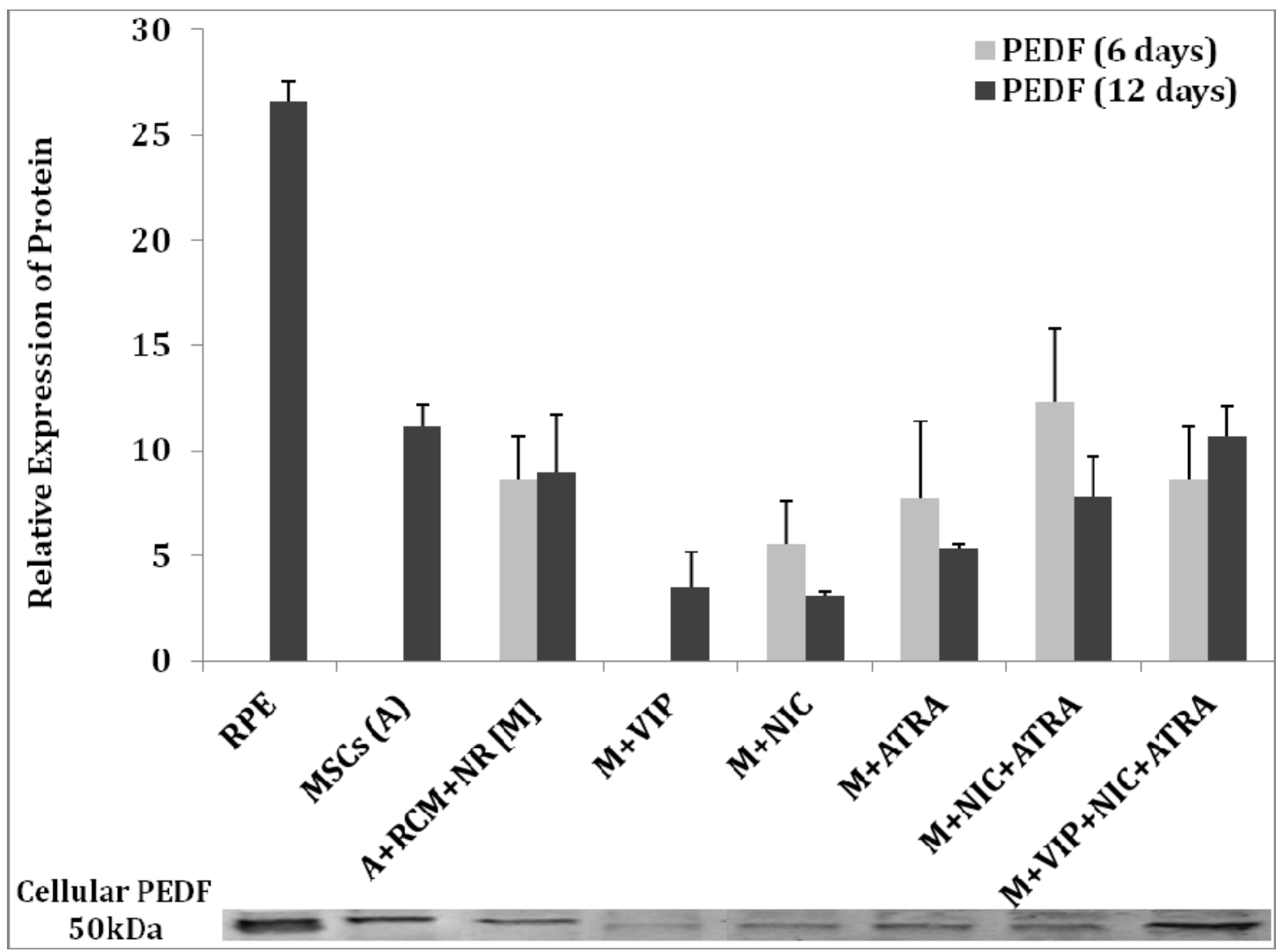

Fig.36. The WB analysis of cellular PEDF in stimulated and unstimulated hAD-MSCs under indirect co-culture with human NR explants in presence of factors (VIP, NIC and ATRA) and their combinations (NIC+ATRA and VIP+NIC+ATRA) in 50\% RCM at 6 and 12 days period. Error bar is indicated as $\pm \mathrm{SD}(\mathrm{n}=2)$.

Table 9. Summary of WB analysis (from Fig. $35 \&$ 36) of co-culture of hAD-MSCs with human neuroretina (hNR) along with combination of factors for 12 days period Note: Here the increase in number of plus sign indicates the difference at statistical significance $(\mathrm{P} \leq 0.05, \mathrm{n}=3$ ) for 12 days period except for PEDF (only indicative difference).

\begin{tabular}{|l|l|l|l|l|l|l|l|l|l|}
\hline WB analysis & CD90 & RPE65 & $\begin{array}{l}\text { Bestro } \\
\text { phin }\end{array}$ & Ezrin & $\begin{array}{l}\text { CK8/ } \\
18\end{array}$ & PEDF & MerTK & MiTF & Pax6 \\
\hline MSCs (A) & +++++ & + & + & + & + & +++ & $+/-$ & $+/$ & + \\
\hline A+RCM+hNR (M) & ++ & ++ & ++ & +++ & ++ & +++ & ++ & ++ & ++ \\
\hline M+VIP & +++ & +++ & +++ & ++ & +++ & ++ & +++ & ++ & ++ \\
\hline M+NIC & ++ & +++ & +++ & ++++ & +++ & ++ & ++ & ++ & ++ \\
\hline M+ATRA & + & ++ & +++ & ++++ & +++ & ++ & ++ & ++ & ++ \\
\hline M+NIC+ATRA & + & +++ & +++ & +++++ & +++ & +++ & ++ & ++ & ++ \\
\hline M+VIP+NIC+ATRA & + & +++ & +++ & +++++ & +++ & +++ & ++++ & ++ & ++ \\
\hline
\end{tabular}




\section{Status of neuroretinal explants cultivated with hAD-MSCs along with factors (VIP, NIC and ATRA) and their combinations in 50\% RCM}

The human neuroretinal (hNR) explants cultivated above under indirect co-culture experiments for one week with hAD-MSCs in 50\% RCM supplemented with factors (VIP, NIC, and ATRA) and their combinations (VIP+NIC, VIP+ATRA, NIC+ATRA, and VIP+NIC+ATRA). These hNR explants were analyzed for general morphology and cellular status of neuroretinal cells of neuroretina by toluidine blue staining and for specific changes in the photoreceptors and synapses and glial cells like Müller cells and astrocytes status by immunohistochemical staining against Calbindin (CB), Synaptophysin (SYP), rhodopsin (RHO), cellular retinaldehyde binding protein (CRALBP) and glial fibrillary acidic protein (GFAP) (Fig. 37 panel set 1 and 2, Fig. 38).

Antibody against Calbindin and Rhodopsin were used to examine the cone and rod PRs integrity. Antibody against Synaptophysin was used to see the synapses between different retinal neuron especially outer and inner synaptic layers (OPL and IPL). Antibody against CRALBP was used to observe the functional status of Müller cells and anti-GFAP was used to examine the pathological changes like reactive gliosis in the Müller cells and the cellular dynamics of astrocytes. In all the immunostaining of retinal explants, nuclei of retinal cells were counterstained with DAPI.

The 0 day samples of fresh neuroretinal explants (Fig. 37 A, B \& C and Fig. 38 A) are positive reference sample and other two control groups (non-treatment groups) are 'RCM only' (Fig. 37 D, E \& F and Fig. 38 B) and 'RCM+MSCs' (Fig. 37 G, H \& I and Fig. 38 C) and the rest panels are for treatment groups described at the beginning of each row of the panel set (Fig. 37 and Fig. 38). The rod PRs of neuroretina explants cultivated in different experiments were studied by immunostaining the hNR explants against rhodopsin (Fig. 38).

\section{Reference retina (0 day samples hNR explants) (Fig. 37 A, B \& C, Fig. 38 A)}

The neuroretinal explants had lots of damages in PRs and other neuroretinal cells to different extent (Fig. 37 A) probably due to pre or post-mortem ischemic injury, cut off of nutrient supply and injury during the processing of explants. However, the general structure of the retina was well preserved with visible outer and inner segments of photoreceptors. The OLM is clearly visible. The outer segments (OS) of PRs were intact. Outer segment of cone PRs were clearly visible and intact (shown by white arrow head) (Fig. 37 B). Photoreceptors' nuclei were well preserved but some of them were swollen. Inner segments (IS) of the PRs were well preserved. Few empty cell body spaces were 
seen in the ONL and INL. The OPL is clearly distinguishable. The synaptic connections between the pedicles of cone PRs and horizontal cells and cone bipolar cells were clearly visible by the dense band of yellow fluorescence i.e. combination of red (Calbindin) and green (Synaptophysin) in the OPL (Fig. 37 B). The INL have different degree of swelling in their cytoplasm. The neuronal processes of IPL were swollen and the dense cytoplasm of Müller cells could be distinguished among them. The ganglion cells were relatively well preserved. Optic nerve fibre also showed significant grade of swelling and vacuolization. Some astrocytes could be distinguished in this layer. The ILM was clearly defined.

\section{Control Retina 1 (1 week samples of hNR explants with RCM only) (Fig. 37 D,E\&F, Fig. 38B)}

The general retinal structure was badly affected and disorganized. The PRs are reduced or completely destroyed. Almost all the cells and retinal structure were degenerated with intense swelling and their nuclei were nuclear pyknosis (irreversible chromatin condensation during cell death), karyorrhexis (destructive fragmentation of nuclei) and karyolysis (complete lysis of chromatin by DNase). The OPL was almost disappeared and ONL and INL were merged. The ganglion cells were also degenerated. The ILM was intact. In lots of samples of explants, the retinal structure was completely disorganized.

\section{Control Retina 2 ( 1 week samples of hNR explants with RCM and hAD-MSCs)} (Fig. 37G,H\&I, Fig. 38C)

The general structure of retina was better preserved than the control retina 1 . In the external part of retina, in some places the PR cells formed rosette like cavity towards the inner part. The PRs cells still had outer and inner segments but PRs nuclei were pyknotic and many of them had karyorrhexis and karyolysis. Many PRs had swollen cytoplasms and the nuclear fragments were dispersed in the cytoplasm. The OPL was lost and the INL showed pyknosis, karyolysis and karyorrhexis. The IPL was clearly visible but the neuronal processes were practically degenerated. The optic nerve fibre layer showed general high degree of degeneration and in this layer Müller cell processes were visible. Some part of OLM was still there. The ILM was clearly defined. 
Test Retina (1 week samples of hNR explants with different factors and their combinations along with 50\% RCM and hAD-MSCs) (Fig. 37J, K, L...Z, AA, AB, AC, AD, Fig. 38D, E, F, G, H, I, J)

In the samples with treatment groups there were different grades of damages in comparison to the non-treatment group with hAD-MSCs. There were lots of variations in the status of the retinal explants depending upon the initial status of the neuroretina at 0 day. In case of VIP treatment there seems to have pathological condition of reactive gliosis evident from the intense staining with GFAP (Fig. 30L red fluorescence), where Müller cells show hypertrophy to fill the space created by the dead and degenerated retinal neurons inside the neural retina. VIP did not seem to show any improvement over the non-treatment group with MSCs, rather in some case it made hNR worse, while NIC and ATRA seemed to show slightly positive protective effects over the preservation of general structure hNR explants. The only sample with VIP+NIC, where the some intact outer segments (OS) of cone PRs were observed, which are shown by white arrows in the panel T of Figure 30. The samples with $(\mathrm{RCM}+\mathrm{MSCs}+\mathrm{VIP}+\mathrm{NIC})$ and $(\mathrm{RCM}+\mathrm{MSC}+\mathrm{VIP}+\mathrm{NIC}+\mathrm{ATRA})$ had relatively good preservation of structure of retina in comparison to other treatment groups. The samples with hAD-MSCs and RCM showed constant preservation of neuroretina in comparison to the hNR explants of control 1.

Immunohistological and histochemical analysis showed that the neuroretinal explants with hAD-MSCs were better preserved than those explants without hAD-MSCs. In the treatment groups, NIC and ATRA seem to have positive effect over the preservation of retinal structure but not significantly better than the hNR explants with hAD-MSCs alone. The significant preservation of neuroretina was observed in the case of VIP+NIC and VIP+NIC+ATRA treatment groups among other treatments groups. The condition of neuroretinal explants in the former was slightly better preservation than the later one. This study also established a novel in vitro method to test the neuroprotective effects of any cell or other biological entity over the degenerating neuroretinal explants. 


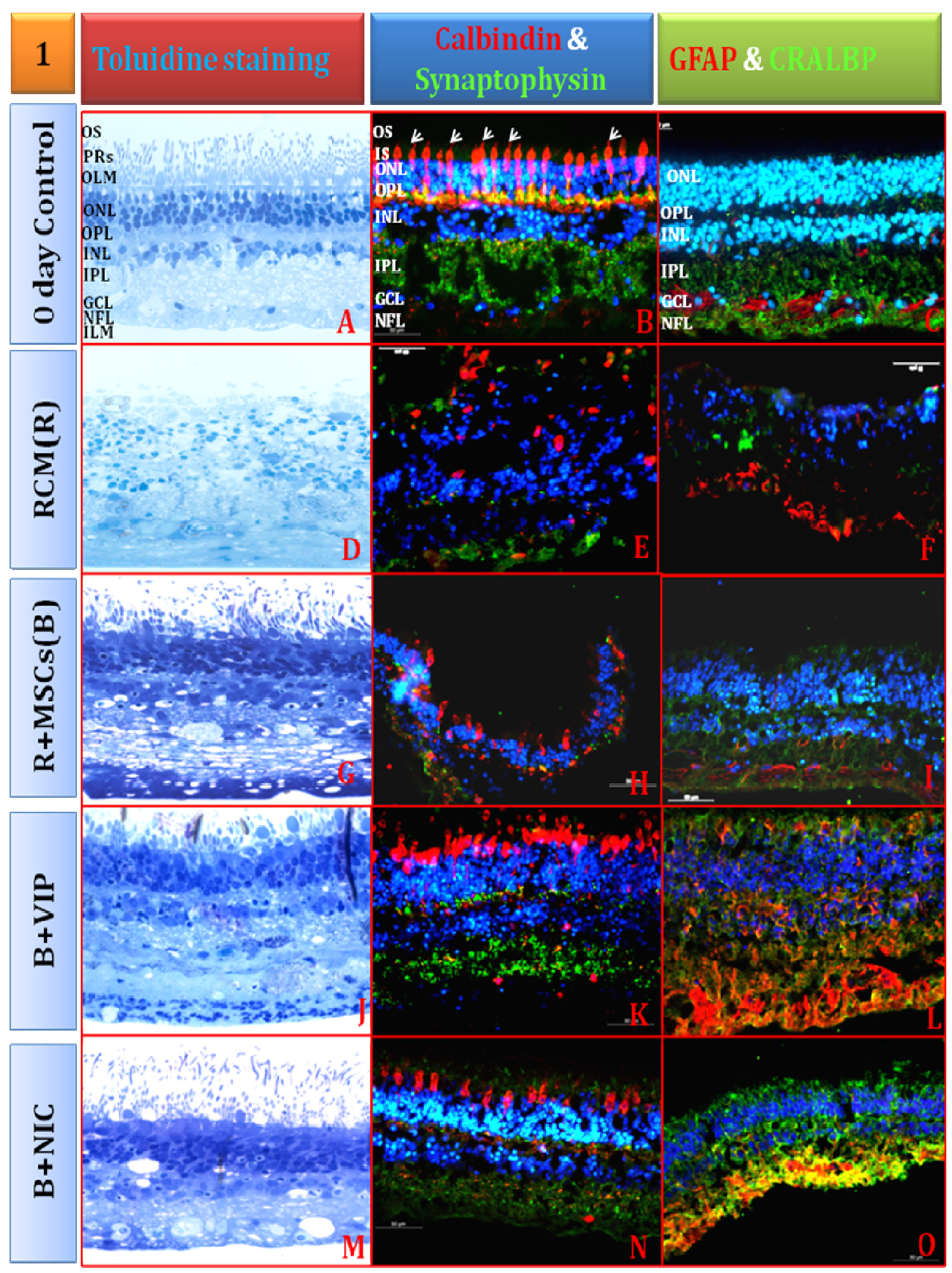

\section{Continued...}

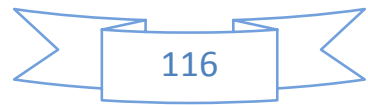




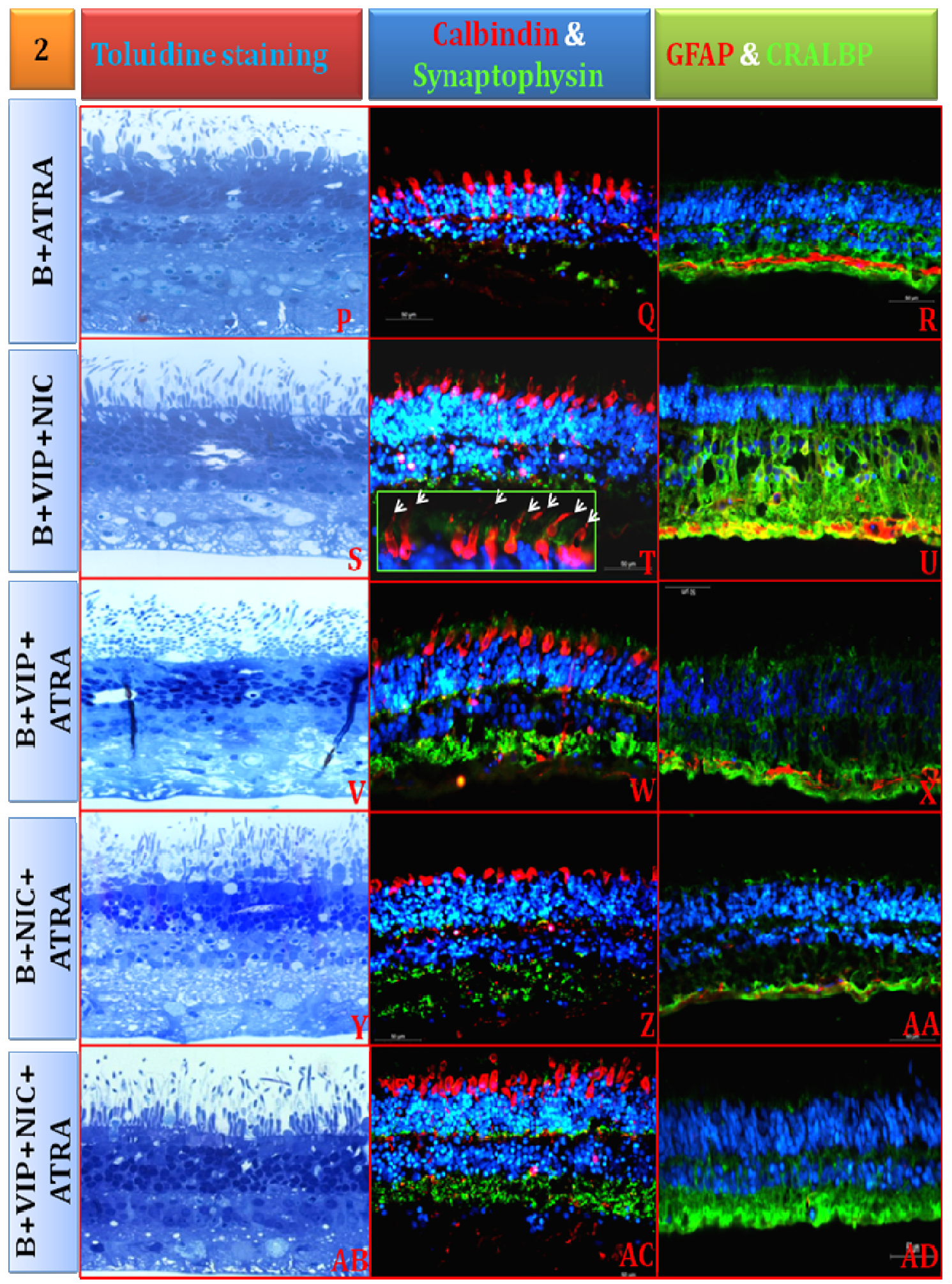

Fig.37. Status of hNR explants co-cultivated with hAD-MSCs for 7 days in the growth medium supplemented with 50\% RCM and factors (VIP, NIC, and ATRA) and their combinations by histochemical staining (toluidine blue-first column) and immunohistochemical staining methods ( $2^{\text {nd }}$ and $3^{\text {rd }}$ column). Calbindin and GFAP staining are red fluorescent in colour and CRALBP and Synaptophysin staining are green fluorescent in colour and nuclei are counterstained with DAPI (blue). All the images were taken at 40x magnification (scale bar-50 $\mu \mathrm{m}$ ).

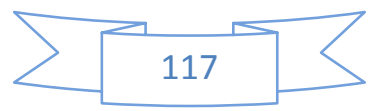




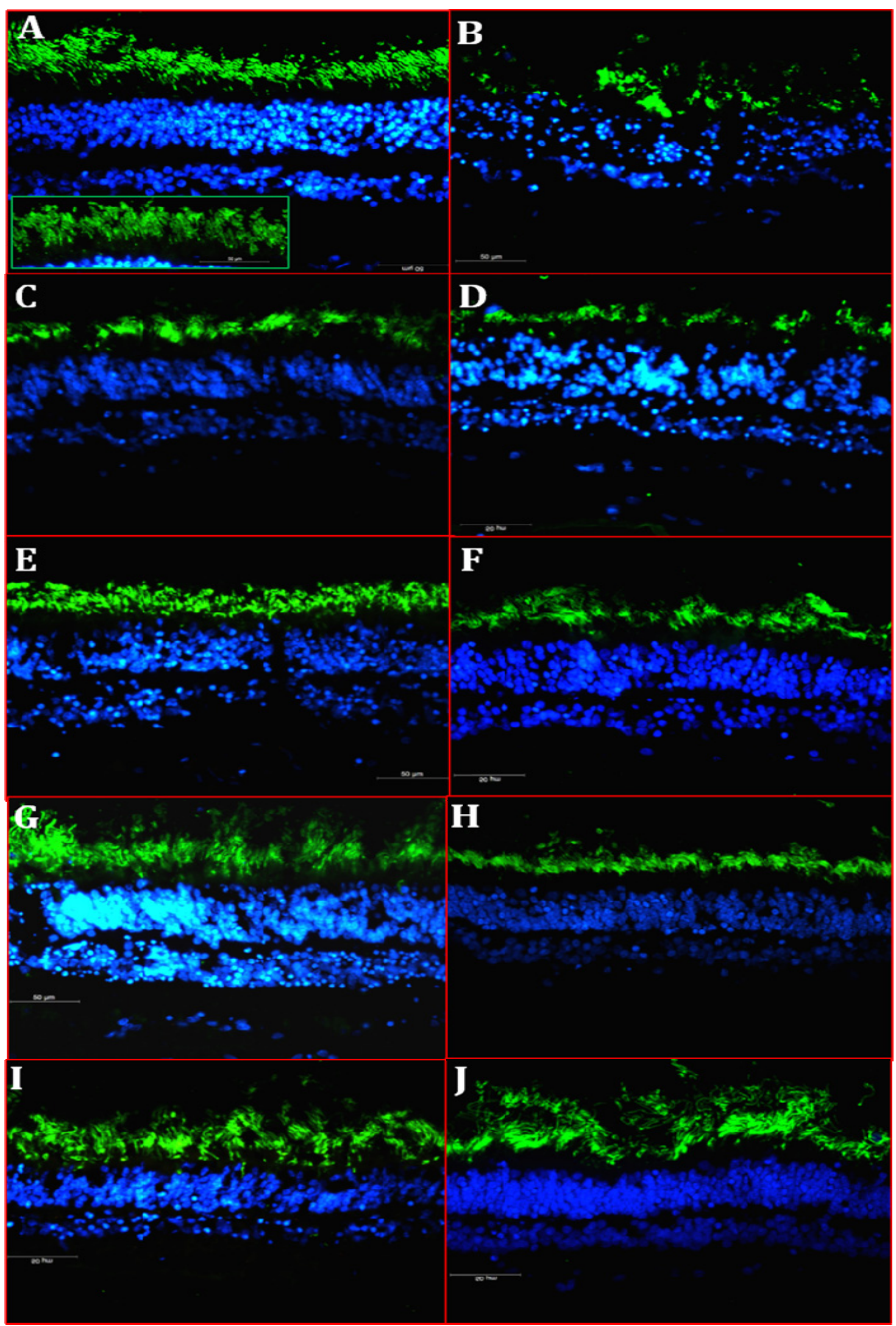

Fig.38. Status of hNR explants co-cultivated with hAD-MSCs in the growth medium supplemented with $50 \%$ RCM and factors (VIP, NIC, and ATRA) and their combination by immunohistochemical staining method. Immunostaining against rhodopsin (green) and counterstaining with DAPI (blue) of hNR explants at 0 day (reference retinal explants) (A) and hNR explants at 7 days with RCM only (control-1) (B), RCM+hAD-MSCs (control-2) (C), VIP (D), NIC (E), ATRA (F), VIP+NIC (G), VIP+ATRA (H), NIC+ATRA (I) and VIP+NIC+ATRA (J). The satellite panel of A has magnified image of rod PRs. All the images were taken at 40x magnification (scale bar $50 \mu \mathrm{m}$ ).

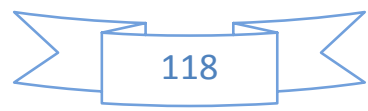


The evaluation of overall preservation status of all the samples of hNR explants from all six experiments was done by three different experts by giving the numerical values from 0 to 10 depending on the grade of preservation status of the neuroretinal explants. The 0 stands for the completely disorganized and destroyed retina (like cell mass without any tissue structure) and 10 stands for the perfectly well-preserved retina with all the tissue structure in good state. The intermediate numbers depend over the comparative status of neuroretinal explants. The evaluation of the effects of all the factors and their combinations was summarized and plotted in the graph with statistical analysis (Fig. 39). The neuroretinal explants with hAD-MSCs are clearly have significant preservation $(\mathrm{P}<0.05)$ than that of control-1 (RCM only). The hNR explants with hAD-MSCs and factors (NIC and ATRA) did not show much significant preservation over those explants with hAD-MSCs alone. VIP alone with hAD-MSCs did not preserve the hNR explants, while in some cases, it also showed reactive gliosis based on intense staining of GFAP. The neuroretinal explants grown in 50\% RCM and hAD-MSCs in combinations with VIP+NIC and VIP+NIC+ATRA had significantly $(\mathrm{P}<0.05)$ better preservation status than those explants with other combinations of factors.

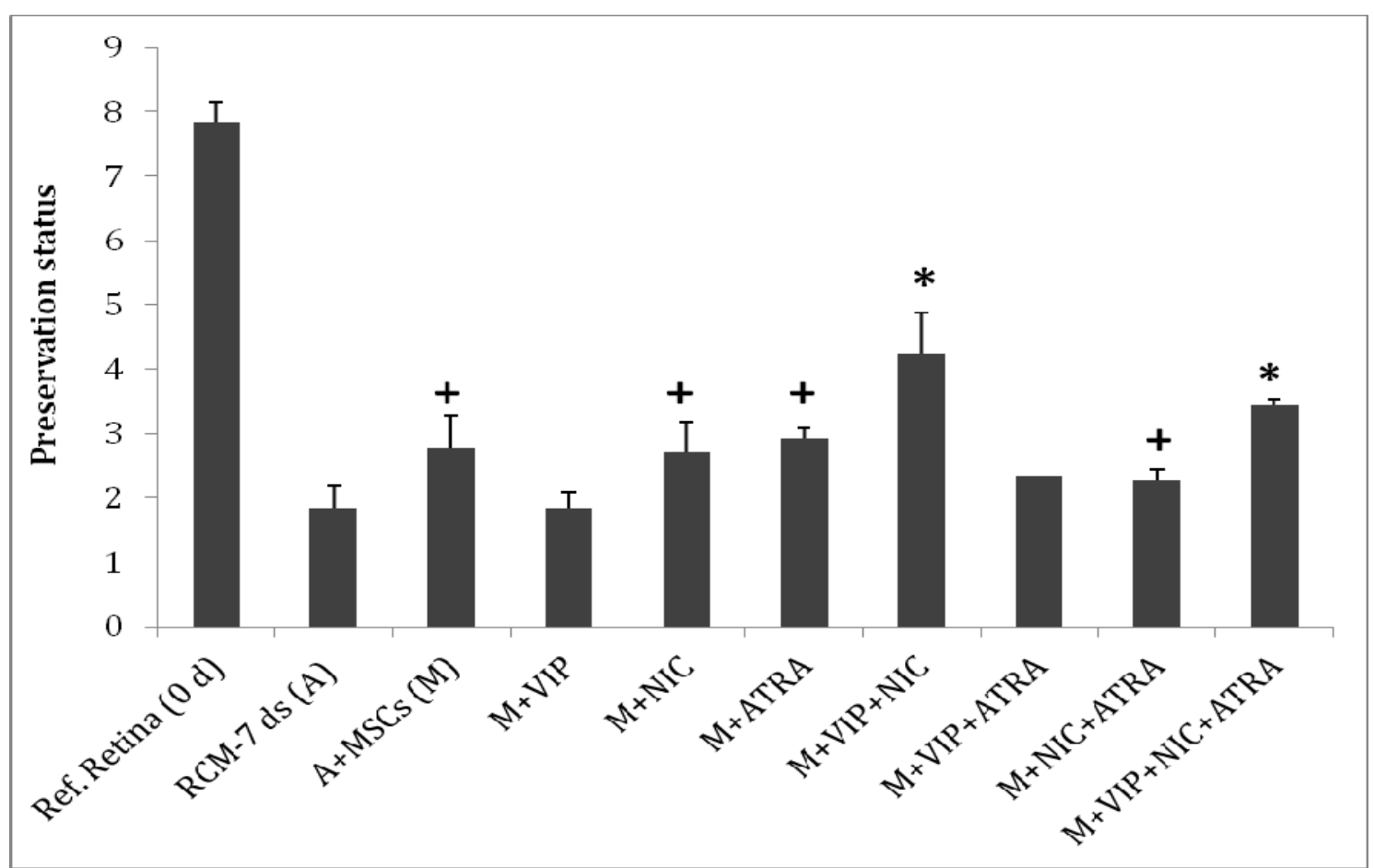

Fig.39. Analysis of preservation status of hNR explants based on visual observation of photomicrographs of histochemical and immunohistochemical staining (Fig. $30 \& 31$ ). The status of preservation of retinal explants were assessed by 3 different experts and the assessment was based on the arbitrary point scale from zero (0) to ten (10), where 0 is for the retinal explants which are completely destroyed/disorganized resembling to cell mass without any retinal tissue structure and 10 is for the retinal explants having perfect cyto-structure with well-organized layers. The statistical significance is set at $\mathrm{P} \leq 0.05\left({ }^{*}\right) /(+)$. Plus sign indicates the significant difference between the hNR explants cultivated in $50 \%$ RCM at one week (A-control-1) and the hNR explants cultivated in 50\% RCM along with hADMSCs plus factors and their combinations (all for one week) while the asterisk show the significant difference between the hNR explants cultivated in 50\% RCM plus hAD-MSCs (M-Control-2) and the hNR explants cultivated in 50\% RCM plus hAD-MSCs with factors and their combinations. Error bar is indicated as $\pm \mathrm{SD}$ ( $\mathrm{n}=3$ to 6 ). 


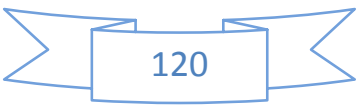




\section{Discussion}

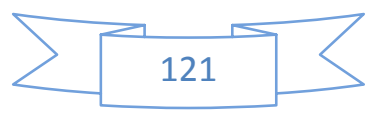




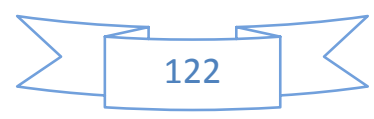


This work is aimed to use the hAD-MSCs as a future candidate for tissue repair and for neuroprotection in cellular therapy for retinal degenerative diseases, especially AMD. In this work, hAD-MSCs had been subjected to many different environments slightly similar to the in vivo subretinal environment present in the affected eye of the AMD patients where both RPE and neural retina are under degeneration process; further these in vitro environments were supplemented with different differentiation factors (VIP, NIC and ATRA) and their combinations to analyze their influences on cell behavior. For this purpose, the hAD-MSCs had been cultivated directly and indirectly with the inactivated and degenerating RPE and with degenerating hNR in the presence and absence of factors and their combinations. In all these different conditions of differentiation for hAD-MSCs to RPE phenotypes, the fate of hAD-MSCs along with their effects on degenerating RPE cells and neural retina had been investigated by using different methods.

Limitation of works: I would like to discuss some of the limitations in these works which we found relevant to mention for having better discussion. Although many limitations could be there in this work, some of them have been identified and mentioned here as follows: few numbers of analytical methods were used to analyze the status of differentiation of hAD-MSCs to RPE phenotypes in some of the experimental modules, selection of type and source of stem cells used, heterogeneity of mesenchymal stem cells, heterogeneity in the health status of human neuroretinal explants among different donors, dependence on random and sudden availability of human eyes, selection of factors used and their combinations, their exact time and period of treatment (time window), lack of functional assay of differentiated RPE like cells from hAD-MSCs, use of only one method (MMC only) to induce degeneration in RPE cells and other unidentified limitations.

This work contains a number of interesting results. Ideally, the results should be verified by various methods to avoid any false positive, which is lacking in few modules of experiments in this work. The capacity of hAD-MSCs to differentiate into non-adipogenic pathways appears to be partially restricted. DNA methylation analyses of lineage-specific promoters in hAD-MSCs suggest an epigenetic programming of hAD-MSCs for adipogenesis, preferentially over differentiation hAD-MSCs to other lineages (Noer et al. 2006, Noer et al. 2007, Boquest et al. 2007). Adult stem cells seem to show more resistance to differentiation to particular cell types (lineage restricted) other than that what it was meant for in vivo probably due to the fact that they are already at the advance stage of differentiation. In case of pluripotent stem cells (ESCs and iPSCs) they are friendlier to get any type of cells probably due to being at the earlier stage of differentiation. Probably this may be one of the various reasons that ESCs and iPSCs have 
been spontaneously transformed into RPE cells (Bharti et al. 2011, Buchholz et al. 2009). The population of MSCs usually has some small but different set of stem cells each having slightly different propensity of differentiation towards particular cell types (Mabuchi et al. 2013, Phinney, Prockop 2007). The differentiated RPE like cells can replace the dead and damaged RPE in AMD and bring some curative effects only when they are capable of doing functions of RPE such as phagocytosis of shed POS, barrier functions, secretion of PEDF and other RPE secretary molecules etc. But in this study we were not able to perform any functional studies over the differentiated hAD-MSCs to RPE like cells.

The main hurdle in these experiments was the availability of postmortem human eyes donor, which was completely random, sudden and scarce. It was really very difficult to set up the experiment in short period to minimize the interval between the enucleation and the processing of human eyes for the use in the experiments. This time window in our experiment was between 2 to 3 days, which affected the RPE cells as well as retina badly probably due to postmortem ischemic injury and loss of nutritional supply and others. There is a report that it is always easier to cultivate the cells (or tissue) if the cells (or tissues) are isolated within 24 hours of postmortem (Cai et al. 2000). Although, the degenerative changes in retinal tissue observed in organotypic co-culture with hAD-MSCs could be partially comparable to those observed under in vivo condition of some retinal degenerative diseases, there were some limitations of this culture system such as retinal explants taken out from these postmortem eyes already had early degeneration inside the globes. This retinal degeneration may be due to axotomy of optic nerve fibres, absence of retinal and choroidal blood supply and absence of RPE layer, that induce acute ischemia in neuroretinal tissue (Fernandez-Bueno et al. 2012). The cellular modifications especially at the inner retina under in vitro condition could differ from in vivo conditions. Nevertheless, this model can reproduce some of the cellular changes revealed in experimental retinal degeneration processes (Fernandez-Bueno et al. 2012). Moreover the retina usually appeared to have in vivo local damages probably due to higher age or other eye diseases (not reported) of different donors ( $\geq 65$ years). So we analyzed the comparative preservation of retinal explants among different co-culture conditions in the experiments rather than absolute damage which might have number of unknown reasons.

In this work, we tried to produce partially similar degenerating environment as of in AMD by using degenerating RPE cells and retinal explants. Ideally the in vivo degenerating condition is influenced by number of factors due to having very complex in vivo pathological dynamics inside the subretinal space and is not possible to generate the same under in vitro condition but at least it could be used as a different approach by using RPE cells under oxidative stress and degenerating retinal explants for producing slightly 
similar conditions for in vitro study. The RPE has number of damages in AMD due to number of reasons and one of the dominant reasons is oxidative stress induced damage (Cai et al. 2000). There are number of published reports, in which researchers have described different methods to induce RPE damage e.g. heat shock method ( $42 \stackrel{\circ}{\circ} \mathrm{C}), \mathrm{H}_{2} \mathrm{O}_{2}, \mathrm{t}-$ Butyl hydroperoxide, $\mathrm{NaIO}_{3}$ treatments etc. Most of them induce ROS inside the cells further leading to cell death depending upon the period and concentration of treatment. Mitomycin C (MMC) has also been reported to produce ROS e.g. superoxide anion and $\mathrm{H}_{2} \mathrm{O}_{2}$, leading to apoptotic cell death by DNA strand damage (Koedrith, Seo 2011, Kennedy et al. 1985). MMC has been reported to induce apoptosis and growth arrest in hRPE in dose dependent manner (Kang et al. 2001). In our experiment the time and concentration of MMC treatment was optimized not only to inhibit the proliferation of RPE cells but also it could later induce the RPE cell death in the long run. The mechanism of degeneration of RPE cells may not be exactly the same as that of AMD but it could be presumed to imitate RPE degeneration in AMD partially. This does not rule out the possibility that imitation could be improved by using other methods.

We used three differentiating factors in these experiments based on previous articles and researches over the use of factors for differentiation of different types of stem cells to RPE like cells. It cannot be ruled out that other factors and other combinations might fetch better results. Although each one is showing some effects at different extent but the precise time window for each factors and the exact combination could be investigated further. Another limitation of these studies is the lack of study of percentage and degree of differentiated population of hAD-MSCs under different conditions used. This is very important to judge the effectiveness between different methods used. It could be done further to elucidate the degree and percentage of differentiation along with functional assay.

Under the study of effects of the differentiating environments over the neuroretinal explants, there were lots of variations in the results of each experiment probably due to the local heterogeneity in the health status of retinal explants in the same experiments and due to global health status of retina obtained from different donors between different experiments.

The hAD-MSCs have been shown to rescue and delay the degeneration of RPE and neuroretinal explants under various in vitro conditions but we could not study the profile of secreted molecules (growth factors and cytokines) under these conditions and their effects, which could be studied further. Another limitation in these studies is that the conditions used for the differentiation of hAD-MSCs are similar for inducing differentiation 
towards the retinal neurons (neuro-ectodermal differentiation). NIC and ATRA have been used in inducing neuronal differentiation from stem cells (Buchholz et al. 2013, Bi et al. 2013, Huang et al. 2012b, Li et al. 2012b). In spite of these facts, in none of the experiments, neuronal markers have been used to check any neuronal differentiation in hAD-MSCs subjected to differentiation. Some of limitations are arises just due to lack of time and human resources. Most of the limitations could be solved further extending the work to the next step in future.

This study does not clarify whether combinations of factors alone could preserve the retina or they show their synergistic effects with hAD-MSCs. All these factors have been reported to have neuroprotective properties in various published works, so there is a chance they may themselves could preserve the retinal explants to some extent. One thing is clearly shown in this study that they alone have anti-proliferative effects over the RPE cells under stress. Thus, further study would be needed to obtain the exact effects of these combinations alone without the use of RCM or hAD-MSCs to claim some pharmaceutical/industrial use of these factors or their combinations.

In spite of being some hurdles and limitations, the clinical importance of these works cannot be denied. The results in this study advocate the strong candidature of hAD-MSCs along with the addition of external factors for their use in subretinal transplantation in AMD patients taking together the advantage of their differentiation potential and paracrine effects as well. Further it needs to be investigated how to get the RPE sheet with correct cytoarchitecture with functional characters.

\section{Characterization of RPE cells and undifferentiated hAD-MSCs}

Our experiences say that the hRPE cells isolated from younger and healthier donors have high viability, pigmentation and proliferative capacity than that of older and diseased one. The hRPE cells lose their pigmentation and epithelial characteristics very fast just after first passage. Further they adopt fibroblastic morphology during further passages after P6-P8. This may be probably due to normal wound healing response of epithelial cells (epithelial-mesenchymal transition-EMT) which appears similar to the disruption of epithelial sheet at every passage by the trypsin treatment (Leopold et al. 2012). The primary culture of RPE seemed to be very vulnerable to the slight change in $\mathrm{pH}$ of the growth medium. Therefore it needs to take extra caution to maintain primary hRPE cells in better condition in cell culture. Our experiences indicate that the seeding density is very important in maintaining hRPE morphology. In my opinion, the epithelial cells may need

minimum cell to cell contacts to grow and maintain their characteristics well and very low 
cell density could probably be sensed as wound like situation where they have to stretch and change to more fibroblastic phenotype to make contacts with other cells in the cell culture. The FC analysis shows that hRPE cells in culture maintain the expression of RPE65 protein (a 65-kDa cytoplasmic protein involved retinoid cycle) expression at higher passages (P10) but there is gradual loss in the intensity of their expression during subculturing. Further results also show that there is no contamination of fibroblastoid cells in hRPE cell culture.

RPE cells also demonstrate good growth, adhesion and viability over the tissue culture treated surface of polystyrene of culture flasks or plates. They also demonstrated to maintain their growth, adhesion and viability over the ELR-RGD polymers. They also demonstrated to maintain their phenotypes well over the bio-compatible polymer (Singh et al. 2013b, Srivastava et al. 2011a). It could be used to test further the characteristics of differentiated RPE like cells over these polymers or others, which could be the next step in cell-substrate development for transplanting differentiated RPE cells from stem cell for future investigation.

RPE cells usually emits lots of autofluorescence in the immunological studies due to presence of pigment molecules inside the cells, which makes them sometimes difficult to distinguish the test fluorescence signal in FC analysis or immunofluorescence studies. The results from the method developed in our study indicate that incubation of the cultured RPE (pRPE) cells and the retinal tissue specimens with TB $(20 \mu \mathrm{g} / \mathrm{mL})$ after immunolabelling (post-treatment) and before paraffin embedding (pre-treatment) respectively quenched the autofluorescence of RPE cells effectively. Thus it can facilitate the detection of expressed cellular proteins in experimental as well as in pathological conditions. It fulfills the requirement for developing a method which can serve to eliminate the autofluorescence of the cells, not only in cell cultures but also in tissues samples. This method significantly increases the quality and value of RPE cell protein analysis, as well as other cell protein analysis performed by FC and Immunofluorescence analysis.

The culture and maintenance of hAD-MSCs are very essential features for maintaining their stemness (potency). The hAD-MSCs are very susceptible to lose or modify their potency if they are not maintained with precautions with standard cell culture practices. It is essential to maintain the hAD-MSCs always under sub-confluence $(<90 \%)$ level. The growth medium with use of antifungal agents was avoided in the culture of hAD-MSCs, as they induce differentiation in the cells (Phinney et al. 1999). The cryoprotective agent, dimethyl sulfoxide ( $\mathrm{Me}_{2} \mathrm{SO} / \mathrm{DMSO}$ ) has been used for cryopreservation of hAD-MSCs, 
although it has been reported that to be toxic at $>4 \stackrel{\circ}{\circ} \mathrm{C}$ and to cause uncontrolled differentiation (Hegner et al. 2005, Ji et al. 2004, Zyuz'kov et al. 2007). The hAD-MSCs have been grown till passage 30 in our study and the higher passage cells show slower growth and lesser differentiation capacity than that of lower one.

Both hRPE and hAD-MSCs including other cell lines (ARPE19 and HFF1) had been maintained and tested phenotypically during the experiments to minimize the variations between the experiments. Immunostaining and WB results obtained in optimization experiments including experimental results, showed something very interesting that almost all the markers were expressed in hAD-MSCs in basal level and some at significant high level (data not shown). Interestingly, the immunological studies indicate significant level of PEDF expression in hAD-MSCs, which is a specific secretary molecule of RPE. Based on the article search online, there is no any article that explains these characteristics of MSCs. It points to an interesting and novel idea that hAD-MSCs might have propensity to differentiate to RPE due to having expression of several RPE markers in them. However, further investigations need to be done to increase and localize the RPE markers in hAD-MSCs. Furthermore hAD-MSCs have lots of paracrine properties similar to RPE secretion profile as mentioned in the introduction section (table 1), which may be made use along its differentiation properties in the retinal degeneration diseases.

The hAD-MSCs have been characterized according to the criteria laid by ISCT. They have been proved up to the marks. They demonstrated positive for all the MSCs specific cell surface markers (CD90, CD73, CD105) and negative for cell surface markers which are specifically absent in MSCs e.g. CD14, CD19, CD34, CD45 and HLA-DR. They have demonstrated the capacity to differentiate into three mesodermal lineages e.g. chondrocytes, osteocytes and adipocytes.

The percentage of hAD-MSCs expressing MSCs-specific cell surface markers was increasing at higher passages (P16 and P21) according to immunophenotypic analysis by FC. It indicates that they become more homogeneous during sub-culturing, however they show lesser potency of differentiation into three mesodermal lineages (chondrocytes, osteocytes and adipocytes) at higher passages. They were not able to form even firm mass of chondrocytes at higher passage in chondrocyte differentiation studies. They show slower growth at higher passages which might be an indicator of cell senescence. 
The most relevant findings from this part are as follows:

RPE cells and hAD-MSCs have been isolated from their sources and well characterized and their culture and maintenance have been established well.

- The hRPE cells lose their characteristics such as pigmentation and cobblestone morphology etc. rapidly during their sub-culturing.

- A protocol based on FC analysis has been established to test the purity of RPE cells (contamination of fibroblastoid cells in RPE culture) -published (Srivastava et al. 2013).

- A protocol for quenching autofluorescence in immunological studies (e.g. FC analysis and immunofluorescence) of RPE cells has been established by using Trypan blue - published (Srivastava et al. 2011b).

- The RPE cells maintain their growth, adhesion and morphological characteristics on biocompatible ELR-RGD polymers - published (Singh et al. 2013b, Srivastava et al. 2011a).

- The hAD-MSCs slowly become more homogeneous during sub-culturing in cell culture but they gradually lose the differential potential at higher passages.

\section{The effects of hAD-MSCs over MMC-treated RPE cells (ARPE19 cells) under indirect co-culture condition -Published Excerpts (Singh et al. 2013a)}

This study was done to see the possibility of use of hAD-MSCs as a candidate for subretinal transplantation in AMD patient to rescue the degenerating RPE. For this purpose, the effects of hAD-MSCs on MMC induced RPE cell death was evaluated under in vitro condition.

MMC is a bioreductive DNA alkylating agent (Rockwell et al. 1982), which was used to inhibit cell proliferation and to induce cell death. It has been demonstrated to induce cell death by apoptosis as well as necrosis in several reports (Wu et al. 2008, Wu et al. 2002, Park et al. 2002, Hoffman et al. 1998). It has been reported that MMC induced cellular apoptosis in corneal endothelial cells was mediated through caspase-8, caspase-9, and the mitochondrial regulated pathways as well as through upregulation of p53-dependent and p21-dependent signal transduction pathways (Wu et al. 2008). Oxidative stress during ageing has been considered one of the main reasons for the degeneration in RPE in older people leading to the development of AMD. Oxidative stress targets directly to mitochondrial-dependent apoptosis that require release of cytochrome $\mathrm{C}$ from mitochondria and subsequent activation of a specific class of cytoplasmic proteases known 
as caspases (Takahashi et al. 2004). Mitomycin C (MMC) has also been reported to produce ROS e.g. superoxide anion and $\mathrm{H}_{2} \mathrm{O}_{2}$, leading to apoptotic cell death by DNA strand damage (Koedrith, Seo 2011, Kennedy et al. 1985). MMC has been reported to induce apoptosis and growth arrest in hRPE in dose dependent manner (Kang et al. 2001). Thus, it seems that MMC in this study induced apoptosis in ARPE19 cells is quite similar to that apoptosis in the degenerating RPE cells of the AMD patients. AB assay showed that MMC inhibited the RPE cells proliferation significantly which is in line with its properties.

Calcein AM/EthD-III probes of cell viability/cytotoxicity kit measures two recognized parameters of cell viability, intracellular esterase activity and plasma membrane integrity. The results obtained using these probes clearly showed that after 3 weeks of MMC treatment, RPE cells were completely dead. The hAD-MSCs rescued the RPE cells partially from entering into death process through secreting some growth factors in the shared growth medium. The proliferative activity of MMC treated RPE cells with hAD-MSCs was significantly higher than that of MMC treated RPE cells without hAD-MSCs. The supernatants of hAD-MSCs demonstrated the similar effects of encouraging proliferation of MMC treated RPE cells. This indicates that hAD-MSCs secrete some protective growth factors in the common shared medium in the indirect co-culture, which probably prevented MMC treated RPE cells entering into cell death. These protective growth factors could be CNTF, BDNF, IGF1, NGF and bFGF etc. The results also showed that hAD-MSCs were maintaining their viability after 3 weeks on track etched PET trans-wells. It indicates that there is no adverse effects of MMC treated degenerating RPE cells over the hAD-MSCs on trans-well. It has been also demonstrated that fibroblast also show some positive effects over the MMC treated RPE cells but this was significantly lower than that of hADMSCs.

In vivo condition, in dry AMD patients, RPE cells are under degenerating stage and releasing many factors. Transplanting hAD-MSCs in subretinal space might create a microenvironment, which could rescue partially the degenerating RPE cells, thereby helping to prevent AMD progression in the old age populations. The results suggest that hAD-MSCs have the capacity to rescue the degenerating RPE cells, thus encouraging their further utilization for in vivo study. However, fibroblasts have also shown lower grade of encouraging effects over the proliferative activity of MMC treated RPE cells, they have different characteristics than that of AD-MSCs (Alt et al. 2011) and involved in producing PVR complications (de Souza et al. 1995). 
The most relevant findings from this part are as follows:

The hAD-MSCs partially rescue the dying RPE cells by inhibiting them to enter into cell death under stressed condition

- MMC treatment at higher dose for longer period induces apoptotic cell death and almost all cells die during the long run after treatment.

- The hAD-MSCs as well as their supernatant encourage the proliferation and metabolic activity in RPE cells under stress induced by MMC.

\section{Cultivation of hAD-MSCs in RCM supplemented with factors and their combinations}

The hAD-MSCs have been induced to RPE phenotypes by culturing them in 50\% hRCM (hRPE condition medium) supplemented with factors (VIP, NIC, and ATRA) and their combinations (VIP+NIC, and NIC+ATRA) for 21 days. RCM contains a number of factors secreted by RPE cells under in vitro culture condition that have pleiotropic roles in stabilizing retina. The inclusion of RCM in this module of experiment exposed hAD-MSCs to RPE secreted factor molecules along with the combinations of added factors, which induces the differentiation of hAD-MSCs towards RPE phenotypes. The presence of RCM in this experiment is also an effort to create a partially similar environment to that of subretinal space. RCM induces the hAD-MSCs to RPE phenotypes partially and this result is in line with findings by others (Huang et al. 2012a, Vossmerbaeumer et al. 2009). The identification of RPE secreted factors involved in the induction of differentiation in hADMSCs could be studied further. To analyze the RPE phenotypes in induced hAD-MSCs, the expression of RPE65, Bestrophin (a calcium ion activated chloride channel transmembrane protein present in the basal side of RPE) and epithelial markers of RPE (CK8/18) and PCK (Pancytokeratin) were assessed by WB and immunocytofluorescence methods. The results indicate that the expression of all the RPE markers used is enhanced significantly $(\mathrm{P}<0.01)$ in the hAD-MSCs grown in 50\% RCM while this effect of differentiation is more potentiated in the presence of combinations of factors (VIP+NIC and NIC+ATRA). Out of these, the VIP+NIC combination has more inductive effects than that of NIC+ATRA. In the case of induction of expression of epithelial marker of RPE (CK8/18 and PCK), ATRA is more effective than that of other factors. As ATRA is already a reported differentiation factor for epithelial characteristics in MSCs (Wan et al. 2012, Li et al. 2012a, Brzoska et al. 2005), so this effect of ATRA is similar to the previous reports. However, the results indicate that VIP or NIC alone does not seem to induce the increase in RPE marker expression significantly, which is not in coherence with other reports 
(Vossmerbaeumer et al. 2009, Vossmerbaeumer et al. 2008). Interestingly, VIP in combination with NIC increases the RPE markers significantly which indicates that the differentiation signaling induced by each of them is complementary. The exact mechanism behind the effect of this combination needs to be elucidated further.

Further immunostaining results for RPE markers (RPE65 and Bestrophin) in induced hAD-MSCs in different conditions also demonstrated the similar results as obtained in WB. The NIC in combination with ATRA and VIP is found to induce the differentiation in hADMSCs better in comparison to that when it is used alone. NIC is already reported to induce differentiation in induced pluripotent cells and embryonic stem cells towards RPE phenotype (Buchholz et al. 2013, Buchholz et al. 2009, Idelson et al. 2009a). Although the differentiation process in embryonic stem cells is different from that of adult stem cells because the adult stem cell is already at advance stage of differentiation in the differentiation path, but it may give the similar cues for particular differentiation pathway.

The most relevant findings from this part are as follows:

$>$ The hAD-MSCs partially differentiate towards RPE phenotypes when induced by NIC, ATRA, VIP+NIC, and NIC+ATRA in the presence of RCM.

- The combinations VIP+NIC and NIC+ATRA perform better than that of their individual effects on differentiation of hAD-MSCs to RPE phenotypes.

- ATRA helps to potentiate epithelial characteristics in differentiating hADMSCs to RPE phenotypes in line with its epithelial differentiation capacities.

- VIP or NIC alone does not show much significant change to RPE phenotypes in hAD-MSCs in contrast to its reported RPE inducing effects in other stem cells.

\section{Induction of differentiation of hAD-MSCs to RPE phenotypes with BMP-4 and POS and fRCM (This part of the work was done at the University of Liverpool)}

In this preliminary work, the phase contrast microscopic study of the hAD-MSCs culture in induction medium demonstrates that fRCM induces the pigmented sphere formation in hAD-MSCs at 15 days and this tendency appears to be potentiated by the presence of BMP4 in fRCM. The formation of pigmented spheres might be one of the characteristics in the differentiation of stem cells to RPE like cells (Gong et al. 2008a). The immunocytochemical study also shows that BMP-4 enhances the expression of RPE protein markers (RPE65 and CK8/18) in the induced hAD-MSCs in comparison to other controls such as uninduced and induced hAD-MSCs with 50\% fRCM. The POS used in the experiment did not fetch any 
results as all the cells grown with POS died. The reason of cell death was unknown but it may be probably due to toxicity produced from POS. BMP-4 is expressed in the optic vesicle during the early embryonic stage and has been reported to play crucial role in the specification of RPE and other eye components in the eye formation (Haynes et al. 2007, Müller et al. 2007, Du et al. 2010, Furuta, Hogan 1998). Thus based on the preliminary results obtained in this module of experiment and the published reports describing the crucial role of BMP-4 in the eye development, its role in adult stem cells differentiation towards RPE phenotype cannot be ruled out without further investigation. Further study needs to be done to confirm the effect of BMP-4 in the differentiation of hAD-MSCs to RPE phenotypes. This study uses fRCM, which was found potentially more effective than that of hRCM (data not shown), it may be probably due to having richer in all the factors released by fresh and healthy fRPE in the medium (Kolomeyer et al. 2011)(data not shown). The fRCM was not used in other modules of experiments because of limitation of fRPE cells. Instead, the aRCM or hRCM was used in other modules of this work.

The most relevant findings from this part are as follows:

$>$ fRCM seems to induce RPE phenotypes and formation of pigmented cell spheres in hAD-MSCs.

$>$ BMP-4 together with fRCM appears to enhance RPE phenotypes and pigmented sphere formation in hAD-MSCs.

\section{Direct co-culture of hAD-MSCs with inactivated RPE cells}

In this direct co-culture condition the hAD-MSCs have been subjected to differentiation towards RPE phenotypes by growing them with inactivated RPE cells. It has been previously described that the higher dose of MMC treatment for two hours not only inhibits the proliferation of the RPE cells but also leads to degeneration in RPE cell by cell death during long culture (after 3 weeks) (Singh et al. 2013a). This condition may be slightly similar to the situation of subretinal RPE in AMD, where RPE is under degeneration due to genetic and environmental abuse.

To analyze the induction of expression of RPE proteins in induced hAD-MSCs co-cultivated with MMC treated RPE cells, RPE65 protein (retinoid cycle component), ZO-1 (epithelial marker of RPE), CK8/18 (RPE specific cytokeratin) and Ezrin (apical marker protein of RPE) were used. The immunofluorescence study showed that all these markers of RPE are increasing significantly in the differentiated hAD-MSCs in comparison to undifferentiated hAD-MSCs. ZO-1 is one of the epithelial markers of RPE and is a component of tight 
junction (zonula occludens), its expression is slightly enhanced in the hAD-MSCs when they are super-confluent but their localization is limited to the cytoplasm. While under induced condition both the localization and expression of ZO-1 are enhanced in hAD-MSCs. The localization of ZO-1 to the cell membrane in induced hAD-MSCs is clearly visible in immunofluorescence studies. Ezrin is a member of ERM (Ezrin, Radixin and Moesin) protein family and functions as a linker between the plasma membrane and actin cytoskeleton and involve in apical microvilli formation in RPE (Huang et al. 2009, Bonilha et al. 1999, Bonilha, Rodriguez-Boulan 2001, Kivela et al. 2000). Although the expression of ezrin is enhanced significantly in induced hAD-MSCs but their pattern is not similar to the expression in the RPE (positive control). It shows clearly their localization is different. It is obvious for any protein the expression is followed by the further processing and localization to their respective sites. The decrease in CD-90 expression in the induced hAD-MSCs demonstrated that they are differentiating or losing the MSCs characteristics thus supporting the evidence of differentiation of hAD-MSCs to RPE phenotypes in direct co-culture.

When MMC treated RPE cells were marked with EdU to track them in the direct co-culture, they were found below the hAD-MSCs layer. Some of them (MMC treated RPE cells) have their nuclei under pyknosis (irreversible chromatin condensation) and karyorrhexis process (final events in the apoptosis), as are demonstrated by the intense red fluorescent bodies or particles in the immunocytostaining analysis. This tracker study demonstrated that the RPE markers are expressed largely in the hAD-MSCs including few of remaining live RPE cells. As already described that the MMC treated RPE cells undergo cell death if they are cultivated alone, while they are partially rescued from cell death by the antiapoptotic paracrine effects of hAD-MSCs over the MMC treated RPE cells in co-culture system (Singh et al. 2013a).

It has been found in this study that hAD-MSCs form darker pigmented cell spheres under induced condition in comparison to uninduced one in which cell mass spheres are less pigmented and frequent. This condition resembles the pigmented neurosphere formation by embryonic stem cells (Gong et al. 2008a). These cell mass spheres formed by hAD-MSCs shows thy-1 (CD90 - an MSCs-specific marker) positive but under induced condition the expression of CD90 reduced significantly in those cells. These cell mass spheres were positive for the RPE65 protein expression in the induced condition as well. These results certainly show that under direct co-culture the hAD-MSCs are differentiating towards RPE like cells, however it requires further study to confirm these results of pigmented sphere formation by hAD-MSCs. 
The differentiation of hAD-MSCs towards RPE phenotypes is further confirmed by the WB analysis. The hAD-MSCs increase significantly $(\mathrm{P}<0.001)$ the expression of RPE65 and ZO1 proteins under direct co-culture. They have significant higher expression of RPE protein markers $(\mathrm{P}<0.01)$ than that of under indirect co-culture thus it shows that cell to cell contacts play crucial roles in stem cell differentiation. MSCs are involved in the cell and tissue homeostasis inside the body. In the case of tissue damage, MSCs usually get some signals (chemokines) from the damaged tissue and migrate to the damage site (homing) and they repair the damage either by replacing the dead and damaged cells by differentiating to the corresponding cells or by secreting some anti-apoptotic factors or other factors or by both ways. In this case MSCs probably get some signal from the damaged tissues to differentiate to replace them, which are confirmed by these experimental results. Furthermore, these results are similar to the results obtained in one of the published studies in which mouse BM-MSCs have been differentiated to RPE phenotype by directly co-cultivating them over the MMC treated RPE cells (Li et al. 2007).

The most relevant findings from this part are as follows:

The hAD-MSCs differentiate to RPE like cells significantly when they are cultured over the inactivated RPE cells.

- MMC treatment induces the apoptotic cell death in RPE cells. It is re-confirmed by nuclear fluorescence of MMC treated RPE cells in the direct co-culture.

- Direct co-culture induces darker pigmented sphere formation in hAD-MSCs with slight characteristic of RPE.

- The degree of differentiation in hAD-MSCs in direct co-culture with RPE cells is significantly greater than that of indirect co-culture.

- This study indicates the importance of cell to cell contact in differentiation process.

\section{Indirect co-culture of hAD-MSCs with MMC treated RPE cells along with factors and their combinations}

It is previously described that in indirect co-culture of hAD-MSCs with MMC treated RPE cells, hAD-MSCs shows the encouraging effect on the proliferative activity of MMC treated RPE cells. Further study shows that the effects of all the factors (VIP, NIC and ATRA) and their different combinations on the proliferative activity of MMC treated RPE cells are suppressive but in the presence of hAD-MSCs these effects are significantly reversed especially at later stage of growth (at 5 days). This shows that hAD-MSCs in combination 
with all these factors stimulate the viability and proliferation of MMC treated cells. This also shows that the importance of the use of hAD-MSCs along with combinations of factors for their effectiveness in increasing the proliferation activity and rescuing the dying RPE cells and simultaneously inducing the differentiation of hAD-MSCs to RPE phenotypes. However there are number of issue need to know further such as identification of factors secreted by uninduced and induced hAD-MSCs and by MMC treated RPE cells, the mechanism involved in this rescue effects etc. In case of NIC and VIP+ATRA, the antiproliferative effect is not very pronounced in comparison to other factors and their combinations. When hAD-MSCs have been exposed to NIC only, fewer cells of hAD-MSCs have been observed in this case, which could be either due to cell death or due to decrease in proliferation of hAD-MSCs. The similar decrease in proliferation has previously been reported upon exposure of hESCs to NIC (Vaca et al. 2008). These results suggest a role of NIC away from the cell survival.

The differentiation of hAD-MSCs towards RPE phenotype in induced conditions is demonstrated by WB analysis of the battery of RPE protein markers (RPE65, Ezrin, CK8/18, Tyrosinase, MerTK, MiTF, Pax6) and an MSCs-specific marker Thy-1 (CD90). The significant decrease in CD90 expression in the induced hAD-MSCs shows that the hADMSCs are differentiating to other cell types while the significant increase in expression of RPE markers shows their differentiation towards RPE phenotypes. In all the cases, there is a significant increase in the RPE markers and a significant decrease in CD90 in the induced hAD-MSCs in comparison to the uninduced hAD-MSCs but especially the combinations of factors appears to potentiate these effects significantly. The retinoid cycle protein of RPE (RPE65) is significantly increased in case of VIP+NIC in comparison to other combinations. The epithelial marker of RPE (CK8/18) and apical marker of RPE (Ezrin) are enhanced significantly in all the combinations and ATRA, while in case of NIC, there is a decrease in expression of Ezrin in comparison to that of induced hAD-MSCs without factors. Tyrosinase, which is involved in the pigment synthesis in RPE cells, is enhanced significantly in the presence of NIC, VIP+ATRA and NIC+ATRA. Immature markers of RPE such as Pax6 and MiTF are more pronounced in the case of VIP and VIP+ATRA. MerTK which is involved in the phagocytosis of shed POS by the RPE, is enhanced in all the combinations. The differential expression of all these markers in case of each factor and their combinations indicate their roles at different stage and point of differentiation signaling pathway. The combination VIP+ATRA is overall better than other combinations while the same combination has more anti-proliferative effects over the MMC treated RPE cells. But the overall picture from this experiment comes out that the combinations of factors work better than that of when they are used alone, which explains complementary 
role of each of them in inducing the differentiation signaling of hAD-MSCs towards RPE phenotypes.

The most relevant findings from this part are as follows:

The hAD-MSCs partially differentiate to RPE phenotypes when induced by the combinations of factors (VIP+NIC, VIP+ATRA, NIC+ATRA, and VIP+NIC+ATRA).

- All the factors and their combinations (except VIP and VIP+ATRA) show antiproliferative effects over the MMC treated RPE cells and these effects are annulled by the presence of hAD-MSCs.

- VIP, NIC, and ATRA show significant effects in enhancing RPE characteristics in hAD-MSCs but their combinations give significant synergistic effects in inducing RPE phenotypes in hAD-MSCs in comparison to that of individual effects.

\section{Indirect co-culture of hAD-MSCs with hNR explants in RCM along with differentiating factors (VIP, NIC and ATRA) and their combinations}

Along with RPE, the neuroretina (especially PRs) is also affected in retinal degenerative diseases. Therefore it is a logical approach to evaluate the effects of hAD-MSCs along with different factors and their combinations on degenerating neuroretina. Indirect co-culture of hAD-MSCs with hNR explants in 50\% RCM demonstrates that there is a significant increase in the expression of RPE protein markers in the induced hAD-MSCs but the differentiation towards RPE phenotype is more potentiated by the presence of factors (VIP, NIC, and ATRA) and their combinations (VIP+NIC, NIC+ATRA, and VIP+NIC+ATRA). The battery of RPE markers for different functions such as retinoid cycle component (RPE65), epithelial markers of RPE (CK8/18), basal marker of RPE (Bestrophin), apical marker of RPE (Ezrin), immature marker of RPE (MiTF and Pax6), phagocytosis marker of RPE (MerTK), secretary protein marker of RPE (PEDF) and MSCs-specific marker CD90 have been used to assess the differentiation status in the uninduced and induced hADMSCs towards RPE phenotypes. The MSCs-specific marker CD90 is significantly decreased in the induced hAD-MSCs especially in case of combinations, which indicates the changing of mesenchymal phenotypes of hAD-MSCs to another cell types. It is interesting to note that in our experiments, the hAD-MSCs are found to express PEDF very significantly, which is a neuroprotective factor secreted by RPE. This is one of the strong evidences about hADMSCs, which encourages to move forward to study about the potential of hAD-MSCs in the subretinal transplantation to rescue the early degeneration of PRs in AMD. PEDF 
expression in hAD-MSCs is maintained in the condition containing combinations of factors, while its expression in hAD-MSCs decreases significantly in the case of individual factor. There are no any significant changes in the immature marker of RPE (MiTF and Pax6) including PEDF expression between induced hAD-MSCs with and without factors. In this setup of experiment, VIP as well as NIC is significantly inducing RPE phenotypes in the induced hAD-MSCs in comparison to the induced hAD-MSCs without any factors. This is in line with the previous reports that VIP induces the partial differentiation of stem cells to RPE phenotypes (Vossmerbaeumer et al. 2009, Vossmerbaeumer et al. 2008). Especially the combinations VIP+NIC and VIP+NIC+ATRA have more potentiating effects over the differentiation of hAD-MSCs towards RPE phenotypes including other combination NIC+ATRA. This indicates the importance of more than one factor molecule during the process of differentiation. This condition could be slightly similar to the subretinal environments of AMD, where the retina is under degeneration process. These results confirm again the importance of the use of hAD-MSCs along with the combination of factors for their effectiveness on rescuing and repairing the retinal damages in retinal degeneration diseases.

All the factors used in this study have pleiotropic roles in differentiation and maturation of RPE from stem cells. VIP has been reported to induce differentiation of stem cells to RPE like cells (Buchholz et al. 2013, Koh 2000, Vossmerbaeumer et al. 2009, Vossmerbaeumer et al. 2008). The concentration of VIP used for differentiation towards RPE phenotypes is in the range of $10^{-6}-10^{-3} \mathrm{M}$. Similarly NIC has been reported to induce differentiation in induced pluripotent stem cells and embryonic stem cells towards RPE phenotype (Buchholz et al. 2013, Buchholz et al. 2009, Idelson et al. 2009a). NIC also inhibits NADdependent histone deacetylase (Peled et al. 2012, Imai et al. 2000), which play important role in differential gene expression and silencing, genome stability, signal transduction, cell growth and cell death. ATRA plays important role in stem cell differentiation by altering epigenetic changes in DNA as well as histone protein (Gudas 2013). ATRA has also been reported to induce epithelial phenotypes in MSCs (Wan et al. 2012, Li et al. 2012a, Brzoska et al. 2005). ATRA regulates the expression profile of stem cell by interacting with nuclear receptors RARs and RXRs. Both NIC and ATRA are probably involved in initiating differentiation process while VIP is involved in both differentiation and maturation process based on the finding that VIP plays important roles in the maturation of developing RPE (Koh 2000).

The conditions of differentiation in this setup are very complex due to numerous player molecules present in the growth medium secreted by cells and the extrinsic factors used. Although it is really difficult to explain the exact roles of each factor in the differentiation 
process, results show that multiple molecules and factors complement each other to initiate the process of differentiation signaling in the induced hAD-MSCs towards RPE phenotypes and help in further maturation of their phenotypes.

The most relevant findings from this part are as follows:

The hAD-MSCs partially differentiate to RPE phenotypes when they are induced by RCM with factors and their combinations (VIP, VIP+NIC, NIC+ATRA, and $\mathrm{VIP}+\mathrm{NIC}+\mathrm{ATRA}$ ) in the presence of human neuroretinal explants under indirect coculture.

- VIP, NIC and ATRA show significant effects in enhancing RPE characteristics in hAD-MSCs in the presence of degenerating neuroretinal explants.

- The combinations (VIP+NIC, NIC+ATRA, and VIP+NIC+ATRA) have been found to potentiate the differentiation capacity of each other significantly.

\section{Differentiation of hAD-MSCs into RPE like cells}

Several methods (five) have been used in this study for differentiating hAD-MSCs to RPE like cells. Among all other methods used, the direct co-culture method appears more robust based on the results obtained. This clearly defines the crucial role of cell to cell contact response (signaling) or cell to ECM signaling apart from roles of exogenous molecules or factors in the differentiation process. Further studies need to be done to elucidate the detailed mechanism of induction of all the factors used. The hAD-MSCs have been differentiated to RPE phenotypes in each experiment at different extent when they are grown with RPE cells or with the medium conditioned by them. This result is in line with other previous report of differentiation of MSCs towards RPE phenotypes (Vossmerbaeumer et al. 2009, Huang et al. 2012a). As it is described previously, all the factors used in this study have pleiotropic roles in various differentiation processes from stem cells. ATRA potentiates the epithelial characteristics of differentiating hAD-MSCs towards RPE. In this study, VIP has been inconsistently shown to produce variable results in different module of experiments and NIC alone does not show much significant effects over the differentiation process. In all the cases of differentiation methods used here, the combinations are found to potentiate the differentiation inducing capacity of each other and fetched better results than those of individual effects. The presence of neuroretinal explants is found to enhance the differentiating capacity of factors slightly. 


\section{Status of neuroretinal explants cultivated with hAD-MSCs along with factors (VIP, NIC and ATRA) and their combinations}

The hAD-MSCs have been found to induce RPE phenotypes by growing them in the bottom of the well with hNR explants over the trans-well in 50\% RCM supplemented with factors (VIP, NIC, and ATRA) and their combinations (VIP+NIC, VIP+ATRA, NIC+ATRA, and VIP+NIC+ATRA). The hNR explants from each conditions of the same setup have been studied to assess their health status by observing the general structure (toluidine blue staining) and specific structure (immunostaining against different specific retinal proteins) of neuro-retinal layers. Toluidine blue staining is used to assess the general global retinal tissue structure while specific immunostaining of freshly embedded retina was used to see the specific component of the neuroretina. Calbindin is a calcium-binding protein that marks cone photoreceptor, horizontal cells, bipolar cells, and amacrine cells. Synaptophysin is a synaptic-vesicle protein present in axon terminals of cone pedicles and rod spherules and in presynaptic vesicles of second order neurons. Rhodopsin is an opsin protein present in rod photoreceptors. CRALBP is retinaldehyde binding protein present in RPE and müller cells. Glial fibrillary acidic protein (GFAP) is an intermediate filament protein present in glial cells (Fernandez-Bueno et al. 2012).

The method developed in this study could be very useful to check the neuroprotective effects of biological (including cells) or chemical substances by incubating them with neuroretinal explants co-culture with RPE cells. This method is developed by extending the method previously developed in the lab for organotypic culture of neuroretina (Fernandez-Bueno et al. 2012, Fernandez-Bueno et al. 2013, Fernandez-Bueno et al. 2008).

The results clearly demonstrate that hAD-MSCs preserved the neuroretinal explants better than that of control-1 (50\% RCM only). In case of VIP, pathological events such as müller cell hypertrophy and reactive astrogliosis were observed by intense GFAP staining. This event could be explained by the finding that VIP is involved in inducing reactive astrocytosis in the CNS (Nishimoto et al. 2011). Although all the factors (VIP, NIC, and ATRA) used have been reported to show neuroprotective effects but they did not show any significant preservation than that of hAD-MSCs alone. In one of the samples of hNR explants of VIP+NIC there are lots of still intact OS of PRs that really gives a potent evidence of neuroprotection of VIP+NIC combination with hAD-MSCs. The combinations of VIP+NIC and VIP+NIC+ATRA have demonstrated significant preservations than other treatments. This confirms again that the neuroprotective roles of each factor may be complementing to each other to produce synergistic effects together. 
All of the factors used for differentiation have also been reported to have neuroprotective roles in various studies. VIP has been considered to have established roles in neuroprotection in vivo and in vitro. The in vivo neuroprotective effect of VIP has been found to be mediated by BDNF (Rangon et al. 2006). VIP has also been found a potent secretagogue that promotes release of astroglia-derived factors (chemokines) such as IL-1, IL-6, NT-3, protease nexin-1 (PN-1), RANTES, macrophage inflammatory protein-1 (MIP1), activity dependent neuroprotective factor (ADNF) and activity dependent neuroprotective protein (ADNP) (Dejda et al. 2005). The VIP receptors (VPAC-1, VPAC-2 and PAC-1) have been reported in neuron and glial cells (Masmoudi-Kouki et al. 2007). The expression of VIP receptor PAC-1 has been shown to increase in the pathological states such as in ischemic injury (Shioda et al. 2006). VIP has been shown to effectively protect the retina at nanomolar concentration in Wistar rat under ischemic retinal

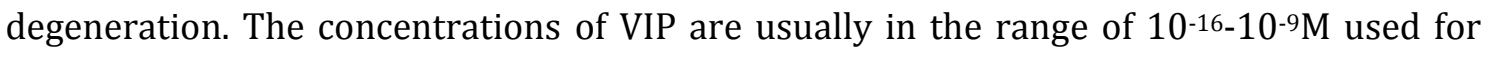
neuroprotection in different studies. The concentration used in our experiments including its previous use in differentiation by others was at micro molar level $\left(5 \times 10^{-6}\right)$, which is higher dose than that used previously for neuroprotection. Nevertheless, in our case, VIP alone in combination with hAD-MSCs has not been found effective to preserve the neuroretinal explants significantly. It was found to worsen the status of neuroretina in few cases.

Similarly, NIC plays several roles in inhibiting cell death and having neuroprotective functions. NIC acts as an inhibitor of the enzyme poly (ADP-ribose) polymerase (PARP), which plays important role in DNA repair in cell death and retinal cell death as well (Paquet-Durand et al. 2007, Uehara et al. 2006, Surjana et al. 2010a). NIC manifests several effects on cell culture, including PARP inhibition, which can protect cells from oxidative stress (Idelson et al. 2009a, Zahabi et al. 2012a). The damage in retinal explants is spread all over the retina in all the layers at different extent. PARP activation plays important role in certain apoptotic pathway (Virag 2005, Moncada, Bolanos 2006) and its upregulation has been suggested to involve in certain type of retinal cell death (Ji et al. 2008). Activation of PARP is dependent upon $\mathrm{NAD}^{+}$and during process of poly-ADP ribosylation, PARP dependent mechanism is activated and leading to the apoptosis (Yu et al. 2006b, PaquetDurand et al. 2007). NIC may be involved in inhibiting cell death or reducing retinal damage in culture by inhibiting PARP, modulating the NAD+ level in the cells (SadanagaAkiyoshi et al. 2003, Gale 1996, Ayoub et al. 1999, Sakakibara et al. 2002, Uehara et al. 2006), reducing ATP depletion (Ayoub et al. 1999), inhibiting lipid peroxidation (Klaidman et al. 1996, Klaidman et al. 2001, Lindner et al. 1998) or reducing inflammation (Ungerstedt et al. 2003a, Ungerstedt et al. 2003b). These multiple effects of NIC may be 
due to being essential substrate for NADH and NADPH. In our case, NIC does not manifest the significant neuroprotective effects over the neuroretinal explants in comparison to the neuroretinal explants with hAD-MSCs alone (control-2).

Similarly, ATRA is also involved in neuroprotection in vivo as well as in vitro (Ahlemeyer et al. 2000, Cheung et al. 2009, Li et al. 2008, Mey 2006, Sato et al. 2008). It has been shown that ATRA reduces oxidative stress in embryonic neurons by increasing the activity of superoxide dismutase, catalase and glutathione reductase (Ahlemeyer et al. 2001). ATRA has been reported to potentiate the neuroprotective effects of NGF (Ahlemeyer et al. 2000). ATRA has been reported to have potent anti-apoptotic effect in spontaneous eosinophil death (Ueki et al. 2008). In our case ATRA did not show any significant neuroprotective effects over the neuroretinal explants in comparison to the neuroretinal explants with hAD-MSCs alone.

The neuroprotective role of MSCs is well established in various studies which is mediated by the numerous neuroprotective factors secreted (paracrine factors) by them. The neuroprotective effects of VIP, NIC, and ATRA on hNR explants might be covered by the neuroprotective effects of hAD-MSCs and does not seem to be prominent but in the case of combinations of VIP+NIC and VIP+NIC+ATRA each factor appears to potentiate the neuroprotective effects of each other.

The most relevant findings from this part are as follows:

$>$ The hAD-MSCs with RCM delay the degeneration of hNR explants significantly under in vitro condition and the neuroprotective effects are greatly enhanced by the presence of two combinations of factors e.g. VIP+NIC and VIP+NIC+ATRA.

- The hAD-MSCs clearly show their neuroprotective effects over the degenerating neuroretinal explants.

- The combination VIP+NIC along with hAD-MSCs and RCM enhances preservation of hNR explants (or delay in degeneration of hNR explants) significantly.

- The combination of VIP+NIC+ATRA shows similar neuroprotective effects as that of VIP+NIC.

- This indirect co-culture of neuroretinal tissue explants with cells (RPE cells and hAD-MSCs) has been established well in this study, which could be a novel method to check the effect of cells or other biological entities on the degeneration of neuroretinal explants and RPE under in vitro condition. 
Conclusions and Future perspectives 


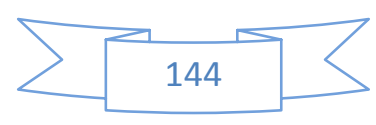




\section{Conclusions}

1. This study concludes that the hAD-MSCs in combination with the factors of differentiation (VIP, NIC and ATRA) along with molecules secreted by the RPE cells not only can partially delay or rescue the degeneration of RPE and neural retina but also can induce the differentiation in hAD-MSCs to RPE phenotypes and hence could replace the diseased and degenerated RPE cells to some extent.

2. RPE cells and hAD-MSCs maintain their growth, viability and phenotypes well in cell culture under proper methods of cell cultivation, however there are gradual loss in these properties of RPE cells during sub-culturing. Different protocols are established for routine testing of different parameters of RPE cells.

3. The differentiation in hAD-MSCs is more effective in direct co-culture with RPE cells than that of indirect co-culture suggesting that cell to cell contacts play very important role in differentiation process of hAD-MSCs to RPE phenotypes.

4. The environments created by the factors (VIP, NIC and ATRA) and their combinations along with RCM not only stimulate the differentiation of hADMSCs to RPE phenotypes but also preserve the neuroretinal explants to some extents under in vitro culture condition.

5. The combination of factors (especially VIP+NIC) is more effective to rescue the degenerating RPE cells and neuroretinal explants as well as to stimulate the differentiation of hAD-MSCs to RPE phenotypes than that of individual factors. 


\section{Future perspectives}

This work has been performed in an applied institute, therefore future steps should be directed towards applications (direct benefits) to the patients, especially to those affected with retinal degenerative diseases. This study investigates the response and effects of hAD-MSCs in different environments constituted by three differentiating factors (VIP, NIC and ATRA) individually or their different combinations along with the molecules secreted by RPE cells under in vitro conditions. At the beginning of the work, a protocol was established to check the purity of RPE cells, which could be a valuable methodological tool in good manufacturing practices for transplantation of fresh or differentiated RPE cells in retinal disease. At the same time, methods were established to test different biocompatible polymers for the maintenance of RPE cells, which could be used to explore new biocompatible polymers which can maintain the differentiated state of RPE like cells derived from any stem cells. This could be a very important step in the development of RPE cells-substrate complex or stem cells-substrate complex, which could be used in RPEsheet subretinal transplantation in case of dry form of AMD or other retinal degenerative diseases. This application of the tissue engineering has been a challenge for researches during the last 10 years and it is still unsolved.

This study finds that hAD-MSCs rescue the degenerating PRE cells under in vitro condition through the secretion of some molecules. The next step of this work could be to find the out the exact molecule(s), which exert(s) the anti-apoptotic effects over the dying and degenerating RPE cells. Further it was also found that hAD-MSCs differentiate to RPE like cells when directly cultivated over the inactivated RPE cells. It shows that hAD-MSCs takes some cues from inactivate degenerating RPE to replace them. Because the MSCs are considered to migrate or home to the damage site and try to repair the damage either by replacing the damaged cells or secreting some growth factors or by both ways. Thinking about this application, the site of injection of these cells became a critical point because the existence of the inner limiting membrane prevents stem cells to migrate from the vitreous cavity to the retinal tissue. All the results over hAD-MSCs obtained in this study advocates the candidature of hAD-MSCs alone or hAD-MSCs pretreated with the combination of factors for their subretinal injection in retinal degenerative disease. The study also finds that under influence of some combinations of different factors with RPE secreted molecules, hAD-MSCs not only have capacity of differentiation towards RPE like cells but also protect the degenerating retina under in vitro conditions. These capabilities, that should be verified, could represent a new approach to some retinal diseases. This is a very interesting finding, which can be used for cell therapy against retinal disease in near 
future. Next logical step could be to study the response and effects of hAD-MSCs in an animal model of retinal degenerative diseases.

There are a large number of animal models such as rodents (mice and rats), rabbits, pig and non-human primates (macaques) developed for AMD but none of them recapitulates all of the features of human AMD. While rodent models have offered the advantage of relative cost-effectiveness, accelerated time scale, and ease of genetic manipulation, they are still limited by the size and the anatomical lack of macula. Rabbit eye is considerably larger than rat eye and is more akin to human eye in size but retina is supplied by a superior central ray of vessels and there is no macula in it. There are non-human primates eye, which are substantially identical to the human eye but they are very costly to maintain, have a slow time course for disease development and are difficult to manipulate genetically. The pig eye is, of the non-primate model, probably the most similar to human eye in size and due to the presence of an area of increased cone density arranged in a central horizontal band analogous to the human macula. Due to the anatomical qualities and intermediate cost, rabbit and pig eyes could be considered as an animal model to test the product of this work in future.

This study also established the method of co-culture of cell and neuroretinal explants in co-culture condition. This is an extension of the organotypic culture of neuroretinal explants developed previously by our colleagues (Fernandez-Bueno et al. 2008). Although in these experiments the system have both RPE cells (on the bottom of well) and neuroretinal explants (over the trans-membrane) under degeneration process, the cellular modifications especially at the inner retina under in vitro condition could differ from in vivo conditions. This retinal degeneration may be due to axotomy of optic nerve fibres, absence of retinal and choroidal blood supply and absence of RPE layer, that induce acute ischemia in neuroretinal tissue (Fernandez-Bueno et al. 2012). Nevertheless, this in vitro model can reproduce some of the cellular changes revealed in experimental retinal degeneration processes and could be similar to some extent to the in vivo pathological subretinal environment of AMD where both neuroretina and RPE are under degeneration. This in vitro environment of cell-organotypic co-culture system in trans-well plates could be a good economic tool in testing the effect of different molecules over the neuroretina as well as on RPE cells for their neuroprotective and anti-apoptotic effects.

About $3.4 \%$ of Spanish elderly population ( $>65$ years) is suffering from the AMD and this percentage is going to increase in the coming decade due to increase in old age population. This work is largely contributed by the Spanish tax payers, which in return it gives bright prospects in terms of increasing their quality of life by providing ray of hope in stem cell therapy against a non-treatable form of AMD. This work offers a preliminary solution as a concoction of hAD-MSCs and combination of factors, which could in future be used to stop the AMD progression or other retinal degenerative diseases. 


\section{Spanish Summary}




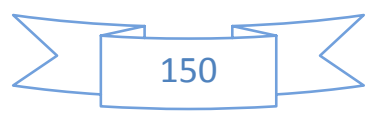


La degeneración macular asociada a la edad (DMAE) supondrá una amenaza de ceguera para un porcentaje importante de las personas de edad avanzada en todo el mundo durante las próximas décadas. Actualmente se reconocen dos formas básicas de DMAE: seca y humeda, la forma seca de la DMAE constituye alrededor del $60 \%$ al $90 \%$ de la población total con DMAE. La forma seca, posee un tamaño de mercado de unos 25-30 mil millones de dólares en EEUU y Europa, y representa un fuerte impacto negativo, cuantificado en alrededor de 30 mil millones de dólares anuales, sobre el producto interior bruto (PIB) estadounidense. (Brown 2010). El envejecimiento de la población en los países industrializados durante las próximas décadas, supondrá una carga adicional para la seguridad social, debido al consecuente incremento de los problemas de salud y de socioeconómicos que conlleva dicho envejecimiento. Por lo tanto, la DMAE constituye un importante problema de salud pública que puede tener un efecto devastador en la calidad de vida de los pacientes y que ya está originando a consecuencias económicas negativas en los países industrializados. Además, existen otras enfermedades degenerativas de la retina como la retinitis pigmentaria, la enfermedad de Stargardt, que también tienen un impacto negativo en la calidad de vida de la población mundial.

Las actuales opciones terapéuticas para la DMAE han mostrado un modesto éxito, principalmente en el tratamiento de la forma húmeda. En el caso de la DMAE seca, no existe en la actualidad ninguna opción de tratamiento disponible.

El epitelio pigmentario de la retina (EPR) juega un papel extremadamente importante en la función visual normal, así como en la preservación de la integridad estructural de la retina. El EPR está considerado como uno de los principales implicados en la patogénesis de la DMAE, debido a los cambios estructurales y bioquímicos que sufren estas células a lo largo de la vida. Todos estos cambios perjudiciales en el EPR afectan progresivamente al estado normal de la retina, de modo que la retina neural principalmente los fotorreceptores (PRs), comienzan a degenerar progresivamente y de forma simultánea con el mal funcionamiento del EPR.

Parece lógico pensar que si en esta enfermedad, el EPR dañado fuera reemplazado por EPR sano, sobre todo en fases tempranas de la DMAE cuando los PRs aún mantienen su funcionalidad, podría obtenerse un gran beneficio en forma de ralentización o prevención de la deterioro de PRs. Existen varios trabajos donde diferentes grupos de investigación han intentado trasplantar EPR y EPI (epitelio pigmentario del iris) utilizando modelos animales, a partir de diferentes fuentes, tanto autólogas, como homólogas o 
heterólogas. Se ha observado que las fuentes homólogas y heterólogas provocan un fuerte rechazo inmunológico, sin embargo, las fuentes autólogas no presentan este problema. Y aunque las fuentes autólogas muestran una gran tolerancia inmunológica, no producen ningún cambio significativo en las funciones visuales, debido a que estas células poseen la misma huella genética y ambiental que las del EPR dañado.

Existen tres estructuras importantes que se ven afectadas muy gravemente en la DMAE: la membrana de Bruch (MB), el EPR y la retina neural, especialmente los PRs. Las terapias avanzadas pretenden alcanzar un diseño conjunto donde se integren ambas partes, la celular/tisular (EPR y retina neural), y la no celular (un sustrato que pueda imitar la MB). Es bien conocida la capacidad de auto-renovación perpetua que poseen las células madre y su capacidad de diferenciación hacia cualquier tipo celular madura o tejido en presencia de las señales apropiadas. Así, las células madre se presentan como una posible fuente de la parte celular/tisular, es decir del EPR o de los PRs e incluso de la retina completa. Así pues Las células madre suponen una enorme esperanza en la generación de distintos tipos celulares retinianos. Existen importantes avances en la obtención de EPR fresco y funcional e incluso PRs, a partir de células madre de origen embrionario (ESCs) y de células madre pluripotentes inducidas (iPSCs). Existen varios ensayos clínicos, en fase de reclutamiento en la actualidad, para el trasplante de EPR derivado de ESCs en pacientes con enfermedades degenerativas de la retina (NCT01345006, NCT01344993, NCT01469832, NCT01625559 y NCT01691261). El Riken Centre for Developmental Biology en Japón, ha lanzado un estudio piloto (Masayo Takahashi) para evaluar la seguridad y la viabilidad del trasplante de capas de células de EPR derivadas de iPSC en pacientes con DMAE exudativa, en Agosto de 2013 (Sipp, Takahashi 2013). La utilización de las ESCs en investigación está obviamente obstaculizada debido tanto a la existencia de problemas éticos que supone como a la posibilidad de teratogénesis, y en el caso de las iPSCs, por problema de seguridad, y el riesgo de formación de tumores que son las principales preocupaciones a tener en cuenta.

En el caso de la utilización de células madre de origen adulto, como las MSCs, dichos problemas se reducen al mínimo. Aunque existen diversas fuentes para la obtención de las MSCs, las dos principales son la médula ósea (BM) y el tejido adiposo (AD) (lipoaspirado). A partir de los detalles descritos posteriormente en la "Introducción", se deduce que el tejido adiposo muestra varias ventajas respecto a la médula ósea como fuente de MSCs, en lo concerniente a su facilidad para el cultivo y la disponibilidad de un gran número de células, sin que exista ninguna otra diferencia significativa entre las BMMSCs y las AD-MSCs. Las AD-MSCs han demostrado su capacidad de diferenciación hacia células de origen neuroectodérmico (Zuk et al. 2001, Zuk et al. 2002) y más recientemente, 
hacia células con fenotipo de células del EPR y células progenitoras de la retina (Vossmerbaeumer y col. 2009a, Huang et al. 2012a, Moviglia et al. 2012a). En varias publicaciones, se ha descrito, la capacidad de las MSCs para rescatar PRs en degeneración y para mejorar las funciones visuales utilizando modelos animales. La prueba de concepto ha sido ya establecida utilizando el trasplante autólogo intravitreo de células mononucleares derivadas de médula ósea (Jonas et al. 2010, Jonas et al. 2008, Siqueira et al. 2011). Uno de los estudios realizados por el Grupo de Retina del IOBA, se ha comprobado in vitro como las hAD-MSCs pueden el rescatar las células del EPR sometidas a condiciones de muerte celular (Singh et al. 2013a). El uso de MSCs en enfermedades degenerativas de la retina no sólo se limita a su diferenciación hacia células del EPR o PRs, sino que se extiende al posible efecto beneficioso derivado de los factores neurotróficos, inmunomoduladores y antiinflamatorios que secretan. Existen varios factores de crecimiento que desempeñan un papel importante en el mantenimiento de la neurorretina así como del EPR, entre los que se incluyen el PEDF, el BDNF, el VEGF, el CNTF, el HGF, el NGF, el LIF, etc. Se ha descrito que dichos factores son secretados por las MSCs incluso después de su diferenciación parcial. En recientes ensayos clínicos (NCT00062765, NCT00447954, NCT00447980, NCT00447993, NCT01530659) las propiedades paracrinas de células recombinantes del EPR (NTC-201 humanas derivadas de la línea celular ARPE19) se han utilizado en un implante intraocular de células encapsuladas (ECT) en enfermedades degenerativas de la retina (RP y GA-Atrofia geográfica) por Neurotech (Sieving et al. 2006, Kauper et al. 2012, Thanos et al. 2004, Zhang et al. 2011). En este implante ECT, el uso de MSCs recombinantes supone una ventaja sobre las células del EPR. De esta forma, la terapia celular con MSCs ofrece una gran oportunidad en la búsqueda de nuevos tratamientos de la DMAE.

Finalmente, en este trabajo se propone la utilización del potencial de diferenciación hacia células con fenotipo EPR que poseen las hAD-MSCs, simultáneamente con sus beneficiosos efectos paracrinos sobre el EPR y la degeneración retiniana in vitro. Este estudio intenta crear un microambiente compuesto por diferentes biomoléculas con la capacidad de guiar la vía de diferenciación de las MSCs hacia fenotipos de EPR y al mismo tiempo, de proteger la neurorretina (especialmente los PRs), mediante la utilización de sistemas de co-cultivo indirectos. Los resultados derivados podrían proporcionar importantes avances en campos que permitirían continuar en la línea de investigación hacia experimentos in vivo antes de iniciar futuros ensayos clínicos en la DMAE. 


\section{Introducción}

\section{Degeneración macular asociada a la edad (DMAE)}

1.1. Definición: La DMAE es una enfermedad ocular en personas mayores de 50 años en las que la mácula, área de la retina requerida para la visión aguda y detallada, empieza a deteriorarse progresivamente con el paso del tiempo. Los síntomas en el desarrollo de la DMAE comienzan con desenfoque leve o distorsión, seguido de la pérdida de la visión central de forma irreversible, pero manteniendo una visión periférica (lateral). Los casos bilaterales con una agudeza visual de 20/200 o menor son considerados como ceguera legal, y los afectados no pueden realizar actividades como conducir, leer, reconocer las caras, etc.

1.2. Prevalencia: Actualmente la DMAE, que fue descrita por primera vez hace 85 años, (Holloway, Verhoeff 1928), es la principal causa de pérdida irreversible de la visión entre las personas mayores de 50 años en países industrializados (Ratnapriya, Chew 2013, Pascolini et al. 2004, Ambati et al. 2003, Ambati, Fowler 2012) y está aumentando de manera exponencial (Rein et al. 2009, Cheung, Eaton 2013). La prevalencia de la enfermedad aumenta con la edad (Klein et al. 2010, Klein et al. 2004, Klein et al. 2007, Friedman et al. 2004), y depende de la raza y de la etnicidad de la población (composición genética de la población) (Klein et al. 2006). Alrededor del 3,4 \% de la población anciana española de más de 65 años sufre DMAE. La DMAE es la principal causa de ceguera en los países desarrollados y la tercera en todo el mundo después de la catarata y el glaucoma, constituyendo el 8-7\% de los casos de ceguera legal en todo el mundo (Resnikoff et al. 2004).

1.3. Formas: Hay dos formas de DMAE: seca, también conocida como atrófica, y húmeda, también conocida como exudativa (Vélez-Montoya et al 2012). La forma seca es más frecuente (60-90\%) y se asocia su frecuencia a pequeños depósitos amarillos denominados drusas. La forma más severa y avanzada de la forma seca de la DMAE también se denomina atrofia geográfica (Mata, Vogel 2010). La forma húmeda es menos frecuente (10-40\%) pero más agresiva.

1.4. Patogenia: A pesar de los recientes avances en investigación clínica, la patogénesis de la DMAE aún es poco conocida. Se basa en una complicada interacción de factores genéticos, medioambientales, metabólicos y funcionales (Nowak 2006). Los factores de riesgo para el desarrollo de la enfermedad como la edad avanzada, el tabaquismo, la ausencia de una 
dieta equilibrada y la obesidad, parecen estar relacionados con el aumento del estrés oxidativo (Klein et al. 2007, Klein et al. 2010), causando un daño progresivo en las células del epitelio pigmentario de la retina (EPR) y afectando a la integridad funcional de la membrana de Bruch (BM), situada debajo del EPR (Beatty et al. 2000, Cai et al. 2000, Boulton et al. 2004b, Liang, Godley 2003). Este daño desencadena directamente la atrofia progresiva de la retina que conduce al desarrollo de enfermedades debilitantes de la misma, como la DMAE (Mata, Vogel 2010, Munoz et al. 2000). El modelo de la patogénesis de la DMAE, basado en observaciones clínicas, patológicas y experimentales, se muestra en la Figura 1 (página 15).

Varios factores de crecimiento contribuyen al desarrollo de la DMAE. En la forma húmeda, parece que un desequilibrio entre los factores pro-angiogénicos y anti-angiogénicas produce neovascularización coroidea (CNV) (Gao et al. 2001, Tong, Yao 2006). Principalmente existen dos factores importantes en la patogénia de la DMAE, el factor de crecimiento del endotelio vascular A (VEGF-A) y el factor derivado del epitelio pigmentario (PEDF). El VEGF-A es un mitógeno, así como un factor quimiotáctico que promueve la supervivencia (inhibe la apoptosis) de las células endoteliales y gliales, mejora la permeabilidad vascular y, por lo tanto, juega un papel muy importante en la angiogénesis fisiológica y patológica (Ferrara et al. 2003, Alon et al. 1995). Bajo condiciones de normoxia, la secreción del VEGF por parte del RPE hacia la parte basal (coriocapilar) es de 2 a 7 veces mayor que en el lado apical (fotoreceptores) (Blaauwgeers et al. 1999). Esta disparidad se exacerba bajo condiciones de hipoxia, así los productos finales de glicación avanzada y los productos intermedios de oxígeno reactivo conducen a la CNV patológica (Lu et al. 1998, Kuroki et al. 1996, Beerepoot et al. 1996, Shima et al. 1995). El PEDF es un factor anti-angiogénico secretado por la parte apical del EPR y su papel es estabilizar la neurorretina mediante la inhibición de la apoptosis y reducir la angiogénesis patológica en la DMAE (Ogata et al. 2001, Cayouette et al. 1999, Cai et al. 2006, Becerra et al. 2004, Dawson 1999). In vitro e in vivo, los resultados muestran que el EPR senescente disminuye drásticamente la secreción de PEDF (Tombran-Tink et al. 1995, Holekamp et al. 2002, Matsunaga et al. 1999). En individuos sanos los factores proangiogénicos y anti-angiogénicos se encuentran en equilibrio, pero este equilibrio se altera en la DMAE, conduciendo a la producción en masa de VEGF y, por lo tanto, a la CNV (Mousa, Mousa 2010, Velez-Montoya et al. 2012, Velez-Montoya et al. 2010, Tong, Yao 2006, Gao et al. 2001). La patogénesis de la forma seca es mucho menos conocida, pero probablemente estén implicados los mismos factores actuando sobre distintos sustratos. 
Varios genes están involucrados directa e indirectamente en la patogénesis de las dos formas de la DMAE. Al menos el 25\% de los pacientes afectados presentan una predisposición genética (Recalde et al. 2008, Garcia Layana et al. 2011, Meyers et al. 1995). Los polimorfismos de un solo nucleótido (SNPs) y sus variaciones alélicas que están ligadas a la DMAE (Gold et al. 2006).

En la actualidad hay algunos tratamientos efectivos contra la forma húmeda de la DMAE basados en anti-angiogénicos tales como: aflibercept, pegaptanib, ranibizumab, bevacizumab, etc. Por el contrario, hasta la fecha no existe ningún tratamiento eficaz contra la forma seca, excepto el uso de algunas medidas preventivas antes del comienzo de los síntomas de la enfermedad, como dejar de fumar y el uso de suplementos minerales, vitaminas antioxidantes y ácidos $\omega$-3 de cadena larga poliinsaturados en la dieta.

2. Retina: La retina es un tejido foto-sensorial de múltiples capas situado en la parte posterior del ojo que contiene varios tipos de neuronas interconectadas por sinapsis (Fig. 2 página18). Se puede dividir en dos partes, la parte neuronal (9 capas) y la parte no neuronal (EPR).

2.1. Neurorretina: Se compone de dos tipos de células fotosensoriales (fotorreceptores) denominados conos y bastones. Los fotorreceptores (PRs) tienen dos partes en el espacio subretiniano: el segmento externo (POS) anexo a las microvellosidades del EPR, y el segmento interno (IS). Los bastones son más abundantes en la retina periférica y son responsables del movimiento y luz de la visión. Por el contrario, los conos son más abundantes en la retina central y son responsables de la visión fina, su detalle y en color. Además de los PRs, algunas células ganglionares son también fotosensibles (Provencio et al. 2000, Web Science News por Carl Zimmer 2012). La retina tiene tres capas con cuerpos celulares, denominadas, capa nuclear externa (ONL) que contiene los cuerpos celulares de los conos y bastones, capa nuclear interna (INL) que contiene los cuerpos celulares de las células bipolares, células horizontales y amacrinas, y capa de células ganglionares (GCL) que contiene los somas de las células ganglionares y las células amacrinas desplazadas (Fig. 2 página 18). Entre ellas se encuentran dos capas sinápticas, que contienen las sinapsis entre neuronas, la primera se denomina llama capa plexiforme externa (OPL), donde los conos y los bastones, las células bipolares y horizontales hacen sinapsis y la segunda se llama la capa plexiforme interna (IPL), donde las células bipolares y algunas células amacrinas conectan con las células ganglionares (Fig. 2 página 18). Durante el proceso de la visión, la luz tiene que atravesar todo el espesor de la retina hasta llegar a los PRs, donde la luz es absorbida por los pigmentos visuales y convertida en una señal 
eléctrica que viaja desde los PRs a las células bipolares y de las bipolares a las células ganglionares, las cuales trasmiten finalmente la señal a la corteza visual del cerebro a través del nervio óptico.

2.2. Epitelio pigmentario de la retina (EPR): El EPR es la parte más externa de la retina, y está localizado entre los PRs y la coroides, como se muestra en el diagrama (Fig. 2 página18). Esta monocapa celular está compuesta por células epiteliales hexagonales, pigmentadas y altamente especializadas. El EPR tiene una morfología polarizada con gran densidad de microvellosidades apicales pigmentadas, que se acoplan a los PRs (Fig. 2 página 18). En la zona basolateral presentan repliegues hacia la capa coriocapilar que ayudan en la adhesión del EPR con la membrana de Bruch (BM) y que facilita el transporte molecular entre el EPR y la circulación sanguínea. Una célula del EPR está conectada a unos 20 PRs (Sparrow et al. 2010). Las células del EPR están interconectadas a través de uniones estrechas (zonula occludens; ZOs) haciendo a esta lámina impermeable a cualquier macromolécula, y por lo tanto ayudando en el mantenimiento del privilegio inmune del ojo (Rizzolo et al. 2011, Rizzolo 2007). El EPR desempeña varios papeles cruciales que son indispensables para el mantenimiento de la estructura, la función y la supervivencia de la retina neural, de la coriocapilar y del endotelio (Boulton, DayhawBarker 2001, Strauss 2009, Strauss 2005). El EPR contiene pigmentos, como la melanina entre otros, que se encargan de absorber la luz extra dispersada para mejorar la resolución, así como para proteger la retina contra la foto-oxidación. El EPR es el encargado de mantener la excitabilidad de los PRs mediante la digestión de los POS, e incorporando el todo-trans retinol, para su conversión a 11-cis retinal y su transporte hasta los PRs (Strauss et al. 1998, Nandrot et al. 2012, Finnemann 2003, Gal et al. 2000, Karl et al. 2008, Kevany, Palczewski 2010). También tiene la función de transporte de nutrientes desde la sangre a los PRs (Ban, Rizzolo 2000, Bazan et al. 1994), e iones, agua y productos finales, desde el espacio subretiniano a la sangre, manteniendo la homeostasis química en el espacio subretiniano y el estado funcional de los PRs. Además, es el responsable de la secreción de citoquinas, factores de crecimiento y moduladores inmunes, responsables de mantener el privilegio inmune en el interior del ojo (Kehrl et al. 1986a, Kehrl et al. 1986b, Ishida et al. 2003, Streilein et al. 2002, Streilein 2003) y de estabilizar los PRs, la coroides y las células endoteliales (Holtkamp et al. 2001, Holtkamp et al. 1998, Bharti et al. 2006, Matsui et al. 2001, Campochiaro et al. 1996, Dawson 1999, Ogata et al. 2001).

2.3. Envejecimiento fisiológico del EPR: El EPR humano es una capa de células diferenciadas que no se dividen y que con la edad acumulan daños, debidos a diversas agresiones 
ambientales (Beatty et al. 2000, Cai et al. 2000, Boulton, Dayhaw-Barker 2001, Boulton et al. 2004b). Los cambios estructurales en el EPR incluyen pérdida de la forma (polaridad), hiperplasia, atrofia y disminución de la densidad celular. La acumulación de lipofuscina (marcador de senescencia del EPR) en los lisosomas celulares, a partir de la quinta década de la vida, origina la incapacidad por parte del EPR de llevar a cabo la digestión completa de los POS, debido al estrés oxidativo (Eldred, Katz 1988, Feeney-Burns et al. 1984, Feeney 1978, Sparrow, Boulton 2005, Weiter et al. 1986b, Kennedy et al. 1995, Boulton et al. 2004a, Boulton et al. 2004b). El aumento de los complejos de pigmento está asociado con la disminución en el número de melanosomas en el EPR a lo largo de la vida (FeeneyBurns et al. 1984, Weiter et al. 1986a, Boulton et al. 1990). El envejecimiento del EPR también se ha asociado con la aparición de drusas, depósitos patológicos extracelulares, amarillentos y redondeados, por debajo del EPR y en la BM, consideradas un signo de DMAE (Gass 1972, Abdelsalam et al. 1999, Mullins et al. 2000, Russell et al. 2000, Gass 2003, Buschini et al. 2011). Además de estos cambios, también se han observado disminución en los niveles de vitamina E, disminución de la fagocitosis, aumento de actividad de la catalasa debido al estrés oxidativo inducido por $\mathrm{H}_{2} \mathrm{O}_{2}$, pérdida de los telómeros, disminución en el contenido de melanina, alteración de la expresión génica, debido a la acumulación de mutaciones, deleciones y reorganizaciones en el ADN mitocondrial, e interacción alterada entre células y matriz extracelular (ECM) (Yu et al. 1997, Robert 1998, Wallace et al. 1999, Wassell et al. 1999, Holz et al. 1999, Ambati et al. 2003). Todos estos cambios relacionados con la edad, hacen perder la funcionalidad al EPR y promueven la muerte celular, al mismo tiempo también afectan negativamente a la $\mathrm{BM}, \mathrm{y}$, en conjunto, conducen a la patogénesis progresiva de la DMAE.

3. Células madre: En 1908, el término "célula madre" fue propuesto para uso científico por el histólogo ruso Alexander Maksimov. Las células madre son células presentes en todos los organismos multicelulares, que tienen la capacidad de auto-renovación perpetua y de diferenciarse para formar un tejido maduro en presencia de las señales apropiadas. Una célula madre produce, mediante división asimétrica, una célula madre y otra célula más diferenciada hasta llegar a las células terminales diferenciadas como se muestra en la figura 3 (página 19). Las células madre pueden clasificarse según su capacidad de diferenciación. a) Las células totipotentes/omnipotentes están presentes en el embrión hasta el estadío de 8 células o cigoto y pueden dar lugar a un embrión completo, por tanto, pueden formar nuevo individuo completo si se les proporciona apoyo materno adecuado. b) Las células pluripotentes están presentes en la masa celular interna del blastocisto o etapa de mórula y pueden diferenciarse en todos los tipos celulares de las 3 capas 
germinales embrionarias (ectodermo, mesodermo o endodermo). c) Las células multipotentes están presentes en todos los tejidos del organismo adulto, pudiendo generar cualquier tipo celular de la misma capa embrionaria de la que proceden. d) células madre oligopotentes pueden diferenciarse en sólo unas pocas células, tales como células madre mieloides y linfoides. e) Las células madre unipotentes pueden dar lugar a un solo tipo celular. f) Y por último, las células nulipotentes no tienen capacidad de diferenciación a ningún tipo celular, pero pueden auto-renovarse.

Las células madre también pueden clasificarse según su origen en tres categorías: a) células madre embrionarias (ESCs) derivadas de la masa celular interna del blastocisto de un embrión de etapa temprana; b) células madre adultas derivadas de cualquier parte del cuerpo; y c) células madre pluripotentes inducidas (iPSCs) obtenidas al transformar células somáticas por reprogramación genética. Las iPSCs son equivalentes a los ESCs en cuanto a su capacidad pluripotente.

3.1. Terapia celular: La terapia celular es considerada como el cuarto y más reciente pilar terapéutico emergente de atención sanitaria a nivel mundial (Mason, Dunnill 2008). La terapia celular se puede dividir en dos ramas: 1) terapia celular somática o terapia de reemplazo celular, en la que se utiliza cualquier célula somática sana diferenciada para reemplazar las células dañadas del cuerpo; y 2) terapia de células madre o medicina regenerativa, en la cual se emplea cualquier tipo de célula madre. Por lo general hay dos tipos principales de estrategias en la terapia celular: (1) la que se centra en la restauración de la estructura y la función de las células o tejidos específicos mediante el uso de células; y (2) la dirigida a restaurar la homeostasis funcional, mediante la capacidad trófica, inmunomoduladora u otra resultante de los efectos bioquímicos de las células trasplantadas. Sin embargo, ambas estrategias pueden ser llevadas a cabo conjuntamente en la misma terapia.

3.2. El progreso de terapia celular en oftalmología: Actualmente hay un creciente interés por el uso de terapia celular en la DMAE, que incluye del uso de células progenitoras de la retina, ESCs, iPSCs y células madre adultas. Teóricamente estas células podrían reemplazar las células del EPR y los PRs en pacientes con DMAE, y varios ensayos in vitro e in vivo han demostrado el éxito de estas terapias.

Una de las principales causas de la degeneración retiniana en la DMAE es la senescencia y el daño del EPR, por lo que la terapia celular se centra en la sustitución del EPR deteriorado por células sanas. Se ha demostrado el retraso en la degeneración de los PRs y la mejora de la función visual tras el trasplante del EPR en los modelos animales (Gouras 
et al. 1984, Li, Turner 1988, Gouras et al. 1989, Lane et al. 1989, Lopez et al. 1989, Sheedlo et al. 1989, Yamamoto et al. 1993, Sauve et al. 1998, Girman et al. 2003, Girman et al. 2005, Gias et al. 2007). Estos estudios finalmente condujeron a la realización de varios ensayos clínicos para el trasplante de EPR (Peyman et al. 1991, Algvere et al. 1994, Gouras, Algvere 1996, Algvere et al. 1997, Algvere et al. 1999, Weisz et al. 1999, Valtink et al. 1999, Del Priore et al. 2001), pero en casi todos los casos se observó un rechazo. Para evitar problemas de rechazo inmunológico, varios grupos estudiaron el trasplante de células adultas autólogas del EPR y del epitelio pigmentario del iris (EPI), con resultados prometedores en cuanto a efectos beneficiosos temporales (Thumann et al. 2000, Abe et al. 2000a, Abe et al. 2000b, Stanga et al. 2001, Binder et al. 2002, Stanga et al. 2002, van Meurs, Van Den Biesen 2003, Binder et al. 2004, Binder et al. 2007, Abe et al. 2007, Krebs et al. 2008, Ma et al. 2009, Caramoy et al. 2010). En el caso de la fuente autóloga, el EPR está igualmente afectado por los factores genéticos y ambientales en todo el individuo, por lo que la fuente autóloga está muy limitada. Algunos grupos también han realizado trasplantes de retina fetal completa, incluyendo el RPE, con éxito en modelos animales e incluso en ensayos en humanos, consiguiendo algunos resultados alentadores (Aramant et al. 1999, Aramant, Seiler 2002a, Aramant, Seiler 2002b, Radtke et al. 1999, Aramant, Seiler 2004, Radtke et al. 2008, Radtke et al. 2002, Seiler, Aramant 2012). Debido a las limitaciones en la obtención de tejido retiniano fresco, sobre todo si es de origen fetal, la terapia celular para el tratamiento de la DMAE está cambiando hacia el trasplante de células madre. La terapia con células madre ha sido estudiada en varios modelos animales de enfermedades degenerativas de la retina (Lund et al. 2001b, Lund et al. 2001a, Arnhold et al. 2006, Lund et al. 2007, Arnhold et al. 2007).

Las ESCs humanas han sido inducidas para su diferenciación hacia células de EPR por varios grupos de investigación, obteniendo células del EPR, células progenitoras de la retina y PRs funcionales (Haruta 2004, Klimanskaya et al. 2004, Lamba et al. 2006, Vugler et al. 2008, Idelson et al. 2009, Corneo, Temple 2009, Carr et al. 2009, Meyer et al. 2009, Clarke et al. 2012). Las células del EPR derivadas de las ESCs se han estudiado en animales de experimentación con resultados prometedores en el tratamiento de la retinitis pigmentosa (RP) y en la mejora de las funciones visuales (Lund et al. 2006, Vugler et al. 2007, Lu et al. 2009). Actualmente están registrados siete ensayos clínicos (consultado en clinicaltrials.gov a fecha 18 de noviembre de 2013 por el término de búsqueda "Embryonic stem cells + AMD"), en los cuales se están realizando trasplantes de células del EPR derivadas de ESCs, tres de ellos se están llevando a cabo en pacientes con DMAE (NCT01344993, NCT01674829 y NCT01691261 (Schwartz et al. 2012)). Algunos 
investigadores han estudiado las iPSCs para la obtención de células del EPR, de células progenitoras de la retina y PRs, con resultados prometedores (Bharti et al. 2011, Barbara 2009, Boucherie et al. 2011, Buchholz et al. 2013, Jin et al. 2009, John et al. 2013, Kuroda et al. 2012, Mekala et al. 2013, Kokkinaki et al. 2011, Lamba et al. 2010, Parameswaran et al. 2010, Meyer et al. 2011). Debido a los problemas éticos y teratogénicos asociados al uso de ESCs e iPSCs, las células madre adultas, como las células madre mesenquimales (MSCs), las cuales no poseen estos inconvenientes, y podrían ser una fuente prometedora para la obtención de un EPR sano.

4. Células madre de mesenquimales (MSCs): Las MSCs han sido definidas por la Sociedad Internacional de Terapia Celular (Horwitz et al. 2005): (i)por su capacidad de adherencia al plástico en condiciones de cultivo estándar; (ii) por expresar en su superficie los marcadores de células madre CD105 (endoglin), CD73 (5'-nucleotidase/ecto-5'nucleotidase-5'-NT) y CD90 (Thy1), y por la ausencia de expresión de marcadores de células hematopoyéticas CD34, CD45 (proteína tirosina fosfato de tipo receptor C-PTPRC), CD14, CD11b (Integrin alfa M-ITGAM ), CD19, CD79a y HLA-DR (molécula de receptor de superficie de MHC de clase II); (iii) y por su capacidad de diferenciación in vitro, bajo estímulos específicos, hacia osteocitos, adipocitos y condrocitos (Dominici et al. 2006, Horwitz et al. 2005, Gimble et al. 2007). Las MSCs también expresan otros marcadores típicos de células madre, como Oct4, Rex1 y Sox2 (Izadpanah et al. 2006). Las MSCs residen en el tejido conectivo de la mayoría de los órganos y tienen capacidad de migrar al sitio de la lesión, especialmente hacia áreas hipóxicas, apoptóticas e inflamadas (capacidad de homing), llevando a cabo su acción en dicho microambiente a través de la secreción de factores solubles.

4.1. Fuentes de MSCs: La mayoría de los órganos contienen un nicho de MSCs para mantener la homeostasis celular en el interior de los órganos (da Silva Meirelles Lindolfo et al. 2006). Las MSCs fueron aisladas por primera vez de la médula ósea de cobaya por Friedenstein y colaboradores en 1970 (Friedenstein et al. 1970). Hasta la fecha, se han aislado MSCs de forma exitosa de diversas fuentes, como la médula ósea, tejido adiposo (lipoaspirado), pulpa dental de los dientes deciduos, periostio, placenta, líquido amniótico, sangre del cordón umbilical, folículo piloso, músculo esquelético, membrana sinovial, cerebro, timo, hígado, bazo, páncreas, aorta, vena cava, riñones, glomérulos renales, pulmones, córnea, retina y cuerpo ciliar entre otros (In 't Anker et al. 2004, Bieback et al. 2004, Bieback, Brinkmann 2010, Kern et al. 2006, Wagner et al. 2005, Sethe et al. 2006, Prockop 1997, Beyer Nardi, da Silva Meirelles 2006, Bianco, Gehron Robey 2000, Zarnett, Salter 1989, Wickham et al. 2003, Tuli et al. 2003, Noth et al. 2002, Noort et al. 2002, 
Nakahara et al. 1990, O'Driscoll et al. 2001, Miura et al. 2003, Sottile et al. 2002, De Bari et al. 2001, De Ugarte et al. 2003, Dragoo et al. 2003, Fukumoto et al. 2003, Gronthos et al. 2001, Jankowski et al. 2002). Aunque la médula ósea sigue siendo la principal fuente de MSCs para la mayoría de los estudios preclínicos y clínicos, los lipoaspirados están ganando importancia como fuente de MSCs debido a su fácil de obtención y aislamiento, y a la abundancia de células obtenidas.

4.2. Tejido adiposo (AD): El tejido adiposo comprende aproximadamente el $10-29 \%$ de la masa corporal en un humano adulto normal (Kahn 2008). Hay dos formas principales: el tejido adiposo marrón (BAT- grasa buena), que es termogénico, responsable de la generación de calor, presente principalmente en los recién nacidos y el tejido adiposo blanco (WAT-grasa mala) que sirve como almacén de energía, protección mecánica y aislamiento, y representa una fuente de múltiples factores autocrinos, paracrinos y endocrinos, tales como proteínas (adipoquinas), ácidos grasos, hormonas esteroides y prostaglandinas (Gimble et al. 2013, Guerre-Millo 2002). A nivel celular, WAT consiste en adipocitos maduros (que contienen grandes gotas de lípidos que ocupan hasta el $90 \%$ del citoplasma), células endoteliales, pericitos, células del músculo liso, fibroblastos, células progenitoras adiposas (preadipocitos) y MSCs. Las MSCs y preadipocitos poseen una mayor capacidad de proliferación y diferenciación bajo condiciones apropiadas (BauerKreisel et al. 2010).

4.3. Aspectos terapéuticos de las MSCs: Las MSCs han recibido mucha atención en los últimos años debido a sus enormes posibilidades en el campo de la terapia celular por su capacidad de diferenciación a múltiples tipos celulares y por sus efectos paracrinos e inmunomoduladores. Además, se ha descrito el papel neuroprotector de las MSCs que se llevan a cabo mediante la secreción de factores de crecimiento tales como CNTF, BDNF, IGF1, bFGF, NGF, que protegen tanto la neurorretina como el EPR de la degeneración (Labouyrie et al., 1999; Lin et al., 2009 Doorn et al., 2011; Levkovitch-Verbin et al., 2010). Las MSCs están siendo utilizadas actualmente en 409 ensayos clínicos en diferentes fases (409 ensayos clínicos registrados con término de búsqueda "Mesenchymal", y 379 ensayos clínicos registrados con término de búsqueda "mesenchymal stem cells OR mesenchymal stromal cells" en ClinicalTrial.gov a fecha 18 de noviembre de 2013) y algunos de ellos están aprobados para su aplicación final. Entre estos ensayos clínicos, 14 están relacionados con enfermedades oculares directa o indirectamente y 5 concretamente con enfermedades de la retina (dos para RP). Un ensayo clínico (NCT01920867) está estudiando el uso de MSCs autólogas de médula ósea en enfermedades de la retina (DMAE, distrofia retiniana hereditaria, enfermedad del nervio óptico y glaucoma). La fuente más frecuente de las MSCs utilizada en estos ensayos clínicos es la médula ósea seguida por el 
tejido adiposo y el cordón umbilical. Las características actuales en favor del uso de las AD-MSCs para aplicaciones clínicas incluyen: la abundancia relativa de la fuente, la facilidad de aislamiento en números clínicamente significativos, la facilidad de cultivo y mantenimiento, su alta capacidad proliferativa, su plasticidad, sus propiedades inmunomoduladoras, su escasa inmunogenicidad (permiten el trasplante alogénico), los efectos paracrinos, su capacidad de migrar hasta el tejido dañado, la facilidad de transfección celular y la ausencia de preocupaciones éticas. Debido a la alta capacidad de proliferación y la supervivencia a largo plazo en comparación con otras células somáticas, la modificación genética de las MSCs es un objetivo atractivo para la terapia celular combinada con terapia génica.

4.4. Factores paracrinos de las MSCs: Las MSCs secretan gran cantidad de factores tales como citocinas, quimioquinas, factores de crecimiento, factores neurotróficos, y proteínas de ECM, que desempeñan diversas funciones importantes y tienen un gran potencial terapéutico (Salgado et al. 2010, Doorn et al. 2012, Meirelles Lda et al. 2009). Estos factores tróficos solubles juegan un papel muy importante en la regeneración y la reparación de tejidos, inhibición de fibrosis y apoptosis, inducción de angiogénesis, estimulación endógena de reclutamiento de células madre y proliferación, y en la regulación de la respuesta inmune (Maumus et al. 2013). La secreción por parte de las MSCs incluye factores angiogénicos (VEGF, HGF, Ang-1, bFGF, IGF-1, PDGF, PIGF, IL6, EPO, MCP1), factores antiangiogénicos (PAI-s, angiostatina, y la trombospondina), factores antiapoptóticos (IGF-1, IL-6, GM-CSF, TGF- $\beta$, bFGF, HGF), factores hematopoyéticos (MCSF, líquidos iónicos, Ang-1, -2, SCF, TPO, LIF, SDF-1, TGF- $\beta$ ), inmunomoduladores (IDO, PGE2, HGF, LIF, HLA-G, el TGF- $\beta$, TSG-6, NO, HO-1, HLA-G5, IL-10, IL-6), factores quimioatrayentes/migratorias (SDF-1, HGF, VEGF, CCL-2 a -6, -20, LIF, IGF, G-CSF, M-CSF, CXCL-2, -3, -5, -8, -10, -11), factores anti-fibróticos (HGF, bFGF, Ang-1, KGF, MMP-2/9, TIMP-1/2), y factores neuroprotectores (BDNF, NGF, GDNF, gelectin-1) (Meirelles Lda et al. 2009, Salgado et al. 2010, Doorn et al. 2012, Maumus et al. 2013, Lopatina et al. 2011, Rubina et al. 2009, Cai et al. 2009, Uccelli et al. 2011b, Uccelli et al. 2011a, Puissant et al. 2005, Di Nicola et al. 2002, Aggarwal, Pittenger 2005, Uccelli et al. 2008, Kogler et al. 2005, Sato et al. 2007, Meisel et al. 2004, Selmani et al. 2008, Le Blanc, Ringden 2005, Paul, Anisimov 2013).

4.5. Potencial de diferenciación de las MSCs: Las MSCs tienen capacidad para diferenciarse en linajes mesenquimales incluyendo condrocitos, osteocitos y adipocitos, cuando son sometidas a unas determinadas condiciones, tanto in vivo como in vitro (Dan et al. 2006, Muraglia et al. 2000, Pittenger et al. 1999, Bruder et al. 1998a, Bruder et al. 1998b, Barry et al. 2001, Digirolamo et al. 1999, Johnstone et al. 1998). Además, las MSCs han sido 
diferenciadas hacia otros tipos celulares tales como hepatocitos, cardiomiocitos, músculo liso, miocitos esqueléticos, células endoteliales, células pancreáticas, células neuronales, células progenitoras de la retina, células del RPE, y células del epitelio corneal, entre otros (Banas et al. 2007, Hong et al. 2005, Drosos, Kolios 2013, Chiou et al. 2005, Ong et al. 2006, Sanchez-Ramos et al. 2000, Sato et al. 2005, Seo et al. 2005, Vossmerbaeumer et al. 2009, Nieto-Miguel et al. 2013, Gupta et al. 2013, Tombran-Tink, Johnson 1989, Anghileri et al. 2008, Zuk et al. 2001, Zuk et al. 2002, Krampera et al. 2007, Timper et al. 2006, Safford, Rice 2005, Talens-Visconti et al. 2007, Talens-Visconti et al. 2006, Mizuno et al. 2002, Lee, Kemp 2006, Moviglia et al. 2012, Zhang, Dong 2008, Huang et al. 2012, Cao et al. 2005, Banas et al. 2009, Konno et al. 2010, Tan et al. 2010, Park et al. 2011).

4.6. MSCs en oftalmología: Como se ha mencionado existen en este momento 14 ensayos están relacionados con el uso de las MSCs en enfermedades oculares. Uno de ellos (NCT01562002), realizado por el IOBA, está estudiando el uso de MSCs alogénicas de médula ósea para el tratamiento del síndrome de insuficiencia límbica. Se han realizado diversos estudios sobre el uso de MSCs para el tratamiento de enfermedades de la superficie ocular (Arnalich-Montiel et al. 2008, McIntosh Ambrose et al. 2010, De Miguel et al. 2010). Además, se ha demostrado la capacidad de curación de quemaduras epiteliales corneales por parte de las MSCs, principalmente debido a sus efectos antiinflamatorios y anti-angiogénicos (Ma et al. 2006, Oh et al. 2008, Oh et al. 2009). Recientemente, se ha estudiado la capacidad de diferenciación de las AD-MSCs hacia epitelio corneal (NietoMiguel et al. 2013).

Respeto a las enfermedades de la retina actualmente, se están llevando a cabo 8 ensayos clínicos. Varios trabajos demuestran que las MSCs, incluyendo las AD-MSCs, puede diferenciarse parcialmente hacia los fenotipos de células retiniana como el EPR, células progenitoras de la retina y PRs, tanto in vitro como in vivo (Arnhold et al. 2006, Vossmerbaeumer et al. 2009, Huang et al. 2012, Moviglia et al. 2012, Kicic et al. 2003, Tomita et al. 2002, Tomita et al. 2006, Zhang, Dong 2008). Se ha descrito la expresión de la proteína rodopsina en MSCs tras ser inyectadas en el espacio subretiniano en ratas y su relación con el factor de crecimiento epidérmico (EGF)(Gong et al. 2008, Castanheira et al. 2008). Las MSCs inyectadas en la cavidad vítrea pueden rescatar y retrasar la degeneración de la retina y de los PRs mediante la liberación de factores neuroprotectores como CNTF, BDNF y bFGF (Inoue et al. 2007, Li et al. 2009). Se ha observado la integración de las MSCs en la retina tras su inyección sistémica en un modelo de RP en rata, rescatando parcialmente la función visual (Wang et al. 2010) y demostrando la capacidad de homing de las MSCs (pueden migrar de una parte a otra parte del cuerpo, 
especialmente hacia el tejido dañado). El perfil de secreción neurotrófico, incluyendo otros factores anti-infamatorios e inmunomoduladores, por parte de las MSCs aboga por su uso potencial en todas las enfermedades relacionadas con la degeneración neuronal o neurorretiniana, incluyendo neuroinflamación, como panuveítis, glaucoma, y enfermedades de la retina como DMAE y RP (Joe, Gregory-Evans 2010, Paul, Anisimov 2013). La inyección intravítrea de MSCs de médula ósea en un modelo de glaucoma en ratas Wistar mostró un efecto de neuroprotección significativo al aumentar la supervivencia de las células ganglionares (Yu et al. 2006). El potencial terapéutico de las MSCs en general, y en concreto en oftalmología, se atribuye sobre todo a su capacidad de neuroprotección y de inmunomodulación. 


\section{Hipótesis}

Es posible crear un microambiente artificial, utilizando hAD-MSCs y diferentes factores o biomoléculas (VIP, NIC, ATRA, y BMP-4) y sus combinaciones, junto con células del EPR o mediante el medio de cultivo condicionado por ellas, que puede inducir la diferenciación de las hAD-MSCs hacia fenotipos de EPR y al mismo tiempo proteger la retina neural en degeneración y las células del EPR en proceso de muerte celular, fenómenos clave en la patogénesis de la forma seca de la DMAE que afecta a la población de edad avanzada. 


\section{Objetivos}

El objetivo general de este trabajo es evaluar el efecto de diferentes factores $\mathrm{o}$ biomoléculas y sus combinaciones, junto con células del EPR o medio de crecimiento condicionado por ellas (medio condicionado de EPR-RCM), sobre la diferenciación de las hAD-MSCs hacia un fenotipo de EPR y sobre la degeneración de la neurorretina y la muerte de las células del EPR en condiciones in vitro.

Los objetivos específicos son los siguientes:

1. Aislamiento, expansión y caracterización de hAD-MSCs y de células del EPR procedentes respectivamente de lipoaspirados humanos donados y de globos oculares post-mortem

a) Extracción, expansión y mantenimiento de las células madre mesenquimales procedentes de lipoaspirados humanos donados.

b) Caracterización de las células madre mesenquimales derivadas de tejido adiposo humano (hAD-MSCs).

i) Inmunofenotipado de las hAD-MSCs mediante citometría de flujo.

ii) Evaluación de la capacidad de diferenciación in vitro en adipocitos, condrocitos, osteocitos.

c) Extracción, ampliación y mantenimiento de las células del EPR de globos oculares humanos y porcinos.

d) Caracterización de las células hRPE por citometría de flujo, inmunofluorescencia y western blot.

2. Determinación del efecto de las hAD-MSCs sobre la proliferación y muerte de las células del EPR

a) Optimización de la concentración y tiempo del tratamiento con mitomicina C (MMC) para provocar un proceso lento de degeneración en la línea celular de EPR (ARPE19) se someta lentamente al proceso de degeneración.

b) Co-cultivo indirecto de hAD-MSCs con células del EPR tratadas con MMC y análisis de su efecto sobre la viabilidad y proliferación de las células del EPR tratadas. 
3. Inducción de la diferenciación de las hAD-MSCs hacia fenotipos de EPR y su caracterización en distintas condiciones

A) La inducción de diferenciación de las hAD-MSCs a fenotipos de EPR por diferentes métodos.

a) Cultivo de hAD-MSCs en un 50\% de hRCM junto con VIP, NIC, ATRA en diferentes combinaciones.

b) Cultivo de hAD-MSCs en un 50\% RCM fetal (fRCM) junto con la proteína morfogénica ósea 4 (BMP-4) y extractos de neurorretina humanos.

c) Co-cultivo directo de hAD-MSCs con células ARPE19 tratadas con MMC para analizar la posible inducción de fenotipos de EPR en hAD-MSCs.

d) Co-cultivo indirecto de las hAD-MSCs junto con una combinación de diferentes factores, como el péptido intestinal vasoactivo (VIP), la nicotinamida (NIC) y el ácido trans-retinoico (ATRA), en un $50 \%$ de medio condicionado de hEPR.

e) Co-cultivo indirecto de los explantes de neurorretina humanos con hAD-MSCs, en un 50\% de RCM tanto solo como con diferentes combinaciones de VIP, NIC y ATRA.

B) Determinación de los fenotipos del EPR en los cultivos de hAD-MSCs bajo las condiciones de cultivo antes mencionados, mediante estudios de inmunofluorescencia y western blot.

4. Análisis y evaluación de explantes de neurorretina cultivados con hAD-MSCs, junto con los factores (VIP, NIC y ATRA) y sus combinaciones en 50\% RCM (3Ae)

a) Evaluación de los cambios estructurales (daño y degeneración) de las diferentes capas de los explantes de neurorretina humanos presentes en los co-cultivos anteriormente mencionados, mediante técnicas histoquímicas.

b) Evaluación de los cambios funcionales y estructurales de las diferentes capas de los explantes de neurorretina humanos presentes en los co-cultivos anteriormente citados, mediante técnicas inmunohistoquímicas. 


\section{Metodología}

Cultivos celulares: Todas los tipos celulares empleados (AEPR19, HFF1, hAD-MSC, hEPR, pEPR) fueron cultivadas bajo condiciones estándar, $37^{\circ} \mathrm{C}$ y atmósfera con $5 \%$ de $\mathrm{CO}_{2}$, renovando el medio de cultivo cada 2-3 días, en los respectivos medios que figuran en la tabla 2 (en la sección de metodología de la parte en inglés). Cuando los cultivos celulares alcanzaron el 90\% de confluencia, las células se levantaron siendo incubadas con 0,05\% de tripsina y 0,02\% de ácido etilendiaminotetraacético (Tripsina-EDTA, Gibco, Invitrogen, Paisley, Reino Unido), o con TrypLE Express (Gibco) durante 5-10 minutos y se resuspendieron en medio de cultivo. Las células se utilizaron para su posterior subcultivo en una proporción de 1:3 a 1:5 o para crioconservarse en medio de cultivo suplementado con un 25\% de suero bovino fetal (FBS) (Gibco) y 5\% de DMSO (Sigma, EE.UU.). El número de células, y la viabilidad de las mismas se determinaron mediante el ensayo estándar de exclusión con azul tripán.

\section{Aislamiento de células y tejidos}

hEPR/pEPR, neuroretina: hEPR y neuroretinas se aislaron cuidadosamente bajo un ambiente estéril en campana de flujo laminar, como se describe en la sección de metodología de la parte en inglés de la tesis. Se retiró la retina periférica (aprox $5 \mathrm{~mm}$ ) y se cortó la retina central para generar explantes de un tamaño aproximado de 7X5 mm para los experimentos de co-cultivo. Después de retirar la neuroretina, el hEPR se aisló del polo posterior mediante su incubación con una mezcla de 0,05\% de tripsina y 0,02 \% de EDTA durante $30-45$ minutos a $37^{\circ} \mathrm{C}$. La solución de tripsina presente en la copa ocular se pipeteó con fuerza varias veces para separar el EPR de la coroides. Las células del EPR se centrifugaron a $1200 \mathrm{rpm}$ durante 5 minutos. Después de centrifugarlas, se resuspendieron en medio de cultivo completo y se colocaron en frascos de $25 \mathrm{~cm}^{2}$ (Nunc, Fisher Scientific, EE.UU.) e incubaron a $37^{\circ} \mathrm{C}$ durante una semana. Estas células pigmentadas cultivadas en el frasco, conocidas como células de cultivo primario, se trataron con tripsina cuando su confluencia alcanzó el 85 a 95\% y se sub-cultivaron desde el pase cero (P-0). En total se obtuvieron 12 ojos de 6 personas fallecidas sin enfermedad ocular concomitante conocida. El procedimiento cuenta con la aprobación explícita del Comité Ético del Hospital Clinico Universitario de Valladolid.

Células madre mesenquimales derivadas de tejido adiposo: El procedimiento para la extracción, la expansión y la caracterización de las MSC a partir de lipoaspirados (LPA) fue idéntica a la utilizada por Nieto-Miguel y colaboradores (Nieto-Miguel et al. 2013). Los LPA se obtuvieron a partir de tejido adiposo subcutáneo humano de seis donantes diferentes, como subproductos de los procedimientos de liposucción después de la firma 
de los consentimientos informados correspondientes aprobado por el Comité Ético. Los LPA se centrifugaron para separar las fracciones solubles y se lavaron dos veces con PBS para eliminar el suero residual y el líquido anestésico. Entonces, los LPA fueron tratados con 0,075\% (0.75mg/mL) de colagenasa tipo I (Gibco -Invitrogen, EE.UU.) durante 45-60 minutos en un agitador orbital, a $37^{\circ} \mathrm{C}$. Los gránulos de alta densidad de los fracciones vasculares estromales (SVF) se obtuvieron después de la neutralización de la actividad de la enzima con FBS y tras su centrifugación a 1200 rpm durante 5 minutos. Después de un lavado con PBS, se incubaron las SVF con 160mM NH4Cl (Sigma-Aldrich, EE.UU.) durante 10 minutos para lisar los glóbulos rojos (eritrocitos). Las SVF se recogieron por centrifugación y se resuspendieron en medio de cultivo, para posteriormente filtrarse a través de unos filtros de nylon de 100 y $40 \mu \mathrm{m}$ (BD Bioscience, EE.UU.) para eliminar los restos de tejido. Después del recuento celular, incluyendo la determinación de la viabilidad, las células se sembraron con una densidad celular de $2 \times 10^{7}$ células en un frasco de $75 \mathrm{~cm}^{2}$ y se incubaron a $37^{\circ} \mathrm{C}$ durante 2-3 días. A continuación, las células adherentes se lavaron con PBS para eliminar las células no adherentes. Estas células adherentes, también denominadas cultivo primario, fueron subcultivadas desde el pase cero (P0) hasta el pase tres (P3). Las hAD-MSCs desde el P3 al P5, incluyendo pases más altos, se emplearon además para su caracterización con el panel de marcadores de superficie celular por citometría de flujo (inmunofenotipado) y para el análisis de su potencial de diferenciación multilinaje. Las hAD-MSCs se cultivaron en condiciones estándar, y cuando alcanzaron una confluencia del 90\%, fueron sub-cultivadas a una densidad celular de 3.000 a 6.000 células $/ \mathrm{cm}^{2}$. Los pases de las hAD-MSCs utilizados en los diferentes experimentos fueron de P3 a P6. Para evitar la diferenciación espontánea las hAD-MSCs se mantuvieron en subconfluencia, es decir, a menos del 95\% de confluencia.

Inmunofenotipado de las hAD-MSCs: Debido a la falta de un marcador específico para las MSCs, se acepta que las células han de ser positivas en más del $95 \%$ para CD105, CD73, y CD90 y negativas para CD34, CD45, CD14 y CD19 en el análisis de citometría de flujo de acuerdo con la ISCT (Dominici et al. 2006, Horwitz et al. 2005). Para el análisis de citometría de flujo se recogieron 200.000 hAD-MSCs (de P3 a P5) en cada tubo, en un total de 6 tubos, y se lavaron dos veces con tampón de lavado frío o PBS. Se tomaron dos tubos adicionales para el análisis de la viabilidad celular y del ciclo celular. Las células fueron bloqueadas con $40 \mu \mathrm{g} / \mathrm{mL}$ de IgG (Sigma-Aldrich, EE.UU.) durante 15 minutos a temperatura ambiente (TA) y, a continuación, las células se incubaron con $10 \mu \mathrm{l}$ de los anticuerpos monoclonales anti-humanos y se combinaron con controles de isotipo a $4^{\circ} \mathrm{C}$ durante 30 minutos en oscuridad, como describe: 
Controles de isotipo

Tubo 1: IgG2a- FITC / IgG1- PE / IgG2a- APC (5 $\mu$ l de cada uno)

Tubo 2: IgG1- FITC / IgG1- PE / IgG1- PC7 (5 $\mu$ l de cada uno)

Tubo 3: IgG2a- FITC / IgG1- PE/ IgG1- PC7 (5 $\mu$ l de cada uno)

Anticuerpos de prueba (panel de anticuerpos para las MSCs)

Tubo 1:CD19-FITC/CD105- PE/ HLADR-APC (10 $\mu$ l de cada uno)

Tubo 2: CD45- FITC/ CD90- PE/ CD34- PC7 (10 $\mu$ l de cada uno)

Tubo 3: CD14- FITC/ CD73- PE/ CD45- PC7 (10 $\mu$ l de cada uno)

Después se lavaron las células con PBS frío, se resuspendieron en $500 \mu \mathrm{l}$ de PBS y se analizaron en citómetro de flujo (CF) (FC Citómica 500 citómetro, Beckman Coulter, Inc., Fullerton, CA).

Diferenciación multi-linaje de las hAD-MSCs in vitro: Las hAD-MSC (de pases entre P3P5 y superiores) se sembraron en cuatro placas Permanox de dos pocillos (LabTek, EE.UU.). Dos de ellas se incubaron con medio de diferenciación adipogénico y osteogénico, (StemPro Adipogenesis Differentiation Kit y StemPro Osteogenesis Differentiation Kit; Gibco, Invitrogen) durante 15 días y durante 29 días, respectivamente, según las instrucciones del fabricante. El resto de placas se emplearon como controles, y fueron incubadas con medio basal. Tras el período de incubación, tanto las placas control como las experimentales, se tiñeron con Oil Red 0 (Sigma 00625), para detectar los triglicéridos y los lípidos en las gotas de aceite de los adipocitos, o con la tinción von Kossa para precipitar las sales de fosfato presente en forma de fosfato de calcio en osteocitos. Para la diferenciación a condrocitos, las hAD-MSCs se dividieron entre dos tubos de $15 \mathrm{~mL}\left(1 \times 10^{6}\right.$ células en cada uno) y se incubaron en StemXVivoTM Chondrogenic Base Media y StemXVivo $^{\mathrm{TM}}$ Chondrogenic Supplement (RD Systems, Minneapolis, EE.UU.) y, el otro, en medio completo normal bajo en glucosa durante 27 días. Después los sedimentos celulares se fijaron con paraformaldehído (PFA) al 4\% a temperatura ambiente (TA) durante 3 horas, se lavaron dos veces con agua destilada, y se colocaron a $4{ }^{\circ} \mathrm{C}$, en una solución al $30 \%$ de sacarosa durante la noche (ON). El día siguiente se incubó con una nueva solución al 30\% de sacarosa a TA durante 30 minutos y se incluyó en un medio criosolidificable (OCT-Compound) (Aname, Madrid, España) en hielo seco o a $-20{ }^{\circ} \mathrm{C}$. Finalmente fueron 
crio-seccionadas, y se tiñeron con azul de alcián. El azul de alcián se utiliza para teñir polisacáridos ácidos tales como glicosaminoglicanos presentes en condrocitos o en cartílagos. En todos los casos, las células se contratiñeron con hematoxilina Gill III (SigmaAldrich, EE.UU.).

Obtención del segmento externo de los fotorreceptores (POS): El procedimiento para preparar una suspensión de POS de ojos de cerdo fue similar al utilizado previamente por otros grupos de investigación (Hall 1978, Huang et al. 2012). Los ojos de cerdo se obtuvieron del matadero local de Laguna de Duero, Valladolid. En su preparación, se extrajo la neuroretina y se transfirió a HBSS o PBS. El tejido neurorretiniano se incubó en $0,25 \%$ de tripsina y $0,02 \%$ de EDTA en un agitador magnético durante 30 minutos a $4{ }^{\circ} \mathrm{C}$. Los grandes fragmentos que pudieron quedar de tejido se eliminaron por precipitación durante $15-20$ minutos (a $4{ }^{\circ} \mathrm{C}$ ) y el sobrenadante se centrifugó a $800 \mathrm{xg}$ durante 10 minutos. El sedimento de la muestra se lavó tres veces con HBSS/PBS y las células se resuspendieron en medio completo DMEM/F12 con una densidad celular de $1 \times 10^{8}$ POS/mL. Se mantuvieron a $-80{ }^{\circ} \mathrm{C}$ hasta su utilización.

Preparación del medio condicionado de EPR (RCM): Las células del EPR se sembraron en frascos de $75 \mathrm{~cm}^{2}$ y se cultivaron hasta su confluencia. El medio condicionado de hEPR (P1 a P3), AEPR19 y EPR fetal (fEPR) se recogió a las 48 horas. Estos medios se centrifugaron a $1000 \mathrm{rpm}$ durante 10 minutos para eliminar los residuos de células y los sobrenadantes se almacenaron a $-80{ }^{\circ} \mathrm{C}$ para su uso posterior. Los medios obtenidos de hEPR, AEPR19 y fEPR se denominan como hRCM, aRCM y fRCM, respectivamente.

Tratamiento de las células del EPR con Mitomicina C (MMC): Las células AEPR19 se sembraron a una densidad celular de 60.000 células $/ \mathrm{cm}^{2}$ y se incubaron durante 24 horas. A continuación, se incubaron durante 2 horas con distintas concentraciones de MMC (25, 50,100 y $200 \mu \mathrm{g} / \mathrm{mL}$ ) en el medio de cultivo para optimizar los experimentos y encontrar la concentración más apropiada de MMC. Tras los tratamientos, las células se lavaron 3 veces con PBS para eliminar cualquier resto de MMC en el medio. Se eligió la concentración de $50 \mu \mathrm{g} / \mathrm{mL}$ para inactivar las células, así como para inducir la muerte celular a largo plazo.

\section{Métodos de diferenciación de las hAD-MSCs a EPR}

Cultivo de las hAD-MSCs en hRCM suplementado con factores de diferenciación: Las hAD-MSCs se sembraron a una densidad celular de 30.000 células $/ \mathrm{cm}^{2}$ en placas de 6 pocillos, así como en placas Permanox de 8-pocillos (Permanox®, LabTek®, EE.UU.). Tras 
24 horas, las células adheridas se lavaron y se cultivaron en DMEMF/12 con un $50 \%$ de hRCM y siete combinaciones diferentes de factores de crecimiento, excluyendo el control. Las siete combinaciones fueron las siguientes: VIP, NIC, ATRA, VIP+NIC, VIP+ATRA, NIC+ATRA, VIP+NIC+ATRA. VIP, NIC y ATRA se utilizaron a una concentración de $5 \mu \mathrm{M}$, $10 \mathrm{mM}$ y $5 \mu \mathrm{M}$, respectivamente.

Cultivos de las hAD-MSCs en fRCM y POS suplementado con BMP-4: Las hAD-MSCs se sembraron a una densidad celular de 30.000 células $/ \mathrm{cm}^{2}$ en placas de 6 pocillos, así como en placas Permanox de 8 pocillos (Permanox®, LabTek®, EE.UU.). Después de 24 horas, las células adheridas se lavaron y se cultivaron en DMEM/F12 con un 50\% fRCM y POS $\left(1 \times 10^{5} \mathrm{POS} / \mathrm{mL}\right)$ suplementado con $200 \mathrm{ng} / \mathrm{mL}$ de BMP-4, durante dos semanas. Se emplearon 8 combinaciones: 1. Medio de crecimiento basal (DMEM/F12 completo, sin

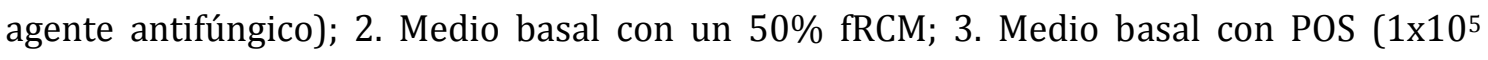
POS $/ \mathrm{mL})$, 4. Medio basal con 50\% fRCM y POS (1x105 POS $/ \mathrm{mL})$ y, estas mismas combinaciones pero suplementadas con BMP-4. El medio de cultivo se cambió cada 2 días. Este experimento se realizó en el Departamento "Eye and Vision Science", de la Universidad de Liverpool.

Diseño experimental (placas de Permanox de 8 pocillos)

\begin{tabular}{|l|l|l|l|l|}
\hline \multirow{3}{*}{ - BMP-4 } & DMEM/F12 & $50 \%$ fRCM & POS $\left(1 \times 10^{5} / \mathrm{mL}\right)$ & $50 \%$ fRCM+POS \\
\cline { 2 - 5 } & DMEM/F12 & $50 \%$ fRCM & POS $\left(1 \times 10^{5} / \mathrm{mL}\right)$ & $50 \%$ fRCM+POS \\
\hline
\end{tabular}

\begin{tabular}{|l|l|l|l|l|}
\hline+ BMP-4 & DMEM/F12 & $50 \%$ fRCM & POS $\left(1 \times 10^{5} / \mathrm{mL}\right)$ & $50 \%$ fRCM+POS \\
\cline { 2 - 5 } & DMEM/F12 & $50 \%$ fRCM & POS $\left(1 \times 10^{5} / \mathrm{mL}\right)$ & $50 \%$ fRCM+POS \\
\hline
\end{tabular}

Co-cultivo directo: Las células del EPR (ARPE19 o hRPE P2) se sembraron en co-cultivo directo en portas de Permanox con una densidad de 60.000 células/ $\mathrm{cm}^{2}$ y se dejaron durante 24 horas en incubación. Al día siguiente, las células de EPR adheridas se lavaron y se incubaron con $50 \mu \mathrm{g} / \mathrm{mL}$ de MMC durante 2 horas. Después, las células se lavaron con PBS o medio de crecimiento tres veces y, a continuación, se sembraron las hAD-MSCs, con una densidad de 30.000 células $/ \mathrm{cm}^{2}$, sobre las células de EPR tratadas con MMC. Las células del EPR tratadas con MMC y hAD-MSCs fueron co-cultivadas directamente en una proporción 2:1, como se muestra en el diagrama (Fig. 2C y D). El tamaño de las células del EPR es mucho más pequeño que la de hAD-MSCs. El medio utilizado para el co-cultivo directo fue DMEM/F12 completo como se muestra en la tabla 1. Para distinguir los dos tipos celulares, las células de EPR se marcaron con EdU (Click-iT EdU imagen Kit, 
Invitrogen, EE.UU.) previamente al tratamiento con MMC, siguiendo las instrucciones del fabricante.

Co-cultivo indirecto: las células ARPE19 y hAD-MSCs se sembraron en las mismas condiciones que en co-cultivo directo pero separadas físicamente por una transmembrana de cultivo celular (BD Falcon ${ }^{\mathrm{TM}}$ ) como se muestra en el diagrama (Fig. 2 A y D). Las células ARPE 19 fueron cultivadas en la parte inferior de las placas de 24 pocillos (BD Falcon) y las hAD-MSCs se cultivaron en soportes permeables de tereftalato de polietileno (PET; BD Falcon) con un tamaño de poro de $0,4 \mu \mathrm{m}$ (densidad de poros de 2,0 $\pm 0,2 \times 10^{6} / \mathrm{cm}^{2}$ ). En otra serie de experimentos, las células hAD-MSC se co-cultivaron indirectamente con las

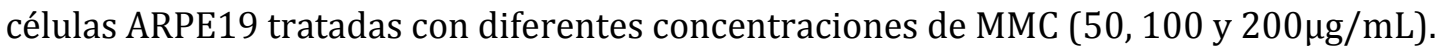

Co-cultivo indirecto de las hAD-MSCs con explantes de hNR: las hAD-MSCs se sembraron en la parte inferior de placas de 6 pocillos al menos 24 horas antes de poner los explantes de retina neural humana. Los explantes de retina neural (tamaño $8 \times 7 \mathrm{~mm}$ ) se colocaron con los fotorreceptores (PRs) hacia abajo sobre la transmembrana (Corning Inc., Nueva York, EE.UU.) como se muestra en el diagrama (Fig. 2B). El medio de cultivo con el $50 \%$ de hRCM se reemplazó cada 2 días. Este tejido de células de co-cultivo se incubó durante una y dos semanas con diferentes factores y sus combinaciones.

Ensayo de viabilidad celular: La viabilidad de las células ARPE19 después de tres semanas de tratamiento con MMC se determinó por el método de exclusión con azul tripán o con el kit de viabilidad/citotoxicidad para células vivas y muertas (Biotium Inc., EE.UU.) siguiendo las indicaciones del fabricante. El kit proporciona una tinción con dos fluorocromos: ester de acetoximetilo de calceína (calceína AM) para teñir las células vivas y homodímero de etidio III (EthD-III) para teñir las células muertas y dañadas. Las células se visualizaron utilizando un microscopio equipado con lámpara de fluorescencia (Leica Microsystems, Mannheim, Alemania).

Ensayo de proliferación celular con alamarBlue (AB): La actividad celular como resultado de la proliferación y la viabilidad se determinó con el ensayo de $\mathrm{AB}$ ( $\mathrm{AbD}$ Serotec, Oxford, Reino Unido) siguiendo las instrucciones del fabricante. Brevemente, las células se lavaron con PBS para después añadir una disolución de $\mathrm{AB}$ al $10 \%$ en medio DMEM/F12. Las células se dejaron en incubación durante 5 horas. Finalmente, se midió la intensidad de fluorescencia a $590 \mathrm{~nm}$ con un espectrofotómetro (Spectra Max M5 MultiMode Microplate Reader, Molecular Devices LLC, EE.UU.). Los resultados obtenidos se expresaron como el valor medio de la intensidad de fluorescencia en unidades arbitrarias $(\mathrm{AFU}) \pm$ el error estándar de la media (SEM). 
Western blot (WB): la expresión de marcadores de proteínas de EPR se determinó mediante WB. En primer lugar, la cantidad total de proteína se determinó con el método del ácido bicinconínico (Thermo Scientific Pierce BCA Protein Assay Kit, USA). Posteriormente, las muestras se cargaron en los pocillos de SDS-PAGE y se separaron por peso molecular. Finalmente, se realizó la transferencia y el marcaje por inmunoquímica. La proteína específica, contra la que se utilizó el anticuerpo primario, se detectó mediante la incubación de la membrana en solución de quimioluminiscencia (ECL; Santa Cruz Biotech Inc., EE.UU.) y las señales fueron capturadas con una cámara CCD en ChemiDoc (BioRad Inc., EE.UU.).

Inmunocitoquímica: Después de la inducción de la diferenciación de la hAD-MSCs, se analizaron los marcadores de EPR por inmunofluorescencia. Las células fueron marcadas por los anticuerpos contra CRALBP, BEST1, RPE65, CK8/18/19, pan-citoqueratina, Ezrin, PAX6 y Z01. Las células fueron también marcadas con CD90 (Thy1) para mostrar las características residuales de las células mesenquimales. Se realizó una contratinción con rodamina faloidina y DAPI para visualizar la morfología normal de las células (mediante la tinción de F-actina) y de los núcleos, respectivamente. El marcaje no específico se probó por omisión de los anticuerpos primarios. Las imágenes de las células se obtuvieron por microscopía de fluorescencia con un microscopio invertido (Leica, Germany) y se analizaron con el software Leica Application Suite Advanced fluorescence (LASAF).

Inmunohistoquímica: Los explantes de neurorretina fueron incluidos en OCT. A continuación, se realizaron cortes de 4 micras de espesor y se colocaron sobre portaobjetos para su posterior procesamiento. Estas secciones se marcaron CRALBP, GFAP, Calbindin, sinaptofisina, y rodopsina y después con anticuerpos secundarios complementarios conjugados con Alexa Fluor 488 o 568 (Invitrogen, EE.UU.). Todos los anticuerpos se utilizan en las diluciones indicadas en la tabla 2 (página54).

Tinción con azul de toluidina: Los explantes de neurorretina co-cultivados con hADMSCs en experimentos anteriores fueron fijados en el fijador de Palay e incluidos en resina epoxi Araldite 506 (Sigma-Aldrich, EE.UU.). Las secciones se realizaron con un microtomo LKB, modelo Ultratome III, y se tiñeron con una solución al 1\% de toluidina en tetraborato de sodio. Las muestras se evaluaron con un microscopio óptico (Axiophot, Zeiss). Se procesaron 128 muestras de explantes de hNR a partir de 6 experimentos diferentes.

Método de evaluación arbitraria del estado de los explantes de neuroretina: Los explantes de neurorretina se evaluaron mediante la observación de las fotomicrografías obtenidas mediante tinción con toluidina e inmunotinción contra CRALBP, GFAP, 
calbindina, sinaptofisina y rodopsina. Las imágenes se graduaron en una escala arbitraria de 0 a 10, dependiendo del grado de conservación y del estado global de los explantes neurorretinianos, incluyendo todas las capas. Los valores de 0-0,5 se dieron a los explantes de retina completamente destruidos y desorganizados (sin estructura tisular definidas) y el valor de 10 se dio a los explantes de retina bien estructurados y conservados. Se evaluaron 2 fotomicrografías representativas de cada muestra, procedentes de 6 experimentos independientes. La valoración de las imágenes fue realizada por 3 observadores experimentados.

Análisis estadístico: Todos los experimentos se han repetido entre tres y seis veces con muestras duplicadas o triplicadas. Los datos obtenidos se expresaron como el valor medio \pm la desviaciones estándar (SD) o \pm el error estándar de la media (SEM). Los datos se analizaron con los programas MS Excel y GraphPad Software en línea (versión), utilizando el test estadístico t de Student (emparejado/dos colas). Se consideraron estadísticamente significativos los valores de $\mathrm{P}<0.05, \mathrm{P}<0,01$ y $\mathrm{P}<0,001$. 


\section{Resultados}

\section{Caracterización de las hAD-MSCs no diferenciadas y de las células del EPR}

Prioramente al comienzo del trabajo experimental se trabajó con las células empleadas en este estudio (hEPR, ARPE19 y hAD-MSCs). Se estableció su correcto aislamiento a partir de órganos o tejidos de origen humano, así como su expansión y mantenimiento. También se estudiaron sus características morfológicas y celulares, optimizando los métodos y protocolos llevados a cabo. Además, se realizaron pruebas para determinar su pureza, viabilidad y otras características celulares inherentes durante la división y el sub-cultivo.

\subsection{Las células de EPR}

\section{Aislamiento, expansión y mantenimiento}

Las células de EPR fueron aisladas de ojos humanos donados post-mortem. Mediante el empleo microscopía de contraste de fases, se demostró que las células del EPR entre los pases P0-P1 adquirieron la morfología propia de células epiteliales, con una gran cantidad de pigmento en el citoplasma tras la confluencia del cultivos (Fig. 8A \& B página76). Sin embargo, en posteriores subcultivos, las células del EPR mostraron una disminución de la pigmentación citoplasmatica y la pérdida de la morfología propia de las células epiteliales. La tasa de crecimiento de las células del EPR fue significativamente menor en comparación con otras células en estudio, por ejemplo las ARPE19 y las hAD-MSCs, y el tiempo de duplicación se situó entorno a 4-5 días (Fig.8D página76). En otros estudios, las células del EPR cultivadas en la superficie de un polímero de elastina integrado con arginina-glicinaaspartato (ELR-RGD) mostraron una adherencia y una proliferación significativas, mantuvieron su morfología y expresaron RPE65 en comparación con las células cultivadas sobre un polímero sin ninguna secuencia específica (Singh et al. 2013, Srivastava et al. 2011a).

\section{Evaluación de la pureza del EPR}

Se observó que las células del EPR comienzan a perder sus características propias, tales como la pigmentación, la expresión de la proteína RPE65 y su morfología tras ser subcultivadas. Se estableció un método para probar la pureza de las células del EPR empleando citometría de flujo, además de otros ensayos de inmmunofluorescencia empleando, por ejemplo, el azul tripán (Srivastava et al. 2013, Srivastava et al. 2011b). 


\subsection{Las hAD-MSCs}

\section{Aislamiento, expansión y mantenimiento}

Las MSCs fueron aisladas a partir de lipoaspirados de tejido adiposo obtenidos de donaciones tras liposucción (Nieto-Miguel et al. 2013) de seis donantes diferentes. Se estableció el procedimiento de aislamiento, expansión, crecimiento y mantenimiento de los pases más bajos hasta los más altos al comenzar el trabajo. El tiempo de duplicación celular de las hAD-MSCs se determinó entre 2 y 4 días, que coincidió con el referido publicaciones previas (Mitchell et al. 2006, Izadpanah et al. 2006). Aunque las hAD-MSCs llegaron al pase 30, en todos los experimentos de diferenciación los pases empleados fueron entre P3-P6.

\section{Caracterización de las hAD-MSCs indiferenciadas (no inducidas)}

Se emplearon pases entre P3-P5 para la caracterización de las células, de acuerdo con los criterios establecidos por la ISCT (Horwitz et al. 2005). En cambio, se continuó trabajando con pases más altos para conocer las características de las hAD-MSCs indiferenciadas, y decidir si emplearlas para experimentos de diferenciación.

\section{Determinación del inmunofenotipo de las hAD-MSCs}

Los resultados mostraron que la gran mayoría de las hAD-MSCs (>99\% población) eran negativas para los marcadores de superficie celular, por ejemplo, CD14, CD19, CD34, CD45 y HLA-DR (Fig. 13B, C, D, E y F página84). Sin embargo, eran positivas (>97\% población) para los marcadores específicos de las MSCs, como por ejemplo, CD73, CD90, CD105 y mostraron una MFI (del inglés, Mean Fluorescence Index) relativa alrededor de 38, 32, y 6, respectivamente (Fig. 13G, H e I página 84).

\section{Potencial de diferenciación de las hAD-MSCs}

Las hAD-MSCs se sometieron a procedimientos de diferenciación en tres linajes mesodérmicos: adipocitos, osteocitos y condrocitos (como se establece en virtud de los criterios mínimos de la ISCT). Se obtuvo una diferenciación exitosa en adipocitos, observando un número significativo de lípidos intracelulares (en rojo) mediante su tinción con "Oil Red"; en condrocitos, con la formación de nódulos densos teñidos con azul alcián; y en osteocitos, observando depósitos intracelulares de sales de calcio (en negro) mediante tinción con von Kossa (Fig.14 página 85). 


\section{Comparación entre pases de las hAD-MSCs indiferenciadas}

Las hAD-MSCs entre los pases P16-P22 se caracterizaron de un modo similar al indicado para las células de pases bajos para evaluar si los parámetros analizados se mantenían durante el subcultivo de las células. Mediante citometria de flujo se obtuvo que la expresión de los marcadores de superficie ocular, como por ejemplo CD14, CD19, CD34, CD45 y HLA-DR fue similar entre células de pases altos y bajos. Sin embargo, las hAD-MSCs (p16-p22) mostraron un mayor MFI relativo, de alrededor de 58, 83 y 7 para la expresión de CD73, CD90 y CD105, respectivamente, en comparación con los de los pases inferiores (43, 44 y 7) (Fig.15B página 86). Durante su cultivo y expansión, mostraron mayor homogeneidad ya que se obtuvieron porcentajes mayores de expresión de marcadores específicos de las MSCs (Fig. 15A página 86).

A partir de los resultados experimentales, se parece claro que la capacidad de diferenciación de las hAD-MSCs de P22 en tres linajes mesodérmicos (adipocitos, osteocitos y condrocitos) fue menor que la de las hAD-MSCs de P6 (Fig.16 página 86).

Los análisis de proliferación celular mostraron que las hAD-MSCs de pases más altos (P16 y P22) tenían una tasa de crecimiento más lento en comparación con la obtenida de las hAD-MSCs de pases inferiores (P2-P8) (Fig. 8D página 76). Incluso las células de estos pases (P2-P8) mostraron una tasa de proliferación similar a la de las células ARPE19 (datos no mostrados). Se necesitaron entre 4-6 días para obtener cultivos confluentes, en función de la densidad de las células sembradas y de las condiciones de cultivo.

\section{Evaluación de los efectos de las hAD-MSCs sobre las células de EPR tratadas con MMC bajo condiciones de co-cultivos indirecto}

Las hAD-MSCs se co-cultivaron en soportes permeables Transwell@ con las células del EPR tratadas con MMC en las placas de 6 pocillos (sistema de co-cultivo indirecto) para probar el efecto de las hAD-MSCs sobre las células del EPR tratadas con MMC.

\section{La MMC provoca la muerte y reduce la proliferación de las células del EPR}

Se observó que el tratamiento con MMC provocó la muerte celular programada (apoptosis) en las células del EPR con la dosis más alta ( $>50 \mu \mathrm{g} / \mathrm{mL}$ ), como se ve en las observaciones microscópicas de las células de EPR tratadas con MMC. La evaluación microscópica mostró la formación de vesículas apoptóticas (Fig. 17A página 89) (las flechas azules indican cuerpos fluorescentes verdes oscuro), la contracción de las células y la formación de vesículas en las células del EPR tratadas con MMC a las dos semanas (Fig. 
17A página 89). Las células estaban prácticamente muertas después de 3 semanas (Fig. 17B página 89). La microscopía de fluorescencia mostró que las células del EPR en el control, sin ningún tratamiento de MMC, eran $100 \%$ viables (Fig. 18A página 90), mientras que casi todas las células del EPR tratadas con MMC (50 $\mu \mathrm{g} / \mathrm{mL})$ estaban muertas después de 3 semanas de tratamiento (Fig. 18B página 90). Los resultados del ensayo alamarBlue (para medir la actividad de proliferación de las células) mostraron que el tratamiento con MMC (50 $\mu \mathrm{g} / \mathrm{mL}, 100 \mu \mathrm{g} / \mathrm{mL}$ y $200 \mu \mathrm{g} / \mathrm{mL})$ redujo significativamente la proliferación de las células del EPR en 3 días $(\mathrm{p}<0,05 ; \mathrm{n}=3)$. La intensidad de la fluorescencia disminuyó significativamente $(\mathrm{p}<0,05 ; \mathrm{n}=3)$ con el aumento de la concentración de MMC $(50 \mu \mathrm{g} / \mathrm{mL}, 100 \mu \mathrm{g} / \mathrm{mL}$ y $200 \mu \mathrm{g} / \mathrm{mL}$ ) (Fig. 18E página 90). Después de 3 días, se encontró una alta intensidad de fluorescencia $(\mathrm{p}<0,05, \mathrm{n}=3)$ en las células del EPR no tratadas en comparación con las tratadas con MMC (Fig. 18E, F y G página 90).

\section{hAD-MSCs rescatan parcialmente las células del EPR (ARPE19) tratadas con MMC de la muerte celular}

El ensayo de viabilidad/toxicidad celular demostró que las células del EPR tratadas con MMC $(50 \mu \mathrm{g} / \mathrm{mL})$ después de tres semanas conservan una viabilidad significativamente mejor que el grupo control ( $\sin$ las hAD-MSCs) ( $p<0,05 ; n=3)$, cuando se co- cultivan con las hAD-MSCs (Fig. 18C página 90). No se observaron efectos adversos sobre las hAD-MSCs después de tres semanas de cultivo en el Transwell ${ }^{\circledR}$, debido a la muerte de las células del EPR por el tratamiento con MMC en el pocillo (Fig. 18D página 90).

El análisis de alamrBlue ${ }^{\circledR}$ mostró que la intensidad de la fluorescencia fue significativamente mayor $(\mathrm{p}<0,05 ; \mathrm{n}=3)$ en las células del EPR cultivadas con hAD-MSCs en comparación con las células del EPR cultivadas solas. Además, en todos los grupos de tratamiento, las células del EPR co-cultivadas con hAD-MSCs y tratadas con MMC mostraron una intensidad de fluorescencia significativamente mayor $(p<0,05 ; n=3)$ que las del EPR tratadas con MMC (Fig. 18E página 90). Los resultados del ensayo $\mathrm{AB}$ mostraron que las células del EPR tratadas con MMC y co-cultivadas con hAD-MSCs tenían una intensidad de fluorescencia significativamente mayor $(\mathrm{p}<0,05 ; \mathrm{n}=3)$ que aquellas cultivadas con fibroblastos (HFF1) (Fig. 18F página 90). El medio condicionado de las hAD-MSCs produjo el mismo efecto estimulante sobre el crecimiento de las células del EPR tratadas con MMC (Fig.18F página 90) y estimuló significativamente $(\mathrm{p}<0,05 ; \mathrm{n}=3$ ) la actividad de proliferación de las células del EPR tratadas con MMC en comparación con HFF1 en los períodos de 3 y 6 días (Fig. 18G página 90). 
Se observó que la proliferación de las células del EPR tratadas con MMC disminuía cuando se aumentó la concentración de MMC de $50 \mu \mathrm{g} / \mathrm{mL}$ a $200 \mu \mathrm{g} / \mathrm{mL}$. El co-cultivo de estas células del EPR tratadas con MMC y cultivadas con hAD-MSCs mostró que la capacidad proliferativa de estas células fue significativamente mayor $(\mathrm{p}<0,05 ; \mathrm{n}=3$ ) que aquellas células del EPR tratadas con MMC y sin hAD-MSCs, pero menor que las de las células del EPR no tratadas y cultivadas con o sin co-cultivo con hAD-MSCs.

Este hallazgo parece indicar que las células del hAD-MSCs secretan factores de protección en el medio de co-cultivo que crean un microambiente que podría ser propicio para rescatar parcialmente las células del EPR de la muerte celular. La MMC provocó la muerte celular programada (apoptosis) en las células del EPR. Las hAD-MSCs rescataron parcialmente a las células del EPR tratadas con MMC de entrar en la muerte celular en el sistema de co-cultivo indirecto y estimularon la proliferación de las células del EPR. Se observó el efecto similar cuando se utilizó un sobrenadante de las hAD-MSCs en el cultivo de las células EPR tratadas con MMC.

\section{Evaluación de la diferenciación de hAD-MSCs hacia los fenotipos del EPR}

Se utilizaron cinco métodos diferentes para estimular la diferenciación de las hAD-MSCs a fenotipo de EPR:

1. Cultivo de hAD-MSCs en el medio de crecimiento con un $50 \%$ de hRCM en presencia de tres factores diferentes (VIP, NIC y ATRA) y sus combinaciones.

2. Cultivo de hAD-MSCs en el medio de crecimiento con un $50 \%$ de fRCM (fetal RCM) y POS (del inglés, Photoreceptor Outer Segment) $(1 \times 105 / \mathrm{mL})$ en presencia de BMP-4 (Department of Eye and Vision Science, Universidad de Liverpool).

3. Co-cultivo directo de hAD-MSCs con células del EPR inactivadas por MMC.

4. Co-cultivo indirecto de hAD-MSCs con las células del EPR inactivadas por MMC en presencia de tres factores diferentes (VIP, NIC y ATRA) y sus combinaciones.

5. Co-cultivo indirecto de hAD-MSCs con explantes neurorretinianos humanos (hNR) en medio DMEM/F12 con $50 \%$ de RCM en presencia de tres factores diferentes (VIP, NIC y ATRA) y sus combinaciones. 


\subsection{El cultivo de las hAD-MSCs en hRCM suplementado con factores y sus combinaciones:}

Las hAD-MSCs fueron estimuladas durante la diferenciación hacia fenotipos EPR mediante el cultivo en medio DMEM/F12 con un 50 \% de hRCM suplementado con factores (VIP, NIC y ATRA) y sus combinaciones (VIP+NIC y NIC+ATRA) durante 21 días. El análisis por WB mostró que hubo un aumento significativo en la expresión de RPE65 en hAD-MSCs cuando se estimularon con las combinaciones de VIP+NIC y VIP+ATRA en comparación con otros factores por sí solos, incluyendo RCM (Tabla 5 página 93). Los marcadores epiteliales CK8/18 y PCK mejoraron significativamente en presencia de ATRA.

El estudio por inmunofluorescencia de las hAD-MSCs estimuladas con factores y sus combinaciones mostró que las combinaciones (VIP+NIC y NIC+ATRA) indujeron un aumento significativo en la expresión de proteínas del EPR (RPE65 y bestrofina) en comparación con otras condiciones, incluyendo las condiciones basales de estimulación solo con hRCM (Fig.20 página 94).

Según los resultados obtenidos por WB y los estudios de inmunocitofluorescencia, las hAD-MSCs aumentaron la expresión de marcadores del EPR cuando fueron suplementadas con NIC, ATRA, VIP+NIC, y NIC+ATRA junto con un $50 \%$ de hRCM. Estos hallazgos indican que se diferenciaron parcialmente hacia el fenotipo EPR.

\subsection{Inducción de la diferenciación de las hAD-MSCs hacía fenotipo EPR con BMP-4 y POS y fRCM (Esta parte del estudio se realizó en el Department of Eye and Vision Science del Instituto de Enfermedades Crónicas y Envejecimiento de la Universidad de Liverpool)}

Las hAD-MSCs se estimularon para diferenciarse hacia los fenotipos de EPR mediante el cultivo con un $50 \%$ de fRCM y POS con y sin BMP-4 (200 ng/mL) durante 15 días, como se describe en el apartado de material y métodos. Curiosamente las combinaciones con POS mostraron algunos efectos tóxicos sobre las proliferaciones de las hAD-MSCs, por lo que las combinaciones con POS no funcionaron. Sin embargo, otras combinaciones solo con fRCM y fRCM con BMP-4 dieron algunos resultados preliminares interesantes. Se encontró que las hAD-MSCs formaron esferas pigmentadas de masas celulares cuando son estimuladas con fRCM y BMP-4, solos o conjuntamente (Fig. 21B y C página 95) en comparación con el control (Fig. 21A página 95). Los estudios de inmunocitoquímica mostraron un aumento significativo en la expresión de los marcadores del EPR (RPE65 y CK8/18) en hAD-MSCs cultivadas en fRCM y fRCM suplementado con BMP-4 (Fig.22 
página 96). Los datos preliminares del análisis por WB no mostraron ningún cambio en la expresión de los marcadores del EPR (tirosinasa y CK8/18) (Fig.23 página 96).

Es necesario repetir estos experimentos para obtener conclusiones a cerca de la diferenciación de las hAD-MSC hacia el fenotipo de EPR en estas condiciones, pero la visualización de los cultivos mediante microscopio y los estudios inmunocitoquímicos indican un aumento del fenotipo de EPR en las hAD-MSCs estimuladas con fRCM, con BMP4 o con ambos simultáneamente.

\subsection{Co-cultivo directo de las hAD-MSCs y las células del EPR inactivadas con MMC}

En este caso, las hAD-MSCs fueron co-cultivadas directamente sobre las células del EPR inactivadas (ARPE19/hEPR) durante un mes, en una proporción de 1:2. La observación con el microscopio óptico mostró que las hAD-MSCs habían formado más esferas celulares pigmentadas en co-cultivo directo con las células de EPR inactivadas, qué los controles.

Los estudios de inmunofluorescencia de las hAD-MSCs cultivadas con células EPR inactivadas mostraron un aumento de la expresión de los marcadores de EPR (RPE65, Z01, CK8/18 y Ezrin) y la reducción de la expresión del marcador específico de las MSCs, el Thy-1 (CD90) (Fig. 26 y 28 páginas 99 and 101). Como resultaba muy difícil distinguir en el co-cultivo los dos tipos de células, las del EPR se marcaron con 5-etinil-2'desoxiuridina (EdU) antes de la inactivación con MMC para localizarlas en el co-cultivo (Fig. 26 página 99). Por lo tanto, la imagen de inmunofluorescencia tomadas a lo largo de diferentes planes en el eje z, muestra los núcleos de las células del EPR inactivadas marcados con el rojo fluorescente de EdU, están por debajo de los núcleos de las hADMSCs (Fig.28G página 101), lo que demuestra la localización de las células, así como la expresión del marcador de EPR en ellas. El análisis inmunocitológico de las hAD-MSCs en el co-cultivo directo con las células del EPR inactivadas mostró la diferenciación de las hAD-MSCs hacia las células del EPR. Esto fue confirmado mediante el análisis por WB en los experimentos de co-cultivo directo. Los resultados confirmaron que no hubo un aumento significativo de la expresión de marcadores del EPR (RPE65 y Z01) en las hADMSCs co-cultivadas con células de hEPR inactivadas ( $\mathrm{P} \leq 0,001)$ (Fig.29 página 102). El aumento de la expresión de los marcadores del EPR (RPE65 y ZO1) en las hAD-MSCs estimuladas en el co-cultivo directo fue mayor que la encontrada en el co-cultivo indirecto ( $\mathrm{P} \leq 0,001)$ (Fig.29 página 102). 
Los resultados basados en estudios de inmunocitoquímica y western blot mostraron que las hAD-MSCs se diferenciaron hacia células del EPR cuando son co-cultivadas con células del EPR inactivadas.

\subsection{Co-cultivo indirecto de las hAD-MSCs con las células del EPR tratadas con MMC junto con los factores}

Las hAD-MSCs se estimularon durante la diferenciación hacia fenotipo EPR cocultivándolas indirectamente en el sistema de Transwell® de 6 pocillos con las células del EPR (parte inferior del pocillo) en presencia de factores (VIP, NIC y ATRA) y sus combinaciones (VIP+NIC, VIP+ATRA, NIC+ATRA y VIP+NIC+ATRA) durante 12 días.

\section{Evaluación de la proliferación de las células del EPR inactivadas}

Se encontró que todos los factores y sus combinaciones tienen efectos antiproliferativos significativos $(\mathrm{P} \leq 0,01)$ en comparación con el control (tratados con $\mathrm{MMC}$, pero sin factores) (Fig.30 página 103). El aumento de los efectos anti-proliferativos fue más pronunciado o significativo $(\mathrm{P} \leq 0,01)$ a los 5 días, en comparación con los de 3 días (indicado por el signo más en el diagrama de barras), excepto en el caso de VIP y VIP+ATRA (Fig. 30 página 103). Al emplear las mismas combinaciones con las hAD-MSCs en co-cultivo, los efectos anti-proliferativos fueron anulados por la presencia de las hADMSCs (Fig. 31 página 104). Curiosamente, se observó que en el caso del co-cultivo con las hAD-MSCs, a los 5 días, la actividad de proliferación de las células del EPR fue mayor que a los 3 días (Fig. 31 página 104), mientras que en el cultivo sin las hAD-MSCs, sucedió al contrario (Fig. 30 página 103).

\section{Evaluación del estado de diferenciación de las hAD-MSCs hacia fenotipos del EPR}

Los resultados del WB (Fig. 32 página 105) y el resumen de la tabla 6 muestran que la expresión del marcador específico de las MSCs, el Thy- 1 (CD90) disminuyó significativamente en las hAD-MSCs estimuladas. Esta disminución fue más notable en el caso de las combinaciones de factores. La expresión de las proteínas RPE65, Ezrin, CK8/18, tirosinasa, MerTK, MiTF y Pax6 aumentó significativamente en las hAD-MSCs estimuladas, en comparación de las hAD-MSCs no estimuladas.

Se encontró que todos los factores mostraron efectos antiproliferativos sobre las células del EPR tratadas con MMC en el método de co-cultivo indirecto, mientras que estos efectos negativos se anularon por la presencia de las hAD-MSCs. Además, las hAD-MSCs en el cocultivo indirecto con el EPR inactivado junto con factores, se diferenciaron hacia fenotipos 
del EPR en diferente grado, al ser estimuladas por diferentes combinaciones de factores. Se encontró que las combinaciones de factores, son mejores para la estimulación de la diferenciación, que los factores utilizados individualmente.

\subsection{Co-cultivo indirecto de las hAD-MSCs con neurorretina humana (hNR) en RCM junto con factores y sus combinaciones}

Las hAD-MSCs se estimularon durante la diferenciación hacia fenotipos del EPR mediante el cultivo indirecto de ellas (parte inferior del pocillo) con explantes de hNR de 6x8mm en un sistema de Transwell de placas de 6-pocillos. Los explantes se pusieron sobre la membrana del Transwell. El medio de cultivo empleado fue suplementado con $50 \%$ de hRCM con tres factores (VIP, NIC y ATRA) y sus combinaciones (VIP+NIC, VIP+ATRA, NIC+ATRA y VIP+NIC+ATRA). Se mantuvieron los cultivos durante 15 días. Más tarde se realizaron otros experimentos a 6 y 12 días.

Las hAD-MSCs mejoraron la expresión de marcadores de EPR significativamente cuando se co-cultivaron con hNR en 50 \% de RCM, pero este efecto se potenció por la presencia de los factores y sus combinaciones. Las combinaciones de factores tales como VIP+NIC, NIC+ATRA, y VIP+NIC+ATRA fueron, en general, más eficaces en la inducción de la expresión de proteínas del EPR en las hAD-MSCs que cuando se emplearon individualmente (Fig. 33, 34, 35 y 36 y en la Tabla 7, 8 y 9 página 108 - 112). La tercera serie de experimentos demostró que la expresión de los marcadores de EPR aumentó significativamente a los 12 días en comparación con los de 6 días, salvo en algunos casos (Fig. 35 página 111). Es interesante observar que las hAD-MSCs expresan PEDF citoplasmático en cantidades significativas, siendo éste un factor neuroprotector muy importante secretado por las células del EPR.

Los resultados del análisis de WB mostraron que las hAD-MSCs fueran inducidas parcialmente hacia fenotipos del EPR por la presencia de combinaciones de factores (VIP, VIP+NIC, NIC+ATRA y VIP+NIC+ATRA), en el co-cultivo indirecto con explantes de hNR con medio de crecimiento suplementado con $50 \%$ de RCM.

\section{Estado de los explantes de hNR cultivados con las hAD-MSCs junto con los factores (VIP, NIC y ATRA) y sus combinaciones}

Los explantes neuroretinianos anteriormente descritos se tiñeron con azul de toluidina para observar la morfología general y el estado de las células neurorretinianas. Los cambios específicos en fotorreceptores, sinápsis, células gliales (células de Müller) y astrocitos se analizaron mediante marcaje inmunohistoquímica contra la calbindina (CB), 
la rodopsina (RHO), la sinaptofisina (SYP), la proteína de unión al retinaldehído celular (CRALBP) y la proteína acídaica glial fibrilar (GFAP) (Fig.37 Panel set 1 y 2, Fig.38 página 116-118). La evaluación de los efectos de todos los factores y sus combinaciones se resumió y se representa en el gráfico con el análisis estadístico (Fig.39 página 119). Los explantes del hNR con las hAD-MSCs (control 2) mostraron un grado de preservación significativamente mayor $(\mathrm{P}<0.05)$ que el del grupo control 1 (sólo $\mathrm{RCM}$ ). Los explantes de hNR con hAD-MSCs y factores (NIC y ATRA) no mostraron un mantenimiento significativo frente a los cultivados solo con las hAD-MSCs. Los explantes cultivados solo con VIP y las hAD-MSCs no se preservaron adecuadamente, mostrando signos evidente de gliosis reactiva, una señal patológica de la degeneración retiniana. Los explantes de hNR cultivados en $50 \%$ RCM y hAD-MSCs en combinaciones con VIP+NIC y VIP+NIC+ATRA tuvieron significativamente $(\mathrm{P}<0.05)$ un mejor estado de conservación que los explantes cultivados con las combinaciones de otros factores.

El análisis inmunohistoquímico e histoquímico reveló que los explantes de hNR con las hAD-MSCs se conservan mejor que los explantes sin estas células. En los grupos de tratamiento, NIC y ATRA parecen tener efectos positivos en el mantenimiento de la estructura de la retina, pero no significativamente mejores que los explantes con solo las hAD-MSCs. Se observó una mejor preservación de la neurorretina en el caso de los grupos de tratamiento VIP+NIC y VIP+NIC+ATRA respecto a otros grupos de tratamiento. 


\section{Discusión}

Este trabajo tiene como objetivo probar la utilidad de las hAD-MSCs como futuras candidatas para la reparación de tejidos y para la neuroprotección en la terapia celular de enfermedades degenerativas de la retina, especialmente en la DMAE. En este trabajo las hAD-MSCs se cultivaron en diferentes ambientes que podrían ser similares al entorno subretiniano presente en el ojo de los pacientes afectados por DMAE, en el cual, tanto el EPR como la retina neural se encuentran bajo procesos de degeneración. Estos ambientes in vitro fueron complementados con diferentes factores de diferenciación (VIP, NIC y ATRA) y sus combinaciones, para analizar su influencia en el comportamiento celular. Además, las hAD-MSCs fueron co-cultivadas directa e indirectamente con el EPR inactivado y con hNR en degeneración en presencia y ausencia de los factores y sus combinaciones. En todas estas condiciones diferentes se estudiaron la diferenciación de las hAD-MSCs hacia fenotipos de EPR, así como los efectos de estos microambientes sobre las células del EPR y sobre la retina neural en degeneración.

Existen algunas limitaciones en este trabajo discutidas anteriormente (página 123). A pesar de ellas, creemos que los resultados pueden tener una importancia clínica. Los resultados en este estudio abogan por el uso de las hAD-MSCs junto con factores externos para el trasplante subretiniano en pacientes con DMAE.

\section{Caracterización de las células del EPR y hAD-MSCs}

En nuestra experiencia, las células de hEPR procedentes de donantes jóvenes y sanos en cultivo, tienen más alta viabilidad, pigmentación y capacidad proliferativa que las procedentes de donantes de más edad y enfermos. Las células del EPR muestran una buena adherencia, crecimiento y viabilidad sobre la superficie de poliestireno tratada que sobre los frascos de cultivo y las placas. Además, se demostró que pueden mantener su adhesión, crecimiento y viabilidad sobre el polímero ELR-RGD manteniendo sus características fenotípicas (Singh et al. 2013b, Srivastava et al. 2011a). Por lo tanto este polímero y otros pueden ser utilizados como sustrato de células en el trasplante de EPR diferenciado a partir de células madre. Las células del hEPR pierden su pigmentación y características epiteliales muy rápido, justo después del primer pase. Además, adoptan morfología fibroblástica después de los pases 6-8 la cual va aumentando a medida que se realizan mas pases. Esto puede ser debido a la respuesta de cicatrización de las células epiteliales, pues es un proceso similar al que tiene lugar en cada pase por el tratamiento con tripsina (Leopold et al. 2012). El cultivo primario de hEPR parece ser muy vulnerable a un ligero cambio en el pH del medio de crecimiento. Nuestra experiencia indica que la 
densidad de siembra es muy importante en el mantenimiento de la morfología del hEPR. El análisis de CF muestra que el hEPR en cultivo mantiene la expresión de la proteína RPE65 en los pases más altos (P10) pero hay una pérdida gradual en la intensidad de su expresión durante el subcultivo. Además, los resultados también muestran que no hay contaminación de las células fibroblastoides en el cultivo de las células del hEPR.

Las células de EPR por lo general, emiten mucha autofluorescencia en los estudios inmunológicos debido a la presencia de moléculas de pigmento dentro de ellas, lo que a veces dificulta analizar la señal de fluorescencia mediante el análisis de CF o en estudios de inmunofluorescencia. Los resultados del método desarrollado en nuestro estudio indican, que al incubar con TB tanto las células de pEPR cultivadas como los explantes de retina, se inactiva la auto-fluorescencia de las células del EPR. Por lo tanto, esto puede facilitar la detección con métodos fluorescentes de las proteínas de dichas células pues este método aumenta significativamente la calidad y el valor del análisis, disminuyendo los falsos positivos derivados de la autofluorescencia de estas células en el análisis por FC e inmunofluorescencia (Srivastava et al. 2013, Srivastava et al. 2011).

Tanto el hEPR, las hAD-MSCs como las líneas celulares (AEPR19 y HFF1) se mantuvieron en cultivo y se comprobó que mantenían sus características fenotípicas durante los experimentos. Los resultados de inmunotinción y WB obtenidos en nuestros experimentos de optimización, muestran resultados interesantes: casi todos los marcadores del EPR se expresan en las hAD-MSCs pero algunos lo hacen de forma significativamente mayor. Curiosamente, los estudios inmunológicos indican niveles significativos de la expresión de PEDF en las hAD-MSCs. Esta molécula es secretada específicamente por el EPR. Por lo tanto, puede ser que las hAD-MSCs tengan tendencia a diferenciarse a EPR. Parece que no hay nada descrito anteriormente por otros autores relacionado con este hallazgo, por lo tanto, se deberían de realizar más investigaciones para localizar más proteínas de EPR en las hAD-MSCs así como lograr aumentar su expresión. Además, las hAD-MSCs secretan ciertos factores que también son secretados por el EPR como se mencionará más adelante (Tabla 1 página), esta característica puede ser beneficiosa para el uso de estas células en el tratamiento de las enfermedades de degeneración de la retina.

Las hAD-MSCs se caracterizaron de acuerdo con los criterios establecidos por el ISCT. Las hAD-MSCs demostraron positividad para los marcadores de superficie celular de las MSCs tales como CD90, CD73, CD105 y negatividad para los CD14, CD19, CD34, CD45 y HLA-DR. También demostraron la capacidad de diferenciarse en tres linajes mesodérmicos: condrocitos, osteocitos y adipocitos. 
El porcentaje de células que expresan marcadores de superficie celular específicos de MSCs aumenta en las hAD-MSCs en pases más altos (P16 y P21) según el análisis inmunofenotípico de FC. Este análisis indica que se vuelven más homogéneas durante el sub-cultivo, sin embargo, muestran menor potencial de diferenciación hacia los tres linajes mesodérmicos (condrocitos, osteocitos y adipocitos) en pases más altos. También muestran un crecimiento más lento en estos pasajes, lo que podría ser un indicador de la senescencia celular.

Los efectos de las hAD-MSCs sobre las células del EPR tratadas con MMC en el cocultivo indirecto- Extracto Publicado (Singh et al. 2013a)

Este estudio se realizó para evaluar por el uso de las hAD-MSCs como candidatas en el trasplante subretiniano y así frenar la degeneración del EPR en pacientes con DMAE. Para este propósito se analizaron, en condiciones in vitro, los efectos de las hAD-MSCs sobre la muerte celular inducida por MMC en las células del EPR (ARPE19).

La MMC es un agente alquilante de ADN biorreductor (Rockwell et al. 1982), que se utilizó para inhibir la proliferación celular e inducir la muerte celular. Está demostrado que la MMC produce ROS (por ejemplo, anión superóxido y $\mathrm{H}_{2} \mathrm{O}_{2}$ ) lo que lleva a la muerte celular apoptótica por daños en la cadena de ADN (Koedrith, Seo 2011, Kennedy et al. 1985). También se utilizó para inducir la apoptosis y detener el crecimiento celular en el cultivo del hEPR de modo dosis dependiente (Kang et al. 2001). Por lo tanto, en este estudio, parece que la apoptosis inducida por MMC en células del EPR es similar a la apoptosis que se produce en las células del EPR en degeneración, como en los pacientes con DMAE. Los ensayos de $\mathrm{AB}$ mostraron que la MMC inhibe significativamente la proliferación de las células del EPR.

Mediante un kit de viabilidad/citotoxicidad se midieron dos parámetros de la viabilidad celular reconocidos, por un lado, la actividad esterasa intracelular, y por otro, la integridad de la membrana plasmática. Los resultados mostraron que después de tres semanas de tratamiento con MMC, las células del EPR estaban completamente muertas. Las hAD-MSCs rescataron parcialmente las células del EPR al entrar en proceso de muerte a través de la secreción de algunos factores de crecimiento en el medio de cultivo compartido. La actividad proliferativa de las células del EPR tratadas con MMC y co-cultivadas con las hAD-MSCs fue significativamente mayor que la de las células del EPR tratadas con MMC sin las hAD-MSCs. El medio en el que se cultivaron las hAD-MSCs también demostró que estas células fomentan la proliferación de las células del EPR tratadas con MMC. Estos hallazgos parecen indicar que las hAD-MSCs secretan algunos factores de crecimiento con efecto de 
protección en el medio compartido en el co-cultivo indirecto, que probablemente evitan que las células del EPR tratadas con MMC entren en muerte celular. Estos factores de crecimiento o de protección podrían ser CNTF, BDNF, IGF1, NGF, bFGF y otros. También se demostró que los fibroblastos muestran algunos efectos positivos sobre las células del EPR tratadas con MMC pero estos fueron significativamente menores que los mostrados por las hAD-MSCs. En condiciones in vivo, en pacientes con DMAE seca, las células EPR están en fase de degeneración y liberan varios factores. La inyección de las hAD-MSCs en el espacio subretiniano puede crear un microambiente beneficioso, lo que podría rescatar parcialmente las células degeneradas del EPR. Esto podría ayudar a prevenir la progresión de la DMAE en la población adulta. Los resultados sugieren que las hAD-MSCs tienen la capacidad de rescatar a las células degeneradas del EPR, fomentando así su posterior utilización en el estudio in vivo.

\section{El cultivo de las hAD-MSCS en RCM suplementado con factores y sus combinaciones}

Las hAD-MSCs fueron inducidas a fenotipos de EPR mediante el cultivo en hRCM (medio condiciónado por hEPR) suplementado con factores (VIP, NIC y ATRA) y sus combinaciones (VIP+NIC y NIC+ATRA) durante 21 días. RCM contiene varios factores secretados por las células del EPR en cultivo que tienen papeles pleiotrópicos en la estabilización de la retina. La presencia de RCM ayuda aun mas a crear un entorno similar al del espacio subretiniano. Estos experimentos hacen que las hAD-MSCs se expongan a los factores secretados por el EPR junto con las combinaciones de factores añadidos, lo que induce a la diferenciación de las hAD-MSCs hacia fenotipos de EPR. Este resultado está en línea con otros trabajos (Huang et al. 2012, Vossmerbaeumer et al. 2009). Los resultados obtenidos por WB indican que la expresión de todos los marcadores de EPR detectados, mejora significativamente $(\mathrm{P}<0,01)$ en las hAD-MSCs cultivadas en $50 \%$ RCM, y este efecto es aun mayor $(\mathrm{p}<0.001)$ en presencia de combinaciones de factores (VIP+NIC y NIC+ATRA). La combinación de VIP+NIC tiene más efectos inductivos que la del NIC+ATRA. En el caso de la inducción a fenotipos epiteliales (CK8/18 y PCK), ATRA es más eficaz que los otros factores. Estos resultados son similares a los descritos en otros artículos (Wan et al. 2012, Li et al. 2012, Brzoska et al. 2005). Sin embargo, los resultados indican que el VIP y NIC por sí solos no parecen inducir significativamente el aumento de la expresión de marcadores de EPR, lo que no está en coherencia con otros trabajos (Vossmerbaeumer et al. 2009, Vossmerbaeumer et al. 2008). Curiosamente, VIP en combinación con NIC aumenta significativamente la expresión de los marcadores propios de EPR lo que indica que la diferenciación inducida por la señalización de cada uno de ellos probablemente es complementaria. 
Estos resultados descritos anteriormente y obtenidos mediante WB se confirmaron con técnicas de inmunomarcaje. La expresión de los marcadores del EPR (RPE65 y bestrofina) en las hAD-MSCs inducidas en diferentes condiciones, son similares. Para inducir la diferenciación en las hAD-MSCs, la combinación de NIC con ATRA y VIP funciona mejor que los factores por separado. Ya se demostró que NIC induce a las células pluripotentes inducidas y a las células madre embrionarias a la diferenciación hacia fenotipos de EPR (Buchholz et al. 2013, Buchholz et al. 2009, Idelson et al. 2009). Aunque el proceso de diferenciación en las células madre embrionarias es diferente al de las células madre adultas, ya que estas últimas están en una etapa avanzada de la ruta de diferenciación y esto puede dar señales similares en la vía de diferenciación.

\section{La inducción de la diferenciación de las hAD-MSCs hacia los fenotipos del EPR con BMP-4 y el POS y fRCM (Esta parte del trabajo se realizó en la Universidad de Liverpool)}

En este trabajo preliminar, los resultados demuestran que la fRCM induce la formación de esferas pigmentadas en las hAD-MSCs a los 15 días y que esta tendencia parece estar potenciada por la presencia de BMP-4 en fRCM. La formación de esferas pigmentadas puede ser una de las características en el camino a la diferenciación de las células madre hacia células de EPR (Gong et al. 2008). El estudio inmunocitoquímico también muestra que la BMP-4 aumenta la expresión de marcadores de proteínas del EPR (RPE65 y CK8/18) en las hAD-MSCs inducidas, en comparación con otros controles tales como las hAD-MSCs inducidas y no inducidas con 50\% fRCM. El POS utilizado en el experimento no muestra ningún resultado pues todas las células cultivadas con POS murieron. La razón de la muerte celular no es conocida pero se cree que pudo ser debida a la toxicidad producida por el propio POS. BMP-4 se expresa en la vesícula óptica durante la etapa embrionaria temprana y se ha demostrado que juega un papel crucial en la especificación del EPR y otros componentes del ojo (Haynes et al. 2007, Müller et al. 2007, Du et al. 2010, Furuta, Hogan 1998). Por lo tanto, en base a los resultados preliminares obtenidos en estos experimentos y en otros trabajos publicados, el papel del BMP-4 en la diferenciación de células madre adultas hacia fenotipos de EPR no se puede descartar. Este estudio utiliza fRCM, que parece ser más efectivo que el de hRCM. Esto puede ser debido, probablemente, a que este medio es más rico en algunos de los factores liberados por las células de fEPR frescas y sanas (Kolomeyer et al. 2011). 


\section{Co-cultivo directo de las hAD-MSCs con las células del EPR inactivadas}

Las hAD-MSCs se diferenciaron hacia los fenotipos de EPR en un cultivo con las células del EPR inactivadas. Se ha descrito previamente que la dosis más alta de tratamiento de MMC durante dos horas no sólo inhibe la proliferación de las células del EPR, sino también conduce a la degeneración de las células del EPR por la muerte celular durante el cultivo a largo plazo (después de 3 semanas) (Singh et al. 2013a). Esta condición puede ser ligeramente similar a la situación del EPR subretiniano en la DMAE, donde el EPR está en degeneración debido a influencias ambientales genéticas.

Los estudios de inmunofluorescencia muestran que la expresión de los marcadores del EPR (RPE65, CK8/18, Z01 y Ezrin) está aumentada significativamente en las hAD-MSCs inducidas, en comparación con las hAD-MSCs indiferenciadas. La expresión de ZO-1 está ligeramente aumentada en las hAD-MSCs cuando están muy confluentes pero su localización se limita al citoplasma, mientras que en la condición de diferenciación, tanto la localización como la expresión de ZO-1 mejora en las hAD-MSCs. La localización de ZO-1 en la membrana celular en las hAD-MSCs inducidas es claramente visible en los estudios de inmunocitofluorescencia. Sin embargo, la expresión de ezrina (implicada en la formación de microvellosidades apicales en EPR) es significativamente mayor en las hADMSCs inducidas, pero su patrón no es similar a la expresión en el EPR (control positivo). Se observa claramente que su localización en las hAD-MSCs es diferente al encontrado en las células del EPR. La disminución de la expresión del CD-90 en el las hAD-MSCs inducidas demuestra que se están diferenciando, o perdiendo las características de las MSCs, apoyando así la evidencia de la diferenciación de las hAD-MSCs hacia los fenotipos del EPR en co-cultivo directo.

Cuando las células del EPR tratadas con MMC se marcaron con EdU para distinguirlas en el co-cultivo directo, se observó que se encuentran por debajo de la capa de las hAD-MSCs. Algunas de ellas (las células EPR tratadas con MMC) tenían sus núcleos bajo picnosis (condensación de la cromatina irreversible) y en proceso de cariorrexis (eventos finales de la apoptosis), como se demuestra por los cuerpos o fragmentos fluorescentes rojos intensos en el análisis de inmunocitomarcaje. Este estudio demuestra que los marcadores del EPR se expresan principalmente en las hAD-MSCs y además en las células del EPR que quedan vivas. Como ya se ha descrito, las células del EPR tratadas con MMC entran en proceso de muerte celular si se cultivan solas, mientras que esto no ocurre de manera tan significativa en el co-cultivo con las hAD-MSCs, debido a sus efectos paracrinos antiapoptóticos (Singh et al. 2013a). 
Se observó que las hAD-MSCs en cultivo bajo condiciones inducidas forman esferas oscuras de células pigmentadas. Esto no ocurre con tanta claridad con las no inducidas, pues las esferas de masas celulares son menos pigmentadas y menos frecuentes. Este hecho se asemeja a la formación de neuroesferas pigmentadas por parte de las células madre embrionarias (Gong et al. 2008). Estas esferas de masas celulares formadas por las hAD-MSCs expresan Thy-1 (un marcador específico de las MSCs), pero en la condición inducida, la expresión de Thy-1 se reduce significativamente. Además, son positivas para la expresión de RPE65 en la condición inducida. Este resultado probablemente muestra que las hAD-MSCs en co-cultivo directo con las céulas del EPR inactivadas se diferencian hacia células de EPR, sin embargo, se requieren estudios adicionales para confirmar este resultado de formación de esferas pigmentadas de hAD-MSCs.

La diferenciación de las hAD-MSCs hacia los fenotipos de EPR se confirmó también por el análisis de WB. Las hAD-MSCs aumentan la expresión de las proteínas RPE65 y ZO-1 significativamente $(\mathrm{P}<0,001)$ en el co-cultivo directo comparado con el control. Además las hAD-MSCs expresan mas significativamente RPE65 y ZO-1 $(\mathrm{P}<0,01)$ que las cocultivadas con el EPR en el co-cultivo indirecto. Esto muestra que los contactos intercelulares desempeñan un papel crucial en la diferenciación de las células madre. Estos resultados son similares a los obtenidos en un artículo, en el cual las BM-MSCs de ratón fueron diferenciadas hacia células parecidas al EPR, al co-cultivarlas directamente con las células de EPR tratadas con MMC (Li et al. 2007).

\section{Co-cultivo indirecto de las hAD-MSCs con las células del EPR tratadas con MMC, junto con los factores de diferenciación}

Se ha descrito previamente que en el co-cultivo indirecto, las hAD-MSCs aumentan la actividad proliferativa de las células del EPR tratadas con MMC. Estudio adicional demuestra que los efectos de todos los factores (VIP, NIC y ATRA), y sus diferentes combinaciones tienen efecto supresor sobre la actividad proliferativa de las células de EPR tratadas con MMC, pero en presencia de las hAD-MSCs, estos efectos se invierten de manera significativa, especialmente en una etapa posterior de crecimiento (a los 5 días). Esto demuestra que las hAD-MSCs en combinación con todos estos factores estimulan la viabilidad y la proliferación de las células del EPR tratadas con MMC. También demuestra la importancia de la utilización de las hAD-MSCs junto con combinaciones de factores, para una mayor eficacia en el aumento de la actividad de proliferación y del rescate de las células del EPR que mueren y, al mismo tiempo, en la diferenciación de las hAD-MSCs a los fenotipos de EPR. Sin embargo, hay varios temas en los que se necesita profundizar más, tales como la identificación de los factores secretados por las hAD-MSCs (no inducidas e 
inducidas) y por las células del EPR tratadas con MMC, el mecanismo implicado en estos efectos de rescate, etc. En caso del NIC y VIP+ATRA, el efecto antiproliferativo no es muy pronunciado en comparación con otros factores y sus combinaciones. Cuando las hADMSCs estuvieron expuestas solo a NIC, se observaron menos células hAD-MSCs, lo que podría ser debido a la muerte celular, o a una disminución en la proliferación. Estos hallazgos son similares a los observados en la exposición de las hESCs a NIC (Vaca et al. 2008). Estos resultados sugieren que el NIC tiene un papel fuera de la supervivencia de la célula.

La disminución significativa en la expresión de CD90 en las hAD-MSCs inducidas, muestra que las hAD-MSCs están diferenciándose a otros tipos de células, mientras que el aumento significativo en la expresión de los marcadores de EPR (RPE65, Ezrin, CK8/18, tirosinasa, MerTK, MITF, Pax6) muestra su diferenciación hacia los fenotipos de EPR. En todos los casos, hay un aumento significativo en la expresión de marcadores de EPR y una disminución significativa en la expresión de CD90 en las hAD-MSCs inducidas, en comparación con las hAD-MSCs no inducidas, pero especialmente las combinaciones de factores parecen potenciar significativamente estos efectos. La expresión diferencial de los marcadores del EPR con cada factor y sus combinaciones indican sus funciones en las diferentes etapas de la vía de señalización de la diferenciación. La combinación VIP+ATRA es en general, mejor que otras combinaciones, mientras que la misma combinación tiene más efectos antiproliferativos sobre las células del EPR tratadas con MMC. Sin embargo, el resumen general de este experimento, muestra que las combinaciones de los factores funcionan mejor que el uso de estos factores por separado, lo que explica la función complementaria de cada uno de ellos en la señalización de la diferenciación de las hADMSCs hacia los fenotipos de EPR.

\section{Co-cultivo indirecto de las hAD-MSCs con explantes del hNR en RCM, junto con los factores diferenciadores y sus combinaciones}

Junto con EPR, la neurorretina (especialmente PRs) también se ve afectada en las enfermedades degenerativas de la retina. Por lo tanto, se planteo al evaluar los efectos de las hAD-MSCs junto con diferentes factores y sus combinaciones sobre la degeneración retiniana. El co-cultivo indirecto de las hAD-MSCs con explantes de hNR en 50\% de RCM demuestra que hay un aumento significativo en la expresión de marcadores de EPR en las hAD-MSCs inducidas, pero la diferenciación hacia los fenotipos de EPR está potenciada por la presencia de factores (VIP, NIC y ATRA) y sus combinaciones (VIP+NIC, NIC+ATRA y VIP+NIC+ATRA). La expresión del marcador específico de las MSCs (CD90) se reduce significativamente en las hAD-MSCs inducidas, especialmente en el caso de las 
combinaciones de factores, lo que indica el cambio del fenotipo mesenquimal de las hADMSCs hacia otros tipos de células. Es interesante observar que en nuestros experimentos, las MSCs expresan PEDF (un factor neuroprotector secretado por el EPR) de forma muy significativa. Este es uno de los hallazgos acerca de las hAD-MSCs que anima a seguir adelante para estudiar el potencial de las hAD-MSCs en el trasplante subretiniano y ralentizar el proceso de la degeneración de PRs en las fases tempranas de la DMAE. La expresión de PEDF en las hAD-MSCs se mantiene en el cultivo que contiene combinaciones de factores, mientras que su expresión disminuye significativamente cuando solo hay un factor. Entre las hAD-MSCs inducidas con y sin factores, no hay cambios significativos en la expresión de los marcadores específicos del EPR inmaduro (Mitf y Pax6) ni en el PEDF. En este experimento, VIP y NIC inducen significativamente a las hAD-MSCs inducidas a fenotipos de EPR si se comparan con las hAD-MSCs inducidas sin ningún tipo de factores. Esto está en consonancia con los artículos anteriores que dicen que VIP induce la diferenciación parcial de las células madre a fenotipos de EPR (Vossmerbaeumer et al. 2009, Vossmerbaeumer et al. 2008). Las combinaciones VIP+NIC, NIC+ATRA y VIP+NIC+ATRA tienen más efectos sobre la diferenciación de las hAD-MSCs hacia los fenotipos de EPR que los factores por separado. Esto indica que hay varios factores importantes durante el proceso de la diferenciación. Esta condición podría ser ligeramente parecida al entorno subretiniano en la DMAE, donde la retina está en proceso de degeneración. Estos resultados confirman de nuevo la importancia de la utilización de hAD-MSCs junto con la combinación de factores en la investigación del rescate y la reparación de los daños en la retina en determinadas enfermedades degenerativas.

Todos los factores utilizados en este estudio juegan papeles pleiotrópicos en la diferenciación y maduración del EPR y de las células madre. Se ha descrito que VIP induce la diferenciación de las células madre adultas a células de EPR (Buchholz et al. 2013, Koh 2000, Vossmerbaeumer et al. 2009, Vossmerbaeumer et al. 2008). La concentración de VIP utilizada para la diferenciación hacia los fenotipos de EPR está en el rango de 10-6-10-3 M. Del mismo modo se ha descrito la utilización del NIC para inducir la diferenciación de células madre pluripotentes inducidas y células madre embrionarias hacia los fenotipos de EPR (Buchholz et al. 2013, Buchholz et al. 2009, Idelson et al. 2009). NIC también inhibe la histona desacetilasa dependiente de NAD (Peled et al. 2012, Imai et al. 2000), que desempeña un papel importante en la expresión diferencial de genes, el silenciamiento, la estabilidad del genoma, la trasducción de señales, el crecimiento celular y la muerte celular. ATRA juega un papel importante en la diferenciación de células madre mediante la alteración de los cambios epigenéticos en el ADN, así como en la proteína histona (Gudas 2013). También se ha descrito que induce fenotipos epiteliales en las MSCs (Wan et al. 
2012, Li et al. 2012, Brzoska et al. 2005) y que regula el perfil de expresión de células madre mediante la interacción con receptores nucleares RAR y RXR. Tanto NIC como ATRA están probablemente implicados en la iniciación del proceso de diferenciación, mientras que VIP está implicado en la diferenciación y maduración del EPR (Koh 2000).

Las condiciones de diferenciación en estos experimentos son muy complejas debido a los factores extrínsecos utilizados y a las numerosas moléculas presentes en el medio de crecimiento que son secretadas por las células. Aunque es muy difícil explicar el papel exacto de cada factor en el proceso de diferenciación, los resultados muestran que múltiples moléculas y factores se complementan entre sí, para iniciar el proceso de señalización de la diferenciación en las hAD-MSCs inducidas hacia los fenotipos de EPR y ayudar en la maduración posterior del sus fenotipos.

\section{La diferenciación de las hAD-MSCs hacía las células de EPR}

En este estudio se utilizaron varios métodos para diferenciar las MSCs hacía las células del EPR. Entre los diversos métodos utilizados, en el método de co-cultivo directo se obtuvieron mejores resultados. Esto demuestra claramente el papel crucial en el proceso de diferenciación de la comunicación entre las células y/o de las células con el sustrato, además de las funciones de las moléculas exógenas o factores. Deben hacerse nuevos estudios para aclarar detalladamente el mecanismo de inducción a la diferenciación de los factores utilizados. Las hAD-MSCs se diferenciaron a los fenotipos de EPR en diferentes grados cuando se cultivaron con las células del EPR o con el medio condicionado por ellas. Este resultado está en línea con otros artículos anteriores sobre la diferenciación de las hAD-MSCs hacia los fenotipos de EPR (Vossmerbaeumer et al. 2009, Huang et al. 2012). Como se ha descrito anteriormente, todos los factores utilizados en este estudio tienen papeles pleiotrópicos en varios procesos de diferenciación de las células madre. ATRA potencia las características epiteliales en la diferenciación de las hAD-MSCs hacia el EPR. En este estudio, se demostró que VIP produce resultados variables en diferentes experimentos y NIC por sí solo no muestra efectos significativos en el proceso de diferenciación. De todos los métodos de diferenciación utilizados, se observó que en los que había combinaciones de los factores se obtuvieron mejores resultados que en los que se utilizaron factores individuales. En el primer caso, los factores se potencian el uno al otro. La presencia de explantes neurorretinianos mejora ligeramente la capacidad de diferenciación de los factores. 
Estado de los explantes neurorretinianos cultivados con hAD-MSCs junto con los factores (VIP, NIC y ATRA) y sus combinaciones

Los explantes hNR cultivados en las mismas condiciones utilizadas para la diferenciación de las hAD-MSCs se estudiaron para evaluar su estado de preservación mediante la observación de la estructura general (tinción con azul de toluidina) y específica (inmunomarcaje contra diferentes proteínas específicas de la retina).

El método desarrollado en este estudio podría ser muy útil para comprobar los efectos neuroprotectores de sustancias biológicas (incluidas las células) o químicas, mediante su incubación con explantes neurorretinianos y co-cultivados con células del EPR. Este método es el resultado de la modificación del cultivo organotípico de neurorretina previamente desarrollado en nuestro laboratorio (Fernandez-Bueno et al. 2012, Fernandez-Bueno et al. 2013, Fernandez-Bueno et al. 2008).

Los resultados demuestran que los explantes co-cultivados con las hAD-MSCs se conservan mejor que los cultivados solamente con 50\% RCM (control 1). Los resultados encontrados mediante tinción de GFAP en los explantes co-cultivados con hAD-MSCs y VIP revelan astrogliosis reactiva intensa. Este evento podría deberse a que el VIP está involucrado en la inducción de astrocitosis reactiva en el sistema nervioso central (Nishimoto et al. 2011). A pesar de que todos los factores utilizados (VIP, NIC y ATRA) se han descrito como neuroprotectores, en nuestros experimentos no se encontraron diferencias significativas entre los explantes co-cultivados con hAD-MSCs y los cocultivados, además de con estas células, con estos factores (individualmente). En una de las muestras de explantes de hNR cultivadas con VIP+NIC muchos de los segmentos externos de los PRs quedaron intactos. Esto siguiere que la combinación de VIP+NIC con las hAD-MSCs muestra una mayor capacidad de neuroprotección. Con las combinaciones de VIP+NIC y VIP+NIC+ATRA se observaron neurorretinas mejor conservadas que con otros tratamientos. Esto confirma una vez más, que las funciones neuroprotectoras de cada factor probablemente están produciendo efectos sinérgicos.

Se ha descrito en varios estudios que todos los factores utilizados para la diferenciación tienen un papel neuroprotector. El papel neuroprotector de las MSCs (bien establecido en diversos estudios) está mediado por los numerosos factores neuroprotectores secretados (factores paracrinos) por ellas. Los efectos neuroprotectores de VIP, NIC y ATRA utilizados por separado en explantes del hNR pueden ser encubiertos por los efectos neuroprotectores de las hAD-MSCs. En el caso de las combinaciones VIP+NIC y el 
VIP+NIC+ATRA parece que cada factor potencia los efectos neuroprotectores de los demás. 


\section{Conclusiones}

1. Las células del EPR y las hAD-MSCs mantienen su crecimiento, viabilidad y sus fenotipos en el cultivo celular utilizando métodos adecuados de cultivo, sin embargo existe una pérdida gradual de dichas propiedades durante los consiguientes sub-cultivos. Se establecieron diferentes protocolos para la comprobación rutinaria de diferentes parámetros de las células del EPR.

2. La diferenciación en hAD-MSCs es más efectiva en co-cultivo directo con células del EPR que en co-cultivo indirecto, sugiriendo que el contacto célula-célula juega un papel muy importante en el proceso de diferenciación de las hAD-MSCs hacia fenotipos de EPR.

3. Los microambientes creados por los factores (VIP, NIC y ATRA) y sus combinaciones con RCM no sólo estimulan la diferenciación de las hAD-MSCs hacia fenotipos de EPR, sino que también ayudan al mantenimiento de los explantes de retina neural en condiciones de cultivo in vitro.

4. La combinación de factores (especialmente VIP+NIC) son más efectivas en el rescate de las células del EPR en proceso de degeneración y de los explantes de neurorretina, así como en la estimulación de la diferenciación de las hAD-MSCs hacia fenotipos de EPR, que los factores utilizados individualmente.

Las hAD-MSCs y los factores de diferenciación (VIP, NIC y ATRA) junto con las moléculas secretadas por las células del EPR, lo que indica que podría ser útiles para no sólo sustituir parcialmente el EPR enfermo y en degeneración, sino que también pueden retrasar y rescatar al EPR en proceso degenerativo y a la retina neural. 


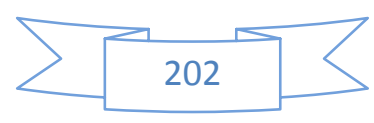




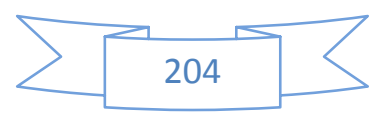


ABDELSALAM, A.; DEL PRIORE, L.and ZARBIN, M. A. Drusen in Age-Related Macular Degeneration: Pathogenesis, Natural Course, and Laser Photocoagulation-Induced Regression. Survey of Ophthalmology, Jul-Aug, 1999, vol. 44, no. 1, pp. 1-29. ISSN 0039-6257; 0039-6257.

ABE, T., et al. Autologous Iris Pigment Epithelial Cell Transplantation in Monkey Subretinal Region. Current Eye Research, Apr, 2000a, vol. 20, no. 4, pp. 268-275. ISSN 0271-3683; 02713683 .

ABE, T., et al. Auto Iris Pigment Epithelial Cell Transplantation in Patients with Age-Related Macular Degeneration: Short-Term Results. The Tohoku Journal of Experimental Medicine, May, 2000b, vol. 191, no. 1, pp. 7-20. ISSN 0040-8727; 0040-8727.

ABE, T., et al. Iris Pigment Epithelial Cell Transplantation for Degenerative Retinal Diseases. Progress in Retinal and Eye Research, 20070117, May, 2007, vol. 26, no. 3, pp. 302-321. ISSN 1350-9462; 1350-9462.

ADAMIS, A. P., et al. Synthesis and Secretion of Vascular Permeability Factor/Vascular Endothelial Growth Factor by Human Retinal Pigment Epithelial Cells. Biochemical and Biophysical Research Communications, Jun 15, 1993, vol. 193, no. 2, pp. 631-638. ISSN 0006-291X; 0006-291X.

ADLER, R.; and BELECKY-ADAMS, T. L. The Role of Bone Morphogenetic Proteins in the Differentiation of the Ventral Optic Cup. Development (Cambridge, England), Jul, 2002, vol. 129 , no. 13 , pp. 3161-3171. ISSN 0950-1991; 0950-1991.

Age-Related Eye Disease Study Research Group. A Randomized, Placebo-Controlled, Clinical Trial of High-Dose Supplementation with Vitamins C and $\mathrm{E}$ and Beta Carotene for Age-Related Cataract and Vision Loss: AREDS Report no. 9. Archives of Ophthalmology, Oct, 2001a, vol. 119, no. 10, pp. 1439-1452. ISSN 0003-9950; 0003-9950.

Age-Related Eye Disease Study Research Group. A Randomized, Placebo-Controlled, Clinical Trial of High-Dose Supplementation with Vitamins C and E, Beta Carotene, and Zinc for Age-Related Macular Degeneration and Vision Loss: AREDS Report no. 8. Archives of Ophthalmology, Oct, 2001b, vol. 119, no. 10, pp. 1417-1436. ISSN 0003-9950; 0003-9950.
Age-Related Eye Disease Study Research Group, et al. The Relationship of Dietary Carotenoid and Vitamin A, E, and C Intake with Age-Related Macular Degeneration in a Case-Control Study: AREDS Report no. 22. Archives of Ophthalmology, Sep, 2007, vol. 125, no. 9, pp. 1225-1232. ISSN 0003-9950; 0003-9950.

AGGARWAL, S.; and PITTENGER, M. F. Human Mesenchymal Stem Cells Modulate Allogeneic Immune Cell Responses. Blood, 20041019, Feb 15, 2005, vol. 105, no. 4, pp. 18151822. ISSN 0006-4971; 0006-4971.

AHLEMEYER, B., et al. Retinoic Acid Reduces Apoptosis and Oxidative Stress by Preservation of SOD Protein Level. Free Radical Biology \& Medicine, May 15, 2001, vol. 30, no. 10, pp. 1067 1077. ISSN 0891-5849; 0891-5849.

AHLEMEYER, B.; HUHNE, R.and KRIEGLSTEIN, J. Retinoic Acid Potentiated the Protective Effect of NGF Against StaurosporineInduced Apoptosis in Cultured Chick Neurons by Increasing the trkA Protein Expression. Journal of Neuroscience Research, Jun 15, 2000, vol. 60, no. 6, pp. 767-778. ISSN 0360-4012; 0360-4012.

AISENBREY, S., et al. Macular Translocation with 360 Degrees Retinotomy for Exudative AgeRelated Macular Degeneration. Archives of Ophthalmology, Apr, 2002, vol. 120, no. 4, pp. 451-459. ISSN 0003-9950; 0003-9950.

ALGVERE, P. V., et al. Transplantation of Fetal Retinal Pigment Epithelium in Age-Related Macular Degeneration with Subfoveal Neovascularization. Graefe's Archive for Clinical and Experimental Ophthalmology $=$ Albrecht Von Graefes Archiv Fur Klinische Und Experimentelle Ophthalmologie, Dec, 1994, vol. 232, no. 12, pp. 707-716. ISSN 0721-832X; 0721-832X.

ALGVERE, P. V., et al. Transplantation of RPE in Age-Related Macular Degeneration: Observations in Disciform Lesions and Dry RPE Atrophy. Graefe's Archive for Clinical and Experimental Ophthalmology = Albrecht Von Graefes Archiv Fur Klinische Und Experimentelle Ophthalmologie, Mar, 1997, vol. 235, no. 3, pp. 149-158. ISSN 0721-832X; 0721-832X.

ALGVERE, P. V.; GOURAS, P.and DAFGARD KOPP, E. Long-Term Outcome of RPE Allografts in Non-Immunosuppressed Patients with AMD. European Journal of Ophthalmology, Jul-Sep, 
1999, vol. 9, no. 3, pp. 217-230. ISSN 1120-6721; $1120-6721$

ALLAN, B. Closer to Nature: New Biomaterials and Tissue Engineering in Ophthalmology. The British Journal of Ophthalmology, Nov, 1999, vol. 83 , no. 11 , pp. 1235-1240. ISSN 0007-1161; 0007 1161 .

ALLAY, J. A., et al. LacZ and Interleukin-3 Expression in Vivo After Retroviral Transduction of Marrow-Derived Human Osteogenic Mesenchymal Progenitors. Human Gene Therapy, Aug 10, 1997, vol. 8, no. 12, pp. 1417-1427. ISSN 1043-0342; 1043-0342.

ALON, T., et al. Vascular Endothelial Growth Factor Acts as a Survival Factor for Newly Formed Retinal Vessels and has Implications for Retinopathy of Prematurity. Nature Medicine, Oct, 1995, vol. 1, no. 10, pp. 1024-1028. ISSN 1078$8956 ; 1078-8956$.

ALT, Eckhard, et al. Fibroblasts Share Mesenchymal Phenotypes with Stem Cells, but Lack their Differentiation and Colony-Forming Potential. Biology of the Cell / Under the Auspices of the European Cell Biology Organization, apr, 2011, vol. 103, no. 4, pp. 197-208. ISSN 1768$322 \mathrm{X}$.

AMBATI, Jayakrishna, et al. Age-Related Macular Degeneration: Etiology, Pathogenesis, and Therapeutic Strategies. Survey of Ophthalmology, may, 2003, vol. 48, no. 3, pp. 257-293. ISSN 00396257.

AMBATI, Jayakrishna; and FOWLER, Benjamin J. Mechanisms of Age-Related Macular Degeneration. Neuron, jul, 2012, vol. 75, no. 1, pp. 26-39. ISSN 1097-4199.

ANGHILERI, E., et al. Neuronal Differentiation Potential of Human Adipose-Derived Mesenchymal Stem Cells. Stem Cells and Development, Oct, 2008, vol. 17, no. 5, pp. 909 916. ISSN 1557-8534; 1547-3287.

ARAMANT, R. B.; SEILER, M. J.and BALL, S. L. Successful Cotransplantation of Intact Sheets of Fetal Retina with Retinal Pigment Epithelium. Investigative Ophthalmology I\& Visual Science, jun, 1999, vol. 40, no. 7, pp. 1557-1564. ISSN 0146-0404.

ARAMANT, Robert B.; and SEILER, Magdalene J. Progress in Retinal Sheet Transplantation. Progress in Retinal and Eye Research, sep, 2004, vol. 23, no. 5, pp. 475-494. ISSN 1350-9462.
ARAMANT, Robert B.; and SEILER, Magdalene J. Retinal Transplantation--Advantages of Intact Fetal Sheets. Progress in Retinal and Eye Research, jan, 2002a, vol. 21, no. 1, pp. 57-73. ISSN 1502852012; 1350-9462.

ARAMANT, Robert B.; and SEILER, Magdalene J. Transplanted Sheets of Human Retina and Retinal Pigment Epithelium Develop Normally in Nude Rats. Experimental Eye Research, aug, 2002b, vol. 75, no. 2, pp. 115-125. ISSN 00144835 .

ARNALICH-MONTIEL, F., et al. AdiposeDerived Stem Cells are a Source for Cell Therapy of the Corneal Stroma. Stem Cells (Dayton, Ohio), 20071206, Feb, 2008, vol. 26, no. 2, pp. 570-579. ISSN 1549-4918; 1066-5099.

ARNHOLD, S., et al. Transplantation of Bone Marrow-Derived Mesenchymal Stem Cells Rescue Photoreceptor Cells in the Dystrophic Retina of the Rhodopsin Knockout Mouse. Graefe's Archive for Clinical and Experimental Ophthalmology = Albrecht Von Graefes Archiv F|\{U\}R Klinische Und Experimentelle Ophthalmologie, mar, 2007, vol. 245, no. 3, pp. 414-422. ISSN 0721-832X.

ARNHOLD, S., et al. Neurally Selected Embryonic Stem Cells Induce Tumor Formation After Long-Term Survival Following Engraftment into the Subretinal Space. Investigative Ophthalmology \& Visual Science, Dec, 2004, vol. 45 , no. 12 , pp. 4251-4255. ISSN 0146-0404; 01460404.

ARNHOLD, Stefan, et al. Adenovirally Transduced Bone Marrow Stromal Cells Differentiate into Pigment Epithelial Cells and Induce Rescue Effects in RCS Rats. Investigative Ophthalmology I\& Visual Science, sep, 2006, vol. 47, no. 9, pp. 4121-4129. ISSN 0146-0404.

ASARI, S., et al. Mesenchymal Stem Cells Suppress B-Cell Terminal Differentiation. Experimental Hematology, May, 2009, vol. 37, no. 5, pp. 604-615. ISSN 1873-2399; 0301-472X.

ASLAM, T., et al. Micronutrients in Age-Related Macular Degeneration. Ophthalmologica.Journal International D'Ophtalmologie.International Journal of Ophthalmology.Zeitschrift Fur Augenheilkunde, 20121120, 2013, vol. 229, no. 2, pp. 75-79. ISSN 1423-0267; 0030-3755.

ATOUI, R.; SHUM-TIM, D.and CHIU, R. C. Myocardial Regenerative Therapy: Immunologic Basis for the Potential "Universal Donor Cells". The Annals of Thoracic Surgery, Jul, 2008, vol. 86, no. 1 , pp. 327-334. ISSN 1552-6259; 0003-4975. 
AUGELLO, A., et al. Bone Marrow Mesenchymal Progenitor Cells Inhibit Lymphocyte Proliferation by Activation of the Programmed Death 1 Pathway. European Journal of Immunology, May, 2005, vol. 35, no. 5, pp. 1482-1490. ISSN 00142980; 0014-2980.

AUGOOD, C., et al. Oily Fish Consumption, Dietary Docosahexaenoic Acid and Eicosapentaenoic Acid Intakes, and Associations with Neovascular Age-Related Macular Degeneration. The American Journal of Clinical Nutrition, Aug, 2008, vol. 88, no. 2, pp. 398-406. ISSN 1938-3207; 0002-9165.

AUQUIER, P., et al. Comparison of Anxiety, Pain and Discomfort in Two Procedures of Hematopoietic Stem Cell Collection: Leukacytapheresis and Bone Marrow Harvest. Bone Marrow Transplantation, Oct, 1995, vol. 16, no. 4, pp. 541-547. ISSN 0268-3369; 0268-3369.

AYOUB, I. A., et al. Nicotinamide Reduces Infarction Up to Two Hours After the Onset of Permanent Focal Cerebral Ischemia in Wistar Rats. Neuroscience Letters, Jan 4, 1999, vol. 259, no. 1, pp. 21-24. ISSN 0304-3940; 0304-3940.

BAIGUERA, S., et al. Tissue Engineered Human Tracheas for in Vivo Implantation. Biomaterials, 20100825, Dec, 2010, vol. 31, no. 34, pp. 89318938. ISSN 1878-5905; 0142-9612.

BAIGUERA, S., et al. Mesenchymal Stromal Cells for Tissue-Engineered Tissue and Organ Replacements. Transplant International : Official Journal of the European Society for Organ Transplantation, 20120116, Apr, 2012, vol. 25, no. 4, pp. 369-382. ISSN 1432-2277; 0934-0874.

BAKALL, B., et al. Aflibercept Therapy for Exudative Age-Related Macular Degeneration Resistant to Bevacizumab and Ranibizumab. American Journal of Ophthalmology, 20130522, Jul, 2013, vol. 156, no. 1, pp. 15-22.e1. ISSN 1879-1891; 0002-9394.

BALEMANS, W.; and VAN HUL, W. Extracellular Regulation of BMP Signaling in Vertebrates: A Cocktail of Modulators. Developmental Biology, Oct 15, 2002, vol. 250, no. 2, pp. 231-250. ISSN 0012-1606; 0012-1606.

BAN, Y.; and RIZZOLO, L. J. Regulation of Glucose Transporters during Development of the Retinal Pigment Epithelium. Brain Research.Developmental Brain Research, may, 2000, vol. 121, no. 1, pp. 89-95. ISSN 0165-3806.
BANAS, A., et al. Rapid Hepatic Fate Specification of Adipose-Derived Stem Cells and their Therapeutic Potential for Liver Failure. Journal of Gastroenterology and Hepatology, 20080625, Jan, 2009, vol. 24, no. 1, pp. 70-77. ISSN 1440-1746; 0815-9319.

BANAS, Agnieszka, et al. Adipose TissueDerived Mesenchymal Stem Cells as a Source of Human Hepatocytes. Hepatology (Baltimore, Md.), jul, 2007, vol. 46, no. 1, pp. 219-228. ISSN 0270-9139.

BARBARA, Santa. E MBRYONIC S TEM C ELLS / I NDUCED P LURIPOTENT S TEM C ELLS Derivation of Functional Retinal Pigmented Epithelium from Induced, 2009, pp. 2427-2434.

BARRY, F., et al. Chondrogenic Differentiation of Mesenchymal Stem Cells from Bone Marrow: Differentiation-Dependent Gene Expression of Matrix Components. Experimental Cell Research, Aug 15, 2001, vol. 268, no. 2, pp. 189-200. ISSN 0014-4827; 0014-4827.

BARRY, F. P., et al. Immunogenicity of Adult Mesenchymal Stem Cells: Lessons from the Fetal Allograft. Stem Cells and Development, Jun, 2005, vol. 14, no. 3, pp. 252-265. ISSN 1547-3287; 1547-3287.

BAUER-KREISEL，P.; GOEPFERICH，A.and BLUNK, T. Cell-Delivery Therapeutics for Adipose Tissue Regeneration. Advanced Drug Delivery Reviews, 20100413, Jun 15, 2010, vol. 62 , no. 7-8, pp. 798-813. ISSN 1872-8294; 0169$409 \mathrm{X}$.

BAZAN, N. G.; RODRIGUEZ DE TURCO, E. B.and GORDON, W. C. Docosahexaenoic Acid Supply to the Retina and its Conservation in Photoreceptor Cells by Active Retinal Pigment Epithelium-Mediated Recycling. World Review of Nutrition and Dietetics, 1994, vol. 75, pp. 120123. ISSN 0084-2230; 0084-2230.

BEATTY, S., et al. The Role of Oxidative Stress in the Pathogenesis of Age-Related Macular Degeneration. Survey of Ophthalmology, Sep-Oct, 2000, vol. 45, no. 2, pp. 115-134. ISSN 00396257; 0039-6257.

BECERRA, S. P., et al. Pigment EpitheliumDerived Factor in the Monkey Retinal Pigment Epithelium and Interphotoreceptor Matrix: Apical Secretion and Distribution. Experimental Eye Research, Feb, 2004, vol. 78, no. 2, pp. 223-234. ISSN 0014-4835; 0014-4835. 
BEEREPOOT, L. V., et al. Up-Regulation of Vascular Endothelial Growth Factor Production by Iron Chelators. Cancer Research, Aug 15, 1996, vol. 56, no. 16, pp. 3747-3751. ISSN 0008-5472; 0008-5472.

BELL, E., et al. Living Tissue Formed in Vitro and Accepted as Skin-Equivalent Tissue of Full Thickness. Science (New York, N.Y.), Mar 6, 1981, vol. 211, no. 4486, pp. 1052-1054. ISSN 0036$8075 ; 0036-8075$.

BERMAN, D. M., et al. Mesenchymal Stem Cells Enhance Allogeneic Islet Engraftment in Nonhuman Primates. Diabetes, 20100709, Oct, 2010, vol. 59, no. 10, pp. 2558-2568. ISSN 1939327X; 0012-1797.

BEYER NARDI, N.; and DA SILVA MEIRELLES, L. Mesenchymal Stem Cells: Isolation, in Vitro Expansion and Characterization. Handbook of Experimental Pharmacology, 2006, vol. (174), no. 174, pp. 249-282. ISSN 0171-2004; 0171-2004.

BEYTH, S., et al. Human Mesenchymal Stem Cells Alter Antigen-Presenting Cell Maturation and Induce T-Cell Unresponsiveness. Blood, 20041028, Mar 1, 2005, vol. 105, no. 5, pp. 22142219. ISSN 0006-4971; 0006-4971.

BHARTI, K.; MILLER, S. S.and ARNHEITER, H. The New Paradigm: Retinal Pigment Epithelium Cells Generated from Embryonic Or Induced Pluripotent Stem Cells. Pigment Cell \& Melanoma Research, 20101007, Feb, 2011, vol. 24, no. 1 , pp. 21-34. ISSN 1755-148X; 1755-1471.

BHARTI, K., et al. The Other Pigment Cell: Specification and Development of the Pigmented Epithelium of the Vertebrate Eye. Pigment Cell Research / Sponsored by the European Society for Pigment Cell Research and the International Pigment Cell Society, Oct, 2006, vol. 19, no. 5, pp. 380-394. ISSN 0893-5785; 0893-5785.

BI, Y., et al. Adenovirus-Mediated RAR-Beta Over-Expression Enhances ATRA-Induced Neuronal Differentiation of Rat Mesenchymal Stem Cells. Archives of Medical Science : AMS, 20121106, Apr 20, 2013, vol. 9, no. 2, pp. 314322. ISSN 1734-1922; 1734-1922.

BIANCO, P.; and GEHRON ROBEY, P. Marrow Stromal Stem Cells. The Journal of Clinical Investigation, Jun, 2000, vol. 105, no. 12, pp. 1663-1668. ISSN 0021-9738; 0021-9738.

BIARNÉS, Marc, et al. Update on Geographic Atrophy in Age-Related Macular Degeneration.
Optometry and Vision Science : Official Publication of the American Academy of Optometry, jul, 2011, vol. 88, no. 7, pp. 881-889. ISSN 1538-9235.

BIEBACK, Karen; and BRINKMANN, Irena. Mesenchymal Stromal Cells from Human Perinatal Tissues: From Biology to Cell Therapy. World Journal of Stem Cells, aug, 2010, vol. 2, no. 4, pp. 81-92. ISSN 1948-0210.

BIEBACK, Karen, et al. Critical Parameters for the Isolation of Mesenchymal Stem Cells from Umbilical Cord Blood. Stem Cells (Dayton, Ohio), jan, 2004, vol. 22, no. 4, pp. 625-634. ISSN 10665099.

BIEBACK, Karen, et al. Mesenchymal Stromal Cells (MSCs): Science and F(R)Iction. Journal of Molecular Medicine (Berlin, Germany), jul, 2012, vol. 90, no. 7, pp. 773-782. ISSN 1432-1440.

BINDER, S., et al. Outcome of Transplantation of Autologous Retinal Pigment Epithelium in AgeRelated Macular Degeneration: A Prospective Trial. Investigative Ophthalmology \& Visual Science, Nov, 2004, vol. 45, no. 11, pp. 41514160. ISSN 0146-0404; 0146-0404.

BINDER, S., et al. Transplantation of Autologous Retinal Pigment Epithelium in Eyes with Foveal Neovascularization Resulting from Age-Related Macular Degeneration: A Pilot Study. American Journal of Ophthalmology, Feb, 2002, vol. 133, no. 2, pp. 215-225. ISSN 0002-9394; 0002-9394.

BINDER, Susanne, et al. Transplantation of the $\mathrm{RPE}$ in AMD. Progress in Retinal and Eye Research, sep, 2007, vol. 26, no. 5, pp. 516-554. ISSN 1350-9462.

BINDEWALD, A., et al. Classification of Fundus Autofluorescence Patterns in Early Age-Related Macular Disease. Investigative Ophthalmology \& Visual Science, Sep, 2005, vol. 46, no. 9, pp. 33093314. ISSN 0146-0404; 0146-0404.

BLAAUWGEERS, H. G., et al. Polarized Vascular Endothelial Growth Factor Secretion by Human Retinal Pigment Epithelium and Localization of Vascular Endothelial Growth Factor Receptors on the Inner Choriocapillaris. Evidence for a Trophic Paracrine Relation. The American Journal of Pathology, Aug, 1999, vol. 155, no. 2, pp. 421428. ISSN 0002-9440; 0002-9440.

BLUM, Barak; and BENVENISTY, Nissim. The Tumorigenicity of Human Embryonic Stem Cells. Advances in Cancer Research, jan, 2008, vol. 100, no. 08, pp. 133-158. ISSN 0065-230X. 
BLUM, Barak; and BENVENISTY, Nissim. Clonal Analysis of Human Embryonic Stem Cell Differentiation into Teratomas. Stem Cells (Dayton, Ohio), aug, 2007, vol. 25, no. 8, pp. 1924-1930. ISSN 1066-5099.

BONILHA, V. L.; FINNEMANN, S. C.and RODRIGUEZ-BOULAN, E. Ezrin Promotes Morphogenesis of Apical Microvilli and Basal Infoldings in Retinal Pigment Epithelium. The Journal of Cell Biology, dec, 1999, vol. 147, no. 7, pp. 1533-1548. ISSN 0021-9525.

BONILHA, V. L.; and RODRIGUEZ-BOULAN, E. Polarity and Developmental Regulation of Two PDZ Proteins in the Retinal Pigment Epithelium. Investigative Ophthalmology I\& Visual Science, dec, 2001, vol. 42, no. 13, pp. 3274-3282. ISSN 0146-0404.

BOQUEST, A. C., et al. CpG Methylation Profiles of Endothelial Cell-Specific Gene Promoter Regions in Adipose Tissue Stem Cells Suggest Limited Differentiation Potential Toward the Endothelial Cell Lineage. Stem Cells (Dayton, Ohio), 20061214, Apr, 2007, vol. 25, no. 4, pp. 852-861. ISSN 1066-5099; 1066-5099.

BOQUEST, Andrew C., et al. Isolation and Transcription Profiling of Purified Uncultured Human Stromal Stem Cells : Alteration of Gene Expression After in Vitro Cell Culture $\hat{A}-i, 2005$, vol. 16, no. March, pp. 1131-1141.

BOUCHERIE, Cl'edric; SOWDEN, Jane C.and ALI, Robin R. Induced Pluripotent Stem Cell Technology for Generating Photoreceptors. Regenerative Medicine, jul, 2011, vol. 6, no. 4, pp. 469-479. ISSN 1746-076X.

BOULTON, M.; and DAYHAW-BARKER, P. The Role of the Retinal Pigment Epithelium: Topographical Variation and Ageing Changes. Eye (London, England), Jun, 2001, vol. 15, no. Pt 3, pp. 384-389. ISSN 0950-222X; 0950-222X.

BOULTON, M., et al. Age-Related Changes in the Morphology, Absorption and Fluorescence of Melanosomes and Lipofuscin Granules of the Retinal Pigment Epithelium. Vision Research, jan, 1990, vol. 30, no. 9, pp. 1291-1303. ISSN 00426989.

BOULTON, M., et al. The Photoreactivity of Ocular Lipofuscin. Photochemical \& Photobiological Sciences : Official Journal of the European Photochemistry Association and the European Society for Photobiology, 20040706, Aug, 2004a, vol. 3, no. 8, pp. 759-764. ISSN 1474905X; 1474-905X.
BOULTON, Mike, et al. Ageing of the Retinal Pigment Epithelium: Implications for Transplantation. Graefe's Archive for Clinical and Experimental Ophthalmology = Albrecht Von Graefes Archiv $F \mid\{U\} R \quad$ Klinische Und Experimentelle Ophthalmologie, jan, 2004b, vol. 242, no. 1, pp. 76-84. ISSN 4429208751; 0721$832 \mathrm{X}$.

BRESSLER, N. M. Age Related Macular Degeneration. New Hope for a Common Problem Comes from Photodynamic Therapy. BMJ (Clinical Research Ed.), Dec 9, 2000, vol. 321, no. 7274, pp. 1425-1427. ISSN 0959-8138; 0959$535 \mathrm{X}$.

BRESSLER, N. M.; and Treatment of Age-Related Macular Degeneration with Photodynamic Therapy (TAP) Study Group. Photodynamic Therapy of Subfoveal Choroidal Neovascularization in Age-Related Macular Degeneration with Verteporfin: Two-Year Results of 2 Randomized Clinical Trials-Tap Report 2. Archives of Ophthalmology, Feb, 2001, vol. 119, no. 2, pp. 198-207. ISSN 0003-9950; 0003-9950.

BROWN, D. M., et al. Primary Endpoint Results of a Phase II Study of Vascular Endothelial Growth Factor Trap-Eye in Wet Age-Related Macular Degeneration. Ophthalmology, Jun, 2011, vol. 118 , no. 6 , pp. 1089-1097. ISSN 1549-4713; 0161-6420.

BROWN, G. C., et al. The Burden of Age-Related Macular Degeneration: A Value-Based Medicine Analysis. Transactions of the American Ophthalmological Society, 2005, vol. 103, pp. 17384 ; discussion 184-6. ISSN 1545-6110; 00659533.

BROWNING, D. J., et al. Aflibercept for AgeRelated Macular Degeneration: A Game-Changer Or Quiet Addition?. American Journal of Ophthalmology, Aug, 2012, vol. 154, no. 2, pp. 222-226. ISSN 1879-1891; 0002-9394.

BRUDER, S. P., et al. The Effect of Implants Loaded with Autologous Mesenchymal Stem Cells on the Healing of Canine Segmental Bone Defects. The Journal of Bone and Joint Surgery.American Volume, Jul, 1998a, vol. 80, no. 7, pp. 985-996. ISSN 0021-9355.

BRUDER, S. P., et al. Bone Regeneration by Implantation of Purified, Culture-Expanded Human Mesenchymal Stem Cells. Journal of Orthopaedic Research : Official Publication of the Orthopaedic Research Society, Mar, 1998b, vol. 16, no. 2, pp. 155-162. ISSN 0736-0266; 07360266 . 
BRZOSKA, Martin, et al. Epithelial Differentiation of Human Adipose Tissue-Derived Adult Stem Cells. Biochemical and Biophysical Research Communications, apr, 2005, vol. 330, no. 1 , pp. 142-150. ISSN 0006-291X.

BUCHHOLZ, D. E., et al. Derivation of Functional Retinal Pigmented Epithelium from Induced Pluripotent Stem Cells. Stem Cells (Dayton, Ohio), Oct, 2009, vol. 27, no. 10, pp. 2427-2434. ISSN 1549-4918; 1066-5099.

BUCHHOLZ, D. E., et al. Rapid and Efficient Directed Differentiation of Human Pluripotent Stem Cells into Retinal Pigmented Epithelium. Stem Cells Translational Medicine, 20130418, May, 2013, vol. 2, no. 5, pp. 384-393. ISSN 21576564; 2157-6564.

BUSCHINI, Elisa, et al. Age Related Macular Degeneration and Drusen: Neuroinflammation in the Retina. Progress in Neurobiology, sep, 2011, vol. 95, no. 1, pp. 14-25. ISSN 1873-5118.

CAI, J., et al. Pigment Epithelium-Derived Factor Inhibits Angiogenesis Via Regulated Intracellular Proteolysis of Vascular Endothelial Growth Factor Receptor 1. The Journal of Biological Chemistry, 20051208, Feb 10, 2006, vol. 281, no. 6, pp. 36043613. ISSN 0021-9258; 0021-9258.

CAI, J., et al. Oxidative Damage and Protection of the RPE. Progress in Retinal and Eye Research, Mar, 2000, vol. 19, no. 2, pp. 205-221. ISSN 13509462; 1350-9462.

CAI, L., et al. IFATS Collection: Human Adipose Tissue-Derived Stem Cells Induce Angiogenesis and Nerve Sprouting Following Myocardial Infarction, in Conjunction with Potent Preservation of Cardiac Function. Stem Cells (Dayton, Ohio), Jan, 2009, vol. 27, no. 1, pp. 230-237. ISSN 15494918; 1066-5099.

CAMPOCHIARO, Peter a.; HACKETT, Sean F.and VINORES, Stanley a. Growth Factors in the Retina and Retinal Pigmented Epithelium. Progress in Retinal and Eye Research, jan, 1996, vol. 15, no. 2, pp. 547-567. ISSN 13509462.

CAO, F., et al. Spatial and Temporal Kinetics of Teratoma Formation from Murine Embryonic Stem Cell Transplantation. Stem Cells and Development, Dec, 2007, vol. 16, no. 6, pp. 883891. ISSN 1547-3287; 1547-3287.

CAO, Y., et al. Transplantation of Chondrocytes Utilizing a Polymer-Cell Construct to Produce Tissue-Engineered Cartilage in the Shape of a Human Ear. Plastic and Reconstructive Surgery,
Aug, 1997, vol. 100, no. 2, pp. 297-302; discussion 303-4. ISSN 0032-1052.

CAO, Ying, et al. Human Adipose Tissue-Derived Stem Cells Differentiate into Endothelial Cells in Vitro and Improve Postnatal Neovascularization in Vivo. Biochemical and Biophysical Research Communications, jul, 2005, vol. 332, no. 2, pp. 370-379. ISSN 0006-291X.

CARAMOY, A., et al. Autologous Translocation of Choroid and Retinal Pigment Epithelium in Geographic Atrophy: Long-Term Functional and Anatomical Outcome. The British Journal of Ophthalmology, 20090818, Aug, 2010, vol. 94, no. 8, pp. 1040-1044. ISSN 1468-2079; 0007-1161.

CARR, Amanda-Jayne, et al. Protective Effects of Human iPS-Derived Retinal Pigment Epithelium Cell Transplantation in the Retinal Dystrophic Rat. PloS One, jan, 2009a, vol. 4, no. 12, pp. e8152. ISSN 1932-6203.

CARR, Amanda-Jayne, et al. Molecular Characterization and Functional Analysis of Phagocytosis by Human Embryonic Stem CellDerived RPE Cells using a Novel Human Retinal Assay. Molecular Vision, jan, 2009b, vol. 15, no. January, pp. 283-295. ISSN 2076084010; 10900535.

CASAROLI-MARANO, R. P.; NIETONICOLAU, N.and MARTINEZ-CONESA, E. M. Progenitor Cells for Ocular Surface Regenerative Therapy. Ophthalmic Research, 20121218, 2013, vol. 49, no. 3, pp. 115-121. ISSN 1423-0259; 0030-3747.

CASIRAGHI, F., et al. Pretransplant Infusion of Mesenchymal Stem Cells Prolongs the Survival of a Semiallogeneic Heart Transplant through the Generation of Regulatory $\mathrm{T}$ Cells. Journal of Immunology (Baltimore, Md.: 1950), Sep 15, 2008 , vol. 181, no. 6, pp. 3933-3946. ISSN 15506606; 0022-1767.

CASTANHEIRA, Paula, et al. Retinal Incorporation and Differentiation of Mesenchymal Stem Cells Intravitreally Injected in the Injured Retina of Rats. Arquivos Brasileiros De Oftalmologia, 2008, vol. 71, no. 5, pp. 644-650. ISSN 1678-2925.

CASTRO-MALASPINA, H., et al. Characterization of Human Bone Marrow Fibroblast Colony-Forming Cells (CFU-F) and their Progeny. Blood, Aug, 1980, vol. 56, no. 2, pp. 289-301. ISSN 0006-4971; 0006-4971. 
CAWTHORN, W. P.; SCHELLER, E. L.and MACDOUGALD, O. A. Adipose Tissue Stem Cells: The Great WAT Hope. Trends in Endocrinology and Metabolism: TEM, 20120312, Jun, 2012, vol. 23, no. 6, pp. 270-277. ISSN 18793061; 1043-2760.

CAYOUETTE, M., et al. Pigment EpitheliumDerived Factor Delays the Death of Photoreceptors in Mouse Models of Inherited Retinal Degenerations. Neurobiology of Disease, Dec, 1999, vol. 6, no. 6, pp. 523-532. ISSN 0969-9961; 0969-9961.

CHAUDHRY, G. R., et al. Fate of Embryonic Stem Cell Derivatives Implanted into the Vitreous of a Slow Retinal Degenerative Mouse Model. Stem Cells and Development, Mar, 2009, vol. 18, no. 2, pp. 247-258. ISSN 1557-8534; 1547-3287.

CHEN, L., et al. Effects of Human Mesenchymal Stem Cells on the Differentiation of Dendritic Cells from CD34+ Cells. Stem Cells and Development, Oct, 2007, vol. 16, no. 5, pp. 719731. ISSN 1547-3287; 1547-3287.

CHEUNG, Chui Ming Gemmy; and WONG, Tien Y. Treatment of Age-Related Macular Degeneration. Lancet, jul, 2013, vol. 6736, no. 13, pp. 13-14. ISSN 1474-547X.

CHEUNG, L. K.; and EATON, A. Age-Related Macular Degeneration. Pharmacotherapy, 20130411, Aug, 2013, vol. 33, no. 8, pp. 838-855. ISSN 1875-9114; 0277-0008.

CHEUNG, Y. T., et al. Effects of all-TransRetinoic Acid on Human SH-SY5Y Neuroblastoma as in Vitro Model in Neurotoxicity Research. Neurotoxicology, 20081114, Jan, 2009, vol. 30, no. 1, pp. 127-135. ISSN 0161-813X; 0161-813X

CHIOU, Shih-Hwa, et al. A Novel in Vitro Retinal Differentiation Model by Co-Culturing Adult Human Bone Marrow Stem Cells with Retinal Pigmented Epithelium Cells. Biochemical and Biophysical Research Communications, jan, 2005, vol. 326, no. 3, pp. 578-585. ISSN 0006-291X.

CIOMBOR, K. K.; BERLIN, J.and CHAN, E. Aflibercept. Clinical Cancer Research : An Official Journal of the American Association for Cancer Research, 20130226, Apr 15, 2013, vol. 19, no. 8, pp. 1920-1925. ISSN 1078-0432; 1078 0432 .

CLARKE, L.; BALLIOS, B. G.and VAN DER KOOY, D. Generation and Clonal Isolation of Retinal Stem Cells from Human Embryonic Stem
Cells. The European Journal of Neuroscience, 20120516, Jul, 2012, vol. 36, no. 1, pp. 1951-1959. ISSN 1460-9568; 0953-816X.

COLTER, D. C., et al. Rapid Expansion of Recycling Stem Cells in Cultures of PlasticAdherent Cells from Human Bone Marrow. Proceedings of the National Academy of Sciences of the United States of America, Mar 28, 2000, vol. 97 , no. 7, pp. 3213-3218. ISSN 0027-8424; 00278424.

COMBADIERE, C., et al. CX3CR1-Dependent Subretinal Microglia Cell Accumulation is Associated with Cardinal Features of Age-Related Macular Degeneration. The Journal of Clinical Investigation, Oct, 2007, vol. 117, no. 10, pp. 2920-2928. ISSN 0021-9738; 0021-9738.

COOK, H. L.; PATEL, P. J.and TUFAIL, a. AgeRelated Macular Degeneration: Diagnosis and Management. British Medical Bulletin, jan, 2008, vol. 85, pp. 127-149. ISSN 1471-8391.

CORNEO, Barbara; and TEMPLE, Sally. Sense and Serendipity Aid RPE Generation. Cell Stem Cell, oct, 2009, vol. 5, no. 4, pp. 347-348. ISSN 1875-9777.

CREECH KRAFT, J., et al. Isotretinoin (13-CisRetinoic Acid) Metabolism, Cis-Trans Isomerization, Glucuronidation, and Transfer to the Mouse Embryo: Consequences for Teratogenicity. Teratogenesis, Carcinogenesis, and Mutagenesis, 1991, vol. 11, no. 1, pp. 21-30. ISSN 0270-3211; 0270-3211.

CREECH KRAFT, J., et al. Teratogenicity and Placental Transfer of all-Trans-, 13-Cis-, 4-Oxoall-Trans-, and 4-Oxo-13-Cis-Retinoic Acid After Administration of a Low Oral Dose during Organogenesis in Mice. Toxicology and Applied Pharmacology, Aug, 1989, vol. 100, no. 1, pp. 162-176. ISSN 0041-008X; 0041-008X.

CROSSNO, J. T.,Jr, et al. Rosiglitazone Promotes Development of a Novel Adipocyte Population from Bone Marrow-Derived Circulating Progenitor Cells. The Journal of Clinical Investigation, Dec, 2006, vol. 116, no. 12, pp. 3220-3228. ISSN 0021-9738; 0021-9738.

da Silva Meirelles Lindolfo; CHAGASTELLES, Pedro Cesarand NARDI, Nance Beyer. Mesenchymal Stem Cells Reside in Virtually all Post-Natal Organs and Tissues. Journal of Cell Science, jun, 2006, vol. 119, no. Pt 11, pp. 22042213. ISSN 0021-9533. 
DAAR, A. S.; and GREENWOOD, H. L. A Proposed Definition of Regenerative Medicine. Journal of Tissue Engineering and Regenerative Medicine, May-Jun, 2007, vol. 1, no. 3, pp. 179184. ISSN 1932-6254; 1932-6254.

DAHL, John-Arne, et al. Genetic and Epigenetic Instability of Human Bone Marrow Mesenchymal Stem Cells Expanded in Autologous Serum Or Fetal Bovine Serum. The International Journal of Developmental Biology, jan, 2008, vol. 52, no. 8, pp. 1033-1042. ISSN 0214-6282.

DAN, Y. Y., et al. Isolation of Multipotent Progenitor Cells from Human Fetal Liver Capable of Differentiating into Liver and Mesenchymal Lineages. Proceedings of the National Academy of Sciences of the United States of America, 20060616, Jun 27, 2006, vol. 103, no. 26, pp. 9912-9917. ISSN 0027-8424; 0027-8424.

DAVIS, M. W.; and VACANTI, J. P. Toward Development of an Implantable Tissue Engineered Liver. Biomaterials, Feb, 1996, vol. 17, no. 3, pp. 365-372. ISSN 0142-9612; 0142-9612.

DAWSON, D. W. Pigment Epithelium-Derived Factor: A Potent Inhibitor of Angiogenesis. Science, jul, 1999, vol. 285, no. 5425, pp. 245-248. ISSN 00368075.

DE BARI, C., et al. Multipotent Mesenchymal Stem Cells from Adult Human Synovial Membrane. Arthritis and Rheumatism, Aug, 2001, vol. 44, no. 8, pp. 1928-1942. ISSN 0004-3591; 0004-3591.

DE MIGUEL, M. P., et al. Cornea and Ocular Surface Treatment. Current Stem Cell Research \& Therapy, Jun, 2010, vol. 5, no. 2, pp. 195-204. ISSN 1574-888X.

DE SOUZA, O. F., et al. Inhibition of Experimental Proliferative Vitreoretinopathy in Rabbits by Suramin. Ophthalmologica.Journal International D'Ophtalmologie.International Journal of Ophthalmology.Zeitschrift Fur Augenheilkunde, 1995, vol. 209, no. 4, pp. 212 216. ISSN 0030-3755; 0030-3755.

De Ugarte Daniel a., et al. Comparison of MultiLineage Cells from Human Adipose Tissue and Bone Marrow. Cells Tissues Organs, 2003, vol. 174, no. 3, pp. 101-109. ISSN 1422-6421.

DE UGARTE, D. A., et al. Comparison of MultiLineage Cells from Human Adipose Tissue and Bone Marrow. Cells, Tissues, Organs, 2003, vol. 174 , no. 3 , pp. 101-109. ISSN 1422-6405; 14226405 .
DEFER, G. L., et al. All-Trans Retinoic Acid in Relapsing Malignant Gliomas: Clinical and Radiological Stabilization Associated with the Appearance of Intratumoral Calcifications. Journal of Neuro-Oncology, Sep, 1997, vol. 34, no. 2, pp. 169-177. ISSN 0167-594X; 0167-594X.

DEJDA, A.; SOKOLOWSKA, P.and NOWAK, J. Z. Neuroprotective Potential of Three Neuropeptides PACAP, VIP and PHI. Pharmacological Reports : PR, May-Jun, 2005, vol. 57, no. 3, pp. 307-320. ISSN 1734-1140; 1734-1140.

DEL PRIORE, L. V., et al. Retinal Pigment Epithelial Cell Transplantation After Subfoveal Membranectomy in Age-Related Macular Degeneration: Clinicopathologic Correlation. American Journal of Ophthalmology, Apr, 2001, vol. 131, no. 4, pp. 472-480. ISSN 0002-9394; 0002-9394.

DELORI, F. C., et al. In Vivo Fluorescence of the Ocular Fundus Exhibits Retinal Pigment Epithelium Lipofuscin Characteristics. Investigative Ophthalmology \& Visual Science, Mar, 1995, vol. 36, no. 3, pp. 718-729. ISSN 01460404; 0146-0404.

DELORI, F. C.; GOGER, D. G.and DOREY, C. K. Age-Related Accumulation and Spatial Distribution of Lipofuscin in RPE of Normal Subjects. Investigative Ophthalmology \& Visual Science, Jul, 2001, vol. 42, no. 8, pp. 1855-1866. ISSN 0146-0404; 0146-0404.

DI NICOLA, M., et al. Human Bone Marrow Stromal Cells Suppress T-Lymphocyte Proliferation Induced by Cellular Or Nonspecific Mitogenic Stimuli. Blood, May 15, 2002, vol. 99, no. 10, pp. 3838-3843. ISSN 0006-4971; 00064971.

DIGIROLAMO, C. M., et al. Propagation and Senescence of Human Marrow Stromal Cells in Culture: A Simple Colony-Forming Assay Identifies Samples with the Greatest Potential to Propagate and Differentiate. British Journal of Haematology, Nov, 1999, vol. 107, no. 2, pp. 275281. ISSN 0007-1048; 0007-1048.

DING, Y., et al. Mesenchymal Stem Cells Prevent the Rejection of Fully Allogenic Islet Grafts by the Immunosuppressive Activity of Matrix Metalloproteinase-2 and -9. Diabetes, 20090609, Aug, 2009, vol. 58, no. 8, pp. 1797-1806. ISSN 1939-327X; 0012-1797.

DOMINICI, M., et al. Minimal Criteria for Defining Multipotent Mesenchymal Stromal Cells. 
the International Society for Cellular Therapy Position Statement. Cytotherapy, 2006, vol. 8, no. 4 , pp. 315-317. ISSN 1465-3249; 1465-3249.

DOORN, Joyce, et al. Therapeutic Applications of Mesenchymal Stromal Cells : Paracrine Effects and Potential Improvements, 2012, vol. 18, no. 2.

DRAGOO, J. L., et al. Tissue-Engineered Cartilage and Bone using Stem Cells from Human Infrapatellar Fat Pads. The Journal of Bone and Joint Surgery.British Volume, Jul, 2003, vol. 85, no. 5, pp. 740-747. ISSN 0301-620X; 0301-620X.

DROSOS, Ioannis; and KOLIOS, George. Stem Cells in Liver Regeneration and their Potential Clinical Applications. Stem Cell Reviews, mar, 2013. ISSN 1558-6804.

DU, Yang, et al. Regulation of Retinal Progenitor Cell Differentiation by Bone Morphogenetic Protein 4 is Mediated by the Smad/Id Cascade. Investigative Ophthalmology $\mid \&$ Visual Science, jul, 2010, vol. 51, no. 7, pp. 3764-3773. ISSN 1552-5783.

DUNN, K. C., et al. ARPE-19, a Human Retinal Pigment Epithelial Cell Line with Differentiated Properties. Experimental Eye Research, Feb, 1996, vol. 62, no. 2, pp. 155-169. ISSN 0014-4835; 0014-4835.

DYLEWSKI, D. P.; NANDY, S.and NANDY, K. Effects of centrophenoxine on lipofuscin in the retinal Pigment Epithelium of old mice. Neurobiology of Aging. http://www.ncbi.nlm.nih.gov/pubmed/?term=Dyle wski+DP,+Nandy $+\mathrm{S},+\mathrm{Nandy}+\mathrm{K} .+$ Effects + of + cent rophenoxine + on + lipofuscin + in + the + retinal + pigme $\mathrm{nt}+$ epithelium + of + old + mice. + Neurobiol+Aging ed., 1983, vol. 4, no. 1, pp. 89-95.

EGGENHOFER, E., et al. Mesenchymal Stem Cells Together with Mycophenolate Mofetil Inhibit Antigen Presenting Cell and $\mathrm{T}$ Cell Infiltration into Allogeneic Heart Grafts. Transplant Immunology, 20101229, Apr 15, 2011, vol. 24, no. 3, pp. 157-163. ISSN 1878-5492; 0966-3274.

ELDRED, G. E.; and KATZ, M. L. Fluorophores of the Human Retinal Pigment Epithelium: Separation and Spectral Characterization. Experimental Eye Research, Jul, 1988, vol. 47, no. 1, pp. 71-86. ISSN 0014-4835; 0014-4835.

ELNER, S. G. Gradual Painless Visual Loss: Retinal Causes. Clinics in Geriatric Medicine, Feb, 1999, vol. 15, no. 1, pp. 25-46, v-vi. ISSN 07490690; 0749-0690.
ENGLISH, K., et al. Cell Contact, Prostaglandin E(2) and Transforming Growth Factor Beta 1 Play Non-Redundant Roles in Human Mesenchymal Stem Cell Induction of CD4+CD25(High) Forkhead Box P3+ Regulatory T Cells. Clinical and Experimental Immunology, 20090203, Apr, 2009 , vol. 156 , no. 1, pp. 149-160. ISSN 13652249; 0009-9104.

European Commission News. Medicinal Products for Human use - Advanced Therapies | Public Health, European Commission. , 2013aAvailable from: $<$ http://ec.europa.eu/health/humanuse/advanced-therapies/ $>$.

European Commission News. News and Updates on Pharmaceuticals - EudraLex - Volume 1 Pharmaceutical Legislation Medicinal Products for Human use | Public Health, European Commission. 2013bAvailable from: $<$ http://ec.europa.eu/health/documents/eudral ex/vol-1/\#reg $>$.

European Commission News. News and Updates on Pharmaceuticals - EudraLex - Volume 2 Pharmaceutical Legislation Notice to Applicants and Regulatory Guidelines Medicinal Products for Human use. | Public Health, European Commission. 2013cAvailable from: $<$ http://ec.europa.eu/health/documents/eudral ex/vol-2/>.

FDA. FDA Approves Aflibercept (Zaltrap)... [Oncology (Williston Park). 2012] - PubMed NCBI. Oncology (Williston Park). http://www.ncbi.nlm.nih.gov/pubmed/23061342 ed., 2012, vol. 26, no. 9, pp. 842-873.

FEENEY, L. Lipofuscin and Melanin of Human Retinal Pigment Epithelium. Fluorescence, Enzyme Cytochemical, and Ultrastructural Studies. Investigative Ophthalmology \& Visual Science, Jul, 1978, vol. 17, no. 7, pp. 583-600. ISSN 01460404; 0146-0404.

FEENEY-BURNS, L.; HILDERBRAND, E. S.and ELDRIDGE, S. Aging Human RPE: Morphometric Analysis of Macular, Equatorial, and Peripheral Cells. Investigative Ophthalmology \& Visual Science, Feb, 1984, vol. 25, no. 2, pp. 195-200. ISSN 0146-0404; 0146-0404.

FERNANDEZ-BUENO, I., et al. Time Course Modifications in Organotypic Culture of Human Neuroretina. Experimental Eye Research, 20120926, Nov, 2012, vol. 104, pp. 26-38. ISSN 1096-0007; 0014-4835.

FERNANDEZ-BUENO, I., et al. Adalimumab (Tumor Necrosis Factor-Blocker) Reduces the 
Expression of Glial Fibrillary Acidic Protein Immunoreactivity Increased by Exogenous Tumor Necrosis Factor Alpha in an Organotypic Culture of Porcine Neuroretina. Molecular Vision, 20130417, Apr 17, 2013, vol. 19, pp. 894-903. ISSN 1090-0535; 1090-0535.

FERNANDEZ-BUENO, Ivan, et al. Müller and Macrophage-Like Cell Interactions in an Organotypic Culture of Porcine Neuroretina. Molecular Vision, jan, 2008, vol. 14, no. November, pp. 2148-2156. ISSN 1090-0535.

FERRARA, N.; GERBER, H. P.and LECOUTER, J. The Biology of VEGF and its Receptors. Nature Medicine, Jun, 2003, vol. 9, no. 6, pp. 669-676. ISSN 1078-8956; 1078-8956.

FIGLIUZZI, M., et al. Bone Marrow-Derived Mesenchymal Stem Cells Improve Islet Graft Function in Diabetic Rats. Transplantation Proceedings, Jun, 2009, vol. 41, no. 5, pp. $1797-$ 1800. ISSN 1873-2623; 0041-1345.

FINNEMANN, S. C. Focal Adhesion Kinase Signaling Promotes Phagocytosis of IntegrinBound Photoreceptors. The EMBO Journal, Aug 15 , 2003, vol. 22, no. 16, pp. 4143-4154. ISSN 0261-4189; 0261-4189.

FISCHER, Andy J., et al. BMP4 and CNTF are Neuroprotective and Suppress Damage-Induced Proliferation of M $\backslash$ uller Glia in the Retina. Molecular and Cellular Neurosciences, dec, 2004, vol. 27, no. 4, pp. 531-542. ISSN 1044-7431.

FRANQUESA, Marcella, et al. Kidney Regeneration and Repair After Transplantation. Current Opinion in Organ Transplantation, apr, 2013, vol. 18, no. 2, pp. 191-196. ISSN 15317013.

FRASER, J. K., et al. Fat Tissue: An Underappreciated Source of Stem Cells for Biotechnology. Trends in Biotechnology, 20060220, Apr, 2006, vol. 24, no. 4, pp. 150-154. ISSN 0167-7799; 0167-7799.

FRIEDENSTEIN, A. J.; CHAILAKHJAN, R. K.and LALYKINA, K. S. The Development of Fibroblast Colonies in Monolayer Cultures of Guinea-Pig Bone Marrow and Spleen Cells. Cell and Tissue Kinetics, Oct, 1970, vol. 3, no. 4, pp. 393-403. ISSN 0008-8730; 0008-8730.

FRIEDENSTEIN, A. J., et al. Precursors for Fibroblasts in Different Populations of Hematopoietic Cells as Detected by the in Vitro Colony Assay Method. Experimental Hematology,
1974, vol. 2, no. 2, pp. 83-92. ISSN 0301-472X; $0301-472 X$.

FRIEDENSTEIN, A. J.; GORSKAJA, J. F.and KULAGINA, N. N. Fibroblast Precursors in Normal and Irradiated Mouse Hematopoietic Organs. Experimental Hematology, Sep, 1976, vol. 4, no. 5, pp. 267-274. ISSN 0301-472X; 0301$472 \mathrm{X}$.

FRIEDMAN, D. S., et al. Prevalence of AgeRelated Macular Degeneration in the United States. Archives of Ophthalmology, Apr, 2004, vol. 122, no. 4, pp. 564-572. ISSN 0003-9950; 00039950.

FU, X., et al. Enhanced Wound-Healing Quality with Bone Marrow Mesenchymal Stem Cells Autografting After Skin Injury. Wound Repair and Regeneration : Official Publication of the Wound Healing Society [and] the European Tissue Repair Society, May-Jun, 2006, vol. 14, no. 3, pp. 325335. ISSN 1067-1927; 1067-1927.

FUKUMOTO, T., et al. Combined Effects of Insulin-Like Growth Factor-1 and Transforming Growth Factor-Beta1 on Periosteal Mesenchymal Cells during Chondrogenesis in Vitro. Osteoarthritis and Cartilage / OARS, Osteoarthritis Research Society, Jan, 2003, vol. 11, no. 1, pp. 55-64. ISSN 1063-4584; 1063-4584.

FURUTA, Y.; and HOGAN, B. L. BMP4 is Essential for Lens Induction in the Mouse Embryo. Genes \& Development, Dec 1, 1998, vol. 12, no. 23, pp. 3764-3775. ISSN 0890-9369; 0890-9369.

GAL, a., et al. Mutations in MERTK, the Human Orthologue of the RCS Rat Retinal Dystrophy Gene, Cause Retinitis Pigmentosa. Nature Genetics, nov, 2000, vol. 26, no. 3, pp. 270-271. ISSN 1061-4036.

GALE, E. A. Theory and Practice of Nicotinamide Trials in Pre-Type 1 Diabetes. Journal of Pediatric Endocrinology \& Metabolism : JPEM, May-Jun, 1996, vol. 9, no. 3, pp. 375-379. ISSN 0334-018X; 0334-018X.

GAO, G., et al. Unbalanced Expression of VEGF and PEDF in Ischemia-Induced Retinal Neovascularization. FEBS Letters, Feb 2, 2001, vol. 489, no. 2-3, pp. 270-276. ISSN 0014-5793; 0014-5793.

GARCIA LAYANA, A. Vitamin and Zinc Supplementation are Useful in Age-Related Macular Degeneration. Archivos De La Sociedad Espanola De Oftalmologia, Feb, 2002, vol. 77, no. 2, pp. 57-58. ISSN 0365-6691; 0365-6691. 
GARCIA LAYANA, A. Photodynamic Therapy. Archivos De La Sociedad Espanola De Oftalmologia, Oct, 2000, vol. 75, no. 10, pp. 655 656. ISSN 0365-6691; 0365-6691.

GARCIA LAYANA, A., et al. Genetics and ARMD: From the Laboratory to the Consulting Room. Archivos De La Sociedad Espanola De Oftalmologia, Apr, 2011, vol. 86, no. 4, pp. 101102. ISSN 1989-7286; 0365-6691.

GARCIA-LAYANA, A., et al. Effects of Lutein and Docosahexaenoic Acid Supplementation on Macular Pigment Optical Density in a Randomized Controlled Trial. Nutrients, 20130215, Feb 15, 2013, vol. 5, no. 2, pp. 543-551. ISSN 2072-6643; 2072-6643.

GASS, J. D. Drusen and Disciform Macular Detachment and Degeneration. 1972. Retina (Philadelphia, Pa.), Dec, 2003, vol. 23, no. 6 Suppl, pp. 409-436. ISSN 0275-004X; 0275-004X.

GASS, J. D. Drusen and Disciform Macular Detachment and Degeneration. Transactions of the American Ophthalmological Society, 1972, vol. 70, pp. 409-436. ISSN 0065-9533; 0065-9533.

GELISKEN, F., et al. Full Macular Translocation Versus Photodynamic Therapy with Verteporfin in the Treatment of Neovascular Age-Related Macular Degeneration: 1-Year Results of a Prospective, Controlled, Randomised Pilot Trial (FMT-PDT). Graefe's Archive for Clinical and Experimental Ophthalmology = Albrecht Von Graefes Archiv Fur Klinische Und Experimentelle Ophthalmologie, Aug, 2007, vol. 245, no. 8, pp. 1085-1095. ISSN 0721-832X; 0721-832X.

GESTA, S.; TSENG, Y. H.and KAHN, C. R. Developmental Origin of Fat: Tracking Obesity to its Source. Cell, Oct 19, 2007, vol. 131, no. 2, pp. 242-256. ISSN 0092-8674; 0092-8674.

GIAS, C., et al. Preservation of Visual Cortical Function Following Retinal Pigment Epithelium Transplantation in the RCS Rat using Optical Imaging Techniques. The European Journal of Neuroscience, Apr, 2007, vol. 25, no. 7, pp. 19401948. ISSN 0953-816X; 0953-816X.

GIMBLE, Jeffrey M., et al. Adipose-Derived Stromal/Stem Cells, 2013, no. March, pp. 3-10.

GIMBLE, Jeffrey M.; KATZ, Adam J.and BUNNELL, Bruce a. Adipose-Derived Stem Cells for Regenerative Medicine. Circulation Research, may, 2007, vol. 100, no. 9, pp. 1249-1260. ISSN $1524-4571$
GIRMAN, S. V.; WANG, S.and LUND, R. D. Time Course of Deterioration of Rod and Cone Function in RCS Rat and the Effects of Subretinal Cell Grafting: A Light- and Dark-Adaptation Study. Vision Research, Feb, 2005, vol. 45, no. 3, pp. 343-354. ISSN 0042-6989; 0042-6989.

GIRMAN, S. V.; WANG, S.and LUND, R. D. Cortical Visual Functions can be Preserved by Subretinal RPE Cell Grafting in RCS Rats. Vision Research, aug, 2003, vol. 43, no. 17, pp. 18171827. ISSN 00426989.

GLENNIE, S., et al. Bone Marrow Mesenchymal Stem Cells Induce Division Arrest Anergy of Activated T Cells. Blood, 20041209, Apr 1, 2005, vol. 105 , no. 7, pp. 2821-2827. ISSN 0006-4971; 0006-4971.

GOLD, B., et al. Variation in Factor B (BF) and Complement Component 2 (C2) Genes is Associated with Age-Related Macular Degeneration. Nature Genetics, 20060305, Apr, 2006, vol. 38, no. 4, pp. 458-462. ISSN 1061$4036 ; 1061-4036$.

GONG, Jie, et al. Effects of Extracellular Matrix and Neighboring Cells on Induction of Human Embryonic Stem Cells into Retinal Or Retinal Pigment Epithelial Progenitors. Experimental Eye Research, jun, 2008a, vol. 86, no. 6, pp. 957-965. ISSN 0014-4835.

GONG, Lihua, et al. Differentiation of Rat Mesenchymal Stem Cells Transplanted into the Subretinal Space of Sodium Iodate-Injected Rats. Clinical I\& Experimental Ophthalmology, oct, 2008b, vol. 36, no. 7, pp. 666-671. ISSN 14429071.

GOURAS, P.; and ALGVERE, P. Retinal Cell Transplantation in the Macula: New Techniques. Vision Research, Dec, 1996, vol. 36, no. 24, pp. 4121-4125. ISSN 0042-6989; 0042-6989.

GOURAS, P.; FLOOD, M. T.and KJELDBYE, H. Transplantation of Cultured Human Retinal Cells to Monkey Retina. Anais Da Academia Brasileira De Ciencias, Dec, 1984, vol. 56, no. 4, pp. 431443. ISSN 0001-3765; 0001-3765.

GOURAS, P., et al. Transplantation of Retinal Epithelium Prevents Photoreceptor Degeneration in the RCS Rat. Progress in Clinical and Biological Research, 1989, vol. 314, pp. 659-671. ISSN 0361-7742; 0361-7742.

GRAYSON, W. L., et al. Bioreactor Cultivation of Functional Bone Grafts. Methods in Molecular 
Biology (Clifton, N.J.), 2011, vol. 698, pp. 231241. ISSN 1940-6029; 1064-3745.

GRONTHOS, S., et al. Surface Protein Characterization of Human Adipose TissueDerived Stromal Cells. Journal of Cellular Physiology, oct, 2001, vol. 189, no. 1, pp. 54-63. ISSN 0021-9541.

GRUBER, Karl. Europe Gives Gene Therapy the Green Light. The Lancet, 11-17, 2012, vol. 380, no. 9855, pp. e10. Available from $<$ http://www.thelancet.com/journals/lancet/article/ PIIS0140-6736(12)61992-8/fulltext?rss=yes $>$. ISSN 0140-6736.

GUDAS, L. J. Retinoids Induce Stem Cell Differentiation Via Epigenetic Changes. Seminars in Cell \& Developmental Biology, 20130821, Aug 21, 2013. ISSN 1096-3634; 1084-9521.

GUERRE-MILLO, M. Adipose Tissue Hormones. Journal of Endocrinological Investigation, Nov, 2002, vol. 25, no. 10, pp. 855-861. ISSN 03914097; 0391-4097.

GUPTA, Kunal; CHANDRAN, Siddharthanand HARDINGHAM, Giles E. Human Stem CellDerived Astrocytes and their Application to Studying Nrf2-Mediated Neuroprotective Pathways and Therapeutics in Neurodegeneration. British Journal of Clinical Pharmacology, apr, 2013, vol. 75, no. 4, pp. 907-918. ISSN 13652125 .

HAINES, J. L., et al. Functional Candidate Genes in Age-Related Macular Degeneration: Significant Association with VEGF, VLDLR, and LRP6. Investigative Ophthalmology \& Visual Science, Jan, 2006, vol. 47, no. 1, pp. 329-335. ISSN 01460404; 0146-0404.

HALL, M. O. Phagocytosis of Light- and DarkAdapted Rod Outer Segments by Cultured Pigment Epithelium. Science (New York, N.Y.), nov, 1978, vol. 202, no. 4367, pp. 526-528. ISSN 0036-8075.

HAMANN, S. Molecular Mechanisms of Water Transport in the Eye. International Review of Cytology, 2002, vol. 215, pp. 395-431. ISSN 00747696; 0074-7696.

HARALAMPUS-GRYNAVISKI, N. M., et al. Spectroscopic and Morphological Studies of Human Retinal Lipofuscin Granules. Proceedings of the National Academy of Sciences of the United States of America, 20030228, Mar 18, 2003, vol. 100 , no. 6 , pp. 3179-3184. ISSN 0027-8424; 00278424.
HARLAND, R. Neural Induction. Current Opinion in Genetics \& Development, Aug, 2000, vol. 10, no. 4, pp. 357-362. ISSN 0959-437X; 0959-437X.

HARUTA, M. In Vitro and in Vivo Characterization of Pigment Epithelial Cells Differentiated from Primate Embryonic Stem Cells. Investigative Ophthalmology $\mid \&$ Visual Science, mar, 2004, vol. 45, no. 3, pp. 1020-1025. ISSN 0146-0404.

HAUSMAN, G. J.; and HAUSMAN, D. B. Search for the Preadipocyte Progenitor Cell. The Journal of Clinical Investigation, Dec, 2006, vol. 116, no. 12 , pp. 3103-3106. ISSN 0021-9738; 0021-9738.

HAYNES, Tracy, et al. BMP Signaling Mediates Stem/Progenitor Cell-Induced Retina Regeneration. Proceedings of the National Academy of Sciences of the United States of America, dec, 2007, vol. 104, no. 51, pp. 2038020385. ISSN 1091-6490.

HEGNER, B., et al. Differential Regulation of Smooth Muscle Markers in Human Bone MarrowDerived Mesenchymal Stem Cells. Journal of Hypertension, Jun, 2005, vol. 23, no. 6, pp. 11911202. ISSN 0263-6352; 0263-6352.

HERRERA, M. B., et al. Mesenchymal Stem Cells Contribute to the Renal Repair of Acute Tubular Epithelial Injury. International Journal of Molecular Medicine, Dec, 2004, vol. 14, no. 6, pp. 1035-1041. ISSN 1107-3756; 1107-3756.

HOFFMAN, S., et al. Verapamil Inhibits Proliferation, Migration and Protein Kinase C Activity in Human Retinal Pigment Epithelial Cells. Experimental Eye Research, jul, 1998, vol. 67 , no. 1, pp. 45-52. ISSN 0014-4835.

HOLEKAMP, N. M.; BOUCK, N.and VOLPERT, O. Pigment Epithelium-Derived Factor is Deficient in the Vitreous of Patients with Choroidal Neovascularization due to Age-Related Macular Degeneration. American Journal of Ophthalmology, Aug, 2002, vol. 134, no. 2, pp. 220-227. ISSN 0002-9394; 0002-9394.

HOLLOWAY, T. B.; and VERHOEFF, F. H. Disc-Like Degeneration of the Macula with Microscopic Report Concerning a Tumor-Like Mass in the Macular Region. Transactions of the American Ophthalmological Society, 1928, vol. 26, pp. 206-228. ISSN 0065-9533; 0065-9533.

HOLTKAMP, G. M., et al. Retinal Pigment Epithelium-Immune System Interactions: Cytokine Production and Cytokine-Induced Changes. 
Progress in Retinal and Eye Research, jan, 2001, vol. 20, no. 1, pp. 29-48. ISSN 3110408945; 13509462.

HOLTKAMP, G. M., et al. Polarized Secretion of IL-6 and IL-8 by Human Retinal Pigment Epithelial Cells. Clinical and Experimental Immunology, apr, 1998, vol. 112, no. 1, pp. 34-43. ISSN 0009-9104.

HOLZ, F. G., et al. Fundus Autofluorescence and Development of Geographic Atrophy in AgeRelated Macular Degeneration. Investigative Ophthalmology \& Visual Science, Apr, 2001, vol. 42, no. 5, pp. 1051-1056. ISSN 0146-0404; 01460404 .

HOLZ, F. G., et al. Inhibition of Lysosomal Degradative Functions in RPE Cells by a Retinoid Component of Lipofuscin. Investigative Ophthalmology \& Visual Science, Mar, 1999, vol. 40, no. 3, pp. 737-743. ISSN 0146-0404; 01460404 .

HONG, Seung Hyun, et al. In Vitro Differentiation of Human Umbilical Cord Blood-Derived Mesenchymal Stem Cells into Hepatocyte-Like Cells. Biochemical and Biophysical Research Communications, may, 2005, vol. 330, no. 4, pp. 1153-1161. ISSN 0006-291X.

HORNAN, D. M., et al. Novel Retinal and Cone Photoreceptor Transcripts Revealed by Human Macular Expression Profiling. Investigative Ophthalmology \& Visual Science, Dec, 2007, vol. 48 , no. 12 , pp. 5388-5396. ISSN 0146-0404; 01460404 .

HORWITZ, E. M., et al. Clarification of the Nomenclature for MSC: The International Society for Cellular Therapy Position Statement. Cytotherapy, 2005, vol. 7, no. 5, pp. 393-395. ISSN 1465-3249; 1465-3249.

HOU, H. Y.; LIANG, H. L.and WANG, Y. S. Bone Marrow-Derived Cells in Neovascular AgeRelated Macular Degeneration: Contribution and Potential Application. Ophthalmic Research, 20100811, 2011, vol. 45, no. 1, pp. 1-4. ISSN 1423-0259; 0030-3747.

HOU, H. Y., et al. A Therapeutic Strategy for Choroidal Neovascularization Based on Recruitment of Mesenchymal Stem Cells to the Sites of Lesions. Molecular Therapy : The Journal of the American Society of Gene Therapy, 20100720 , Oct, 2010, vol. 18, no. 10, pp. 18371845. ISSN 1525-0024; 1525-0016.
HSIAO, S. T., et al. Comparative Analysis of Paracrine Factor Expression in Human Adult Mesenchymal Stem Cells Derived from Bone Marrow, Adipose, and Dermal Tissue. Stem Cells and Development. http://www.ncbi.nlm.nih.gov/pubmed/?term=S.T.+ Hsiao, + A.+Asgari, +Z.+Lokmic, + R. + Sinclair, + G.J. + Dusting, + S.Y.+Lim, +R.J.+Dilley, + Comparative+ analysis + of + paracrine + factor + expression + in + hum an+adult + mesenchymal + stem + cells + derived + from + bone + marrow,,+ adipose, + and + dermal + tissue,$+\mathrm{St}$ em+Cells+Dev. $+21+(2012)$ ed., 2012, vol. 21, no. 12, pp. 2189-2203.

HUANG, Chen, et al. Combination of Retinal Pigment Epithelium Cell-Conditioned Medium and Photoreceptor Outer Segments Stimulate Mesenchymal Stem Cell Differentiation Toward a Functional Retinal Pigment Epithelium Cell Phenotype. Journal of Cellular Biochemistry, feb, 2012a, vol. 113 , no. 2, pp. 590-598. ISSN 10974644.

HUANG, Jing; POSSIN, Daniel E.and SAARI, John C. Localizations of Visual Cycle Components in Retinal Pigment Epithelium. Molecular Vision, jan, 2009, vol. 15, no. December 2008, pp. 223234. ISSN 1090-0535.

HUANG, Q., et al. Culture and Induced Multilineage Differentiation of Mesenchymal Stem Cells Derived from Human Nasal Mucosa. Lin Chuang Er Bi Yan Hou Tou Jing Wai Ke Za Zhi= Journal of Clinical Otorhinolaryngology, Head, and Neck Surgery, Jun, 2012b, vol. 26, no. 11, pp. 490-3, 498. ISSN 1001-1781.

HUILLARD, E.; LAUGIER, D.and MARX, M. Defects in Chicken Neuroretina Misexpressing the BMP Antagonist Drm/GremLin. Developmental Biology, Jul 15, 2005, vol. 283, no. 2, pp. 335-344. ISSN 0012-1606; 0012-1606.

HUMES, H. D. Tissue Engineering of a Bioartificial Kidney: A Universal Donor Organ. Transplantation Proceedings, Aug, 1996, vol. 28, no. 4, pp. 2032-2035. ISSN 0041-1345; 00411345 .

IDELSON, M., et al. Directed Differentiation of Human Embryonic Stem Cells into Functional Retinal Pigment Epithelium Cells. Cell Stem Cell, Oct 2, 2009a, vol. 5, no. 4, pp. 396-408. ISSN 1875-9777.

IDELSON, Maria, et al. Directed Differentiation of Human Embryonic Stem Cells into Functional Retinal Pigment Epithelium Cells. Cell Stem Cell, oct, 2009b, vol. 5, no. 4, pp. 396-408. ISSN 18759777. 
IMAI, S., et al. Transcriptional Silencing and Longevity Protein Sir2 is an NAD-Dependent Histone Deacetylase. Nature, Feb 17, 2000, vol. 403, no. 6771, pp. 795-800. ISSN 0028-0836; 0028-0836.

IN 'T ANKER, P. S., et al. Isolation of Mesenchymal Stem Cells of Fetal Or Maternal Origin from Human Placenta. Stem Cells (Dayton, Ohio), 2004, vol. 22, no. 7, pp. 1338-1345. ISSN 1066-5099; 1066-5099.

INOUE, Y., et al. Subretinal Transplantation of Bone Marrow Mesenchymal Stem Cells Delays Retinal Degeneration in the RCS Rat Model of Retinal Degeneration. Experimental Eye Research, 20070506, Aug, 2007, vol. 85, no. 2, pp. 234-241. ISSN 0014-4835; 0014-4835.

ISHIDA, K., et al. Participation of Pigment Epithelium in Ocular Immune Privilege. 3. Epithelia Cultured from Iris, Ciliary Body, and Retina Suppress T-Cell Activation by Partially Non-Overlapping Mechanisms. Ocular Immunology and Inflammation, Jun, 2003, vol. 11, no. 2, pp. 91-105. ISSN 0927-3948; 0927-3948.

IZADPANAH, Reza, et al. Biologic Properties of Mesenchymal Stem Cells Derived from Bone Marrow and Adipose Tissue. Journal of Cellular Biochemistry, dec, 2006, vol. 99, no. 5, pp. 12851297. ISSN 0730-2312.

JANKOWSKI, R. J.; DEASY, B. M.and HUARD, J. Muscle-Derived Stem Cells. Gene Therapy, May, 2002, vol. 9, no. 10, pp. 642-647. ISSN 0969-7128; 0969-7128.

JI, Dan; LI, Guang-Yuand OSBORNE, Neville N. Nicotinamide Attenuates Retinal Ischemia and Light Insults to Neurones. Neurochemistry International, 2008, vol. 52, no. 4-5, pp. 786-798. ISSN 0197-0186.

JI, L.; DE PABLO, J. J.and PALECEK, S. P. Cryopreservation of Adherent Human Embryonic Stem Cells. Biotechnology and Bioengineering, Nov 5, 2004, vol. 88, no. 3, pp. 299-312. ISSN 0006-3592; 0006-3592.

JIN, Zi-Bing, et al. Induced Pluripotent Stem Cells for Retinal Degenerative Diseases: A New Perspective on the Challenges. Journal of Genetics, dec, 2009, vol. 88, no. 4, pp. 417-424. ISSN 0973-7731.

JOE, Aaron W.; and GREGORY-EVANS, Kevin. Mesenchymal Stem Cells and Potential Applications in Treating Ocular Disease. Current
Eye Research, nov, 2010, vol. 35, no. 11, pp. 941952. ISSN 1460-2202.

JOHN, S., et al. Choice of Cell Source in CellBased Therapies for Retinal Damage due to AgeRelated Macular Degeneration: A Review. Journal of Ophthalmology, 20130422, 2013, vol. 2013, pp. 465169. ISSN 2090-004X; 2090-004X.

JOHNSON, E. J., et al. The Influence of Supplemental Lutein and Docosahexaenoic Acid on Serum, Lipoproteins, and Macular Pigmentation. The American Journal of Clinical Nutrition, May, 2008, vol. 87, no. 5, pp. 15211529. ISSN 1938-3207; 0002-9165.

JOHNSTONE, B., et al. In Vitro Chondrogenesis of Bone Marrow-Derived Mesenchymal Progenitor Cells. Experimental Cell Research, Jan 10, 1998, vol. 238, no. 1, pp. 265-272. ISSN 0014-4827; 0014-4827.

JONAS, J. B., et al. Intravitreal Autologous BoneMarrow-Derived Mononuclear Cell Transplantation. Acta Ophthalmologica, 20090714, Jun, 2010, vol. 88, no. 4, pp. e131-2. ISSN 1755-3768; 1755-375X.

JONAS, J. B., et al. Intravitreal Autologous Bone Marrow-Derived Mononuclear Cell Transplantation: A Feasibility Report. Acta Ophthalmologica, 20070926, Mar, 2008, vol. 86, no. 2 , pp. $225-226$. ISSN $1755-3768$; 1755-375X.

JORGENSON, E., et al. B4-4: Genome-Wide Association Study of Macular Degeneration: Early Results from the Kaiser Permanente Research Program on Genes, Environment, and Health (RPGEH). Clinical Medicine \& Research, Sep, 2013, vol. 11, no. 3, pp. 146-147. ISSN 1554$6179 ; 1539-4182$.

KACHGAL, S.; and PUTNAM, A. J. Mesenchymal Stem Cells from Adipose and Bone Marrow Promote Angiogenesis Via Distinct Cytokine and Protease Expression Mechanisms. Angiogenesis, 20101121, Mar, 2011, vol. 14, no. 1, pp. 47-59. ISSN 1573-7209; 0969-6970.

KAHN, C. R. Medicine. can we Nip Obesity in its Vascular Bud?. Science (New York, N.Y.), Oct 24, 2008, vol. 322, no. 5901, pp. 542-543. ISSN 10959203; 0036-8075.

KAISER, P. K. Emerging Therapies for Neovascular Age-Related Macular Degeneration: Drugs in the Pipeline. Ophthalmology, May, 2013, vol. 120, no. 5 Suppl, pp. S11-5. ISSN 1549-4713; 0161-6420. 
KALININA, N. I., et al. Mesenchymal Stem Cells in Tissue Growth and Repair. Acta Naturae, Oct, 2011, vol. 3, no. 4, pp. 30-37. ISSN 2075-8251; 2075-8251.

KANG, S. G., et al. Mechanism of Growth Inhibitory Effect of Mitomycin-C on Cultured Human Retinal Pigment Epithelial Cells: Apoptosis and Cell Cycle Arrest. Current Eye Research, Mar, 2001, vol. 22, no. 3, pp. 174-181. ISSN 0271-3683; 0271-3683.

KARL, M. O., et al. Endogenous Gas6 and Ca2+ Channel Activation Modulate Phagocytosis by Retinal Pigment Epithelium. Cellular Signalling, 20080216, Jun, 2008, vol. 20, no. 6, pp. 11591168. ISSN 0898-6568; 0898-6568.

KASSIS, I.; VAKNIN-DEMBINSKY, A.and KARUSSIS, D. Bone Marrow Mesenchymal Stem Cells: Agents of Immunomodulation and Neuroprotection. Current Stem Cell Research \& Therapy, Mar, 2011, vol. 6, no. 1, pp. 63-68. ISSN 1574-888X.

KAUPER, K., et al. Two-Year Intraocular Delivery of Ciliary Neurotrophic Factor by Encapsulated Cell Technology Implants in Patients with Chronic Retinal Degenerative Diseases. Investigative Ophthalmology \& Visual Science, 20121101, Nov 1, 2012, vol. 53, no. 12, pp. 74847491. ISSN 1552-5783; 0146-0404.

KEHRL, J. H., et al. Transforming Growth Factor Beta is an Important Immunomodulatory Protein for Human B Lymphocytes. Journal of Immunology (Baltimore, Md.: 1950), Dec 15, 1986a, vol. 137 , no. 12 , pp. 3855-3860. ISSN 0022-1767; 0022-1767.

KEHRL, J. H., et al. Production of Transforming Growth Factor Beta by Human T Lymphocytes and its Potential Role in the Regulation of T Cell Growth. The Journal of Experimental Medicine, May 1, 1986b, vol. 163, no. 5, pp. 1037-1050. ISSN 0022-1007; 0022-1007.

KENNEDY, C. J.; RAKOCZY, P. E.and CONSTABLE, I. J. Lipofuscin of the Retinal Pigment Epithelium: A Review. Eye (London, England), 1995, vol. 9 ( Pt 6), no. Pt 6, pp. 763771. ISSN 0950-222X; 0950-222X.

KENNEDY, K. A., et al. pH Dependence of Mitomycin C-Induced Cross-Linking Activity in EMT6 Tumor Cells. Cancer Research, Aug, 1985, vol. 45, no. 8, pp. 3541-3547. ISSN 0008-5472; 0008-5472.
KENNEDY, K. A., et al. Retinoic Acid Enhances Skeletal Muscle Progenitor Formation and Bypasses Inhibition by Bone Morphogenetic Protein 4 but Not Dominant Negative BetaCatenin. BMC Biology, 20091008, Oct 8, 2009, vol. 7, pp. 67-7007-7-67. ISSN 1741-7007; 17417007.

KERN, Susanne, et al. Comparative Analysis of Mesenchymal Stem Cells from Bone Marrow, Umbilical Cord Blood, Or Adipose Tissue. Stem Cells (Dayton, Ohio), may, 2006, vol. 24, no. 5, pp. 1294-1301. ISSN 1066-5099.

KEVANY, B. M.; and PALCZEWSKI, K. Phagocytosis of Retinal Rod and Cone Photoreceptors. Physiology (Bethesda, Md.), Feb, 2010, vol. 25, no. 1, pp. 8-15. ISSN 1548-9221; 1548-9221.

KICIC, A., et al. Differentiation of Marrow Stromal Cells into Photoreceptors in the Rat Eye. The Journal of Neuroscience : The Official Journal of the Society for Neuroscience, Aug 27, 2003 , vol. 23, no. 21, pp. 7742-7749. ISSN 15292401; 0270-6474.

KILROY, G. E., et al. Cytokine Profile of Human Adipose-Derived Stem Cells: Expression of Angiogenic, Hematopoietic, and Pro-Inflammatory Factors. Journal of Cellular Physiology, Sep, 2007, vol. 212, no. 3, pp. 702-709. ISSN 00219541; 0021-9541.

KIVELA, T., et al. Ezrin, a Membrane-Organizing Protein, as a Polarization Marker of the Retinal Pigment Epithelium in Vertebrates. Cell and Tissue Research, Aug, 2000, vol. 301, no. 2, pp. 217-223. ISSN 0302-766X; 0302-766X.

KLAIDMAN, L. K.; MUKHERJEE, S. K.and ADAMS, J. D. Oxidative Changes in Brain Pyridine Nucleotides and Neuroprotection using Nicotinamide. Biochimica Et Biophysica Acta, feb, 2001, vol. 1525 , no. 1-2, pp. 136-148. ISSN 00063002 .

KLAIDMAN, L. K., et al. Nicotinamide as a Precursor for NAD+ Prevents Apoptosis in the Mouse Brain Induced by TertiaryButylhydroperoxide. Neuroscience Letters, Mar 8, 1996, vol. 206, no. 1, pp. 5-8. ISSN 0304-3940; 0304-3940.

KLEIN, R., et al. The Prevalence of Age-Related Macular Degeneration and Associated Risk Factors. Archives of Ophthalmology, Jun, 2010, vol. 128, no. 6, pp. 750-758. ISSN 1538-3601; 0003-9950. 
KLEIN, R., et al. Fifteen-Year Cumulative Incidence of Age-Related Macular Degeneration: The Beaver Dam Eye Study. Ophthalmology, Feb, 2007, vol. 114 , no. 2, pp. 253-262. ISSN 15494713; 0161-6420.

KLEIN, R., et al. The Epidemiology of AgeRelated Macular Degeneration. American Journal of Ophthalmology, Mar, 2004, vol. 137, no. 3, pp. 486-495. ISSN 0002-9394; 0002-9394.

KLEIN, Ronald, et al. Prevalence of Age-Related Macular Degeneration in 4 Racial/Ethnic Groups in the Multi-Ethnic Study of Atherosclerosis. Ophthalmology, mar, 2006, vol. 113, no. 3, pp. 373-380. ISSN 1549-4713.

KLIMANSKAYA, Irina, et al. Derivation and Comparative Assessment of Retinal Stem Cells using Transcriptomics, 2004, vol. 6, no. 3 .

KOEDRITH, P.; and SEO, Y. R. Enhancement of the Efficacy of Mitomycin C-Mediated Apoptosis in Human Colon Cancer Cells with RNAi-Based Thioredoxin Reductase 1 Deficiency. Experimental and Therapeutic Medicine, 20110630, Sep, 2011, vol. 2, no. 5, pp. 873-878. ISSN 1792-0981; 1792-0981.

KOGLER, G., et al. Cytokine Production and Hematopoiesis Supporting Activity of Cord BloodDerived Unrestricted Somatic Stem Cells. Experimental Hematology, May, 2005, vol. 33, no. 5, pp. 573-583. ISSN 0301-472X; 0301-472X.

$\mathrm{KOH}$, S. M. VIP Enhances the Differentiation of Retinal Pigment Epithelium in Culture: From cAMP and pp60(C-Src) to Melanogenesis and Development of Fluid Transport Capacity. Progress in Retinal and Eye Research, nov, 2000, vol. 19 , no. 6, pp. 669-688. ISSN 1350-9462.

KOKKINAKI, M.; SAHIBZADA, N.and GOLESTANEH, N. Human Induced Pluripotent Stem-Derived Retinal Pigment Epithelium (RPE) Cells Exhibit Ion Transport, Membrane Potential, Polarized Vascular Endothelial Growth Factor Secretion, and Gene Expression Pattern Similar to Native RPE. Stem Cells (Dayton, Ohio), May, 2011, vol. 29, no. 5, pp. 825-835. ISSN 15494918; 1066-5099.

KOLOMEYER, Anton M.; SUGINO, Ilene K.and ZARBIN, Marco a. Characterization of Conditioned Media Collected from Aged Versus Young Human Eye Cups. Investigative Ophthalmology I\& Visual Science, jul, 2011, vol. 52, no. 8, pp. 5963-5972. ISSN 1552-5783.
KONNO, M., et al. Efficiently Differentiating Vascular Endothelial Cells from Adipose TissueDerived Mesenchymal Stem Cells in Serum-Free Culture. Biochemical and Biophysical Research Communications, 20100812, Oct 1, 2010, vol. 400, no. 4, pp. 461-465. ISSN 1090-2104; 0006-291X.

KRAMPERA, Mauro, et al. Induction of NeuralLike Differentiation in Human Mesenchymal Stem Cells Derived from Bone Marrow, Fat, Spleen and Thymus. Bone, feb, 2007, vol. 40, no. 2, pp. 382390. ISSN 8756-3282.

KREBS, I., et al. Subretinal Surgery and Transplantation of Autologous Pigment Epithelial Cells in Retinal Angiomatous Proliferation. Acta Ophthalmologica, Aug, 2008, vol. 86, no. 5, pp. 504-509. ISSN 1755-3768; 1755-375X.

KULESSA, H.; TURK, G.and HOGAN, B. L. Inhibition of Bmp Signaling Affects Growth and Differentiation in the Anagen Hair Follicle. The EMBO Journal, Dec 15, 2000, vol. 19, no. 24, pp. 6664-6674. ISSN 0261-4189; 0261-4189.

KURODA, T., et al. Highly Sensitive in Vitro Methods for Detection of Residual Undifferentiated Cells in Retinal Pigment Epithelial Cells Derived from Human iPS Cells. PloS One, 20120517, 2012, vol. 7, no. 5, pp. e37342. ISSN 1932-6203; 1932-6203.

KUROKI, M., et al. Reactive Oxygen Intermediates Increase Vascular Endothelial Growth Factor Expression in Vitro and in Vivo. The Journal of Clinical Investigation, Oct 1, 1996, vol. 98, no. 7, pp. 1667-1675. ISSN 0021-9738; 0021-9738.

KWIDZINSKI, E., et al. Indolamine 2,3Dioxygenase is Expressed in the CNS and DownRegulates Autoimmune Inflammation. FASEB Journal : Official Publication of the Federation of American Societies for Experimental Biology, 20050606, Aug, 2005, vol. 19, no. 10, pp. 13471349. ISSN 1530-6860; 0892-6638.

LAMBA, D. A., et al. Efficient Generation of Retinal Progenitor Cells from Human Embryonic Stem Cells. Proceedings of the National Academy of Sciences of the United States of America, 20060814, Aug 22, 2006, vol. 103, no. 34, pp. 12769-12774. ISSN 0027-8424; 0027-8424.

LAMBA, D. A., et al. Generation, Purification and Transplantation of Photoreceptors Derived from Human Induced Pluripotent Stem Cells. PloS One, 20100120, Jan 20, 2010, vol. 5, no. 1, pp. e8763. ISSN 1932-6203; 1932-6203. 
LANDRUM, J. T.; and BONE, R. A. Lutein, Zeaxanthin, and the Macular Pigment. Archives of Biochemistry and Biophysics, Jan 1, 2001, vol. 385, no. 1, pp. 28-40. ISSN 0003-9861; 00039861.

LANE, C.; BOULTON, M.and MARSHALL, J. Transplantation of Retinal Pigment Epithelium using a Pars Plana Approach. Eye (London, England), 1989, vol. 3 ( Pt 1), no. Pt 1, pp. 27-32. ISSN 0950-222X; 0950-222X.

LANGER, R. S.; and VACANTI, J. P. Tissue Engineering: The Challenges Ahead. Scientific American, Apr, 1999, vol. 280, no. 4, pp. 86-89. ISSN 0036-8733; 0036-8733.

Le Blanc K, et al. Mesenchymal Stem Cells Inhibit and Stimulate Mixed Lymphocyte Cultures and Mitogenic Responses Independently of the Major Histocompatibility Complex. Scandinavian Journal of Immunology, jan, 2003, vol. 57, no. 1, pp. 11-20. ISSN 0300-9475.

LE BLANC, K., et al. Mesenchymal Stem Cells for Treatment of Steroid-Resistant, Severe, Acute Graft-Versus-Host Disease: A Phase II Study. Lancet, May 10, 2008, vol. 371, no. 9624, pp. 1579-1586. ISSN 1474-547X; 0140-6736.

LE BLANC, K., et al. Treatment of Severe Acute Graft-Versus-Host Disease with Third Party Haploidentical Mesenchymal Stem Cells. Lancet, May 1, 2004, vol. 363, no. 9419, pp. 1439-1441. ISSN 1474-547X; 0140-6736.

LE BLANC, K.; and RINGDEN, O. Immunobiology of Human Mesenchymal Stem Cells and Future use in Hematopoietic Stem Cell Transplantation. Biology of Blood and Marrow Transplantation : Journal of the American Society for Blood and Marrow Transplantation, May, 2005, vol. 11, no. 5, pp. 321-334. ISSN 1083$8791 ; 1083-8791$.

LEE, Hyunju, et al. Cleavage of the Retinal Pigment Epithelium-Specific Protein RPE65 Under Oxidative Stress. International Journal of Biological Macromolecules, aug, 2010, vol. 47, no. 2, pp. 104-108. ISSN 1879-0003.

LEE, J. H.; and KEMP, D. M. Human AdiposeDerived Stem Cells Display Myogenic Potential and Perturbed Function in Hypoxic Conditions. Biochemical and Biophysical Research Communications, 20060123, Mar 17, 2006, vol. 341 , no. 3, pp. 882-888. ISSN 0006-291X; 0006291X.
LEE, R. H., et al. Characterization and Expression Analysis of Mesenchymal Stem Cells from Human Bone Marrow and Adipose Tissue. Cellular Physiology and Biochemistry : International Journal of Experimental Cellular Physiology, Biochemistry, and Pharmacology, 2004a, vol. 14, no. 4-6, pp. 311-324. ISSN 1015-8987; 1015-8987.

LEE, Ryang Hwa, et al. Cellular Physiology and Biochemistr Y Biochemistry Characterization and Expression Analysis of Mesenchymal Stem Cells from Human Bone Marrow and Adipose Tissue, 2004b, vol. 739, pp. 311-324.

LEE, S. T., et al. Slowed Progression in Models of Huntington Disease by Adipose Stem Cell Transplantation. Annals of Neurology, Nov, 2009, vol. 66, no. 5, pp. 671-681. ISSN 1531-8249; 0364-5134.

LEOPOLD, P. L.; VINCENT, J.and WANG, H. A Comparison of Epithelial-to-Mesenchymal Transition and Re-Epithelialization. Seminars in Cancer Biology, 20120731, Oct, 2012, vol. 22, no. 5-6, pp. 471-483. ISSN 1096-3650; 1044-579X.

L'HEUREUX, N., et al. A Completely Biological Tissue-Engineered Human Blood Vessel. FASEB Journal : Official Publication of the Federation of American Societies for Experimental Biology, Jan, 1998, vol. 12, no. 1, pp. 47-56. ISSN 0892-6638; 0892-6638.

LI, H., et al. Effects of Multiple Agents on Epithelial Differentiation of Rabbit AdiposeDerived Stem Cells in 3D Culture. Tissue Engineering.Part A, 20120613, Sep, 2012a, vol. 18, no. 17-18, pp. 1760-1770. ISSN 1937-335X; 1937-3341.

LI, L., et al. The Effects of Retinoic Acid on the Expression of Neurogranin After Experimental Cerebral Ischemia. Brain Research, 20080620, Aug 21, 2008, vol. 1226, pp. 234-240. ISSN 00068993; 0006-8993.

LI, L. X.; and TURNER, J. E. Inherited Retinal Dystrophy in the RCS Rat: Prevention of Photoreceptor Degeneration by Pigment Epithelial Cell Transplantation. Experimental Eye Research, Dec, 1988, vol. 47, no. 6, pp. 911-917. ISSN 00144835; 0014-4835.

LI, N.; LI, X. R.and YUAN, J. Q. Effects of BoneMarrow Mesenchymal Stem Cells Transplanted into Vitreous Cavity of Rat Injured by Ischemia/Reperfusion. Graefe's Archive for Clinical and Experimental Ophthalmology = Albrecht Von Graefes Archiv Fur Klinische Und Experimentelle Ophthalmologie, 20081216, Apr, 
2009, vol. 247 , no. 4, pp. 503-514. ISSN $1435-$ 702X; 0721-832X.

LI, X., et al. Human Cord Blood-Derived Multipotent Stem Cells (CB-SCs) Treated with allTrans-Retinoic Acid (ATRA) Give Rise to Dopamine Neurons. Biochemical and Biophysical Research Communications, 20120204, Mar 2, 2012b, vol. 419, no. 1, pp. 110-116. ISSN 10902104; 0006-291X.

LI, Yang, et al. Endogenous Bone Marrow Derived Cells Express Retinal Pigment Epithelium Cell Markers and Migrate to Focal Areas of RPE Damage. Investigative Ophthalmology I\& Visual Science, sep, 2007, vol. 48, no. 9, pp. 4321-4327. ISSN 0146-0404.

LIANG, F. Q.; and GODLEY, B. F. Oxidative Stress-Induced Mitochondrial DNA Damage in Human Retinal Pigment Epithelial Cells: A Possible Mechanism for RPE Aging and AgeRelated Macular Degeneration. Experimental Eye Research, Apr, 2003, vol. 76, no. 4, pp. 397-403. ISSN 0014-4835; 0014-4835.

LIECHTY, K. W., et al. Human Mesenchymal Stem Cells Engraft and Demonstrate Site-Specific Differentiation After in Utero Transplantation in Sheep. Nature Medicine, Nov, 2000, vol. 6, no. 11, pp. 1282-1286. ISSN 1078-8956; 1078-8956.

LIM, D. A., et al. Noggin Antagonizes BMP Signaling to Create a Niche for Adult Neurogenesis. Neuron, Dec, 2000, vol. 28, no. 3, pp. 713-726. ISSN 0896-6273; 0896-6273.

LIM, Laurence S., et al. Age-Related Macular Degeneration. Lancet, may, 2012, vol. 379, no. 9827, pp. 1728-1738. ISSN 1474-547X.

LIN, S. C., et al. Endogenous Retinoic Acid Regulates Cardiac Progenitor Differentiation. Proceedings of the National Academy of Sciences of the United States of America, 20100503, May 18, 2010, vol. 107, no. 20, pp. 9234-9239. ISSN 1091-6490; 0027-8424.

LINDNER, M. D., et al. Dissociable Long-Term Cognitive Deficits After Frontal Versus Sensorimotor Cortical Contusions. Journal of Neurotrauma, Mar, 1998, vol. 15, no. 3, pp. 199216. ISSN 0897-7151; 0897-7151.

LIU, J.; WILSON, S.and REH, T. BMP Receptor $1 \mathrm{~b}$ is Required for Axon Guidance and Cell Survival in the Developing Retina. Developmental Biology, Apr 1, 2003, vol. 256, no. 1, pp. 34-48. ISSN 0012-1606; 0012-1606.
LIU, T. M., et al. Identification of Common Pathways Mediating Differentiation of Bone Marrow- and Adipose Tissue-Derived Human Mesenchymal Stem Cells into Three Mesenchymal Lineages. Stem Cells (Dayton, Ohio), 20061109, Mar, 2007, vol. 25, no. 3, pp. 750-760. ISSN 10665099; 1066-5099.

LONGONI, B., et al. Mesenchymal Stem Cells Prevent Acute Rejection and Prolong Graft Function in Pancreatic Islet Transplantation. Diabetes Technology \& Therapeutics, Jun, 2010, vol. 12, no. 6, pp. 435-446. ISSN 1557-8593; $1520-9156$.

LOPATINA, T., et al. Adipose-Derived Stem Cells Stimulate Regeneration of Peripheral Nerves: BDNF Secreted by these Cells Promotes Nerve Healing and Axon Growth De Novo. PloS One, 20110314, Mar 14, 2011, vol. 6, no. 3, pp. e17899. ISSN 1932-6203; 1932-6203.

LOPEZ, R., et al. Transplanted Retinal Pigment Epithelium Modifies the Retinal Degeneration in the RCS Rat. Investigative Ophthalmology \& Visual Science, Mar, 1989, vol. 30, no. 3, pp. 586588. ISSN 0146-0404; 0146-0404.

LOSKUTOVA, E., et al. Macular Pigment and its Contribution to Vision. Nutrients, 20130529, May 29,2013 , vol. 5 , no. 6 , pp. 1962-1969. ISSN 2072$6643 ; 2072-6643$.

LOTAN, R.; and LOTAN, D. Stimulation of Melanogenesis in a Human Melanoma Cell Line by Retinoids. Cancer Research, sep, 1980a, vol. 40, no. 9, pp. 3345-3350. ISSN 0008-5472.

LOTAN, Reuben; and LOTAN, Dafna. Stimulation of Melanogenesis in a Human Melanoma Cell Line by Retinoids Stimulation of Melanogenesis in a Human Melanoma Cell Line by Retinoids1, 1980b, pp. 3345-3350.

LU, Bin, et al. Long-Term Safety and Function of RPE from Human Embryonic Stem Cells in Preclinical Models of Macular Degeneration. Stem Cells (Dayton, Ohio), sep, 2009, vol. 27, no. 9, pp. 2126-2135. ISSN 1549-4918.

LU, L.; YASZEMSKI, M. J.and MIKOS, a. G. Retinal Pigment Epithelium Engineering using Synthetic Biodegradable Polymers. Biomaterials, dec, 2001, vol. 22, no. 24, pp. 3345-3355. ISSN $1713348535 ; 0142-9612$.

LU, M., et al. Advanced Glycation End Products Increase Retinal Vascular Endothelial Growth Factor Expression. The Journal of Clinical 
Investigation, Mar 15, 1998, vol. 101, no. 6, pp. 1219-1224. ISSN 0021-9738; 0021-9738.

LUND, R. D., et al. Subretinal Transplantation of Genetically Modified Human Cell Lines Attenuates Loss of Visual Function in Dystrophic Rats. Proceedings of the National Academy of Sciences of the United States of America, Aug 14, 2001a, vol. 98, no. 17, pp. 9942-9947. ISSN $0027-$ $8424 ; 0027-8424$.

LUND, R. D., et al. Cell Transplantation as a Treatment for Retinal Disease. Progress in Retinal and Eye Research, Jul, 2001b, vol. 20, no. 4, pp. 415-449. ISSN 1350-9462; 1350-9462.

LUND, R. D., et al. Human Embryonic Stem CellDerived Cells Rescue Visual Function in Dystrophic RCS Rats. Cloning and Stem Cells, Fall, 2006, vol. 8, no. 3, pp. 189-199. ISSN 15362302; 1536-2302.

LUND, R. D., et al. Cells Isolated from Umbilical Cord Tissue Rescue Photoreceptors and Visual Functions in a Rodent Model of Retinal Disease. Stem Cells (Dayton, Ohio), 20061019, Mar, 2007, vol. 25, no. 3, pp. 602-611. ISSN 1066-5099; 1066-5099.

MA, Y., et al. Reconstruction of Chemically Burned Rat Corneal Surface by Bone MarrowDerived Human Mesenchymal Stem Cells. Stem Cells (Dayton, Ohio), 20050818, Feb, 2006, vol. 24, no. 2, pp. 315-321. ISSN 1066-5099; 10665099 .

MA, Z., et al. Autologous Transplantation of Retinal Pigment Epithelium-Bruch's Membrane Complex for Hemorrhagic Age-Related Macular Degeneration. Investigative Ophthalmology \& Visual Science, 20081230, Jun, 2009, vol. 50, no. 6, pp. 2975-2981. ISSN 1552-5783; 0146-0404.

MABUCHI, Y., et al. LNGFR $(+)$ THY1(+)VCAM-1(Hi+) Cells Reveal Functionally Distinct Subpopulations in Mesenchymal Stem Cells. Stem Cell Reports, 20130711, Jul 11, 2013, vol. 1, no. 2, pp. 152-165. ISSN 2213-6711; 22136711 .

Macular Photocoagulation Study Group. Argon Laser Photocoagulation for Neovascular Maculopathy. Five-Year Results from Randomized Clinical Trials. Macular Photocoagulation Study Group. Archives of Ophthalmology, Aug, 1991, vol. 109, no. 8, pp. 1109-1114. ISSN 0003-9950; $0003-9950$

Macular Photocoagulation Study Group. Argon Laser Photocoagulation for Neovascular
Maculopathy. Three-Year Results from Randomized Clinical Trials. Macular Photocoagulation Study Group. Archives of Ophthalmology, May, 1986, vol. 104, no. 5, pp. 694-701. ISSN 0003-9950; 0003-9950.

MADEN, M. Role and Distribution of Retinoic Acid during CNS Development. International Review of Cytology, 2001, vol. 209, pp. 1-77. ISSN 0074-7696; 0074-7696.

MAHAFFY, H., et al. Advanced Glycation Endproducts Accumulate in RPE Lysosomal Compartments and Induce Widespread Alteration in Gene Expression: Possible Role in Age-Related Dysfunction. Investigative Ophthalmology \& Visual Science. http://abstracts.iovs.org/cgi/content/abstract/44/5/2 274 ed., 2003, vol. 44, no. 5, pp. 2274.

MANGELSDORF, D. J.; and EVANS, R. M. The RXR Heterodimers and Orphan Receptors. Cell, Dec 15, 1995, vol. 83, no. 6, pp. 841-850. ISSN 0092-8674; 0092-8674.

MARMORSTEIN, a. D. The Polarity of the Retinal Pigment Epithelium. Traffic (Copenhagen, Denmark), dec, 2001, vol. 2, no. 12, pp. 867-872. ISSN 1398-9219.

MARMORSTEIN, a. D., et al. Apical Polarity of N-CAM and EMMPRIN in Retinal Pigment Epithelium Resulting from Suppression of Basolateral Signal Recognition. The Journal of Cell Biology, aug, 1998, vol. 142, no. 3, pp. 697710. ISSN 0021-9525.

MARTINEZ-OSORIO, H., et al. Characterization and Short-Term Culture of Cells Recovered from Human Conjunctival Epithelium by Minimally Invasive Means. Molecular Vision, 20091027, Oct 27, 2009, vol. 15, pp. 2185-2195. ISSN 10900535; 1090-0535.

MASMOUDI-KOUKI, O., et al. Role of PACAP and VIP in Astroglial Functions. Peptides, 20070623, Sep, 2007, vol. 28, no. 9, pp. 17531760. ISSN 0196-9781; 0196-9781.

MASON, C.; and DUNNILL, P. A Brief Definition of Regenerative Medicine. Regenerative Medicine, Jan, 2008a, vol. 3, no. 1, pp. 1-5. ISSN 1746-076X; 1746-0751.

MASON, C.; and DUNNILL, P. A Brief Definition of Regenerative Medicine. Regenerative Medicine, Jan, 2008b, vol. 3, no. 1, pp. 1-5. ISSN 1746-076X; 1746-0751. 
MATA, N. L.; and VOGEL, R. Pharmacologic Treatment of Atrophic Age-Related Macular Degeneration. Current Opinion in Ophthalmology, May, 2010, vol. 21, no. 3, pp. 190-196. ISSN $1531-7021 ; 1040-8738$.

MATSUI, H., et al. Lens Epithelium-Derived Growth Factor: Increased Survival and Decreased DNA Breakage of Human RPE Cells Induced by Oxidative Stress. Investigative Ophthalmology \& Visual Science, Nov, 2001, vol. 42, no. 12, pp. 2935-2941. ISSN 0146-0404; 0146-0404.

MATSUNAGA, H., et al. The mRNA Phenotype of a Human RPE Cell Line at Replicative Senescence. Molecular Vision, dec, 1999, vol. 5, no. July, pp. 39. ISSN 1090-0535.

Matthew L. Brown. ACT Seeks FDA Approval for Stem Cell Study | MetroWest495 Biz. , Nov 30, 2010Available

from:<http://www.wbjournal.com/article/2010113 0/METROWEST01/311309992>.

MAUMUS, Marie; JORGENSEN, Christianand NO $\backslash E L$, Dani`ele. Mesenchymal Stem Cells in Regenerative Medicine Applied tô̂ rheumatic Diseases: Role of Secretome and Exosomes. Biochimie, may, 2013. ISSN 1638-6183.

MCINTOSH AMBROSE, W.; SCHEIN, O.and ELISSEEFF, J. A Tale of Two Tissues: Stem Cells in Cartilage and Corneal Tissue Engineering. Current Stem Cell Research \& Therapy, Mar, 2010, vol. 5, no. 1, pp. 37-48. ISSN 1574-888X.

MEIRELLES LDA, S., et al. Mechanisms Involved in the Therapeutic Properties of Mesenchymal Stem Cells. Cytokine \& Growth Factor Reviews, 20091118, Oct-Dec, 2009, vol. 20, no. 5-6, pp. 419-427. ISSN 1879-0305; 1359 6101.

MEISEL, R., et al. Human Bone Marrow Stromal Cells Inhibit Allogeneic T-Cell Responses by Indoleamine 2,3-Dioxygenase-Mediated Tryptophan Degradation. Blood, 20040304, Jun 15,2004 , vol. 103, no. 12, pp. 4619-4621. ISSN 0006-4971; 0006-4971.

MEKALA, Subba Rao, et al. Derivation, Characterization and Retinal Differentiation of Induced Pluripotent Stem Cells. Journal of Biosciences, jan, 2013, vol. 38, no. 1, pp. 123-134. ISSN 0250-5991.

MENDEZ-FERRER, S., et al. Mesenchymal and Haematopoietic Stem Cells Form a Unique Bone Marrow Niche. Nature, Aug 12, 2010, vol. 466, no. 7308 , pp. 829-834. ISSN 1476-4687; 00280836 .

MEY, J. New Therapeutic Target for CNS Injury? the Role of Retinoic Acid Signaling After Nerve Lesions. Journal of Neurobiology, Jun, 2006, vol. 66 , no. 7, pp. 757-779. ISSN 0022-3034; 00223034 .

MEYER, J. S., et al. Optic Vesicle-Like Structures Derived from Human Pluripotent Stem Cells Facilitate a Customized Approach to Retinal Disease Treatment. Stem Cells (Dayton, Ohio), Aug, 2011, vol. 29, no. 8, pp. 1206-1218. ISSN 1549-4918; 1066-5099.

MEYER, J. S., et al. Modeling Early Retinal Development with Human Embryonic and Induced Pluripotent Stem Cells. Proceedings of the National Academy of Sciences of the United States of America, 20090825, Sep 29, 2009, vol. 106, no. 39 , pp. 16698-16703. ISSN 1091-6490; 00278424.

MEYERS, S. M.; GREENE, T.and GUTMAN, F. A. A Twin Study of Age-Related Macular Degeneration. American Journal of Ophthalmology, Dec, 1995, vol. 120, no. 6, pp. 757-766. ISSN 0002-9394; 0002-9394.

MEZA-ZEPEDA, Leonardo a., et al. HighResolution Analysis of Genetic Stability of Human Adipose Tissue Stem Cells Cultured to Senescence. Journal of Cellular and Molecular Medicine, apr, 2008, vol. 12, no. 2, pp. 553-563. ISSN 1582-1838.

MITCHELL, James B., et al. Immunophenotype of Human Adipose-Derived Cells: Temporal Changes in Stromal-Associated and Stem Cell-Associated Markers. Stem Cells (Dayton, Ohio), feb, 2006, vol. 24, no. 2, pp. 376-385. ISSN 1066-5099.

MIURA, M., et al. SHED: Stem Cells from Human Exfoliated Deciduous Teeth. Proceedings of the National Academy of Sciences of the United States of America, 20030425, May 13, 2003, vol. 100 , no. 10 , pp. 5807-5812. ISSN 0027-8424; 0027-8424.

MIZUNO, H., et al. Myogenic Differentiation by Human Processed Lipoaspirate Cells. Plastic and Reconstructive Surgery, Jan, 2002, vol. 109, no. 1, pp. 199-209; discussion 210-1. ISSN 0032-1052.

MONCADA, S.; and BOLANOS, J. P. Nitric Oxide, Cell Bioenergetics and Neurodegeneration. Journal of Neurochemistry, Jun, 2006, vol. 97, no. 6, pp. 1676-1689. ISSN 0022-3042; 0022-3042. 
MORIGI, M., et al. Mesenchymal Stem Cells are Renotropic, Helping to Repair the Kidney and Improve Function in Acute Renal Failure. Journal of the American Society of Nephrology : JASN, Jul, 2004, vol. 15 , no. 7, pp. 1794-1804. ISSN 1046$6673 ; 1046-6673$.

MORIKAWA, S., et al. Prospective Identification, Isolation, and Systemic Transplantation of Multipotent Mesenchymal Stem Cells in Murine Bone Marrow. The Journal of Experimental Medicine, 20091019, Oct 26, 2009, vol. 206, no. 11, pp. 2483-2496. ISSN 1540-9538; 0022-1007.

MOUSA, S. A.; and MOUSA, S. S. Current Status of Vascular Endothelial Growth Factor Inhibition in Age-Related Macular Degeneration. BioDrugs : Clinical Immunotherapeutics, Biopharmaceuticals and Gene Therapy, Jun, 2010, vol. 24, no. 3, pp. 183-194. ISSN 1173-8804; 1173-8804.

MOVIGLIA, G. A., et al. In Vitro Differentiation of Adult Adipose Mesenchymal Stem Cells into Retinal Progenitor Cells. Ophthalmic Research, 20120821, 2012a, vol. 48 Suppl 1, pp. 1-5. ISSN 1423-0259; 0030-3747.

MOVIGLIA, Gustavo a., et al. In Vitro Differentiation of Adult Adipose Mesenchymal Stem Cells into Retinal Progenitor Cells. Ophthalmic Research, jan, 2012b, vol. 48 Suppl 1, no. suppl 1, pp. 1-5. ISSN 1423-0259.

MÜLLER, Frank; ROHRER, Hermannand VOGEL-H $\backslash$ OPKER, Astrid. Bone Morphogenetic Proteins Specify the Retinal Pigment Epithelium in the Chick Embryo. Development (Cambridge, England), oct, 2007, vol. 134, no. 19, pp. 34833493. ISSN 0950-1991.

MULLINS, R. F., et al. Drusen Associated with Aging and Age-Related Macular Degeneration Contain Proteins Common to Extracellular Deposits Associated with Atherosclerosis, Elastosis, Amyloidosis, and Dense Deposit Disease. FASEB Journal : Official Publication of the Federation of American Societies for Experimental Biology, may, 2000, vol. 14, no. 7, pp. 835-846. ISSN 0892-6638.

MUNDRA, Vaibhav; GERLING, Ivan C.and MAHATO, Ram I. Mesenchymal Stem Cell-Based Therapy. Molecular Pharmaceutics, jan, 2013, vol. 10, no. 1, pp. 77-89. ISSN 1543-8392.

MUNOZ, B., et al. Causes of Blindness and Visual Impairment in a Population of Older Americans: The Salisbury Eye Evaluation Study. Archives of Ophthalmology, Jun, 2000, vol. 118, no. 6, pp. 819-825. ISSN 0003-9950; 0003-9950.
MURAGLIA, A.; CANCEDDA, R.and QUARTO, R. Clonal Mesenchymal Progenitors from Human Bone Marrow Differentiate in Vitro According to a Hierarchical Model. Journal of Cell Science, Apr, 2000, vol. 113 ( Pt 7), no. Pt 7, pp. 1161-1166. ISSN 0021-9533; 0021-9533.

MURALI, Deepa, et al. Distinct Developmental Programs Require Different Levels of Bmp Signaling during Mouse Retinal Development. Development (Cambridge, England), mar, 2005, vol. 132, no. 5, pp. 913-923. ISSN 0950-1991.

NAKAGAWA, H., et al. Human Mesenchymal Stem Cells Successfully Improve Skin-Substitute Wound Healing. The British Journal of Dermatology, Jul, 2005, vol. 153, no. 1, pp. 29-36. ISSN 0007-0963; 0007-0963.

NAKAHARA, H., et al. Bone and Cartilage Formation in Diffusion Chambers by Subcultured Cells Derived from the Periosteum. Bone, 1990, vol. 11, no. 3, pp. 181-188. ISSN 8756-3282; 1873-2763.

NAKANISHI, C., et al. Gene and Protein Expression Analysis of Mesenchymal Stem Cells Derived from Rat Adipose Tissue and Bone Marrow. Circulation Journal : Official Journal of the Japanese Circulation Society, 20110712, 2011, vol. 75, no. 9, pp. 2260-2268. ISSN 1347-4820; 1346-9843.

NANDROT, E. F., et al. Retinal Pigment Epithelial Cells use a MerTK-Dependent Mechanism to Limit the Phagocytic Particle Binding Activity of Alphavbeta5 Integrin. Biology of the Cell / Under the Auspices of the European Cell Biology Organization, 20120305, Jun, 2012, vol. 104, no. 6, pp. 326-341. ISSN 1768-322X; 0248-4900.

NAU, H. Teratogenicity of Isotretinoin Revisited: Species Variation and the Role of all-TransRetinoic Acid. Journal of the American Academy of Dermatology, Nov, 2001, vol. 45, no. 5, pp. S183-7. ISSN 0190-9622; 0190-9622.

NAUTA, A. J.; and FIBBE, W. E. Immunomodulatory Properties of Mesenchymal Stromal Cells. Blood, 20070730, Nov 15, 2007, vol. 110 , no. 10 , pp. 3499-3506. ISSN 0006-4971; 0006-4971.

NIETO-MIGUEL, T., et al. In Vitro Simulation of Corneal Epithelium Microenvironment Induces a Corneal Epithelial-Like Cell Phenotype from Human Adipose Tissue Mesenchymal Stem Cells. Current Eye Research, 20130614, Sep, 2013, vol. 
38, no. 9, pp. 933-944. ISSN 1460-2202; 02713683 .

NISHIMOTO, M., et al. Activation of the VIP/VPAC2 System Induces Reactive Astrocytosis Associated with Increased Expression of Glutamate Transporters. Brain Research, 20110131, Apr 6, 2011, vol. 1383, pp. 43-53. ISSN 1872-6240; 0006-8993.

NOER, A.; BOQUEST, A. C.and COLLAS, P. Dynamics of Adipogenic Promoter DNA Methylation during Clonal Culture of Human Adipose Stem Cells to Senescence. BMC Cell Biology, 20070529, May 29, 2007, vol. 8, pp. 18. ISSN 1471-2121; 1471-2121.

NOER, A., et al. Stable CpG Hypomethylation of Adipogenic Promoters in Freshly Isolated, Cultured, and Differentiated Mesenchymal Stem Cells from Adipose Tissue. Molecular Biology of the Cell, 20060607, Aug, 2006, vol. 17, no. 8, pp. 3543-3556. ISSN 1059-1524; 1059-1524.

NOHE, A., et al. Signal Transduction of Bone Morphogenetic Protein Receptors. Cellular Signalling, Mar, 2004, vol. 16, no. 3, pp. 291-299. ISSN 0898-6568; 0898-6568.

NOORT, W. A., et al. Mesenchymal Stem Cells Promote Engraftment of Human Umbilical Cord Blood-Derived CD34(+) Cells in NOD/SCID Mice. Experimental Hematology, Aug, 2002, vol. 30, no. 8, pp. 870-878. ISSN 0301-472X; 0301$472 \mathrm{X}$.

NOTH, U., et al. Multilineage Mesenchymal Differentiation Potential of Human Trabecular Bone-Derived Cells. Journal of Orthopaedic Research: Official Publication of the Orthopaedic Research Society, Sep, 2002, vol. 20, no. 5, pp. 1060-1069. ISSN 0736-0266; 0736-0266.

NOWAK, J. Z. Age-Related Macular Degeneration (AMD): Pathogenesis and Therapy. Pharmacological Reports : PR, May-Jun, 2006, vol. 58, no. 3, pp. 353-363. ISSN 1734-1140; 1734-1140.

NUSSBAUM, Jeannette, et al. Transplantation of Undifferentiated Murine Embryonic Stem Cells in the Heart: Teratoma Formation and Immune Response. FASEB Journal : Official Publication of the Federation of American Societies for Experimental Biology, may, 2007, vol. 21, no. 7, pp. 1345-1357. ISSN 1530-6860.

O'DRISCOLL, S. W., et al. The Chondrogenic Potential of Periosteum Decreases with Age. Journal of Orthopaedic Research : Official
Publication of the Orthopaedic Research Society, Jan, 2001, vol. 19, no. 1, pp. 95-103. ISSN 07360266; 0736-0266.

OGATA, N., et al. Pigment Epithelium Derived Factor as a Neuroprotective Agent Against Ischemic Retinal Injury. Current Eye Research, Apr, 2001, vol. 22, no. 4, pp. 245-252. ISSN 0271$3683 ; 0271-3683$.

OGAWA, M. Differentiation and Proliferation of Hematopoietic Stem Cells. Blood, Jun 1, 1993, vol. 81 , no. 11 , pp. 2844-2853. ISSN 0006-4971; 0006-4971.

$\mathrm{OH}, \mathrm{J}$. Y., et al. The Anti-Inflammatory and AntiAngiogenic Role of Mesenchymal Stem Cells in Corneal Wound Healing Following Chemical Injury. Stem Cells (Dayton, Ohio), 20080110, Apr, 2008, vol. 26, no. 4, pp. 1047-1055. ISSN 15494918; 1066-5099.

$\mathrm{OH}$, J. Y., et al. Cytokine Secretion by Human Mesenchymal Stem Cells Cocultured with Damaged Corneal Epithelial Cells. Cytokine, 20090214, Apr, 2009, vol. 46, no. 1, pp. 100-103. ISSN 1096-0023; 1043-4666.

OHR, M.; and KAISER, P. K. Aflibercept in Wet Age-Related Macular Degeneration: A Perspective Review. Therapeutic Advances in Chronic Disease, Jul, 2012, vol. 3, no. 4, pp. 153-161. ISSN 2040-6223; 2040-6223.

OKADA, Y., et al. Retinoic-Acid-ConcentrationDependent Acquisition of Neural Cell Identity during in Vitro Differentiation of Mouse Embryonic Stem Cells. Developmental Biology, Nov 1, 2004, vol. 275, no. 1, pp. 124-142. ISSN 0012-1606; 0012-1606.

OKANO, T.; and MATSUDA, T. Tissue Engineered Skeletal Muscle: Preparation of Highly Dense, Highly Oriented Hybrid Muscular Tissues. Cell Transplantation, Jan-Feb, 1998, vol. 7, no. 1, pp. 71-82. ISSN 0963-6897; 0963-6897.

ONG, Shin-Yeu; DAI, Huiand LEONG, Kam W. Inducing Hepatic Differentiation of Human Mesenchymal Stem Cells in Pellet Culture. Biomaterials, aug, 2006, vol. 27, no. 22, pp. 40874097. ISSN 0142-9612.

ORFANOS, C. E.; EHLERT, R.and GOLLNICK, H. The Retinoids. A Review of their Clinical Pharmacology and Therapeutic Use. Drugs, Oct, 1987, vol. 34, no. 4, pp. 459-503. ISSN 00126667; 0012-6667. 
PALAY, Sanford L.; and CHAN-PALAY, Victoria. Cerebeller Cortex: Cytology and Organization. . Springer-Verlag, ed., Berlin, Heidelberg, New York: , 1974.

PANDA-JONAS, S.; JONAS, J. B.and JAKOBCZYK-ZMIJA, M. Retinal Pigment Epithelial Cell Count, Distribution, and Correlations in Normal Human Eyes. American Journal of Ophthalmology, Feb, 1996, vol. 121, no. 2, pp. 181-189. ISSN 0002-9394; 0002-9394.

PAQUET-DURAND, F., et al. Excessive Activation of Poly(ADP-Ribose) Polymerase Contributes to Inherited Photoreceptor Degeneration in the Retinal Degeneration 1 Mouse. The Journal of Neuroscience : The Official Journal of the Society for Neuroscience, Sep 19, 2007, vol. 27, no. 38, pp. 10311-10319. ISSN $1529-2401 ; 0270-6474$.

PARAMESWARAN, S., et al. Induced Pluripotent Stem Cells Generate both Retinal Ganglion Cells and Photoreceptors: Therapeutic Implications in Degenerative Changes in Glaucoma and AgeRelated Macular Degeneration. Stem Cells (Dayton, Ohio), Apr, 2010, vol. 28, no. 4, pp. 695703. ISSN 1549-4918; 1066-5099.

PARK, Bong-Wook, et al. Peripheral Nerve Regeneration using Autologous Porcine SkinDerived Mesenchymal Stem Cells. Journal of Tissue Engineering and Regenerative Medicine, 02-21, 2011, vol. 5, no. 10, pp. n/a-n/a. Available from $<$ http://dx.doi.org/10.1002\%2Fterm.404>. ISSN 1932-6254.

PARK, H. K., et al. Mitomycin C-Induced Cell Death in Mouse Lens Epithelial Cells. Ophthalmic Research, Jul-Aug, 2002, vol. 34, no. 4, pp. 213 219. ISSN 0030-3747; 0030-3747.

PASCOLINI, D., et al. Review Article 2002 Global Update of a Vailable Data on Visual Impairment : A Compilation of Population-Based Prevalence Studies, 2004.

PATRICK, C. W.,Jr, et al. Preadipocyte Seeded PLGA Scaffolds for Adipose Tissue Engineering. Tissue Engineering, Apr, 1999, vol. 5, no. 2, pp. 139-151. ISSN 1076-3279; 1076-3279.

PATRICK, C. W.,Jr, et al. Long-Term Implantation of Preadipocyte-Seeded PLGA Scaffolds. Tissue Engineering, Apr, 2002, vol. 8, no. 2, pp. 283-293. ISSN 1076-3279; 1076-3279.

PAUL, G.; and ANISIMOV, S. V. The Secretome of Mesenchymal Stem Cells: Potential Implications for Neuroregeneration. Biochimie,
20130719, Jul 19, 2013. ISSN 1638-6183; 03009084.

PELED, T., et al. Nicotinamide, a SIRT1 Inhibitor, Inhibits Differentiation and Facilitates Expansion of Hematopoietic Progenitor Cells with Enhanced Bone Marrow Homing and Engraftment. Experimental Hematology, 20111220, Apr, 2012, vol. 40, no. 4, pp. 342-55.e1. ISSN 1873-2399; 0301-472X.

PEYMAN, G. A., et al. A Technique for Retinal Pigment Epithelium Transplantation for AgeRelated Macular Degeneration Secondary to Extensive Subfoveal Scarring. Ophthalmic Surgery, Feb, 1991, vol. 22, no. 2, pp. 102-108. ISSN 0022-023X; 0022-023X.

PHINNEY, D. G., et al. Plastic Adherent Stromal Cells from the Bone Marrow of Commonly used Strains of Inbred Mice: Variations in Yield, Growth, and Differentiation. Journal of Cellular Biochemistry, Mar 15, 1999, vol. 72, no. 4, pp. 570-585. ISSN 0730-2312; 0730-2312.

PHINNEY, Donald G.; and PROCKOP, Darwin J. Concise Review: Mesenchymal Stem/Multipotent Stromal Cells: The State of Transdifferentiation and Modes of Tissue Repair--Current Views. Stem Cells (Dayton, Ohio), nov, 2007, vol. 25, no. 11, pp. 2896-2902. ISSN 5049887755; 1549-4918.

PITTENGER, M. F., et al. Multilineage Potential of Adult Human Mesenchymal Stem Cells. Science (New York, N.Y.), apr, 1999, vol. 284, no. 5411, pp. 143-147. ISSN 0036-8075.

POPP, F. C., et al. Mesenchymal Stem Cells can Affect Solid Organ Allograft Survival. Transplantation, May 15, 2009, vol. 87, no. 9 Suppl, pp. S57-62. ISSN 1534-6080; 0041-1337.

POPP, F. C., et al. Mesenchymal Stem Cells can Induce Long-Term Acceptance of Solid Organ Allografts in Synergy with Low-Dose Mycophenolate. Transplant Immunology, 20080830, Nov, 2008, vol. 20, no. 1-2, pp. 55-60. ISSN 0966-3274; 0966-3274.

PROCKOP, D. J. Marrow Stromal Cells as Stem Cells for Nonhematopoietic Tissues. Science (New York, N.Y.), Apr 4, 1997, vol. 276, no. 5309, pp. 71-74. ISSN 0036-8075; 0036-8075.

PROCTOR, B. L.; and GAULDEN, M. E. Chemical stability of mitomycin $\mathrm{C}$ in culture medium with and without fetal calf serum as determined by high pressure liquid chromatography and mass spectrometry.. Archives of Environmental Contamination and 
Toxicology.

http://www.ncbi.nlm.nih.gov/pubmed/?term=Che mical + stability + of + mitomycin $+\mathrm{C}+$ in + culture + med ium + with + and + without + fetal + calf + serum + as + det ermined+by+high+pressure+liquid + chromatograp hy+and + mass + spectrometry. ed., 1986, vol. 15, no. 2 , pp. 235-240.

PROVENCIO, I., et al. A Novel Human Opsin in the Inner Retina. The Journal of Neuroscience : The Official Journal of the Society for Neuroscience, Jan 15, 2000, vol. 20, no. 2, pp. 600-605. ISSN 1529-2401; 0270-6474.

PUISSANT, $\quad \mathrm{B} \backslash$ 'enl'edicte, et al. Immunomodulatory Effect of Human Adipose Tissue-Derived Adult Stem Cells: Comparison with Bone Marrow Mesenchymal Stem Cells. British Journal of Haematology, apr, 2005, vol. 129, no. 1, pp. 118-129. ISSN 0007-1048.

QUILLEN, D. A. Common Causes of Vision Loss in Elderly Patients. American Family Physician, Jul, 1999, vol. 60, no. 1, pp. 99-108. ISSN 0002838X; 0002-838X.

RADTKE, N. D., et al. Preliminary Report: Indications of Improved Visual Function After Retinal Sheet Transplantation in Retinitis Pigmentosa Patients. American Journal of Ophthalmology, sep, 1999, vol. 128, no. 3, pp. 384-387. ISSN 0002-9394.

RADTKE, Norman D., et al. Vision Improvement in Retinal Degeneration Patients by Implantation of Retina Together with Retinal Pigment Epithelium. American Journal of Ophthalmology, aug, 2008, vol. 146, no. 2, pp. 172-182. ISSN $0002-9394$

RADTKE, Norman D., et al. Transplantation of Intact Sheets of Fetal Neural Retina with its Retinal Pigment Epithelium in Retinitis Pigmentosa Patients. American Journal of Ophthalmology, apr, 2002, vol. 133, no. 4, pp. 544-550. ISSN 0002-9394.

Randomized Clinical Trial. Argon Laser Photocoagulation for Senile Macular Degeneration. Results of a Randomized Clinical Trial. Archives of Ophthalmology, Jun, 1982, vol. 100, no. 6, pp. 912-918. ISSN 0003-9950; 00039950 .

RANGON, C. M., et al. Mechanisms of VIPInduced Neuroprotection Against Neonatal Excitotoxicity. Annals of the New York Academy of Sciences, Jul, 2006, vol. 1070, pp. 512-517. ISSN 0077-8923; 0077-8923.
RASMUSSON, I. Immune Modulation by Mesenchymal Stem Cells. Experimental Cell Research, 20060424, Jul 15, 2006, vol. 312, no. 12, pp. 2169-2179. ISSN 0014-4827; 0014-4827.

RASMUSSON, I., et al. Mesenchymal Stem Cells Inhibit the Formation of Cytotoxic $\mathrm{T}$ Lymphocytes, but Not Activated Cytotoxic $\mathrm{T}$ Lymphocytes Or Natural Killer Cells. Transplantation, Oct 27, 2003, vol. 76, no. 8, pp. 1208-1213. ISSN 0041-1337; 0041-1337.

RATNAPRIYA, R.; and CHEW, E. Y. AgeRelated Macular Degeneration-Clinical Review and Genetics Update. Clinical Genetics, aug, 2013, vol. 84, no. 2, pp. 160-166. ISSN 1399-0004.

RAYMOND, S. M; and JACKSON, I. J. The Retinal Pigmented Epithelium is Required for Development and Maintenance of the Mouse Neural Retina. Current Biology : CB, Nov 1, 1995, vol. 5, no. 11, pp. 1286-1295. ISSN 0960-9822; 0960-9822.

RECALDE, S., et al. Age-Related Macular Degeneration Genetics. Ophthalmology, May, 2008 , vol. 115 , no. 5, pp. 916-916.e1. ISSN 15494713; 0161-6420.

REIN, D. B., et al. Forecasting Age-Related Macular Degeneration through the Year 2050: The Potential Impact of New Treatments. Archives of Ophthalmology, Apr, 2009, vol. 127, no. 4, pp. 533-540. ISSN 1538-3601; 0003-9950.

REINOSO, R., et al. Differential Cell Proliferation, Apoptosis, and Immune Response in Healthy and Evaporative-Type Dry Eye Conjunctival Epithelia. Investigative Ophthalmology \& Visual Science, 20110701, Jul 1, 2011, vol. 52, no. 7, pp. 4819-4828. ISSN 15525783; 0146-0404.

RESNIKOFF, Serge, et al. Policy and Practice Global Data on Visual Impairment in the Year 2002, 2004, vol. 012831, no. 04.

RICHER, S., et al. Double-Masked, PlaceboControlled, Randomized Trial of Lutein and Antioxidant Supplementation in the Intervention of Atrophic Age-Related Macular Degeneration: The Veterans LAST Study (Lutein Antioxidant Supplementation Trial). Optometry (St.Louis, Mo.), Apr, 2004, vol. 75, no. 4, pp. 216-230. ISSN 1529-1839; 1558-1527.

RIZZOLO, L. J. Development and Role of Tight Junctions in the Retinal Pigment Epithelium. International Review of Cytology, 2007, vol. 258, pp. 195-234. ISSN 0074-7696; 0074-7696. 
RIZZOLO, L. J. Polarity and the Development of the Outer Blood-Retinal Barrier. Histology and Histopathology, Oct, 1997, vol. 12, no. 4, pp. 1057-1067. ISSN 0213-3911; 0213-3911.

RIZZOLO, L. J., et al. Integration of Tight Junctions and Claudins with the Barrier Functions of the Retinal Pigment Epithelium. Progress in Retinal and Eye Research, 20110617, Sep, 2011, vol. 30, no. 5, pp. 296-323. ISSN 1873-1635; 1350-9462.

ROBERT, L. Mechanisms of Aging of the Extracellular Matrix: Role of the Elastin-Laminin Receptor. Gerontology, 1998, vol. 44, no. 6, pp. 307-317. ISSN 0304-324X; 0304-324X.

ROCKWELL, S.; KENNEDY, K. A.and SARTORELLI, A. C. Mitomycin-C as a Prototype Bioreductive Alkylating Agent: In Vitro Studies of Metabolism and Cytotoxicity. International Journal of Radiation Oncology, Biology, Physics, Mar-Apr, 1982, vol. 8, no. 3-4, pp. 753-755. ISSN 0360-3016; 0360-3016.

RUBINA, K., et al. Adipose Stromal Cells Stimulate Angiogenesis Via Promoting Progenitor Cell Differentiation, Secretion of Angiogenic Factors, and Enhancing Vessel Maturation. Tissue Engineering.Part A, Aug, 2009, vol. 15, no. 8, pp. 2039-2050. ISSN 1937-335X; 1937-3341.

RUSSELL, S. R., et al. Location, Substructure, and Composition of Basal Laminar Drusen Compared with Drusen Associated with Aging and Age-Related Macular Degeneration. American Journal of Ophthalmology, Feb, 2000, vol. 129, no. 2, pp. 205-214. ISSN 0002-9394; 0002-9394.

SADANAGA-AKIYOSHI, F., et al. Nicotinamide Attenuates Focal Ischemic Brain Injury in Rats: With Special Reference to Changes in Nicotinamide and NAD+ Levels in Ischemic Core and Penumbra. Neurochemical Research, Aug, 2003, vol. 28, no. 8, pp. 1227-1234. ISSN 03643190; 0364-3190.

SAFFORD, K. M.; and RICE, H. E. Stem Cell Therapy for Neurologic Disorders: Therapeutic Potential of Adipose-Derived Stem Cells. Current Drug Targets, Feb, 2005, vol. 6, no. 1, pp. 57-62. ISSN 1389-4501; 1389-4501.

SAKAKIBARA, Y., et al. Delayed Treatment with Nicotinamide (Vitamin B3) Reduces the Infarct Volume Following Focal Cerebral Ischemia in Spontaneously Hypertensive Rats, Diabetic and Non-Diabetic Fischer 344 Rats. Brain Research, Mar 22, 2002, vol. 931, no. 1, pp. 68-73. ISSN 0006-8993; 0006-8993.
SAlGADO, A. J., et al. Adipose Tissue Derived Stem Cells Secretome: Soluble Factors and their Roles in Regenerative Medicine. Current Stem Cell Research \& Therapy, Jun, 2010, vol. 5, no. 2, pp. 103-110. ISSN 1574-888X.

SANCHEZ-RAMOS, J., et al. Adult Bone Marrow Stromal Cells Differentiate into Neural Cells in Vitro. Experimental Neurology, aug, 2000, vol. 164, no. 2, pp. 247-256. ISSN 0014-4886.

SANGIOVANNI, J. P., et al. Omega-3 LongChain Polyunsaturated Fatty Acid Intake Inversely Associated with 12-Year Progression to Advanced Age-Related Macular Degeneration. Archives of Ophthalmology, Jan, 2009a, vol. 127, no. 1, pp. 110-112. ISSN 1538-3601; 0003-9950.

SANGIOVANNI, J. P., et al. ?-3 Long-Chain Polyunsaturated Fatty Acid Intake and 12-Y Incidence of Neovascular Age-Related Macular Degeneration and Central Geographic Atrophy: AREDS Report 30, a Prospective Cohort Study from the Age-Related Eye Disease Study. , 2009bAvailable

from:<http://ajcn.nutrition.org/content/90/6/1601.1 ong $>$.

SANGIOVANNI, J. P., et al. The Relationship of Dietary Omega-3 Long-Chain Polyunsaturated Fatty Acid Intake with Incident Age-Related Macular Degeneration: AREDS Report no. 23. Archives of Ophthalmology, Sep, 2008, vol. 126, no. 9, pp. 1274-1279. ISSN 1538-3601; 00039950.

SANGIOVANNI, J. P., et al. The Relationship of Dietary Lipid Intake and Age-Related Macular Degeneration in a Case-Control Study: AREDS Report no. 20. Archives of Ophthalmology, May, 2007, vol. 125, no. 5, pp. 671-679. ISSN 00039950; 0003-9950.

SATO, K., et al. Nitric Oxide Plays a Critical Role in Suppression of T-Cell Proliferation by Mesenchymal Stem Cells. Blood, 20060919, Jan 1, 2007, vol. 109, no. 1, pp. 228-234. ISSN 00064971; 0006-4971.

SATO, Y., et al. Stereo-Selective Neuroprotection Against Stroke with Vitamin A Derivatives. Brain Research, 20080918, Nov 19, 2008, vol. 1241, pp. 188-192. ISSN 1872-6240; 0006-8993.

SATO, Yasushi, et al. Human Mesenchymal Stem Cells Xenografted Directly to Rat Liver are Differentiated into Human Hepatocytes without Fusion, 2005, vol. 106, no. 2, pp. 756-763. 
SAUVE, Y., et al. Visual Field Loss in RCS Rats and the Effect of RPE Cell Transplantation. Experimental Neurology, Aug, 1998, vol. 152, no. 2, pp. 243-250. ISSN 0014-4886; 0014-4886.

SCHIPPER, B. M., et al. Regional Anatomic and Age Effects on Cell Function of Human AdiposeDerived Stem Cells. Annals of Plastic Surgery, May, 2008, vol. 60, no. 5, pp. 538-544. ISSN 1536-3708; 0148-7043.

SCHUTT, F., et al. Proteins Modified by Malondialdehyde, 4-Hydroxynonenal, Or Advanced Glycation End Products in Lipofuscin of Human Retinal Pigment Epithelium. Investigative Ophthalmology \& Visual Science, Aug, 2003, vol. 44, no. 8, pp. 3663-3668. ISSN 0146-0404; 0146-0404.

SCHWARTZ, Steven D., et al. Embryonic Stem Cell Trials for Macular Degeneration: A Preliminary Report. Lancet, mar, 2012, vol. 379, no. 9817 , pp. $713-720$. ISSN 1474-547X.

Science Web News by Carl Zimmer. The Brain: Our Strange, Important, Subconscious Light Detectors | DiscoverMagazine.Com. http://discovermagazine.com/2012/jan-feb/12-thebrain-our-strange-light-detector\#.UgaFhn-K_3Q ed. , 2012, 15 January, 2012.

SEDDON, J. M., et al. Dietary Carotenoids, Vitamins A, C, and E, and Advanced Age-Related Macular Degeneration. Eye Disease Case-Control Study Group. JAMA : The Journal of the American Medical Association, Nov 9, 1994, vol. 272, no. 18, pp. 1413-1420. ISSN 0098-7484; 0098-7484.

SEDDON, J. M.; COTE, J.and ROSNER, B. Progression of Age-Related Macular Degeneration: Association with Dietary Fat, Transunsaturated Fat, Nuts, and Fish Intake. Archives of Ophthalmology, Dec, 2003, vol. 121, no. 12, pp. 1728-1737. ISSN 0003-9950; 00039950.

SEDDON, J. M., et al. Dietary Fat and Risk for Advanced Age-Related Macular Degeneration. Archives of Ophthalmology, Aug, 2001, vol. 119, no. 8, pp. 1191-1199. ISSN 0003-9950; 00039950 .

SEDDON, Johanna M., et al. Risk Models for Progression to Advanced Age-Related Macular Degeneration using Demographic, Environmental, Genetic, and Ocular Factors. Ophthalmology, nov, 2011, vol. 118 , no. 11 , pp. 2203-2211. ISSN 15494713.
SEILER, Magdalene J.; and ARAMANT, Robert B. Cell Replacement and Visual Restoration by Retinal Sheet Transplants. Progress in Retinal and Eye Research, nov, 2012, vol. 31, no. 6, pp. 661687. ISSN 1873-1635.

SELMANI, Z., et al. Human Leukocyte AntigenG5 Secretion by Human Mesenchymal Stem Cells is Required to Suppress $\mathrm{T}$ Lymphocyte and Natural Killer Function and to Induce CD4+CD25highFOXP3+ Regulatory T Cells. Stem Cells (Dayton, Ohio), 20071011, Jan, 2008, vol. 26, no. 1, pp. 212-222. ISSN 1549-4918; 10665099 .

SEO, Min Jeong, et al. Differentiation of Human Adipose Stromal Cells into Hepatic Lineage in Vitro and in Vivo. Biochemical and Biophysical Research Communications, mar, 2005, vol. 328, no. 1 , pp. 258-264. ISSN 0006-291X.

SETHE, S.; SCUTT, A.and STOLZING, A. Aging of Mesenchymal Stem Cells. Ageing Research Reviews, 20051128, Feb, 2006, vol. 5, no. 1, pp. 91-116. ISSN 1568-1637; 1568-1637.

SHEEDLO, H. J.; LI, L.and TURNER, J. E. Photoreceptor Cell Rescue in the RCS Rat by RPE Transplantation: A Therapeutic Approach in a Model of Inherited Retinal Dystrophy. Progress in Clinical and Biological Research, 1989, vol. 314, pp. 645-658. ISSN 0361-7742; 0361-7742.

SHIMA, D. T.; DEUTSCH, U.and D'AMORE, P. A. Hypoxic Induction of Vascular Endothelial Growth Factor (VEGF) in Human Epithelial Cells is Mediated by Increases in mRNA Stability. FEBS Letters, Aug 21, 1995, vol. 370, no. 3, pp. 203-208. ISSN 0014-5793; 0014-5793.

SHINOKA, T., et al. Tissue-Engineered Heart Valves. Autologous Valve Leaflet Replacement Study in a Lamb Model. Circulation, Nov 1, 1996, vol. 94, no. 9 Suppl, pp. II164-8. ISSN 0009-7322; $0009-7322$.

SHIODA, S., et al. Pleiotropic Functions of PACAP in the CNS: Neuroprotection and Neurodevelopment. Annals of the New York Academy of Sciences, Jul, 2006, vol. 1070, pp. 550-560. ISSN 0077-8923; 0077-8923.

SIEVING, P. A., et al. Ciliary Neurotrophic Factor (CNTF) for Human Retinal Degeneration: Phase I Trial of CNTF Delivered by Encapsulated Cell Intraocular Implants. Proceedings of the National Academy of Sciences of the United States of America, 20060227, Mar 7, 2006, vol. 103, no. 10, pp. 3896-3901. ISSN 0027-8424; 0027-8424. 
SILVA, C., et al. Expression Profile of Male Germ Cell-Associated Genes in Mouse Embryonic Stem Cell Cultures Treated with all-Trans Retinoic Acid and Testosterone. Molecular Reproduction and Development, Jan, 2009, vol. 76, no. 1, pp. 11-21. ISSN 1098-2795; 1040-452X.

SINGH, A. K., et al. Adipose Derived Mesenchymal Stem Cells Partially Rescue Mitomycin C Treated ARPE19 Cells from Death in Co-Culture Condition. Histology and Histopathology, 20130530, May 30, 2013a. ISSN 1699-5848; 0213-3911.

SINGH, Amar K., et al. Bioactive Substrates for Human Retinal Pigment Epithelial Cell Growth from Elastin-Like Recombinamers. Journal of Biomedical Materials Research.Part A, mar, 2013b, pp. 1-8. ISSN 1552-4965.

SIOUD, M. New Insights into Mesenchymal Stromal Cell-Mediated T-Cell Suppression through Galectins. Scandinavian Journal of Immunology, Feb, 2011, vol. 73, no. 2, pp. 79-84. ISSN 1365-3083; 0300-9475.

SIPP, Douglas; and TAKAHASHI, Masayo. $<b r$ >Pilot Clinical Study into iPS Cell Therapy for Eye Disease Starts in Japan. , 30th July 2013, 2013.

SIQUEIRA, R. C., et al. Intravitreal Injection of Autologous Bone Marrow-Derived Mononuclear Cells for Hereditary Retinal Dystrophy: A Phase I Trial. Retina (Philadelphia, Pa.), Jun, 2011, vol. 31 , no. 6 , pp. 1207-1214. ISSN 1539-2864; 0275$004 X$.

SITTINGER, M., et al. Tissue Engineering and Autologous Transplant Formation: Practical Approaches with Resorbable Biomaterials and New Cell Culture Techniques. Biomaterials, Feb, 1996, vol. 17, no. 3, pp. 237-242. ISSN 01429612; 0142-9612.

SITU, R., et al. Effects of all-Trans-Retinoic Acid on Melanocyte Adhesion and Motility. Dermatology.

http://www.ncbi.nlm.nih.gov/pubmed/?term=Situ, + R.,+Dermatology, $+186,+38,+199$ ed., 1993, vol. 186 , no. 1 , pp. $38-44$.

SIVAPRASAD, S.; and HYKIN, P. What is New in the Management of Wet Age-Related Macular Degeneration?. British Medical Bulletin, 20130207, 2013, vol. 105, pp. 201-211. ISSN $1471-8391 ; 0007-1420$

SNODDERLY, D. M. Evidence for Protection Against Age-Related Macular Degeneration by
Carotenoids and Antioxidant Vitamins. The American Journal of Clinical Nutrition, Dec, 1995, vol. 62, no. 6 Suppl, pp. 1448S-1461S. ISSN 0002-9165; 0002-9165.

SOTIROPOULOU, P. A., et al. Interactions between Human Mesenchymal Stem Cells and Natural Killer Cells. Stem Cells (Dayton, Ohio), 20050811, Jan, 2006, vol. 24, no. 1, pp. 74-85. ISSN 1066-5099; 1066-5099.

SOTTILE, V., et al. Stem Cell Characteristics of Human Trabecular Bone-Derived Cells. Bone, May, 2002, vol. 30, no. 5, pp. 699-704. ISSN 8756-3282; 1873-2763.

SPAGGIARI, G. M., et al. Mesenchymal Stem Cells Inhibit Natural Killer-Cell Proliferation, Cytotoxicity, and Cytokine Production: Role of Indoleamine 2,3-Dioxygenase and Prostaglandin E2. Blood, 20071019, Feb 1, 2008, vol. 111, no. 3, pp. 1327-1333. ISSN 0006-4971; 0006-4971.

SPAGGIARI, G. M., et al. Mesenchymal Stem Cell-Natural Killer Cell Interactions: Evidence that Activated NK Cells are Capable of Killing MSCs, Whereas MSCs can Inhibit IL-2-Induced NK-Cell Proliferation. Blood, 20051020, Feb 15, 2006, vol. 107 , no. 4, pp. 1484-1490. ISSN 0006-4971; 00064971.

Spanish Eyes Epidemiological (SEE) Study Group. Prevalence of Age-Related Macular Degeneration in Spain. British Journal of Ophthalmology, jul, 2011, vol. 95, no. 7, pp. 931936. ISSN 1468-2079.

SPARROW, J. R.; and BOULTON, M. RPE Lipofuscin and its Role in Retinal Pathobiology. Experimental Eye Research, May, 2005, vol. 80, no. 5, pp. 595-606. ISSN 0014-4835; 0014-4835.

SPARROW, J. R.; HICKS, D.and HAMEL, C. P. The Retinal Pigment Epithelium in Health and Disease. Current Molecular Medicine, Dec, 2010, vol. 10, no. 9, pp. 802-823. ISSN 1875-5666; $1566-5240$

SRIVASTAVA, Girish K., et al. Elastin-Like Recombinamers as Substrates for Retinal Pigment Epithelial Cell Growth. Journal of Biomedical Materials Research.Part A, jun, 2011a, vol. 97, no. 3, pp. 243-250. ISSN 1552-4965.

SRIVASTAVA, Girish K., et al. Trypan Blue Staining Method for Quenching the Autofluorescence of RPE Cells for Improving Protein Expression Analysis. Experimental Eye Research, dec, 2011b, vol. 93, no. 6, pp. 956-962. ISSN 1096-0007. 
SRIVASTAVA, Girish K., et al. Flow Cytometry Assessment of the Purity of Human Retinal Pigment Epithelial Primary Cell Cultures. Journal of Immunological Methods, mar, 2013, vol. 389, no. 1-2, pp. 61-68. ISSN 1872-7905.

STANGA, P. E., et al. Functional Assessment of the Native Retinal Pigment Epithelium After the Surgical Excision of Subfoveal Choroidal Neovascular Membranes Type II: Preliminary Results. International Ophthalmology, 2001, vol. 23, no. 4-6, pp. 309-316. ISSN 0165-5701; 0165 5701.

STANGA, P. E., et al. Retinal Pigment Epithelium Translocation After Choroidal Neovascular Membrane Removal in Age-Related Macular Degeneration. Ophthalmology, Aug, 2002, vol. 109 , no. 8, pp. 1492-1498. ISSN 0161-6420; 01616420 .

STEINDL-KUSCHER, K., et al. Activation of the Beta-Catenin Signaling Pathway and its Impact on RPE Cell Cycle. Investigative Ophthalmology \& Visual Science, 20090415, Sep, 2009, vol. 50, no. 9, pp. 4471-4476. ISSN 1552-5783; 0146-0404.

STEWART, M. W. Aflibercept (VEGF Trap-Eye): The Newest Anti-VEGF Drug. The British Journal of Ophthalmology, 20120323, Sep, 2012, vol. 96, no. 9, pp. 1157-1158. ISSN 1468-2079; $0007-$ 1161 .

STEWART, M. W.; GRIPPON, S.and KIRKPATRICK, P. Aflibercept. Nature Reviews.Drug Discovery, 20120330, Mar 30, 2012, vol. 11, no. 4, pp. 269-270. ISSN 1474$1784 ; 1474-1776$.

STONE, E. M.; SHEFFIELD, V. C.and HAGEMAN, G. S. Molecular Genetics of AgeRelated Macular Degeneration. Human Molecular Genetics, Oct 1, 2001, vol. 10, no. 20, pp. 22852292. ISSN 0964-6906; 0964-6906.

STRAUSS, O. [Retinal Pigment Epithelium]. Der Ophthalmologe : Zeitschrift Der Deutschen Ophthalmologischen Gesellschaft, apr, 2009, vol. 106, no. 4, pp. 297-298. ISSN 9783540878933; $1433-0423$

STRAUSS, O., et al. The Royal College of Surgeons Rat: An Animal Model for Inherited Retinal Degeneration with a Still Unknown Genetic Defect. Acta Anatomica, 1998, vol. 162, no. 2-3, pp. 101-111. ISSN 0001-5180; 0001-5180.

STRAUSS, Olaf. The Retinal Pigment Epithelium in Visual Function. Physiological Reviews, jul,
2005, vol. 85, no. 3, pp. 845-881. ISSN 00319333.

STREILEIN, J. Wayne. Ocular Immune Privilege : The Eye Takes a Dim but Practical View of Immunity and Inflammation, 2003, vol. 74, no. August, pp. 179-185.

STREILEIN, J. W., et al. Immunobiology and Privilege of Neuronal Retina and Pigment Epithelium Transplants. Vision Research, Feb, 2002, vol. 42, no. 4, pp. 487-495. ISSN 00426989; 0042-6989.

SUESSKIND, D., et al. Full Macular Translocation Following Photodynamic Therapy in Neovascular Age-Related Macular Degeneration. Eye (London, England), 20070330, Jun, 2008, vol. 22, no. 6, pp. 834-837. ISSN 0950-222X; 0950$222 \mathrm{X}$.

SUGII, S., et al. Feeder-Dependent and FeederIndependent iPS Cell Derivation from Human and Mouse Adipose Stem Cells. Nature Protocols, 20110224, Mar, 2011, vol. 6, no. 3, pp. 346-358. ISSN 1750-2799; 1750-2799.

SUGII, S., et al. Human and Mouse AdiposeDerived Cells Support Feeder-Independent Induction of Pluripotent Stem Cells. Proceedings of the National Academy of Sciences of the United States of America, 20100203, Feb 23, 2010, vol. 107 , no. 8 , pp. $3558-3563$. ISSN 1091-6490; 00278424.

SUNDELIN, S. P.; and NILSSON, S. E. Lipofuscin-Formation in Retinal Pigment Epithelial Cells is Reduced by Antioxidants. Free Radical Biology \& Medicine, Jul 15, 2001, vol. 31, no. 2, pp. 217-225. ISSN 0891-5849; 0891-5849.

SURJANA, D.; HALLIDAY, G. M.and DAMIAN, D. L. Role of Nicotinamide in DNA Damage, Mutagenesis, and DNA Repair. Journal of Nucleic Acids, 20100725, Jul 25, 2010a, vol. 2010 , pp. 10.4061/2010/157591. ISSN 2090021X; 2090-0201.

SURJANA, Devita; HALLIDAY, Gary M.and DAMIAN, Diona L. Role of Nicotinamide in DNA Damage, Mutagenesis, and DNA Repair. Journal of Nucleic Acids, jan, 2010b, vol. 2010. ISSN 2090-021X.

SWENOR, B. K., et al. The Impact of Fish and Shellfish Consumption on Age-Related Macular Degeneration. Ophthalmology, 20100713, Dec, 2010 , vol. 117 , no. 12 , pp. $2395-2401$. ISSN 1549 4713; 0161-6420. 
SYEDAIN, Z. H., et al. Implantable Arterial Grafts from Human Fibroblasts and Fibrin using a MultiGraft Pulsed Flow-Stretch Bioreactor with Noninvasive Strength Monitoring. Biomaterials, 20101008, Jan, 2011, vol. 32, no. 3, pp. 714-722. ISSN 1878-5905; 0142-9612.

TAKAHASHI, A., et al. Oxidative Stress-Induced Apoptosis is Associated with Alterations in Mitochondrial Caspase Activity and Bcl-2Dependent Alterations in Mitochondrial $\mathrm{pH}$ (pHm). Brain Research Bulletin, Feb 15, 2004, vol. 62, no. 6, pp. 497-504. ISSN 0361-9230; 0361-9230.

TALENS-VISCONTI, R., et al. Human Mesenchymal Stem Cells from Adipose Tissue: Differentiation into Hepatic Lineage. Toxicology in Vitro : An International Journal Published in Association with BIBRA, 20060905, Mar, 2007, vol. 21, no. 2, pp. 324-329. ISSN 1879-3177; 0887-2333.

TALENS-VISCONTI, R., et al. Hepatogenic Differentiation of Human Mesenchymal Stem Cells from Adipose Tissue in Comparison with Bone Marrow Mesenchymal Stem Cells. World Journal of Gastroenterology : WJG, Sep 28, 2006, vol. 12 , no. 36 , pp. 5834-5845. ISSN 1007-9327; $1007-9327$

TALLONE, T., et al. Adult Human Adipose Tissue Contains several Types of Multipotent Cells. Journal of Cardiovascular Translational Research, 20110215, Apr, 2011, vol. 4, no. 2, pp. 200-210. ISSN 1937-5395.

TAN, G., et al. Differential Effect of Myocardial Matrix and Integrins on Cardiac Differentiation of Human Mesenchymal Stem Cells. Differentiation; Research in Biological Diversity, 20100321, AprJun, 2010, vol. 79, no. 4-5, pp. 260-271. ISSN 1432-0436; 0301-4681.

TAN, J. S., et al. Dietary Antioxidants and the Long-Term Incidence of Age-Related Macular Degeneration: The Blue Mountains Eye Study. Ophthalmology, 20070730, Feb, 2008, vol. 115, no. 2, pp. 334-341. ISSN 1549-4713; 0161-6420.

THANOS, C. G., et al. Sustained Secretion of Ciliary Neurotrophic Factor to the Vitreous, using the Encapsulated Cell Therapy-Based NT-501 Intraocular Device. Tissue Engineering, Nov-Dec, 2004, vol. 10, no. 11-12, pp. 1617-1622. ISSN 1076-3279; 1076-3279.

THOMAS, M.; MOUSA, S. S.and MOUSA, S. A. Comparative Effectiveness of Aflibercept for the Treatment of Patients with Neovascular Age-
Related Macular Degeneration. Clinical Ophthalmology (Auckland, N.Z.), 20130308, 2013, vol. 7, pp. 495-501. ISSN 1177-5467; 1177-5467.

THUMANN, G., et al. Transplantation of Autologous Iris Pigment Epithelium After Removal of Choroidal Neovascular Membranes. Archives of Ophthalmology, Oct, 2000, vol. 118, no. 10, pp. 1350-1355. ISSN 0003-9950; 00039950 .

TIMPER, Katharina, et al. Human Adipose TissueDerived Mesenchymal Stem Cells Differentiate into Insulin, Somatostatin, and Glucagon Expressing Cells. Biochemical and Biophysical Research Communications, mar, 2006, vol. 341, no. 4, pp. 1135-1140. ISSN 0006-291X.

TOMBRAN-TINK, J.; and JOHNSON, L. V. Neuronal Differentiation of Retinoblastoma Cells Induced by Medium Conditioned by Human RPE Cells. Investigative Ophthalmology $\mid \&$ Visual Science, aug, 1989, vol. 30, no. 8, pp. 1700-1707. ISSN 0146-0404.

TOMBRAN-TINK, J., et al. Expression, Secretion, and Age-Related Downregulation of Pigment Epithelium-Derived Factor, a Serpin with Neurotrophic Activity. The Journal of Neuroscience : The Official Journal of the Society for Neuroscience, Jul, 1995, vol. 15, no. 7 Pt 1, pp. 4992-5003. ISSN 0270-6474; 0270-6474.

TOMITA, M., et al. Bone Marrow-Derived Stem Cells can Differentiate into Retinal Cells in Injured Rat Retina. Stem Cells (Dayton, Ohio), 2002, vol. 20 , no. 4, pp. 279-283. ISSN 1066-5099; 10665099 .

TOMITA, M., et al. A Comparison of Neural Differentiation and Retinal Transplantation with Bone Marrow-Derived Cells and Retinal Progenitor Cells. Stem Cells (Dayton, Ohio), Oct, 2006 , vol. 24 , no. 10 , pp. $2270-2278$. ISSN 10665099; 1066-5099.

TONG, J. P.; and YAO, Y. F. Contribution of VEGF and PEDF to Choroidal Angiogenesis: A Need for Balanced Expressions. Clinical Biochemistry, 20060110, Mar, 2006, vol. 39, no. 3, pp. 267-276. ISSN 0009-9120; 0009-9120.

TOTH, C. A.; and FREEDMAN, S. F. Macular Translocation with 360-Degree Peripheral Retinectomy Impact of Technique and Surgical Experience on Visual Outcomes. Retina (Philadelphia, Pa.), 2001, vol. 21, no. 4, pp. 293303. ISSN 0275-004X; 0275-004X. 
TOUBAI, T., et al. Mesenchymal Stem Cells for Treatment and Prevention of Graft-Versus-Host Disease After Allogeneic Hematopoietic Cell Transplantation. Current Stem Cell Research \& Therapy, 20090901, Dec, 2009, vol. 4, no. 4, pp. 252-259. ISSN 1574-888X.

TRAYNOR, K. Aflibercept Approved for Macular Degeneration. American Journal of Health-System Pharmacy : AJHP : Official Journal of the American Society of Health-System Pharmacists, Jan 1, 2012, vol. 69, no. 1, pp. 6. ISSN 1535-2900; 1079-2082.

TROUSSE, F.; ESTEVE, P.and BOVOLENTA, P. Bmp4 Mediates Apoptotic Cell Death in the Developing Chick Eye. The Journal of Neuroscience : The Official Journal of the Society for Neuroscience, Feb 15, 2001, vol. 21, no. 4, pp. 1292-1301. ISSN 1529-2401; 0270-6474.

TSE, W. T., et al. Suppression of Allogeneic TCell Proliferation by Human Marrow Stromal Cells: Implications in Transplantation. Transplantation, Feb 15, 2003, vol. 75, no. 3, pp. 389-397. ISSN 0041-1337; 0041-1337.

TUCKER, B. A., et al. Transplantation of Adult Mouse iPS Cell-Derived Photoreceptor Precursors Restores Retinal Structure and Function in Degenerative Mice. PloS One, 20110429, Apr 29, 2011, vol. 6, no. 4, pp. e18992. ISSN 1932-6203; $1932-6203$

TULI, R., et al. A Simple, High-Yield Method for Obtaining Multipotential Mesenchymal Progenitor Cells from Trabecular Bone. Molecular Biotechnology, Jan, 2003, vol. 23, no. 1, pp. 37-49. ISSN 1073-6085; 1073-6085.

U.S.Food and Drug Administration. FDA Approved Trametinib (MEKINIST Tablet, GlaxoSmithKline, LLC), for the Treatment of Patients with Unresectable Or Metastatic Melanoma with BRAF V600E Or V600K Mutation as Detected by an FDA-Approved Test. , May29, 2013Available

from: $<$ http://www.fda.gov/Drugs/InformationOnD rugs/ApprovedDrugs/ucm354478.htm>.

U.S.Food and Drug Administration. FDA Approved Dabrafenib (TAFINLAR Capsule, GlaxoSmithKline, LLC), for the Treatment of Patients with Unresectable Or Metastatic Melanoma with BRAF V600E Mutation as Detected by an FDA-Approved Test. , May 29, 2013Available

from:<http://www.fda.gov/Drugs/InformationOnD rugs/ApprovedDrugs/ucm354477.htm>.
U.S.Food and Drug Administration. FDA Approved Dabrafenib (TAFINLAR Capsule, GlaxoSmithKline, LLC), for the Treatment of Patients with Unresectable Or Metastatic Melanoma with BRAF V600E Mutation as Detected by an FDA-Approved Test. , 2013Available

from: $<$ http://www.fda.gov/drugs/informationondru gs/approveddrugs/ucm279174.htm\#updates>.

UCCELLI, A., et al. Neuroprotective Features of Mesenchymal Stem Cells. Best Practice \& Research.Clinical Haematology, 20110225, Mar, 2011 a, vol. 24, no. 1, pp. 59-64. ISSN 1532-1924; $1521-6926$

UCCELLI, A.; LARONI, A.and FREEDMAN, M. S. Mesenchymal Stem Cells for the Treatment of Multiple Sclerosis and Other Neurological Diseases. Lancet Neurology, Jul, 2011b, vol. 10, no. 7 , pp. 649-656. ISSN 1474-4465; 1474-4422.

UCCELLI, A.; MANCARDI, G.and CHIESA, S. Is there a Role for Mesenchymal Stem Cells in Autoimmune Diseases?. Autoimmunity, Dec, 2008a, vol. 41, no. 8, pp. 592-595. ISSN $1607-$ 842X; 0891-6934.

UCCELLI, A.; MORETTA, L.and PISTOIA, V. Mesenchymal Stem Cells in Health and Disease. Nature Reviews.Immunology, Sep, 2008b, vol. 8, no. 9 , pp. 726-736. ISSN 1474-1733; 1474-1733.

UCCELLI, A.; MORETTA, L.and PISTOIA, V. Immunoregulatory Function of Mesenchymal Stem Cells. European Journal of Immunology, Oct, 2006a, vol. 36, no. 10, pp. 2566-2573. ISSN 0014-2980; 0014-2980.

UCCELLI, A.; PISTOIA, V.and MORETTA, L. Mesenchymal Stem Cells: A New Strategy for Immunosuppression?. Trends in Immunology, 20070402, May, 2007, vol. 28, no. 5, pp. 219-226. ISSN 1471-4906; 1471-4906.

UCCELLI, A.; and PROCKOP, D. J. Why should Mesenchymal Stem Cells (MSCs) Cure Autoimmune Diseases?. Current Opinion in Immunology, 20101117, Dec, 2010, vol. 22, no. 6, pp. 768-774. ISSN 1879-0372; 0952-7915.

UCCELLI, A., et al. Stem Cells in Inflammatory Demyelinating Disorders: A Dual Role for Immunosuppression and Neuroprotection. Expert Opinion on Biological Therapy, Jan, 2006b, vol. 6, no. 1, pp. 17-22. ISSN 1744-7682; 1471-2598.

UEHARA, N., et al. Nicotinamide Blocks NMethyl-N-Nitrosourea-Induced Photoreceptor Cell Apoptosis in Rats through Poly (ADP-Ribose) 
Polymerase Activity and Jun N-Terminal Kinase/Activator Protein-1 Pathway Inhibition. Experimental Eye Research, 20051005, Mar, 2006, vol. 82, no. 3, pp. 488-495. ISSN 0014-4835; 0014-4835.

UEKI, S., et al. Retinoic Acids are Potent Inhibitors of Spontaneous Human Eosinophil Apoptosis. Journal of Immunology (Baltimore, Md.: 1950), Dec 1, 2008, vol. 181, no. 11, pp. 7689-7698. ISSN 1550-6606; 0022-1767.

UEKI, T., et al. A Novel Secretory Factor, Neurogenesin-1, Provides Neurogenic Environmental Cues for Neural Stem Cells in the Adult Hippocampus. The Journal of Neuroscience : The Official Journal of the Society for Neuroscience, Dec 17, 2003, vol. 23, no. 37, pp. 11732-11740. ISSN 1529-2401; 0270-6474.

UMETSU, Y., et al. Structural Difference of Vasoactive Intestinal Peptide in Two Distinct Membrane Mimicking Environments. Biochimica Et Biophysica Acta, 20110322, Mar 22, 2011. ISSN 0006-3002; 0006-3002.

UNGERSTEDT, J. S.; BLOMBACK, M.and SODERSTROM, T. Nicotinamide is a Potent Inhibitor of Proinflammatory Cytokines. Clinical and Experimental Immunology, Jan, 2003a, vol. 131, no. 1, pp. 48-52. ISSN 0009-9104; 00099104.

UNGERSTEDT, J. S., et al. Nicotinamide Inhibits Endotoxin-Induced Monocyte Tissue Factor Expression. Journal of Thrombosis and Haemostasis : JTH, Dec, 2003b, vol. 1, no. 12, pp. 2554-2560. ISSN 1538-7933; 1538-7836.

VACA, P., et al. Nicotinamide Induces Differentiation of Embryonic Stem Cells into Insulin-Secreting Cells. Experimental Cell Research, 20071204, Mar 10, 2008, vol. 314, no. 5, pp. 969-974. ISSN 0014-4827; 0014-4827.

VALTINK, M., et al. Physiological Features of Primary Cultures and Subcultures of Human Retinal Pigment Epithelial Cells before and After Cryopreservation for Cell Transplantation. Graefe's Archive for Clinical and Experimental Ophthalmology = Albrecht Von Graefes Archiv Fur Klinische Und Experimentelle Ophthalmologie, Dec, 1999, vol. 237, no. 12, pp. 1001-1006. ISSN 0721-832X; 0721-832X.

VAN MEURS, J. C.; and VAN DEN BIESEN, P. R. Autologous Retinal Pigment Epithelium and Choroid Translocation in Patients with Exudative Age-Related Macular Degeneration: Short-Term Follow-Up. American Journal of Ophthalmology,
Oct, 2003, vol. 136, no. 4, pp. 688-695. ISSN 0002-9394; 0002-9394.

VELEZ-MONTOYA, R., et al. Intraocular and Systemic Levels of Vascular Endothelial Growth Factor in Advanced Cases of Retinopathy of Prematurity. Clinical Ophthalmology (Auckland, N.Z.), 20100907, Sep 7, 2010, vol. 4, pp. 947-953. ISSN 1177-5483; 1177-5467.

VELEZ-MONTOYA, Raul, et al. CURRENT KNOWLEDGE AND TRENDS IN AGERELATED MACULAR DEGENERATION: Today's and Future Treatments. Retina (Philadelphia, Pa.), dec, 2012, no. C, pp. 2013. ISSN 1539-2864.

VERNER-COLE, E. A.; DAVIS, S. J.and LAUER, A. K. Aflibercept for the Treatment of Neovascular Age-Related Macular Degeneration. Drugs of Today (Barcelona, Spain : 1998), May, 2012, vol. 48, no. 5, pp. 317-329. ISSN 1699$3993 ; 1699-3993$.

VIRAG, L. Structure and Function of Poly(ADPRibose) Polymerase-1: Role in Oxidative StressRelated Pathologies. Current Vascular Pharmacology, Jul, 2005, vol. 3, no. 3, pp. 209214. ISSN 1570-1611; 1570-1611.

VOGT, Rhonda R., et al. Bone Morphogenetic Protein-4 Enhances Vascular Endothelial Growth Factor Secretion by Human Retinal Pigment Epithelial Cells. Journal of Cellular Biochemistry, aug, 2006, vol. 98, no. 5, pp. 1196-1202. ISSN 0730-2312.

VON RUCKMANN, A.; FITZKE, F. W.and BIRD, A. C. Distribution of Fundus Autofluorescence with a Scanning Laser Ophthalmoscope. The British Journal of Ophthalmology, May, 1995, vol. 79, no. 5, pp. 407-412. ISSN 0007-1161; 0007-1161.

VOSSMERBAEUMER, U., et al. Induction of Retinal Pigment Epithelium Properties in Ciliary Margin Progenitor Cells. Clinical \& Experimental Ophthalmology, May, 2008, vol. 36, no. 4, pp. 358-366. ISSN 1442-9071; 1442-6404.

VOSSMERBAEUMER, U., et al. Retinal Pigment Epithelial Phenotype Induced in Human Adipose Tissue-Derived Mesenchymal Stromal Cells. Cytotherapy, 2009, vol. 11, no. 2, pp. 177-188. ISSN 1477-2566; 1465-3249.

VUGLER, Anthony, et al. Elucidating the Phenomenon of HESC-Derived RPE: Anatomy of Cell Genesis, Expansion and Retinal Transplantation. Experimental Neurology, dec, 
2008, vol. 214 , no. 2 , pp. $347-361$. ISSN $1090-$ 2430 .

VUGLER, Anthony, et al. Embryonic Stem Cells and Retinal Repair. Mechanisms of Development, 2007, vol. 124, no. 11-12, pp. 807-829. ISSN 2076084010; 0925-4773.

WAGNER, Wolfgang, et al. Comparative Characteristics of Mesenchymal Stem Cells from Human Bone Marrow, Adipose Tissue, and Umbilical Cord Blood. Experimental Hematology, nov, 2005, vol. 33, no. 11, pp. 1402-1416. ISSN 0301-472X.

WALLACE, D. C.; BROWN, M. D.and LOTT, M. T. Mitochondrial DNA Variation in Human Evolution and Disease. Gene, Sep 30, 1999, vol. 238, no. 1, pp. 211-230. ISSN 0378-1119; 03781119.

WAN, J. X., et al. Bone Marrow-Derived Mesenchymal Stem Cells Differentiation into Tubular Epithelial-Like Cells in Vitro. Cell Biochemistry and Function, 20111129, Mar, 2012, vol. 30, no. 2, pp. 129-138. ISSN 1099-0844; 0263-6484

WANG, N. K., et al. Transplantation of Reprogrammed Embryonic Stem Cells Improves Visual Function in a Mouse Model for Retinitis Pigmentosa. Transplantation, Apr 27, 2010a, vol. 89, no. 8, pp. 911-919. ISSN 1534-6080; 00411337.

WANG, Shaomei, et al. Non-Invasive Stem Cell Therapy in a Rat Model for Retinal Degeneration and Vascular Pathology. PloS One, jan, 2010b, vol. 5, no. 2, pp. e9200. ISSN 1932-6203.

WASSELL, J., et al. The Photoreactivity of the Retinal Age Pigment Lipofuscin. The Journal of Biological Chemistry, Aug 20, 1999, vol. 274, no. 34, pp. 23828-23832. ISSN 0021-9258; 00219258.

WATT, F. M.; and HOGAN, B. L. Out of Eden: Stem Cells and their Niches. Science (New York, N.Y.), Feb 25, 2000, vol. 287, no. 5457, pp. $1427-$ 1430. ISSN 0036-8075; 0036-8075.

WEALE, R. A. Guest Editorial: Notes on the Macular Pigment. Ophthalmic \& Physiological Optics : The Journal of the British College of Ophthalmic Opticians (Optometrists), Jan, 2007, vol. 27, no. 1, pp. 1-10. ISSN 0275-5408; 02755408 .

WEI, X., et al. Adipose Stromal Cells-Secreted Neuroprotective Media Against Neuronal
Apoptosis. Neuroscience Letters, 20090621, Oct 2, 2009, vol. 462, no. 1, pp. 76-79. ISSN 1872-7972; 0304-3940.

WEI, Xin, et al. Mesenchymal Stem Cells: A New Trend for Cell Therapy. Acta Pharmacologica Sinica, jun, 2013, vol. 34, no. 6, pp. 747-754. ISSN 1745-7254.

WEISZ, J. M., et al. Allogenic Fetal Retinal Pigment Epithelial Cell Transplant in a Patient with Geographic Atrophy. Retina (Philadelphia, Pa.), 1999, vol. 19, no. 6, pp. 540-545. ISSN 0275-004X; 0275-004X.

WEITER, J. J., et al. Retinal Pigment Epithelial Lipofuscin and Melanin and Choroidal Melanin in Human Eyes. Investigative Ophthalmology $\mid \&$ Visual Science, feb, 1986a, vol. 27, no. 2, pp. 145152. ISSN 0146-0404.

WEITER, J. J., et al. Retinal Pigment Epithelial Lipofuscin and Melanin and Choroidal Melanin in Human Eyes. Investigative Ophthalmology \& Visual Science, Feb, 1986b, vol. 27, no. 2, pp. 145152. ISSN 0146-0404; 0146-0404.

WHITEHEAD, A. J.; MARES, J. A.and DANIS, R. P. Macular Pigment: A Review of Current Knowledge. Archives of Ophthalmology, Jul, 2006, vol. 124, no. 7, pp. 1038-1045. ISSN 0003-9950; 0003-9950.

WICKHAM, M. Q., et al. Multipotent Stromal Cells Derived from the Infrapatellar Fat Pad of the Knee. Clinical Orthopaedics and Related Research, Jul, 2003, vol. (412), no. 412, pp. 196212. ISSN 0009-921X; 0009-921X.

WONG, T. Y.; LIEW, G.and MITCHELL, P. Clinical Update: New Treatments for Age-Related Macular Degeneration. Lancet, Jul 21, 2007, vol. 370, no. 9583, pp. 204-206. ISSN 1474-547X; 0140-6736.

WOOTEN, B. R.; and HAMMOND, B. R. Macular Pigment: Influences on Visual Acuity and Visibility. Progress in Retinal and Eye Research, Mar, 2002, vol. 21, no. 2, pp. 225-240. ISSN 13509462; 1350-9462.

WORMALD, R., et al. Photodynamic Therapy for Neovascular Age-Related Macular Degeneration. The Cochrane Database of Systematic Reviews, 20070718, Jul 18, 2007, vol. (3), no. 3, pp. CD002030. ISSN 1469-493X; 1361-6137.

WORMALD, R., et al. Photodynamic Therapy for Neovascular Age-Related Macular Degeneration. The Cochrane Database of Systematic Reviews, 
20051019, Oct 19, 2005, vol. (4), no. 4, pp. CD002030. ISSN 1469-493X; 1361-6137.

WORMALD, R., et al. Photodynamic Therapy for Neovascular Age-Related Macular Degeneration. The Cochrane Database of Systematic Reviews, 2003, vol. (2), no. 2, pp. CD002030. ISSN 1469493X; 1361-6137.

WORMALD, R., et al. Photodynamic Therapy for Neovascular Age-Related Macular Degeneration. The Cochrane Database of Systematic Reviews, 2001, vol. (3), no. 3, pp. CD002030. ISSN 1469493X; 1361-6137.

WU, K. Y.; WANG, H. Z.and HONG, S. J. Mechanism of Mitomycin-Induced Apoptosis in Cultured Corneal Endothelial Cells. Molecular Vision, 20080915, Sep 15, 2008, vol. 14, pp. 17051712. ISSN 1090-0535; 1090-0535.

WU, W. C.; KAO, Y. H.and HU, D. N. A Comparative Study of Effects of Antiproliferative Drugs on Human Retinal Pigment Epithelial Cells in Vitro. Journal of Ocular Pharmacology and Therapeutics : The Official Journal of the Association for Ocular Pharmacology and Therapeutics, Jun, 2002, vol. 18, no. 3, pp. 251 264. ISSN 1080-7683; 1080-7683.

YAMAMOTO, S., et al. Retinal Pigment Epithelial Transplants and Retinal Function in RCS Rats. Investigative Ophthalmology \& Visual Science, Oct, 1993, vol. 34, no. 11, pp. 3068-3075. ISSN 0146-0404; 0146-0404.

YANNAS, I. V., et al. Wound Tissue can Utilize a Polymeric Template to Synthesize a Functional Extension of Skin. Science (New York, N.Y.), Jan 8, 1982, vol. 215, no. 4529, pp. 174-176. ISSN 0036-8075; 0036-8075.

YLA-HERTTUALA, S. Endgame: Glybera Finally Recommended for Approval as the First Gene Therapy Drug in the European Union. Molecular Therapy: The Journal of the American Society of Gene Therapy, Oct, 2012, vol. 20, no. 10, pp. 1831-1832. ISSN 1525-0024; 1525-0016.

YU, C. E., et al. Mutations in the Consensus Helicase Domains of the Werner Syndrome Gene. Werner's Syndrome Collaborative Group. American Journal of Human Genetics, Feb, 1997, vol. 60, no. 2, pp. 330-341. ISSN 0002-9297; 0002-9297.

YU, S., et al. Effects of Bone Marrow Stromal Cell Injection in an Experimental Glaucoma Model. Biochemical and Biophysical Research Communications, 20060427, Jun 16, 2006a, vol.
344, no. 4, pp. 1071-1079. ISSN 0006-291X; 0006-291X.

YU, S. W., et al. Apoptosis-Inducing Factor Mediates Poly(ADP-Ribose) (PAR) PolymerInduced Cell Death. Proceedings of the National Academy of Sciences of the United States of America, 20061120, Nov 28, 2006b, vol. 103, no. 48, pp. 18314-18319. ISSN 0027-8424; $0027-$ 8424.

ZAHABI, A., et al. A New Efficient Protocol for Directed Differentiation of Retinal Pigmented Epithelial Cells from Normal and Retinal Disease Induced Pluripotent Stem Cells. Stem Cells and Development, 20120203, Aug 10, 2012a, vol. 21, no. 12 , pp. $2262-2272$. ISSN $1557-8534$; $1547-$ 3287.

ZAHABI, A., et al. A New Efficient Protocol for Directed Differentiation of Retinal Pigmented Epithelial Cells from Normal and Retinal Disease Induced Pluripotent Stem Cells. Stem Cells and Development, 20120203, Aug 10, 2012b, vol. 21, no. 12 , pp. $2262-2272$. ISSN $1557-8534$; $1547-$ 3287.

ZARBIN, M.; and SZIRTH, B. Current Treatment of Age-Related Macular Degeneration. Optometry and Vision Science : Official Publication of the American Academy of Optometry, Jul, 2007, vol. 84, no. 7, pp. 559-572. ISSN 1040-5488; 10405488.

ZARBIN, Marco a. Current Concepts in the Pathogenesis of Age-Related Macular Degeneration. Archives of Ophthalmology, apr, 2004, vol. 122, no. 4, pp. 598-614. ISSN 00039950.

ZARNETT, R.; and SALTER, R. B. Periosteal Neochondrogenesis for Biologically Resurfacing Joints: Its Cellular Origin. Canadian Journal of Surgery.Journal Canadien De Chirurgie, May, 1989, vol. 32, no. 3, pp. 171-174. ISSN 0008428X; 0008-428X.

ZHANG, B., et al. Mesenchymal Stem Cells Induce Mature Dendritic Cells into a Novel Jagged-2-Dependent Regulatory Dendritic Cell Population. Blood, 20081002, Jan 1, 2009, vol. 113 , no. 1, pp. 46-57. ISSN 1528-0020; 00064971.

ZHANG, Kang, et al. Ciliary Neurotrophic Factor Delivered by Encapsulated Cell Intraocular Implants for Treatment of Geographic Atrophy in Age-Related Macular Degeneration. Proceedings of the National Academy of Sciences of the United 
States of America, apr, 2011, vol. 108, no. 15, pp. 6241-6245. ISSN 1091-6490.

ZHANG, W., et al. Effects of Mesenchymal Stem Cells on Differentiation, Maturation, and Function of Human Monocyte-Derived Dendritic Cells. Stem Cells and Development, Jun, 2004, vol. 13, no. 3, pp. 263-271. ISSN 1547-3287; 1547-3287.

ZHANG, Z. Q.; and DONG, F. T. In Vitro Differentiation of Rat Mesenchymal Stem Cells into Photoreceptors. [Zhonghua Yan $\mathrm{Ke} \mathrm{Za} \mathrm{Zhi]}$ Chinese Journal of Ophthalmology, Jun, 2008, vol. 44, no. 6, pp. 540-544. ISSN 0412-4081; 04124081 .

ZHAO, J., et al. A Novel Strategy to Engineer Small-Diameter Vascular Grafts from MarrowDerived Mesenchymal Stem Cells. Artificial Organs, 20110725, Jan, 2012, vol. 36, no. 1, pp. 93-101. ISSN 1525-1594; 0160-564X.

ZUK, P. a., et al. Multilineage Cells from Human Adipose Tissue: Implications for Cell-Based Therapies. Tissue Engineering, apr, 2001, vol. 7, no. 2 , pp. 211-228. ISSN 1076-3279.

ZUK, Patricia A., et al. Human Adipose Tissue is a Source of Multipotent Stem Cells Â-i, 2002, vol. 13, no. December, pp. 4279-4295.

ZYUZ'KOV, G. N., et al. Effect of Dimethylsulfoxide on the Functions of Mesenchymal and Hemopoietic Precursors. Bulletin of Experimental Biology and Medicine, Apr, 2007, vol. 143, no. 4, pp. 535-538. ISSN 0007-4888; 0007-4888. 


\title{
Adipose derived mesenchymal stem cells partially rescue mitomycin C treated ARPE19 cells from death in co-culture condition
}

\author{
Amar K. Singh ${ }^{1}$, Girish K. Srivastava ${ }^{1,2,3}$, María T. García-Gutiérrez ${ }^{1,2}$ and J. Carlos Pastor ${ }^{1}$ \\ ${ }^{1}$ IOBA-Eye Institute, University of Valladolid, Valladolid, Spain, ${ }^{2}$ Regenerative Medicine and Cell Therapy Networking Center of \\ "Castilla y León", Spain and ${ }^{3}$ Networking Research Center on Bioengineering, Biomaterials and Nanomedicine (CIBER-BBN), \\ Valladolid, Spain
}

Summary. Age-related macular degeneration is a retinal disease with important damage at the RPE layer. This layer is considered a target for therapeutical approaches. Stem cell transplantation is a promising option for retinal diseases. Adipose derived mesenchymal stem cells secret growth factors which might play a significant role in RPE maintenance. This study aimed to evaluate human AD-MSCs ability to rescue mitomycin C treated dying ARPE19 cells in co-culture condition.

ARPE19 cells were treated with MMC $(50 \mu \mathrm{g} / \mathrm{ml}$, $100 \mu \mathrm{g} / \mathrm{ml}$ and $200 \mu \mathrm{g} / \mathrm{ml}$ ) for 2 hours to induce cell death. These treated cells were co-cultured with hADMSCs in indirect co-culture system for 3 days and 3 weeks. Then the viability, growth and proliferation of these ARPE19 cells were evaluated by a cell viability/cytotoxicity assay kit and Alamar Blue (AB) assay. Untreated ARPE19 cells and human skin fibroblasts (HSF) were used as controls.

MMC blocked ARPE19 cell proliferation significantly in 3 days and cells were almost completely dead after 3 weeks. Cell toxicity of MMC increased significantly with concentration. When these cells were co-cultured with hAD-MSCs, a significant growth difference was observed in treated cells compared to untreated cells. hAD-MSCs rescue capacity was also significantly higher than HSF for treated ARPE19 cells.

This study showed that hAD-MSCs rescued MMC treated ARPE19 cells from death. It probably occurred

Offprint requests to: Girish K. Srivastava, Ph.D., Institute of Applied Ophthalmobiology-Eye Institute (IOBA), University of Valladolid, Campus Miguel Delibes, Paseo de Belén, 17, Valladolid, 47011, Spain. e-mail: girish@ioba.med.uva.es due to undefined growth factors secreted by hAD-MSCs in the medium, shared by treated ARPE19 cells in coculture conditions. This study supports further evaluation of the effect of hAD-MSCs subretinal transplantation over the RPE degeneration process in AMD patients.

Key words: Human adipose derived mesenchymal stem cells, Age-related macular degeneration, Cell death, ARPE19 cell, Mitomycin C

\section{Introduction}

Age related macular degeneration (AMD) is the most common cause of irreversible legal blindness in older people over 65 years in the developed countries (Ambati et al., 2003) and about 3.4\% of Spanish old age population of 65 years or older is suffering from AMD (Spanish Eyes Epidemiological Study Group, 2011). There are two forms of AMD: dry (also known as atrophic) which is the most frequent and wet (also known as neovascular or exudative) (Gottlieb, 2002). The wet form has some effective treatments based on intraocular injections of anti-VEGF agents, but to date there is no treatment for the dry forms of AMD. One of the main events of dry AMD is the degeneration and disappearance of the retinal pigment epithelium (RPE) layer of the retina. RPE plays a crucial role in maintaining the adequate functions of neuroretina. If the degeneration of this trophic layer, RPE, of the retina could be prevented somehow or at least delayed, AMD could probably be halted. Several growth factors which 
play important roles in maintaining the health of the neuroretina as well as the RPE, which include PEDF, BDNF, VEGF and others, have been identified (Kolomeyer et al., 2011).

New advanced treatments like genetic and stem cell therapy are under investigation, out of which mesenchymal stem cell (MSC) therapy represents a promising alternative (Binder et al., 2007). MSCs are present in several tissues which renew the mesenchymal lineages both under normal and pathological conditions. In addition to replacing the dead and damaged cells the MSC may provide a source of trophic and biochemical positive effects due to having the capacity to secret many immunomodulatory and neuroprotective growth factor molecules (Levkovitch-Verbin et al., 2010; Doorn et al., 2012). Several growth factors like CNTF, BDNF, IGF1, NGF and bFGF secreted by MSC (Labouyrie et al., 1999; Lin et al., 2009) might protect neuroretina and RPE from further degeneration.

Therefore, the secretion of those neurotrophic growth factors by adipose derived MSCs (AD-MSCs) could have positive effects over the survival of ageing RPE and hence might help in slowing the retinal degeneration process in AMD patients. The purpose of this work is to investigate the possibility of rescuing mitomycin $\mathrm{C}$ triggered ARPE19 cell death by coculturing them with human AD-MSCs.

\section{Materials and methods}

\section{Cell culture}

ARPE19 cells, hAD-MSCs and human skin fibroblast (HSF, ATCC name: BJ cell line) were cultured under standard culture conditions of $5 \% \mathrm{CO}_{2}$ at $37^{\circ} \mathrm{C}$ in the humidified cell culture incubator in their respective standard culture mediums. The mediums were renewed at 2-3 day intervals. The standard culture mediums for ARPE19 cells, hAD-MSCs and HSF were complete DMEM/F12, DMEM low glucose with glutamine (Gibco, Invitrogen, Paisley, UK) and Eagle's Minimum Essential Medium (EMEM, ATCC formulated cat no30-2003) respectively, each containing $1 / 100$ penicillinstreptomycin solution supplemented with $10 \%$ FBS (Gibco, Invitrogen, Paisley, UK). DMEM/F12 medium also contained 1/100 Amphotericin B solution. On obtaining the confluency ( $>90 \%$ ) cells were trypsinized with $0.05 \%$ trypsin-tetrasodium ethylenediaminetetraacetate (Trypsin-EDTA, Gibco, Invitrogen, Paisley, UK), washed and re-suspended in phosphate-buffered saline (PBS; Gibco, Invitrogen, Paisley, UK). Cell numbers and viability for seeding the cells for each experiment were determined by standard Trypan Blue exclusion assay. ARPE19 cell line and HSF was purchased from the American Type Culture Collection (ATCC, Manassas, VA, USA). hAD-MSCs were gifted by Dr. Teresa Nieto from the Ocular Surface group of IOBA after their routine characterization. The hAD-MSCs used in this study fulfil the minimum criteria for multipotent human mesenchymal stromal cells, as defined by the International Society for Cellular Therapy (Horwitz et al., 2005), such as their multipotent differentiation capacity towards osteoblasts, adipocytes and chondrocytes and the flow cytometry analysis of the panel of MSC specific cell marker (CD73, CD90, CD105, CD45, CD34, CD14, CD19, HLA-DR) expressions (Nieto et al., 2009).

\section{Mitomycin C treatment of ARPE19 cells}

Mitomycin C (MMC from Streptomyces caespitosus) was purchased from Sigma Chemicals, USA. ARPE19 cells were seeded at the density of 60,000 cells $/ \mathrm{cm} 2$ in the 24 well plate in standard culture conditions, incubated for 24 hours and allowed to adhere to polystyrene surface properly and to adopt proper morphology. ARPE19 cells were then incubated with MMC in three different concentrations, $50 \mu \mathrm{g} / \mathrm{ml}, 100$ $\mu \mathrm{g} / \mathrm{ml}$ and $200 \mu \mathrm{g} / \mathrm{ml}$ dissolved in the growth medium, in four wells for each concentration for 2 hours. After MMC incubation ARPE19 cells were washed with PBS three times to remove any residual MMC over the cell monolayer. These treated ARPE19 cells were used for co-culturing with hAD-MSCs and HSF.

\section{Indirect co-culture of hAD-MSCs with ARPE19 cells}

ARPE19 cells and hAD-MSCs were co-cultivated in the same wells in 1:1 ratio for 3 days as well as for 3 weeks, sharing the same growth medium (complete DMEM/F12 medium) but physically separated by transmembrane of BD Falcon ${ }^{\mathrm{TM}}$ cell culture inserts. The ARPE19 cells were cultured in the bottom of the 24-well plates (BD Falcon) and the hAD-MSCs were cultured on the transwell inserts (BD Falcon), the tissue culture treated track-etched polyethylene terephthalate (PET) having a membrane of $0.4 \mu \mathrm{m}$ pore size (pore density of $2.0 \pm 0.2 \times 10^{6} / \mathrm{cm}^{2}$ ). The hAD-MSCs were also cocultivated with ARPE19 cells treated with different concentrations of MMC. In another set of experiments, treated and untreated ARPE19 cells were co-cultured with HSF.

\section{Cell viability assay by viability/cytotoxicity assay kit for determining live and dead cells}

The cell viability/cytotoxicity assay kit for live \& dead cells (Biotium Inc., USA) was used to evaluate cell viability after three weeks of co-culture in accordance with the manufacturer's protocol. The kit provides a two-color fluorescent staining of live (green) and dead (red) cells using two probes; Calcein acetoxymethyl ester (calcein AM) stains live cells green while EthD-III (Ethidium homodimer III) stains dead and damaged cells red. After staining, both cells, APRE19 and hAD-MSCs, were visualized using a fluorescence microscope (Leica Microsystems, Mannheim, Germany) and were photographed at random per well. 
Alamar Blue (AB) assay for evaluating cell viability and proliferation

After 3 days of co-culture growth conditions, AB assay was used for evaluating cell viability along with cell proliferation activity. Cells were washed with PBS and complete medium with $10 \%$ Alamar Blue (AbD Serotec, Oxford, UK) was added to each well and incubated under standard incubation conditions for 5 hours. The fluorescence intensity of $\mathrm{AB}$ was measured (Spectra Max M5 Multi-Mode Microplate Reader, Molecular Devices LLC, US) with excitation wavelength at $560 \mathrm{~nm}$ and emission wavelength at 590 $\mathrm{nm}$. The degree of $A B$ reduction corresponded to the number of live cells and cell proliferation activity. The negative control for the measurement of fluorescence intensity was taken as the medium with $\mathrm{AB}$ reagent without any cells. The viability and proliferation activity of cells in different culture conditions is directly correlated with the degree of reduction of $\mathrm{AB}$, which is reflected by the fluorescence intensity measured for each sample. The experiments were performed three times each with duplicates. The mean value of arbitrary fluorescence intensity unit (AFU) \pm standard error of mean (SEM) were plotted against all the different samples of the experiments. The diagrammatic presentation of the experiment is shown in Fig. 1.

\section{Statistical analysis of $A B$ fluorescence readings}

All the samples are quadruplicated in the microplate reader to avoid any pipetting errors. All the experiments were repeated three times in duplicates to check the reproducibility of the trends observed. The readings obtained from three times repetition of the experiments were subjected to statistical analysis through MS Excell programme and online GraphPad Software. Standard deviations (SD), standard errors of means (SEM), means and $\mathrm{p}$-values were calculated and the observed statistical significance was adjusted to $\mathrm{P}<0.05$ and $\mathrm{P}<0.01$. T-test (paired/two tailed) was used to compare the different means of each treatment group obtained by the three experiments.

\section{Results}

\section{Mitomycin C treatment triggers ARPE19 cell death}

Fluorescence microscopy as well as $\mathrm{AB}$ assay analysis results showed that MMC $(50 \mu \mathrm{g} / \mathrm{ml}, 100 \mu \mathrm{g} / \mathrm{ml}$ and $200 \mu \mathrm{g} / \mathrm{ml}$ ) treatment reduced ARPE19 cell proliferation in 3 days significantly $(\mathrm{p}<0.05, \mathrm{n}=3)$ and almost all ARPE19 cells died completely after 3 weeks (Figs. 2, 3). The results obtained after cell viability/ toxicity assay at 3 weeks in Fig. 2 show the pattern of
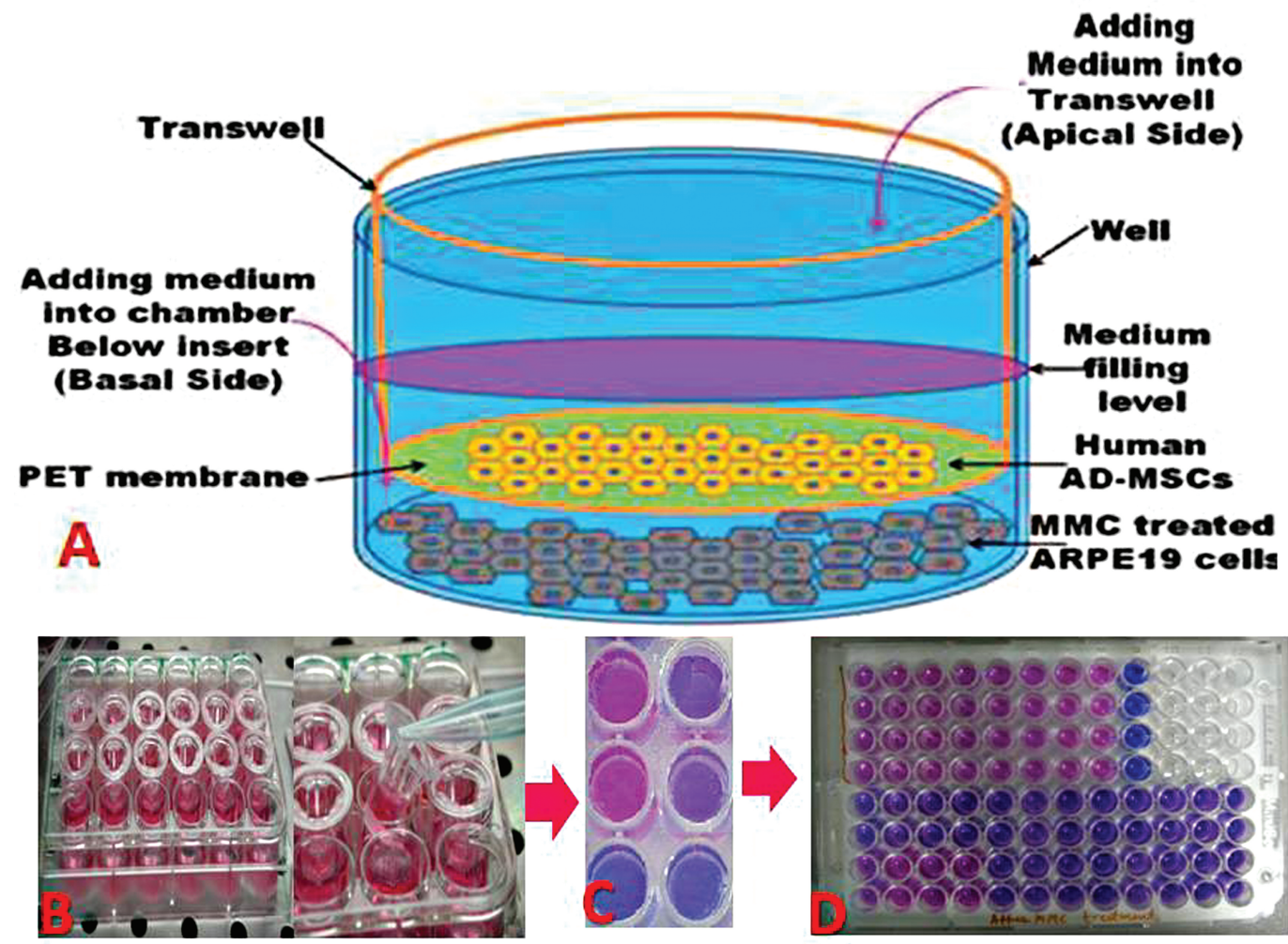
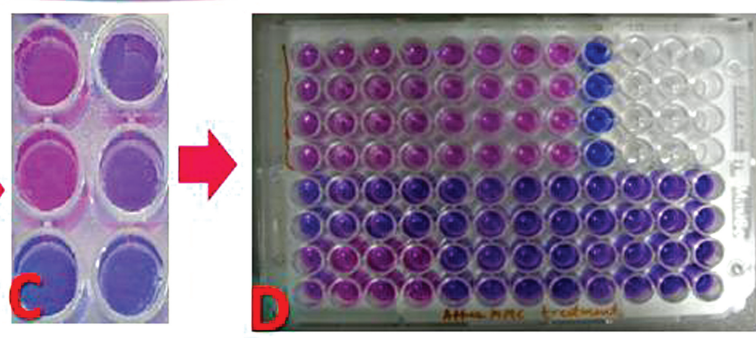

Fig. 1. Diagrammatic representation of the experiments carried out.

A. Schematic representation of indirect co-culture system B. Indirect co-culture system in which MMC treated ARPE19 cells were co-cultivated with AD-MSCs. C. Incubation of ARPE19 cells with Alamar Blue $\mathbf{D}$. Reading of

fluorescence of incubated $A B$ medium in quadruplets using microplate reader. 
cell survival and death under different culture conditions by green and red fluorescence, respectively. The ARPE19 cells in control without any treatment showed $100 \%$ viability (Fig. 2A) while ARPE19 cells with 50 $\mu \mathrm{g} / \mathrm{ml} \mathrm{MMC} \mathrm{treatment} \mathrm{were} \mathrm{almost} \mathrm{dead} \mathrm{(Fig.} \mathrm{2B)} \mathrm{after}$ 3 weeks.

When proliferation activity of ARPE19 cells cultured for 3 days was measured by $A B$ assay, a significant high fluorescence intensity $(\mathrm{p}<0.05, \mathrm{n}=3)$ was found in the wells with untreated ARPE19 cells compared to the wells with MMC treated ARPE19 cells (table 1). Fluorescence intensity decreased significantly $(\mathrm{p}<0.05, \mathrm{n}=3)$ with the increase of concentration of MMCs $(50 \mu \mathrm{g} / \mathrm{ml}, 100 \mu \mathrm{g} / \mathrm{ml}$ and $200 \mu \mathrm{g} / \mathrm{ml}$ ) (Fig. 3,

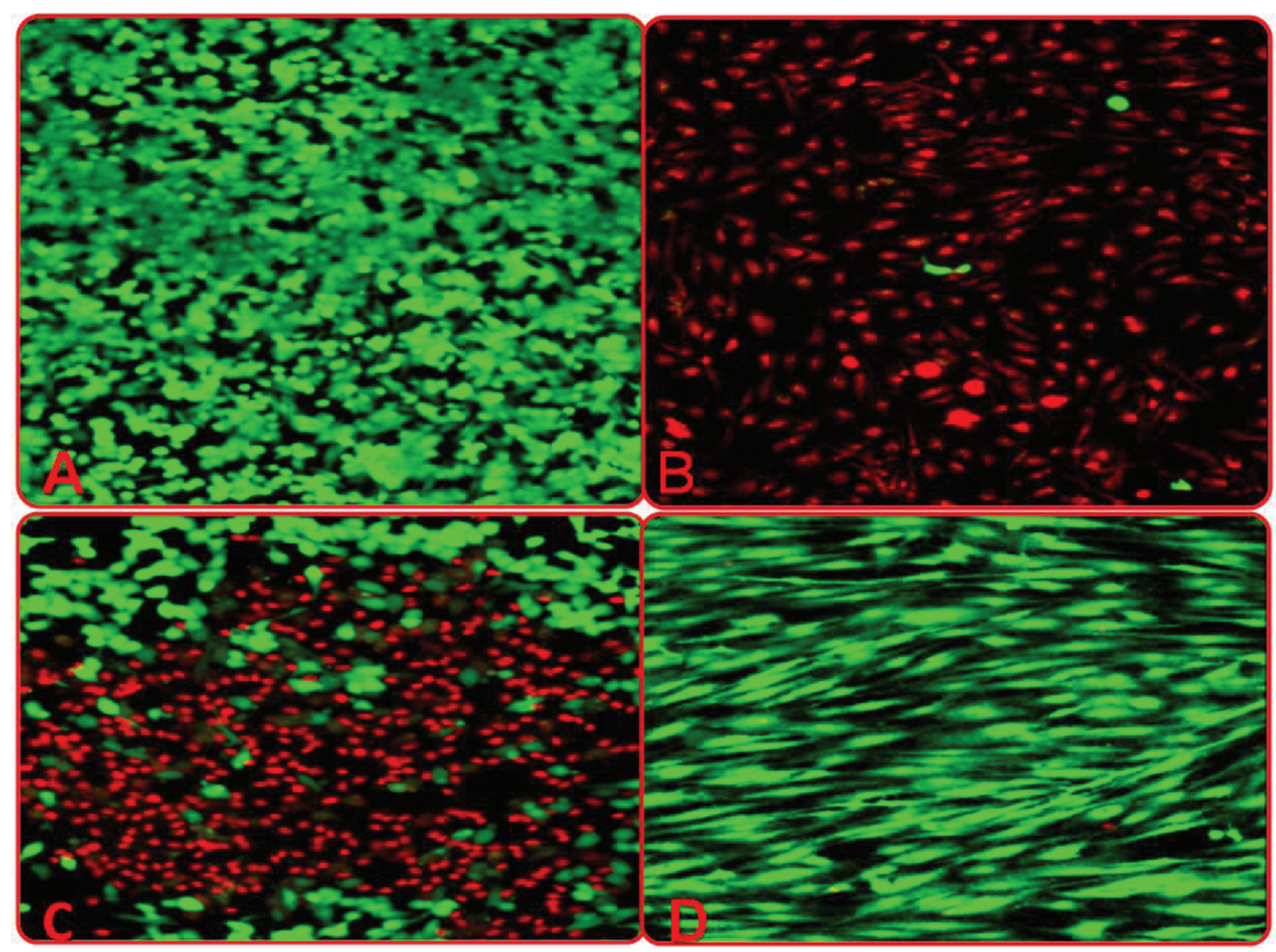

Fig. 2. Viability /Cytotoxicity assay of MMC treated and untreated ARPE19 cells cocultivated with hAD-MSCs for 3 weeks in standard culture condition. Green fluorescence indicates live cells and red fluorescence indicates dead cells A. Untreated ARPE19 cells. B. ARPE19 cells treated with $50 \mu \mathrm{g} / \mathrm{ml} \mathrm{MMC}$. C. ARPE19 cells treated with $M M C$ and co-cultured with hAD-MSCs. D. hAD-MSCs on Falcon transwell.

Table 1. Statistical data analysis of proliferation of MMC treated $(50 \mu \mathrm{g} / \mathrm{ml}, 100 \mu \mathrm{g} / \mathrm{ml}$ and $200 \mu \mathrm{g} / \mathrm{ml})$ and untreated ARPE19 cells co-cultivated with hAD-MSCs or HSF for 3 days in standard culture conditions.

\begin{tabular}{|c|c|c|c|c|c|}
\hline Culture conditions & Mean AFU $(n=3)$ & SD & $\operatorname{SEM}(n=3)$ & P-value $\left(^{*}\right)$ & P-value $\left({ }^{* *}\right)$ \\
\hline ARPE19 cells+MSCs on TW & $18,625.14$ & 885.29 & 511.14 & 0.0437 & \\
\hline ARPE19 cells+50 ugMMC+MSCs on TW & $17,106.12$ & $1,803.09$ & $1,041.09$ & 0.0312 & \\
\hline ARPE19 cells+100 ugMMC & $10,088.56$ & 2580.21 & $1,489.68$ & & 0.0153 \\
\hline ARPE19 cells+100 ug MMC+MSCs on TW & $14,838.51$ & 2304.66 & $1,330.60$ & 0.0194 & \\
\hline ARPE19 cells+200 ugMMC & $5,238.20$ & 647.97 & 374.11 & & 0.0492 \\
\hline
\end{tabular}

Mean AFU reflects the means of AFU values obtained from AB assay. SD and SEM are standard deviation and standard error mean respectively, associated with corresponding mean AFU values. Single asterisk $\left(^{*}\right)$ represents the significant P-value between MMC treated or untreated ARPE19 cells vs MMC treated or untreated ARPE19 cells co-cultivated with AD-MSCs or HSF. Double asterisk $\left({ }^{* *}\right)$ represents the significant P-value between untreated vs MMC treated ARPE19 cells. Statistical significance is adjusted at $\mathrm{P}<0.05\left(^{*}\right)$. 


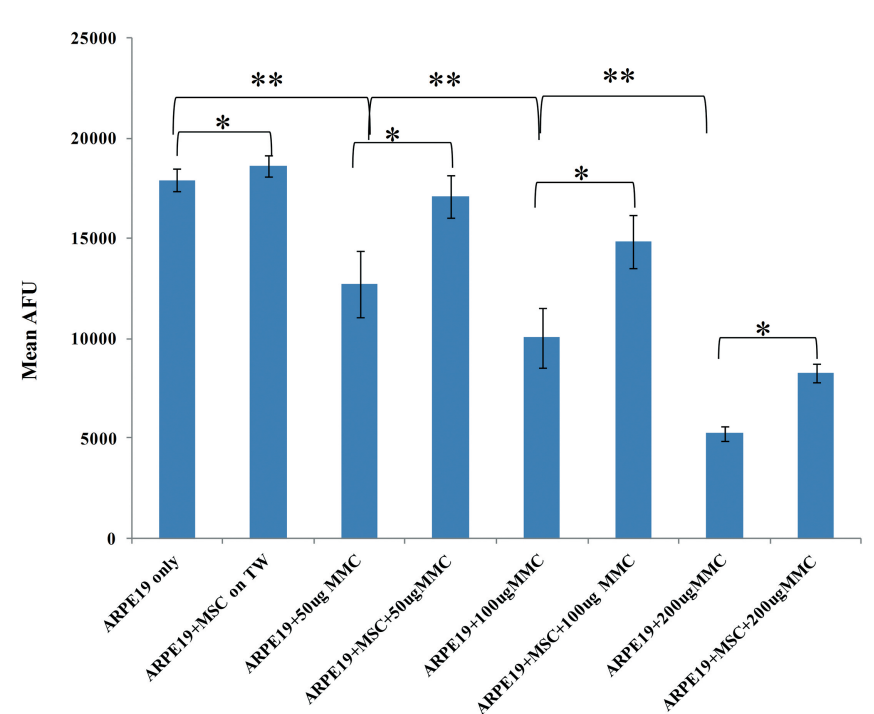

Fig. 3. MMC treated $(50 \mu \mathrm{g} / \mathrm{ml}, 100 \mu \mathrm{g} / \mathrm{ml}$ and $200 \mu \mathrm{g} / \mathrm{ml})$ and untreated ARPE19 cells proliferation in co-culture conditions with hAD-MSCs at 3 days. Alamar Blue assay was used to detect the cell number as well as cell proliferation activity and data is presented as mean arbitrary fluorescence unit (AFU) vs cell culture conditions. All values shown are corrected with background fluorescence of the reagent in medium alone. The bar represents mean value of fluorescence intensity (AFU) of three different experiments in duplicated wells \pm SEM. Single asterisk $\left(^{*}\right)$ and double asterisk $\left(^{* *}\right)$ represent the significant P-value between different culture conditions as presented in graph. Statistical significance is adjusted at $\mathrm{P}<0.05$.

table 1)

\section{MSCs partially rescue the MMC-treated ARPE19 cells} from death

Cell viability/toxicity assay demonstrated that 50 $\mu \mathrm{g} / \mathrm{ml}$ MMC treated ARPE19 cells after three weeks retained significant viability, when co-cultured with hAD-MSCs (Fig. 2C) and there was no adverse effect after three weeks on the hAD-MSCs grown on transwell due to death of MMC treated ARPE19 cells in the well (Fig. 2D).

$\mathrm{AB}$ analysis showed that in each treatment group the ARPE19 cells co-cultured with hAD-MSCs expressed significantly higher fluorescence intensity $(\mathrm{p}<0.05, \mathrm{n}=3)$ than ARPE19 cells alone (Fig. 3, Table 1).

As a control, in a further experiment a comparative study using $\mathrm{AB}$ assay kit was done to determine the effects of hAD-MSCs and HSF separately on $50 \mu \mathrm{g} / \mathrm{ml}$ MMC treated ARPE19 cells. The results showed that the wells with hAD-MSCs had significantly higher fluorescence intensity $(\mathrm{p}<0.05, \mathrm{n}=3)$ than the wells containing HSF (Fig 4).

\section{Discussion}

Cell therapy approaches using differentiated human

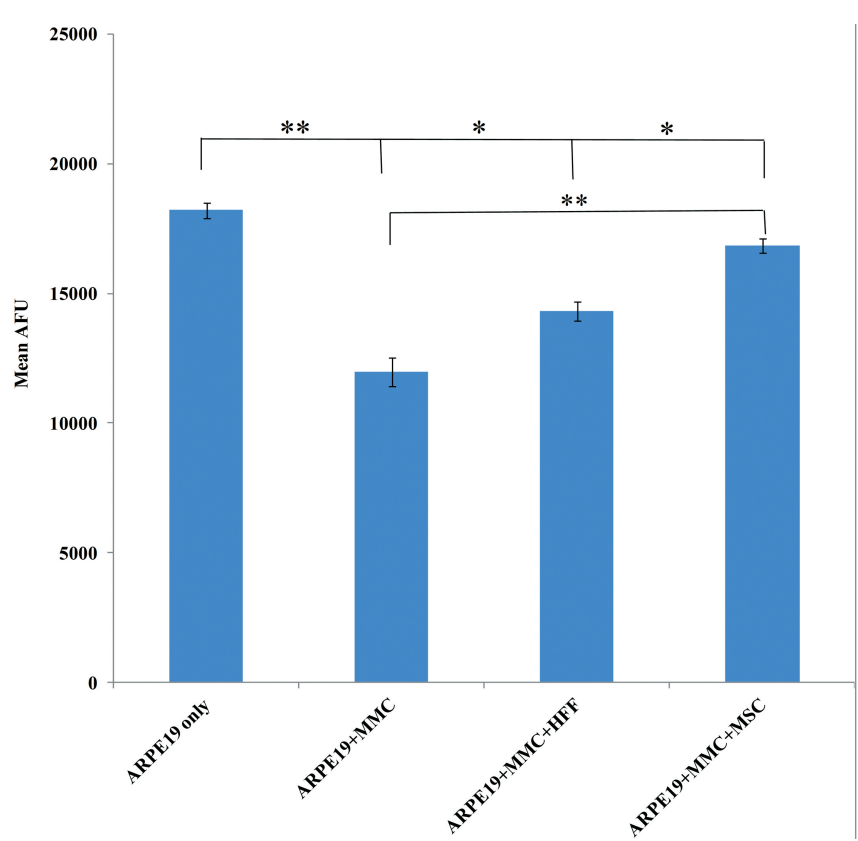

Fig. 4. MMC treated $(50 \mu \mathrm{g} / \mathrm{ml})$ and untreated ARPE19 cells proliferation in co-culture conditions with hAD-MSCs and HSF separately at 3 days. Alamar Blue assay was used to detect the cell numbers as well as cell proliferation activity and data is presented as mean arbitrary fluorescence unit (AFU) vs cell culture conditions. All values shown are corrected with background fluorescence of the reagent in medium alone. The bar represents mean value of fluorescence intensity (AFU) of three different experiments in duplicated wells \pm SEM. Single asterisk $\left({ }^{*}\right)$ and $\left(^{* *}\right)$ represent the significant P-value between deferent culture conditions as presented in graph. Statistical significance is adjusted at $\mathrm{P}<0.05$.

embryonic stem cells (hESCs) are currently under evaluation as a treatment for dry AMD patients (Klimanskaya et al., 2004). A clinical trial is undergoing in this respect by Advanced Cell Technology, USA (ClinicalTrials.gov identifier: NCT01344993). However, apart from ethical issues, it seems to be a complicated procedure due to a number of issues; obtaining hESCs in sufficient quantity, implanting hESCs-RPE cells with a suitable cell carrier in patients, control over the need of using immune-suppression, a possible graft rejection, postsurgical complications for graft integration as well as securing implanted cell survival in vivo in sufficient amounts for detaining dry AMD progression in the patients. Even more, the high cost of implementing this procedure in each patient makes it far from the access of millions of people suffering from dry AMD all over the world. Therefore, it would be worth looking for some other procedures that can utilize the protective capacity of patients' own cells for stopping or delaying the progress of RPE cell degeneration at initial stage, thus delaying AMD progression and which can be applicable effectively in all AMD patients.

Published reports showed that sub retinal implanted bone marrow derived MSCs could integrate and 
differentiate partially into RPE cells in animal models such as mice (Atmaca-Sonmez et al., 2006). This supports the feasibility of using mesenchymal stem cells as a potential candidate for transplantation in suretinal space. However there are no reports evaluating hADMSCs rescue capacity on dying RPE cells as has been achieved in this study.

MSCs can be obtained from different sources and are used in clinics routinely for cell based treatment for replacing dead and damaged cells, such as skin treatment, due to their capacity to differentiate into different lineages (Sasaki et al., 2008). MSCs also secret a number of immunomodulatory and neuroprotective trophic factors that might protect degenerating retina and RPE cells. MSCs derived from patients' own tissues provide autologous nature to the cells and support to overcome the problems generated due to immunoreactivity (Hilfiker et al., 2011). Out of different tissue sources of MSCs, adipose tissue seems to be one of the most appropriate because of easy procedures to obtain it in sufficient amount (De Ugarte et al., 2003).

MMC is a bioreductive DNA alkylating agent (Rockwell et al., 1982) which was used to inhibit cell proliferation and to induce cell death as well. It has been used to induce cell death by apoptosis as well as necrosis in several publications (Hofman et al., 1998; Wu et al., 2002, 2008; Park et al., 2002). A recent paper showed that MMC-induced cellular apoptosis in corneal endothelial cells was mediated through Caspase-8, Caspase-9, and the mitochondrial regulated pathways, as well as through upregulation of p53-dependent and p21dependent signal transduction pathways (Wu et al., 2008). Oxidative stress over time has been hypothesized to underlie the development of AMD. Oxidative stress directly targets mitochondrial-dependent apoptosis that requires release of cytochrome $\mathrm{c}$ from mitochondria and subsequent activation of a specific class of cytoplasmic proteases known as caspases (Takahashi et al., 2004). Thus, it seems that MMC induced apoptosis in ARPE19 cells is quite similar to apoptosis in the degenerating RPE cells of the AMD patients.

$\mathrm{AB}$ assay analysis results obtained by treating ARPE19 cells with MMC at different concentrations of $50 \mu \mathrm{g} / \mathrm{ml}, 100 \mu \mathrm{g} / \mathrm{ml}$ and $200 \mu \mathrm{g} / \mathrm{ml}$ for 2 hours in standard culture condition followed by growing them for three days showed that MMC was able to partially inhibit ARPE19 cell proliferation (Fig. 3). Calcein $\mathrm{AM} / \mathrm{EthD}$-III probes of cell viability/cytotoxicity kit could measure two recognized parameters of cell viability; intracellular esterase activity and plasma membrane integrity, respectively, in ARPE19 cells which were under MMC triggered cell death process. The results obtained using these probes clearly showed that after three weeks cells were completely dead if treated with $50 \mu \mathrm{g} / \mathrm{ml}$ MMC (Fig. 2B). This confirmed the suitability of using this concentration of MMC for further study.

In a further study, the hAD-MSCs were co-cultivated for three days and three weeks with MMC treated and untreated ARPE19 cells using transwells. AB assay at three days demonstrated that there was significant growth difference between ARPE19 cells co-cultured with hAD-MSCs and ARPE19 cells in MMC treated or untreated group. This result confirmed that the hADMSCs support the ARPE19 cells proliferation as well as partially rescuing the ARPE19 cells triggered for cell death. The fluorescence microscopic observations using viability/toxicity assay kit at three weeks further confirmed that hAD-MSCs are capable of rescuing partially the dying ARPE19 cells (Fig. 2C). It is hypothesized that the several factors secreted by hADMSCs are involved in this partial rescue process. These putative protective factors could be CNTF, BDNF, IGF1, NGF and bFGF etc according to the literature (Labouyrie et al., 1999; Lin et al., 2009). The identification and measurement of these putative protective factors present in supernatants collected in different incubation conditions are still under development. The approach used for this development would be to block reciprocal influences of these putative protective factors on hAD-MSCs and ARPE19 cells. The results also showed that hAD-MSCs maintained their viability for three weeks on track-etched PET transwells. This showed that factors released by dying ARPE19 cells do not affect the viability of hAD-MSCs but their effects on the differentiation towards a cell lineage could not be ruled out and it is still under evaluation.

As evident from the graph in Fig. 3, the proliferation of MMC treated ARPE19 cells decreased as the concentration of MMC increased from $50 \mu \mathrm{g} / \mathrm{ml}$ to 200 $\mu \mathrm{g} / \mathrm{ml}$. Co-culturing of these MMC treated ARPE19 cells with hAD-MSCs showed that treated ARPE19 cells in co-culture condition were able to proliferate significantly higher than those cells that were not in coculture condition. However, they proliferated less than the ARPE19 cells under untreated conditions. This indicates that the putative factors released in co-culture medium were creating a microenvironment which might be conducive to rescuing the ARPE19 cells from the cell death process. In in vivo conditions, in dry AMD patients, RPE cells are under degenerating stage and implanting hAD-MSCs in the subretinal space might create a microenvironment as observed in in vitro conditions, to rescue partially the degenerating RPE cells, thus helping to prevent AMD progression.

To confirm further that this rescue capacity is only associated with hAD-MSCs, and that other types of cells such as fibroblasts can have different grades of rescue capacity, we compared the effects of hAD-MSCs and HSF over MMC treated ARPE19 cells in co-culture conditions. The results in Fig. 5 confirmed that hADMSCs have a higher capacity to rescue ARPE19 cells than the HSF. However, fibroblasts have different characteristics from hAD-MSCs (Alt et al., 2011) and are involved in producing some retinal diseases, such as proliferative vitroretinopathy (PVR) complications (de Souza et al., 1995), thus it encourages further hADMSCs in vivo study. 


\section{$A D$-MSCs partially rescue ARPE19 cells from MMC induced cell death}

Thus, it is concluded that the hAD-MSCs partially rescue MMC treated dying ARPE19 cells in in vitro conditions, probably by creating a microenvironment containing many factors released in co-culture conditions. The results strongly support making a strategy for future hAD-MSC therapy for dry AMD patients.

Acknowledgements. This work was supported by grants from Castilla and Leon Regenerative Medicine and Cell Therapy Network Center, National Plan of I+D+I 2008-2011 and ISCIII-Subdireccion General de Evaluación y Fomento de la Investigación (PS09/00938) (MICNN) cofinanced by FEDER, JCYL BIO/39/VA26/10 and VA386A12-2, Junta de Castilla y León, Spain to JCP and GKS. A.K. Singh is a Pre-doctoral research scholar supported by $\mathrm{AECl}$, Spanish Ministry of Foreign Affairs and Cooperation.

Declaration of interest. The authors do not have any conflicts of interest. Author Contributions. Amar Kumar and Girish Kumar contributed equally to the work, Maria T. Garcia provided technical support and $\mathrm{J}$ Carlos Pastor supported in article writing.

\section{References}

Alt E., Yan Y., Gehmert S., Song Y.H., Altman A., Gehmert S., Vykoukal D. and Bai X. (2011). Fibroblasts share mesenchymal phenotypes with stem cells, but lack their differentiation and colony-forming potential. Biol. Cell 103, 197-208.

Ambati J., Ambati B.K., Yoo S.H., Sakurai E., Lynn B.C. and Kuziel W.A. (2003). Age-related macular degeneration: etiology, pathogenesis, and therapeutic strategies. Surv. Ophthalmol. 48, 257-293.

Atmaca-Sonmez P., Li Y., Yamauchi Y., Schanie C.L., Ildstad S.T. and Kaplan H.J. (2006). Systemically transferred hematopoietic stem cells home to the subretinal space and express RPE-65 in a mouse model of retinal pigment epithelium damage. Exp. Eye Res. 83, 1295-1302.

Binder S, Stanzel B.V., Krebs I. and Glittenberg C. (2007). Transplantation of the RPE in AMD. Prog. Retin. Eye Res. 26, 516-554.

de Souza O.F., Sakamoto T., Kimura H., Koda R.P., Gabrielian K., Spee C. and Ryan S.J. (1995). Inhibition of experimental proliferative vitreoretinopathy in rabbits by suramin. Ophthalmologica 209, 212216.

De Ugarte D.A., Morizono K., Elbarbary A., Alfonso Z., Zuk P.A. and Zhu M. (2003). Comparison of multi-lineage cells from human adipose tissue and bone marrow. Cells Tissues Organs 174, 101109.

Doorn J., Moll G., Le Blanc K., van Blitterswijk C. and de Boer J. (2012). Therapeutic applications of mesenchymal stromal cells: paracrine effects and potential improvements. Tissue Eng. Part B Rev. 18, 101-115.

Gottlieb J.L. (2002). Age-related macular degeneration. JAMA. 288, 2233-2236.

Hilfiker A., Kasper C., Hass R.. and Haverich A. (2011). Mesenchymal stem cells and progenitor cells in connective tissue engineering and regenerative medicine: is there a future for transplantation?. Langenbecks Arch. Surg. 396, 489-497.

Hoffman S., Gopalakrishna R., Gundimeda U., Murata T., Spee C. and
Ryan S.J. (1998). Verapamil inhibits proliferation, migration and protein kinase $\mathrm{C}$ activity in human retinal pigment epithelial cells. Exp Eye Res. 67, 45-52.

Horwitz E.M., Le Blanc K., Dominici M., Mueller I., Slaper-Cortenbach I., Marini F.C., Deans R.J., Krause D.S. and Keating A. (2005). International Society for Cellular Therapy. Clarification of the nomenclature for MSC: The International Society for Cellular Therapy position statement. Cytotherapy 7, 393-395.

Klimanskaya I., Hipp J., Rezai K.A., West M., Atala A. and Lanza R. (2004). Derivation and comparative assessment of retinal pigment epithelium from human embryonic stem cells using transcriptomics. Cloning Stem Cells 6, 217-245.

Kolomeyer A.M., Sugino I.K. and Zarbin M.A. (2011). Characterization of conditioned media collected from cultured adult versus fetal retinal pigment epithelial cells. Invest. Ophthalmol. Vis. Sci. 52, 5973-5986.

Labouyrie E., Dubus P. and Groppi A. (1999). Expression of neurotrophinsand their receptors in human bone marrow. Am. J. Pathol. 154, 405-415.

Levkovitch-Verbin H., Sadan O., Vander S., Rosner M., Barhum Y. and Melamed E. (2010). Intravitreal injections of neurotrophic factors secreting mesenchymal stem cells are neuroprotective in rat eyes following optic nerve transection. Invest. Ophthalmol. Vis. Sci. 51, 6394-6400.

Lin N., Hu K. and Chen S. (2009). Nerve growth factor-mediated paracrine regulation of hepatic stellate cells by multipotent mesenchymal stromal cells. Life Sci. 85, 291-295.

Nieto T., Corrales R.M., Sáez V., Corell A., Martino M., Pérez-Simón J.A., García-Montes M.T. and Calonge M. (2009). Corneal epithelium cell marker acquisition by human adipose tissue mesenchymal stem cells (hAT-MSC). Invest. Ophthalmol. Vis. Sci. 50, ARVO E-abstract 2424 2424/A512.

Park H.K., Lee K.W., Choi J.S. and Joo C.K. (2002). Mitomycin Cinduced cell death in mouse lens epithelial cells. Ophthalmic Res. 34, 213-219.

Rockwell S., Kennedy K.A. and Sartorelli A.C. (1982). Mitomycin-C as a prototype bioreductive alkylating agent: in vitro studies of metabolism and cytotoxicity. Int. J. Radiat. Oncol. Biol. Phys. 8, 753755.

Sasaki M., Abe R., Fujita Y., Ando S., Inokuma D. and Shimizu H. (2008). Mesenchymal stem cells are recruited into wounded skin and contribute to wound repair by transdifferentiation into multiple skin cell type. J. Immunol. 180, 2581-2587.

Spanish Eyes Epidemiological (SEE) Study Group. (2011). Prevalence of age-related macular degeneration in Spain. Br. J. Opthalmol. 95, 931-936.

Takahashi A., Masuda A., Sun M., Centonze V.E. and Herman B. (2004). Oxidative stress-induced apoptosis is associated with alterations in mitochondrial caspase activity and Bcl-2-dependent alterations in mitochondrial pH (pHm). Brain Res. Bull. 62, 497-504.

Wu W.C., Kao Y.H. and Hu D.N. (2002). A Comparative study of effects of antiproliferative drugs on human retinal pigment epithelial cells in vitro. J. Ocul. Pharmacol. Ther. 18, 251-264.

Wu K.Y., Wang H.Z. and Hong S.J. (2008). Mechanism of mitomycin induced apoptosis in cultured corneal endothelial cells. Mol. Vis. 14, 1705-1712.

Accepted May 16, 2013 


\title{
Bioactive substrates for human retinal pigment epithelial cell growth from elastin-like recombinamers
}

\author{
Amar K. Singh, ${ }^{1 \dagger}$ Girish K. Srivastava, ${ }^{1,2,3 \dagger}$ Laura Martín, ${ }^{4 \dagger}$ Matilde Alonso, ${ }^{4}$ J. Carlos Pastor ${ }^{1,3}$ \\ ${ }^{1}$ IOBA-Eye Institute, University of Valladolid, Valladolid, Spain \\ ${ }^{2}$ Networking Research Center on Bioengineering, Biomaterials and Nanomedicine (CIBER-BBN), Valladolid, Spain \\ ${ }^{3}$ Regenerative Medicine and Cell Therapy Networking Center of "Castilla y León", Spain \\ ${ }^{4}$ BIOFORGE Group, University of Valladolid, Valladolid, Spain
}

Received 13 July 2012; revised 6 February 2013; accepted 4 March 2013

Published online in Wiley Online Library (wileyonlinelibrary.com). 10.1002/jbm.a.34726

\begin{abstract}
The aim of this study was to investigate the use of bioactive RGD-containing elastin-like recombinamers (ELRRGDs) as a substrate that can maintain human retinal pigment epithelial cell (hRPE) phenotype and growth pattern. Results obtained are compared with previously published behavior of ARPE19 cells. The extension of these results to hRPE is required because ARPE19 cells cannot be used clinically to treat age-related macular degeneration. hRPE cells were isolated, cultured, seeded, and grown on surface of glass, treated polystyrene (TCP), and solvent-cast ELR-RGD and ELR-IK film with no specific sequence. Cells were analyzed to study cell adhesion, proliferation, morphology, and RPE65 protein expression by staining with diamidino-2-phenylindole, Rhodamine-Phalloidin, and anti-RPE65 antibody at $12,24,72,120,168$, and $360 \mathrm{~h}$. hRPE cells always grew better on ELR-RGD than on glass and ELR-IK but not on TCP. The kinetic hRPE growth curves confirmed that growth differences
\end{abstract}

started to appear at $24 \mathrm{~h}$ for these surfaces in ascending order of cell growths, namely glass, ELR-IK, ELR-RGD, and TCP. There was a clear difference at $360 \mathrm{~h}$. ELR-RGD maintained hRPE cells stable morphology and RPE65 protein expression. ELR-RGD seems to be a good substrate for growing hRPE cells with stable morphology and RPE65 protein expression. As such, this work confirms our hypothesis regarding ELR-RGD substrates viability, which can be used as a Bruch's membrane prosthesis for further studies in animals. However, these results must subsequently be extrapolated to use of hRPE cells in animals to evaluate them as a transplantation vehicle in human. (c) 2013 Wiley Periodicals, Inc. J Biomed Mater Res Part A: 00A: 000-000, 2013.

Key Words: biomaterial, elastin-like recombinamers, hRPE cells

How to cite this article: Singh AK, Srivastava GK, Martín L, Alonso M, Pastor JC. 2013. Bioactive substrates for human retinal pigment epithelial cell growth from elastin-like recombinamers. J Biomed Mater Res Part A 2013: 00A: 000-000.

\section{INTRODUCTION}

Age-related macular degeneration (AMD) is a progressive disorder of the macula that affects central vision, thereby producing irreversible blindness in people older than 65 years in developed countries. ${ }^{1}$ AMD has two forms, namely wet and dry, the latter of which still lacks a treatment despite affecting $8 \%$ of the elderly population. Degeneration of the retinal pigment epithelium (RPE), an external layer of the retina, which in theory could be repaired using tissue engineering, is one of the key events that occurs in AMD. ${ }^{2}$

Bruch's membrane (BrM), an underlying layer of the RPE, helps to maintain the RPE in a healthy, polarized, and functional state. ${ }^{3}$ Although RPE cell transplantation, such as subretinal injection of stem cells, adult RPE cells, photoreceptors, nasal to macular RPE patch translocation and many others, has proved only partial successful, ${ }^{4-12}$ these studies have highlighted the need for healthy autologous RPE cells grown on an appropriate extracellular matrix (ECM) that can maintain the polarity, phenotype, and functional characteristics of RPE cells under in vivo conditions. A failure to implement these requirements in transplantation leads to poor results.

Adult RPE cell culture may offer an alternative that could lead to partial reversal of the aging influence. ${ }^{1314151617}$ Thus, a combination of these partially renewed cells with appropriate substrates might find a use in RPE cell transplantation. However, although numerous studies have reported a number of natural or synthetic ECMs, most of them failed in either in vitro or in vivo studies. ${ }^{18}$

In a previous study, ${ }^{19}$ we described the substrate characteristics required to ensure success in RPE transplantation. As such, our search for new RPE transplantation substrates led us to test elastin-like recombinamers (ELRs). Solvent-cast ELR films on glass are $652.8 \mathrm{~nm}$ thick, flexible, biocompatible, and biodegradable and could be used as porous scaffolds. These ELRs were prepared using genetic 
engineering techniques that allow various different functionalities that mimic the complex properties of the ECM to be incorporated. Our previous study involved solvent-casting ELR films containing an RGD sequence (ELR-RGD), a wellknown sequence found in numerous ECM proteins for promoting cell attachment, profusion, and survival, and another ELR without any sequence (ELR-IK) as a control.

ARPE19 cells are spontaneous immortalized RPE cells and, as such, could be used to overcome several problems, such as sufficient cell supply and growth rate. Furthermore, they are relatively easy to culture and homogeneous, thus meaning that they could be used to perform initial experiments and obtain preliminary results that could support further studies with primary cells. ${ }^{20}$ Our previous results showed that solvent-cast ELR-RGD films support ARPE19 cell viability (>90\%), adhesion (>90\%), and good cell growth, with the cells retaining their epithelial morphology, intercellular tight and gap junctions, and specific RPE65 and Z01 protein expression. These findings suggested an extension of the study to primary RPE cells.

Although ARPE19 cells belong to an RPE cell line, their difference with respect to primary RPE cells make them unsuitable for performing in vivo studies. ${ }^{2122}$ Therefore, in this extended study, we evaluated the behavior of fresh human RPE (hRPE) cells on ELR-RGD and compared the results with ARPE19 cells to determine the suitability of ELR-RGD for application in further subretinal transplantation.

\section{MATERIALS AND METHODS}

\section{Solvent-cast ELR film preparation}

The solvent-cast ELR films used in this study were prepared according to a previously reported method. ${ }^{19}$ In brief, plasmid constructs containing two ELR genes, namely ELR-RGD and ELR-IK, the former of which contains the well-known bioactive arginine-glycine-aspartic acid (RGD) adhesion sequence and the latter of which contains no specific sequence, were prepared using genetic engineering techniques. ELR production was performed using Escherichia coli cell systems. The ELRs produced were subsequently collected and purified. Protein purity and molecular weight were verified using sodium dodecyl sulfate polyacrylamide gels and mass spectrometry (MALDI-TOF/MS), whereas the correctness of the sequence was checked by amino acid analysis and amino acid sequencing.

The amino acid sequences of the ELRs are shown below: ELR-RGD: MGSS-H ${ }_{6}$-SSGLVPRGSHMESLLP- $\left\{\left[(\mathrm{VPGIG})_{2}\right.\right.$ (VPGKG) $\left.(\text { VPGIG) }]_{2}\right]_{2}$ AVTGRGDSPASS[(VPGIG) $\left.\left.{ }_{2}(\text { VPGKG)(VPGIG) })_{2}\right]_{2}\right\}_{6}$

$$
\left(M_{\mathrm{w}}=60,661 \mathrm{Da}\right)
$$

ELR-IK: MESLLP-(VPGIG-VPGIG-VPGKG-VPGIG-VPGIG) $24-\mathrm{V}$ $\left(M_{\mathrm{w}}=51,980 \mathrm{Da}\right)$

ELR films were obtained by solvent-casting onto glass cover-slips (Fisher Scientific SL, ref no: W2755B) and subsequent cross-linking with hexamethylene diisocyanate (HDI), a lysine-targeted homobifunctional crosslinker, as described elsewhere. $^{23}$ In brief, an aqueous ELR solution $(80 \mu \mathrm{L}, 50$ $\mathrm{mg} / \mathrm{mL}$ ) was deposited on circular glass cover slips and incubated for $8 \mathrm{~h}$ at $60^{\circ} \mathrm{C}$. The dry, solvent-cast films were cross-linked by immersion in $10 \% \mathrm{HDI}$ /acetone solution overnight and then washed exhaustively with type I water. All reagents were purchased from Sigma Aldrich (Madrid, Spain).

Costar $^{\circledR} 24$-well cell culture plates with a tissue culture treated polystyrene (TCP) surface were used (Corning, Life Sciences, Europe) in this study. Solvent-cast ELR films on glass and untreated glass cover slips were placed and retained at the center of a 24-well culture plate. The solvent-cast ELR films and controls (glass, TCP) were subsequently exposed to UV light for $12 \mathrm{~h}$ and then incubated in Dulbecco's modified eagle medium (DMEM; Invitrogen, Paisley, UK) with antibiotics for $2 \mathrm{~h}$ to sterilize them before seeding the hRPE cells.

\section{Cell culture}

This study followed the tenets of the declaration of Helsinki and was approved by the Institutional Research Committee of the University of Valladolid. Human eyes from donors with no known concomitant ocular diseases who had provided specific research consent were obtained from the Hospital Clínico Universitario (Valladolid, Spain) postmortem after the removal of corneas for transplantation. Eyes were wrapped in gauze saturated with $0.1 \mathrm{M}$ sodium phosphate buffer (PB) and transported to the laboratory in a humid chamber. One eye from each of two donors (aged 57 and 59) were obtained for this study and processed 3-4 h postmortem.

hRPE cells were isolated from these eyes and maintained in DMEM medium in accordance with previously established protocols. ${ }^{24}$ The DMEM medium was also supplemented with $10 \%$ fetal bovine serum (FBS), antibiotics [penicillin (100 U/mL)-streptomycin $(0.1 \mathrm{mg} / \mathrm{mL})], 1 \mathrm{mM}$ sodium pyruvate, and $2 \mathrm{mM}$ L-glutamine (Invitrogen-Gibco).

Confluent hRPE cells were detached using trypsin (0.1\%) and tetrasodium ethylenediaminetetraacetate (EDTA; 0.04\%) (Invitrogen, Paisley, UK) and then washed with phosphatebuffered saline (PBS). The percentage of the hRPE population expressing RPE65, epithelial and fibroblast proteins, hRPE cell morphology, pigment presence, cell cycle, and viability in cultures were checked using flow cytometry, immunostaining techniques, and standard trypan blue exclusion assay in accordance with our previously published protocol. ${ }^{25}$ Trypan blue was used to quench the autofluorescence during immunostaining of hRPE cells against RPE marker proteins, in accordance with our previous protocol. ${ }^{26}$

Cultures showing healthy, viable, and pigmented hRPE cells with more than 90\% RPE65, epithelial protein expression, and free from fibroblast contamination were selected for use in this study. These hRPE cells $\left(1 \times 10^{5} /\right.$ well $)$ were seeded on sterile glass, TCP, ELR-IK, and ELR-RGD film surfaces and incubated under standard cell culture conditions (humidified atmosphere at $37^{\circ} \mathrm{C}$ with $5 \% \mathrm{CO}_{2}$ ). The cells were analyzed at 12, 24, 72, 120168 , and $360 \mathrm{~h}$ to determine their morphology and growth.

\section{Cell number quantification}

Substrate surfaces were washed with PBS to remove nonadhered cells at each time point studied $(12,24,72,120168$, 


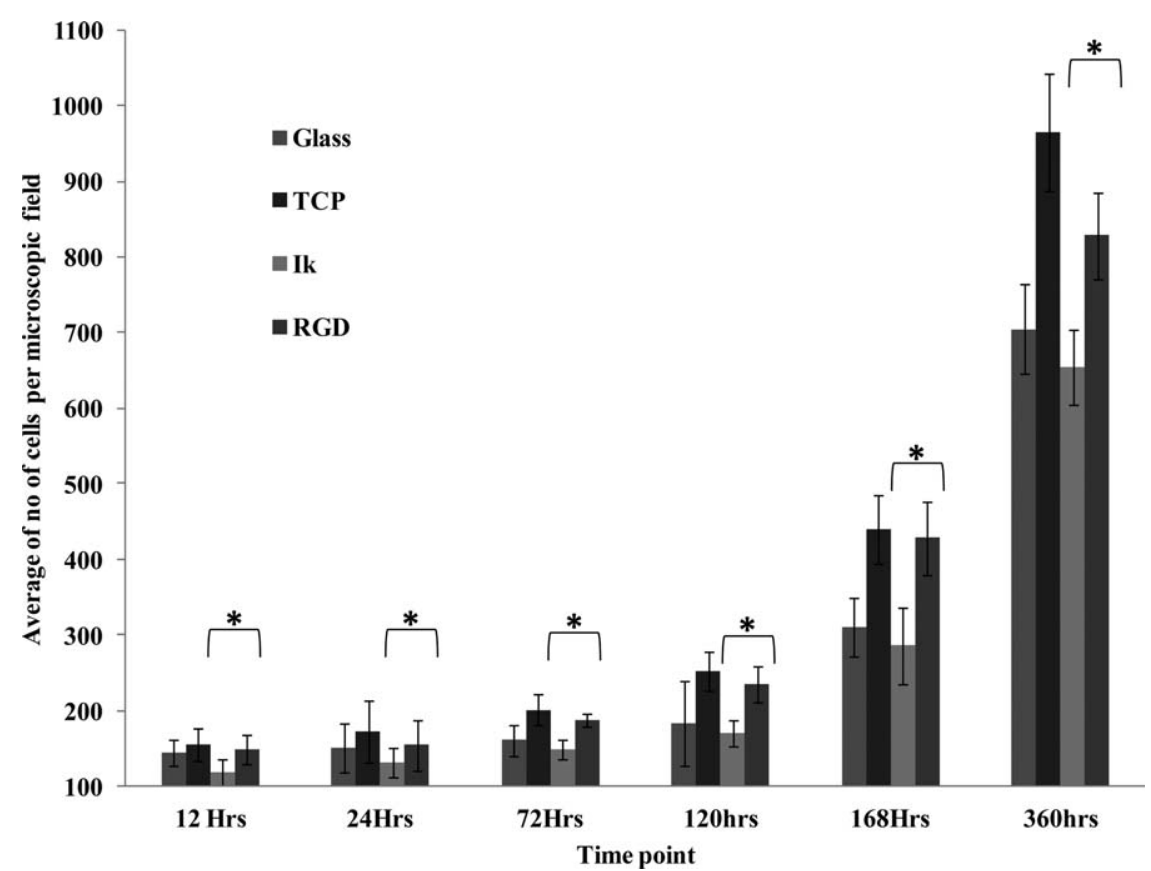

FIGURE 1. hRPE cell growth on each substrate studied. The histogram shows the mean number of cells counted using phase-contrast microscopy for different substrates (glass, TCP, ELR-IK, and ELR-RGD) at different time intervals $(12,24,72,120$, 168, and 360 h). The data are presented as mean number of cells per field $(10 \times) \pm$ SD. The ELR-RGD substrate is more favorable for cell growth than ELR-IK. $P<0.05$ is considered significant for comparing the number of cells growing on different surfaces.

and $360 \mathrm{~h}$ ) and the cell numbers determined by manual counting using a phase-contrast microscope (Leica Microsystems, Mannheim, Germany). A total of 20 random fields $(10 \times)$ were photographed for each substrate, and the cells contained in each field counted using Adobe Photoshop Elements software. The mean number of cells per field of view $(10 \times)$ was calculated for each time point studied and for each substrate and presented as a histogram showing the average cell count per field of view \pm 1 standard deviation (SD) versus time.

A line plot graph of cell growth kinetics for each substrate was plotted by normalizing the average number of cells per microscopic field at each time point with respect to the initial time $\left(N_{\mathrm{t}} / N_{0}\right)$, multiplied by 100 to make the figures more visible on the $y$ axis, versus time $\left(N_{\mathrm{t}} / N_{0} \times\right.$ 100 vs. time) using MS Excel 2007.

\section{Cell morphology and RPE65 protein expression}

The cell morphology on each substrate was determined by staining cytoskeleton actin filaments with Rhodamine-Phalloidin (RP) (Molecular Probes-Invitrogen, Paisley, UK), which emits red fluorescence, whereas cell specificity was studied by an immunostaining technique against the RPE65 protein, a specific marker of RPE, which emits green fluorescence, as described previously. ${ }^{26}$ In brief, at the 120 -h time point, the cells were washed with PBS (3-5 min), fixed with paraformaldehyde (4\%) for $10 \mathrm{~min}$, permeabilized with PBT $(0.2 \%$ TritonX-100 in PBS) for $10 \mathrm{~min}$, washed once again, and blocked for $1 \mathrm{~h}$ in antibody blocking buffer $(10 \%$ normal goat serum, $1 \%$ bovine serum albumin in
PBT). The resulting cells were incubated overnight with an anti-RPE65 mouse monoclonal antibody (MoAb, Novus Biologicals, Littleton, $\mathrm{CO})(1: 100)$ at $4^{\circ} \mathrm{C}$ and then washed again and incubated with a goat anti-mouse IgG-FITC antibody (1:100) (Jackson Immunoresearch Laboratories, West Grove, $\mathrm{PA}$ ) for $1 \mathrm{~h}$ at room temperature. The cells were also costained with RP stain (1:40) (200 U/mL) for $20 \mathrm{~min}$, and finally, the nuclei were counterstained with diamidino-2phenylindole (DAPI) then mounted and observed under a fluorescence microscope.

\section{Statistical analysis}

The results obtained for each substrate was expressed as mean number of cells per field of view $(10 \times) \pm$ standard deviation (SD), as calculated using MS excel 2007. Data were tested for normality and investigated for statistical significance using Student's unpaired $t$ test using the online GraphPad software. Statistical significance was set at $P<0.05$.

\section{RESULTS}

hRPE cell adhesion and growth on different substrates The number of cells on each surface was counted at 12, 24, 72, 120 168, and $360 \mathrm{~h}$ and analyzed by determining the mean cell number, standard deviation, and $P$ value. The results obtained, which are presented as a histogram in Figure 1, show that cells grew better on ELR-RGD than on ELR-IK at all time points studied (12, 24, 72, 120 168, and $360 \mathrm{~h}$ ). Similarly, the number of cells on glass was always higher than that observed on ELR-IK but lower than that found on ELR-RGD. The number of cells on TCP was always 


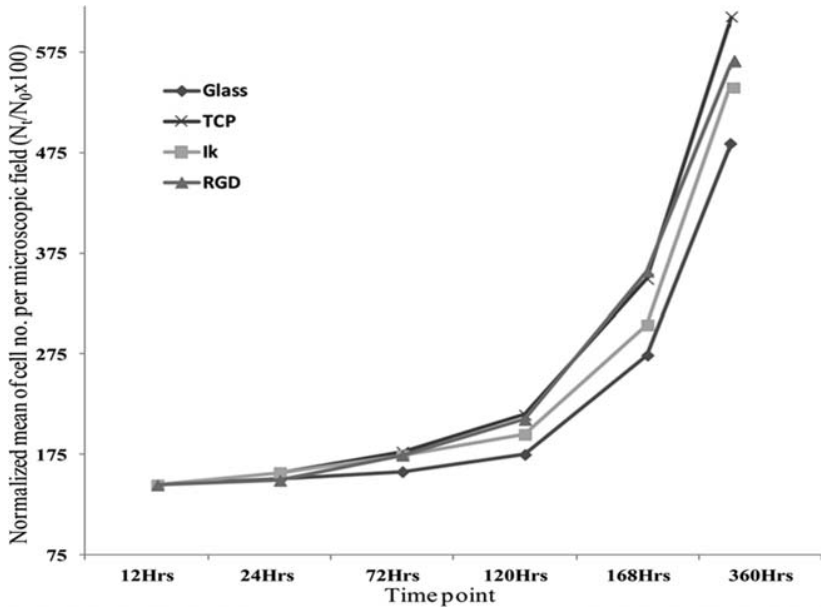

FIGURE 2. The growth of hRPE cells as normalized number of average cells per microscopic field at $10 \times$ magnification against the initial number of adhered cells at $12 \mathrm{~h}$ multiplied by $100\left(N_{\mathrm{t}} / N_{0} \times 100\right)$ vs. time (h), where $N_{0}$ is the average number of cells per microscopic field at $12 \mathrm{~h}$ and $N_{\mathrm{t}}$ the average number of cells per microscopic field at different time points $(12,24,72,120,168$, and 360 h). TCP and ELRRGD show similar cell growth curves. ELR-RGD supports cell growth better than ELR-IK, and the glass surface is the poorest for cell growth.

higher than that for all other substrates (glass, ELR-IK, and ELR-RGD; Fig. 1).

The differences between the number of cells on the substrates (glass, TCP, ELR-IK, and ELR-RGD) was low until 72 $h$, after which the number of cells on TCP and ELR-RGD increased significantly in comparison with the number on glass and ELR-IK, as observed at $168 \mathrm{~h}$ (Fig. 1). A significant difference was subsequently found between the number of cells on TCP and ELR-RGD at $360 \mathrm{~h}$ (Fig. 1). The histogram shows that the cells growing on ELR-IK need more time to become confluent than all other substrates studied, as can also be seen at $120 \mathrm{~h}$ in Figure 3 .

The growth curves in the kinetic cell growth plot (Fig. 2) clearly show that the ELR-RGD growth curve is similar to that for TCP, thereby suggesting that the ELR-RGD film surface stimulates hRPE cell proliferation in a similar manner to TCP. The ELR-RGD growth curve is clearly different to that for ELR-IK, thus confirming the difference between cell growth on these two surfaces. The growth curve for glass shows that hRPE cell growth on this surface is poorest.

\section{Cell morphology}

Microscopic observation of hRPE cell growth on the substrates after $120 \mathrm{~h}$ showed that the cells did not reach confluence in any case, although there was a clear difference in cell density on the different substrates (Fig. 3), decreasing in the order: TCP, ELR-RGD, glass, and ELR-IK. The cells growing on the TCP surface were found to be more closely packed than those on the ELR surface and to form cell patches with a mosaic morphology. The cells on ELR-IK were dispersed, rounded, or with fibroblast-like morphology (Fig. 3).

The microscopic observations of hRPE cell morphology were further confirmed by using a red fluorescent stain
(RP), which demonstrated well-formed hexagonal circumferential actin fibers in the cells growing on glass and ELRRGD [Fig. 4(D,G)]. DAPI staining showed a rounded cell nucleus in each cell in all cases in merged panels [Fig. $4(\mathrm{~B}, \mathrm{E}, \mathrm{H})]$. hRPE cells growing on a TCP surface stained for RPE65 protein were taken as a positive control [Fig. 4(A)], and the same cells with no primary antibody but with addition of a secondary antibody used as a negative staining control [Fig. 4(B)] to rule out any nonspecific staining by the FITC-conjugated secondary antibody.

\section{RPE65 protein assessment}

A specificity assessment of RPE65 protein expression in hRPE cells by immunostaining showed that hRPE cells expressed RPE65 protein on both glass and ELR-RGD substrates, thus meaning that hRPE cells maintained their RPEtype characteristics on both surfaces [Fig. 4(C,F)].

\section{DISCUSSION}

Although the dry form of AMD affects $8 \%$ of the elderly population older than 65 years, it is currently untreatable. ${ }^{12}$ Initial cell transplantation studies using a suitable substrate as a cell carrier achieved only partial success. ${ }^{18}$ As a result, numerous subsequent studies have been performed to find a suitable substrate for use as an hRPE cell carrier. The substrates tested to date include mesh-supported submicron parylene-C membranes, silk fibroin, polyimide membranes, cryoprecipitated membranes, anterior lens capsules, cadaver Bruch's membranes, Descemet's membranes, natural or synthetic biodegradable or nonbiodegradable polymers films, and collagens, to name but a few. ${ }^{27282930313233}$ However, most of these proved unsuitable for various reasons, including their cytotoxic nature, poor RPE cell adhesion, growth, and behavior under in vitro and in vivo conditions, handling difficulties, etc. In a previous study, ${ }^{19}$ we found that ARPE19 cells (an RPE cell line) showed an improved response in terms of stability, adhesion, growth, epithelial morphology, intercellular junction formation, and RPE65 and Z01 protein expression on an ELR-RGD film surface. This led us to further study this system to determine primary hRPE cell behavior on the surface of this film, which would be a key step toward the development of an RPE cell-ELR scaffold as part of a regenerative medicine strategy. We were also interested in determining other aspects such as ELR permeability, film thickness for handling and transplantation in the subretinal space in animal, posttransplantation output, etc.

hRPE cells were grown on different substrate surfaces (glass, TCP, ELR-IK, and ELR-RGD) and number of cells present quantified at different time points using a manual counting method. Biochemical testing methods were not used for this study because a small peripheral area around the glass cover slip and the bottom surface of the 24-well tissue culture plate was not covered with the solvent-cast ELR films. ELR-coated round glass cover slips were prepared, then placed and retained at the center of each well on a 24-well tissue culture plate. It proved difficult to 


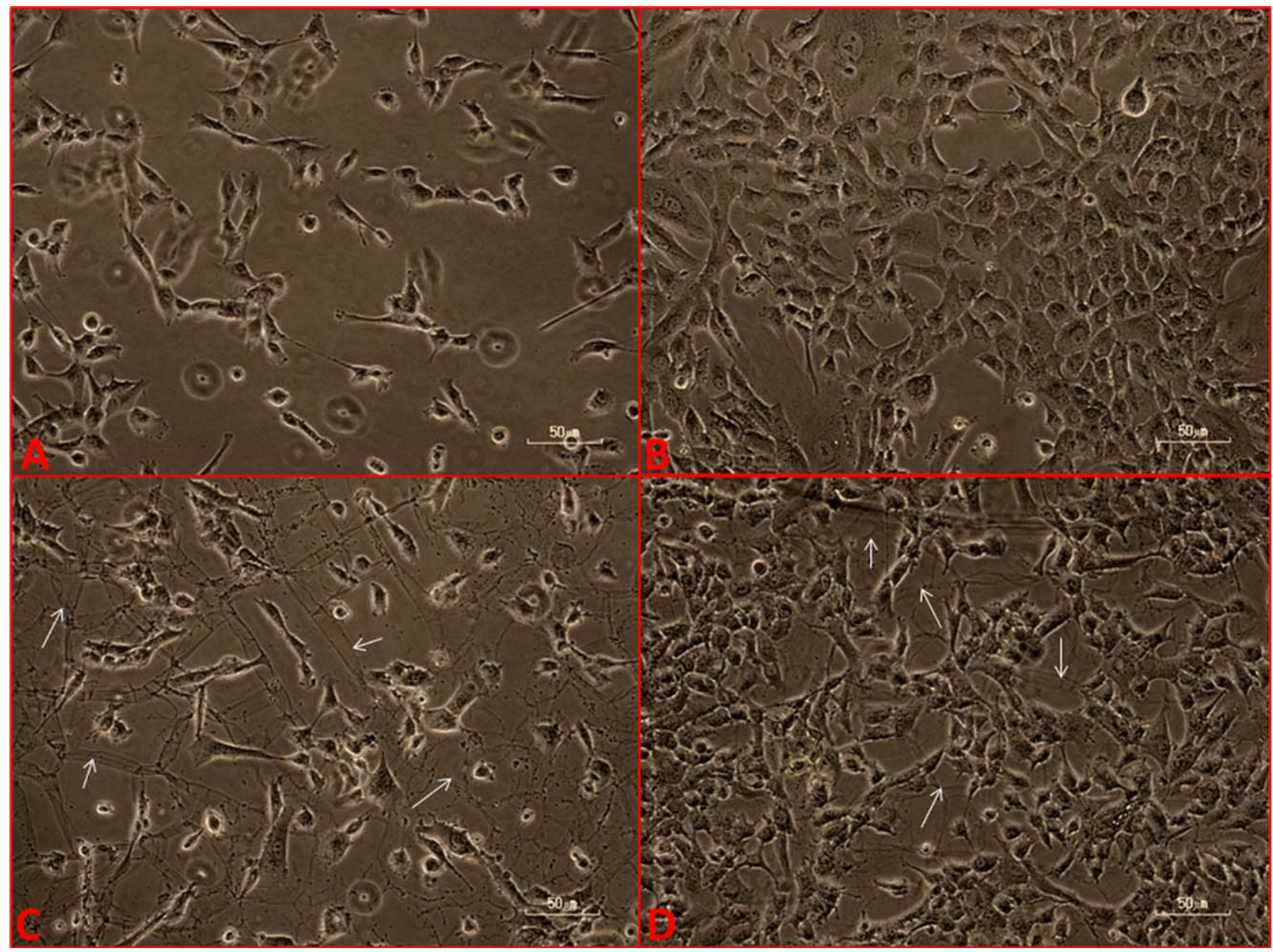

FIGURE 3. Phase-contrast microscopy demonstrating the cell density, cellular morphology, and cell patch formation on each substrate studied for $120 \mathrm{~h}$. ELP-IK does not support hRPE cells as well as ELR-RGD. (A) Glass; (B) TCP; (C) ELR-IK; (D) ELR-RGD. The white arrows in panels C and $D$ indicate the wrinkles of solvent-cast ELR films on the surface of glass cover slips. [Color figure can be viewed in the online issue, which is available at wileyonlinelibrary.com.]

exclude cell proliferation readings for that part of the well not coated with ELR whether MTT, Alamar blue, or any other biochemical methods were used for the same well. As such, a manual counting method was used for this study. ${ }^{2734}$ In addition, this method allows us to compare the data with our previously published work.

The histogram results (Fig. 1) clearly show better hRPE cell growth on ELR-RGD than on ELR-IK at all time points studied (12, 24, 72, 120 168, and 360 h). This observation was further confirmed by Figure 2 and strongly supports our proposal that insertion of the RGD sequence into the ELR improves its surface properties for hRPE cell adhesion and proliferation. Because the ELR films studied herein were prepared on glass cover slip, a glass cover slip was used as one of the control. Although, to the best of our knowledge, this cover slip was not treated, both controls and experimental surfaces were incubated in a medium supplemented with serum (FBS). Previous reports have suggested that serum proteins may influence glass surfaces, ${ }^{35}$ probably because of the deposition of serum proteins that could favor cell attachment on them. Despite this, glass surfaces are usually considered to be hydrophobic. It is also known that RPE cells secret matrix proteins that favor cell proliferation during growth. ${ }^{36}$ In addition, the kinetic growth graph (Fig. 2) shows that RPE cells growing on the glass surface proliferate less than on all other surfaces. Figure 3 also shows that there are fewer RPE cells on the glass and that these cells form few cell patches containing more rounded cells than on all other surfaces except ELR-IK. Thus, our findings suggest that glass surface seems to partially support RPE cell growth but not TCP, ELR-IK, and ELR-RGD surfaces.

Cell growth on TCP was always more significant than on any of other substrates (glass, ELR-RGD, and ELR-IK). It is evident that, although glass and TCP support hRPE cell adhesion and proliferation, they cannot be used in hRPE cell transplantation in the subretinal space because of their rigidity and nondegradable nature. The hRPE cell adhesion and proliferation trend on these substrates was similar to that for ARPE19 cell growth on these surfaces reported previously by our group. ${ }^{19}$ As a result, this study greatly supports our previous findings as regards the behavior of 


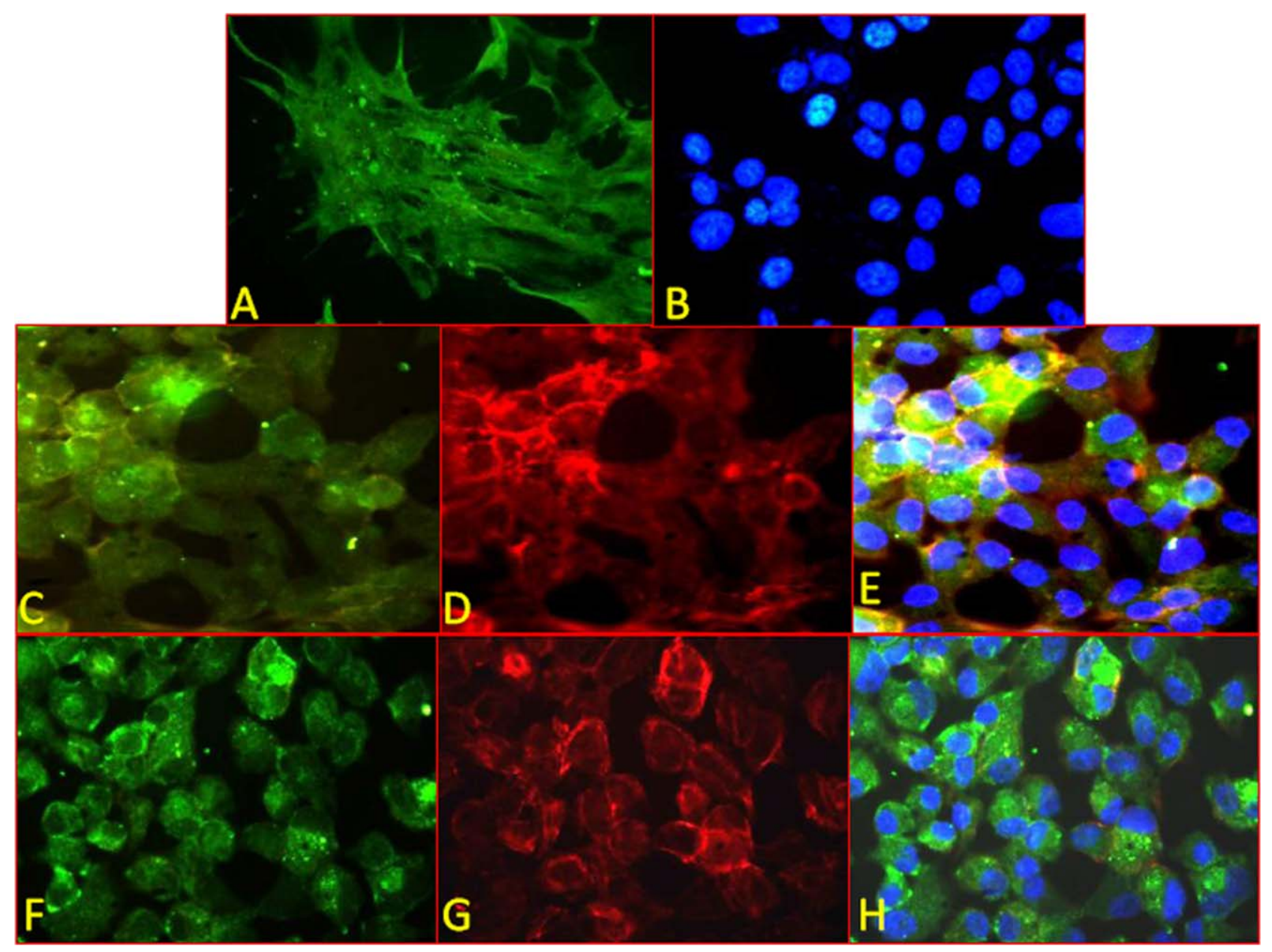

FIGURE 4. Immunostaining of hRPE cells grown on TCP, glass, and ELR-RGD for $120 \mathrm{~h}$ to evaluate RPE65 protein expression (green) and Rhodamine-Phalloidin (red) staining for morphology. (A) hRPE cells grown on TCP detected by RPE65 MoAb. (B) hRPE cells grown on TCP used as a negative control, with omission of the primary antibody and addition of only the secondary Ab, counterstained with DAPI (cell nuclei appear in blue). (C, D) RPE65 protein expression (green) and morphology (red) of hRPE cells, respectively, growing on glass. (E) Merged photo of (C) and (D) with DPAl-stained nuclei (blue). (F, G) RPE65 protein expression and morphology of hRPE cells, respectively, growing on ELR-RGD. (H) Merged photo of (F) and (G) counterstained with DAPI (blue). [Color figure can be viewed in the online issue, which is available at wileyonlinelibrary.com.]

ARPE19 cells on ELR-RGD and could therefore lead to further practical benefits in the future for hRPE cell-substrate transplantation for the treatment of retinal degenerative diseases.

The growth rates of both cell types (hRPE and ARPE19) on glass, TCP, ELR-IK, and ELR-RGD differed because of the fact that, in our case, the hRPE cells extracted from the donated human eye (donors usually older than 50 years) grow more slowly than ARPE19 under normal growth conditions in the tissue culture flask. During the early stages $(24 \mathrm{~h})$, the hRPE cells grown on glass, ELR-IK, and ELR-RGD presented similar growth patterns to those observed in the case of APRE19, with the exception of growth on TCP. Thus, although ARPE19 and hRPE cells always grew well on TCP, ARPE19 cell growth was always significantly higher than that for hRPE cells at all time points studied $(12,24,72$, 120 168, and $360 \mathrm{~h}$ ). This is probably because of the more adaptable and proliferative nature of ARPE19 cells (an established but nonimmortalized hRPE cell line) in comparison with hRPE primary cells. ${ }^{3738}$ The use of a nonimmortalized human RPE cell line allows us to compare the surface cell response with a high level of control because of the reproducibility of such cells in culture and the extensive literature regarding their "normal" behavior. ${ }^{20}$ The results from this study confirmed that ARPE19 cells do not necessarily respond in the same way as primary hRPE cells, a finding which has also been confirmed by other authors. ${ }^{39}$ Thus, it is important to note that primary cells must be used when testing cell supports in any eventual surgical treatment.

Although hRPE cells maintained a similar growth pattern for the first $72 \mathrm{~h}$, there was a significant difference in ARPE19 cell growth patterns on solvent-cast ELR-RGD and ELR-IK films over the same period. Despite this, ELR-RGD was better able to support hRPE and ARPE19 cell growth than ELR-IK in both studies. These results show that hRPE cells require $72 \mathrm{~h}$ to adapt to, and interact with, the ELR-IK and ELR-RGD surfaces to generate signals for subsequent 
proliferation, with growth rates for ELR-RGD and TCP increasing thereafter. ARPE19 cells required only $24 \mathrm{~h}$ to adapt to the surfaces and interact with ELR-IK and ELRRGD. These results confirm our initial hypothesis whereby cell lines and primary cells behave differently in terms of certain properties such as adherence, proliferation, appropriate morphology acquisition and, in this case, proliferation on different surfaces over $72 \mathrm{~h}$.

The number of hRPE cells on all the substrates tested (glass, TCP, ELR-IK, and ELR-RGD) remained low for the first $72 \mathrm{~h}$ but subsequently increased significantly on TCP and ELR-RGD in comparison with glass and ELR-IK, as can be seen at 168 and $360 \mathrm{~h}$. The difference between the number of cells on TCP and ELR-RGD increased markedly at $360 \mathrm{~h}$ (Fig. 1), thus suggesting that the hRPE growth rate on all substrates is relatively constant for the first $72 \mathrm{~h}$ but then begins to increase for TCP and ELR-RGD (168 h), subsequently increasing significantly for TCP (360 h). This suggests that both a surface response and cell-to-cell contact are involved in inducing proliferation. These results were further confirmed by plotting a growth curve (Fig. 2). Thus, the growth curve for hRPE cells on the ELR-RGD surface followed the same pattern as that on TCP, whereas that for ELR-IK showed slower growth and growth on glass being the slowest. These findings confirm that the ELR-RGD substrate provides a better surface for hRPE cell growth than either glass or ELR-IK. The growth curve for glass started to separate from those for ELR-RGD and TCP after $24 \mathrm{~h}$, whereas the growth curve for ELR-IK started to separate after $72 \mathrm{~h}$, thereby confirming that the glass surface is by far the worst surface for hRPE cell proliferation and that TCP and ELR-RGD provide the best surfaces for hRPE cell proliferation. The genetic engineering technology used to produce the ELR-RGD substrate has an advantage over TCP in that it offers the possibility to prepare a biodegradable, flexible, and porous membrane support vehicle for RPE-substrate transplantation that can mimic the behavior of BrM in the affected eye of patients with AMD. Although the simpler synthetic material (TCP) is better able to support hRPE cell adhesion and growth, its rigid and nondegradable nature make it unsuitable for clinical use.

A comparison of the hRPE and ARPE19 cell density on all substrates at $120 \mathrm{~h}$ clearly showed that hRPE cells (Fig. 3) needed more time to become confluent, except in the case of those hRPE and ARPE19 cells growing on ELR-IK, where it can clearly be seen that the cell density was higher for ARPE19 cells than for hRPE cells on ELR-IK. This finding suggests that the ELR is more favorable for ARPE19 than hRPE cell growth but that the former can only be used to obtain preliminary data in vitro and in vivo that might not be applicable in clinical trials.

Microscopic examination of the hRPE cell morphology on the substrates showed that hRPE cells growing on TCP and ELR-RGD for $120 \mathrm{~h}$ showed a packed mosaic epithelial morphology (patches of cells), although the number and cellular compactness of these hRPE cellular patches was lower on ELR-RGD than on TCP at all time points studied. Similar results were observed in the case of ARPE19 cells. The growth kinetics on ELR-RGD was similar to those on TCP, as can be seen from the graph in Figure 2. However, more dispersed rounded and fibroblast-like hRPE cells were present on ELR-IK than was the case for ARPE19 cells. This finding confirms that ELR-RGD is more favorable for the acquisition of a packed mosaic epithelial morphology by hRPE cells than ELR-IK, as also confirmed by RP staining [Fig. 4(D,G)].

As hRPE cells lose RPE65 protein, a specific marker of RPE-type characteristics, during culture, ${ }^{25}$ an evaluation of RPE65 protein expression from the beginning is important. Our previously published FAC evaluation results showed that more than $90 \%$ of seeded hRPE cells expressed RPE65 proteins. $^{25}$ In light of this, we undertook immunostaining studies with the cells growing on both glass and ELR-RGD as the ELR films are cast on glass coverslips and ELR-RGD was found to be superior to ELR-IK. As such, it seemed important to evaluate RPE65 protein expression in cells growing on both these substrates, especially because it had already been confirmed that cell growth, morphology, and patch formation were different on the different surfaces (glass, TCP, ELR-IK, and ELR-RGD,). RPE65 protein expression was maintained (100\%) on ELR-RGD during $120 \mathrm{~h}$ of study. DAPI nuclear staining showed the presence of rounded nuclei in all hRPE and ARPE19 cells grown on all substrates. This reflects the fact that hRPE and ARPE19 cells can adapt to the ELR-RGD without any loss of RPE-type characteristics, thus supporting the use of dual hRPE and ELR-RGD layers in further in vivo studies.

In summary, RGD-containing ELRs have shown their ability to support the growth of hRPE cells in good numbers while maintaining their RPE-type characteristics. As such, they could be considered to be a suitable carrier for RPE cell transplantation in the future.

\section{REFERENCES}

1. Gehrs KM, Anderson DH, Johnson LV, Hageman GS. Age-related macular degeneration-Emerging pathogenetic and therapeutic concepts. Ann Med 2006;38:450-471.

2. Green WR. Histopathology of age-related macular degeneration. Mol Vis 1999;5:27.

3. Curcio CA, Johnson M, Huang JD, Rudolf M. Apolipoprotein Bcontaining lipoproteins in retinal aging and age-related macular degeneration. J Lipid Res 2010;51:451-467.

4. MacLaren R, Pearson R, MacNeil A, Douglas R, Salt T, Akimoto M, Swaroop A, Sowden J, Ali R. Retinal repair by transplantation of photoreceptor precursors. Nature 2006;444:203-207.

5. Wang S, Girman S, Lu B, Bischoff N, Holmes T, Shearer R, Wright LS, Svendsen CN, Gamm DM, Lund RD. Long-term vision rescue by human neural progenitors in a rat model of photoreceptor degeneration. Invest Ophthalmol Vis Sci 2008;49:3201-3206.

6. Seiler MJ, Thomas B, Chen Z, Wu R, Sadda SR, Aramant RB. Retinal transplants restore visual responses: Trans-synaptic tracings from visually responsive sites labels transplant neurons. Eur $\mathrm{J}$ Neurosci 2008;28:208-220.

7. Wang S, Lu B, Holmes T, Bischoff N, Lund RD. Morphological and functional rescue in RCS rats after RPE cell line transplantation at a later stage of degeneration. Invest Ophthalmol Vis Sci 2008;49:416-421.

8. Girman SV, Wang S, Lund RD. Cortical visual functions can be preserved by subretinal RPE cell grafting in RCS rats. Vision Res 2003;43:1817-1827.

9. Coffey PJ, Girman S, Wang SM, Hetherington L, Keegan DJ, Adamson P, Greenwood J, Lund RD. Long-term preservation of 
cortically dependent visual function in RCS rats by transplantation. Nat Neurosci 2002;5:53-56.

10. Sagdullaev BT, Aramant RB, Seiler MJ, Woch G, McCall MA. Retinal transplantation-induced recovery of retinotectal visual function in a rodent model of retinitis pigmentosa. Invest Ophthalmol Vis Sci 2003;44:1686-1695.

11. Kwan AS, Wang S, Lund RD. Photoreceptor layer reconstruction in a rodent model of retinal degeneration. Exp Neurol 1999;159: 21-33.

12. van Zeeburg EJ, Maaijwee KJ, Missotten TO, Heimann $\mathrm{H}$, van Meurs JC. A free retinal pigment epithelium-choroid graft in patients with exudative age-related macular degeneration: Results up to 7 years. Am J Ophthalmol 2012;153:120-127.

13. Ishida M, Lui GM, Yamani A, Sugino IK, Zarbin MA. Culture of human retinal pigment epithelial cells from peripheral scleral flap biopsies. Curr Eye Res 1998;17:392-402.

14. Burke JM, Skumatz CM. Autofluorescent inclusions in long-term postconfluent cultures of retinal pigment epithelium. Invest Ophthalmol Vis Sci 1998;39:1478-1486.

15. Gullapalli VK, Sugino IK, Zarbin MA. Culture-induced increase in alpha integrin subunit expression in retinal pigment epithelium is important for improved resurfacing of aged human Bruch's membrane. Exp Eye Res 2008;86:189-200.

16. Sugino IK, Sun Q, Wang J, Nunes CF, Cheewatrakoolpong N Rapista A, Johnson AC, Malcuit C, Klimanskaya I, Lanza R, Zarbin MA. Comparison of FRPE and human embryonic stem cellderived RPE behavior on aged human Bruch's membrane. Invest Ophthalmol Vis Sci 2011;52:4979-4997.

17. Bian Q, Gao S, Zhou J, Qin J, Taylor A, Johnson EJ, Tang G, Sparrow JR, Gierhart D, Shang F. Lutein and zeaxanthin supplementation reduces photo-oxidative damage and modulates the expression of inflammation-related genes in retinal pigment epithelial cells. Free Radic Biol Med 2012;53:1298-1307.

18. Hynes SR, Lavik EB. A tissue-engineered approach towards retina repair: Scaffolds for cell transplantation to the subretinal space. Graefes Arch Clin Exp Ophthalmol 2010;248:763-778.

19. Srivastava GK, Martín L, Singh AK, Fernandez-Bueno I, Gayoso MJ, Garcia-Gutierrez MT, Girotti A, Alonso M, Rodríguez-Cabello JC, Pastor JC. Elastin-like recombinamers as substrates for retinal pigment epithelial cell growth. J Biomed Mater Res A 2011;97: 243-250.

20. Dunn KC, Aotaki-Keen AE, Putkey FR, Hjelmeland LM. ARPE-19, a human retinal pigment epithelial cell line with differentiated properties. Exp Eye Res 1996;62:155-169.

21. Alge CS, Hauck SM, Priglinger SG, Kampik A, Ueffing M. Differential protein profiling of primary versus immortalized human RPE cells identifies expression patterns associated with cytoskeletal remodeling and cell survival. J Proteome Res 2006;5:862-878.

22. Gupta SK, Jollimore CA, McLaren MJ, Inana G, Kelly ME. Mammalian retinal pigment epithelial cells in vitro respond to the neurokines ciliary neurotrophic factor and leukemia inhibitory factor. Biochem Cell Biol 1997;75:119-125.

23. Martín L, Alonso M, Girotti A, Arias FJ, Rodríguez Cabello JC. Synthesis and characterization of macroporous thermo sensitive hydrogels from recombinant elastin-like polymers. Biomacromolecules 2009;10:3015-3022.
24. Zhang L, Li X, Zhao M, He P, Yu W, Dong J, Liu G, Li C, Shi X. Antisense oligonucleotide targeting c-fos mRNA limits retinal pigment epithelial cell proliferation: A key step in the progression of proliferative vitreoretinopathy. Exp Eye Res 2006;83:1405-1411.

25. Srivastava GK, Reinoso $R$, Singh AK, Fernandez-Bueno I, Martino M, Garcia-Gutierrez MT, Pastor JC, Corell A. Flow cytometry assessment of the purity of human retinal pigment epithelial primary cell cultures. J Immunol Methods 2013;389:61-68.

26. Srivastava GK, Reinoso $R$, Singh AK, Fernandez-Bueno I, Hileeto D, Martino M, Garcia-Gutierrez MT, Merino JM, Alonso NF, Corell A, Pastor JC. Trypan Blue staining method for quenching the autofluorescence of RPE cells for improving protein expression analysis. Exp Eye Res 2011;93:956-962.

27. Kearns V, Mistry A, Mason S, Krishna Y, Sheridan C, Short R, Williams RL. Plasma polymer coatings to aid retinal pigment epithelial growth for transplantation in the treatment of age related macular degeneration. J Mater Sci: Mater Med 2012;23:20132021.

28. Sheridan C, Krishna $Y$, Williams R, Mason S, Wong D, Heimann $H$, Kent D, Grierson I. Transplantation in the treatment of agerelated macular degeneration: Past, present and future directions. Expert Rev Ophthalmol 2007;2:1-14.

29. Ma Z, Han L, Wang C, Dou H, Hu Y, Feng $X, X u$ Y, Wang Z, Yin Z, Liu $Y$. Autologous transplantation of retinal pigment epitheliumBruch's membrane complex for hemorrhagic age-related macular degeneration. Invest Ophthalmol Vis Sci 2009;50:2975-2981.

30. Lu B, Zhu D, Hinton D, Humayun MS, Tai YC. Mesh-supported submicron parylene-C membranes for culturing retinal pigment epithelial cells. Biomed Microdevices 2012;14:659-667.

31. Shadforth AM, George KA, Kwan AS, Chirila TV, Harkin DG. The cultivation of human retinal pigment epithelial cells on Bombyx mori silk fibroin. Biomaterials 2012;33:4110-4117.

32. Mazumder MA, Fitzpatrick SD, Muirhead B, Sheardown H. Cell-adhesive thermogelling PNIPAAm/hyaluronic acid cell delivery hydrogels for potential application as minimally invasive retinal therapeutics. J Biomed Mater Res A 2012;100:1877-1887.

33. Subrizi A, Hiidenmaa $H$, Ilmarinen $T$, Nymark $S$, Dubruel $P$, Uusitalo H, Yliperttula M, Urtti A, Skottman H. Generation of hESCderived retinal pigment epithelium on biopolymer coated polyimide membranes. Biomaterials 2012;33:8047-8054.

34. Tezcaner A, Bugra K, Hasirci V. Retinal pigment epithelium cell culture on surface modified poly(hydroxybutyrate-co-hydroxyvalerate) thin films. Biomaterials 2003;24:4573-4583.

35. Schwartz JR, Roy SK. In vitro culture of hamster ovarian primary interstitial cells: Effect of serum. Biol Reprod 1998;59:1187-1194.

36. Campochairo PA, Jerdan JA, Glaser BM. The extracellular matrix of human retinal pigment epithelial cells in vivo and its synthesis in vitro. Invest Ophthalmol Vis Sci 1986;27:1615-1621.

37. Al-Hussaini H, Kam JH, Vugler A, Semo M, Jeffery G. Mature retinal pigment epithelium cells are retained in the cell cycle and proliferate in vivo. Mol Vis 2008;14:1784-1791.

38. Kaur G, Dufour JM. Cell lines: Valuable tools or useless artifacts. Spermatogenesis 2012;2:1-5.

39. Hornof M, Toropainen E, Urtti A. Cell culture models of the ocular barriers. Eur J Pharm Biopharm 2005;60:207-225. 
Research paper

\title{
Flow cytometry assessment of the purity of human retinal pigment epithelial primary cell cultures
}

\author{
Girish K. Srivastava a,b,d,*,1, Roberto Reinoso a,b,1 , Amar K. Singh a, Ivan Fernandez-Bueno a,b,d, \\ Mario Martino a,b, Maria T. Garcia-Gutierrez ${ }^{\mathrm{a}, \mathrm{b}}$, J. Carlos Pastor ${ }^{\mathrm{a}, \mathrm{d}}$, Alfredo Corell ${ }^{\mathrm{a}, \mathrm{b}, \mathrm{c}}$ \\ a Institute of Applied Ophthalmobiology (IOBA), University of Valladolid, Valladolid, Spain \\ b Networking Research Center on Bioengineering, Biomaterials and Nanomedicine (CIBER-BBN), Valladolid, Spain \\ c Department of Immunology, University of Valladolid, Valladolid, Spain \\ ${ }^{\mathrm{d}}$ Regenerative Medicine and Cell Therapy Networking Center of "Castilla y León", Spain
}

\section{A R T I C L E I N F O}

\section{Article history:}

Received 27 August 2012

Received in revised form 12 December 2012

Accepted 3 January 2013

Available online 11 January 2013

\section{Keywords:}

Flow cytometry

hRPE

Purity

RPE65

Epithelial and fibroblast proteins

\begin{abstract}
A B S T R A C T
Culturing of human retinal pigment epithelial cells (hRPE) is the initial step in cell therapy of some retinal diseases. To transfer these cells into clinical use, it is necessary to guarantee that they are well differentiated and contamination free. Fluorescence microscopy is the easiest method to do this, but it is associated with operator subjectivity, and the results are highly variable. The aim of this study was to demonstrate the practicality of implementing flow cytometry (FC) analysis to determine the purity of human RPE primary cell cultures. An ARPE19 cell line, human skin fibroblasts, hRPE, and human corneal epithelial cells were analysed by FC to determine the percentage of the hRPE population expressing RPE65 and epithelial and fibroblast proteins. The cell viability and DNA content also were determined. FC analysis showed that the hRPE cells were healthy, stable, and expressed RPE65 protein in the study working conditions. The density of RPE65 protein expression decreased during passages 2 to 10 , which was confirmed using a Western blot technique. However, the hRPE cells did not express the 112-kDa epithelial and fibroblast proteins in the current working conditions. These findings suggested that FC facilitates a detailed analysis of human RPE primary cell cultures, a necessary step in developing new cell therapies for retinal diseases.
\end{abstract}

(c) 2013 Elsevier B.V. All rights reserved.

\section{Introduction}

Retinal pigment epithelial (RPE) cells are of great interest to investigators who are doing research in the fields of retinal tissue engineering and cell transplantation because of the crucial roles these cells play in maintaining normal retinal functions (Engelmann and Valtink, 2004; Valtink and Engelmann, 2009). Improper functioning of the RPE leads to photoreceptor degeneration and consequent visual impairment. Currently, some therapeutic strategies are promising,

\footnotetext{
* Corresponding author at: Institute of Applied Ophthalmobiology (IOBA), University of Valladolid, Campus Miguel Delibes, Paseo de Belen, 17, Valladolid, 47011, Spain.

E-mail address: girish@ioba.med.uva.es (G.K. Srivastava).

1 Authors equally contributed in this work.
}

such as transplantation of fresh RPE cells or genetically manipulated RPE cells that secrete trophic factors, which might cure or delay the course of photoreceptor degenerative diseases (Majji and De Juan, 2000).

Different techniques have been used previously to isolate RPE cells, such as mechanical scraping or enzymatic digestion of the RPE monolayer after removing the vitreous and peeling the retina (Singhal and Vemuganti, 2005). However, it is difficult to define the degree of purity of the RPE population in cell cultures (Wiencke et al., 2003). Furthermore, an investigation based on RPE cell cultures can be successful only if the cell culture has an appropriate number of healthy, vital, proliferative RPE cells with most of their characteristics retained, such as pigmentation and phagocytic capacity. The cultures must not undergo cellular transdifferentiation to cells with fibroblast-like morphology and they must not have components from the 
underlying choroidal connective tissue, such as fibroblasts, endothelial, and blood cells (Fröhlich et al., 2003). Thus, the development of a method that can define the degree of purity of the RPE population in in vitro cell cultures would facilitate the planning of future therapeutic strategies.

Flow cytometry (FC) is an easy, quick, robust, and reliable technology for classifying cell populations according to size, cytoplasmic complexity, and differential expression of surface, cytoplasmic, and nuclear markers detected by specific staining of the markers with fluorochrome-combined reagents (Othmer and Zepp, 1992; Dent et al., 1989). Most current research work related to human RPE (hRPE) primary cell culture has been performed using established RPE cell lines such as ARPE19, D407, and others (Steindl-Kuscher et al., 2009; Tan et al., 2010; Thomson et al., 2010; Fujii et al., 2010), but studies have reported differences in expression of different proteins in RPE-derived cell lines and human primary RPE cells (Alge et al., 2006; Gupta et al., 1997); therefore, some cellular properties of the hRPE cells might differ among RPE cell lines. However, various reports have shown that ARPE19 cells express RPE65 proteins (SteindlKuscher et al., 2009). Thus, it is essential to develop primary cultures of hRPE cells for experimental purposes and to use more appropriate techniques for quick and reliable analysis of purity. To the best of our knowledge, no report has been published about the use of the FC technique to evaluate the purity of hRPE primary cell cultures for experimental purposes.

The aim of this study was to demonstrate the practicality of routinely implementing FC to determine the purity and health of the hRPE primary cells in their in vitro cell cultures before using them for further experiments.

\section{Methods}

\subsection{Reagents}

Purified anti-RPE65 mouse monoclonal antibody (MoAb) for detecting RPE cells (recognizes a 65-kDa surface molecule) was purchased from Abcam, Cambridge, UK. Mouse antihuman epithelial/fibroblasts MoAb conjugated with R phycoerythrin (R-PE) for detecting epithelial and fibroblast cells (recognizes a 112-kDa surface molecule) was purchased from RayBiotech, Inc., Norcross, GA, USA. Anti-human fibroblast $\mathrm{MoAb}$ for detecting fibroblasts by immunohistochemistry was purchased from Sigma-Aldrich, St. Louis, MO, USA. Fluorescein isothiocyanate (FITC) and horseradish peroxidase (HRP) conjugated goat anti-mouse IgG antibodies were purchased from Jackson ImmunoResearch Laboratories, Inc., West Grove, PA, USA. Unspecific mouse MoAbs as isotype negative controls were purchased from Beckman-Coulter, Fullerton, CA, USA. Rhodamine-phalloidin (RP) was purchased from Invitrogen, Barcelona, Spain. For Fontana-Masson staining, silver nitrate and sodium thiosulfate were obtained from Panreac, Barcelona, Spain. Gold chloride and nuclear solution were purchased from Sigma-Aldrich and BDH Chemical (UK), respectively. Annexin V-FITC and a propidium iodide (PI) commercial kit were purchased from Beckman Coulter.

\subsection{Cell culture}

hRPE cells, ARPE19 cells, human skin fibroblasts (HSF), and human corneal epithelial (HCE) cells were used in this study.
hRPE cells were isolated from the eyes of three cadaver donors gifted by the University Hospital Clinic of Valladolid and maintained in Dulbecco's modified Eagle medium (DMEM) (Invitrogen-Gibco) in accordance with previous established protocols (Zhang et al., 2006). An ARPE19 cell line was obtained from the American Type Culture Collection and grown in DMEM/ F12 medium (Invitrogen-Gibco). The ARPE19 cell line was selected as a positive control for RPE65 expression. HCE and HSF were gifts from the Ocular Surface Group of Institute of Applied Ophthalmobiology and Bioforge Group of the University of Valladolid, Spain, respectively. HCE cells were grown in supplemented DMEM/F12. HSF was maintained in RPMI1640 medium (Invitrogen-Gibco). HSF cells were used as a negative control for RPE65 expression and a positive control for fibroblast MoAb expression.

\subsection{Immunofluorescence assay}

Primary hRPE cells $\left(1 \times 10^{6} /\right.$ well $)$ were cultured on six-well polystyrene cell culture plates (Nunc, Roskilde, Denmark) for 7 days, stained with anti-RPE65 MoAb, anti-human fibroblast MoAb, RP, and Fontana-Masson and analyzed by a phasecontrast and fluorescence microscope (Leica Microsystems, Mannheim, Germany). Briefly, cultured cells were permeabilized with a solution of $1 \times$ phosphate buffered saline (PBS) with $0.1 \%$ Triton-X (PBT) for 10 minutes. Cells were incubated overnight at $4{ }^{\circ} \mathrm{C}$ with diluted $(1: 100)$ primary antibodies (anti-RPE65 and anti-human fibroblast) in an antibody blocking solution. Cells then were rinsed with PBT and incubated for 1 hour at room temperature (RT) with anti-mouse IgG-FITC, which was diluted previously in PBS (1:100). Cells also were co-stained with 1:40 dilution of RP (200 unit/ml) in PBS for 20 minutes, mounted, and observed under fluorescence microscope. For Fontana-Masson staining, cells were stained with an ammoniacal silver solution as previously described (Randhawa et al., 2009).

\subsection{FC assays}

Cells obtained by trypsinization of each cell culture were analyzed by FC to determine the cell viability, cell cycle (DNA content) and expression of different proteins. All FC assays were performed in triplicate, although only a representative experiment is shown. For each assay, at least 5000 cells were analyzed. FC analysis was performed with a cytometer (Cytomics FC 500) using 488 nm excitation with an argon-ion laser for FITC, PE, and PI. The data were analyzed with a software program (Cytomics RXP, Beckman-Coulter). Controls included cross-reactivity of the fluorescence signals of each channel and isotype-matched unspecific MoAbs used as negative controls. The mean fluorescence intensities (MFIs) were quantified and expressed as arbitrary units. The MFI value is directly related to the density of a given protein per cell. The MFI index was calculated as the ratio of MFI $(+) /$ MFI $(-)$, where MFI $(+)$ was the MFI value of the cells stained with the corresponding MoAb and MFI $(-)$ was the MFI value of the cells stained with the isotype-matched unspecific negative controls.

For cell viability analysis, the cells were stained with an annexin V-FITC and PI commercial kit (Beckman-Coulter) as previously described (Reinoso et al., 2011; Martínez-Osorio et 
al., 2009). hRPE cells exposed to UV-B light for 48 hours were used as positive controls of apoptosis. For cell-cycle analysis, the DNA content of the cells was determined by staining with PI after cell permeabilization (Coulter DNA Prep Reagents Kit, Beckman-Coulter) as previously described (Reinoso et al., 2011; Martínez-Osorio et al., 2009).

For protein staining, cells were incubated with irrelevant immunoglobulins (Sigma-Aldrich) to block Fc receptors for nonspecific binding and then resuspended in $250 \mu \mathrm{l}$ of a solution of fixation/permeabilization (BD Cytofix/Cytoperm) and incubated for 20 minutes at $4{ }^{\circ} \mathrm{C}$. The cells then were incubated in the dark for 30 minutes at $4{ }^{\circ} \mathrm{C}$ with $1 \mu \mathrm{l}$ of neat anti-RPE65 (IgG1) primary MoAb or with $5 \mu$ of anti-human epithelial/fibroblasts primary MoAB conjugated with R-PE (IgG2a). The cellular pellet then was resuspended and incubated in the dark for 30 minutes at $4{ }^{\circ} \mathrm{C}$ with $20 \mu$ of secondary antibody conjugated with FITC previously diluted 1:50 in washing buffer (BD Perm/Wash). At this step, cells undergoing staining with anti-human epithelial/fibroblasts primary MoAb did not need incubation with a secondary antibody. The cells were washed $(2 \times 5 \mathrm{~min} \times 1200 \mathrm{rpm})$ with $1 \mathrm{ml}$ of $1 \%$ PBS-BSA followed by resuspension of pellet in $500 \mu \mathrm{l}$ of PBS containing 1\% p-formaldehyde and the cell fluorescence was measured.

ARPE19, HSF, and HCE cells were used as controls to demonstrate the specificity of the antibodies. The autofluorescence observed in the early passages of the hRPE cell primary cultures was quenched using Trypan blue as previously described (Srivastava et al., 2011).

\subsection{Electrophoresis and Western blot analysis}

hRPE cells from passages 2 and 9 and ARPE19 cells were homogenized separately in ice-cold radioimmunoprecipitation assay buffer plus protease inhibitors (Santa Cruz Biotechnology, Inc., Santa Cruz, CA, USA). ARPE19 cellular proteins were used as a positive control for determining the protein expression. The total protein level was measured using the bicinchoninic acid method (Pierce Biotechnology, Rockford, IL, USA). The proteins in each sample were separated by sodium dodecyl sulfatepolyacrylamide gel electrophoresis on $8 \%$ polyacrylamide gels, transferred to the nitrocellulose membranes, and blocked in Tris buffered saline (5\% dried milk, 4\% FBS and 0.05\% Tween-20 in Tris; TBS-T) for 1 hour. The membranes were incubated overnight at $4{ }^{\circ} \mathrm{C}$ with the primary anti-RPE65 (1:1000) and anti- $\beta$-actin (1:1000, Antibodies-online, Inc., Atlanta, GA, USA) MoAbs, then washed with TBS-Tween-20 for 10 minutes and incubated with secondary HRP-conjugated Abs (1:3000) at RT for 1 hour. Immunoreactive bands were visualized by a chemiluminescence method using the ChemiDoc gel documentation system (Bio-Rad, Hercules, CA, USA), and images were analyzed with the Quantity One software (Bio-Rad). Equal loading was proved by $\beta$-actin detection.

\section{Results}

\subsection{Immunofluorescence assays}

Isolated hRPE cells showed normal morphologic characteristics in cell culture after becoming confluent. At the beginning, cells appeared rounded with pigment but when they became attached to the surface of the culture plate, they acquired the fibroblast-like morphology with pigment. As the culture became confluent, cells adapted a hexagonal morphology seen on phase contrast and fluorescence microscopy after staining of actin with RP (Fig. 1A, C). Immunostaining with anti-RPE65 MoAb showed that the cells in culture (passage 1 ) were positive for this protein (Fig. 1B). Fontana-Masson staining results showed the presence of pigment in these hRPE cells (Fig. 1D).

\subsection{Cell viability and cell-cycle FC analysis}

For analysis of cell viability and cell-cycle phases, a homogeneous cell population was selected (Fig. 2A) based on the previous morphologic analysis of the total population. FC analysis showed that the vast majority (99.5\%) of the hRPE cells (passage 5) were viable. The percentages of early and late apoptotic cells and dead cells were $0.1 \%, 0 \%$, and $0.3 \%$, respectively (Fig. 2B). However, cell-cycle analysis showed that $86.3 \%$ of cells were in the $G_{0} / G_{1}$ phase $(2 n)$. Cells in the S-phase of mitosis accounted for $3.5 \%$ of cells, while $7.4 \%$ of cells were in the $\mathrm{G}_{2} / \mathrm{M}$ phase ( $4 \mathrm{n}$ ) (Fig. 2C). Similar results were seen for other passages studied (data not shown). Higher percentages of early and late apoptotic cells and dead cells were found in the hRPE cells exposed to UV-B light for 48 hours (positive control) compared with unstimulated hRPE cells (Fig. 2D).

\subsection{Analysis of RPE65 and epithelial and fibroblast protein expression}

The FC results showed that most of the ARPE19 cells (>90\%; MFI index, 6) expressed the cytoplasmic RPE65 protein (Fig. 3A, Table 1). The best results were obtained after blocking the Fc receptors of the cells, incubating them with anti-RPE65 $\mathrm{MoAb}$ at $4{ }^{\circ} \mathrm{C}$, and using an FITC-conjugated secondary antibody at RT for 1 hour. Similar results were obtained in five independent analyses of ARPE19 cells (data not shown). Our findings also showed that the ARPE19 cells did not express the epithelial/fibroblast protein ( $<1 \%$; Fig. 3B). In contrast, most HCE cells (88.2\%; MFI index, 4.6) expressed this protein (Fig. 3C, Table 1). A similar pattern was seen in the HSF, which were positively labeled with the anti-human epithelial/ fibroblasts MoAb (99.8\%; MFI index, 21.4) (Fig. 3D, Table 1), and negatively labeled with the RPE65 MoAb (<1\%; Fig. 3E).

FC analysis showed that most hRPE cells expressed RPE65 protein. In contrast, these cells did not express the epithelial/ fibroblast protein (Fig. 4, Table 1). Our results also showed that RPE65 expression decreased over passages from 93.6\% (passage 5 ) to $85.3 \%$ (passage 10 ). However, a stronger loss of protein density (MFI index) was observed from 6.3 (passage 5) to 3.0 (passage 10) (Fig. 4, Table 1 ).

Western blot results also showed that hRPE cultured cells in passages 2 and 9 expressed RPE65 protein; however, the expression level was lower in cells in passage 9 compared with cells in passage 2 (Fig. 5). These results were consistent in three different hRPE primary cell cultures obtained from different donors.

\section{Discussion}

RPE is considered a primary target in AMD therapies (Nowak, 2006), because current available reports have shown the 


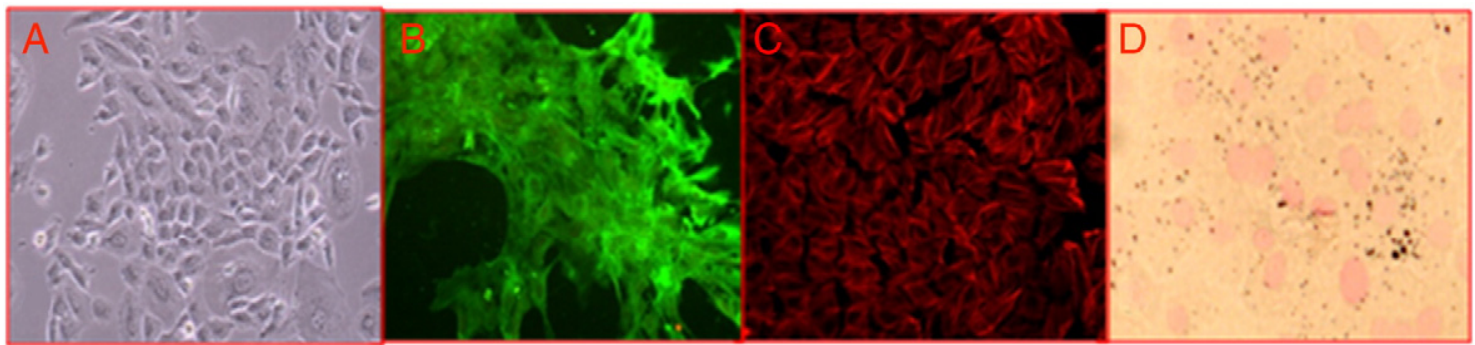

Fig. 1. Phase contrast and fluorescence microscopy study of hRPE cells in in vitro culture (passage 1). (A) Hexagonal morphology acquired by hRPE cells. (B) hRPE cellular RPE65 protein expression. (C) hRPE cellular actin detection by rhodamin-phalloidin staining. (D) hRPE cellular pigments detection by Fontana-Masson staining.

possibility of stopping retinal atrophy by transplanting the RPE cells (Binder et al., 2007; Del Priore et al., 2004; Coffey et al., 2002). RPE cell lines, e.g., ARPE19, show some differences from fresh RPE cells. ARPE19 cells express RPE65 protein (Steindl-Kuscher et al., 2009). hRPE cells express some specific proteins including RPE65 and CRALBP; however, CRALBP also is expressed by retinal Müller cells (Martin et al., 2009). Thus, the selection of RPE65 as a marker of RPE cells and their detection in complex mixture cultures could be considered appropriate. In addition, CRALBP in vivo protein expression could be more
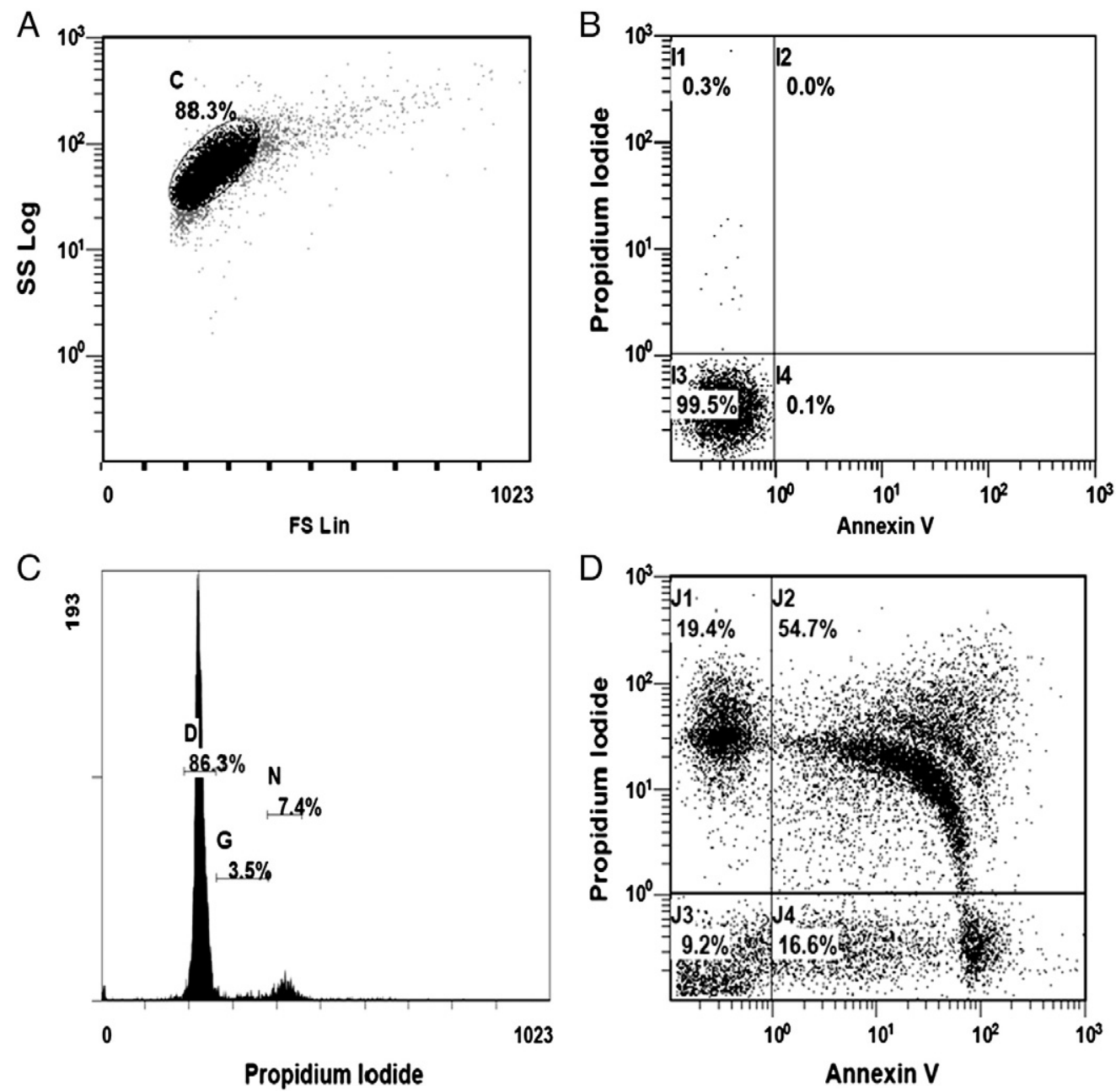

Fig. 2. Cell viability and cell-cycle analysis of hRPE cells in primary cell culture (passage 5) using FC. (A) Selection of homogenous hRPE cell population of primary cell culture for FC analysis. (B) Detection of apoptotic cells in hRPE primary cell culture by PI and annexin V staining. (C) Detection of cell cycle in hRPE primary cell culture by PI staining. (D) Cell viability analysis in hRPE cells stimulated with UV-B light for 48 hours. Three independent experiments were performed. All FC histograms correspond to a representative experiment. Similar data were obtained from other hRPE cell passages. 
A

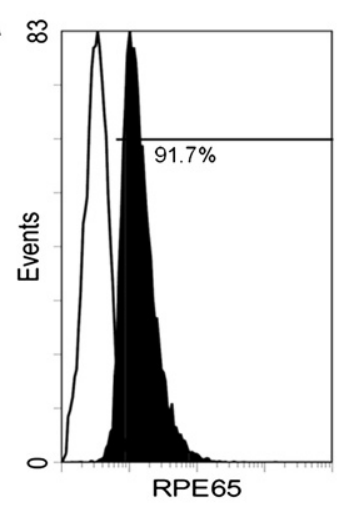

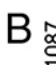

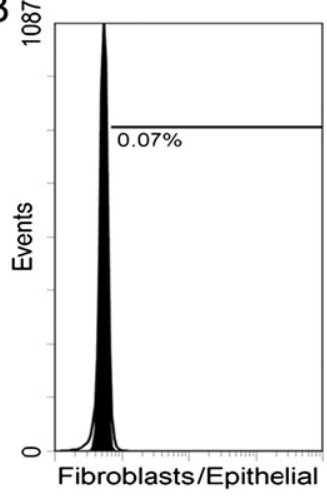

C

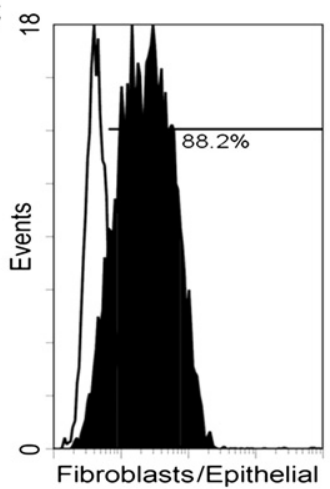

$D_{\stackrel{\circ}{\circ}}$

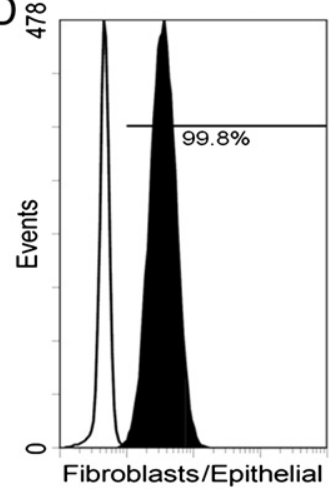

$E$ \%

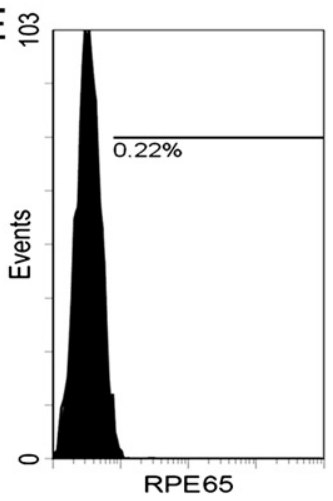

Fig. 3. RPE65 and epithelial and fibroblastic protein expression analysis in ARPE19, HCE, and HSF cells using FC after staining with anti-RPE65 MoAb and antihuman epithelial/fibroblast MoAb. (A) Percentage of ARPE19 cells expressing RPE65 protein. (B) Percentage of ARPE19 cells expressing epithelial and fibroblastic proteins. (C) Percentage of HCE cells expressing epithelial protein. (D) Percentage of HSF expressing fibroblastic protein. (E) Percentage of HSF expressing RPE65 protein.

prominent or stable, but both RPE65 and CRALBP protein expressions are down-regulated in vitro (Liggett et al., 2009; Alge et al., 2003).

Establishing reliable, pure, healthy hRPE primary cell cultures is of primary importance for RPE-related studies. Currently, most laboratories use fluorescence microscopy to determine the types of cells present in hRPE primary cell culture (Geisen et al., 2006; Hu and Bok, 2001; Zhang et al., 2006; Ohno-Matsui et al., 2001; Maminishkis et al., 2006). However, it is difficult to use this technology for quick, reliable, and reproducible quantitative measurement of changes such as the status of the cell cycle, the percentage of the population undergoing cell death or expressing specific cell molecules, dedifferentiation, and contamination of other cell types in a bulk series of primary hRPE cell cultures if that is needed for the investigation.
In the current study, FC was used to determine the number of viable hRPE cells in primary cell cultures after staining with annexin V and PI stains. The results showed that few $(<0.5 \%)$ hRPE cells in the cultures were apoptotic or dead. The hRPE cells in the cultures were mostly viable, and the results obtained were compatible with a healthy culture. The minimal percentage of annexin $\mathrm{V}$ staining was consistent with the beginning of culture confluency. It is well known that hRPE cells in vivo are quiescent, non-dividing cells resting in the $\mathrm{G}_{0}$-phase of the cell cycle (Ts'o and Friedman, 1967) and forcing them into $G_{1}$ phase by exposing them to pathological or culture conditions (Okada, 1980) would induce an active cell cycle resulting in proliferation and loss of accumulated materials in the adult RPE cells. Detection of most cells in $G_{0} / G_{1}$ phase by DNA staining clearly confirmed that cultured hRPE cells resembled those in vivo. Moreover, the

Table 1

RPE65 and epithelial and fibroblast antigen expressions in cultures of hRPE cells, ARPE19, HCE, and HSF.

\begin{tabular}{|c|c|c|c|c|c|c|c|c|}
\hline \multirow[t]{2}{*}{ Cell type } & \multicolumn{4}{|c|}{ RPE65 } & \multicolumn{4}{|c|}{ Epithelial/fibroblast } \\
\hline & $\%$ & $\operatorname{MFI}(-)$ & $\operatorname{MFI}(+)$ & MFI Index & $\%$ & $\operatorname{MFI}(-)$ & $\operatorname{MFI}(+)$ & MFI Index \\
\hline ARPE19 & 91.7 & 0.3 & 1.8 & 6.0 & 0.1 & 0.4 & 0.4 & 1.0 \\
\hline $\mathrm{HCE}$ & - & - & - & - & 88.2 & 0.5 & 2.3 & 4.6 \\
\hline HSF & 0.2 & 0.3 & 0.3 & 1.0 & 99.8 & 0.5 & 10.7 & 21.4 \\
\hline hRPE (p5) & 93.6 & 0.3 & 1.9 & 6.3 & 0.1 & 0.5 & 0.5 & 1.0 \\
\hline hRPE (p8) & 90.1 & 0.3 & 1.0 & 3.3 & 0.1 & 0.4 & 0.4 & 1.0 \\
\hline hRPE (p10) & 85.3 & 0.3 & 0.9 & 3.0 & 0.1 & 0.4 & 0.4 & 1.0 \\
\hline
\end{tabular}

MFI: Mean Fluorescence intensity. 
A
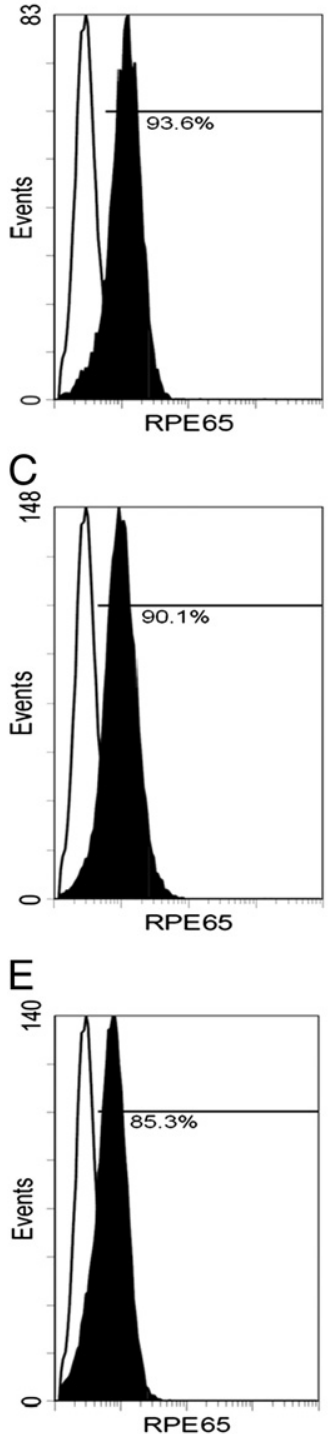

$\mathrm{B}$

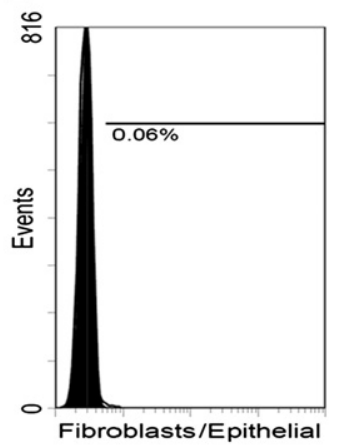

D

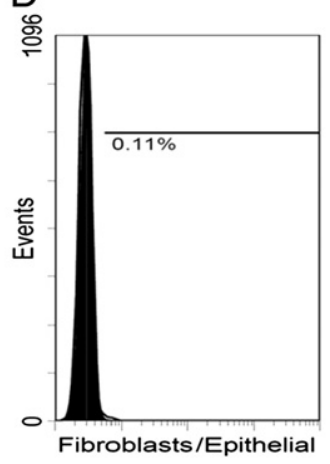

F

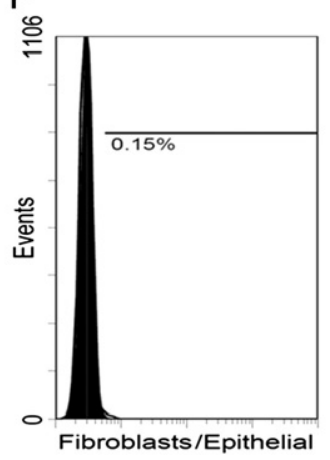

Fig. 4. RPE65 and epithelial and fibroblastic protein expression analysis in hRPE cells of the primary cell cultures of passages 5, 8, and 10 using FC after staining with anti-RPE65 and antihuman epithelial/fibroblast MoAb. (A, C, E) Percentage of hRPE cells expressing RPE65 protein. (B, D, F) Percentage of hRPE cells expressing epithelial and fibroblast proteins.

cultures showed an important capacity to proliferate, with about $10 \%$ of the cells either in the $S$ or $G_{2}$ phase.

The hRPE cells in the early phases of culture contained pigments that were responsible for cellular autofluorescence signal emission in a wide nanometer range of the spectra when these cells were analyzed by FC. In these cases, Trypan blue was used to quench the autofluorescence (Srivastava et al., 2011).

Another step to consider in the FC protocol was the combination of intracellular and extracellular markers, together with the secondary antibody selection in the case of indirect immunofluorescence staining protocols. In our particular method, it was difficult to combine the simultaneous labeling with the anti-epithelial/fibroblast and anti-RPE65 MoAbs. The difficulties arose for a few reasons.

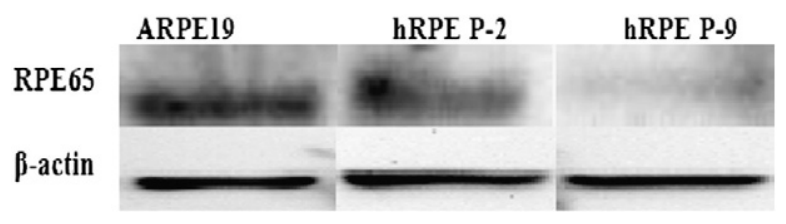

Fig. 5. RPE65 protein expression analysis in hRPE cells of primary cell cultures of passages 2 and 9 using a Western blot technique. ARPE19 cells are used as a control for detecting RPE65 protein and $\beta$-actin is used to determine equal loading of proteins in each well of stacking gel in the Western blot procedure.

The first reason is the above-mentioned autofluorescence emission of these RPE cells. Second, the permeabilizing cells could somehow alter some surface protein expression and permeabilization was necessary for RPE65 indirect labeling; antiepithelial/fibroblast MoAb detects 112-kDa epithelial and fibroblast protein by direct labeling, thus, increasing the possibility of detecting two types of cell contamination in the same sample while antifibroblast MoAb detects only fibroblast protein by indirect labeling. Third, the use of two indirect labelings causes higher background noise in any FC study; for this reason, we used direct labeling with antiepithelial/fibroblast MoAb and indirect labeling with anti-RPE65 MoAb.

In addition to acquiring proliferative capacity, RPE cells can transdifferentiate into fibroblast-like cells after retinal detachment and vitreous retraction resulting in proliferative vitreoretinopathy (Saika et al., 2004). Thus, it seems that loss of cell-cell contact, cell substrate, or both in RPE cells initiates proliferation and epithelial fibroblast transition, producing deviation from the characteristics of RPE cells and resulting in contamination and low cell yields in in vitro RPE primary cell cultures (Stevens et al., 2005). Establishing a reliable FC protocol to successfully detect contamination in the culture was achieved during the current study. FC analysis clearly showed in excess of $90 \%$ of hRPE cells expressing the RPE65 protein, a specific marker of RPE cells, in in vitro primary cultures. However, the cells began to lose the RPE65 protein expression as the cell cultures were trypsinized and underwent subsequent passages from 1 to 10 . These results also were confirmed using a Western blot technique, which showed that the cells in passage 9 had far less RPE protein expression compared with passage 2 . The Western blot assay detected a marked decrease in RPE65 protein expression between passages 2 and 9, while FC showed only an 8\% decrease (93.6\% of hRPE cells at passage 5 vs. $85.3 \%$ at passage 10 ). However, there was a significant loss of RPE65 protein density per cell, i.e., between passages (as detected by a $50 \%$ reduction in the MFI index value): at passage 5, the MFI index of the hRPE cells was 6.3, which decreased at passages 8 (MFI index, 3.3) and passages 10 (MFI index, 3). This significant reduction in MFI together with the $8 \%$ loss with respect to relative number of positive cells parallels the reduced values detected by the Western blot assay.

Detection of the 112-kDa surface epithelial/fibroblast protein in HCE but not in ARPE19 indicated that the antihuman epithelial/fibroblast MoAb cannot be used to detect this surface-bound epithelial protein in hRPE cell cultures but can be used to detect contamination of other epithelial cells (corneal) in in vitro primary cultures of hRPE cells. Detection of the 112-kDa surface protein in the HSF indicated that the 
antiepithelial/human fibroblast MoAb is a perfect choice for detecting any potential contamination of fibroblasts in the cell cultures and is a potential transdifferentiation marker from hRPE cells to fibroblasts. The results showed the total absence of fibroblast protein expression by hRPE cells in the cell cultures of different passages; therefore, the hRPE primary cell cultures established for the current study could be considered free of contamination.

In the current study, a novel FC procedure was successfully developed to analyze hRPE cell cultures: the expression of hRPE65 seems a suitable marker of RPE purity and the lack of epithelial/fibroblast 112-kDa protein expression confirms the lack of transdifferentiation in the successive assayed passages. This procedure was successfully tested in hRPE primary cultures and in ARPE19, HSF, and HCE cell lines. In our hands, this protocol can be used routinely to discriminate cells expressing these different proteins in cell cultures.

\section{Conclusions}

In summary, FC can be used for quick, sensitive, robust, reliable, reproducible, and thus, routine detection of RPE cell purity in primary hRPE cell cultures and to test the cell viability and cycle and the presence of contaminating fibroblasts or other cell types in a series of RPE primary cell cultures. Thus, implementation of FC in the regular evaluation of the stability of hRPE primary cell cultures is advantageous over fluorescence microscopy methods.

\section{Acknowledgments}

This work was supported by grants from Castilla and Leon Regenerative Medicine and Cell Therapy Network Center and from National Plan of I + D + I 2008-2011 and ISCIIISubdirectorate General for Evaluation and Research Promotion (PS09/00938) (MICNN) with co-sponsorship by FEDER, and JCYL BIO/39/VA26/10, Junta de Castilla y León, Spain, to GKS and JCP. A.K. Singh is supported by pre-doctoral scholarships from AECI, the Spanish Ministry of Foreign Affairs. The authors thank Alicia Rodríguez Gascón, University of País Vasco, (Spain) for providing ARPE19 cells; Ana de la Mata San Pedro and Dr. Teresa Nieto Miguel of Ocular Surface group of IOBA, University of Valladolid (Spain) for providing HCE cells; the Bioforge Group of University of Valladolid (Spain) for providing HSF; and the residents of University Hospital Clinic of Valladolid (Spain) for providing human eye globes.

\section{References}

Alge, C.S., Suppmann, S., Priglinger, S.G., Neubauer, A.S., May, C.A., Hauck, S., Welge-Lussen, U., Ueffing, M., Kampik, A., 2003. Comparative proteome analysis of native differentiated and cultured dedifferentiated human RPE cells. Invest. Ophthalmol. Vis. Sci. 44, 3629.

Alge, C.S., Hauck, S.M., Priglinger, S.G., Kampik, A., Ueffing, M., 2006. Differential protein profiling of primary versus immortalized human RPE cells identifies expression patterns associated with cytoskeletal remodeling and cell survival. J. Proteome Res. 5, 862.

Binder, S., Stanzel, B.V., Krebs, I., Glittenberg, C., 2007. Transplantation of the RPE in AMD. Prog. Retin. Eye Res. 26, 516.

Coffey, P.J., Girman, S., Wang, S.M., Hetherington, L., Keegan, D.J., Adamson, P., Greenwood, J., Lund, R.D., 2002. Long-term preservation of cortically dependent visual function in RCS rats by transplantation. Nat. Neurosci. 5, 53.
Del Priore, L.V., Tezel, T.H., Kaplan, H.J., 2004. Survival of allogeneic porcine retinal pigment epithelial sheets after subretinal transplantation. Invest. Ophthalmol. Vis. Sci. 45, 985.

Dent, G.A., Leglise, M.C., Pryzwansky, K.B., Ross, D.W., 1989. Simultaneous paired analysis by flow cytometry of surface markers, cytoplasmic antigens, or oncogene expression with DNA content. Cytometry 10, 192.

Engelmann, K., Valtink, M., 2004. RPE cell cultivation. Graefes Arch. Clin. Exp. Ophthalmol. 242, 65.

Fröhlich, E., Maier, E., Klessen, C., 2003. Isolation of bovine retinal pigment epithelial cells using adhesion to agarose: demonstration of cellular and regional heterogeneity. J. Histochem. Cytochem. 51, 121.

Fujii, Y., Kachi, S., Terasaki, H., Kawasumi, T., Honda, H., Ito, A., 2010. Transfer of gene to human retinal pigment epithelial cells using magnetite cationic liposomes. Br. J. Ophthalmol. 94, 1074.

Geisen, P., McColm, J.R., King, B.M., Hartnett, M.E., 2006. Characterization of barrier properties and inducible VEGF expression of several types of retinal pigment epithelium in medium-term culture. Curr. Eye Res. 31, 739.

Gupta, S.K., Jollimore, C.A., McLaren, M.J., Inana, G., Kelly, M.E., 1997. Mammalian retinal pigment epithelial cells in vitro respond to the neurokines ciliary neurotrophic factor and leukemia inhibitory factor. Biochem. Cell Biol. 75, 119.

Hu, J., Bok, D., 2001. A cell culture medium that supports the differentiation of human retinal pigment epithelium into functionally polarized monolayers. Mol. Vis. 7, 14.

Liggett, T.E., Griffiths, T.D., Gaillard, E.R., 2009. Isolation and characterization of a spontaneously immortalized bovine retinal pigmented epithelial cell line. BMC Cell Biol. 10, 33.

Majji, A.B., de Juan Jr., E., 2000. Retinal pigment epithelial autotransplantation: morphological changes in retina and choroid. Graefes Arch. Clin. Exp. Ophthalmol. 238, 779 .

Maminishkis, A., Chen, S., Jalickee, S., Banzon, T., Shi, G., Wang, F.E., Ehalt, T., Hammer, J.A., Miller, S.S., 2006. Confluent monolayers of cultured human fetal retinal pigment epithelium exhibit morphology and physiology of native tissue. Invest. Ophthalmol. Vis. Sci. 47, 3612.

Martin, P.M., Ananth, S., Cresci, G., Roon, P., Smith, S., Ganapathy, V., 2009. Expression and localization of GPR109A (PUMA-G/HM74A) mRNA and protein in mammalian retinal pigment epithelium. Mol. Vis. 15, 362.

Martínez-Osorio, $\mathrm{H}$. Calonge, M., Corell, A. Reinoso, R. López, A. Fernández, I. San José, E.G., Diebold, Y., 2009. Characterization and short-term culture of cells recovered from human conjunctival epithelium by minimally invasive means. Mol. Vis. 15, 2185.

Nowak, J.Z., 2006. Age-related macular degeneration (AMD): pathogenesis and therapy. Pharmacol. Rep. 58, 353.

Ohno-Matsui, K., Morita, I., Tombran-Tink, J., Mrazek, D., Onodera, M., Uetama, T., Hayano, M., Murota, S.I., Mochizuki, M., 2001. Novel mechanism for agerelated macular degeneration: an equilibrium shift between the angiogenesis factors VEGF and PEDF. J. Cell. Physiol. 189, 323.

Okada, T.S., 1980. Cellular metaplasia or transdifferentiation as a model for retinal cell differentiation. Curr. Top. Dev. Biol. 16, 349.

Othmer, M., Zepp, F., 1992. Flow cytometric immunophenotyping: principles and pitfalls. Eur. J. Pediatr. 151, 398.

Randhawa, M., Huff, T., Valencia, J.C., Younossi, Z., Chandhoke, V., Hearing, V.J., Baranova, A., 2009. Evidence for the ectopic synthesis of melanin in human adipose tissue. FASEB J. 23, 835.

Reinoso, R., Calonge, M., Castellanos, E., Martino, M., Fernández, I., Stern, M.E., Corell, A., 2011. Differential cell proliferation, apoptosis, and immune response in healthy and evaporative-type dry eye conjunctival epithelia. Invest. Ophthalmol. Vis. Sci. 52, 4819.

Saika, S., Kono-Saika, S., Tanaka, T., Yamanaka, O., Ohnishi, Y., Sato, M., Muragaki, Y., Ooshima, A., Yoo, J., Flanders, K.C., Roberts, A.B., 2004. $\mathrm{Smad} 3$ is required for dedifferentiation of retinal pigment epithelium following retinal detachment in mice. Lab. Invest. 84, 1245.

Singhal, S., Vemuganti, G.K., 2005. Primary adult human retinal pigment epithelial cell cultures on human amniotic membranes. Indian J. Ophthalmol. 53, 109.

Srivastava, G.K., Reinoso, R., Singh, A.K., Fernandez-Bueno, I., Hileeto, D., Martino, M., Garcia-Gutierrez, M.T., Merino, J.M., Alonso, N.F., Corell, A., Pastor, J.C., 2011. Trypan Blue staining method for quenching the autofluorescence of RPE cells for improving protein expression analysis. Exp. Eye Res. 93, 956.

Steindl-Kuscher, K., Krugluger, W., Boulton, M.E., Haas, P., Schrattbauer, K., Feichtinger, H., Adlassnig, W., Binder, S., 2009. Activation of the betacatenin signalling pathway and its impact on RPE cell cycle. Invest. Ophthalmol. Vis. Sci. 50, 4471.

Stevens, M.J., Larkin, D.D., Feldman, E.L., DelMonte, M.A., Greene, D.A., 2005. Establishment, maintenance, and transfection of in vitro cultures of human retinal pigment epithelium. Methods Mol. Med. 107, 343.

Tan, J., Deng, Z.H., Liu, S.Z., Wang, J.T., Huang, C., 2010. TGF-beta2 in human retinal pigment epithelial cells: expression and secretion regulated by cholinergic signals in vitro. Curr. Eye Res. 35, 37. 
Thomson, H.A., Treharne, A.J., Walker, P., Grossel, M.C., Lotery, A.J., 2010 Optimisation of polymer scaffolds for retinal pigment epithelium (RPE) cell transplantation. Br. J. Ophthalmol. 95, 563.

Ts'o, M.O., Friedman, E., 1967. The retinal pigment epithelium. Arch. Ophthalmol. 78,641 .

Valtink, M., Engelmann, K., 2009. Culturing of retinal pigment epithelium cells. Dev. Ophthalmol. 43, 109.
Wiencke, A.K., Kiilgaard, J.F., Nicolini, J., Bundgaard, M., Röpke, C., La Cour, M., 2003. Growth of cultured porcine retinal pigment epithelial cells. Acta Ophthalmol. Scand. 81, 170.

Zhang, L., Li, X., Zhao, M., He, P., Yu, W., Dong, J., Liu, G., Li, C., Shi, X., 2006. Antisense oligonucleotide targeting c-fos mRNA limits retinal pigment epithelial cell proliferation: a key step in the progression of proliferative vitreoretinopathy. Exp. Eye Res. 83, 1405. 
Methods in eye research

\title{
Trypan Blue staining method for quenching the autofluorescence of RPE cells for improving protein expression analysis
}

\author{
Girish K. Srivastava ${ }^{\text {a,b,c,*, Roberto Reinoso }}{ }^{\text {a,b,c }}$, Amar K. Singh a , Ivan Fernandez-Bueno a \\ Denise Hileeto $^{a}$, Mario Martino ${ }^{\text {a,b,c }}$, Maria T. Garcia-Gutierrez ${ }^{\text {a,b,c }}$, Jose M. Pigazo Merino ${ }^{\text {a }}$, \\ Nieves Fernández Alonso ${ }^{a}$, Alfredo Corell ${ }^{a}$, J. Carlos Pastor ${ }^{a}$ \\ ${ }^{a}$ Institute of Applied Ophthalmobiology (IOBA), University of Valladolid, Valladolid, Spain \\ ${ }^{\mathrm{b}}$ Castilla and Leon Regenerative Medicine and Cell Therapy Network Centre, Spain \\ ${ }^{\mathrm{c}}$ CIBER-BBN, Spain
}

\section{A R T I C L E I N F O}

\section{Article history:}

Received 25 February 2011

Accepted in revised form 4 July 2011

Available online 18 July 2011

\section{Keywords:}

autofluorescence

retinal pigment epithelial (RPE) cells

trypan blue

flow cytometry (FC)

immunohistochemistry (IHC)

\begin{abstract}
A B S T R A C T
Retinal pigment epithelial (RPE) cells are currently in the "spotlight" of cell therapy approaches to some retinal diseases. The analysis of the expressed proteins of RPE primary cells is an essential step for many of these approaches. But the emission of autofluorescence by RPE cells produces higher background noise interference thereby creating an impediment to this analysis. Trypan Blue (TB), a routinely used counterstain, has the capacity to quench this autofluorescence, if it is used in optimized concentration. The results from the method developed in our study indicate that incubation of the cultured RPE cells with $20 \mu \mathrm{g} / \mathrm{ml}$ of TB after immunolabelling (post-treatment) as well as incubation of the retinal tissue specimens with same concentration before paraffin embedding, sectioning and immunolabelling (pre-treatment) can be applied to effectively quench the autofluorescence of RPE cells. Thus it can facilitate the evaluation of expressed cellular proteins in experimental as well as in pathological conditions, fulfilling the current requirement for developing a method which can serve to eliminate the autofluorescence of the cells, not only in cell cultures but also in tissues samples. This method should significantly increase the quality and value of RPE cell protein analysis, as well as other cell protein analysis performed by Flow cytometry (FC) and Immunohistochemistry (IHC) techniques.
\end{abstract}

(c) 2011 Elsevier Ltd. All rights reserved.

\section{Introduction}

Retinal pigment epithelium (RPE) plays crucial roles in the normal function of the retina and its alterations cause some severe retinal diseases. The potential possibility to stop or delay these degenerative retinal diseases by transplanting RPE cells is considered a possible promising new therapeutic tool (Valtink and Engelmann, 2009). This approach makes RPE cells interesting for the investigators working in the field of tissue engineering, genetic engineering and cell transplantation (Majji and de Juan, 2000; Carr et al., 2009 and Wang et al., 2010). Recently numerous methods of isolation, cultivation, identification as well as transplantation have been developed. RPE cells retain their ability to proliferate in culture as well as in pathological conditions (Singhal and Vemuganti, 2005; Okada, 1980 and Stevens et al., 2005) but culturing leads to

\footnotetext{
* Corresponding author. Institute of Applied Ophthalmobiology (IOBA), University of Valladolid, Campus Miguel Delibes, Paseo de Belen, 17, Valladolid 47011, Spain. Tel.: +34 9831847 53; fax: +3498318 4762 .

E-mail address: girish@ioba.med.uva.es (G.K. Srivastava).
}

deviation from the normal characteristics of RPE cells (Saika et al., 2004). In in vivo conditions RPE cells retain their stability but in in vitro conditions they are unable to retain their characteristics such as epithelial cubical morphology, polarity, secretion of the different factors on apico-basal ends, light absorption, visual cycle of retinal, junctional barrier for metabolic transportation and phagocytosis for renovation of external segments of photoreceptors (Binder et al., 2007). This fact has motivated the investigators to utilize established RPE cell lines that do not need characterisation. But current studies have shown differences in protein expression between RPE cell lines and RPE primary cells (Alge et al., 2006 and Gupta et al., 1997), therefore, developing appropriate techniques for analysing the primary RPE cell proteins in experimental as well as in control conditions are currently required.

Currently, Flow cytometry (FC) as well as Immunohistochemistry (IHC) are techniques widely used to localize and use specific proteins to separate cells in cell cultures and tissues related studies. FC is an easy handling, quick and reliable technology for classification of the cell population according to its morphological and physiological properties (Othmer and Zepp, 1992). It identifies 
specific cell populations but is unable to analyse the protein expression of tissue bound cells (Dent et al., 1989).

It is well known that all cells have some intrinsic level of autofluorescence, commonly caused by mitochondria, lysosomes, aromatic amino acids and other endogenous fluorophores such as pyridinic (NADPH), flavin and riboflavins coenzymes (Aubin, 1979 and Benson et al., 1979). In addition to this, RPE cells have pigments resulting in intensive emission of autofluorescence over a broad range of spectra and this causes overlaps of emission range of the spectra of commonly used fluorescent dyes. Thus, it interferes with the detection of RPE cell corresponding fluorescence over the background noise.

Trypan Blue (TB), a routinely used laboratory counterstain, has the property to quench autofluorescence (Cowen et al., 1985; Lynch and Derbyshire, 1986). In the current study, we tried to establish an FC as well as IHC method to detect specific fluorescence signals corresponding to expressed cellular proteins over cell background noise after quenching cellular autofluorescence by an optimized concentration of TB for analysing RPE cells in primary cell cultures as well as in retinal tissue.

\section{Materials and methods}

\subsection{Reagents}

Anti-RPE65 mouse monoclonal antibody (MoAb; IgG1) for detecting RPE cells was purchased from Abcam, Cambridge, UK. FITC conjugated goat anti-mouse antibody (IgG) was purchased from Jackson Immunoresearch Laboratories, Inc., West Grove, PA, USA and was used as secondary antibody. IgG from Sigma Aldrich, USA and unspecific mouse MoAbs as isotype negative controls from Beckman-Coulter, Fullerton, CA, USA were purchased.

Paraformaldehyde was purchased from Sigma Aldrich, USA. Cell Wash solution, fixation/permeabilization solution (Cytofix/ Cytoperm $^{\mathrm{TM}}$ ) and Washing solution (Perm/Wash ${ }^{\mathrm{TM}}$ ) were purchased from BD Biosciences, San Jose, CA, USA. PBS from Lonza, Belgium and TB counterstain solution from Lonza, Walkersville, MD USA, were purchased. The FC analysis was performed with a Cytomics FC 500 Cytometer (Beckman-Coulter, Fullerton, CA, USA) using $488 \mathrm{~nm}$ excitation with an argon-ion laser for FITC. The data collected were analyzed using the Cytomics RXP software program (BeckmanCoulter).

\subsection{Cell culture}

Pig RPE (pRPE) cells of passages P1-P6 were used in this study. pRPE cells were isolated (Dintelmann et al., 1999) from the pig's eyes gifted by local slaughter house of Valladolid, Spain and maintained in DMEM medium (Invitrogen-Gibco) supplemented with 10\% foetal bovine serum (FBS), antibiotics penicillin $(100 \mathrm{U} / \mathrm{ml})-$ streptomycin $(0.1 \mathrm{mg} / \mathrm{ml}), 1 \mathrm{mM}$ sodium pyruvate, and $2 \mathrm{mM}$ L-glutamine (Invitrogen-Gibco). Cells were trypsinized after cell cultures became confluent, immunostained and passed through the FC for optimizing the protocol for analysis.

\subsection{Flow cytometry $(F C)$}

Quantitative analysis of cells expressing RPE65 (Lee et al., 2010; Steindl-Kuscher et al., 2009), a specific protein marker of RPE cells, was performed to determine the deviation of cells from its natural characteristics in primary RPE cell cultures.

pRPE cells were washed with $2 \mathrm{ml}$ of Cell Wash Solution and centrifuged at $500 \times g$ for $5 \mathrm{~min}$, the pellet was resuspended and incubated with $50 \mu \mathrm{l}$ of $\operatorname{IgG}(40 \mu \mathrm{g} / \mathrm{ml})$ for $15 \mathrm{~min}$ at room temperature (RT) to block Fc receptors for unspecific binding. Cells were then resuspended in $250 \mu$ l of a fixation/permeabilization solution (Cytofix/Cytoperm ${ }^{\mathrm{TM}}$ ) for $20 \mathrm{~min}$ in the dark at $4{ }^{\circ} \mathrm{C}$ to fix the cells and permeabilizate the cell membrane. After incubation, cells were washed twice with $1 \mathrm{ml}$ of washing solution. Afterwards, the cells were incubated in the dark for $30 \mathrm{~min}$ at $4{ }^{\circ} \mathrm{C}$ with $1 \mu \mathrm{l}$ of anti-RPE65 (IgG1) mouse MoAb. Cells were then washed with washing solution. The cells undergoing RPE65 staining were incubated in the dark for $30 \mathrm{~min}$ at $4{ }^{\circ} \mathrm{C}$ with $20 \mu$ l of goat anti-mouse IgG secondary antibody conjugated with FITC previously diluted 1:50 in washing solution. Cells were washed with $1 \mathrm{ml}$ of $1 \%$ PBSBSA after incubation with secondary antibody and resuspended and incubated in $500 \mu \mathrm{l}(10,20$ and $40 \mu \mathrm{g} / \mathrm{ml}$ solution) ice-cold TB counterstain solution for $10 \mathrm{~min}$ at $4{ }^{\circ} \mathrm{C}$ (post-treatment). Finally the cells were washed, resuspended in $500 \mu \mathrm{l}$ of $1 \times$ PBS and cell fluorescence was measured. Mean Fluorescence Intensity (MFI) that reflects the expression density of a given protein per cell was recorded and expressed as arbitrary units. Controls included cross-reactivity of the fluorescence signals of each channel, as well as isotype-matched unspecific MoAbs used as negative staining controls.

\subsection{Retinal tissue sections (RTSs) and treatment with Trypan Blue (TB)}

Pig ocular globes were collected from local slaughter house and transported in $4 \%$ p-formaldehyde in $48 \mathrm{~h}$. On arrival at IOBA, the anterior segment was removed and the posterior segment of each ocular globe was cut into $3-5 \mathrm{~mm}$ small pieces and rehydrated by washing with PBS. Each piece was incubated with $0.5 \%$ TritonX-100 for $10 \mathrm{~min}$ at RT to permeabilize all the cells. Each piece was again washed and subsequently incubated with $0,20,200$ and $250 \mu \mathrm{g} / \mathrm{ml}$ of counterstain TB for 15 min at RT (pre-treatment). All pieces were washed twice with PBS after incubation, dehydrated and embedded in paraffin wax. Retinal tissue sections (RTSs) of 3- $\mu$ m were cut from each piece, mounted on slide with $50 \%$ glycerol under cover-slip and observed using a microscope (Leica Microsystems, Mannheim, Germany) on wave lengths, A (Blue, excitation filter (EF) BP 360/40), I3 (Green, EF BP 450-490) and N2.1 (Red, EF BP 515-560). Brightness and contrast of each photo were adjusted using Adobe Photoshop 7.0 (Adobe Systems, San Jose, CA).

In another set of experiments, retinal pieces were not treated with TB and were embedded in paraffin. $3-\mu \mathrm{m}$ thick RTSs were cut from these untreated paraffin embedded pieces. Subsequently these RTSs were incubated with different concentrations of TB (post-treatment), mounted and observed using a microscope.

\subsection{Immunofluorescence labelling of $T B$ treated retinal tissue sections (TRTSs)}

For RPE65 antigen immunofluorescence labelling, the RTSs (control vs TB treated) were dewaxed by immersing the slides in xylene (twice for $10 \mathrm{~min}$ ), rehydrated with graded ethanol (5 min in $100 \%$ and $5 \mathrm{~min}$ in $95 \%$ ethanol), and transferred to deionised water and finally washed twice with PBS. Each RTS was pre-treated for antigen retrieval with citrate buffer, $\mathrm{pH} 6$ for $15 \mathrm{~min}$ at $100{ }^{\circ} \mathrm{C}$. It was followed by rinsing in water for 5 min and subsequent washing three times with PBS. And then each RTS was blocked in PBT $(0.5 \%$ TritonX-100 in PBS), 10\% normal goat serum (Sigma-Aldrich), and $1 \%$ BSA (Sigma-Aldrich) during $1 \mathrm{~h}$ at RT. The RTSs were incubated, after blocking, with anti-human RPE65 MoAb, previously diluted 1.50 in PBS, for $30 \mathrm{~min}$ at RT. All RTSs were washed three times in PBS and exposed for $30 \mathrm{~min}$ at RT to a FITC conjugated goat anti-mouse IgG secondary antibody, previously diluted 1:50 in PBS. Slides were then cover-slipped with 1:1 PBS-glycerol. Negative controls included omission of the primary antibody or secondary 
antibody. Microscopic studies were performed for detecting autofluorescence and antigen specific FITC fluorescence.

\section{Results}

\subsection{Flow cytometry (FC) analysis of TB treated RPE cells}

FC analysis (Fig. 1A) showed that pRPE cells of primary cultures (passage 1-6) were emitting intensive autofluorescence. Emission of intensive autofluorescence by pRPE cells produced high background noise (see negative control staining "white" in Fig. 1A), interfering with the detection of specific fluorescence signals ("black" in Fig. 1A) corresponding to expressed cell proteins above background noise.

A protocol was optimized for determining the expression of RPE65 antigen in primary cultures of pRPE cells. Both, the percentage of protein expression and the Mean Fluorescence Intensity (MFI) were analysed. It was difficult to detect RPE65 protein corresponding specific fluorescence signals above background noise without quenching the autofluorescence of the pRPE cells. The pRPE cells were analyzed with or without treatment with different concentrations of TB to optimize and improve the FC protocol. The FC results showed the overlapping of the positive and negative fluorescence staining in the absence of TB treatment (Fig. 1A) while post-
Table 1

RPE65 expression in primary cultures of pRPE cells with different concentrations of Trypan Blue.

\begin{tabular}{lllcc}
\hline \multirow{2}{*}{ Trypan Blue concentration $(\mu \mathrm{g} / \mathrm{ml})$} & \multicolumn{3}{l}{ RPE65 } \\
\cline { 2 - 5 } & $\%$ & MFI negative & MFI positive & MFI Index \\
\hline 0 & $*$ & 7.7 & 14.2 & 1.8 \\
10 & 74.94 & 0.5 & 5.3 & 10.6 \\
20 & 80.01 & 0.5 & 9.6 & 19.2 \\
40 & 53.58 & 0.4 & 2.3 & 5.7 \\
\hline
\end{tabular}

*Not valued.

MFI: Mean Fluorescence Intensities.

treatment with TB clearly separated the positive and negative fluorescence signals (Fig. 1B, C, and D). It detected clearly that $74.94 \%$ $($ MFI index $=10.6), 80.01 \%$ (MFI index $=19.2$ ) and $53.58 \%$ (MFI index $=5.7$ ) of pRPE cells were RPE65 antigen positive with 10 , 20 and $40 \mu \mathrm{g} / \mathrm{ml}$ ice-cold TB counterstain solution respectively (Fig. 1B, C and D). These results (Fig. 1B, C and D), together with the optimum MFI obtained (Table 1), clearly indicated that posttreatment of RPE cells of cell cultures with $20 \mu \mathrm{g} / \mathrm{ml}$ of TB counterstain was the best staining conditions for quenching the pRPE cells autofluorescence for further FC studies.
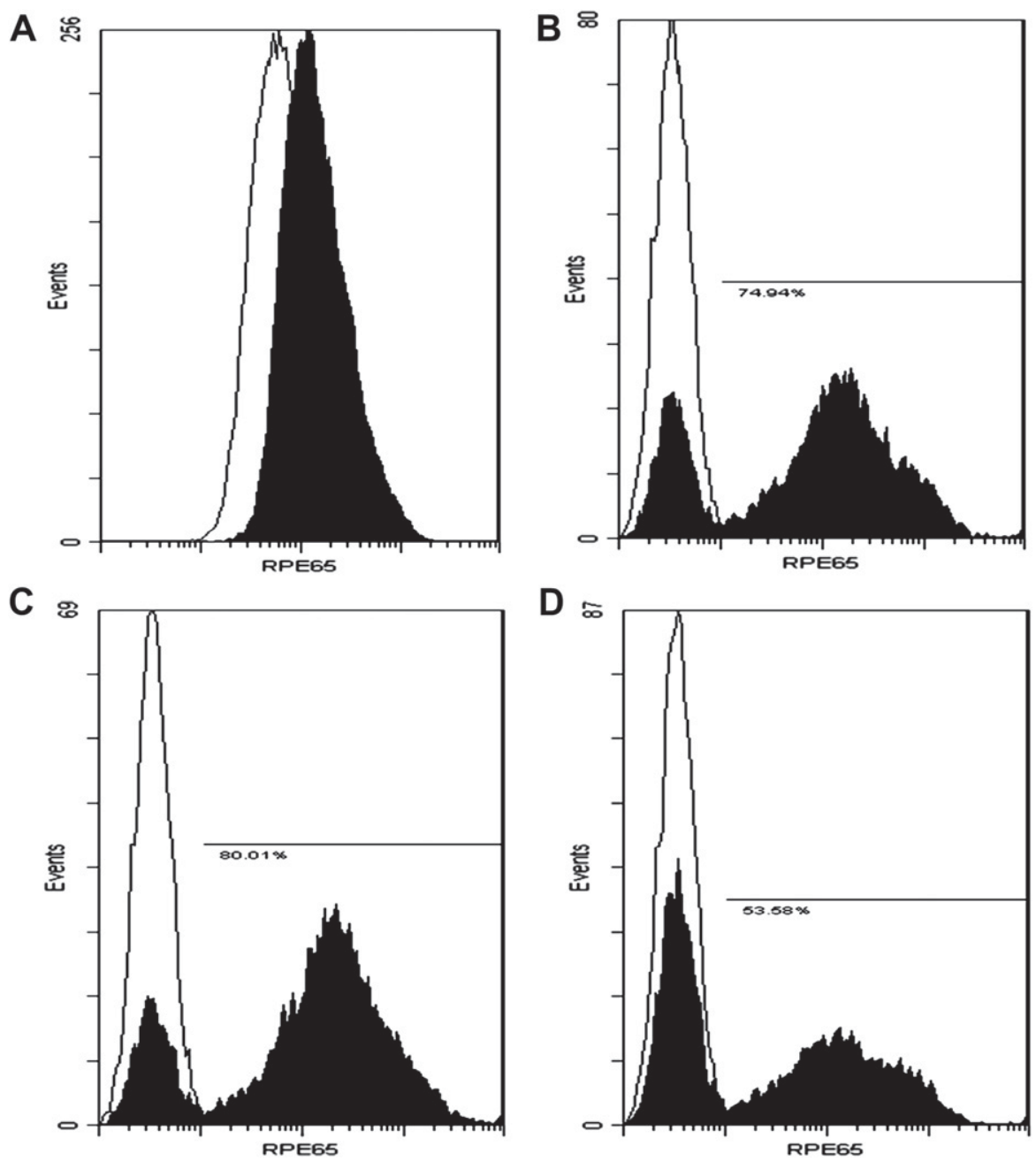

Fig. 1. FC analysis for percentage of pRPE cells of the primary cell culture (Passage 1) expressing RPE65 antigen after staining with anti-RPE65 MoAb followed by incubation with 0 , 10, 20 and $40 \mu \mathrm{g} / \mathrm{ml}$ of TB stain. A) Percentage of pRPE cells detected by anti-RPE65 MoAb in presence of $0 \mu \mathrm{g} / \mathrm{ml}$ of TB with optimum MFI. B) Percentage of pRPE cells detected by anti-RPE65 MoAb in presence of $10 \mu \mathrm{g} / \mathrm{ml}$ of TB with optimum MFI. C) Percentage of pRPE cells detected by anti-RPE65 MoAb in presence of $20 \mu \mathrm{g} / \mathrm{ml}$ of TB with optimum MFI. D) Percentage of pRPE cells detected by anti-RPE65 MoAb in presence of $40 \mu \mathrm{g} / \mathrm{ml}$ of TB with optimum MFI. 

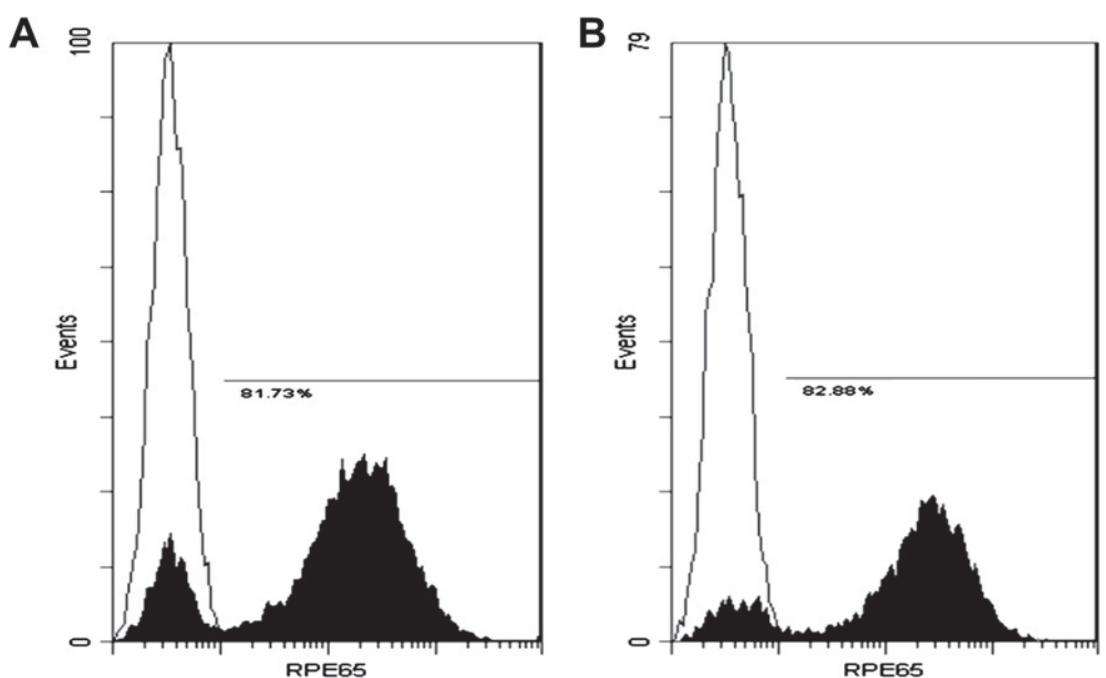

Fig. 2. FC analysis for percentage of pRPE cells of primary cell culture of different passages (Passage 1 and 6 ) expressing RPE65 antigen after staining with anti-RPE65 Ab followed by incubation with $20 \mu \mathrm{g} / \mathrm{ml}$ of TB. A) Percentage of pRPE cells (Passage 1) expressing RPE65 antigen with optimum MFI. B) Percentage of pRPE cells (Passage 6) expressing RPE65 antigen with optimum MFI.

FC analysis of different cell passages showed that $81.73 \%$ and $82.88 \%$ of pRPE cells in passage 1 and 6 were RPE65 antigen positive with the selected post-treatment of $20 \mu \mathrm{g} / \mathrm{ml}$ ice-cold TB counterstain solution respectively (Fig. $2 \mathrm{~A}$ and B).

\subsection{Immunohistochemical (IHC) analysis of TB treated tissue bound RPE cells}

Phase contrast microscopy showed the RPE layers of RTSs were emitting autofluorescence in the range of green fluorescence, to some extent in the range of blue fluorescence but there was no emission of red fluorescence (Fig. 3A, B, C, D). Pre-treatment of retinal tissue pieces with concentration of 20,200, 250 and $2000 \mu \mathrm{g} / \mathrm{ml}$ of TB at RT for 15 min followed by embedding in paraffin and sectioning showed that $\mathrm{TB}$, at minimum concentration of $20 \mu \mathrm{g} / \mathrm{ml}$, had prevented RPE layer of RTSs to emit autofluorescence (Fig. 4). Microscopic observation of RTSs of post-TB treated retinal pieces embedded in paraffin showed that these RTSs were emitting red fluorescence (Data not shown). Immunofluorescence microscopy showed the detection of RPE65 antigens by anti-RPE65 MoAb in Pre-20 $\mu \mathrm{g} / \mathrm{ml}$ TRTSs (Fig. 5A and B). RPE65 antigens were not detected in control sections incubated with
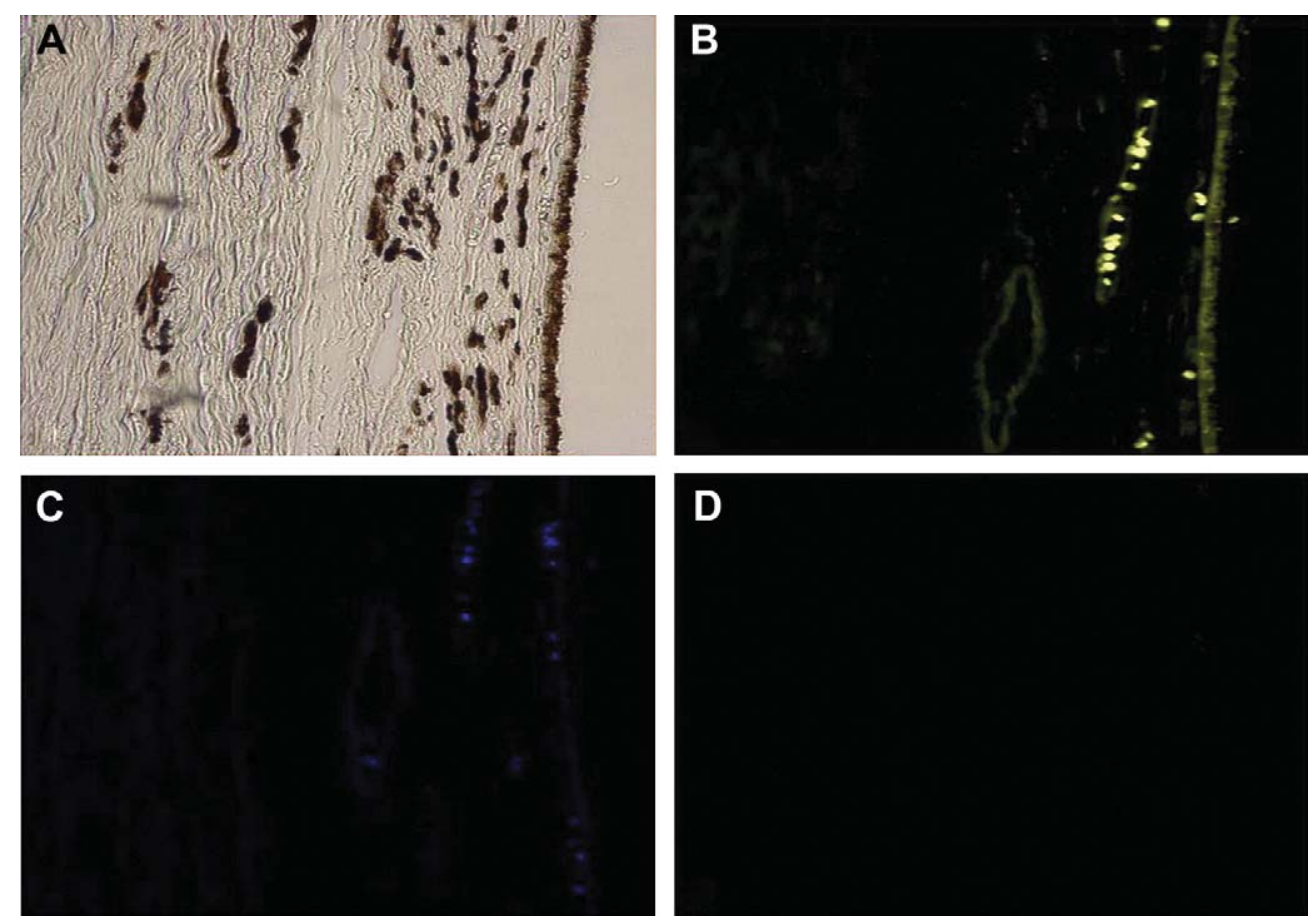

Fig. 3. Detection of autofluorescence of p-formaldehyde fixed paraffin embedded non-TB treated 3- $\mu$ m thick tissue sections of porcine retina. A) Phase contrast microscopic observation of porcine retinal tissue section. B) Detection of green autofluorescence emitted by pigmented RPE layer of porcine retinal tissue section. C) Detection of blue autofluorescence by pigmented RPE layer of porcine retinal tissue section. D) Detection of red autofluorescence by pigmented RPE layer of porcine retinal tissue section. (For interpretation of the references to colour in this figure legend, the reader is referred to the web version of this article.) 


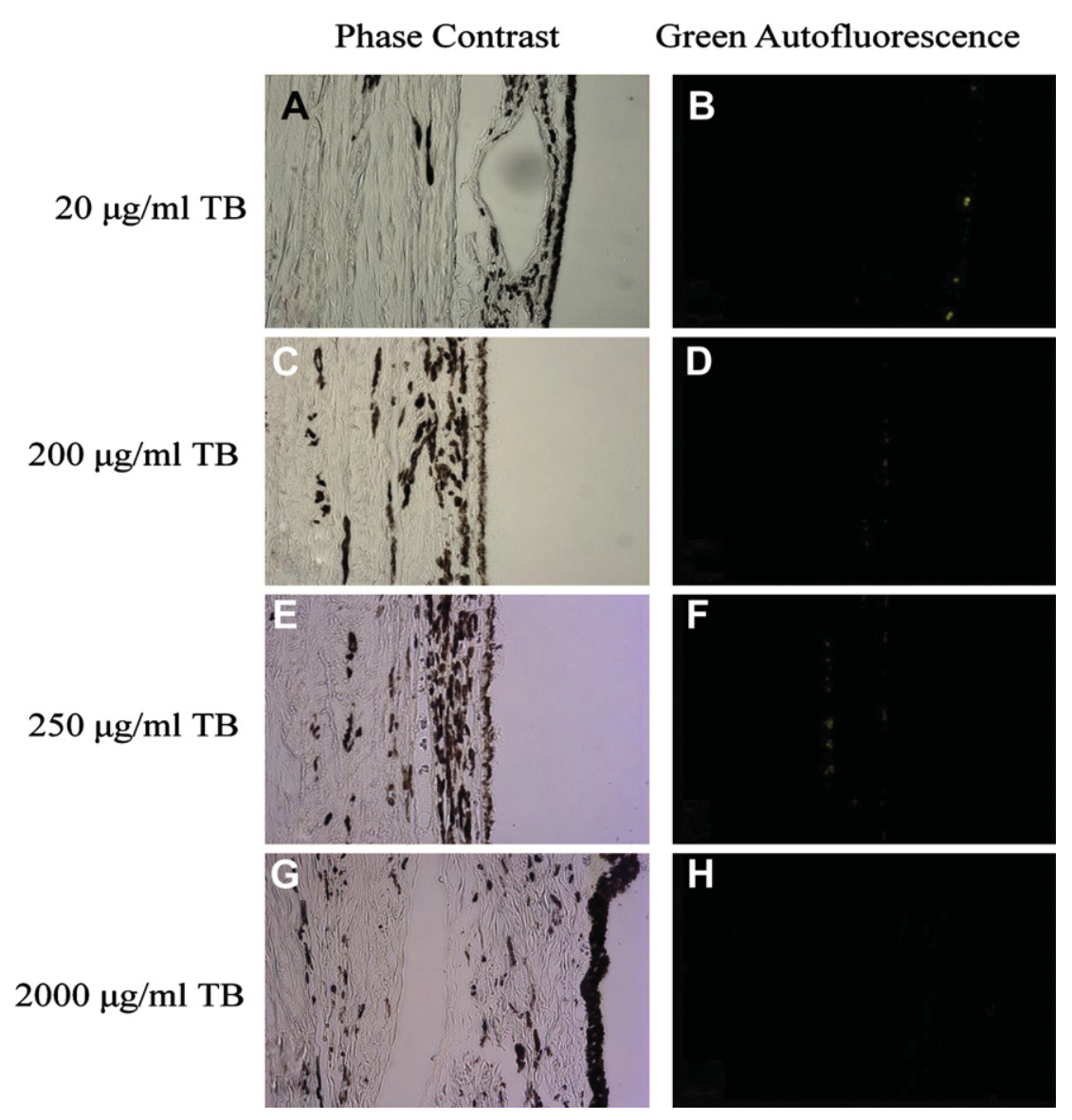

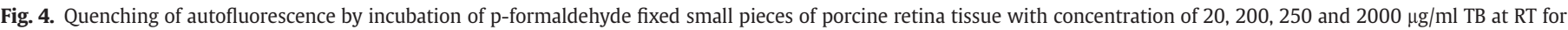
$15 \mathrm{~min}$, followed by washing, embedding in paraffin and cutting into 3- $\mu \mathrm{m}$ thick tissue sections for observation under microscope. $20 \mu \mathrm{g} / \mathrm{ml}(\mathrm{A}$ and $\mathrm{B}) ; 200 \mu \mathrm{g} / \mathrm{ml}(\mathrm{C}$ and $\mathrm{D}) ; 250 \mu \mathrm{g} /$ $\mathrm{ml}(\mathrm{E}$ and $\mathrm{F})$; and $2000 \mu \mathrm{g} / \mathrm{ml}(\mathrm{G}$ and $\mathrm{H})$ TB treated retinal tissue sections showed pigmented RPE layer in phase contrast microscopy (A, C, E and G) but did not emit green autofluorescence in fluorescence microscopy (B, D, F and $\mathrm{H}$ ). (For interpretation of the references to colour in this figure legend, the reader is referred to the web version of this article.)

anti-RPE65 MoAb or with FITC conjugated goat anti-mouse antibody (Fig. 5C, D, E and F).

\section{Discussion}

Current investigation reports for developing treatments for retinal atrophy have shown the importance of the RPE monolayer, functioning as a barrier between the choroid and neural retina, in designing therapeutic strategies (Nowak, 2006; Binder et al., 2007; Del Priore et al., 2004; Coffey et al., 2002). Cellular protein expression is a reflection of the cell phenotype, functional characteristics, and stability in experimental or pathological conditions. This protein expression can be detected using FC and IHC, two well known and widely used protein detection technologies, used not only on a single cell level but also for determining tissue bound immunofluorescence. But RPE cells contain pigments, as shown in Fig. 3, emitting intensive autofluorescence that causes high background noise interfering with the detection of proteins expressed by RPE cell (Figs. 1A and 3). Therefore currently it becomes essential to develop methods for evaluating the expressed proteins in RPE cells to determine the degree of deviation of RPE cells from their native characteristics in experimental or pathological conditions.

Autofluorescence has been a problem for immunofluorescent labelling studies for many years (Kingsley et al., 2001; Monici, 2005) because it results in overlapping area between positive and negative signals that are difficult to separate even after changing the instrument settings (Fig. 1A). In this study, the FC was setup with optimized voltage of PMT for measuring RPE65 protein corresponding fluorescence (FITC, FL1 detector) with the higher possible MFI, above minimum background noise but cells related autofluorescence (Fig. 3) held up to detect the protein bound fluorescence with a high overlapping between the positive and negative signals, as shown in Fig. 1A. This showed that in addition to instrument settings for minimizing background noise, the quenching of autofluorescence of these cells was required.

TB has been used routinely in the laboratories as a counterstain for years (Cowen et al., 1985; Lynch and Derbyshire, 1986). In this study, it has been successfully used to reduce the internal background noise produced by the autofluorescence of pRPE cells. After permeabilization and staining, TB was allowed to diffuse rapidly into the cells resulting into their uniform distribution throughout the cytoplasm and nucleus. This brought dye molecules with proper distance and orientation to autofluorescent or nonspecifically bound fluorescent molecules. That resulted into quenching of the autofluorescence and detection of specific protein bound fluorescence with higher intensity and specificity (The Merk Index, 1983; Wu and Brand, 1992). But this required optimizing the concentration of TB because one pitfall of TB is its potential to quench specifically bound fluorescence if it is not used in optimized concentration. And this particular point has been achieved in this study.

For FC, the results (Table 1 and Fig. 1) clearly showed that posttreatment with $20 \mu \mathrm{g} / \mathrm{ml}$ of ice-cold TB counterstain was the best 


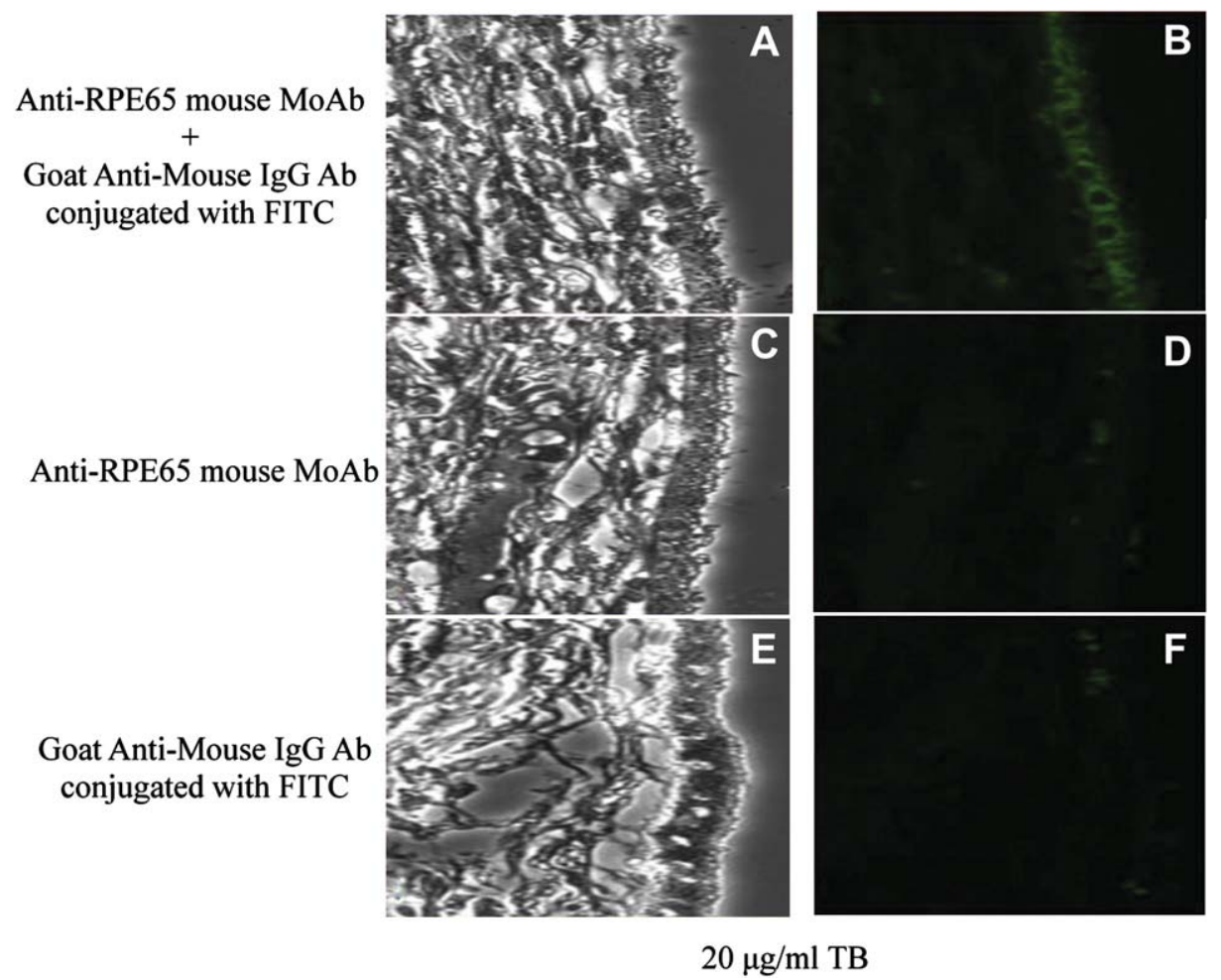

Fig. 5. Detection of Anti-RPE65 antigens of pigmented RPE layer of $20 \mu \mathrm{g} / \mathrm{ml}$ TB treated $3-\mu \mathrm{m}$ thick retinal tissue sections. $20 \mu \mathrm{g} / \mathrm{ml}(\mathrm{A}, \mathrm{B}, \mathrm{C}, \mathrm{D}, \mathrm{E}$ and $\mathrm{F}$ ) TB treated retinal tissue sections showed pigmented RPE layer in phase contrast microscopy (A, C and E) and green fluorescence (B) on incubation with anti-RPE65 mouse MoAb, followed by incubation with goat anti-mouse IgG antibody conjugated with FITC but did not emit green fluorescence (D and F) on incubation with Anti-RPE65 mouse MoAb (D) or goat anti-mouse IgG antibody (F) alone in fluorescence microscopy. (For interpretation of the references to colour in this figure legend, the reader is referred to the web version of this article.)

conditions to minimize autofluorescence and maximize positive to negative MFI signals; in Fig. 1C a clear separation between the RPE65 positive cells and the negative control signals is shown. This experimental approach, together with the optimized FC settings, improved the FC method to detect RPE65 protein bound fluorescence. In addition, this optimized FC method was used to check the RPE65 antigen expression in advanced stage culture (passage 6) and it was found that $82.88 \%$ pRPE cells were maintaining RPE65 protein. Further studies to use this optimized FC method for detecting other expressed RPE cell proteins are currently underway.

The results from the histopathologic evaluation clearly demonstrated that RPE layer of RTSs were emitting intense autofluorescence in absence of TB treatment but pre-treatment of RTSs with 20,200, 250 and $2000 \mu \mathrm{g} / \mathrm{ml}$ TB at RT for 15 min blocked the emission of autofluorescence (Fig. 4). Interestingly the posttreatment of RTSs with 20, 200, 250 and $2000 \mu \mathrm{g} / \mathrm{ml} \mathrm{TB}$ at RT for 15 min showed that in each case, RTS was emitting red fluorescence. The emission of red fluorescence had prevented the use of RTSs for further immunofluorescence labelling (data not shown). The emission of red fluorescence from post-treated RTSs might be due to interaction of paraffin with the TB stain. The results also showed that RPE65 antigens were clearly immunolabelled in RPE layers of pre-TB treated RTSs (Fig. 5).

Thus, in this study, a TB staining method has been successfully used to reduce the internal background noise and to facilitate the analysis of expressed RPE cell proteins both by FC and by IHC techniques.

\section{Conclusions}

In summary, a method of counterstaining with an optimum concentration of TB was successfully implemented to reduce the background noise produced by autofluorescence of RPE cells thereby allowing to accurately measure the intense as well as dim cellular protein related specific fluorescence in FC and IHC RPE cell analysis.

\section{Acknowledgements}

This work was supported by grants from Castilla and Leon Regenerative Medicine and Cell Therapy Network Center, National Plan of I+D+I 2008-2011 and ISCIII-Subdireccion General de Evaluación y Fomento de la Investigación (PS09/00938) (MICNN) with cofinantiation FEDER and JCYL BIO/39/VA26/10, Junta de Castilla y León, Spain to JCP and GKS. A.K. Singh and Ivan Fernandez are Pre-doctoral research scholars supported by AECI, Spanish Ministry of Foreign Affairs and Cooperation and Junta de CastillaLeón, Spain respectively.

\section{References}

Alge, C.S., Hauck, S.M., Priglinger, S.G., Kampik, A., Ueffing, M., 2006. Differential protein profiling of primary versus immortalized human RPE cells identifies expression patterns associated with cytoskeletal remodeling and cell survival. J. Proteome Res. 5, 862-878.

Aubin, J.E., 1979. Autofluorescence of viable cultured mammalian cells. J. Histochem. Cytochem. 27, 36-43.

Benson, R.C., Meyer, R.A., Zaruba, M.E., McKhann, G.M., 1979. Cellular autofluorescence - is it due to flavins? J. Histochem. Cytochem. 27, 44-48.

Binder, S., Stanzel, B.V., Krebs, I., Glittenberg, C., 2007. Transplantation of the RPE in AMD. Prog. Retin. Eye Res. 26, 516-554.

Carr, A.J., Vugler, A.A., Hikita, S.T., Lawrence, J.M., Gias, C., Chen, L.L., Buchholz, D.E., Ahmado, A. Semo, M. Smart, M. Hasan, S. da Cruz, L, Johnson, LV., Clegg, D.O. Coffey, P.J., 2009. Protective effects of human iPS-derived retinal pigment epithelium cell transplantation in the retinal dystrophic rat. PLoS One 4, e8152. Coffey, P.J., Girman, S., Wang, S.M., et al., 2002. Long-term preservation of cortically dependent visual function in RCS rats by transplantation. Nat. Neurosci. 5, 53-56.

Cowen, T., Haven, A.J., Burnstock, G., 1985. Pontamine sky blue: a counterstain for background autofluorescence in fluorescence and immunofluorescence histochemistry. Histochemistry 82 (3), 205-208. 
Del Priore, L.V., Tezel, T.H., Kaplan, H.J., 2004. Survival of allogeneic porcine retinal pigment epithelial sheets after subretinal transplantation. Invest. Ophthalmol. Vis. Sci. 45, 985-992.

Dent, G.A., Leglise, M.C., Pryzwansky, K.B., Ross, D.W., 1989. Simultaneous paired analysis by flow cytometry of surface markers, cytoplasmic antigens, or oncogene expression with DNA content. Cytometry 10, 192-198.

Dintelmann, T.S., Heimann, K., Kayatz, P., Schraermeyer, U., 1999. Comparative study of ROS degradation by IPE and RPE cells in vitro. Graefes Arch. Clin. Exp. Ophthalmol. 237, 830-839.

Gupta, S.K., Jollimore, C.A., McLaren, M.J., Inana, G., Kelly, M.E., 1997. Mammalian retinal pigment epithelial cells in vitro respond to the neurokines ciliary neurotrophic factor and leukemia inhibitory factor. Biochem. Cell Biol. 75, 119-125.

Kingsley, K., Carroll, K., Huff, J.L., Plopper, G.E., 2001. Photobleaching of arterial autofluorescence for immunofluorescence applications. Biotechniques 30 (4), 794-797.

Lee, H., Chung, H., Arnouk, H., Lamoke, F., Hunt, R.C., Hrushesky, W.J., Wood, P.A Lee, S.H., Jahng, W.J., 2010. Cleavage of the retinal pigment epithelium-specific protein RPE65 under oxidative stress. Int. J. Biol. Macromol. 47 (2), 104-108.

Lynch, J.A., Derbyshire, J.B., 1986. Application of a modified indirect fluorescen antibody test to the detection of antibodies to bovine respiratory syncytial virus in Ontario cattle. Can. J. Vet. Res. 50, 384-389.

Majji, A.B., de Juan Jr., E., 2000. Retinal pigment epithelial autotransplantation: morphological changes in retina and choroid. Graefes Arch. Clin. Exp. Ophthalmol. 238, 779-791.

Monici, M., 2005. Cell and tissue autofluorescence research and diagnostic applications. Biotechnol. Annu. Rev. 11, 227-256.

Nowak, J.Z., 2006. Age-related macular degeneration (AMD): pathogenesis and therapy. Pharmacol. Rep. 58, 353-363.
Okada, T.S., 1980. Cellular metaplasia or transdifferentiation as a model for retinal cell differentiation. Curr. Top. Dev. Biol. 16, 349-380.

Othmer, M., Zepp, F., 1992. Flow cytometric immunophenotyping: principles and pitfalls. Eur. J. Pediatr. 151, 398-406.

Saika, S., Kono-Saika, S., Tanaka, T., et al., 2004. Smad3 is required for dedifferentiation of retinal pigment epithelium following retinal detachment in mice. Lab. Invest. 84, 1245-1258.

Singhal, S., Vemuganti, G.K., 2005. Primary adult human retinal pigment epithelial cell cultures on human amniotic membranes. Indian J. Ophthalmol. 53, 109-113.

Steindl-Kuscher, K., Krugluger, W., Boulton, M.E., Haas, P., Schrattbauer, K. Feichtinger, H., Adlassnig, W., Binder, S, 2009. Activation of the beta-catenin signalling pathway and its impact on RPE cell cycle. Invest. Ophthalmol. Vis. Sci. 50, 4471-4476.

Stevens, M.J., Larkin, D.D., Feldman, E.L., DelMonte, M.A., Greene, D.A., 2005. Establishment, maintenance, and transfection of in vitro cultures of human retinal pigment epithelium. Methods Mol. Med. 107, 343-351.

The Merk Index, 1983. An Encyclopedia of Chemicals, Drugs, and Biologicals. Merk and Co., Inc., New Jersey, Monograph number 9592, p 1398.

Valtink, M., Engelmann, K., 2009. Culturing of retinal pigment epithelium cells. Dev. Ophthalmol. 43, 109-119.

Wang, N.K., Tosi, J., Kasanuki, J.M., Chou, C.L., Kong, J., Parmalee, N., Wert, K.J. Allikmets, R., Lai, C.C., Chien, C.L., Nagasaki, T., Lin, C.S., Tsang, S.H., 2010. Transplantation of reprogrammed embryonic stem cells improves visual function in a mouse model for retinitis pigmentosa. Transplantation 89, 911-919.

Wu, P. Brand, L, 1992. Orientation factor in steady-state and time-resolved resonance energy transfer measurements. Biochemistry 31, 7939-7947. 


\title{
Elastin-like recombinamers as substrates for retinal pigment epithelial cell growth
}

\author{
Girish K. Srivastava, ${ }^{1,2}$ Laura Martín, ${ }^{3}$ Amar K. Singh, ${ }^{1}$ Ivan Fernandez-Bueno, ${ }^{1}$ \\ Manuel J. Gayoso, ${ }^{1}$ Maria T. Garcia-Gutierrez, ${ }^{1}$ Alessandra Girotti, ${ }^{3}$ Matilde Alonso, ${ }^{3}$ \\ José C. Rodríguez-Cabello, ${ }^{3}$ José C. Pastor ${ }^{1}$ \\ ${ }^{1}$ Institute of Applied Ophthalmobiology (IOBA), University of Valladolid, Valladolid, Spain \\ ${ }^{2}$ Castilla and Leon Regenerative Medicine and Cell Therapy Network Center, Spain \\ ${ }^{3}$ G.I.R. BIOFORGE Group, University of Valladolid, CIBER-BBN, Valladolid, Spain
}

Received 3 August 2010; revised 17 December 2010; accepted 10 January 2011

Published online 25 March 2011 in Wiley Online Library (wileyonlinelibrary.com). DOI: 10.1002/jbm.a.33050

\begin{abstract}
The aim of this study is to investigate the use of elastin-like recombinamers (ELRs) as a substrate that can maintain the growth, phenotype, and functional characteristics of retinal pigment epithelial (RPE) cells efficiently and as a suitable carrier for the transplantation of autologous RPE cells for treatment of age-related macular degeneration (AMD). ELR films containing a bioactive sequence, RGD (ELR-RGD), and one with no specific sequence (ELR-IK) as control, were obtained by solvent-casting onto glass and subsequent cross-linking. ARPE 19 cells were seeded on sterilized ELR films as well as on the control surfaces. Cells were analysed after 4, 24, 72, and $120 \mathrm{~h}$ to study cell adhesion, proliferation, cell viability, morphology, and specificity by staining with Trypan blue, DAPI, Rhodamin-Phalloidin and RPE65, ZO-1 antibodies and observing under fluorescence as well as electron microscope.
\end{abstract}

ARPE19 cells seeded on both ELR films and controls were $100 \%$ viable and maintained their morphology and set of characteristics at the different time points studied. Cell proliferation on ELR-RGD was significantly higher than that found on ELR-IK at all time points, although it was less than the growth rate on polystyrene. ARPE19 cells grow well on ELR-RGD maintaining their phenotype. These results should be extended to further studies with fresh human RPE cells and in vivo studies to determine whether this ELR-RGD matrix could be used as a Bruch's membrane prosthesis and carrier for transplantation of RPE cells in patients suffering with AMD. (C) 2011 Wiley Periodicals, Inc. J Biomed Mater Res Part A: 97A: 243-250, 2011.

Key Words: regenerative medicine, biomaterial, RPE cells, AMD, elastin-like recombinamers

\section{INTRODUCTION}

Age-related macular degeneration (AMD) is the leading cause of irreversible severe vision loss and legal blindness in patients over 65 years. Furthermore, the prevalence of this disease $(8 \%)$ increases steeply with age. ${ }^{1,2}$

There are basically two clinical forms of AMD: wet (or neovascular) and dry (or atrophic). Wet AMD can currently be treated with anti-vascular endothelial growth factors (anti-VEGF), whereas dry AMD, which accounts for the majority of cases (60-80\%), cannot as yet be treated, even palliatively. ${ }^{3}$ Despite intense basic and clinical research efforts, the pathology of AMD is not completely understood, although it appears to be a multifactorial disease involving a complex interaction between metabolic, functional, genetic, and environmental factors. ${ }^{4}$ It is well known that disease-related alterations, at least in the initial stages of AMD, are limited to the external layers of the retina, which in theory can be repaired using cell therapy approaches. ${ }^{5-8}$ Retinal pigment epithelium (RPE) plays a pivotal role in normal retinal functioning, including the transport of nutrients from the choroidal blood vessel layer to the photoreceptors, elimination of their waste and formation of the blood-retina barrier that controls the transport of substances into the retina. ${ }^{9}$ The accumulation of drusen, basal deposits and advanced glycation end products (AGEs) below the RPE layer as a result of dysfunction of the diseased RPE are typical signs of AMD that probably contribute to damage of the Bruch's membrane (BrM), which supports the RPE cells and maintains their health, polarity and functionality, and photoreceptors. ${ }^{10}$ Over the last 25 years, several approaches, such as patch graft transplant, RPE transplantation and macular translocation, have shown that replacing diseased RPE with healthier autologous RPE in selected cases can rescue photoreceptors, prevent further visual loss, or even promote visual improvement in both humans and animals. $^{11-16}$

It is also known that ex vivo expansion of adult RPE cells offers the potential to partially reverse the influence of aging, although there is a high risk of cell transdifferentiation. ${ }^{17-21}$ The best results are obtained when these adult

Correspondence to: G. K. Srivastava; e-mail: girish@ioba.med.uva.es

Contract grant sponsors: Castilla and Leon Regenerative Medicine and Cell Therapy Network Center, CIBER-BBN, AECI (Part of the Spanish Ministry of Foreign Affairs), The Junta de Castilla-León

Contract grant sponsor: National Plan of I+D+I 2008-2011 and Spanish Institute of Health Carlos III (ISCIII)-Subdireccion General de Evaluacion y Fomento de la Investigacion (MICNN) with confinantiation FEDER; contract grant number: PS09/00938 
autologous RPE cells are in contact with an appropriate extracellular matrix (ECM) that provides appropriate physiological signals to maintain their differentiated state, polarity, phenotype, and functional characteristics. ${ }^{22}$ Numerous biocompatible natural or synthetic ECMs have been investigated as transplant vehicles in the last few years, although the latest studies in this field have shown that unfavorable characteristics of these ECMs lead to neural retinal atrophy. ${ }^{10}$ The development of an appropriate biocompatible substrate is therefore vital. This substrate must be in the form of a flexible thin film so that it can be handled easily during surgical transplantation. It must also be able to support and maintain an organized monolayer of healthy RPE cells and must have bioactive domains that provide physiological signals to cells to ensure they maintain all their characteristics in vivo after transplantation, a porous structure to allow exchange of materials in and out of the RPE monolayer, thereby supporting the survival of other cell layers in the retina, including, photoreceptors, and must not form toxic by-products upon biodegradation. The elastin-like recombinamers (ELRs) developed by a group at our university could be useful in this respect ${ }^{23,24}$ as other ELRs provided by the same group have been shown to be suitable for tissue engineering to restore vision by reconstructing the ocular surface. ${ }^{25}$

Genetic engineering techniques involving protein-based polymers have allowed the design and synthesis of new advanced materials with an unrivalled degree of complexity and control that can incorporate structural and functional domains derived from ECM proteins almost at will. These recombinant polymers offer the possibility to obtain materials that combine some of the complex properties found in natural proteins with functions of particular interest that are not displayed in the living organism. ELRs have been attracting interest because of their excellent biocompatibility, mechanical properties and bioactivity, amongst others. $^{26-28}$

In this study, we investigated the suitability of an ELR film containing bioactive RGD sequence for allowing the growth of a monolayer of RPE with maintaining their characteristics for future subretinal transplantation.

\section{MATERIALS AND METHODS}

\section{ELR expression and purification}

Standard molecular biology techniques were used to construct two ELR genes containing a bioactive domain (ELRRGD and ELR-IK). ELR-RGD contains a peptide loop present in the human fibronectin protein with the well-known arginine-glycine-aspartic acid (RGD) sequence for cell adhesion. $^{29,30}$ ELR-IK, which has no bioactive domain, was used as negative control.

Polymer production was carried out using cellular systems for genetically engineered protein biosynthesis in Escherichia coli. Synthetic oligonucleotides were purchased from IBA GmbH (Goettingen, Germany), and Taq DNA polymerase and the restriction enzyme Eam 1104 I were purchased from Stratagene (La Jolla, CA). The restriction enzymes XbaI, HindIII, DpnI, EcoRI, and SapI were purchased from Fermen- tas (Burlington, ON), and T4 DNA ligase, Shrim Alkaline Phosphatase (S.A.P), and Antartic alkaline phosphatase were purchased from New England Biolabs (Beverly, Ma). PCR amplifications were performed in an Eppendorf AG 22331 thermocycler. Gel photographs were taken with the Gel logic 100 Imaging System running the Kodak 3.6 software. Escherichia coli BLR (DE3) strain and the expression vector pET $(25+)$ from Novagen (Madison WI), and Ampicillin from Apollo Scientific (Bredbury, UK), were used during elastinlike recombinamer (ELR) production, which was performed with several cycles of temperature-dependent reversible precipitation, as described elsewhere. ${ }^{24}$

The purity and molecular weight of the proteins were verified by SDS-PAGE gels and mass spectrometry (MALDITOF/MS), whereas the correctness of the sequence was checked by amino acid analysis and amino acid sequencing. Physical properties were characterized by differential scanning calorimetry (DSC) and turbidity measurements.

The amino-acid sequences of the ELRs used in this work are depicted below:

$$
\begin{aligned}
& \text { ELR-RGD: MGSS-H } 6 \text {-SSGLVPRGSHMESLLP-\{[(VPGIG) })_{2} \text { (VPGKG) } \\
& \text { (VPGIG) } \left.\left.\left.\left.\left.{ }_{2}\right]_{2} \text { AVTGRGDSPASS[(VPGIG) }\right)_{2} \text { (VPGKG)(VPGIG) }\right)_{2}\right]_{2}\right\}_{6} \\
& \left(M_{\mathrm{w}}=60,661 \mathrm{Da}\right) \text {. }
\end{aligned}
$$

\section{ELR-IK: MESLLP-(VPGIG-VPGIG-VPGKG-VPGIG-VPGIG) $24-\mathrm{V}$}

$$
\left(M_{\mathrm{w}}=51,980 \mathrm{Da}\right) \text {. }
$$

\section{Preparation of ELR films}

ELR-films were obtained by solvent-casting onto glass and subsequent cross-linking with hexamethylene diisocyanate (HDI), a lysine-targeted homobifunctional crosslinker, as described elsewhere. ${ }^{31}$

Thus, an aqueous ELR solution ( $80 \mu \mathrm{L}, 50 \mathrm{mg} / \mathrm{mL})$ was deposited on circular cover glasses (diameter: $12 \mathrm{~mm}$; Thermo Scientific, Madrid Spain). After incubation for $8 \mathrm{~h}$ at $60^{\circ} \mathrm{C}$, the dry films were cross-linked by immersion in $10 \%$ HDI/acetone solution overnight and then exhaustively washed with type I water. All reagents were purchased from Sigma Aldrich, Madrid Spain.

The ELR films and controls (glass, polystyrene) were placed in a 24-well cell-culture plate, sterilized for $12 \mathrm{~h}$ under UV light, and then incubated in Dulbecco's Modified Eagle Medium (DMEM; Invitrogen, Paisley, UK) with antibiotics for $2 \mathrm{~h}$ before cell seeding.

\section{Cell culture}

ARPE19 cells were used for all quantitative and qualitative analysis in this study. These cells ${ }^{32}$ were obtained from the American Type Culture Collection ATCC (Manassas, VA) and maintained in a $1: 1(\mathrm{v} / \mathrm{v})$ mixture of DMEM/F12 supplemented with $10 \%$ FBS, $2 \mathrm{mM}$ L-glutamine, $50 \mathrm{U} / \mathrm{mL}$ penicillin $\mathrm{G}, 50 \mathrm{mg} / \mathrm{mL}$ streptomycin, and $2.5 \mathrm{mg} / \mathrm{mL}$ amphotericin $\mathrm{B}$ (Invitrogen-Gibco, Paisley, UK). Cells were maintained in a humidified atmosphere at $37^{\circ} \mathrm{C}$ in the presence of $5 \% \mathrm{CO}_{2}$, and the medium was replaced every 3 days until confluence was reached. Cells were then detached with $0.1 \%$ trypsin and $0.04 \%$ tetrasodium ethylenediaminetetraacetate (EDTA) 


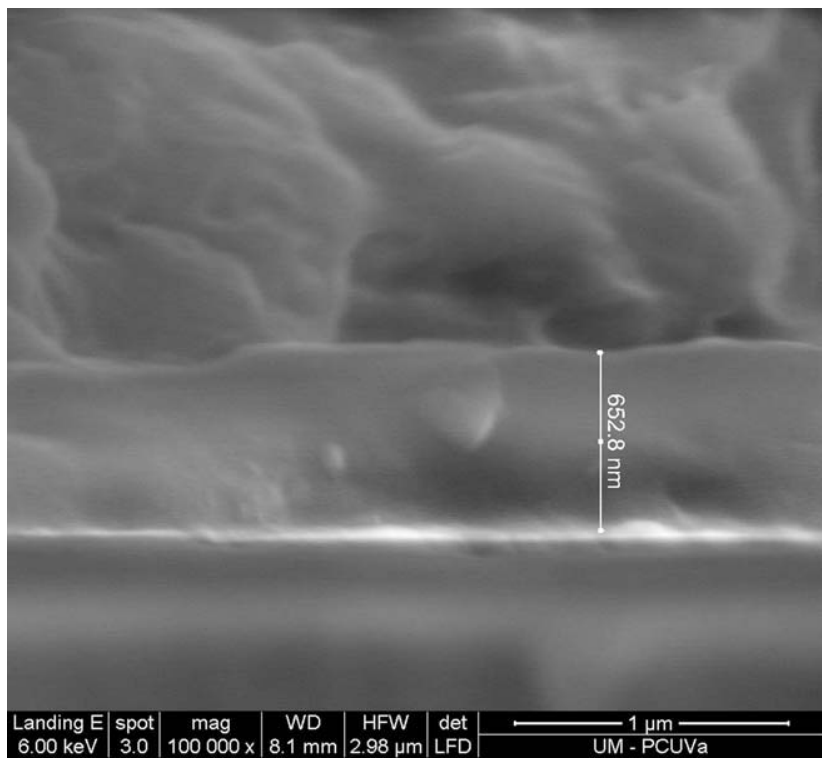

FIGURE 1. SEM of ELR-RGD film showed $652.8 \mathrm{~nm}$ thickness of the polymeric film.

(Invitrogen-Gibco, Paisley, UK), mixed with complete medium to block the trypsin-EDTA activity, and washed and resuspended in phosphate-buffered saline (PBS; InvitrogenGibco, Paisley, UK).

Cell numbers and viability were determined by standard Trypan blue exclusion assay. $1 \times 10^{4}$ ARPE19 cells were seeded on ELR-RGD film scaffold and controls (ELR-IK, the polystyrene substrate of standard tissue culture plates and glass) and incubated under standard conditions for up to $120 \mathrm{~h}$.

\section{Cell number quantification}

At defined time periods after cell seeding, the substrates were washed with PBS to remove nonadhered cells and the cell numbers were determined by manual counting using a

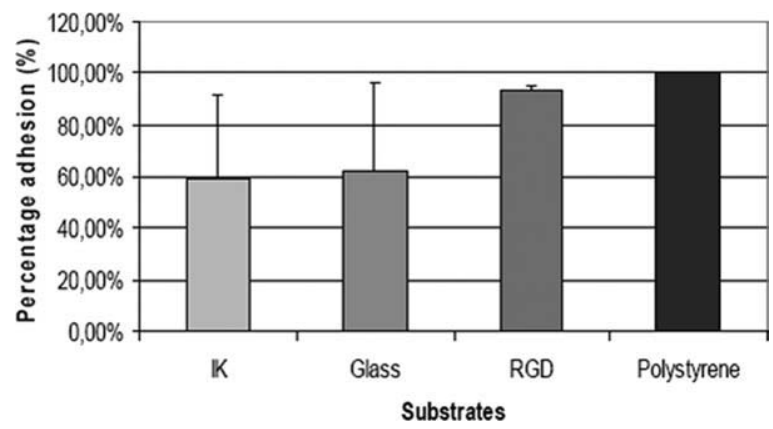

FIGURE 2. Percentage of ARPE19 cells adhered to different substrates at $4 \mathrm{~h}$. The data presented are the mean number of cells (phase-contrast microscopy as well as nuclear counts of cells, assuming $1 \mathrm{nu}$ cleus per cell) per field $(\times 10)$ attached to each surface at $4 \mathrm{~h}$ as a percentage of the control (polystyrene) $\pm 1 \mathrm{SD}$. This histogram shows that the ELR-RGD surface supports adhesion of ARPE19 cells to lesser extent than polystyrene surface but is nevertheless higher than that for ELR-IK and glass. $p<0.05$ is considered significant for comparing the percentage of adhered cells on different surfaces.

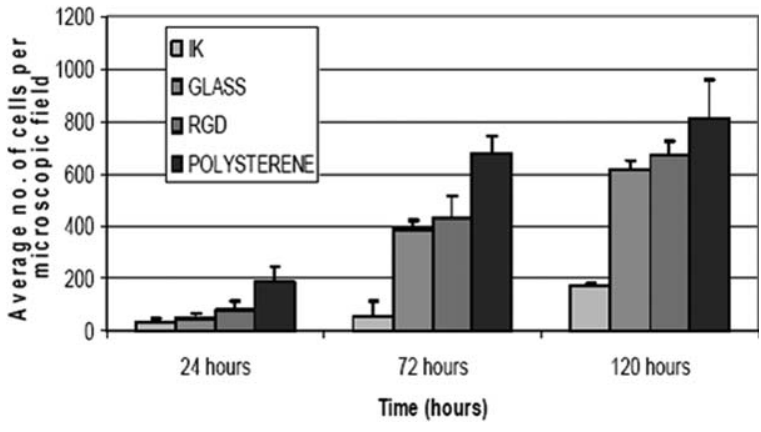

FIGURE 3. Growth of ARPE19 cells on each substrate studied. The histogram shows the mean number of cells (phase-contrast microscopy as well as nuclear counts of cells, assuming 1 nucleus per cell) on different substrates (ELR-RGD, ELR-IK, polystyrene, and glass) at different time intervals $(24,72$, and $120 \mathrm{~h})$. The data are presented as mean number of cells per field $(\times 10) \pm 1$ SD. The ELR-RGD, polystyrene, and glass substrates are more favorable for cell growth than ELR-IK. $p<0.05$ is considered significant for comparing the number of cells growing on different surfaces.

phase contrast and fluorescence microscope (Leica Microsystems, Mannheim, Germany). Cells were nuclear stained with DAPI (Invitrogen, Eugene, OR) for $1 \mathrm{~min}$ at room temperature (RT), mounted in a fluorescent mounting medium (Invitrogen, Paisley, UK), and visualized using a fluorescence microscope. Twenty fields $(\times 10)$ were photographed at random per substrate. The cells, and their nuclei, contained in each field were counted using Adobe Photoshop Elements software. The mean number of nuclei per field of view $(\times 10)$ was calculated for each time interval for each treatment and presented as a histogram showing the average nuclear count per field of view \pm 1 standard deviation (SD) versus time.

\section{Cytotoxicity}

The cytotoxicity of all substrates was analyzed after 4, 24, 72 , and $120 \mathrm{~h}$ incubation. Thus, the substrates were washed with PBS, the cells stained with Trypan blue and viewed immediately under an optical microscope. Twenty fields $(\times 10)$ of each substrate were photographed at random and the mean number of nonstained cells per field of view $(\times 10)$ was taken as the number of surviving cells. The total number of stained and nonstained cells at 4, 24, 72, and $120 \mathrm{~h}$ was calculated as detailed above.

\section{Cell adhesion}

Cells were counted after $4 \mathrm{~h}$, under a phase-contrast microscope, to determine the cell attachment to ELR films and control surfaces, as detailed above. For cell adhesion determination, the average number of cells on the polystyrene substrate (positive control) was set to $100 \%$ and the average number of cells on the other substrates (ELR-RGD, ELRIK, and Glass) calculated as a percentage of the cells growing on the polystyrene surface. For the quantification at $4 \mathrm{~h}$, the values presented are the mean number of cells per field $(\times 10)$ attached to each substrate as a percentage of the polystyrene control $\pm 1 \mathrm{SD}$. 


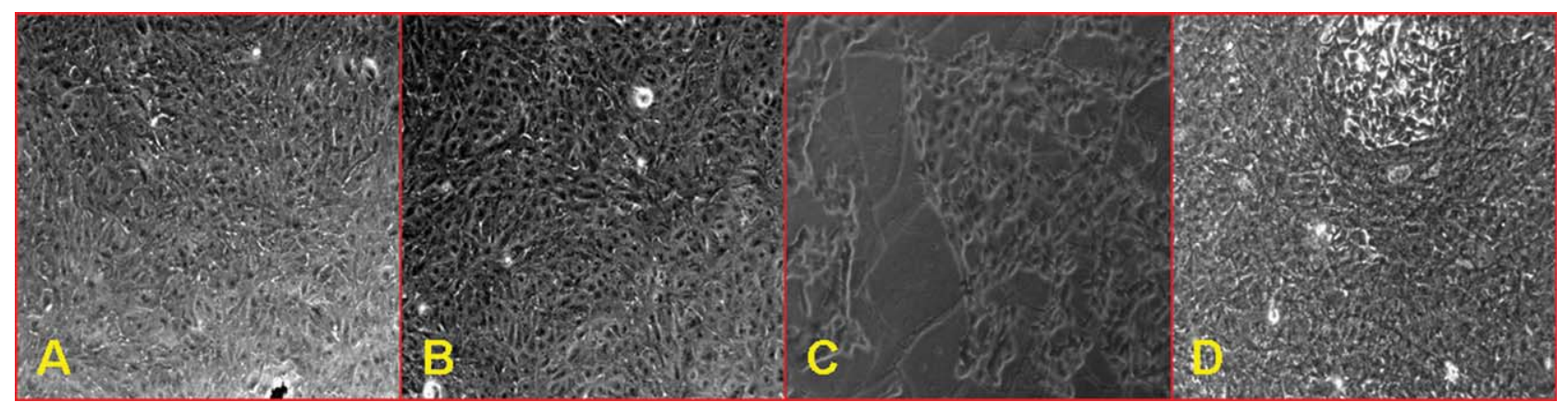

FIGURE 4. Growth of ARPE19 cells on different substrates. Phase-contrast microscopy demonstrates the cell density as well as the hexagonal morphology and the mosaic pattern of ARPE19 cells on each substrate studied at $120 \mathrm{~h}$. ELP-IK does not support the growth of ARPE19 cells. (A) Polystyrene; (B) glass; (C) ELR-IK; (D) ELR-RGD. [Color figure can be viewed in the online issue, which is available at wileyonlinelibrary.com.]

\section{Cellular morphology and specificity assessment}

The epithelial morphology of the cells growing on each substrate was determined by staining cytoskeleton actin filaments with Rhodamine-Phalloidin (Molecular Probes-Invitrogen, Paisley, UK) and tight junction with anti-ZO-1 antibody (Invitrogen, Camarillo, CA), whereas cell specificity was studied by an immunostaining technique against the RPE65 antigen (Novus Biologicals, Inc., Littleton, CO), a specific marker of RPE. ${ }^{32}$ At each time point the cells were washed with PBS $(3 \times 5 \mathrm{~min})$, fixed with $4 \%$ paraformaldehyde for $10 \mathrm{~min}$, permeabilized with PBT $(0.2 \%$ TritonX100 in PBS) for $10 \mathrm{~min}$, washed with PBS $(3 \times 5 \mathrm{~min})$ and blocked for $1 \mathrm{~h}$ in antibody blocking buffer $(10 \%$ Normal goat serum, 1\% Bovine serum albumin in PBT). The cells were then incubated with a 1:100 dilution of anti-RPE65 antibody or with a 1:50 dilution of anti-ZO-1 antibody in PBT overnight at $4^{\circ} \mathrm{C}$, then washed with PBT $(3 \times 5 \mathrm{~min})$ and incubated with a 1:100 dilution of anti-mouse IgG-FITC (Jackson Immunoresearch Laboratories, Inc., West Grove, PA) in PBS for $1 \mathrm{~h}$ at room temperature. The cells were also costained with a 1:40 dilution of Rhodamine-Phalloidin (200 unit/mL) in PBS for $20 \mathrm{~min}$, then mounted and observed under a fluorescence microscope, as described above.

The presence of intercellular tight junction and gap junction between the cells grown on each substrate was confirmed by electron microscopy. Briefly cell cultures were fixed for $2 \mathrm{~h}$ in $0.5 \%$ paraformaldehyde and $0.5 \%$ glutaraldehyde in phosphate buffer $(0.1 M, \mathrm{pH} 7.4)$, washed in the same buffer and postfixed in 1\% 0sO4 in phosphate buffer. After dehydration in alcohols the pieces were embedded in low-viscosity epoxy resin (Spurr resin embedding kit. TAAB Laboratories Equipment Ltd, Berks, UK). Thin sections were examined in a JEOL 1200-E Il electron microscope after staining with uranyl acetate and lead citrate.

\section{Statistical analysis}

The results for each substrate were expressed as mean per field of view $(\times 10) \pm 1$ standard deviation (SD). Data were tested for normality and investigated for statistical significance using Student's $t$-test. Statistical significance was set at $p<0.05$.

\section{RESULTS}

\section{Cell adhesion}

The average number of cells adhered to the ELR-IK, ELRRGD films (each $652.8 \mathrm{~nm}$ thickness, Fig. 1), and glass substrates as a percentage of the polystyrene control after $4 \mathrm{~h}$, as shown in figure 1, demonstrated that the average number of cells adhered to the ELR-RGD and polystyrene substrates was significantly higher $(p<0.05)$ than that attached to the other substrates after $4 \mathrm{~h}$. There were no significant differences $(p>0.05)$ between the number of cells attached to the ELR-IK and glass substrates (Fig. 2).

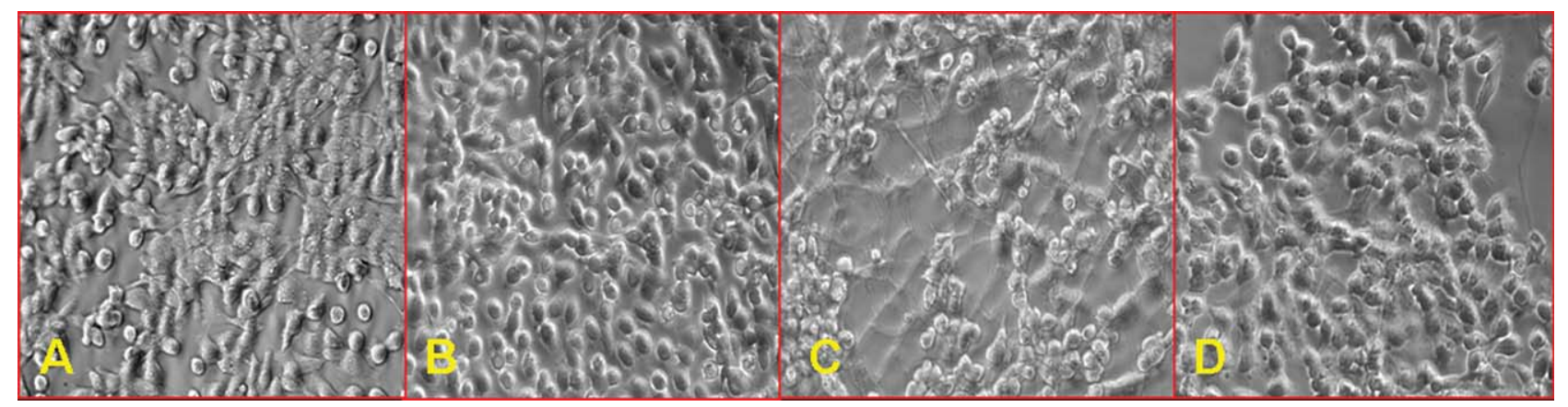

FIGURE 5. Cytotoxicity test using Trypan blue stain. ARPE19 cells growing on different substrates were stained with Trypan blue and observed under a phase-contrast microscope. The cells growing on each substrate are viable at all time points. The above pictures, taken at a field view of $\times 10$, are representative pictures at $120 \mathrm{~h}$. (A) Polystyrene; (B) glass; (C) ELR-IK; (D) ELR-RGD. [Color figure can be viewed in the online issue, which is available at wileyonlinelibrary.com.] 


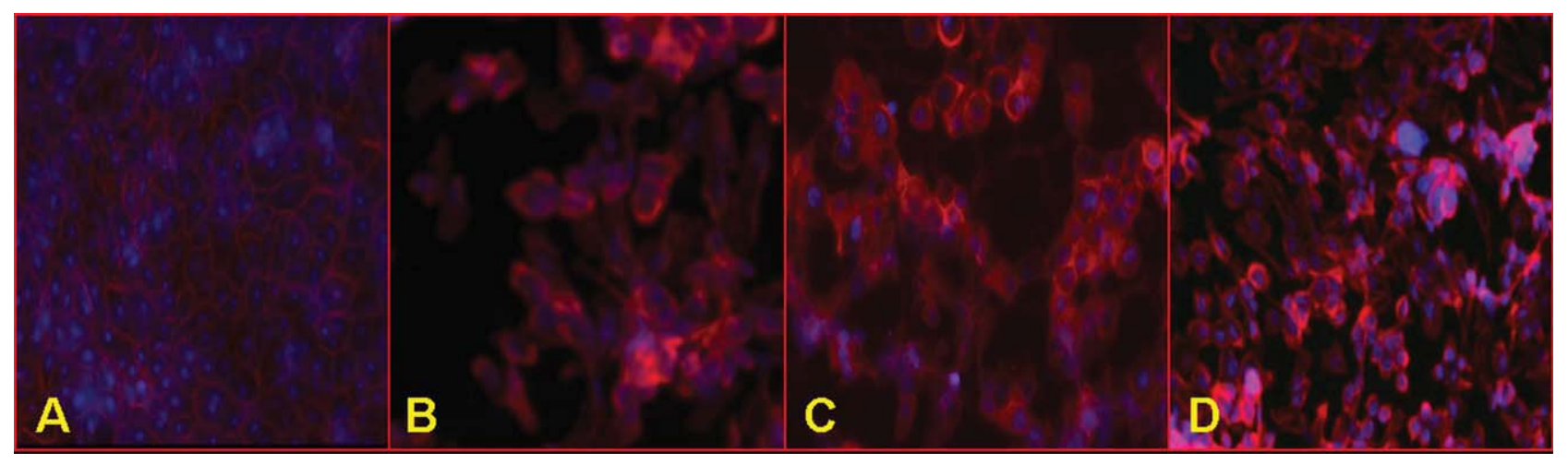

FIGURE 6. Cell morphology of ARPE19 cells on different substrates demonstrated by F-actin staining with fluorescent Rhodamin-Phalloidin stain at $120 \mathrm{~h}$. A more homogenous hexagonal mosaic morphology with higher cell density is seen for polystyrene than for ELR-RGD, glass and ELRIK. (A) Polystyrene; (B) glass; (C) ELR-IK; (D) ELR-RGD. [Color figure can be viewed in the online issue, which is available at wileyonlinelibrary.com.]

\section{Cell proliferation}

Cell proliferation was evaluated by quantifying the number of cells on the surface of all substrates at 24, 72, and $120 \mathrm{~h}$, as shown in Figure 3. The histogram shows that cell growth on ELR-RGD was significantly higher $(p<0.05)$ than that found on ELR-IK at all time points $(24,72$, and $120 \mathrm{~h}$ ). However, both these films showed a significantly lower ( $p<$ 0.05 ) average number of cells on their surface than that observed on the surface of the polystyrene at all time intervals (Fig. 3). At $120 \mathrm{~h}$, the cells growing on the surface of ELR-RGD, polystyrene and glass were nearly confluent, whereas the cells growing on ELR-IK needed more time to become confluent (Fig. 4), thus showing that the growth rates on ELR-RGD, polystyrene and glass are higher than on ELR-IK.

\section{Cell viability}

The Trypan blue exclusion assay was used to detect dead cells before seeding and also after growth of the cells on the different substrates. The results of this assay showed that the cells were $100 \%$ viable at the time of seeding (data not shown) and also after growing on each substrate at different time intervals (Fig. 5).

\section{Cell morphology and RPE65 expression}

Phase-contrast microscopy showed that the cells appeared to have reached confluence on the surface of all substrates, except ELR-IK, after 120 h (Fig. 4), although there was a clear difference in cell density on different substrates from the centre to the periphery. Thus, the cells growing on polystyrene surface were more closely packed and homogenous, showing a more mosaic morphology in both the central and peripheral regions, whereas the cell density and homogeneity of the mosaic pattern decreased from the centre to the periphery on the ELR-RGD, glass, and ELR-IK substrates (data not shown).

Immunofluorescence staining with Rhodamin-Phalloidin demonstrated good hexagonal circumferential actin fibers in the cells growing on all substrates (Fig. 6) while immunostaining with anti-ZO-1 antibody (Fig. 7) and electron microscopy (Fig. 8) study showed the presence of tight and gap junction confirming the epithelial morphology of the cells growing on each substrate. Under electron microscopy tight junctions (zonula occludens) were characterized by groups of union points where the plasma membranes of two adjacent cells were connected. Gap junctions (nexus) were observed as zones of membrane apposition with a dense line between the membranes (Fig. 8). A rounded cell

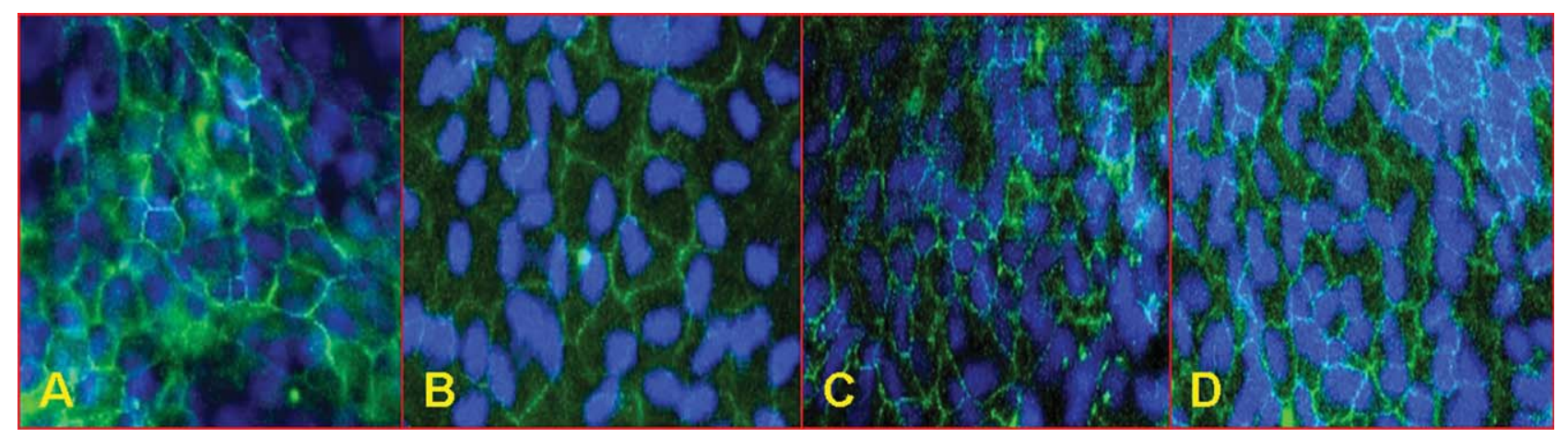

FIGURE 7. Epithelial cell morphology of ARPE19 cells on different substrates demonstrated by ZO-1 staining at $120 \mathrm{~h}$. A more homogenous hexagonal mosaic epithelial morphology with higher cell density is seen for polystyrene than for ELR-RGD, glass and ELR-IK. A: Polystyrene; (B) glass; (C) ELR-IK; (D) ELR-RGD. [Color figure can be viewed in the online issue, which is available at wileyonlinelibrary.com.] 


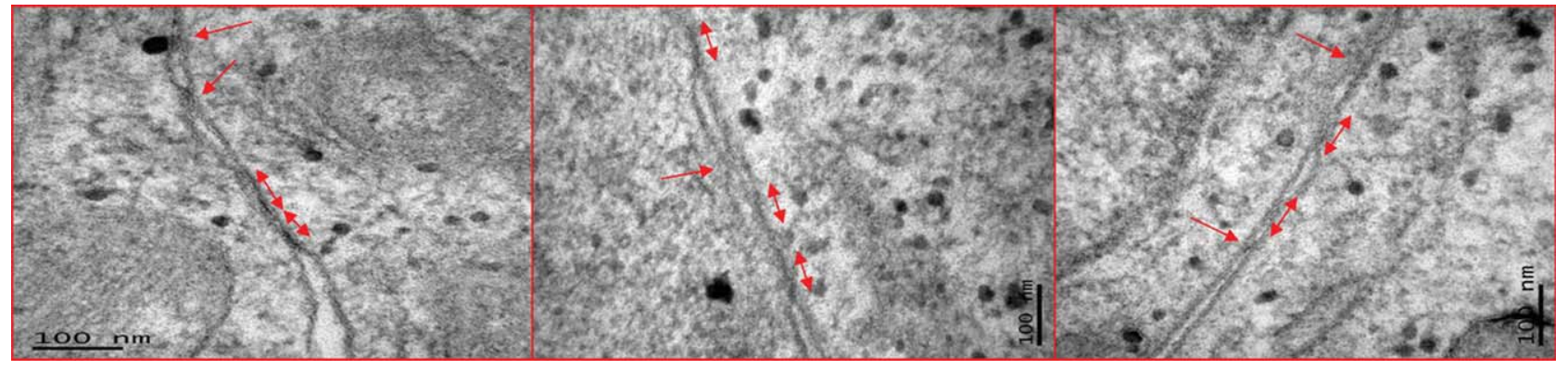

FIGURE 8. Presence of intercellular tight junctions (arrow) and gap junctions (doubleheaded arrow) among the ARPE19 cells growing on different substrates as observed by electron microscopy at $120 \mathrm{~h}$. (A) Polystyrene; (B) ELR-IK; (C) ELR-RGD. [Color figure can be viewed in the online issue, which is available at wileyonlinelibrary.com.]

nucleus was observed in all cases, as detected by DAPI staining (Fig. 6 and 7).

Immunofluorescence staining with RPE65 antibody showed that the cells growing on the different substrates maintained their RPE-type characteristics and that there was no transformation into, or contamination by, other cell types (Fig. 9).

\section{DISCUSSION}

It is well known that the stimuli provided by a substrate to cells influence their behavior. ${ }^{33-35}$ A large number of substrates, including cryoprecipitated membranes, anterior lens capsules, cadaver Bruch's membranes, Descemet's membranes, natural or synthetic biodegradable or nonbiodegradable polymers films and collagens, to name but a few, have been studied for the replacement of RPE cells. ${ }^{10,36-42}$ Most of these, however, have proved to be either very difficult to handle or are unable to deliver and maintain appropriate signals at the level of the RPE-substrate interface. Such signals are required to maintain RPE cells in their differentiated state under in vitro or in vivo conditions. The development of an appropriate synthetic, flexible, biocompatible film with an appropriate pore size for transportation that is easy to handle during surgery and results in minimum postsurgical complications is therefore currently vital for the success of dual-layer biomimetic transplants consisting of a layer of healthy RPE cells cultured on a support membrane. ${ }^{43}$ Such a film must also have appropriate surface characteristics that can provide the stimuli required to maintain the RPE cells in their subretinal localization. The ELR films of $652.8 \mathrm{~nm}$ thickness used in this study, which are composed of polymers containing repeat sequences of elastin, an extracellular elastic protein found in the vertebrate ECM, could be useful in this respect as their elastic and biocompatible characteristics are similar to those of naturally occurring ECMs and the elastic layer of BrM. ${ }^{44}$ The recombinant nature of these polymeric films enables the insertion of peptide sequences through genetic engineering to extend their properties, including bioactive domains. These polymers have previously been used for the formation of hydrogels with different pore sizes using a salt-leaching technique. ${ }^{31}$ ELRs biodegrade into natural amino acids by normal routes, thus allowing enough time for the transplanted cells to synthesize their own substratum and establish the correct pigment cell-neural retina architecture. Thus, the ELR films used in this study are flexible, biocompatible, biodegradable and with appropriate thickness $(652.8 \mathrm{~nm})$, and would appear to be highly suitable for use in RPE cell transplantation in AMD patients.

The RGD sequence is found in a number of ECM structural proteins that promote cell attachment, spreading, differentiated phenotype, and survival. ${ }^{45}$ Indeed, in this study,

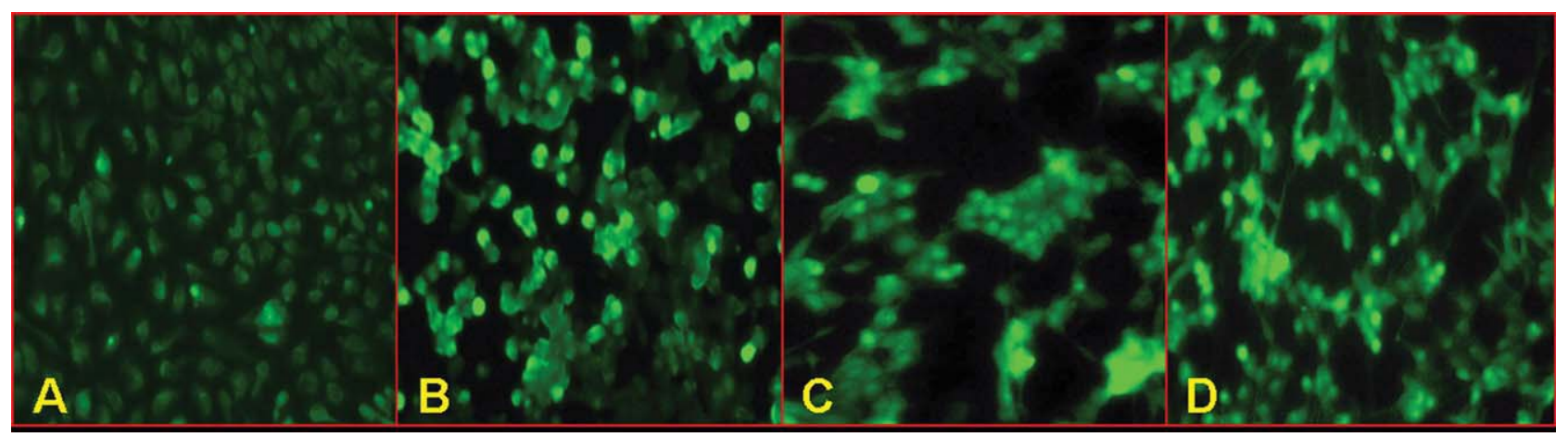

FIGURE 9. Expression of RPE65 by ARPE19 cells on different substrates. At $120 \mathrm{~h}$, immunostaining with RPE65 antibody demonstrates the expression of RPE65 antigen by ARPE19 cells growing on different surfaces. (A) Polystyrene; (B) glass; (C) ELR-IK; (D) ELR-RGD. [Color figure can be viewed in the online issue, which is available at wileyonlinelibrary.com.] 
interactions between RPE and RGD supported stability of RPE. Thus, the ELR must be able to maintain RPE quiescence, adherence, polarity and viability whilst also promoting proliferation after damage and migration into a wound. Therefore, inserting an RGD sequence in the bioactive domain of recombinant ELR and growing ARPE19 cells on these polymers will help to determine the suitability of this substrate for transplantation.

Despite having fewer adherences at $4 \mathrm{~h}$ than the polystyrene surface, ELR-RGD was nevertheless found to be a good support for the growth of ARPE19 cells. Indeed, there was a very clear difference between ELR-RGD and ELR-IK at 4, 24, 72, and $120 \mathrm{~h}$. At $120 \mathrm{~h}$, the difference between the number of cells attached to ELR-RGD, polystyrene and glass and those attached to ELR-IK, which did not support the growth of ARPE19 cells well, was notable. This shows that inserting bioactive sequences into ELR-IK modifies the adhesion and proliferation characteristics of the polymer and that an ELRRGD film promotes the attachment of the cultured cells to the film. Furthermore, the total absence of dead cells, expression of RPE65 protein and presence of intracellular tight and gap junction, which means that the ARPE19 cells maintained their epithelial morphology and specificity for the whole 120 -h growth period, strongly suggests that none of the ELRs used in this study are cytotoxic and that they all deliver optimal signals for promoting stability of the RPE cells including barrier formation for transportation under in vitro conditions. They could therefore be suitable carriers for RPE transplantation in the sub-macular region. A previous study has highlighted differences in the expression of different proteins between RPE-derived cell lines and human primary RPE cells, ${ }^{46}$ therefore the response of human primary RPE cells to these substrates might also differ. ARPE19 cells, spontaneous immortalized RPE cells, ${ }^{32}$ were used in this study to overcome several problems: the supply of cells, heterogeneity, the ease of culture, and the fast growth of cells as well as they have been used previously in several similar studies. ${ }^{41,43}$ These experimental results showed that in contact with ELRs, ARPE19 cells maintained certain properties which could be useful data for further study of interaction of ELRs with primary RPE cells. In addition, as stated previously, ex vivo expansion of primary RPE cells leads into partial reversion of the influence of ageing but with high risk of transdifferentiation which also needs to be ruled out with cells grown on these substrates. Further studies in these respects are currently underway. Furthermore, in vivo studies can also lead to different responses to those observed in vitro. This also needs to be ruled out with further studies before an RPE-ELR transplant can be performed in a clinical setting.

In summary, genetic engineering techniques are highly suitable for the production of complex polymers with a well-defined sequence that mimic the rich complexity of the natural ECM in terms of functionality and bioactivity. Herein, a ELR containing a bioactive sequence (RGD) found in the ECM produced using such genetic engineering techniques showed good adhesion and proliferation while maintaining the phenotype of ARPE19 cells and could therefore be suitable carriers for RPE transplantation.

\section{ACKNOWLEDGMENTS}

The authors would also like to thank Alicia Rodríguez Gascón, University of País Vasco (Spain), for providing the ARPE19 cells, and Cristhian Urzua Salinas and Jesús Gómez Escudero for their help.

\section{REFERENCES}

1. Chopdar A, Chakravarthy U, Verma D. Age related macular degeneration. BMJ 2003;326:485-488.

2. Tasman W, Rovner B. Age-related macular degeneration: Treating the whole patient. Arch Ophthalmol 2004;122:648-649.

3. Friedman DS, O'Colmain BJ, Muñoz B, Tomany SC, McCarty C, de Jong PT, Nemesure B, Mitchell P, Kempen J; Eye Diseases Prevalence Research Group. Prevalence of age-related macular degeneration in the United States. Arch Ophthalmol 2004;122: 564-572.

4. Nowak JZ. Age-related macular degeneration (AMD): Pathogenesis and therapy. Pharmacol Rep 2006;58:353-363.

5. Gass JD. Drusen and disciform macular detachment and degeneration. Arch Ophthamol 1973;90:206-217.

6. da Cruz L, Chen FK, Ahmado A, Greenwood J, Coffey P. RPE transplantation and its role in retinal disease. Prog Retin Eye Res 2007;26:598-635.

7. Limb GA, Daniels JT, Cambrey AD, Secker GA, Shortt AJ, Lawrence JM, Khaw PT. Current prospects fro adult stem cell-based therapies in ocular repair and regeneration. Curr Eye Res 2006;31: 381-390.

8. Gaillard F, Sauvé Y. Cell based therapy for retina degeneration: The promise of a cure. Vision Res 2007;47:2815-2824.

9. Radtke ND, Aramant RB, Petry HM, Green PT, Pidwell DJ, Seiler MJ. Vision improvement in retinal degeneration patients by implantation of retina together with retinal pigment epithelium. Am J Ophthalmol 2008;146:172-182.

10. Binder S, Stanzel BV, Krebs I, Glittenberg C. Transplantation of the RPE in AMD. Prog Retin Eye Res 2007;26:516-554.

11. Del Priore LV, Tezel TH, Kaplan HJ. Survival of allogeneic porcine retinal pigment epithelial sheets after subretinal transplantation. Invest Ophthalmol Vis Sci 2004;45:985-992.

12. Coffey PJ, Girman S, Wang SM, Hetherington L, Keegan DJ, Adamson P, Greenwood J, Lund RD. Long-term preservation of cortically dependent visual function in RCS rats by transplantation. Nat Neurosci 2002;5:53-56.

13. van Meurs JC, ter Averst E, Hofland LJ, van Hagen PM, Mooy CM, Baarsma GS, Kuijpers RW, Boks T, Stalmans P. Autologous peripheral retinal pigment epithelium translocation in patients with subfoveal neovascular membranes. $\mathrm{Br} \mathrm{J}$ Ophthalmol 2004; 88:110-113.

14. Lüke $C$, Alteheld $N$, Aisenbrey $S$, Lüke $M$, Bartz-Schmidt KU, Walter P, Kirchhof B. Electro-oculographic findings after 360 degrees retinotomy and macular translocation for subfoveal choroidal neovascularisation in age-related macular degeneration. Graefes Arch Clin Exp Ophthalmol 2003;241:710-715.

15. Wong D, Stanga $P$, Briggs $M$, Lenfestey $P$, Lancaster E, Li KK, Lim KS, Groenewald C. Case selection in macular relocation surgery for age related macular degeneration. $\mathrm{Br} \mathrm{J}$ Ophthalmol 2004;88:186-190.

16. Binder S, Stolba U, Krebs I, Kellner L, Jahn C, Feichtinger $H$, Povelka M, Frohner U, Kruger A, Hilgers RD, Krugluger W. Transplantation of autologous retinal pigment epithelium in eyes with foveal neovascularization resulting from age-related macular degeneration: A pilot study. Am J Ophthalmol 2002;133:215-225.

17. Rak DJ, Hardy KM, Jaffe GJ, McKay BS. Ca++-switch induction of RPE differentiation. Exp Eye Res 2006;82:648-656.

18. Grisanti S, Guidry C. Transdifferentiation of retinal pigment epithelial cells from epithelial to mesenchymal phenotype. Invest Ophthalmol Vis Sci 1995;36:391-405.

19. Lee SC, Kwon OW, Seong GJ, Kim SH, Ahn JE, Kay ED. Epitheliomesenchymal transfifferentiation of cultured RPE cells. Ophthalmic Res 2001;33:80-86.

20. Hiscott P, Sheridan C, Magee RM, Grierson I. Matrix and the retinal pigment epithelium in proliferative retinal disease. Prog Retin Eye Res 1999;18:167-190. 
21. Knoernschild T, Grasbon T, Wilsch C, Kampik A, Lütjen-Drecoll E. RPE cell transplants to non-immune-privileged sites of the eye transform into fibroblast-like cells. Curr Eye Res 2003;27:25-34.

22. Grierson I, Hiscott P, Hogg P, Robey H, Mazure A, Larkin G. Development, repair and regeneration of the retinal pigment epithelium. Eye (Lond) 1994;8:255-262.

23. Rodríguez-Cabello JC, Martín L, Alonso M, Arias FJ, Testera AM. "Recombinamers" as advanced materials for the post-oil age. Polymer 2009;50:5159-5169.

24. Girotti A, Reguera J, Arias FJ, Alonso M, Testera AM, Rodríguez-Cabello JC. Influence of the molecular weight on the inverse temperature transition of a model genetically engineered elastin-like pH-responsive polymer. Macromolecules 2004;37:3396-3400.

25. Martínez-Osorio $H$, Juárez-Campo M, Diebold $Y$, Girotti A, Alonso M, Arias FJ, Rodríguez-Cabello JC, García-Vázquez C, Calonge M Genetically engineered elastin-like polymer as a substratum to culture cells from the ocular surface. Curr Eye Res 2009;34: 48-56.

26. Di Zio K, Tirrell DA. Mechanical properties of artificial protein matrices engineered for control of cell and tissue behavior. Macromolecules 2003;36:1553-1558.

27. Lee J, Macosko CW, Urry DW. Phase transition and elasticity of protein-based hydrogels. J Biomater Sci Polym Ed 2001;12: 229-242.

28. Urry DW, Parker TM, Reid MC, Gowda DC. Biocompatibility of the bioelastic materials, poly(GVGVP) and its -irradiation cross-linked matrix: Summary of generic biological test results. J Bioact Compat Polymers 1991;6:263-282.

29. Berg MC, Yang SY, Hammond PT, Rubner MF. Controlling mammalian cell interactions on patterned polyelectrolyte multilayer surfaces. Langmuir 2004;20:1362-1368.

30. Ruoslahti E. RGD and other recognition sequences for integrins. Annu Rev Cell Dev Biol 1996;12:697-715.

31. Martín L, Alonso M, Girotti A, Arias FJ, Rodríguez Cabello JC. Synthesis and characterization of macroporous thermo sensitive hydrogels from recombinant elastin-like polymers. Biomacromolecules 2009;10:3015-3022.

32. Dunn KC, Aotaki-Keen AE, Putkey FR, Hjelmeland LM. ARPE-19, a human retinal pigment epithelial cell line with differentiated properties. Exp Eye Res 1996;62:155-169.

33. Berg MC, Yang SY, Hammond PT, Rubner MF. Controlling mammalian cell interactions on patterned polyelectrolyte multilayer surfaces. Langmuir 2004;20:1362-1368.
34. Zarbin MA. Analysis of retinal pigment epithelium integrin expression and adhesion to aged submacular human Bruch's membrane. Trans Am Ophthalmol Soc 2003;101:499-520.

35. Gullapalli VK, Sugino IK, Zarbin MA. Culture-induced increase in alpha integrin subunit expression in retinal pigment epithelium is important for improved resurfacing of aged human Bruch's membrane. Exp Eye Res 2008;86:189-200.

36. Sheridan C, Williams R, Grierson I. Basement membranes and artificial substrates in cell transplantation. Graefes Arch Clin Exp Ophthalmol 2004;242:68-75.

37. Sheridan C, Krishna Y, Williams R, Mason S, Wong D, Heimann $H$, Kent D, Grierson I. Transplantation in the treatment of agerelated macular degeneration: Past, present and future directions. Expert Rev Ophthalmol 2007;2:1-14.

38. Ma Z, Han L, Wang C, Dou H, Hu Y, Feng X, Xu Y, Wang Z, Yin Z, Liu $Y$. Autologous transplantation of retinal pigment epitheliumBruch's membrane complex for hemorrhagic age-related macular degeneration. Invest Ophthalmol Vis Sci 2009;50:2975-2981.

39. Maaijwee K, Van Den Biesen PR, Missotten T, Van Meurs JC. Angiographic evidence for revascularization of an rpe-choroid graft in patients with age-related macular degeneration. Retina. 2008;28:498-503.

40. Neeley WL, Redenti S, Klassen H, Tao S, Desai T, Young MJ, Langer R. A microfabricated scaffold for retinal progenitor cell grafting. Biomaterials 2008;29:418-426.

41. Lu JT, Lee CJ, Bent SF, Fishman HA, Sabelman EE. Thin collagen film scaffolds for retinal epithelial cell culture. Biomaterials 2007; 28:1486-1494.

42. Thumann G, Hueber A, Dinslage $S$, Schaefer $F$, Yasukawa $T$, Kirchhof B, Yafai Y, Eichler W, Bringmann A, Wiedemann P. Characteristics of iris and retinal pigment epithelial cells cultured on collagen type I membranes. Curr Eye Res 2006;31:241-249.

43. Lee CJ, Fishman HA, Bent SF. Spatial cues for the enhancement of retinal pigment epithelial cell function in potential transplants. Biomaterials 2007;28:2192-2201.

44. Booij JC, Baas DC, Beisekeeva J, Gorgels TG, Bergen AA. The dynamic nature of Bruch's membrane. Prog Retin Eye Res 2010;29: $1-18$.

45. Ruoslahti E. RGD and other recognition sequences for integrins. Annu Rev Cell Dev Biol 1996;12:697-715.

46. Alge CS, Hauck SM, Priglinger SG, Kampik A, Ueffing M. Differential protein profiling of primary versus immortalized human RPE cells identifies expression patterns associated with cytoskeletal remodeling and cell survival. J Proteome Res 2006;5:862-878. 